\title{
NUCLEAR SCIENCE DIVISION 1995-1996 ANNUAL REPORT
}

February 1997

\section{Division Director}

Lee S. Schroeder

Division Deputy

Janis M. Dairiki

\section{Editor}

Arthur M. Poskanzer

\section{Assistant Editors}

Georgia Fujikawa

Bill King

Sherrill L. Whyte 


\section{DISCLAIMER}

This report was prepared as an account of work sponsored by an agency of the United States Government. Neither the United States Government nor any agency thereof, nor any of their employees, makes any warranty, express or implied, or assumes any legal liability or responsibility for the accuracy, completeness, or usefulness of any information, apparatus, product, or process disclosed, or represents that its use would not infringe privately owned rights. Reference herein to any specific commercial product, process, or service by trade name, trademark, manufacturer, or otherwise does not necessarily constitute or imply its endorsement, recommendation, or favoring by the United States Government or any agency thereof. The views and opinions of authors expressed herein do not necessarily state or reflect those of the United States Government or any agency thereof. 


\section{DISCLAMIER}

Portions of this document may be illegible in electronic image products. Images are produced from the best available original document. 


\section{Table of Contents}

Introduction

L.S. Schroeder

\section{Overviews}

Nuclear Structure and Reactions Program

L.S. Schroeder

Institute for Nuclear and Particle Astrophysics

R.G. Stokstad

Relativistic Nuclear Collisions Program

H.G. Ritter

Nuclear Theory Program

J. Randrup

Nuclear Data Evaluation Program: Isotopes Project

J.M. Dairiki

88-Inch Cyclotron Operations

C.M. Lyneis

Group Lists

Nuclear Structure and Reactions

Beta-Delayed Proton Decay of ${ }^{27} \mathbf{P}$

T.J. Ognibene, J. Powell, D.M. Moltz, M.W. Rowe, and J. Cerny

Proton Capture on ${ }^{7} \mathrm{Be}$ and the Solar Neutrino Problem

J. Powell, D.M. Moltz, M.W. Rowe, and Joseph Cerny

Search for Proton Decay from an Isomer of ${ }^{77} \mathbf{R b}$ with RAMA

M.W. Rowe, D.M. Moltz, J. Powell, and J. Cerny

Calibration of Low-Energy Particle-Identification Telescopes

60

M.W. Rowe, D.M. Moltz, T.J. Ognibene, J. Powell, and J. Cerny

Gas Phase Chromatography of $\mathrm{FrBr}$ and $\mathrm{BiBr}_{3}$

E.R. Sylwester, D.C. Hoffman, J. Adams, Y.H. Chung, K.E. Gregorich, M.B. Hendricks, M.R. Lane, C. Laue, D.M. Lee, C.A. McGrath, D.A. Shaughnessy, D.A. Strellis, and P.A. Wilk

Gas Phase Chromatography of $\mathrm{HfBr}_{4}$

E.R. Sylwester, D.C. Hoffman, J. Adams, Y.H. Chung, K.E. Gregorich, M.B. Hendricks, M.R. Lane, C. Laue, D.M. Lee, C.A. McGrath, D.A. Shaughnessy, D.A. Strellis, and P.A. Wilk

Gas Phase Chromatography of $\mathrm{WBr}_{6}$

E.R. Sylwester, D.C. Hoffman, J. Adams, Y.H. Chung, K.E. Gregorich, M.B. Hendricks, M.R. Lane, C. Laue, D.M. Lee, C.A. McGrath, D.A. Shaughnessy, D.A. Strellis, and P.A. Wilk 
C.D. Kacher, K.E. Gregorich, D.M. Lee, B. Kadkhodayan, S.A. Kreek, M.R. Lane, M.F. Mohar, M.P. Neu, N.J. Stoyer, E.R. Sylwester, Y. Watanabe, B. Wierczinski, A. Bilewicz, and D.C. Hoffman

First Chemical Separations of Seaborgium (Element 106)

K.E. Gregorich, D.C. Hoffman, E.R. Sylwester, D.M. Lee, and the LBNL, GSI, Mainz, Dresden, Krakow, JAERI, PSI, Bern, Rossendorf, JINR, Oslo, and Chalmers Collaboration

Production and Decay of ${ }^{265} \mathrm{Sg}$ and ${ }^{266} \mathrm{Sg}$

K.E. Gregorich, B. Kadkhodayan, B. Wierczinski, D.M. Lee, C.D. Kacher, D.A. Shaughnessy, M.R. Lane, E.R. Sylwester, M.B. Hendricks, N.J. Stoyer, A.C. Veeck, and D.C. Hoffman

A Search for ${ }^{264} \mathrm{Sg}$

C.A. McGrath, K.E. Gregorich, D.M. Lee, M.R. Lane, E.R. Sylwester, D.A. Shaughnessy, M.B. Hendricks, D.A. Strellis, P.A. Wilk, and D.C. Hoffman

Electronics for On-Line Liquid Scintillation $\alpha$-Particle Spectroscopy

K.E. Gregorich

Improved Half-Life Measurement of ${ }^{224} \mathrm{~Pa}$ and its ${ }^{209} \mathrm{Bi}\left({ }^{18} \mathrm{O}, 3 \mathrm{n}\right){ }^{224} \mathrm{~Pa}$ Production Cross Section

P.A. Wilk, K.E. Gregorich, M.B. Hendricks, M.R. Lane, D.M. Lee, C.A. MacGarth, D.A. Shaughnessy, D.A. Strellis, E.R. Sylwester, and D.C. Hoffman

Off-Line Generation of ${ }^{233} \mathrm{~Pa}$ and the ${ }^{227} \mathrm{~Pa}$ Yield Through SISAK

P.A. Wilk and D.C. Hoffman

Production of ${ }^{211} \mathrm{Bi}$ from the Reaction of ${ }^{209} \mathrm{Bi}$ with ${ }^{22} \mathrm{Ne}$ Projectiles

Y.H. Chung, K.E. Gregorich, D.M. Lee, and D.C. Hoffman

Production Cross Sections for ${ }^{254} \mathrm{Fm}$ and ${ }^{256} \mathrm{Fm}$ in ${ }^{248} \mathrm{Cm}+{ }^{22} \mathrm{Ne}$ Reactions

M.B. Hendricks, R.J. Wallsgrove, K.E. Gregorich, M.R. Lane, D.M. Lee, D.A. Shaughnessy, D.A. Strellis, E.R. Sylwester, P.A. Wilk, and D.C. Hoffman

Production of Nuetron Difficient Plutonium Isotopes

M.B. Hendricks, K.E. Gregorich, M.R. Lane, D.M. Lee, D.A. Shaughnessy, D.A. Strellis, E.R. Sylwester, P.A. Wilk, and D.C. Hoffman

Electron-Capture Delayed Fission of ${ }^{242} \mathrm{Es}$

D.A. Shaughnessy, K.E. Gregorich, M.B. Hendricks, M.R. Lane, D.M. Lee, D.A. Strellis, E.R. Sylwester, P.A. Wilk, and D.C. Hoffman

Electron-Capture Delayed Fission Study of ${ }^{246}$ Es

D.A. Shaughnessy, K.E. Gregorich, M.B. Hendricks, M.R. Lane, D.M. Lee, C.A. McGrath, D.A. Strellis, E.R. Sylwester, P.A. Wilk, and D.C. Hoffman

Design of a Sample Changer/Transport System to be used for Gammasphere Studies on Electron-Capture Delayed Fission

D.A. Strellis, K.E. Gregorich, Y.H. Chung, M.R. Lane, E.R. Sylwester, M.B. Hendricks, D.A. Shaughnessy, C.A. McGrath, P.A. Wilk, D.M. Lee, and D.C. Hoffman

Confirmation of the Assignment of a 1.5-Second Fission Activity to ${ }^{259} \mathrm{Fm}$

C.A. McGrath, K.E. Gregorich, D.M. Lee, M.R. Lane, E.R. Sylwester, D.A. Shaughnessy, M.B. Hendricks, D.A. Strellis, B. Wierczinski, and D.C. Hoffman 
Spontaneous Fission Properties of ${ }^{262} \mathbf{R f}$

M.R. Lane, K.E. Gregorich, D.M. Lee, M.F. Mohar, M. Hsu, C.D. Kacher,

B. Kadkhodayan, M.P. Neu, N.J. Stoyer, E.R. Sylwester, J.C. Yang, and D.C. Hoffman

Spontaneous Fission Reviews

D.C. Hoffman, T.M. Hamilton, and M.R. Lane

Relative Deformations of Superdeformed Bands in ${ }^{131,132} \mathrm{Ce}$.

R.M. Clark, I.Y. Lee, P. Fallon, D.T. Joss, S.J. Asztalos, J.A. Becker, L. Bernstein,

B. Cederwall, M.A. Deleplanque, R.M. Diamond, L.P. Farris, K. Hauschild, W.H. Kelly, A.O. Macchiavelli, P.J. Nolan, N. O'Brien, A.T. Semple, F.S. Stephens, and R. Wadsworth

Low-Spin Termination of the Superdeformed Band in ${ }^{135} \mathrm{Nd}$

M.A. Deleplanque, S. Frauendorf, R.M. Clark, R.M. Diamond, F.S. Stephens, J.A. Becker, M.J. Brinkman, B. Cederwall, P. Fallon, L.P. Farris, E.A. Henry, H. Hübel, J.R. Hughes, W. Korten, I.Y. Lee, A.O. Macchiavelli, M.A. Stoyer, P. Willsau, J.E. Draper, C. Duyar, and E. Rubel

Proton Superdeformed Bands below the $\mathrm{Z}=80 \mathrm{Gap}$ in ${ }^{191} \mathrm{Au}$ : More Evidence for Pseudo-Spin Coupling

C. Schück, E. Gueorguieva, A. Lopez-Martens, F. Hannachi, Ch. Vieu, M. Kaci, J.S. Dionisio, W.H. Kelly, M.A. Deleplanque, F.S. Stephens, B. Kharraja, B.J.P. Gall, A. Korichi, U.J. van Severen, W. Pohler, B. Schulze, H. Hübel, and R. Wadsworth

Confirmation of a Superdeformed Band in ${ }^{192} \mathbf{P b}$

S.J. Asztalos, P. Fallon, J.A. Becker, L.A. Bernstein, R.M. Clark, M.A. Deleplanque, R.M. Diamond, L.P.Farris, E.A. Henry, W.H. Kelly, I.Y. Lee, A.O. Macchiavelli, and F.S. Stephens

Octupole Vibrations and Signature Splitting in Even Mass $\mathbf{H g}$

Superdeformed Bands

P. Fallon, F.S. Stephens, S. Asztalos, B. Busse, R.M. Clark, M.A. Deleplanque, R.M. Diamond, R. Krücken, I.Y. Lee, A.O. Macchiavelli, R.W. MacLeod, G. Schmid, K. Vetter, and T. Nakatsukasa

Relative Quadrupole Moments of ${ }^{192,}{ }^{193} \mathrm{Hg}$ Superdeformed Bands

B.C. Busse, P. Fallon, R. Krücken, D. Ackermann, I. Ahmad, S.J. Asztalos, D.J. Blumenthal, M.P. Carpenter, R.M. Clark, M.A. Deleplanque, R.M. Diamond, S.M. Fischer, F. Hannachi, R.V.F. Janssens, T.L. Khoo, A. Korichi, T. Lauritsen, I.Y. Lee, C.J. Lister, A. Lopez-Martens, A.O. Macchiavelli, R.W. MacLeod, E.F. Moore, D. Nisius, G. Schmid, D. Seweryniak, F.S. Stephens, and K. Vetter

Test of $\Delta \mathrm{I}=2$ Staggering in the Superdeformed Bands of ${ }^{194} \mathbf{H g}$

R. Krücken, G. Hackman, M.A. Deleplanque, R.V.F. Janssens, D. Ackermann, S. Asztalos, R.M. Clark, R.M. Diamond, P. Fallon, B. Herskind, T.L. Khoo, T. Lauritsen, I.Y. Lee, A.O. Macchiavelli, R.W. MacLeod, G.J. Schmid, F.S. Stephens, and $K$. Vetter

Discrete SD-ND Linking Transitions from the Yrast Superdeformed Band in ${ }^{194} \mathrm{~Pb}: \mathrm{J}^{\pi}$ and $\mathrm{E}_{\mathrm{x}}$

K. Hauschild, L.A. Bernstein, J.A. Becker, D.E. Archer, R.W. Bauer, D.P. McNabb, J.A. Cizewski, K.-Y. Ding, W. Younes, R. Krücken, R.M. Diamond, R.M. Clark, P. Fallon, I.-Y. Lee, A.O. Macchiavelli, R. MacLeod, G.J. Schmid, M.A. Deleplanque, F.S. Stephens, and W.H. Kelly 
The Decay of the Superdeformed Band in ${ }^{194} \mathrm{~Pb}$ : Electromagnetic Properties

R. Krücken, S.J. Asztalos, J.A. Becker, B. Busse, R.M. Clark, M.A. Deleplanque, A. Dewald, R.M. Diamond, P. Fallon, K. Hauschild, I.Y. Lee, A.O. Macchiavelli, R.W. MacLeod, R. Peusquens, G.J. Schmid, F.S. Stephens, K. Vetter, and P. von Brentano

Evidence for 'Magnetic Rotation': Lifetimes of States in the M1-Bands of ${ }^{198,199} \mathrm{~Pb}$

R.M. Clark, S.J. Asztalos, G. Baldsiefen, J.A. Becker, L. Bernstein, M.A. Deleplanque, R.M. Diamond, P. Fallon, I.M. Hibbert, H. Hübel, R. Kürcken, I.Y. Lee, A.O. Macchiavelli, R.W. MacLeod, G. Schmid, F.S. Stephens, and R. Wadsworth

Search for Two-Phonon Octupole Vibrational States in ${ }^{208} \mathbf{P b}$

K. Vetter, A.O. Macchiavelli, S.J. Asztalos, R.M. Clark, M.A. Deleplanque, R.M. Diamond, P. Fallon, R. Krücken, I.Y. Lee, R.W. MacLeod, G.J. Schmid, and F.S. Stephens

Study of Neutron-Rich Nuclei Using Deep-Inelastic Reactions

I.Y. Lee, S. Asztalos, M.A. Deleplanque, B. Cederwall, R.M. Diamond, P. Fallon, A.O. Macchiavelli, L. Phair, F.S. Stephens, G.J. Wozniak, S.G. Frauendorf, J.A. Becker, E.A. Henry, P.F. Hua, D.G. Sarantites, J.X. Saladin, and C.H. Yu

A Measurement of the Gamma Ray Polarization Sensitivity of Gammasphere

G.J. Schmid, A.O. Macchiavelli, M.A. Deleplanque, S. Asztalos, R.M. Clark, R.M. Diamond, P. Fallon, R. Krücken, I.Y. Lee, R.W. MacLeod, F.S. Stephens, and K. Vetter

Multiparticle-Rotor Model for Rotational Band Structure of ${ }^{154} \mathrm{Gd}$

S.Y. Chu, J.O. Rasmussen, and M.A. Stoyer

Models for Rotational Spin Distributions of Primary Fission Products

J.O. Rasmussen, S.Y. Chu, D. Strellis, S. Asztalos, R. Donangelo, L.F. Canto, J.D. Cole, M.A. Stoyer, Y.X. Dardenne, and J.H. Hamilton

Multiband Theory for Heavy-Ion Neutron-Pair Transfer among Deformed Gd Nuclei

S.Y. Chu, J.O. Rasmussen, M.A. Stoyer, P. Ring, L.F. Canto, and R. Donangelo

Form Factors for Two-Nucleon Transfer in the Diabolical Region of

Rotating Nuclei

A.R. Farhan, L.F. Canto, J.O. Rasmussen, and P. Ring

The 8th Edition of the Table of Isotopes

R.B. Firestone, V.S. Shirley, C.M. Baglin, S.Y.F. Chu, and J. Zipkin

The Isotope Explorer

S.Y.F. Chu, H. Nordberg, L.P. Ekstrom, and R.B. Firestone

Nuclear Data on the Internet

R.B. Firestone, S.C. Wu, and S.Y.F. Chu

Superdeformed and High-Spin Nuclear Structure Data on the Internet

B. Singh, R.B. Firestone, and S.Y.F. Chu

Reducibility and Thermal Scaling in Nuclear Multifragmentation

L.G. Moretto, R. Ghetti, L. Phair, K. Tso, and G.J. Wozniak

Aspects of Reducibility and Thermal Scaling in Multifragmentation

R. Ghetti, L.G. Moretto, L. Phair, K. Tso, and G.J. Wozniak 
Reducibility, Thermal and Mass Scaling in Angular Correlations from Multifragmentation Reactions

L. Phair, L.G. Moretto, G.J. Wozniak, R.T. de Souza, D.R. Bowman, N. Carlin, C.K. Gelbke, W.G. Gong, Y.D. Kim, M.A. Lisa, W.G. Lynch, G.F. Peaslee, M.B. Tsang, and F. Zhu

Comment on "Circumstantial Evidence for Critical Behavior in Peripheral Au+Au Collisions at $35 \mathrm{MeV} /$ nucleon"

L. Phair, Th. Rubehn, L.G. Moretto, and G.J. Wozniak

Comment on "Probing the Nuclear Liquid-Gas Phase Transition"

L.G. Moretto, R. Ghetti, L. Phair, K. Tso, and G.J. Wozniak

Stable Coulomb Bubbles?

L.G. Moretto, K. Tso, and G.J. Wozniak

Charge Correlations and Dynamical Instabilities in Multifragmentation

107

L.G. Moretto, Th. Rubehn, L. Phair, N. Colonna, G.J. Wozniak, D.R. Bowman,

G.F. Peaslee, N. Carlin, R.T. de Souza, C.K. Gelbke, W.G. Gong, Y.D. Kim, M.A. Lisa, W.G. Lynch, and C. Williams

Scaling Laws, Shell Effects, and Transient Times in Fission Probabilities

L.G. Moretto, K.X. Jing, R. Gatti, G.J. Wozniak, and R.P. Schmitt

Scaling Laws and Transient Times in ${ }^{\mathbf{3}} \mathrm{He}$ Induced Nuclear Fission

Th. Rubehn, K.X. Jing, L.G. Moretto, L. Phair, K. Tso, and G.J. Wozniak

First Chance Fission Probability of Neighboring Po Isotopes

Th. Rubehn, K.X. Jing, L.G. Moretto, L. Phair, K. Tso, and G.J. Wozniak

Transition State Rates and Mass Asymmetric Fission Barriers of Compound Nuclei 90,94,98Mo

K.X. Jing, L.G. Moretto, A.C. Veeck, N. Colonna, I. Lhenry, K. Tso, K. Hanold, W. Skulski, Q. Sui, and G.J. Wozniak

Characterization of Nuclear Physics Targets Using Rutherford Backscattering

Th. Rubehn, G.J. Wozniak, L. Phair, L.G. Moretto, and K.M. Yu

LBNL 88-Inch Cyclotron Improvements

C.M. Lyneis, Z.Q. Xie, and D.J. Clark

Development of Exotic Nuclear Beams at the 88-Inch Cyclotron

D.M. Moltz, P.E. Haustein, J. Powell, E.B. Norman, M.W. Rowe, and J. Cerny

Performance of the Upgraded LBL AECR Source

Z.Q. Xie and C.M. Lyneis

Production of Intense High Charge State Ions with an Aluminum Liner in the Advanced Electron Cyclotron Resonance Ion Source

Z.Q. Xie and C.M. Lyneis

A Microtron Cyclotron - the Slipatron

D.J. Clark

Non-Nuclear Science Programs at the 88-Inch Cyclotron: Part A. Space Applications 
Non-Nuclear Science Programs at the 88-Inch Cyclotron: Part B. Biology, Physics, and New Technologies

M.A. McMahan

\section{Institute for Nuclear and Particle Astrophysics}

Sudbury Neutrino Observatory - PMT Support Structure \& Panel Arrays

K.T. Lesko, Y.D. Chan, M. Dragowsky, M. Isaac, M.E. Moorhead, E.B. Norman, A. Schuelke, and R.G. Stokstad

${ }^{6} \mathrm{Li}$ Neutron Poison for SNO

M.E. Moorhead, Y.D. Chan, M. Dragowsky, M.C. Isaac, R.M. Larimer, K.T. Lesko, E.B. Norman, A. Schuelke, and R.G. Stokstad

A $4 \pi \beta$-tagged Decay Chamber for Gaseous SNO Calibration Sources

Y.D. Chan, M. Dragowsky, M.C. Isaac, K.T. Lesko, M.E. Moorhead, E.B. Norman, A. Schuelke, and R.G. Stokstad

Sudbury Neutrino Observatory, Neutral Current Detectors

K.T. Lesko, Y.D. Chan, M. Dragowsky, M. Isaac, M.E. Moorhead, E.B. Norman, A. Schuelke, and R.G. Stokstad

Sudbury Neutrino Observatory - Contamination Control

R.G. Stokstad, K.T. Lesko, Y.D. Chan, M. Dragowsky, M. Isaac, E. Norman, and A. Schuelke

Sudbury Neutrino Observatory, Monte-Carlo Simulations and Data Analysis

M.E. Moorhead, Y.D. Chan, M. Dragowsky, M. Isaac, K.T. Lesko, E.B. Norman, A. Schuelke, and R.G. Stokstad

The Sudbury Neutrino Observatory: Monitoring the Experiment

Y.D. Chan, M.C. Isaac, M. Dragowsky, K.T. Lesko, E.B. Norman, A. Schuelke, and R.G. Stokstad

Response of the SNO Detector to High Energy Gamma-Rays

M.C. Perillo Isaac, Y.D. Chan, M. Dragowsky, D. Hurley, K.T. Lesko, R.J. McDonald, M.E. Moorhead, E.B. Norman, A. Schuelke, A.R. Smith, and R.G. Stokstad

Measurements of the High Energy Gamma-Ray Flux at SNO

M.C. Perillo Isaac, Y.D. Chan, M. Dragowsky, D. Hurley, K.T. Lesko, R.J. McDonald, M.E. Moorhead, E.B. Norman, A. Schuelke, A.R. Smith, and R.G. Stokstad

Sudbury Neutrino Observatory: Simulation of the Calibration Decay Chamber for ${ }^{16} \mathrm{~N}$ and ${ }^{17} \mathrm{~N}$

M. Dragowsky, Y.D. Chan, M.C. Perillo Isaac, K.T. Lesko, M.E. Moorhead,

E.B. Norman, A. Schuelke, and R.G. Stokstad

Sudbury Neutrino Observatory, Monte-Carlo Simulation and Background Studies of the PSUP

A. Schuelke, K.T. Lesko, M. Dragowsky, Y.D. Chan, M. Isaac, E. Norman, and R. Stokstad

Sudbury Neutrino Observatory, Extending the Detector Geometry in SNOMAN for Background Investigations

A. Schuelke, K.T. Lesko, Y.D. Chan, M. Dragowsky, M. Isaac, E. Norman, and R. Stokstad 
R\&D for a Km3 Neutrino Detector

W. Chinowsky, H. Crawford, R. Fusezy, Y.D. He, S. Jackson, K. Lesko, D. Lowder,

J. Ling, M. Moorhead, D. Nygren, B. Price, J. Przybylski, A. Richards, G. Shapiro,

G. Smoot, L. Stevenson, R. Stokstad, and R. Tripp

Half-Life of ${ }^{44} \mathbf{T i}$

136

E.B. Norman, E. Browne, Y.D. Chan, I.D. Goldman, R.-M. Larimer, K.T. Lesko, M. Nelson, F.E. Wietfeldt, and I. Zlimen

Survival of ${ }^{180} \mathrm{Ta}$ During the s-Process

R.-M. Larimer, Y.D. Chan, M.P.C. Isaac, K.T. Lesko, A.O. Macchiavelli, R.W. Macleod, M.E. Moorhead, E.B. Norman, K.S. Krane, K. Zaerpoor, J. Becker, L.A. Bernstein, and L.P. Ferris

Search for the $\beta^{+}$Decay of ${ }^{144} \mathrm{Pm}$

K. Zaerpoor, M.R. Dragowsky, K.S. Krane, M.M. Hindi, S.J. Robinson,

D.E. DiGregorio, E.B. Norman, Y.D. Chan, M.C.P. Isaac, R.-M. Larimer,

A.O. Macchiavelli, and R. MacCleod

Preliminary Results from a Study of the Double Beta Decay of ${ }^{150} \mathbf{N d}$

139

M.C. Perillo Isaac, Y.D. Chan, D. Hurley, R.J. McDonald, E.B. Norman, and A.R. Smith

Does The Lifetime of ${ }^{40} \mathrm{~K}$ Depend Upon its Chemical State?

E.B. Norman, M.C. Perillo-Isaac, M.E. Moorhead, R.-M. Larimer, R.J. McDonald, A.R. Smith, and E. Browne

Weak Gamma Rays in the Electron-Capture Decay of ${ }^{194} \mathrm{Au}$

R.R.P. Teixeira, I.D. Goldman, M.T.F. da Cruz, E. Browne, I.Y. Lee, A.O. Macchiavelli, and E.B. Norman

Nuclear Astrophysics Data Home Page

J. Dairiki, R. Firestone, R. Stokstad, S. Woosley, and S.C. Wu

Laser Trapping Radioactive Atoms for Precise Weak Interaction Tests

M.A. Rowe, S.J. Freedman, B.K. Fujikawa, G. Gwinner, S.-Q. Shang, P.A. Vetter, and E.G. Wasserman

Measurement of the Partial $0^{+} \rightarrow 0^{+}$Half Life of ${ }^{10} \mathrm{C}$ with GAMMASPHERE

B.K. Fujikawa, S.J. Asztalos, C.J. Bowers, R.M. Clark, M.-A. Deleplanque-Stephens, P. Fallon, S.J. Freedman, J.P. Greene, I.-Y. Lee, L.J. Lising, A.O. Macchiavelli, R.W. MacLeod, J.C. Reich, M.A. Rowe, S.-Q. Shang, F.S. Stephens, and E.G. Wasserman

Test of the Conserved Vector Current Hypothesis in the Beta-Decay of ${ }^{14} \mathrm{C}$ J.L. Mortara, I. Ahmad, S.J. Freedman, B.K. Fujikawa, J.P. Greene, J.P. Schiffer, and A.R. Zeuli

Test of the Conserved Vector Current Hypothesis in the Beta-Decay of ${ }^{14} \mathrm{O}$

S.J. Freedman, B.K. Fujikawa, J.L. Mortara, D.W. Ross, and D. Wutte

$\beta-\gamma$ Directional Correlation in ${ }^{22} \mathrm{Na}$

C.J. Bowers, S.J. Freedman, B.K. Fujikawa, J.C. Reich, S.-Q. Shang, P.A. Vetter, E.G. Wasserman, and the Gammasphere Collaboration

Search for the Beta-Decay of ${ }^{54} \mathbf{M n}$

I. Ahmad, S. Austin, S.J. Freedman, K.T. Lesko, J.L. Mortara, and A. Wuosmaa 
Time Reversal Invariance Violation in Polarized Neutron Beta Decay

L.J. Lising, S.J. Freedman, B.K. Fujikawa, E.G. Wasserman, and the emiT Collaboration

Test of Time Reversal Invariance Violation in the Beta-Decay of ${ }^{56} \mathrm{Co}$

J.L. Mortara, J.C. Davis, and S.J. Freedman

Atomic Parity Nonconservation in Stable $\mathrm{Yb}$ Isotopes

151

C.J. Bowers, D. Budker, E.D. Commins, D. DeMille, S.J. Freedman, G. Gwinner, S.-Q. Shang, and J.E. Stalnaker

Experimental Study of the ${ }^{8} \mathrm{~B}$ Neutrino Spectrum

S.J. Freedman, K.E. Rehm, B.K. Fujikawa, G. Gwinner, B. Harss, C.L. Jiang, M. Paul, R.C. Pardo, J.P. Schiffer, S.Q. Shang, and P. Vetter

Search for Narrow Positron Emission Lines in Heavy Ion Collisions with APEX

S. Freedman, M. Maier, as part of the APEX Collaboration

Studying New Physics Using Ultrahigh-Energy Cosmic-Ray $\gamma$-Family Data Y.D. He

Search for Dirac Magnetic Monopole Production in High Energy Heavy Ion Collisions

Y.D. He and P.B. Price

Measurement of Low Energy Ion Tracks in CR-39 Plastic with an Atomic

Force Microscope

Y.D. He and C.I. Hancox

Low-background Counting Facilities

A.R. Smith, R.J. McDonald, D.L. Hurley, and E.B. Norman

Low-background Gamma-ray Spectroscopy Applied to Measuring the Uptake of Metals by Bacteria

A.R. Smith, R.J. McDonald, D.L. Hurley, and E.B. Norman

Low-Level Measurement of Alpha-Particle Emitters in Ceramics and Metals

A.R. Smith, R.J. McDonald, D.L. Hurley, and E.B. Norman

\section{Relativistic Nuclear Collisions}

$\Delta$ Resonance Production in ${ }^{58} \mathrm{Ni}+\mathrm{Cu}$ Collisions at $\mathrm{E}=1.97 \mathrm{~A} \mathrm{GeV}$

E.L. Hjort and the EOS Collaboration

Dielectron Production in A-A Reactions at $1.0 \mathrm{~A} \mathrm{GeV}$

R.J. Porter and the DLS Collaboration

The E895 experiment at the AGS

G. Rai and the E895 Collaboration

Rare Processes in Relativistic Heavy Ion Collisions-Results from BNL E878

M.J. Bennett, J.B. Carroll, H. Crawford, M. Cronqvist, J. Engelage, I. Flores, L. Greiner, T.J. Hallman, H.H. Heckman, C. Kuo, and P.J. Lindstrom

Development of a Distributed Drift Chamber for BNL E896

J. Engelage, M.J. Bennett, J.B.Carroll, H. Crawford, M. Cronquist, I. Flores, L. Greiner, E. Judd, P.J. Lindstrom, J.Marx, L.Sakrejda, and G.Visser 
Spectroscopy of Scalar Mesons with the Crystal Barrel Detector

K.M. Crowe, T. Case, F.H. Heinsius, P. Kammel, M. Lakata, and the Crystal Barrel Collaboration

Measurement of Rare $\omega \rightarrow \eta \gamma$ Radiative Decay

M. Lakata, K. Crowe, T. Case, P. Kammel, and F.-H. Heinsius

A Precision Measurement of Nuclear Muon Capture on ${ }^{3} \mathrm{He}$

170

K.M. Crowe, T. Case, and P. Kammel

Elliptic Event Shapes at the CERN SPS

171

T. Wienold, I. Huang, and the NA49 Collaboration

Proton and Negative Hadron Spectra from NA49

172

M. Toy, P. Jacobs, and the NA49 Collaboration

A New Method for V0 Reconstruction in NA49 CERN Experiment

173

S. Margetis

$\mathbf{K}_{\mathrm{S}}^{0}$ production in $\mathrm{Pb}+\mathrm{Pb}$ collisions at CERN SPS

174

S. Margetis and the NA49 Collaboration

Projectile Fragmentation in $\mathbf{P b}+\mathbf{P b}$ Collisions at $158 \mathrm{~A} \cdot \mathrm{GeV}$

175

I. Huang, S. Margetis, G. Odyniec, and the NA49 Collaboration

Fragmentation of $160 \mathrm{~A} \mathrm{GeV} \mathrm{Pb}$ in Various Targets

176

Y.D. He and P. B. Price

STAR TPC System Test

W. Betts, F. Bieser, R. Bossingham, M. Botlo, M. Cherney, J. Chrin, P. Colarco, H. Crawford, H. Diaz, K. Dao, D. Greiner, L. Greiner, E. Hjort, S. Jacobson, R.C. Jared, E. Judd, S.R. Klein, A.N. Lebedev, M.J. Levine, V. Lindenstruth, M.A. Lisa, K. Marks, C. McParland, T.S. McShane, J. Meier, M.T. Nguyen, D.L. Olson, I. Sakrejda, J. Schambach, R.A. Scheetz, N.T.B. Stone, C.E. Tull, G. Visser, C. Vu, H. Wieman, E.Yee, and the STAR Collaboration

Front End Electronics for the STAR TPC

F.S. Bieser, J. Hunter, S.R. Klein, C. Vu, and the STAR Collaboration

Triggering in the STAR Experiment at RHIC

E. Judd and the STAR Trigger Group

STAR Analysis Framework

D.L. Olson, C.E. Tull, and D. Prindle

Simulations and Analysis Software for the STAR TPC

P.M. Jacobs, M.A. Lisa, I.M. Sakrejda, N.T.B. Stone, and the STAR Collaboration

The PDSF Computing Facility at LBNL

R.J. Porter

Development of a Micro TPC Vertex Detector

H. Wieman, E. Beuville, M. Burks, D. Fritz, S. Margetis, F. Retiere, S. Trentalange, and R. Wells

Production and Detection of Hyperfragments in High Energy Nucleus-

Nucleus Collisions

Y.D. He and P.B. Price 
Coherent Nuclear Interactions at RHIC

S. Klein and E. Scannapieco

NSD Pre-College Educational Activities

H.S. Matis, Chairman NSD Educational Committee

Nuclear Theory

Study of Medium-induced Parton Energy Loss in $\gamma+$ jet Events of High-Energy

Heavy-Ion Collisions

X.N. Wang and Z. Huang

Jet Quenching in the Direction Opposite to a Tagged Photon in High-Energy

Heavy-Ion Collisions

190

X.N. Wang, Z. Huang, and I. Sarcevic

Dilepton and Photon Production from a Coherent Pion Oscillation

191

Z. Huang and X.N. Wang

Thermal Equilibration in an Expanding Parton Plasma

H. Heiselberg and X.N. Wang

Domain Structure of a Disoriented Chiral Condensate from a Wavelet Perspective

Z. Huang, I. Sarcevic, R. Thews, and X.N. Wang

$\mathrm{J} / \psi$ Suppression in an Equilibrating Parton Plasma

194

X.-M. Xu, D. Kharzeev, H. Satz, and X.N. Wang

Screening of Initial Parton Production in Ultrarelativistic Heavy-ion Collisions

K.J. Eskola, B. Müller, and X.N. Wang

Partial $U(1)_{A}$ Restoration and $\eta$ Enhancement in High-Energy Heavy-Ion

Collisions

196

Z. Huang and X.N. Wang

Study of Parton Energy Loss in High-energy Heavy-ion Collisions

197

X.N. Wang

A pQCD-based Approach to Parton Production and Equilibration in High-Energy Nuclear Collisions

X.N. Wang

Phenomenology of Charm and Bottom Production

199

R. Vogt

Lepton Production from Charm Decay in Nuclear Collisions at $p \sqrt{s}=200 \mathrm{GeV}$ and 5.5 TeV per Nucleon

S. Gavin, P.L. McGaughey, P.V. Ruuskanen, and R. Vogt

Charm and Bottom Quark Production Cross Sections Near Threshold

201

J. Smith and R. Vogt

Resummed Heavy Quark Production Cross Sections to Next-to-Leading Logarithm

N. Kidonakis, J. Smith, and R. Vogt

Reanalysis of the EMC Charm Production Data With Extrinsic and Intrinsic Charm at NLO

B.W. Harris, J. Smith, and R. Vogt 
Charmed Hadron Asymmetries in the Intrinsic Charm Coalescence Model

R. Vogt and S.J. Brodsky

Charmonium Suppression by Comover Scattering in $\mathrm{Pb}+\mathrm{Pb}$ Collisions

S. Gavin and R. Vogt

Transverse Momentum of $\psi$ and Dimuon Production in $\mathrm{Pb}+\mathrm{Pb}$ Collisions

S. Gavin and R. Vogt

Systematics of Quarkonium Production

G.A. Schuler and R. Vogt

Upsilon Suppression in $\mathbf{P b}+\mathbf{P b}$ Collisions at the LHC?

R. Vogt

Determining the Existence and Nature of the Quark-Gluon Plasma by Upsilon Suppression at the LHC

J.F. Gunion and R. Vogt

Aspects of Chiral Symmetry

V. Koch

Properties of Hadrons in the Nuclear Medium

C.M. Ko, V. Koch, and G.Q. Li

Cold Kaons from Hot Fireballs

V. Koch

Thermal Effects on Dilepton Production from Pion-Annihilation

C. Song, V. Koch, S.H. Lee, and C.M. Ko

Dilepton Production in Relativistic Heavy-ion Collisions

$V$. Koch and C. Song

Pion Electromagnetic Formfactor at Finite Temperature

C. Song and V. Koch

Chemical Relaxation Time of Pions in Hot Hadronic Matter

C. Song and V. Koch

Excess of Pions with Chiral Rymmetry Restoration

C. Song and V. Koch

Effective Mass of Phi Mesons at Finite Temperature

C. Song

Dissipation and Decoherence in Mean Field Theory

S. Habib , Y. Kluger, E. Mottola, and J.P. Paz

Anomalous Transverse Distribution of Pions as a Signal for Disoriented

Chiral Condensates

F. Cooper, Y. Kluger, and E. Mottola

Symmetry Breaking in $\lambda \phi^{4}$ Theory

F. Cooper, S. Habib, Y. Kluger, and E. Mottola

Statistical Properties of the Linear Sigma Model

J. Randrup

Amplification of Pionic Instabilities in High-Energy Collisions?

J. Randrup 
Mean-Field Treatment of the Linear Sigma Model in Dynamical Calculations of DCC Observables

J. Randrup

Treatment of Pionic Modes at the Nuclear Surface for Transport Descriptions

J. Helsgesson and J. Randrup

Simplified Treatment of Collective Instabilities in Matter

J. Randrup

Multifragmentation with Brownian One-Body Dynamics

A. Guarnera, Ph. Chomaz, M. Colonna, and J. Randrup

Inclusion of Quantum Fluctuations in Wave Packet Dynamics

A. Ohnishi, and J. Randrup

Quantum Fluctuation Effects on Nuclear Fragment Formation

A. Ohnishi and J. Randrup

Quantum Fluctuations Affect Critical Properties of Noble Gases

A. Ohnishi and J. Randrup

$\mathrm{K}^{+}$Momentum Spectrum from $\left(\mathrm{K}^{-}, \mathrm{K}^{+}\right)$Reactions in the Intranuclear

Cascade Model

Y. Nara, A. Ohnishi, T. Harada, and A. Engel

Four-Alpha Linear-Chain States in ${ }^{16} \mathrm{O}$

N. Itagaki, A. Ohnishi, and K. Katō

Detectability of Strange Matter in Heavy Ion Experiments

J. Schaffner-Bielich, C. Greiner, A. Diener, and H. Stöcker

Quark Phase Transition \& Time-structure of Pulsar Spin-Down

N.K. Glendenning, S. Pei, and F. Weber

Response of Pulsar Braking to Rotation and Internal Structure

N.K. Glendenning, S. Pei, and F. Weber

Crystalline Structure Size in Neutron Stars

N.K. Glendenning and S. Pei

Crystalline Cores of Neutron Stars

N.K. Glendenning

Internal Constitution of Neutron and Strange Stars

N.K. Glendenning

Thermal Evolution of Compact Stars

C. Schaab, F. Weber, M.K. Weigel, and N.K. Glendenning

The Nuclear Thomas-Fermi Model

W.D. Myers and W.J. Swiatecki

The Rotating Nuclear Thomas-Fermi Model

W.D. Myers and W.J. Swiatecki

The Nuclear Thomas-Fermi Model with Angular Momentum: Fission Barriers,

Superdeformations, Moments of Inertia

W.D. Myers and W.J. Swiatecki 
The Congruence Energy: A Contribution to Nuclear Masses, Deformation

Energies and Fission Barriers

W.D. Myers and W.J. Swiatecki

The Excitation of an Independent-Particle Gas-Classical or Quantal-by a Time-

Dependent Potential Well

J. Blocki, J. Skalski, and W.J. Swiatecki

The Excitation of an Independent-Particle Gas by a Time-Dependent

Potential Well. Part II

J. Blocki, J. Skalski, and W.J. Swiatecki

The Effect of Dynamical Correlations in a Slowly Pumped Knudsen Gas

J. Blocki, C. Jarzynski, and W.J. Swiatecki

\section{Seminars}




\section{Introduction}

\section{L.S. Schroeder}

This report describes the activities of the Nuclear Science Division (NSD) for the two-year period, January 1, 1995 to January 1, 1997. This was a time of major accomplishments for all research programs in the Division-many of which are highlighted in the reports of this document.

During this period, several changes affecting the Division took place. In the fall of 1995, James Symons, the Division Director for ten very successful years, returned to full-time research. Much of the success of the Division's activities are due to his foresight and dedicated leadership at the Berkeley Lab, as well as his many contributions on the national scene. In 1996, the NSD office was relocated to the Bldg. 50 complex, along with the Relativistic Nuclear Collisions (RNC) group and the Isotopes Project. While this relocation squeezed us on space, it had the very positive benefit of providing closer ties with the other General Science areas (Accelerator Research and Fusion and Physics Divisions) also housed in the Bldg. 50 complex. Also, in the 1995-96 period, the U.S. nuclear science community revisited its long-range planning process-providing a strong road map for nuclear science research over the next few years. The NSD hosted the Town Meeting on Electoweak Interactions, Astrophysics and Non-accelerator Experiments, as part of this process. Several NSD members participated in the NSAC Long-Range planning activity at $\mathrm{Cal}$ Tech and in the construction of the final report. Finally, it is with great sadness that we also recognize the passing of two of our colleagues during this period-Mike Nitschke, a major force behind the national call for an ISL facility for radioactive beams, and Virginia Shirley, a key member of the Table of Isotopes team. 
Much of the activity at the 88-Inch Cyclotron centers on Gammasphere and its strong research program. This period witnessed a change from the "early implementation" phase, to the "full implementation" phase of the Gammasphere program, with its nearly complete (110 elements) complement of detectors. In addition, several auxiliary detectors, provided by the Gammasphere research community, were added-making the Gammasphere suite of detectors the world's most powerful instrument for studying nuclear structure. A formal Gammasphere Dedication was held in December 1995 at the Berkeley Lab, including a meeting devoted to the early results from Gammasphere. Presently, over 250 researchers, representing universities and national laboratories from around the world, are actively engaged in this research effort, with demand for beam time running extremely high.

To further enhance the 88-Inch's low energy research program, a new instrument, the Berkeley Gas-Filled Separator (BGS) is under construction. This device, designed to provide enhanced separation capabilities, will serve as the centerpiece for a strong program in heavy element research, production of exotic nuclei and nuclear structure studies. Present plans call for BGS operation in the summer/fall of 1997.

Considerable progress has been made in the area of weak interaction studies at the 88-Inch Cyclotron. Activities include the use of Gammasphere, as well as the continued progress in the trapping of radioactive ${ }^{21} \mathrm{Na}$ atoms. The trapping facility continues to be upgraded with the number of trapped atoms reaching about 50,000 - sufficient to initiate an active physics program.

Other major activities at the 88-Inch Cyclotron include: high statistics studies of the systematics (energy, target/projectile dependence) of complex fragment emission, the possibility of light $(C, N, O)$ radioactive beams at the 88-Inch, a nuclear astrophysics program using Gammasphere as well as other devices. Finally, excellent progress has been made on a 3rd generation ECR ion source which will provide the heaviest beams at energies above the coulomb barrier for the 88 -Inch research community.

During this reporting period considerable progress was made on the final assembly of the solar neutrino detector at the Sudbury Neutrino Observatory (SNO). The Berkeley SNO team has been actively involved in this effort and it is anticipated that the heavy water fill will occur in the latter half of 1997. In addition to participating in the construction efforts at Sudbury to complete the SNO detector, NSD researchers have been turning their attention to extracting physics from SNO. Looking to the future, through the focus provided by the Institute for Particle and Nuclear Astrophysics (INPA), a joint activity with the Physics Division, concepts are being developed, and early R\&D carried out, on a next-generation detector $\left(\mathrm{km}^{3}\right)$ to study high energy neutrinos of astrophysical origin.

In the area of relativistic heavy-ion collisions, considerable progress and redirection of effort has occurred in the last few years. The Pb-era at the CERN Super-Proton Synchrotron is in full swing. The NSD's physics activity at CERN continues to be focused on NA49, where we had primary responsibility for the 
electronics associated with the TPC's. The development of electronics for TPC's has been an active part of our efforts through many different experiments-from EOS, to NA35/NA49 and STAR. For the NA49 experiment, attention is now focused on physics results, including observables such as strange particle yields and flow. After a very successful program at the Bevalac, the EOS TPC was moved to the AGS in 1995 and has successfully completed data-taking for $\mathrm{Au}+\mathrm{Au}$ collisions at a range of energies between $2-8 \mathrm{GeV} /$ nucleon, and is the only large-acceptance electronic detector with data in this interesting transition region between Bevalac and full-energy AGS physics. Areas of interest for this program include the energy dependence of flow and strange particle production.

The STAR detector continues on track for physics at RHIC in the summer/fall of 1999. During the last two years, many major reviews of STAR, by both DoE and RHIC management, have been successfully carried out. Locally, the TPC, which is the heart of the STAR detector, is nearing completion and shipment to RHIC is expected in the fall of this year. Systems testing and finally cosmic ray tests of the TPC will be conducted before shipping. As we move from the construction phase of STAR to physics with STAR, our activities on the analysis and software aspects of the STAR program are playing a more prominent role. The recent arrival of the National Energy Research Scientific Computing Center (NERSC) at the Berkeley Lab, with its scientific computing capabilities and expertise, has provided the Division with a substantial opportunity to enhance our ability to extract the physics from STAR. Active collaborations with NERSC and others interested in the high volumes of data that will be generated by detectors like STAR are being formed. Hopefully, the solutions to problems of mutual interest will result from this effort.

The Nuclear Theory program has been very active over the last two years in activities spanning low-energy nuclear structure, studies of astrophysical interest such as neutron stars, and relativistic nuclear collisions with particular emphasis on RHIC physics. Close interaction with experimental groups has continued to grow, including weekly seminars covering the range of research interests of the Division. A strong visitor's theory program continues to enrich the Division.

In 1996 the Isotopes Project produced the 8th Edition of the Table of Isotopes, both in hard copy and CD-ROM. New focus areas in nuclear structure, nuclear astrophysics, and electronic data dissemination have been developed by the group. They continue to be a central player in the U.S. data evaluation and dissemination program supported by the DoE.

Finally, this two-year annual report is being put out in both hardcopy and through electronic publishing on the World Wide Web (WWW). With increased use of the electronic dissemination of information, we do not plan to provide hard copies of future NSD annual reports. The Division has a home page on the WWW (http://user88.lbl.gov/nsd_home.html) or access through the Berkeley Lab home page (http://www.lbl.gov), as do many of our research groups-please visit us! Any comments and suggestions should be addressed to our Editor, Art Poskanzer, who has, with his useful efficiency, put together an excellent document. 


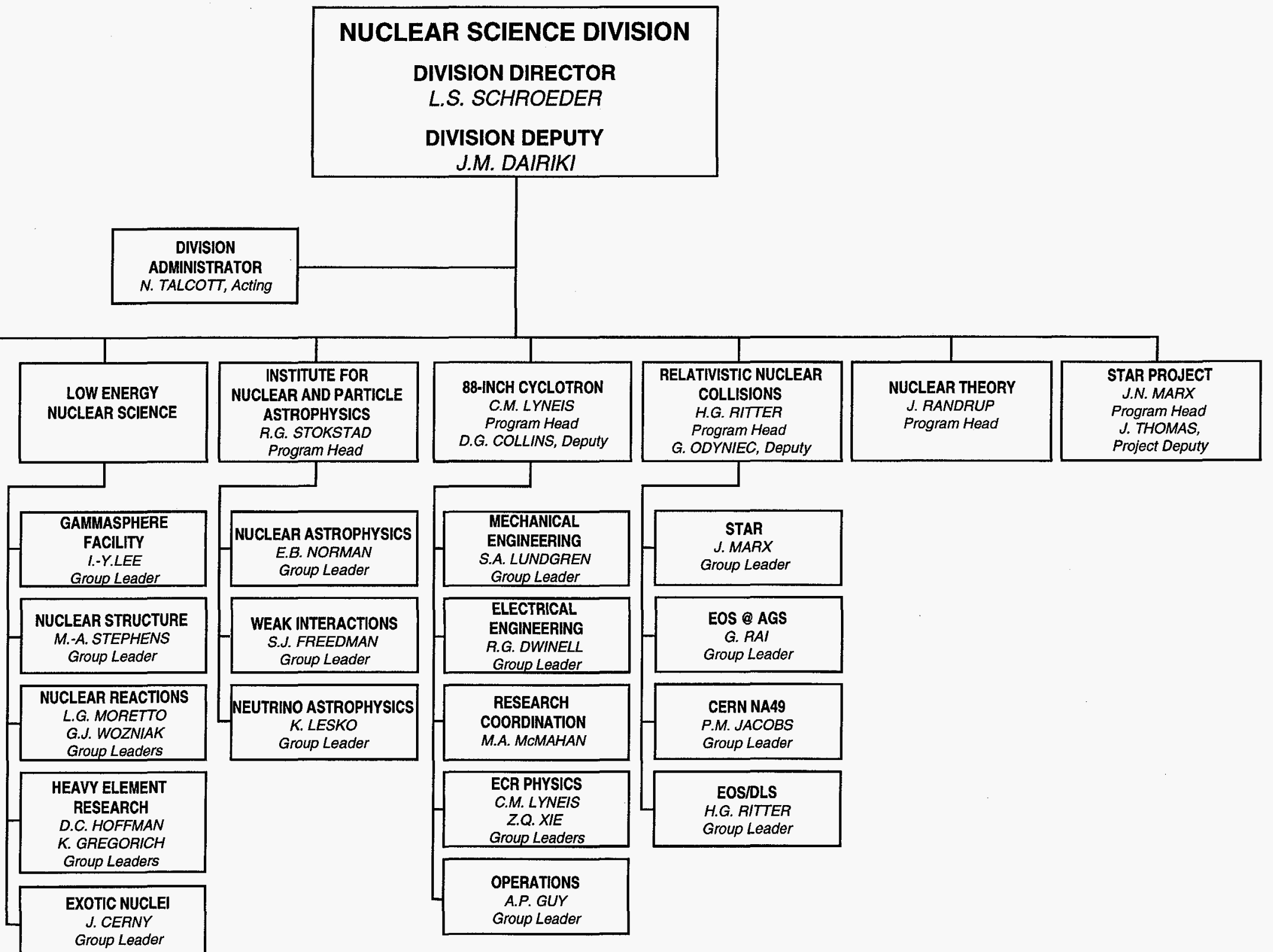





\section{Nuclear Structure and Reactions Program}

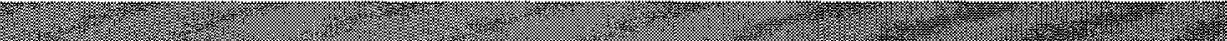

\section{L.S. Schroeder}

The Nuclear Structure and Reactions Program uses a broad range of observables and techniques to study the nuclear medium and address problems of current interest in nuclear physics. This activity focuses on studying nuclear systems at extremes of angular momentum, isospin, neutron and proton number, as well as temperature. A brief overview of the various programs is given below, while a more detailed accounting of individual activities can be found in the "Nuclear Structure and Reactions" contributions later in this report.

Most of these programs are centered at the 88-Inch Cyclotron. The Cyclotron is operated as a national facility, providing a wide range of ion species and energies, for a diverse national and international user community. The high reliability and ease of changing beam species and energy make the 88-Inch Cyclotron ideally suited for nuclear structure and reactions studies.

A broad range of nuclear instrumentation is in place at the 88-Inch to address this research program. The central focus of the research program at the 88-Inch, over the last few years, has been the exploitation of the unique capabilities of Gammasphere. This National $\gamma$-ray Facility, consisting of a $4 \pi$ array of 110 Compton-suppressed high-purity germanium detectors, augmented with auxiliary detectors, utilizes over $50 \%$ of the research time at the Cyclotron. Gammasphere, as well as other detector systems in operation at the Cyclotron, are described below and in other sections of this document. 


\section{Nuclear Structure}

The Nuclear Structure Group has two main focuses: one is to pursue a physics program mainly centered on high-spin studies of the atomic nucleus, and the other is to develop new instrumentation to push the frontiers of nuclear structure and other research. The Nuclear Structure Group has played the leading role in the construction and operation of Gammasphere. Both the Director and the Deputy Director of Gammasphere are members of the Nuclear Structure Group, and other members support the operation of the array, as well as contribute to its construction and maintenance. Gammasphere was dedicated on December 1, 1995 and on that occasion, a "Workshop on Gammasphere Physics" was organized by this group. About 100 scientists attended and highlights of the physics done at the large gamma-ray arrays was reported. The proceedings are published in World Scientific.

This group is interested in the structure of the nucleus, mostly at high angular momentum. One of the main goals is to understand nuclear behavior in terms of the characteristics of a few-body quantal system. Persistent themes reflecting these interests have been identical bands and the $C_{4}$ symmetry suggested for the nuclear shape. Progress on these as yet unresolved issues is described below. More superdeformed bands are discovered as the sensitivity of Gammasphere increases. We found interesting evidence for the contribution of pseudo-spin symmetry in a pair of identical bands. However, the focus is now on understanding the properties of SD bands rather than finding more bands. This is now possible as more precise energy and lifetime measurements can be made. Using these new capabilities, our group found evidence for octupole deformations in some superdeformed nuclei. We could compare deformations of identical bands. We could also learn about the mechanism of the decay of superdeformed bands. In normally deformed nuclei, lifetime measurements confirmed the predictions of the "tilted axis cranking" model, supporting the new concept of "magnetic rotation" as a nuclear excitation. We have been looking for the octupole two-phonon excitation in ${ }^{208} \mathrm{~Pb}$ and have set new limits on its existence. We are interested in neutron-rich nuclei and the possibility that they will reveal new properties of nuclei. We have produced such nuclei at high spins using deep inelastic reactions.

In the future, our group intends to take new directions in addition to pursuing the subjects outlined in the previous paragraph. One of the most tantalizing goals is to find hyperdeformed nuclei; i.e., nuclei even more elongated and probably better rotors than superdeformed nuclei. So far there is no evidence for them but we continue trying new ways to produce them. We are interested in heavy nuclei as they are the most collective nuclei with normal deformation. In particular, it will be very interesting to study the evolution of octupole excitations in these nuclei. The future use of various recoil separators, with and without Gammasphere, should give us access to more exotic nuclei. It would also be interesting to study very light nuclei and observe the various exotic shapes predicted. With Gammasphere, one can study continuum spectra at high spins better than ever before. We intend to pursue this in various aspects of the orderto-chaos transition in nuclei. 
Finally, the group is engaged in a collaboration with S. Freedman and his group to measure the branching ratio of the superallowed $\left(0^{+} \rightarrow 0^{+}\right) \beta^{+}$decay of ${ }^{10} \mathrm{C}$. This work is expected to provide the best test of unitarity of the CKM matrix. We are also interested in pursuing a wider variety of physics, for example looking for strange matter and rare decays of positronium.

The group is also developing a new Gamma-Ray Energy Tracking Array (GRETA), which would have a resolving power a thousand times that of Gammasphere. This array would consist of a solid shell of highly segmented Ge detectors. Research and development is underway. Preliminary cluster recognition studies indicate a large improvement over Gammasphere. Also, the induced signal into the segment adjacent to the "hit" segment has been calculated and compares well to the measured one in the Gammasphere segmented detector. It is essential to measure this signal in order to have position resolution in the measurement of the gamma-ray interaction points. $R \& D$ is progressing on GRETA design.

A recoil separator is also under study as an auxiliary detector for Gammasphere. With a high efficiency and reasonable mass resolution, such a system (e.g. solenoid-dipole) appears quite attractive.

\section{Proton-Rich Nuclei}

Studies near the proton drip line can provide excellent tests of the limitations of nuclear models and stimulate improvements in these models. For nuclides beyond the proton drip line, searches for new ground-state proton emitters are of interest to compare reduced widths to nuclear structure theory, as are searches for the quantum-mechanically interesting decay mode of ground-state twoproton emission $\left({ }^{2} \mathrm{He}\right)$. For nuclides near the drip line, the large available $\beta$-decay energies open up new beta-delayed multi-particle decay channels (e.g., $2 p$ decay). In order to extend the dynamic range for the detection of these betadelayed protons, this group has developed detectors with gas $\Delta \mathrm{E}$-gas $\Delta \mathrm{E}$-silicon $\mathrm{E}$ telescopes with a threshold of $180 \mathrm{keV}$. An array consisting of four of these telescopes was used in observing beta-delayed proton decay branch from ${ }^{23} \mathrm{Al}$ which proceeds via the isobaric analog state (IAS) in ${ }^{23} \mathrm{Mg} .{ }^{23} \mathrm{Al}$ represents the lightest member of the $A=4 n+3, T_{z}=-3 / 2$ mass series for which proton decay of the IAS is possible. Recent measurements have extended the successful search for new low-energy proton groups to ${ }^{27} \mathrm{P}$ and ${ }^{31} \mathrm{Cl}$, but these decays do not proceed via the IAS. This group will attempt to confirm the results from several experiments started during the past year which include confirmation of betadelayed proton decay from the $\mathrm{T}_{z}=-5 / 2$ nuclide ${ }^{23} \mathrm{Si}$ and of beta-delayed twoproton decay of the $T_{z}=-2$ nuclide ${ }^{46} \mathrm{Mn}$. A new search will commence for the beta-delayed proton decay of ${ }^{77} \mathrm{Zr}$. Finally, this group is expending considerable effort in developing the capability of running a few radioactive ion beams at the 88-Inch Cyclotron. The primary production machine is a small medical cyclotron located at LBNL and tests are under way to measure the efficiency of coupling a gas-jet transport system to an ECR source. The first beams will be ${ }^{11} \mathrm{C}$ and ${ }^{14} \mathrm{O}$ accelerated to energies $>15 \mathrm{MeV} /$ nucleon for nuclear reaction studies. 


\section{Heavy Element Nuclear and Radiochemistry Program}

The nuclear and chemical properties of the heavy actinides and transactinide elements at the limits of both nuclear stability and the periodic table are being investigated. Studies to produce and characterize new elements and isotopes, to study nuclear reactions, and to elucidate the chemical properties of the transactinide elements have been conducted at the LBNL 88-Inch Cyclotron as well as at the GSI-UNILAC Facility in Darmstadt, Germany.

Both physical and radiochemical techniques are utilized and a large number of students have been trained in modern nuclear and radiochemical techniques. Currently, the research efforts are concentrated in the following areas: 1) Production and identification of new isotopes and elements; 2) Study of nuclear decay of actinide and transactinide isotopes, especially spontaneous fission (SF) and electron-capture delayed fission (ECDF), and nuclear properties; 3) Development of rapid separation and sensitive detection techniques for study of isotopes with half-lives as short as seconds for definitive studies of both nuclear and chemical properties.

An international collaboration was hosted at LBNL to study the chemical properties of hahnium (105) using the 1.8 -second ${ }^{261} \mathrm{Ha}$. The 1.8 -second isotope ${ }^{225} \mathrm{~Pa}$ of its psuedo-homolog protactinium $(\mathrm{Z}=91)$ was also studied under the same conditions. The automated, continuous liquid-liquid extraction system (SISAK) coupled with the flowing liquid scintillation alpha spectroscopy system (LISSY) enables us to achieve separation times of 1-2 seconds with nearly $100 \%$ detection efficiency.

Recent experimental confirmation of the predicted stabilizing influence in the region of the deformed shells at $N=162$ and $Z=108$ has sparked a renaissance of interest in the production and study of the half-lives and decay properties of isotopes near these shells. (Our measurement of a 2.1-second SF half-life for ${ }^{262} \mathrm{Rf}$ and an alpha half-life around 10 seconds for ${ }^{266} \mathrm{Sg}$ support these predictions.) In order to more sensitively explore and study this extension of the known region of stability, the Berkeley Gas-filled Separator, a new instrument designed for efficient separation of compound nucleus products has been designed, and is scheduled for completion in late 1997. Its sensitivity to half-lives as short as microseconds and cross sections as small as picobarns will facilitate access to this region and a number of exciting studies of nuclear phenomena.

First studies of both the aqueous and gas-phase chemical properties of Seaborgium ( $\mathrm{Sg}$, element 106) were performed as part of an international collaboration. These indicated that $\mathrm{Sg}$ was properly placed under $\mathrm{Mo}$ and $\mathrm{W}$ in group 6 of the periodic table. New information about the production cross sections and nuclear properties of $264,265,266 \mathrm{Sg}$ produced via the reaction of ${ }^{248} \mathrm{Cm}$ with ${ }^{22} \mathrm{Ne}$ projectiles was also obtained. Studies of more complex chemical properties of Sg are planned. Gas-phase studies of bromides of its lighter homologs have already been conducted in preparation for studies with Sg. 
Investigations of electron-capture delayed fission have been extended to the light Es isotopes. A sample changer is being built to transport samples to Gammasphere to permit study of fission barriers and fission shape isomers and their gamma decay back to the ground state in an out-of-beam environment. Our studies of SF half-lives and decay properties are continuing and two more SF reviews have been published.

\section{Nuclear Reactions}

At low energies complex fragment or intermediate mass fragment $(\mathrm{Z}>2)$ emission is a rare process. Increasing the excitation energy brings the emission of these fragments to the forefront, until they dominate the reaction scene. The goal of the Nuclear Reactions group is to characterize the physics of this emission process, both theoretically and experimentally, from its onset as binary compound nucleus decay to its full deployment as multifragmentation.

\section{Scaling Laws, Shell Effects and Transient Times in Compound Nucleus Emission}

Our recent work has demonstrated the compound nucleus emission of complex fragments and characterized it to be a generalized fission process controlled by a set of conditional barriers constrained to a given mass asymmetry. For several systems near $A \sim 100$, we have measured excitation functions and extracted conditional barriers. These barriers compare favorably with those calculated with the finite-range model. As the fission barriers in medium-heavy nuclei were instrumental in defining uniquely the liquid drop model parameters, the conditional barriers offer a unique tool to fix the parameters of its more modern descendants like the finite range models. In particular, the measurement of conditional barriers for several isotopes of the same element, can constrain the large uncertainty in the surface asymmetry coefficient of the droplet model.

Complex fragment excitation functions have also been used to search for atomic number $\mathrm{Z}$ and energy $\mathrm{E}$ dependent deviations from transition-statemethod predictions that would indicate the existence of transient time effects. No, Z- and/or E-dependent effects, that could be attributed to an increased collectivity with increasing mass (charge) of the emitted fragment and associated with transient or stationary solutions of Kramers' diffusion equation, are visible in our data. Over ninety excitation functions, for complex fragments from four different compound nuclei, can be collapsed into a single universal straight line that is consistent with the transition-state predictions. Once the phase space associated with the non-reactive degrees of freedom at the conditional saddle point is removed, the reduced rates are identical for all fragments. This result seems to rule out, for all Z-values, transient effects that should become noticeable with increasing excitation energy and decreasing mass asymmetry.

The scaling reported above for complex fragments is not directly applicable to fission because of the strong ground state shell effects encountered in the ${ }^{208} \mathrm{~Pb}$ 
region. However, because of the simple asymptotic form of the shell correction, we have been able to modify the scaling in a simple way. The remarkable result is that all of the sixteen studied fission excitation functions are exactly scalable. In other words, they are reducible to the same straight line. The scaling extends over more than $100 \mathrm{MeV}$ of excitation energy and over an increase of fission probability of a factor $10^{7}$. No failure of the transition state rate is detectable over these ranges, and fission delay times in crossing the saddle point larger than $10^{-20}$ sec can be ruled out.

As a "by-product" of the above analysis, we obtain the ground state shell corrections. These are in excellent agreement with those extracted from ground state masses. Thus, this procedure can be offered as an alternative method for the extraction of shell effects, which, incidentally, is purely local and independent of the liquid drop fit extended over the entire mass table.

\section{Reducibility and Thermal Scaling in Multifragmentation}

The process of multifragmentation is still sketchy and incomplete. However, we believe that we have succeeded in unveiling important features which may be the key to a deeper understanding.

The pervasive aspect of reducibility indicates that, whatever the mechanism, the fragments are emitted essentially independent of one another. We have shown that the probability $P_{n}$ of emitting $n$ fragments can be reduced to the probability of emitting a single fragment through the binomial equation. Similarly the $n$ fragment charge distributions can be reduced to the one fragment charge distribution. Furthermore, the particle-particle angular correlation can be reduced to the individual particle angular distributions.

In all the above quantities, reducibility is somehow restricted by what we may call "dynamical constraints." For the emission probabilities, the constraint is the binomial parameter $m$, indicative either of a dynamical time window, or of the finite source size. For the charge distributions, reducibility is restricted by the parameter $c$, which seems to indicate some special way of enforcing charge conservation. We speculate that its transition from near zero to a finite value with increasing energy could be an indication of a transition from phase coexistence (liquid-vapor) to a single phase (vapor). Finally, the angular correlations violate reducibility at small relative angle where particle-particle interactions become manifest.

These broad features of reducibility speak to the near independence of fragment emission but not to its mechanism.

Thermal scaling instead makes a clear statement about the fact that the elementary probabilities entering in the $n$ fragment emission probabilities, the $n$ fragment charge distributions, and the two fragment angular correlations are thermal. In other words, these probabilities have the form of a Boltzmann factor and clearly portray its characteristic energy dependence (linear Arrhenius plots). 
Thus, the resulting picture is tantalizingly close, but not quite that of a compound nucleus emission. Apparently sources are dynamically generated which, within dynamical constraints of time and size, emit fragments in a thermal manner. Among the potential fruits that can be reaped from the pursuit of the analysis outlined so far are dynamical features of source formation, size and lifetime, as well as static features like barriers, source sizes and densities.

\section{Stable Coulomb Bubbles?}

The depletion of charge in the central cavity of nuclear bubbles reduces the Coulomb energy significantly and thus stabilizes "Coulomb" bubbles against monopole oscillations. These Coulomb bubbles, however, are at least unstable to perturbation of the quadrupole radial mode. At sufficiently high temperature, the vapor pressure in the central cavity drives the bubble to a thinner configuration that is stable against all radial modes. Since a thin Coulomb bubble behaves like a sheet, it becomes susceptible to the proximity surface instability via the crispation modes and may meet its demise when its thickness is comparable to the range of the proximity interaction.

\section{Heavy Element Collaboration}

J. Alstad ${ }^{4}$, M. Andrassy ${ }^{3}$, U. Becker ${ }^{2}$, R. Binder ${ }^{9}$, W. Brüchle ${ }^{1}$, R. Dressler ${ }^{8}$, K. Eberhard ${ }^{2}$, B. Eichler ${ }^{5}$, St. Fischer ${ }^{8}$, B. Fricke ${ }^{14}$, H. Gäggeler ${ }^{5,6}$, R. Güenther ${ }^{2}$, A. Hofmeister ${ }^{2}$, S. Hübener ${ }^{7}$, E. Jäger ${ }^{1}$, D. Jost ${ }^{5}$, B. Kadkhodayan ${ }^{11}$, J. Kratz ${ }^{2}$, R. Malmhack ${ }^{3}$, M. Mandel ${ }^{2}$, R. Misiak ${ }^{12}$, A. Nähler ${ }^{2}$, Y. Nagame 13, H. Nitsche ${ }^{7,8}$, J.P. Omtvedt ${ }^{4}$, Y. Oura ${ }^{13}$, W. Paulus ${ }^{2}$, V. Pershina ${ }^{14}$, G. Pfrepper ${ }^{10}$, R. Pfrepper ${ }^{10}$, A. Ross ${ }^{7}$, M. Schädel ${ }^{1}$, B. Schausten ${ }^{1}$, E. Schimpf ${ }^{1}$, D. Schumann ${ }^{8}$, G. Skarnemark ${ }^{3}$, A. Seibert ${ }^{2}$, R. Süss ${ }^{9}$, St. Taut ${ }^{8}$, U. Tharun ${ }^{2}$,

N. Trautmann ${ }^{2}$, A. Türler ${ }^{5}$, N. Wiehl ${ }^{2}$, B. Wierczinski ${ }^{3}$, G. Wirth ${ }^{1}$, S. Zauner ${ }^{2}$

${ }^{1}$ Gesellschaft für Schwerionenforschung, Darmstadt,Germany

${ }^{2}$ Institut für Kernchemie, Universität Mainz, Mainz, Germany

${ }^{3}$ Chalmers University of Technology, Göteborg, Sweden

${ }^{4}$ Department of Chemistry, University of Oslo, Norway

${ }^{5}$ Labor für Radiochemie, Paul Scherrer Institut, Switzerland

${ }^{6}$ Institut für anorganische Chemie, Universität Bern, Bern, Switzerland

${ }^{7}$ Institut für Radiochemie, Forschungszentrum Rossendorf, Dresden, Germany

${ }^{8}$ Forschungsgruppe Radiochemie, Technische Universität Dresden, Dresden, Germany

${ }^{9}$ Arbeitsgruppe Kern- und Radiochemie, Universität Leipzig, Leipzig, Germany

${ }^{10}$ Institut für Biophysik, Universität Leipzig, Germany

${ }^{11}$ Glenn T. Seaborg Institute for Transactinum Science, Lawrence Livermore National Laboratory, Livermore, CA USA

12Niewodniczanski Institute of Nuclear Physics, Poland

13Japan Atomic Energy Research Institute, Japan

${ }^{14}$ Theoretische Physik, Kassel, Germany 


\section{Institute for Nuclear and Particle Astrophysics}

R.G. Stokstad

\section{Introduction}

The areas of research at the Institute (INPA) are broad and have a strong interdisciplinary flavor, yet a common purpose connects them-to use the science and the technologies of nuclear physics and particle physics to address fundamental questions bearing on the nature of the universe: past, present, and future. Specific research topics include solar neutrinos, high energy neutrinos, detection of nearby and distant supernovae, weak interactions in atomic and nuclear processes, the cosmic microwave background radiation, direct detection of dark matter, cosmic rays and chronometers, the theory of pulsars and neutron stars, and geoastrophysics. Research and education are combined not only through the participation of students and postdoctoral researchers, but also at the high school level through summer programs for teachers and a major project, the Hands-On Universe, that brings on-line astronomical images to the classroom.

INPA is sponsored by the Nuclear Science Division and the Physics Division at LBNL. While participants in INPA are predominantly from these two Divisions, the Physics Department and the Space Sciences Laboratory at UC Berkeley are well represented. Indeed, the Institute benefits from the rich concentration of astrophysics in the greater Bay Area. A wide range of experimental facilities is used by INPA participants; at LBNL (the 88-Inch Cyclotron, Gammasphere, lowbackground counting facilities, Leuschner Observatory), in North America (Sudbury Neutrino Observatory, the Keck Telescopes, nuclear physics facilities 
at Argonne and Brookhaven national laboratories, university laboratories), throughout the world (Chile, Australia, Antarctica), and in space (HST, COBE).

This overview naturally focuses on research where Nuclear Science Divisionassociated researchers are heavily involved. A few highlights from other areas are mentioned, and the overview concludes with a brief description of INPA's institutional activities.

\section{Neutrino Astrophysics}

The Sudbury Neutrino Observatory (SNO), a 1,000-ton heavy-water Cerenkov detector under construction in a nickel mine in Canada, is back on track after construction delays associated with the fabrication of the acrylic vessel that will contain the $\mathrm{D}_{2} \mathrm{O}$. The most significant milestone has been the completion of the upper half of the detector, with both the photomultiplier support structure (PSUP, with phototubes) and the acrylic vessel (AV) suspended by their cables from the deck above. Construction of the lower half of SNO has begun. LBNL is responsible for the PSUP and has also helped the collaboration with the AV. Contamination control in construction is critical to keep backgrounds to tolerable levels-this aspect of SNO continues to receive attention from the LBNL group. When the detector is filled with water and ready to begin its shake-down period, other activities will become important and are being prepared for now at LBNL as well as at other collaborating institutions. These activities include calibration ( ${ }^{16} \mathrm{~N}$ and ${ }^{17} \mathrm{~N}$ sources), neutron detection ( ${ }^{3} \mathrm{He}$ neutron detectors) and suppression ( ${ }^{6} \mathrm{Li}$ poison), data acquisition (graphical interfaces) and data analysis (Monte Carlo simulation and measurement of $\beta$ and $\gamma$ backgrounds). When SNO begins taking data, the years of planning and construction should bring their reward. By the year 2000, the neutral current to charged current ratio and the shape of the ${ }^{8} \mathrm{~B}$ spectrum-two independent tests for neutrino oscillations-will have been measured and we should have a better perspective on the standard models of particle physics and of the sun.

The same properties of neutrinos that make them a valuable probe of the sun could also make them a unique window on the most energetic objects in the cosmos. Several INPA participants are members of the AMANDA collaboration, which is constructing a water Cherenkov detector in deep Antarctic ice to observe high energy neutrinos. It is in the planning and R\&D toward the next generation neutrino observatory, however, that INPA is making a major contribution. This detector will have dimensions on the order of a square or cubic kilometer, and therefore the sensitivity to detect neutrinos from distant point sources, such as Active Galactic Nuclei.

\section{Nuclear Astrophysics}

Laboratory measurements of nuclear properties are essential in understanding the processes by which heavy nuclei are synthesized from primordial nuclei. Certain individual isotopes can take on key roles. ${ }^{44} \mathrm{Ti}$ is such an example; gamma rays from its decay are observable and can be used to determine the 
amount of ${ }^{44} \mathrm{Ti}$ produced in a supernova if the half life of this nucleus is known. Past measurements place this value in the range 39 to 67 years, which translates into a factor of six uncertainty for the remnant of a supernovae that exploded 300 years ago. (Half lives in the range of tens of years are particularly difficult to measure). Measurements on ${ }^{44} \mathrm{Ti}$ produced at the 88-Inch Cyclotron and counted off-line yield a value of $62 \pm 2$ years and, because they were made relative to other isotopes with well-known half lives, resolve the present discrepancy.

Knowing the half life of unstable (but long-lived) nuclei present in cosmic rays makes it possible to determine the residence time of these nuclei in our galaxy, i.e., they can serve as a cosmic chronometer. In this case, the half lives need to be of the order of $10^{6}$ years. The decay rate of a nucleus in space (where it has no surrounding electrons to capture) can be much longer than when it is housed in an atom or ion on earth. Measurements of very weak decay branches are therefore necessary. ${ }^{144} \mathrm{Pm}$ and ${ }^{54} \mathrm{Mn}$ are two such cases; the latter nucleus is of particular interest because it has recently been possible to measure the relative abundance of the $\mathrm{Mn}$ isotopes in cosmic rays. Experiments are being made both at the 88-Inch Cyclotron and at ANL, using different experimental techniques. The rarest, and one of the most enigmatic unstable nuclei in nature, ${ }^{180} \mathrm{Ta}$, is still the subject of experimental work in the hope of discovering how it was synthesized and why it survived. Finally, geological (as opposed to cosmological) dating techniques can also be affected if there is a dependence of the half life on the chemical composition of the compound in which the radioactive nucleus is located. Such a chemical dependence has been suspected for ${ }^{40} \mathrm{~K}$, which is used in the potassium-argon dating method. Present experiments on ${ }^{40} \mathrm{~K}$ contained in different compounds indicate this is not the case.

\section{Data for Nuclear Astrophysics}

Nuclei heavier than lithium can only be made in stars, and in the later, rapid burning and explosive stages of stellar evolution. The prediction of the abundance of these nuclei is a triumph of nuclear astrophysics, and requires an amount of nuclear information on a similarly grand scale. INPA, the Isotopes Project, and UC Santa Cruz have assembled a number of the data-bases used in nucleosynthesis calculations and made them available to the community through our new Nuclear Astrophysics Data Home Page.

\section{Fundamental Measurements}

The standard model of particle physics is the cornerstone for understanding the origin and development of the universe. Many of the key elements or parameters of the standard model are reflected in nuclear properties and measured in precision low-energy nuclear (or even atomic) experiments. We establish, test, and look for physics beyond the standard model in these nuclear physics experiments. Parity non-conservation, second class currents, time reversal invariance, the conserved vector current theory, double beta decaythese are some of the topics studied in the physics of weak interactions. 
The trapping of radioactive neutral atoms with laser beams offers significant opportunities for increasing precision in tests of weak interaction theory. This is because the atoms are both confined in a vacuum and polarized by the trapping mechanism. During the past two years, steady improvements in the laser trapping facility at the 88-Inch Cyclotron have led to the trapping of $5 \times 10^{4}{ }^{21} \mathrm{Na}$ atoms $\left(t_{1 / 2}=21 \mathrm{~s}\right)$ and a precise measurement of the ground state hyperfine transition. The next steps will be the installation of an in-vacuo beta detector and a measurement of the beta-decay asymmetry.

The branching ratio for the super-allowed beta decay of ${ }^{10} \mathrm{C}\left(\mathrm{g} \cdot \mathrm{s} .0^{+}\right)$to the first $0^{+}$state of ${ }^{10} \mathrm{~B}$ is critical for the determination of the $\mathrm{u}-\mathrm{d}$ element of the CabbiboKobayashi-Maskawa mixing matrix. This matrix is assumed to be unitary in the standard model of electro-weak interactions. The present measurement of this branching ratio uses Gammasphere, a high-resolution gamma-ray detector array; the first of two runs has been analyzed and yielded a value that is in agreement with a unitary CKM matrix but that differs by $2.5 \sigma$ from a recent measurement made at Chalk River Nuclear Laboratories. Gammasphere is also ideal for the measurement of angular correlations, as in the case of the $\beta-\gamma$ directional correlation in the decay of ${ }^{22} \mathrm{Na}$. This quantity enters into higher-order terms that test standard model predictions and can be used to search for second class currents.

The detailed shape of the energy spectrum in beta decay is related in the conserved vector current (CVC) theory to the strength of an associated electromagnetic transition. ${ }^{14} \mathrm{C}$ and ${ }^{14} \mathrm{O}$ are two cases for testing CVC. Measurements on the former nucleus have been completed (at ANL), while the latter will be studied at the 88-Inch Cyclotron using a new, high-efficiency Cusp ion source for the production of the ${ }^{14} \mathrm{O}\left(\mathrm{t}_{1 / 2}=71 \mathrm{~s}\right)$ radioactive source.

Double beta decay is a well-known though relatively rare phenomenon, the most interesting aspect of which would be neutrino-less $\beta \beta$ decay. (The latter has not been observed.) $\beta \beta$ decay to excited $0^{+}$states is also possible, though energetically disfavored. A search for this in ${ }^{150} \mathrm{Nd}$ is being conducted the Oroville low-background counting facility using $5 \mathrm{~kg}$ of natural neodymium oxide.

The breaking of $\mathrm{CP}$ symmetry explains the predominance of matter over antimatter in the universe. The most fundamental theorem in physics, " $\mathrm{CPT}=1$ " implies that Time Reversal Invariance must also be broken at some level. Searches for TRI-violating effects are therefore important for our understanding of how the universe evolved immediately after the Big Bang. Two searches are in progress. The first, called "emiT," is a study of the directional correlations in the beta decay of spin-polarized neutrons. This experiment, a collaboration of several institutions, has been several years in preparation, and is currently taking data with the cold neutron source at NIST. The other experiment uses low temperatures to align nuclei of ${ }^{56} \mathrm{Co}$ and observes the directional correlation of the nuclear spin, the emitted positron, and a subsequent gamma ray. This experiment is scheduled to have results later this year. 
The weak interaction responsible for nuclear beta decay (W-exchange) can induce a small Parity Non Conserving component in electromagnetic transitions in atomic systems through the weak neutral current (Z-exchange). The latter thus complements the study of nuclear beta decay. Atomic PNC experiments are in progress on a range of stable isotopes of $\mathrm{Yb}$ and may yield information on the weak interaction independent of atomic structure.

Materials that register a track when an ionizing particle passes through (emulsions, glasses, plastics, and insulating minerals) have found a wide application in the search for exotic particles. Searches for WIMPS and for magnetic monopoles have employed a variety of track detectors and have also used an Atomic Force Microscope for scanning.

\section{Low Background Counting}

The Low Background Counting Facilities used in the study of $\beta \beta$ decay have also been instrumental in a wide variety of experiments and in support activities for other institutions. The other types of work (done at the facilities at Berkeley and at Oroville) include low-activity materials certification, cosmic ray activation, neutron activation analysis, and environmental health and safety activities.

\section{Past and Future}

We note here two projects that address the early history and the ultimate fate of the universe and which are based in the Physics Division. The cosmic microwave background radiation observed today reflects the state of the universe about $3 \times 10^{5}$ years after the Big Bang, at the time radiation and matter decoupled. The next generation of satellites, to follow COBE in the study of anisotropies in the CMBR, are being planned.

The fate of the universe depends on its matter density, which is expressed as a ratio to a critical density at which the expansion rate of the universe slows to zero at infinite time. The supernova cosmology project searches for (and regularly discovers!) type $1 \mathrm{~A}$ supernovae at very large distances. In essence, the luminosity of a type $1 \mathrm{~A}$ supernova is a constant or "standard candle," which gives its distance, and the red shift of its host galaxy gives its velocity. Thus, the Hubble diagram can be extended to very large distances (or far back in time). Deviations from a linear dependence of recessional velocity on distance would indicate that $\Omega$ (the ratio of the mass density of the universe to the critical value) is different from 1 . 


\section{Institutional Activities}

The purpose of the Institute is to further interdisciplinary work in Nuclear and Particle Astrophysics at LBNL by:

- promoting interaction and communication among its members

- sharing of intellectual, technical, and administrative resources

- planning of new research proposals and development of detector systems

- developing innovative educational outreach programs

- establishing seminar, postdoctoral, and visitor programs

- sponsoring of workshops

The list of active participants has grown to approximately 80 , while the number of people receiving e-mail announcements of the weekly Journal Club is $\sim 200$. Attendance at the Journal Club is typically $30-40$ people. The daily tea has become an established feature of INPA life and attracts usually 15-20 people for conversation and lively argument. The Common Room is heavily used for regularly scheduled group meetings and ad-hoc get-togethers. The list of Journal Club speakers is contained elsewhere in this Annual Report.

New initiatives in which INPA plays an important role are the R\&D for the next generation of high energy neutrino detector and the development of a Nuclear Astrophysics Data Center.

Recent visitors to INPA include B. Balantekin, D. Branch, R. Ellis, J. Frieman, L. Krauss, A. Olinto, G. Savard, and M. Turner.

Workshops sponsored by INPA:

LBNL Workshop on Physics and Simulation Issues for $\mathrm{Km}^{3}$ Neutrino Observatory. Dec. 1, 1994.

NSAC/DNP TOWN MEETING on Electroweak Interactions, Astrophysics, and Non-Accelerator Experiments. February 4-5, 1995.

INPA Km3 Simulation Workshop. August 31, 1995.

US Meeting on Future Prospects for Km-scale Neutrino Detectors. April 18-19, 1996. (Arcadia, CA)

Additional information on the Institute and its activities can be found on the World Wide Web under the URL http:/ / www-inpa.lbl.gov/. 


\section{Relativistic Nuclear Collisions Program}

H.G. Ritter

The Relativistic Nuclear Collisions Program (RNC) conducts experiments studying the collision of nuclei in four energy regimes: (1) the Bevalac, where nuclear matter is compressed sufficiently to study its equation of state; (2) the AGS at Brookhaven National Laboratory (BNL), extending the studies of the Bevalac to an energy range where the maximum pressure from the baryons is likely to occur; (3) the SPS at CERN, where the energy density of the nucleons in the collision of very heavy nuclei may be sufficient to produce a phase transition to a plasma of free quarks and gluons; and (4) RHIC, where the energy density of the produced particles will be sufficiently high that production of the quark-gluon plasma is expected to occur. Understanding the reaction dynamics of nuclear matter and its equation of state is of fundamental interest.

The major efforts at the Bevalac have been the Dilepton Spectrometer (DLS) and the EOS Time Projection Chamber (TPC). Analysis of those experiments is near completion. The EOS TPC has been moved to the AGS to continue its studies at higher energies as experiment E895. Two periods of running have produced a rich sample of data, spanning the energy range from two to eight $\mathrm{AGeV}$. The construction of CERN experiment NA49 has been successfully completed, and the data taking and analysis are under way. The main focus of the high-energy heavy-ion research program at LBNL is the STAR experiment at RHIC, which will begin data-taking in 1999. 


\section{CERN/RHIC Physics}

The collisions of the heaviest nuclei at the highest energies ( $\mathrm{Pb}$ ions at the SPS, $\mathrm{Au}$ ions at RHIC) are expected to create systems whose space-time dynamics are qualitatively different from those of the colliding light ions studied up to now. The heavy systems have significantly higher energy densities over longer time scales. The extremely large number of produced hadrons in such collisions (several thousand in a central Au-Au event at RHIC) presents a real technical challenge and a unique opportunity: nontrivial, statistically significant signals can be extracted from single events, a technique known as "event-by-event" analysis. The correlation of extreme values of several observables sensitive to the quark-gluon plasma phase transition in a single event is a powerful tool for selecting ensembles of interesting events for detailed study. An event-by-event measurement of the produced particles provides the opportunity to select events with extreme values of temperature (particle spectrum), flavor (strangeness content), shape of the flow (particle momentum), and size (two-particle correlations). This technique requires a large acceptance detector that can determine the momentum and identify a large fraction of the particles emitted in the collision.

NA49 is a fixed-target experiment at the SPS designed to study $\mathrm{Pb}-\mathrm{Pb}$ collisions at $160 \mathrm{GeV} /$ nucleon $\left(\sqrt{S_{n n}}=17 \mathrm{GeV}\right)$. Its goal is to simultaneously measure many hadronic signals that are thought to be sensitive to the quark-gluon plasma. To perform event-by-event analysis, it measures and identifies almost all charged particles in the forward half of phase space and carries out detailed ensemble measurements of all the single-event observables as well as strange particle decays, two-particle correlation functions, and other hadronic observables.

STAR is a collider experiment at RHIC designed to study Au-Au collisions at $\sqrt{S_{n n}}=200 \mathrm{GeV}$. Its goal is similar to NA49's, to simultaneously measure many hadronic signals. To perform event-by-event analysis, it will measure and identify almost all charged particles over two units of rapidity, centered at midrapidity. At RHIC there is a high rate of hard processes. Hard-scattered partons are predicted to be sensitive to the medium through which they propagate. The process can be calculated in perturbative quantum chromodynamics. The study of high transverse momentum particles and jets as a function of energy and mass of the colliding system may also be an attractive experimental approach to identify the presence of quark matter.

\section{Bevalac/AGS Physics}

The hot and dense system produced in nuclear collisions can be studied with penetrating probes, dileptons in the case of the DLS . Dileptons provide a unique tool for probing the early phase of the hot, condensed system created in central A-A collisions. The yield and the mass spectrum of dileptons is sensitive to the density and temperature of the early phase of the collision, providing information on the nuclear matter equation of state and on medium effects. 
From the study of the hadronic signals of nuclear collisions with $4 \pi$ detectors, like the EOS TPC, a rather simple picture is emerging, compatible with the formation of a single source that seems to show a high degree of thermalisation and flow in the final state. Part of the flow pattern is established early by geometry and compression (directed flow), the other part is dominated by compression and expansion (radial flow). A careful and systematic comparison of data with microscopic models will lead to a better understanding of inmedium effects and the nuclear matter equation of state. Experiment E895 continues these studies in the AGS energy region with the focus on the study of flow and strange particle production.

\section{Experiments}

\section{The Dilepton Spectrometer (DLS)}

The DLS collaboration, from late 1986 until the closure of the Bevalac, carried out systematic measurements of $\mathrm{e}^{+} \mathrm{e}^{-}$production in $\mathrm{p}-\mathrm{p}, \mathrm{p}-\mathrm{d}$ and $\mathrm{A}-\mathrm{A}$ collisions as a function of beam energy and kinematics of the pair. The DLS results, about 30k pairs, represent the world's only $\mathrm{e}^{+} \mathrm{e}^{-}$data at Bevalac/SIS energies. Important results include: (1) existence of measurable dielectron yields, (2) observation of contributions from mesonic decays $\left(\pi^{0}, \eta, \rho / \omega\right)$, bremsstrahlung, and $\Delta / \mathrm{N}^{*}$ decays, (3) strong energy dependence of the $\mathrm{pd} / \mathrm{pp}$ yield ratios signifying the presence of the $\eta$-meson, (4) absolute value and shape of the mass spectrum at $5 \mathrm{GeV}$ in $\mathrm{p}-\mathrm{p}$ and $\mathrm{p}-\mathrm{d}$ collisions, which shows a need to modify existing N-N model calculations (pp vs. pn contributions, inelasticity), and (5) observation of high mass pairs ( $>500 \mathrm{MeV}$ ) in Ca-Ca collisions, which may be evidence for pionic annihilation. Analysis continues on the high-statistics A-A ( $\mathrm{Ca}-\mathrm{Ca}, \alpha-\mathrm{Ca}$, $\mathrm{d}-\mathrm{Ca}, \mathrm{C}-\mathrm{C}$ ) studies, and evolution of mass and $\mathrm{p}_{\mathrm{t}}$ distributions with projectile/target mass. Recent results from analysis of the A-A systems show that the low-mass region of the spectrum is well fitted by the shapes of the $\pi^{0}$ and $\eta$; in this region the yield for all four data sets is proportional to $A_{P} A_{T}$; at higher masses the ratio of the $\mathrm{CaCa} / \mathrm{CC}$ yields is proportional to $\left(\mathrm{A}_{\mathrm{P}} \mathrm{A}_{\mathrm{T}}\right)^{1.3}$. The fitted yield of $\eta$ 's is several times larger than recent model calculations.

\section{EOS TPC at the Bevalac}

EOS was designed to study heavy-ion reactions over the whole energy range of the Bevalac. The TPC enables the measurement of the production cross-sections for protons, light composite particles, and pions over a large dynamic range. The EOS collaboration performed an extensive series of measurements prior to the shutdown of the Bevalac. Excitation functions of four systems $(\mathrm{Ni}+\mathrm{Cu}, \mathrm{Ni}+\mathrm{Au}$, $\mathrm{La}+\mathrm{La}$, and $\mathrm{Au}+\mathrm{Au}$ ) were measured from $250 \mathrm{MeV}$ per nucleon up to the highest energy. In addition, the multifragmentation of the systems $\mathrm{Au}+\mathrm{C}, \mathrm{Kr}+$ $\mathrm{C}$, and $\mathrm{La}+\mathrm{C}$ at $1 \mathrm{GeV}$ per nucleon were investigated.

The physics analysis of the data is being performed at LBNL and other collaborating institutions. A complete excitation function of directed and radial flow in the $\mathrm{Au}+\mathrm{Au}$ system has been measured. A systematic study of the 
emission of light composite fragments shows that the coalesence mechanism describes composite particle production very well. Preferred out-of-plane emission at mid-rapidity (squeeze-out) has been shown to be compatible with emission from one source with a well defined temperature and higher mean flow velocities in the out-of-plane direction. Systematic comparison of the data with model calculations are in progress. Preliminary results favor models with momentum-dependent interactions and a soft equation of state. In addition, multifragmentation of the $\mathrm{Au}+\mathrm{C}$ system at $1 \mathrm{GeV}$ per nucleon has been analyzed as a critical phenomenon. A method to extract critical indices from the data has been developed. Preliminary results show that the critical indices extracted are compatible with the critical indices of the liquid-gas phase transition.

\section{EOS TPC at the AGS (E895)}

E895 is a major new experiment at the Brookhaven National Laboratory (BNL) designed to carry out a systematic and exclusive measurement of the energy and mass dependence of particle production, correlations, collective flow effects, and strangeness production in $\mathrm{Au}+\mathrm{Au}$ collisions. Theoretical studies indicate maximum baryon density, as high as eight times normal nuclear matter density, is achievable in central Au+Au collisions between 2 and $10 \mathrm{~A} \mathrm{GeV}$ beam energies. Therefore, E895 extends the physics program started at the Bevalac to understand the collision dynamics and obtain information on the nuclear matter equation of state. Additionally, E895 will study the change of medium effects since the baryon densities are high enough to alter the masses and widths of hadrons, which in turn, could indicate the occurrence of chiral symmetry restoration or the formation of baryon rich Quark Gluon Plasma.

The experimental arrangement consists of the EOS Time Projection chamber placed in a large 1 Tesla dipole magnet and the MUSIC detector positioned downstream of the TPC. The detectors are located in the MPS area of the Alternating Gradient Synchrotron (AGS) heavy ion facility. E895 was made operational and, in December 1995, had successfully recorded data using 2 and $4 \mathrm{~A} \mathrm{GeV} \mathrm{Au} \mathrm{beams.} \mathrm{High} \mathrm{statistics} \mathrm{were} \mathrm{obtained} \mathrm{with} \mathrm{a} \mathrm{Au} \mathrm{target,} \mathrm{and} \mathrm{less} \mathrm{with}$ $\mathrm{Ag}, \mathrm{Cu}$ and Be targets. During the second run in November 1996 data was recorded at 6 and $8 \mathrm{~A} \mathrm{GeV}$ using the same targets. A complete off-line data analysis is underway at LBNL utilizing the Parallel Distributed Scientific Farm (PDSF) at NERSC, as well as at other member institutions.

\section{NA49 at the SPS}

NA49 is a large acceptance experiment based on a set of Time Projection Chambers. Particle identification is performed primarily by the measurement of $\mathrm{dE} / \mathrm{dx}$ in the relativistic rise regime (leading to TPCs that are $3.6 \mathrm{~m}$ deep), supplemented by time-of-flight over a part of phase space. Event characterization for triggering is performed by forward calorimetry.

NA49 completed its construction phase and began taking data with the full complement of detectors in Fall 1995. Since then approximately 2 million lead- 
lead collisions at $158 \mathrm{GeV} /$ nucleon and 1 million proton-proton collisions at $158 \mathrm{GeV} / \mathrm{c}$ have been recorded, comprising a data set of about twenty terabytes.

In order to extract the most interesting volume-dependent effects in nucleusnucleus collisions, NA49 data on central collisions of lead ions are compared to NA35 data on central collisions of sulphur ions at about the same energy ( $200 \mathrm{GeV} /$ nucleon). NA49 finds that transverse energy production per participant nucleon increases slightly for the lead collisions, indicating a somewhat higher stopping. The rapidity distributions of primordial protons show the same effect. Produced particle multiplicities (total negative hadrons, which are mostly pions, and neutral and charged kaons) scale roughly with the number of participants. On the other hand, transverse mass spectra show an increase of inverse slope with the mass of the particle, which is indicative of increased radial flow for lead compared to sulphur collisions. Thus, the picture that emerges from transverse energy measurements and inclusive spectra from central collisions of lead ions compared to that of sulphur ions is one of somewhat greater stopping, with the additional available energy going into increased radial flow, while the strangeness enhancement appears to be about the same.

NA49 has also found a novel and unexpected feature in medium impact parameter collisions of lead ions. By means of techniques developed at the Bevalac to study flow phenomena, a quadrupole asymmetry in the azimuthal distribution of transverse energy was found. This apparent memory by the system of the initial reaction geometry is of great interest to the theoretical community. Effort is under way to study this phenomenon in the NA49 TPCs, in addition to the calorimeters.

\section{STAR at RHIC}

The purpose of STAR (Solenoidal Tracker at RHIC) is to discover the Quark Gluon Plasma, a new state of matter which hasn't existed in the universe since a few microseconds after the Big Bang. RHIC will provide colliding beams of gold ions at center-of-mass energies ranging from about $50 \mathrm{GeV}$ up to a maximum of $200 \mathrm{GeV}$ per nucleon. Other beam combinations are possible. STAR's goal is similar to NA49's; to simultaneously measure many hadronic signals of the QGP resulting from ultra-relativistic collisions. But hopefully STAR will explore nuclear collisions with energy densities that are much higher than are available at CERN. STAR will be able to perform event-by-event analysis, it will measure and identify virtually all charged particles over two units of rapidity (centered at mid-rapidity), and it will do an excellent job of characterizing high $p_{t}$ particles and jets that are the result of hard-scattered partons.

The detector will consist of a Time Projection Chamber (TPC) and Silicon Vertex Tracker located inside a 5.2-m-diameter solenoidal magnet to provide tracking, momentum analysis, and particle identification using the $\mathrm{dE} / \mathrm{dx}$ technique. The trigger detector systems include a central scintillator barrel around the TPC, vertex position detectors near the beamline just outside the magnet, and calorimeters located in the region of the beam insertion magnets to selectively 
veto events according to the number of spectators. An electromagnetic calorimeter to trigger on transverse energy and measure jet cross-sections is being added. In addition, two forward time projection chambers are being built by the Max Planck Institute (Munich). The forward TPCs will extend the detector's angular coverage from two units of pseudorapidity up to approximately four.

The TPC is nearing completion at LBNL. The outer field cage and the pressure vessel were recently mated and the detector electronics will soon be added. Complete tests of the TPC, using cosmic rays, are scheduled for the summer of 1997. Simultaneously, LBNL personnel are engaged in preparing the detector hall at Brookhaven to receive the TPC and associated detectors in preparation for full operation in the summer of 1999.

LBNL's Relativistic Nuclear Collisions Program is providing the focus for these activities and will be the intellectual center for STAR during the operations phase of the experiment. Within the STAR organization, the RNC program is providing the Project Management for STAR as well as building a large fraction of the hardware. RNC has primary responsibility for the TPC, TPC electronics, and overall detector integration. RNC also has significant responsibilities within the software efforts in STAR. RNC physicists form the core of the software development team that is focused on tracking and particle identification by $\mathrm{dE} / \mathrm{dx}$ in the TPC. This effort has recently benefited from a successful proposal to move a large computing facility from the SSC to LBNL. RNC physicists are currently proposing an even larger compute facility in association with NERSC. The goal of this larger center is to provide the primary simulation and off-line analysis center for STAR data after the year 2000. Currently, 40 physicists and engineers from LBNL are working on STAR. The STAR collaboration consists of 370 physicists and engineers from 36 institutions around the world.

\section{Development}

\section{Micro TPC Vertex Detector}

We are developing a new Micro TPC vertex detector that will be capable of tracking in the high track density environments which will be encountered in the STAR experiment at RHIC and in the ALICE experiment at LHC. This detector can handle track densities of 10 tracks $/ \mathrm{cm}^{2}$, approaching the capabilities of silicon devices. It has, however, the important advantage that it is essentially massless in comparison. This greatly reduces problems due to multiple scattering and secondary interactions, which is of utmost importance for vertex detectors. This technology could provide a significant improvement over silicon in these experiments particularly at low $\mathrm{p}_{\mathrm{t}}$ where exciting results have been predicted for heavy ion collisions. The Micro TPC will use micro-strip gas chambers (MSGCs) to read out the signal. The fine pitch of these devices is well matched to the low diffusion that can be achieved with a short drift distance in dimethyl-ether (DME). This combination makes it possible to achieve much better two-track resolution than has been possible with other TPCs. We have demonstrated that electrons can be drifted in DME over the planned drift distance of $15 \mathrm{~cm}$ without 
significant attenuation. We have also produced a MSGC on a silicon amplifier chip and demonstrated suitable operation.

\section{DLS Collaboration}

S. Beedoe ${ }^{1}$, R. Bossingham, M. Bougteb ${ }^{3}$, J. Carroll $^{1}$, W. Christie ${ }^{4}$, W. Gong, T. Hallman ${ }^{1}$, L. Heilbron, H.Z. Huang, G. Igo', P. Kirk ${ }^{5}$, G. Krebs, A. LetessierSelvon, L. Madansky4, F. Manso ${ }^{3}$, H.S. Matis, D. Miller6 ${ }^{6}$, J. Miller, C. Naudet, R.J. Porter ${ }^{2}$, M. Prunet ${ }^{3}$, G. Roche ${ }^{3}$, L. Schroeder, P.A. Seidl, Z.F. Wang ${ }^{5}$, R. Welsh" ${ }^{4}$, W.K. Wilson, A. Yegneswaran

${ }^{1}$ University of California at Los Angeles, Los Angeles, CA, USA.

${ }^{2}$ University of California at Davis, Davis, CA, USA.

${ }^{3}$ Université de Clermont II, Aubière, France.

${ }^{4}$ Johns Hopkins University, Baltimore, MD, USA.

${ }^{5}$ Louisiana State University, Baton Rouge, LA, USA.

${ }^{6}$ Northwestern University, Evanston, IL, USA.

\section{EOS Collaboration}

S. Albergo ${ }^{1}$, F. Bieser, F.P. Brady ${ }^{2}$, Z. Caccia ${ }^{1}$, D.A. Cebra ${ }^{2}$, A.D. Chacon ${ }^{6}$, J.L. Chance ${ }^{2}$, Y. Choi ${ }^{5}$, S. Costa ${ }^{1}$, J.B. Elliott ${ }^{5}$, M.L. Gilkes, ${ }^{7}$, J.A. Hauger ${ }^{5}$, A.S. Hirsch, ${ }^{5}$, E.L. Hjort ${ }^{5}$, A. Insolia ${ }^{1}$, M. Justice ${ }^{4}$, D. Keane ${ }^{4}$, J. Kintner ${ }^{2}$, V. Lindenstruth ${ }^{3}$, M.A. Lisa, H. Liu ${ }^{4}$, H.S. Matis, M. McMahan, C. McParland, W.F.J. Mueller ${ }^{3}$, D.L. Olson, M.D. Partlan, N.T. Porile ${ }^{5}$, R. Potenza ${ }^{1}$, G. Rai, J. Rasmussen, H.G. Ritter, J. Romanski ${ }^{1}$, J.L. Romero ${ }^{2}$, G.V. Russo ${ }^{1}$, H. Sann ${ }^{3}$, R.P. Scharenberg ${ }^{5}$, A. Scott ${ }^{4}$, Y. Shao ${ }^{4}$, B.K. Srivastava ${ }^{5}$, T.J.M. Symons, M.L. Tincknell ${ }^{5}$, C. Tuvè ${ }^{1}$, S. Wang ${ }^{4}$, P.G. Warren ${ }^{5}$, H.H. Wieman, T. Wienold, K.L. Wolf 6

${ }^{1}$ University of Catania, Catania, Italy.

${ }^{2}$ University of California at Davis, Davis, CA, USA.

${ }^{3}$ Gesellschaft für Schwerionenforschung (GSI), Darmstadt, Germany.

${ }^{4}$ Kent State University, Kent, OH, USA.

${ }^{5}$ Purdue University, West Lafayette, IN, USA.

${ }^{6}$ Texas A \& M University, College Station, TX, USA.

\section{E895 Collaboration}

N.N. Ajitanand 4 , J. Alexander ${ }^{4}$, D. Best, F. Bieser, F.P. Brady ${ }^{1}$, T. Case, W. Caskey ${ }^{1}$, D.A. Cebra ${ }^{1}$, J. Chance ${ }^{1}$, P. Chung ${ }^{4}$, K. Crowe, A. Das ${ }^{8}$, J. Draper ${ }^{1}$, M. Gilkes ${ }^{3}$, S. Gushue ${ }^{5}$, M. Heffner ${ }^{1}$, A.S. Hirsch ${ }^{3}$, E. Hjort ${ }^{3}$, L. Huo ${ }^{11}$, M.L. Justice ${ }^{2}$, P. Kammel, M. Kaplan ${ }^{6}$, D. Keane ${ }^{2}$, J. Kintner ${ }^{7}$, D. Krofcheck ${ }^{10}$, R. Lacey ${ }^{4}$, H. Liu ${ }^{2}$, Y.M. Liu ${ }^{11}$, M. Lisa ${ }^{8}$, R. McGrath ${ }^{4}$, C.P. McParland, Z. Milosevich ${ }^{6}$, D.L. Olson, C. Pinkenburg ${ }^{4}$, N. Porile ${ }^{3}$, G. Rai, J. Rasmussen, H.G. Ritter, J. Romero ${ }^{1}$, R. Scharenberg ${ }^{3}$, B. Shrivastava ${ }^{3}$, L. Schroeder, N. Stone, T.J.M. Symons, S. Wang ${ }^{2}$, J. Whitfield ${ }^{6}$, T. Wienold, L. Wood ${ }^{1}$, W. Zhang ${ }^{11}$ 
${ }^{1}$ University of California at Davis, Davis, CA, USA.

${ }^{2}$ Kent State University, Kent, OH, USA.

${ }^{3}$ Purdue University, Lafayette, IN, USA.

${ }^{4}$ State University of New York at Stony Brook, Stony Brook, NY, USA.

${ }^{5}$ Brookhaven National Laboratory, Upton, NY, USA.

${ }^{6}$ Carnegie Mellon University, Pittsburg, PA, USA.

${ }^{7}$ St. Mary's College, Moraga, CA, USA.

${ }^{8}$ Ohio State University, Columbus, OH, USA.

${ }^{9}$ Columbia University, Nevis Lab., NY, USA.

${ }^{10}$ The University of Auckland, New Zealand.

${ }^{11}$ Harbin Institute of Technology, Harbin, PR China.

\section{NA49 Collaboration}

S.V. Afanasiev ${ }^{9}$, T. Alber ${ }^{13}$, H. Appelshauser ${ }^{7}$, J. Bachler ${ }^{5}$, L.S. Barnby ${ }^{3}$, J. Bartke ${ }^{6}$, H.Bialkowska ${ }^{14}$, C.O. Blyth ${ }^{3}$, R. Bock ${ }^{7}$, C. Bormann ${ }^{9}$, F.P. Brady ${ }^{8}$, R. Brockmann ${ }^{7}$, N. Buncic 5,10 , P. Buncic ${ }^{5,7}$, H.L. Caines ${ }^{3}$, D. Cebra ${ }^{8}$, P. Chan ${ }^{16}$, G. Cooper ${ }^{2}$, J.G. Cramer ${ }^{16,13}$, P.B. Cramer 16, P. Csato ${ }^{4}$, M. Cyprian ${ }^{12}$, I. Derado ${ }^{13}$, O. Dietz ${ }^{10}$, J. Dunn ${ }^{8}$, V. Eckardt ${ }^{13}$, F. Eckhardt ${ }^{12}$, S. Euler ${ }^{12}$, M.I. Ferguson ${ }^{5}$, H.G. Fischer ${ }^{5}$, D. Flier ${ }^{10}$, Z. Fodor ${ }^{4}$, P. Foka ${ }^{7}$, P. Freund ${ }^{13}$, M. Fuchs ${ }^{7}$, F. Gabler ${ }^{10}$, J. Gal ${ }^{4}$, M. Gazdzicki ${ }^{9}$, E. Gladysz ${ }^{6}$, J. Grebieszkow ${ }^{14}$, J. Günther ${ }^{9}$, J.W. Harris' ${ }^{2}$, S. Hegyi ${ }^{4}$, L.A. Hill ${ }^{3}$, I. Huang ${ }^{8}$, M.A. Howe ${ }^{16}$, H. Hümmler ${ }^{10}$, G. Igo ${ }^{11}$, D. Irmscher ${ }^{2}$, P. Jacobs ${ }^{2}$, P.G. Jones ${ }^{3}$, K. Kadija 17,13, V. I. Kolesnikov ${ }^{9}$, M. Kowalski ${ }^{6}$, B. Lasiuk ${ }^{11}$, P. Levai ${ }^{4}$, A.I. Malakhov ${ }^{9}$, S. Margetis ${ }^{2}$, C. Markert7, G.L.Melkumov ${ }^{9}$, J.W. Mitchell ${ }^{8}$, A. Mock ${ }^{13}$, J. Molnar ${ }^{4}$, J.M. Nelson ${ }^{3}$, G. Odyniec, G. Palla4, A.D. Panagiotou ${ }^{1}$, A. Petridis ${ }^{1}$, A. Piper ${ }^{12}$, A.M. Poskanzer ${ }^{2}$, S. Poziombka ${ }^{10}$, D.J. Prindle ${ }^{16}$, F. Pühlhofer ${ }^{12}$, W. Rauch ${ }^{13}$, R. Renfordt ${ }^{95}$, W. Retyk ${ }^{14}$, H.G. Ritter ${ }^{2,5}$, D. Röhrich ${ }^{9}$, C.Roland 7 , G. Roland ${ }^{10}$, H. Rudolph ${ }^{2}$, A. Rybicki ${ }^{6}$, A. Sandoval ${ }^{7}$, H. Sann ${ }^{7}$, E. Schäfer ${ }^{13}$, D.Schmischke ${ }^{10}$, N. Schmitz ${ }^{13}$, S. Schönfelder ${ }^{13}$, A. Yu. Semenov ${ }^{9}$, P. Seyboth ${ }^{13}$, J. Seyerlein ${ }^{13}$, F. Sikler ${ }^{4}$, E. Skrzypczak ${ }^{15}$, G. T. A.Squier ${ }^{3}$, R. Stock ${ }^{9}$, H. Ströbele ${ }^{9}$, I. Szentpetery ${ }^{4}$, J. Sziklai ${ }^{4}$, M. Toy ${ }^{2,11}$, T.A. Trainor ${ }^{16}$, S. Trentalange ${ }^{11}$, M. Vassiliou ${ }^{1}$, G. Vesztergombi ${ }^{4}$, D. Vranic ${ }^{7,17}$, D. Weerasundara ${ }^{16}$, S. Wenig ${ }^{5}$, C. Whitten ${ }^{11}$, T. Wienold ${ }^{2}$, L. Wood ${ }^{8}$, T.A. Yates ${ }^{3}$, J. Zimanyi ${ }^{4}$, X.Z. Zhu ${ }^{16}$, R. Zybert ${ }^{3}$

${ }^{1}$ Department of Physics, University of Athens, Athens, Greece.

${ }^{2}$ Lawrence Berkeley Laboratory, University of California, Berkeley, CA, USA.

${ }^{3}$ Birmingham University, Birmingham, England.

${ }^{4}$ Institute of Physics, Budapest, Hungary.

${ }^{5} \mathrm{CERN}$, Genève, Switzerland.

${ }^{6}$ Institute of Nuclear Physics, Cracow, Poland.

${ }^{7}$ Gesellschaft für Schwerionenforschung (GSI), Darmstadt, Germany.

${ }^{8}$ University of California at Davis, Davis, CA, USA.

${ }^{9}$ Fachbereich Physik der Universität, Frankfurt, Germany.

${ }^{10}$ Fachbereich Physik der Universität, Freiburg, Germany.

${ }^{11}$ University of California at Los Angeles, Los Angeles, CA, USA

${ }^{12}$ Fachbereich Physik der Universität, Marburg, Germany.

${ }^{13}$ Max-Planck-Institut für Physik, Munich, Germany. 
${ }^{14}$ Institute for Nuclear Studies, Warsaw, Poland.

${ }^{15}$ Institute for Experimental Physics, University of Warsaw, Warsaw, Poland.

${ }^{16}$ University of Washington, Seattle, WA, USA.

${ }^{17}$ Rudjer Boskovic Institute, Zagreb, Croatia.

\section{STAR $\star$ Collaboration}

N. Added ${ }^{27}$, H.N. Agakishiev ${ }^{10}$, S. Ahmad ${ }^{26}$, S.A. Akimenko ${ }^{24}$, A.V. Alyushin ${ }^{19}$, M.V. Alyushin ${ }^{19}$, N. Amelin ${ }^{10}$, J. Amsbaugh ${ }^{32}$, G.T. Anderson', E. Anderssen 15, L. Andreeva 19, A. Aprahamian 21, Yu.I. Arestov 24, E.V.Atkin 19, V. Avdeichikov ${ }^{11}$, G.S. Averichev ${ }^{10}$, R. Badalian ${ }^{10}$, A. Baldwin ${ }^{14}$, K. Barish ${ }^{7}$, V.V. Baublis ${ }^{19}$, N.I. Belikov ${ }^{24}$, R. Bellwied ${ }^{33}$, V.I. Belousov ${ }^{24}$, M. Bennett ${ }^{5}$, S. Bennett ${ }^{33}$, J. Bercovitz ${ }^{15}$, D. Best ${ }^{15}$, W. Betts ${ }^{29}$, H. Bichsel ${ }^{32}$, J. Bielecki ${ }^{33}$, F. Bieser ${ }^{15}$, N.N. Biswas ${ }^{21}$, C. Blyth ${ }^{35}$, B.E. Bonner ${ }^{26}$, R. Bossingham ${ }^{15}$, F.P. Brady 6 , W.J. Braithwaite 2 , C Brown ${ }^{8}$, R. Brown ${ }^{4}$, V. Budilov ${ }^{11}$, C. Byrd2, H. Caines ${ }^{22}$, N. Carlin ${ }^{27}$, J.B. Carroll 7 , D.A. Cebra ${ }^{6}$, A. Chalyguine ${ }^{11}$, J. Chance, W. Chen ${ }^{4}$, S.P. Chernenko ${ }^{10}$, M.G. Cherney ${ }^{9}$, J. Chrin ${ }^{9}$, W. Christie ${ }^{4}$, B.V. Chujko ${ }^{24}$, P.Colarco9 , G. Cooper ${ }^{15}$, T.M. Cormier 33 , J.G. Cramer ${ }^{32}$, H.J. Crawford ${ }^{5}$, M. Cronqvist ${ }^{5}$, D. Crosetto26, A. Darun 22, A.M. Davidenko 24 , J.W. Dawson 1 , D. Dayton ${ }^{4}$ A.A. Derevschikov ${ }^{24}$, P.A. DeYoung ${ }^{8}$, D. Di Massimo ${ }^{4}$, J. Dioguardi ${ }^{4}$, W. Dominik ${ }^{30}$, M. Duff ${ }^{16}$, H. Dyke 22 , J.E. Draper 6 , I. Duck ${ }^{26}$, V. Eckardt 17 , W.R. Edwards ${ }^{15}$, S. Efremov 26, V. Emelianov 19, M. Engelage 5, G. Eppley 26, R. Eremeev ${ }^{11}$, S.V. Erin ${ }^{24}$, A. Etkin ${ }^{4}$, P. Fachini27, N. Fadeev ${ }^{11}$, O. Fateev 10, A. Feshchenko ${ }^{11}$, H. Fessler ${ }^{17}$, I. Flores ${ }^{5}$, K.J. Foley ${ }^{4}$, Z. Fraenkel ${ }^{34}$, A. French ${ }^{33}$, P. Freund ${ }^{17}$, D. Fritz ${ }^{15}$, N. Gagunashvili11, U. Garg ${ }^{21}$, O. Gavrichtchouk ${ }^{11}$, M. Gazdzicki ${ }^{12}$, V. Ghazikhanian ${ }^{7}$, J. Grabski ${ }^{31}$, W. Gong ${ }^{15}$, O.A. Grachov ${ }^{24}$, D. Greiner ${ }^{15}$, L. C. Greiner ${ }^{5}$, V. Grigoriev ${ }^{19}$, V.V. Grushin ${ }^{19}$, V.J. Guarino ${ }^{1}$, Y. Guo ${ }^{33}$, M. Gupta ${ }^{26}$, E. Gushin ${ }^{19}$, W.N. Haberichter ${ }^{1}$, R.W. Hackenburg ${ }^{4}$, J. Hall ${ }^{33}$, T.J. Hallman ${ }^{4}$, D. Hardtke 22, G. Harper ${ }^{32}$, J. Harris ${ }^{36}$, F. Heistermann ${ }^{4}$, C. Henderson ${ }^{15}$, S. Heppelmann 23 , D.A. Hill ${ }^{1}$, N. Hill ${ }^{1}$, A.S. Hirsch ${ }^{25}$, E. Hjort ${ }^{25}$, G.W. Hoffmann ${ }^{29}$, M. Howe ${ }^{32}$, H. Huang ${ }^{25}$, T.J. Humanic ${ }^{22}$, J.B. Hunter ${ }^{15}$, G.J. Igo ${ }^{7}$, A. Ioukaev ${ }^{11}$, P.M. Jacobs ${ }^{15}$, S.R. Jacobson 15 , R. Jamil ${ }^{9}$, R.C. Jared ${ }^{15}$, P. Jensen ${ }^{29}$, P. Jones ${ }^{35}$, E. Judd 5 , M.L. Justice ${ }^{14}$, K. Kadija17, M. Kaplan 8 , V. Kaplin ${ }^{19}$, A. Karakash ${ }^{19}$, A. Karev ${ }^{11}$, P.J. Karol ${ }^{8}$, T. Kasprzyk ${ }^{1}$ ， D. Keane ${ }^{14}$, A.M. Khodinov ${ }^{19}$, S.R. Klein ${ }^{15}$, L. Kolobashkina ${ }^{19}$, A. Kolomyichenko ${ }^{11}$, M. Konrad ${ }^{17}$, A.S. Konstantinov ${ }^{24}$, I. Kossarev 11, L. Kotchenda19， I. Kotov ${ }^{24}$, N. Kouzmine 11, A.D. Kovalenko ${ }^{10}$, M.A. Kramer ${ }^{20}$, H. Kraner ${ }^{4}$, V. Krivokhizh ${ }^{11}$ E. Kukhtin ${ }^{11}$, R. Kutuev ${ }^{11}$, A. Kuznetsov ${ }^{10}$, A. LacKamp ${ }^{9}$, V. Ladygin 10, B. Lasiuk ${ }^{7}$, A.N. Lebedev ${ }^{19}$, T. Lecompte ${ }^{1}$, I. Lee ${ }^{16}$, B. Leonhardt', M.J. LeVine ${ }^{4}$, Q. Li $^{33}$, Y. Li ${ }^{33}$, C .Liaw ${ }^{4}$, V. Lindenstruth ${ }^{5}$, M. Lisa ${ }^{15}$, H. Lie ${ }^{14}$, A. Ljubicic Jr. ${ }^{3}$, W.J. Llope ${ }^{26}$, G. LoCurto ${ }^{22}$, V. Loginov ${ }^{19}$, R.S. Longacre ${ }^{4}$, W.A. Love ${ }^{4}$, D. Lynn ${ }^{4}$, L. Madansky 13, E. Makliaev 19, S. Margetis ${ }^{15}$, K.M. Marks 15, J. Marx ${ }^{15}$, R. Matheus ${ }^{27}$, H.S. Matis ${ }^{15}$, Yu.A. Matulenko ${ }^{24}$, E.A. Matushevsky ${ }^{10}$, C.P. McParland ${ }^{15}$, T.S. McShane ${ }^{9}$ K. Medved ${ }^{11}$, R. Mekhdiyev ${ }^{11}$, A.P. Meschanin 24, B. Miller ${ }^{4}$, Z. Milosevich ${ }^{8}$, N.G. Minaev 24, R.H. Minor ${ }^{15}$, E. Minor ${ }^{23}$, J.W. Mitchell13, V.Mitsyn ${ }^{11}$, L. Mogavero 4 , C.F. Moore ${ }^{29}$, M.G. Munhoz 27, L. Muresan 10, R. Muresan 10, J.J. Musulmanbekov 10, G.S. Mutchler ${ }^{26}$, A.I. Mysnick ${ }^{24}$, A. Nagaitsev ${ }^{11}$, J. Nelson ${ }^{35}$, V.N. Nevolin ${ }^{19}$, 
P. Nevski ${ }^{4}$, V. Nikitin ${ }^{11}$, E. Nikonov ${ }^{10}$, P. Nomokonov ${ }^{10}$, S.B. Nurushev ${ }^{24}$, V. Odintsov ${ }^{11}$, G. Odyniec ${ }^{15}$, C. Ogilvie ${ }^{16}$, D.L. Olson ${ }^{15}$, A.St. Oltchak ${ }^{19}$, E.M. Onischenko ${ }^{19}$, G. Ososkov ${ }^{10}$, E. Paganis ${ }^{29}$, G. Paic ${ }^{3}$, S. Pandey ${ }^{33}$, Yu.A. Panebratsev ${ }^{10}$, J. Passaneau ${ }^{23}$, A.I. Pavlinov 24, A. Pavluk ${ }^{11}$, T. Pawlak ${ }^{31}$, M. Pentia ${ }^{10}$, W. Peryt ${ }^{31}$, D. Peshekhonov ${ }^{11}$, V. Peshekhonov ${ }^{11}$, E. Petereit ${ }^{1}$, D. Pilipenko ${ }^{11}$, N.M. Piskunov ${ }^{10}$, E.D. Platner ${ }^{26}$, J. Pluta ${ }^{31}$, I. Polk ${ }^{4}$, N.T. Porile ${ }^{25}$, A.M. Poskanzer ${ }^{15}$, E. Potrebenikova ${ }^{10}$, L. Price ${ }^{1}$, D.G. Prindle ${ }^{32}$, C. Pruneau ${ }^{33}$, G. Rai ${ }^{15}$, A. Rawlins ${ }^{15}$, R.L. Ray ${ }^{29}$, S.V. Razin ${ }^{10}$, D. $\operatorname{Read}^{29}$, D. Reichhold ${ }^{22}$, R.E. Renfordt ${ }^{12}$, F. Retiere ${ }^{15}$, A. Ridiger ${ }^{19}$, P.J. Riley ${ }^{29}$, J. Riso ${ }^{33}$, H.-G. Ritter ${ }^{15}$, J.B. Roberts ${ }^{26}$, D. Roehrich 12 , H. Roehrig ${ }^{12}$, A.A. Rollefson ${ }^{2}$, J.L. Romero ${ }^{6}$, I. Roufanov ${ }^{11}$, V.L. Rykov ${ }^{24}$, I. Sakrejda ${ }^{15}$, A.C. Saulys ${ }^{4}$, N. Saviljic ${ }^{10}$, I. Savin ${ }^{11}$, E.Schaefer ${ }^{17}$, J.J. Schambach ${ }^{29}$, R.P. Scharenberg ${ }^{25}$, J. Scheblein ${ }^{4}$, B. Scheetz ${ }^{4}$, A. Schiemann ${ }^{9}$, R. Schlueter ${ }^{15}$, D. Schmischke ${ }^{12}$, N. Schmitz ${ }^{17}$, L.S. Schroeder ${ }^{15}$, M. Schulz ${ }^{4}$, J. Sedlmeir ${ }^{4}$, J.E. Seger ${ }^{9}$, J.C. Seyboth ${ }^{17}$, P. Seyboth ${ }^{17}$, D. Seymour ${ }^{32}$, A. Shabunov ${ }^{10}$, M. Shafranov ${ }^{11}$, M. Shafranova ${ }^{11}$, E. Shahaliev ${ }^{10}$, A.V. Shalnov ${ }^{19}$, J. Sheen ${ }^{33}$, K.E. Shestermanov ${ }^{24}$, S.S. Shimanskiy ${ }^{10}$, A.B. Simakov ${ }^{19}$, G. Skoro ${ }^{10}$, N.V.Slavin ${ }^{10}$, N. Smirnoff ${ }^{36}$, G. Smirnov ${ }^{11}$, L.P. Smykov ${ }^{10}$, R. Soja ${ }^{4}$, L.F. Soloviev ${ }^{24}$, S. Somov ${ }^{19}$, H.M. Spinka ${ }^{1}$, B.K. Srivastava ${ }^{25}$, R. Stock ${ }^{12}$, N.T.Stone ${ }^{15,}$ M.N. Strikhanov ${ }^{19}$ ， B. Stringfellow ${ }^{25}$, H. Stroebele ${ }^{12}$, E.A. Strokovskiy ${ }^{10}$, E. Sugarbaker ${ }^{22}$, K. Sulimma ${ }^{12}$, A. Sustich2, D. Swan ${ }^{18}$, T.J.M. Symons ${ }^{15}$, E.M. Szanto ${ }^{27}$, A. Szanto de Toledo 27 , J. Takahashi ${ }^{27}$, J.L. Tang ${ }^{29}$, H. Themann ${ }^{26}$, J. Thomas ${ }^{15}$, V. Tikhonov ${ }^{24}$, M.L. Tincknell ${ }^{25}$, M.V. Tokarev ${ }^{10}$, M. Toy ${ }^{7}$, T.A. Trainor ${ }^{32}$, S. Trentalange ${ }^{7}$, O.D. Tsay ${ }^{24}$, I. Tserruya ${ }^{34}$, C.E. Tull ${ }^{15}$, T. Tustonic ${ }^{3}$, T. Ullrich $^{36}$, D.G. Underwood ${ }^{1}$, Z. Usubov ${ }^{11}$, A. Vander Molen ${ }^{18}$, A.M. Vanyashin ${ }^{19}$, V. Vasendina ${ }^{11}$, A.N. Vasiliev ${ }^{24}$, M.Vidal ${ }^{17}$, G.J. Visser ${ }^{15}$, Yu.A. Volkov ${ }^{19}$, S.V. Voloshin ${ }^{19}$, S. Vorozhtsov ${ }^{11}$, D. Vranic ${ }^{3}$, C.Q. Vu ${ }^{15}$, F. Wang ${ }^{15}$, H.J. Ward ${ }^{29}$, J.W. Watson ${ }^{14}$, D.D. Weerasundara ${ }^{14}$, R.P. Wells ${ }^{15}$, G.D. Westfall ${ }^{18}$, J. Whitfield ${ }^{8}$, C.A. Whitten Jr. ${ }^{7}$, H. Wieman ${ }^{15}$, W.K. Wilson ${ }^{33}$, J.S. Wirth ${ }^{15}$, D.C. Wold ${ }^{2}$, K.L. Wolf ${ }^{28}$, L. Wood ${ }^{6}$, M. Wright ${ }^{26}$, N. Xu1 ${ }^{15}$, E. Yee ${ }^{15}$, P. Yepes ${ }^{26}$, A. Yokosawa ${ }^{1}$, V.I. Yurevich ${ }^{10}$, J. Yurkon ${ }^{18}$, Yu.V. Zanevsky ${ }^{10}$, W. Zhang ${ }^{14}$, N. Zhidkov ${ }^{11}$, V. Zhiltsov ${ }^{11}$, R. Zoulkarneev ${ }^{11}$, R. Zybert ${ }^{35}$

${ }^{1}$ Argonne National Laboratory, Argonne, IL, USA.

2University of Arkansas, Little Rock, AR, USA.

${ }^{3}$ Rudjer Boskovic Institute, Zagreb, Croatia.

${ }^{4}$ Brookhaven National Laboratory, Upton, NY, USA.

${ }^{5}$ University of California at Berkeley, Space Science Laboratory, Berkeley, CA, USA.

${ }^{6}$ University of California at Davis, Davis, CA, USA.

${ }^{7}$ University of California at Los Angeles, Los Angeles, CA, USA.

${ }^{8}$ Carnegie Mellon University, Pittsburgh, PA, USA.

${ }^{9}$ Creighton University, Omaha, NE, USA.

${ }^{10}$ Laboratory of High Energy (JINR), Dubna, Russia.

${ }_{11}$ Particle Physics Laboratory (JINR), Dubna, Russia.

12University of Frankfurt, Frankfurt, Germany.

13Johns Hopkins University, Baltimore, MD, USA.

${ }^{14}$ Kent State University, Kent, OH, USA.

${ }^{15}$ Lawrence Berkeley Laboratory, Berkeley, CA, USA.

${ }^{16}$ Massachusetts Institute of Technology, Cambridge, MA, USA. 
${ }^{17}$ Max-Planck-Institut für Physik, Munich, Germany.

${ }^{18}$ Michigan State University, East Lansing, MI, USA.

${ }^{19}$ Moscow Engineering Physics Institute, Moscow, Russia.

${ }^{20}$ City College of New York, New York, NY, USA.

${ }^{21}$ University of Notre Dame, Notre Dame, IN, USA.

${ }^{22}$ Ohio State University, Columbus, $\mathrm{OH}, \mathrm{USA}$.

23Pennsylvania State University, University Park, PA, USA.

${ }^{24}$ Institute of High Energy Physics, Protvino, Russia.

${ }^{25}$ Purdue University, West Lafayette, IN, USA.

${ }^{26}$ Rice University, Houston, TX, USA.

${ }^{27}$ Universidade de São Paulo, São Paulo, Brazil.

${ }^{28}$ Texas A \& M University, College Station, TX, USA.

${ }^{29}$ University of Texas at Austin, Austin, TX, USA.

${ }^{30}$ Warsaw University, Warsaw, Poland.

${ }^{31}$ Warsaw University of Technology, Warsaw, Poland.

${ }^{32}$ University of Washington, Seattle, WA, USA.

${ }^{33}$ Wayne State University, Detroit, MI, USA.

${ }^{34}$ Weizmann Institute of Science, Rehovot, Israel.

${ }^{35}$ University of Birmingham, Birmingham, England.

${ }^{36}$ Yale University, New Haven, CT, USA. 


\section{Nuclear Theory Program}

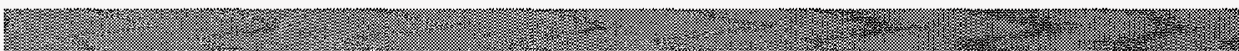

\section{J. Randrup}

The Nuclear Theory Program at Berkeley Lab seeks to address important problems at the frontier of nuclear science to achieve a deeper understanding of the physical nature of quantal many-body systems at and below the hadronic level. This involves developing and applying theories and methods for prediction, analysis, and interpretation of experiments. While the current research program is primarily focused on high-energy nuclear collisions, significant efforts remain in the areas of compact stellar objects, macroscopic nuclear properties, and chaos studies. The main themes are briefly described below.

\section{Parton Dynamics}

On the basis of a QCD-inspired partonic description, a number of critical issues in heavy-ion collisions at RHIC energies and beyond have been addressed: the initial condition of the dense matter formed, the approach of such dense parton matter to equilibrium, and the probes of the early parton dynamics. While the initial parton density is found to be very high, it is also far from equilibrium and the equilibration time depends sensitively on the initial parton density. A few hard probes, such as charm, $J / \psi$, and jets, have been investigated as indicators of the initial parton density and early parton dynamics. Continuing research will seek to reduce the uncertainty in the initial conditions as well as further explore the associated hard probes. 


\section{Hard probes of QCD matter}

A number of studies have been directed towards heavy-quark production, both in the initial nucleon-nucleon interactions and in the plasma phase. Heavy-quark production in $p p$ collisions has been studied using perturbative QCD at leading order, next-to-leading order, and, near the production threshold, all-order resummation at leading log and next-to-leading log. The knowledge gained from $p p$ physics can help us to understand the level of heavy-quark contribution to the dilepton spectrum at RHIC and LHC although medium effects, such as energy loss by the charm quark moving through the nucleus, may play an important role. This latter effect is being examined in more detail, particularly by including the effects of longitudinal expansion, as is the possibility that $e \mu$ coincidence measurements for open charm decay may provide information on the parton energy-loss mechanism.

Quarkonium production and suppression is an especially interesting topic in light of recent CERN SPS data from NA50 and this data has been addressed in terms of hadronic suppression mechanisms. At higher energies, where plasma production is likely, the high initial temperature of the plasma could lead to $r$ suppression or result in the gluons and light quarks gaining an effective mass, thereby enhancing thermal production of heavy quarks in the plasma. Manifestations of higher-twist effects on heavy-quark production are also being pursued, both in the context of the intrinsic charm model and, more recently, in a final-state coalescence model involving the quantum mechanical overlap of the initial- and final-state wave functions on the amplitude level.

\section{Disoriented chiral condensates}

A number of investigations have been carried out within the confines of the linear sigma model. In particular, the conditions for the occurrence of the DCC phenomenon has been studied in various collision scenarios and it has been shown that the soft pionic modes become unstable only when a sufficiently rapid expansion or cooling takes place. A variety of possible DCC signatures have been studied, including the power spectra of the emerging pions and the distribution of the neutral pion fraction. Wavelet-type analysis techniques have also been developed for the study of domain structure of the pion field.

Dileptons may be a very sensitive probe of soft pionic modes and dilepton spectra have been calculated with different models in order to examine the prospects for using this observable as a DCC diagnostic. It is planned to explore this mechanism in detail, with an emphasis on the role of other hadrons in DCC formation and decay.

\section{Chiral restoration and in-medium effects}

Dilepton production at the CERN SPS has been investigated within a hadronic transport model. It was found that although the CERES data can be explained within errors, the present dilepton data do not provide additional sensitivity to 
the initial hadronic configuration. In-medium effects on the pion form factor and the pion dispersion relation lead to changes in the dilepton yield which, however, are smaller than the errors of present data. This calculation will be continued in order to explore the intermediate mass regime.

Studies in the hadronic phase have shown that the chemical equilibration rate is shortened by the presence of vector mesons and also affected by the onset of chiral restoration. Expansion and finite-size effects will be addressed within the transport model, and it is planned to extend these investigations into the strange sector. Furthermore, it is predicted that the $\Lambda(1405)$ has a strongly momentumdependent in-medium mass shift due to the Pauli principle. The prospects for investigating this effect with pion-induced reactions and stopped kaons are currently being assessed.

The collaborative effort to include the collective spin-isospin modes in transport treatments of heavy-ion collisions is being continued. The formal developments made earlier for nuclear matter have been adapted to the finite, non-equilibrium environment of the colliding system and implemented into an existing nuclear Boltzmann transport code. Dynamical simulations are being carried out in order to ascertain how the pionic collectivity affects experimental observables, such as flow patterns and pion yields.

Chiral models of the nuclear force will be extended to describe hypernuclei and the equation of state for both strange and charmed matter, with relevance for both heavy-ion physics and compact stars. Associated precursor signals for the chiral phase transition in dense matter will be sought and the possible transition to a kaon condensed phase in the core of a neutron star will be also studied.

\section{Transport theory}

With a view towards high-energy nuclear collisions, work has been made on developing a suitable transport description from quantum field theory, using a $1 / N_{f}$ expansion, a loop expansion around background fields, and closed time path formalism.

The efforts to take account of quantum effects in dynamical simulations of many-body systems have progressed significantly. Building on earlier work, the quantum fluctuations inherent in wave-packet dynamics have been included into molecular dynamics by means of a quantal Langevin force. The extended description leads to a considerably improved reproduction of the observed intermediate-mass fragment yields for nuclear collisions. The method is quite general and can be employed in other areas of physics as well, for example for atomic clusters where it affects the critical properties of noble gases significantly.

Studies of nuclear dynamics with effective one-body transport theories have recently included the first realistic test application to the multifragmentation of expanding gold nuclei. 


\section{Nuclear Astrophysics}

Gravity binds nucleons in a neutron star an order of magnitude more strongly than the strong force binds them in nuclei. The Pauli principle is therefore brought strongly into play in distribution the baryon number over many species of baryons and quarks. Suggested observational consequences have so far had little specificity (like cooling for which many different scenarios all fall into only several categories). Several avenues have recently been pursued: formation of low-mass black holes triggered by hyperonization or conversion of the core to quark matter; crystalline structure in the mixed confined-deconfined phase and possible effects on pulsar glitches; and structural changes (such as size and moment of inertia) which will reflect themselves in the time structure of pulsar spin down. Such changes are expected because the density profile changes with angular velocity and centrifugal force, ushering in thresholds for the population of new baryonic species. With each threshold the equation of state will be softened. Therefore the transformations will be reflected in the time-dependence of the pulsar rotation. In particular the signal of a first order phase transition is strongly registered in the braking index of pulsars, a measurable quantity. It is estimated that the signal will be present in about ten of the presently know pulsars if the phase transition does take place.

\section{Macroscopic Nuclear Properties}

The long-range aim of this work is to develop a quantitative understanding of the macroscopic properties of nuclei (binding energy, surface energy, density, compressibility, neutron matter, etc.). Using the semi-classical Thomas-Fermi method, we have recently achieved such a description, which yields the binding energy (or mass) of a nucleus as a function of $N, Z$ and the nuclear shape. The model is currently being generalizing to include angular momentum as well. An approximate description of ground-state, super-deformed and fission-isomeric rotational bands, and of the associated moments of inertia has been achieved.

\section{Transition from order to chaos}

The development of a macroscopic theory of nuclear dynamics continues based on the parallel between a transition from ordered to chaotic nucleonic motions and the transition from an elastic to a dissipative collective nuclear response. Recent work concerns a comprehensive three-way comparison between classical and quantal computer simulations and the Wall Formula for nuclear dissipation, as applied to the excitation of independent particles in a time-dependent potential well rippled at a wide range of frequencies. Four of the most important corrections to the basic Wall Formula have been included. The results are being applied to a renewed comparison of theory and experiments on fission and nucleus-nucleus collisions. 


\section{Nuclear Data Evaluation Program: Isotopes Project}

\section{J.M. Dairiki}

The Isotopes Project compiles, evaluates, and disseminates nuclear structure and radioactive decay data for basic and applied research. The group coordinates its activities with both the national and international data networks and, in particular, plays a major leadership role in the U.S. Nuclear Data Network (USNDN). The traditional strong data evaluation effort of the group continues and includes a pioneering role in horizontal data evaluations. The Isotopes Project has had a seminal role in modernizing the current nuclear data information system and has become a lead data center for the development of new electronic dissemination and publication methods for nuclear data. Significant and rapid progress is being made in exploiting the capabilities of the World Wide Web.

This annual report period has been particularly productive for the group with the completion of several major projects, many of them in collaboration with researchers from other institutions.

- Publication of the 8th edition of the Table of Isotopes, both on CD-ROM and in hard copy, April 1996

- Production and release of the Nuclear Data and References CD-ROM, June 1996-LBNL, Lund University, BNL collaboration

- Publication of the 2nd edition of the Table of Superdeformed Nuclear Bands and Fission Isomers —LBNL, McMaster University collaboration 
- Release of version 1.0 of VuENSDF software (subsequently renamed Isotope Explorer), July 1996-LBNL, Lund University collaboration

- Internet access of nuclear data

\section{Data Evaluation}

New research priorities present special opportunities and challenges for the nuclear data program. Three particular areas of opportunity are the needs of the high-spin research community, where new detector arrays such as Gammasphere and Eurogam are producing data at tremendous rates; nuclear astrophysics, where there is a stated need for a central point for the collection and dissemination of laboratory and theoretical nuclear data of direct relevance to nuclear astrophysics; and the needs of both the basic and applied research communities for critically evaluated decay data standards. In addition, a joint proposal to the U.S.-Hungary Science and Technology Program by G. Molnar (Institute of Isotopes, Budapest) and the Isotopes Project has been funded. This provides resources for the horizontal evaluation of capture gamma-ray data. The Isotopes Project has initiated evaluation efforts in these new areas while also continuing its traditional mass-chain evaluations.

\section{High-Spin Data Evaluation}

Following a recommendation at the May 1994 international data network meeting, some of the group's effort has been directed towards the evaluation of high-spin data. B. Singh has coordinated all the U.S. Nuclear Data Network's effort in this field, with evaluators from LBNL, Lund University, McMaster University, BNL, and ORNL. Procedures and guidelines for evaluating high-spin data were developed and communicated to the network. Lists of nuclides of high priority are updated and distributed regularly. Since July 1994, isotope-ordered lists of current nuclear structure experimental papers for both high- and low-spin studies published in ten main journals are prepared by B. Singh and made available (quarterly) to the research community and evaluators via the WWW. Experimental data from many of these recent papers are also being made available via the WWW. This work is ongoing and has gained even more importance since budgetary constraints would not permit hire of an anticipated high-spin data coordinator.

In collaboration with McMaster University, the Isotopes Project has selectively updated data for nuclei in the superdeformed regions with mass numbers $A=81-$ 84, 130-137, 142-154, and 189-198, entered them into the comprehensive Evaluated Nuclear Structure Data File (ENSDF), and published them in the Table of Superdeformed Nuclear Bands and Fission Isomers. These data are updated continuously and are now available via WWW.

The Isotopes Project has compiled/evaluated high-spin data for nuclides with $\mathrm{A}=135,153,163,182,186,188,190,193,194$, and 211 for inclusion in ENSDF. In addition, high-spin data for about 140 isotopes were updated for the Table of 
Isotopes. These data are available on the Isotopes Project webserver and through the National Nuclear Data Center (NNDC) at BNL.

\section{Decay Data Evaluation}

In accordance with another recommendation from the 1994 international meeting, E. Browne (LBNL) and R. Helmer (INEL) have organized an effort to selectively update decay data for radionuclides important for applied research and detector calibration. In collaboration with colleagues at the NNDC and in France (LPRI), Germany (PTB) and the United Kingdom (Imperial College), they prepared a list of about 250 radionuclides in this area. Evaluation of decay data for these nuclides is now underway by the collaboration; 11 completed evaluations are being circulated for review. LBNL has completed 4 decay evaluations- ${ }^{75} \mathrm{Se},{ }^{194} \mathrm{Ir},{ }^{188} \mathrm{Re}$, and ${ }^{192} \mathrm{Ir}$ - as part of this effort. Significant effort was also devoted to developing the guidelines and computer codes necessary to assure consistent evaluation methods and standards. All evaluations will be included in ENSDF and will be submitted to the IAEA for inclusion in its decaydata database.

\section{Nuclear Astrophysics Data Evaluation}

At a nuclear astrophysics data workshop held at Caltech in late 1996, a Steering Committee, chaired by Peter Parker (Yale), was formed and charged with ascertaining and prioritizing the data needs of the nuclear astrophysics community. The Steering Committee submitted a "white paper" to DOE in July 1996, calling for a central location for nuclear astrophysics data where evaluated data of diverse types are brought together and made available in a form that facilitates their dissemination and maximizes their utility. LBNL is submitting a proposal (jointly with the Woosley group at U.C. Santa Cruz) to hire a data coordinator and establish such a center at LBNL. Already, in collaboration with U.C. Santa Cruz and S.C. Wu, National Tsing Hua University (Taiwan), LBNL has developed a prototype nuclear astrophysics home page on the WWW (see separate contribution to this report) and several of the existing important nuclear astrophysics databases are now available on-line for the first time. This website is currently accessed over 500 times per month.

\section{Mass Chain Evaluations}

The Isotopes Project has permanent responsibility for evaluating 43 mass chains with $A=81,83,89-93,167-194,206,210-212,215,219,223$, and 227, and for adapting evaluated data with $A=23-26$, and $33-44$ into the ENSDF format. The group has also accepted temporary responsibility for evaluating mass chains with $A=59,76,79$, and 80, originally assigned to other centers. Since May 1994 nine mass-chain evaluations ( $A=81,93,170,172,179,182,186,191$ and 194) were completed and submitted for publication, five mass-chain evaluations were reviewed, and thirteen mass chains $(A=76,81,168,170,172,173,178,179,180$, $182,185,191$, and 194) were published in Nuclear Data Sheets. 


\title{
Data Dissemination
}

\author{
World Wide Web
}

The evolution of the Internet and, particularly of the World Wide Web, has changed the paradigm for nuclear data dissemination. Information can be provided easily in a variety of ways. During the past two years, the Isotopes Project has made significant and rapid progress in exploiting the capabilities of the Internet.

Linked home pages for access to data from ENSDF and the Nuclear Science References (NSR) databases as well as the Table of Isotopes, high-spin data, nuclear astrophysics data and nuclear masses have been developed. Data are provided in a variety of formats including text, Postscript, and Portable Document Format. Links have also been provided to databases residing at other data centers. Usage of these home pages has increased dramatically since their introduction in April, 1995. Currently about 2500 users log in each month. In 1996 these users made as many as 38,000 link requests per month. The rate of access to each of the home pages is continuing to climb and is summarized in a separate contribution to this report.

\section{Isotope Explorer (VuENSDF)}

Version 1.0 of VuENSDF, developed by a collaborative effort between the Isotopes Project and Lund University, was released in July 1996. VuENSDF is a Windows $\mathrm{C}++$ application designed to retrieve ENSDF and NSR information directly over the Internet from a server, from the Table of Isotopes CD-ROM, or from a local disk file. It displays level scheme drawings, tabular listings of nuclear levels and transitions, and NSR references. Data can be displayed by nuclear band structure and selected by coincidence relationships. Tables can be constructed according to user specifications, readily sorted by any numerical field, and output as tab-delineated records for input into other user codes. VuENSDF can search the NSR file by keynumber or author and display the keyword abstracts. Tables and drawings may be printed, and a print preview option is offered. VuENSDF can be downloaded from the Web. Running VuENSDF directly provides users with Internet access to nuclear data without requiring a WWW browser, or the program can be used as a helper application with a commercial browser.

In 1996 development began on Version 2.0 of this software which was renamed the Isotope Explorer. The new name reflects the much greater capabilities of the new version. New features include a nuclear chart interface which can access the ENSDF file directly to display level schemes or tables. It can also search for level or transition data, filter and retrieve the data, generate functional relationships between data, and display the results on the chart. Isotope Explorer also includes a new plot display mode to graph the relationships between selected variables. New reference search capabilities are being built into the program so that keyword searches of the NSR file can be performed. More details are available in a separate contribution to this report. 


\section{Table of Isotopes, 8th Edition}

The 8th edition of the Table of Isotopes was published by John Wiley \& Sons in April 1996. The 3168-page, 2-volume book is packaged with an interactive CDROM that contains the Table of Isotopes in Adobe Acrobat PDF format for viewing on PC and Macintosh personal computers, and on UNIX workstations. The CD-ROM version contains a chart of the nuclides graphical index and separate indices organized for radioisotope users and nuclear structure physicists. Future editions of the Table of Isotopes will be provided on CD-ROM and also over the Internet (see separate contribution for more details).

\section{Nuclear Data and References CD-ROM}

In 1994 the Lund-LBNL collaboration released Papyrus NSR, a CD-ROM containing the NSR file and the PAPYRUS bibliographic database management system, to facilitate literature searches on PCs. In June 1996, a new CD-ROM, Nuclear Data and References, was released by a Lund/LBNL/BNL collaboration. This CD-ROM, which updates its predecessor (PAPYRUS NSR) includes:

The Nuclear Science References (NSR) database, with over 140,000 references, updated to December 8, 1995. Retrieval of information can be done using the PAPYRUS bibliographic database management system, the same software that was used for the 1994 release.

NuDat, a numerical database of nuclear properties, accessed and managed by PCNuDat (software developed at BNL). This database includes data from ENSDF, the Nuclear Wallet Cards, the 1995 update to the Atomic Mass Evaluation, and from Neutron Cross Sections, Vol. 1 and 2.

\section{Table of Superdeformed Nuclear Bands and Fission Isomers}

The Table of Superdeformed Nuclear Bands and Fission Isomers, first published as an LBNL report in October, 1994, has been updated and published in Nuclear Data Sheets. This publication contains adopted level data for all nuclei with superdeformed bands, moment of inertia plots and level scheme drawings for all superdeformed bands, and related plots for actinide shape (fission) isomers. In addition, a complete reference list is provided for experimental and theoretical superdeformation studies and for fission isomers. Preprints of the 2 nd edition were distributed at the Gammasphere Dedication Workshop held in Berkeley on December 1-2, 1995. This publication, prepared in collaboration with McMaster University, is updated continuously and made available on the Internet (see separate contribution to this report). 


\section{Summary}

The Isotopes Project will continue its leading role in data evaluation and dissemination. Continued development of Internet data dissemination will be a major activity. The goal is to distribute data to the research community using convenient and effective formats, so that the information can be utilized for the advancement of science and technology. The biggest challenge for nuclear data will be to continue to provide quality, up-to-date information. In order to do this, we will work closely with the research community. 


\section{8-Inch Cyclotron Operations}

\section{C.M. Lyneis}

The 88-Inch Cyclotron is a versatile and reliable accelerator of beams from hydrogen to uranium. It is operated by Lawrence Berkeley National Laboratory (LBNL) as a national facility in support of the U.S. Department of Energy programs in nuclear science. Forefront scientific research in nuclear structure, heavy elements, proton-rich nuclei, nuclear astrophysics, fundamental symmetries, and reaction mechanisms is carried out. During FY96, a total of 313 users took part in experiments utilizing 4,942 hours of beam on target. They came from 20 universities, 3 other DOE National Laboratories, 25 foreign institutions and 2 other laboratories. There were 161 scientists and 61 students participating in nuclear science experiments and 88 scientist and engineers participating in non-nuclear science experiments. The Cyclotron also provides beams for the application of nuclear techniques to other areas of research, including biology and medicine and industrial applications. Industrial partners from aerospace and semiconductor corporations, as well as from NASA, DOE, and DOD laboratories, use beams from the Cyclotron to study the interaction of ions in microcircuits, simulating the cosmic-ray environment in space.

The 88-Inch Cyclotron is the site of Gammasphere, a high-resolution gammaray detector array that is a major initiative of the nuclear structure research community. It consists of an array of 110 large Compton-suppressed germanium detectors which make it the most powerful such array in the world. 


\section{Accelerator Use}

Operation of the 88-Inch Cyclotron was at seven days/week in FY96 and will continue at that level through FY97. The Accelerator Operation Summary (Table 1) shows that $80 \%$ of the scheduled time was used for research (beam on target) while the remaining time was divided between tuning $(11 \%)$, machine studies $(3 \%)$, and unscheduled maintenance $(6 \%)$. Nuclear research accounted for 3831 research hours, applied research for 910 hours, biology for 72 hours and high energy physics for 110 hours. The applied research-in partnership with the aerospace industry, NASA, DOE, and DOD laboratories-consisted of two parts: (1) testing of microelectronics by using cyclotron beams to simulate space radiation, and (2) calibrating detectors for use in space flights. The biology research was done primarily in support of the NASA NSCORT program.

\section{Ions, Energies, and Intensities}

The cyclotron fed by the ECR sources provides a wide range of ions, energies, and intensities in support of the experimental program at the 88-Inch Cyclotron. Using the low and high temperature ovens in the ECR sources, most elements can be accelerated. To date 41 elements have been accelerated including every element from hydrogen to zinc. The beams which have been accelerated are listed in Table 2. The isotopes which are run from natural feeds are listed in parentheses. In addition, many ions have been run using isotopically enriched source materials including ${ }^{3} \mathrm{He},{ }^{13} \mathrm{C},{ }^{15} \mathrm{~N},{ }^{18} \mathrm{O},{ }^{21,22} \mathrm{Ne},{ }^{33,34,36} \mathrm{~S},{ }^{44,48} \mathrm{Ca},{ }^{64} \mathrm{Ni},{ }^{70} \mathrm{Ge}$, ${ }^{78,86} \mathrm{Kr},{ }^{136} \mathrm{Xe}$, and ${ }^{154} \mathrm{Sm}$. Two of the new beams, ${ }^{64} \mathrm{Zn}$ and ${ }^{54} \mathrm{Cr}$, developed were both for outside users utilizing Gammasphere. The ${ }^{54} \mathrm{Cr}$ was produced from natural chromium at the required intensity even though its isotopic abundance is only $2.4 \%$. This illustrates the combined power of the ECR ion source and the excellent transmission from ion source to experimenter's target. A $780 \mathrm{MeV}{ }^{162} \mathrm{Dy}$ beam was produced for the study of multinucleon transfer reactions between heavy nuclei, marking the heaviest beam used by Gammasphere to date. We also improved the efficiency of producing ${ }^{36} \mathrm{~S}$ beams from the AECR by reducing handling losses which occur when using the isotopically enriched $\mathrm{CS}_{2}$.

The variety of beams, energies and intensities described in Table 2 has been developed in support of the research programs. Heavy element radiochemistry experiments require intense (several $\mathrm{e} \mu \mathrm{A}$ ) heavy-ion beams up to mass 48 at 6-8 $\mathrm{MeV} /$ nucleon. Groups studying heavy-ion reaction mechanisms and complex fragmentation of highly excited nuclei use higher energy beams such as nitrogen and oxygen at $32 \mathrm{MeV} /$ nucleon, neon at $25 \mathrm{MeV} /$ nucleon, and krypton at 13 $\mathrm{MeV} /$ nucleon as well as light-ion beams of ${ }^{3} \mathrm{He}$ and ${ }^{4} \mathrm{He}$ over a wide energy range. Light-ion beams are also frequently utilized by other groups. For example, the laser trapping of radioactive beams uses $25 \mathrm{MeV}$ proton beams to produce the ${ }^{21} \mathrm{Na}$. The study of $\beta$-delayed proton emission requires several e $\mu \mathrm{A}$ beams of ${ }^{3} \mathrm{He}$ at 40 to $110 \mathrm{MeV}$. The nuclear astrophysics group typically uses beams of protons, deuterons, ${ }^{3} \mathrm{He}$ and ${ }^{4} \mathrm{He}$ at $8-25 \mathrm{MeV} /$ nucleon. 


\section{ECR Ion Source Development}

Development of ECR ion sources in FY96 included completion of the AECR upgrade project and the design and construction of a prototype magnet for a very high performance Third Generation ECR ion source, both described below.

The AECR source was upgraded by increasing its magnetic fields to improve its plasma confinement and thereby enhancing the source performance. After a few months of tailoring the magnetic field configuration to match the twofrequency plasma heating ( 14 and $10 \mathrm{GHz}$ ), the upgraded AECR source (AECR$\mathrm{U})$ with its higher magnetic fields and higher magnetic mirror ratios has demonstrated significantly enhanced performance. For heavy ions at intensity of about $1 \mathrm{e} \mu \mathrm{A}$, the charge state was shifted from $42+$ to $48+$ for the heaviest natural element-uranium, and from $41+$ to $46+$ for bismuth. An order of magnitude enhancement for fully stripped argon ions ( $\mathrm{I} \geq 60 \mathrm{e} \mu \mathrm{A}$ ) also has been achieved. High charge state heavy-ion beams of xenon- 136 and uranium-238 produced by the source were injected into the 88-Inch Cyclotron. After acceleration to energies greater than $10 \mathrm{MeV} /$ nucleon for xenon and $6 \mathrm{MeV} /$ nucleon for uranium, the extracted beam intensities from the cyclotron are $1 \times 10^{7}$ for xenon $41+$ and a few hundreds pps of xenon 46+. Even with very low transmission for the highly charged heavy ions due to beam loss from charge exchange with residual gas in the cyclotron, uranium ions with charge states up to $60+$ were confirmed with a crystal detector. A few particles per second at a total energy of $1.935 \mathrm{GeV}$, the highest beam energy ever produced by the 88 -inch cyclotron, were measured.

A new very high magnetic field superconducting ECR ion source, the Third Generation ECR is under development at the Cyclotron. It will boost the maximum energies and intensities for heavy ions from the cyclotron. Recent progress on ECR ion sources indicates that significantly higher performance can be obtained by incorporating three key ECR source developments. First, the new ECR source will utilize superconducting magnets so that very high mirror ratios can be used to improve the plasma confinement. Second, multiple frequency heating will be used to provide enhanced electron heating and better plasma stability. Finally, the surfaces of plasma chamber walls will have high secondary emission coefficient such as obtained with $\mathrm{Al}_{2} \mathrm{O}_{3}$ which will reduce the operating pressure and subsequent loss of high charge states due to charge exchange.

A prototype superconducting magnet structure including three solenoid coils and six race track coils was developed as an FY96 LDRD project. Its design calls for axial field strength of more than 3 Tesla and a radial field strength of more than 2 Tesla. Sufficient superconducting wire from surplus SSC wire was obtained and has been used to fabricate the solenoid and sextupole coils of the prototype magnet structure which will be tested in April 1997 to determine its training characteristics, field strength and quality. Design of the iron yoke, cryostat, plasma chamber, extraction system of the new source are underway with a goal of operation in two years. In FY98, construction and installation of all the main components including the plasma chamber, extraction system, the vacuum system, helium refrigerator, and high voltage system is planned. During 
the final phase of the project the RF system required to operate the source with multiple frequencies would be procured and installed.

\section{Other Accelerator Improvement Projects (AIP) at the 88-Inch Cyclotron}

The Vacuum Upgrade project is close to completion. The $20^{\circ} \mathrm{K}$ cryopanel and the $77^{\circ} \mathrm{K}$ shield have been completed and the shield has been installed and the CTI-1400 refrigeration system from the Bevalac has been installed and tested. The interconnecting piping between the 2 CTI-1400's is complete. The only remaining task is connecting the helium lines between the refrigerators and the new cryopanel

All of the diagnostic components for the Phase Width Reduction project are installed and working including the two fast Faraday Cups with fast Heliax cables to the cyclotron control room. The multi-turn collimator has been upgraded and reinstalled, a fast oscilloscope has been purchased and a fast scintillator system is in operation as a cross check for the fast Faraday Cup signals. A series of beam development runs demonstrated that phase width can be reduced to 1 to $2 \mathrm{~ns}$ with the system.

Construction of a redundant personnel radiation safety system is complete. The Data Highway, remote I/O system are complete and software for the Process Logic Controller and Lab View for control and monitoring are ready. The wiring to the Vault and experimental cave is complete. Final connection to the micro switches on the cave doors and the beam plugs will be done in the next month followed by system commissioning.

\section{User Support}

The Research Coordination Group provides information, assistance, and coordination to users of the 88-Inch Cyclotron. It is the main contact between the Cyclotron operations staff and outside users. As such, the group is responsible for developing and maintaining experimental facilities at the Cyclotron, and for making these facilities attractive to a diverse group of users from around the country and, in some cases, from around the world.

Our users fall into two classes: (1) scientific users whose experimental proposals are reviewed by a Program Advisory Committee (PAC) and who are awarded time based on the scientific merits of their proposals (or who are awarded discretionary time by the Cyclotron Head), and (2) industrial users who purchase beam time for their own proprietary use.

The Research Coordination Group coordinates the PAC, which meets twothree times a year to review proposals for beam time, and schedules beam time. It sponsors an annual users' meeting at the fall meeting of the Division of Nuclear Physics of the American Physical Society. It supports the Gammasphere Users' Executive Committee through surface mailings and E-mail venues. It 
provides information to users of the 88-Inch Cyclotron as well as the general public through the World Wide Web, brochures, and newsletters.

The Research Coordination Group also maintains the electronics module pool and several data acquisition and analysis computers. Upgrade and modernization of the data acquisition system has been occurring regularly over the course of the last several years.

\section{Non-Nuclear Science Research}

The 88-Inch Cyclotron is a major source of heavy-ion beams for Single-Event Effect (SEE) testing of solid-state components for the U.S. space program. Because of the ability to run "cocktails" of beams, enabling switches from one ion to another in a matter of minutes, it is possible to quickly establish the energy deposition level at which a SEE will occur. The availability of proton beams, used for studying radiation effects on charge-coupled devices, has further increased the demand for use of the Cyclotron.

The Aerospace Corporation, in cooperation with 88-Inch Operations, has installed a specially instrumented scattering chamber on a dedicated beam line in Cave $4 \mathrm{~b}$. A trailer has been installed above Caves $4 \mathrm{a}$ and $4 \mathrm{~b}$ to serve as a counting area for applied programs in those caves. A thin scintillating film transmission detector has been built to measure the beam flux and a beam energy measurement system was adapted from the SuperHILAC for use in the Cyclotron beam lines. These improvements have enabled us to support a large number of small companies and projects who otherwise would not be able to use the Cyclotron.

The $60^{\prime \prime}$ chamber in Cave 4a was modified with parts from the sister chamber at the Bevalac in order to install larger diameter beam pipe, allowing the irradiation of parts up to $6^{\prime \prime}$ diameter. A sample changer was built to sit in the chamber which can irradiate up to 35 4-6" samples without breaking vacuum. Dosimetry was installed to measure the beam flux and uniformity with scintillating fiber detectors. Using this setup, Si substrates are irradiated for eventual use as flat-panel displays.

The radiation biology program continues to use a small amount of beam time annually. The effect of radiation on cells is studied through the use of highenergy protons, helium, and nitrogen. The Irradiation Station built for the biomed studies has also been used extensively by the high energy physics community to study radiation damage in parts being designed for the Atlas detector at the LHC at CERN. 
Table 1. FY96 88-Inch Cyclotron Operating Statistics

Accelerator Operation Summary

Research

Tuning

4942 (hours)

Machine Studies

694

Unscheduled Shutdowns

158

Scheduled Shutdowns

353

2637

Electrical Energy Consumption (GWH)

Cost of Electrical Energy

(Thousands of Dollars)

Financial Support for Accelerator Facility

Operation (thousands of Dollars)

Heavy Ion Physics (KB-02-02)

3880

Biomedical and Environmental Research

Other Sources

Total

Experiment Summary

Beam Utilization for Research

Nuclear Research

Atomic Physics

3831 (hours)

Biology and Medicine

High Energy Physics

110

Other Research

Total

Nuclear Science Research

Universities

Other DOE National Laboratories

Foreign Institutions

Other labs

Number of Experiments

Number of Scientists Participating

Number of Students

Non-nuclear Science Research

Institutions and Companies

Number of scientists and engineers

Total users (all research)

Percentage of Beam Time (all research)

In-House Staff

$40 \%$

Universities

$17 \%$

DOE National Laboratories

$12 \%$

Companies

$5 \%$

Foreign Institutions

$11 \%$

Other government labs

$15 \%$

Total

$100 \%$ 
Table 2. 88-Inch Cyclotron Beam List

\begin{tabular}{|c|c|c|c|}
\hline Ion $^{a}$ & $\begin{array}{c}\text { High } \\
\text { Energy }{ }^{b} \\
(\mathrm{MeV} / \mathrm{u})\end{array}$ & $\begin{array}{c}\text { Typical Current } \\
\text { @ High Energy } \\
(\mathrm{e} \mu \mathrm{A})\end{array}$ & $\begin{array}{c}\text { Typical Current } \\
@ \approx 5-6 \mathrm{MeV} / \mathrm{u} \\
(\mathrm{e} \mu \mathrm{A})\end{array}$ \\
\hline $\mathrm{p}$ & 55 & 6. & 20. \\
\hline p (pol.) & 50 & 0.4 & 0.7 \\
\hline $\mathrm{d}$ & 32 & 0.7 & 20. \\
\hline $\mathrm{d}$ (pol.) & 32 & 0.2 & 0.7 \\
\hline${ }^{3} \mathrm{He}$ & 45 & 10. & 10. \\
\hline${ }^{4} \mathrm{He}$ & 32 & 2. & 8. \\
\hline${ }^{7} \mathrm{Li}$ & 23 & 0.4 & 1. \\
\hline${ }^{9} \mathrm{Be}$ & 25 & 0.15 & 1. \\
\hline${ }^{11} \mathrm{~B}$ & 26 & 0.05 & 1. \\
\hline${ }^{12} \mathrm{C}$ & 32 & 0.01 & 12. \\
\hline${ }^{14} \mathrm{~N}$ & 32 & 0.03 & 5. \\
\hline $16 \mathrm{O}$ & 32 & 2. & 20. \\
\hline${ }^{19} \mathrm{~F}$ & 24 & 0.1 & 3. \\
\hline${ }^{20} \mathrm{Ne}(22)$ & 27 & 0.1 & 4. \\
\hline${ }^{23} \mathrm{Na}$ & 25 & 0.01 & 0.07 \\
\hline${ }^{24} \mathrm{Mg}(25,26)$ & 23 & 0.7 & 3. \\
\hline${ }^{27} \mathrm{Al}$ & 23 & 0.07 & 4.5 \\
\hline${ }^{28} \mathrm{Si}(29,30)$ & 22 & 0.1 & 2. \\
\hline${ }^{31} \mathrm{P}$ & 22 & 0.25 & 2 \\
\hline${ }^{32} \mathrm{~S}(34)$ & 22 & 0.2 & 4. \\
\hline${ }^{35} \mathrm{Cl}(37)$ & 24 & 0.01 & 5. \\
\hline${ }^{40} \mathrm{Ar}$ & 23 & 0.04 & 23. \\
\hline${ }^{39} \mathrm{~K}$ & 22 & 0.03 & 1.8 \\
\hline${ }^{40} \mathrm{Ca}$ & 23 & 0.04 & 2.5 \\
\hline${ }^{45} \mathrm{Sc}$ & 20 & 0.023 & 0.22 \\
\hline${ }^{48} \mathrm{Ti}$ & 20 & 0.03 & 0.6 \\
\hline${ }^{51} \mathrm{~V}$ & 19 & 0.03 & 0.4 \\
\hline${ }^{52} \mathrm{Cr}$ & 18 & 0.03 & 0.6 \\
\hline${ }^{55} \mathrm{Mn}$ & 18 & 0.03 & 0.3 \\
\hline${ }^{56} \mathrm{Fe}(54)$ & 18 & 0.03 & 0.5 \\
\hline${ }^{58} \mathrm{Ni}$ & 18 & 0.02 & 0.3 \\
\hline${ }^{59} \mathrm{Co}$ & 17 & 0.006 & 0.2 \\
\hline${ }^{63} \mathrm{Cu}(65)$ & 17 & 0.04 & 0.4 \\
\hline
\end{tabular}


Table 2. Continued

\begin{tabular}{lccc}
\hline & $\begin{array}{c}\text { High } \\
\text { Energy } \\
(\mathrm{MeV} / \mathrm{u})\end{array}$ & $\begin{array}{c}\text { Typical Current } \\
\text { @ High Energy } \\
(\mathrm{e} \mu \mathrm{A})\end{array}$ & $\begin{array}{c}\text { Typical Current } \\
@ \approx 5-6 \mathrm{MeV} / \mathrm{u} \\
(\mathrm{e} \mu \mathrm{A})\end{array}$ \\
\hline${ }^{64} \mathrm{Zn}$ & 16 & 0.02 & 0.2 \\
${ }^{84} \mathrm{Kr}(78,82,86)$ & 14 & 0.005 & $6 . \mathrm{b}$ \\
${ }^{107} \mathrm{Ag}$ & 11 & 0.007 & 0.2 \\
${ }^{120} \mathrm{Sn}(118)$ & 9 & 0.002 & 0.1 \\
${ }^{132} \mathrm{Xe}(129,131,136)$ & 8 & 0.005 & $0.3^{\mathrm{c}}$ \\
${ }^{139} \mathrm{La}$ & 8 & 0.002 & 0.03 \\
${ }^{154} \mathrm{Sm}$ & 7 & 0.07 & $0.07^{\mathrm{b}}$ \\
${ }^{159} \mathrm{~Tb}$ & 7 & 0.006 & 0.006 \\
${ }^{162} \mathrm{Dy}$ & 7 & .07 & $.07^{\mathrm{b}}$ \\
${ }^{209} \mathrm{Bi}$ & 5 & 0.005 & 0.005 \\
${ }^{238} \mathrm{U}$ & 4 & 0.001 & \\
\hline
\end{tabular}

a) Most abundant isotope is listed. Other isotopes run from natural feed are shown in parentheses. Their intensities are proportional to the isotopic abundance, and their high energy values proportional to $1 / \mathrm{A}^{2}$.

b) Current for isotopically enriched sample.

c) Higher energies beams are available at lower intensities for $M>16$.

The following ions have been run at the 88-Inch Cyclotron using isotopically enriched source material. The intensity is proportional to enrichment factor and currents for the same element in the table:

$$
{ }^{13} \mathrm{C},{ }^{15} \mathrm{~N},{ }^{18} \mathrm{O},{ }^{21,22} \mathrm{Ne}, 33,34,36 \mathrm{~S},{ }^{36} \mathrm{Ar},{ }^{44,48} \mathrm{Ca},{ }^{64} \mathrm{Ni},{ }^{70} \mathrm{Ge},{ }^{78,86} \mathrm{Kr},{ }^{136} \mathrm{Xe}
$$





\section{5-96 Group Lists}

Following are the names of people in the Nuclear Science Division groups. The long-term visitors are included with their home institutions in parenthesis. [Group leaders are in bold face, ${ }^{*}=$ Graduate student, ${ }^{* *}=$ Undergraduate student.]

\author{
Administrative Staff \\ Yvette C. Clinton** \\ Janis M. Dairiki, Deputy \\ Erika Decool \\ Denise Diaz \\ Joy B. Lofdahl \\ Meredith Montgomery, Div. Admin. \\ Barbara E. Phillips \\ Lee S. Schroeder, Division Director \\ Jeanette Shereda \\ Wanda J. Smith-Burnett \\ Jane D. Toby \\ Catherine J. Sterling \\ Sherrill Whyte
}

\section{For Glenn T. Seaborg, \\ Associate Director at Large: \\ Kristin Balder-Froid \\ Carole P. Logie \\ Rachel A. Starbuck \\ Dan L. Tieu}

\section{Crystal Barrel}

David S. Armstrong

Thomas A. Case

Kenneth M. Crowe (Retired Staff)

Fritz H. Heinsius (UC Berkeley)

Peter Kammel (UCB \& Austrian Academy of Sciences)

Mark T. Lakata*

Leteka S. Thomas** (UC Berkeley)

\section{Exotic Nuclei}

Jon C. Batchelder

Joseph Cerny

Dennis M. Moltz

Theodore J. Ognibene*

James D. Powell

Michael Rowe*

\section{Heavy Element Nuclear and Radiochemistry \\ Yong H. Chung (Hallym) \\ Albert Ghiorso (Retired Staff) \\ Kenneth E. Gregorich}

Melissa B. Hendricks*

Darleane C. Hoffman (Retired Staff)

Chris D. Kacher*

Michael R. Lane*

Carola Laue (Feodor Lynen Fellow)

Diana M. Lee

Walter D. Loveland (Oregon State)

Christopher A. McGrath

Victor Ninov

J. Michael Nitschke

Tsutomu Otsuki (Tohoku)

Glenn T. Seaborg

Dawn A. Shaughnessy*

Nancy J. Stoyer

Dan A. Strellis*

Eric R. Sylwester*

Alan C. Veeck*

Jerry C. Yang** (UC Berkeley)

Yasuo Watanabe (Fuji Electric Corp.)

Richard J. Wallsgrove** (UC Berkeley)

Birgit Wierczinski

Philip A. Wilk*

Jeb L. Adams*

Christy J. Keoshian*

Nicole S. Wieland*

Isotopes Project

Coral M. Baglin

Edgardo Browne

S-Y. Frank Chu

Janis M. Dairiki

Peter Ekstrom (Lund)

Richard B. Firestone

Jacob Gilat (Soreq)

Henrik Nordberg** (Lund)

Virginia S. Shirley $\bullet$

Balraj Singh

S-C. Alice Wu (Nat'1 Tsing Hua Univ)

Jean H. Zipkin

\section{Low Background Counting}

Donna L. Hurley

Richard J. McDonald

Eric B. Norman

Alan R. Smith (Retired Staff) 
Neutrino Astrophysics Group

Yuen-Dat Chan

Lawrence H. Friedman* (UC Berkeley)

Maria C. Isaac

Kevin T. Lesko

Martin E. Moorhead

Anett Schuelke (DAAD Fellow)

Paul Sigismondi (Head-Royce H.S.)

Robert G. Stokstad

Nuclear Astrophysics

Michael R. Dragowsky* (Oregon State)

Daniel DiGregorio (TANDAR, Buenos Aires)

Iuda D. Goldman (Sao Paulo)

Munther Hindi (Tennessee Tech.)

Kenneth S. Krane (Oregon State)

Ruth-Mary Larimer (Retired Staff)

Eric B. Norman

Victor Noto (Mandeville H.S., LA)

Koorosh Zaerpoor* (Oregon State)

Nuclear and Astrophysics

Michael J. Bennett

Henry J. Crawford (UCSSL)

Mats Cronquist (UCSSL)

Jon M. Engelage (UCSSL)

Ismael Flores (UCSSL)

Leo C. Greiner (UCSSL)

Harry H. Heckman (Retired Staff)

Tchang F. Hoang

Eleanor G. Judd (UCSSL)

Sean A. Kelly (UCLA)

Peter J. Lindstrom (UCSSL)

Volker Lindenstruth (UCSSL)

Linda J. Miller (UCSSL)

Zoran Milosevich (CMU)

Ed Platner (Rice)

Gerard J. Visser (UCSSL)

James P. Whitfield (CMU)

Nucleus-Nucleus Collisions

Yudong $\mathrm{He}$

P. Buford Price

Austin A. Richards

Ben Weaver

\section{Nuclear Reactions}

Luc Beaulieu

Dimitry E. Breus

Roberta Ghetti* (Lund University)

Kexing Jing*

Luciano G. Moretto

Larry W. Phair

Fabrice Piron* (Univ. of Paris - Orsay)
Thorsten Rubehn

Kin Tso*

Gordon J. Wozniak

Nuclear Structure

Stephen J. Asztalos*

Brian C. Busse* (Oregon State)

Bo W. Cederwall

Roderick M. Clark

Marie-Agnes Deleplanque-Stephens

Richard M. Diamond (Retired Staff)

Paul Fallon

Patrick H.G.M. Hendriks** (Einohoven Technology)

Reiner Kruecken

I-Yang Lee

Augusto O. Macchiavelli

Randy W. MacLeod

Gregory J. Schmid

Frank S. Stephens

Kai Vetter

Stefan G. Frauendorf (Rossendorf)

Relativistic Nuclear Collisions

Aleksandr V. Alyushin (MEPhI)

Mikhail V. Alyushin (MEPhI)

Dieter Best

Wayne J. Betts* (UT Austin)

Fred S. Bieser

Roy Bossingham

Morgan T. Burks* (Arkansas)

Jim B. Carroll (UCLA)

Daniel A. Cebra (UC Davis)

A. Dean Chacon (Texas A\&M)

Michael G. Cherney (Creighton)

Glenn E. Cooper* (UC Berkeley)

Jan T. Chrin (Creighton)

Douglas E. Greiner (Retired Staff)

John W. Harris

Eric L. Hjort (Purdue)

Detlev H.W. Irmscher

Peter M. Jacobs

Susanna R. Jacobson

Marvin L. Justice (Kent State)

Jessoca Kintner

Spencer R. Klein

Anton Konashenok* (UC Berkeley)

Alexei Lebedev (MEPhI)

Michael A. Lisa (Ohio State)

Heng Liu* (Kent State)

Spyridon Margetis

Jay N. Marx

Howard S. Matis

Charles P. McParland

Thomas S. McShane (Creighton) 
John C. Meier ${ }^{*}$ (Creighton)

Grayzna Odyniec

Douglas L. Olson

Martin L. Partlan

R. Jefferson Porter

Arthur M. Poskanzer

Gulshan Rai

Fabrice G. Retiere**

Hans-Georg Ritter

Heinrich Rudolph

Iwona Sakrejda

Lee S. Schroeder

Nathan T.B. Stone

T. James M. Symons

James $\mathrm{H}$. Thomas

Milton Y. Toy* (UCLA)

Stephen Trentalange (UCLA)

Fuqiang Wang

Howard $\mathrm{H}$. Wieman

Thomas Wienold

John D. Wolf

Eugene B. Yee

\section{Special Projects}

Homer E. Conzett (Retired Staff)

Erwin M. Friedlander (Retired Staff)

Bernard G. Harvey (Retired Staff)

John O. Rasmussen (Retired Staff)

Mark A. Stoyer

\section{Subthreshold Kaons and}

Antiprotons

John S. Drewery

Selig N. Kaplan

Victor Perez-Mendez (Retired Staff)

\section{Theory}

Baha Benhassine (Nantes)

Jan Blocki (Swierk)

Sidney Bludman (Philadelphia)

Paul Bonche (Saclay)

Nicolae Carjan (Bordeaux)

Michael Christiansen (Aarhus)

Stefan Frauendorf (Rossendorf)

Norman Glendenning (Retired Staff)

Johan Helgesson (Lund)

Yuval Kluger

Volker Koch
Peter Levai (Budapest)

Ziwei Lin

Bill Myers (Retired Staff)

Akira Ohnishi (Sapporo)

Patrick Palou (Bordeaux)

Jorgen Randrup

Martin Redlich (Visitor)

Jürgen Schaffner

Janusz Skalski (Swierk)

Chungsik Song

Wladek Swiatecki (Retired Staff)

Robert Vinh Mau (Paris)

Ramona Vogt

Xin-Nian Wang

Fridolin Weber (Munich)

Xiaoming $\mathrm{Xu}$ (Shanghai)

Shin-Nan Yang (Taipei)

Jozsef Zimanyi (Budapest)

Weak Interactions

Christopher J. Bowers*

Stuart J. Freedman

Brain K. Fujikawa

Gerald Gwinner

Laura J. Lising*

Justin L. Mortara*

Mary A. Rowe*

Song-Quan Shang

Paul A. Vetter

Eric G. Wasserman

88-Inch Cyclotron Operations

David J. Clark (Retired Staff)

Robert C. Coates

Eduardo I. Diaz

Thomas L. Gimpel

Aran P. Guy

Abraham Katzanck

Ruth-Mary Larimer (Retired Staff)

Claude M. Lyneis

James R. Morel

Margaret A. McMahan Norris

William $\mathrm{H}$. Rathbun

Vicki L. Saling

Catherine R. Siero

$\mathrm{ZuQ}$. Xie

Deceased 




\section{Beta-Delayed Proton Decay of 27P}

\section{T. J. Ognibene, J. Powell, D. M. Moltz, M. W. Rowe, and Joseph Cerny}

The decays of the $A=4 n+3, T_{Z}=-3 / 2$ series nuclei, with their large beta-decay $Q$ values, offer an opportunity to study Gamow-Teller transition strengths up to high excitation energies. The known members of this series in the sd shell, ${ }^{23} \mathrm{Al},{ }^{27} \mathrm{P},{ }^{31} \mathrm{Cl}$ and ${ }^{35} \mathrm{~K}$, all exhibit beta-delayed proton branches. In 1995 we published a $\beta \mathrm{p}$ study of ${ }^{23} \mathrm{Al}$ utilizing our low threshold particle detector telescopes [1]. This work has now been extended to ${ }^{27} \mathrm{P}$ and ${ }^{31} \mathrm{Cl}$ [2].

The isotope ${ }^{27} \mathrm{P}\left(\mathrm{QEC}_{\mathrm{EC}}=11.6 \mathrm{MeV}\right)$ was produced via the ${ }^{28} \mathrm{Si}(\mathrm{p}, 2 \mathrm{n})$ reaction with 45 $\mathrm{MeV}$ protons from the $88^{\prime \prime}$ cyclotron impacting on a series of thin natural silicon targets. Radioactive product nuclei recoiled out of the target foils, were thermalized in helium and were swept away on potassium chloride aerosols. These aerosols were transported through a thin capillary and deposited on a collection spot in the detector station. The transport time in this setup was approximately $200 \mathrm{~ms}$, comparable to the ${ }^{27} \mathrm{P}$ halflife of $260 \pm 80 \mathrm{~ms}$. The collection point was located on a slowly rotating wheel to reduce the buildup of longer-lived radionuclides.

The deposition spot was observed by two low-energy particle detection telescopes, each consisting of two gas $\Delta \mathrm{E}$ detectors backed by a single $300 \mu \mathrm{m}$ silicon detector for measuring the particle energy. The thin $\Delta E^{\prime}$ 's allow the separation of protons from alpha and beta events. The use of two $\Delta \mathrm{E}$ 's further suppresses beta contamination of the identified proton spectrum, necessary in the high beta fluxes typical of helium-jet experiments. These gas detectors are thinner than existing silicon $\Delta E$ 's, leading to a lower detection threshold. In this experiment protons were detectable down to about $240 \mathrm{keV}$ with an energy resolution of approximately $30 \mathrm{keV}$. This improves on the best previous measurement of $27 \mathrm{P}$ [3] where the all-silicon detector telescopes had a low-energy threshold of $700 \mathrm{keV}$ and a resolution of $75 \mathrm{keV}$.

The measured proton spectrum after a 290 $\mathrm{mC}$ bombardment is shown in the accompanying figure. Four lines were identified as belonging to ${ }^{27} \mathrm{P}$, the lower two of which were not seen previously. A partial decay scheme is inset in the figure. Contamination from the betadelayed proton decay of ${ }^{28} \mathrm{P}$, produced via the ${ }^{28} \mathrm{Si}(\mathrm{p}, \mathrm{n})$ reaction, was studied through a separate bombardment at $E_{p}=28 \mathrm{MeV}$, which is below the ${ }^{27} \mathrm{P}$ production threshold. Energy and detection efficiency calibrations were based on the $28 \mathrm{P}$ transitions in addition to those from the well-known $\beta$ p emitter ${ }^{25} \mathrm{Si}$ which was produced via proton bombardment of an aluminum target. The total beta-delayed proton branch of ${ }^{27} \mathrm{P}$ was estimated to be $0.07 \%$.

The measured Gamow-Teller strengths were compared with predictions obtained from shell-model calculations. While a portion of the expected strength was positively identified, a more complete comparison awaits a $\beta \gamma$ measurement of the ${ }^{27} \mathrm{P}$ decay below the proton threshold.

1. R. J. Tighe et al., Phys. Rev. C52 (1995) R2298.

2. T. J. Ognibene et al., Phys. Rev. C54 (1996) 1098. 3. J. Äystö et al., Phys. Rev. C32 (1985) 1700.

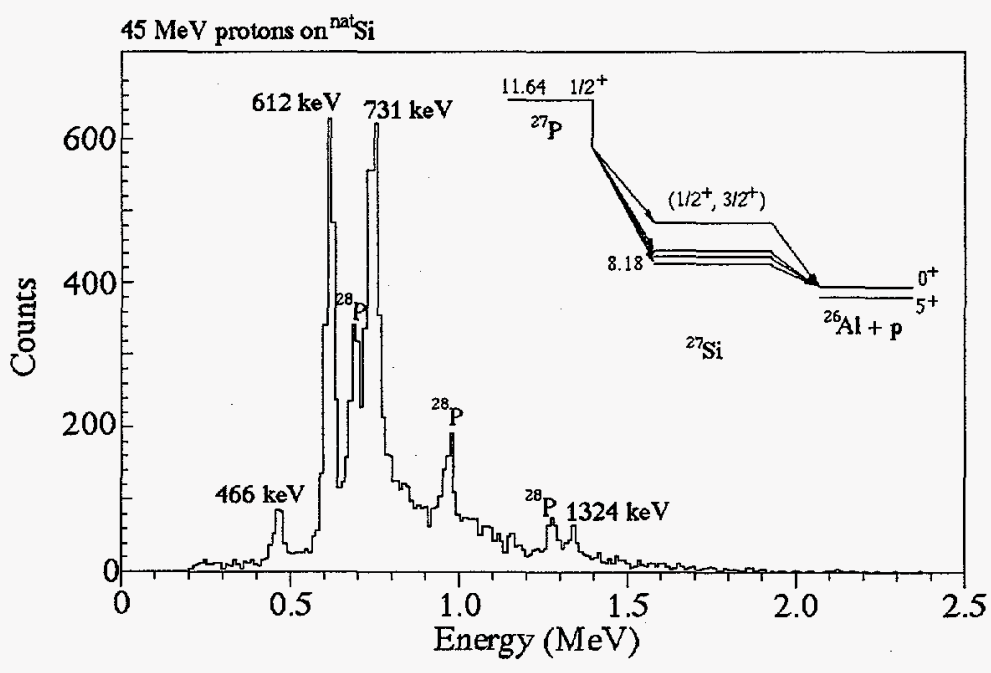




\title{
Proton Capture on ${ }^{7}$ Be and the Solar Neutrino Problem
}

\author{
J. Powell, D. M. Moltz, M. W. Rowe, and Joseph Cerny
}

The observed deficit of neutrinos coming from the sun, in comparison to the calculations of solar models, constitutes the Solar Neutrino Problem. This observed ratio is about $1 / 4$ to $1 / 2$. Of the three types of neutrino measurements reported to date, capture on $37 \mathrm{Cl}$, water Cherenkov, and capture on ${ }^{71} \mathrm{Ga}$, the first two methods are primarily sensitive to neutrinos from beta decay of ${ }^{8} \mathrm{~B}$. Thus, the predicted event rates for these two methods are sensitive to potential inaccuracies in the experimentally determined cross sections of reactions that lead to the creation of this isotope, as these measurements serve as input into the solar model calculations. One significant reaction is the capture of a proton onto a nucleus of ${ }^{7} \mathrm{Be}$ to form ${ }^{8} \mathrm{~B}$. This is the sole reaction creating ${ }^{8} \mathrm{~B}$.

A survey of previous experiments on ${ }^{7} \mathrm{Be}(\mathrm{p}, \gamma)^{8} \mathrm{~B}$ reveals moderate but significant systematic inconsistencies both between separate measurements and in modeled fits to the individual data sets. These differences are on the order of $25 \%$. Although a resolution of the uncertainty in this one reaction rate cannot account for the solar neutrino problem, an accurately determined solar neutrino emission spectrum is necessary in order to relate the results of current and future neutrino experiments to the various models, such as neutrino oscillations, that have been proposed to account for the observed discrepancy.

We are currently working on an improved measurement of the low-energy cross section of ${ }^{7} \mathrm{Be}(\mathrm{p}, \gamma){ }^{8} \mathrm{~B}$, in collaboration with TUNL†. The radionuclide ${ }^{7} \mathrm{Be}\left(t_{1 / 2}=53 \mathrm{~d}\right)$ will be produced via a $(p, n)$ reaction on ${ }^{7} \mathrm{Li}$. The beryllium will be chemically separated and purified, and subsequently electroplated onto a target backing. During the experiment, the ${ }^{8} \mathrm{~B}$ produced by irradiation with the proton beam will be detected by the alpha particles emitted following beta decay to ${ }^{8} \mathrm{Be}^{*}\left(t_{1 / 2}=0.77 \mathrm{~s}\right)$. These alphas will be detected in a large area gas $\Delta \mathrm{E}$-gas $\Delta \mathrm{E}$-silicon $\mathrm{E}$ detector telescope of a design similar to the detectors currently used by our group $[1,2]$. The $\Delta \mathrm{E}$ detectors are necessary to filter out events resulting from the intense flux of $\gamma$ rays emitted by the decaying ${ }^{7} \mathrm{Be}$. The target will be fixed to a wheel that will transfer it, on a time scale of about one second, between the proton beam and the detector. Both the ${ }^{7} \mathrm{Be}$ target and detector system are being developed by LBNL, while the measurement itself will be performed at TUNL, where a special low-energy, high-current accelerator is being built for this experiment.

This experiment will improve on previous work [3] primarily through use of a target containing ten times as much ${ }^{7} \mathrm{Be}$. This, combined with our detector and the new accelerator, will allow the cross section to be accurately determined to lower energies than previously measured.

+ Triangle Universities Nuclear Laboratory, Durham, North Carolina 27708

1. D. Moltz et al., Nuc. Instr. Meth A349 (1994) 210.

2. M. W. Rowe et al., (submitted to Nuc. Instr. Methods).

3. B. W. Filippone et al., Phys. Rev. C28 (1983)

2222. 


\title{
Search for Proton Decay from an Isomer of $77 \mathrm{Rb}$ with RAMA
}

\author{
M. W. Rowe, D. M. Moltz, J. Powell, and Joseph Cerny
}

Direct proton emission was first discovered in a high spin isomer of ${ }^{53} \mathrm{Co} 1,2$. The measured half-life of $53 \mathrm{~m} \mathrm{Co}$ is $247 \mathrm{~ms}$ and this proton decay is a $1.5 \%$ branch. Subsequently, ground state proton emission has been observed in many nuclei from ${ }^{105} \mathrm{Sb}$ to ${ }^{187} \mathrm{Re}$; however, ${ }^{53 \mathrm{~m} C o}$ remains the only example of proton decay from an isomeric state of a nuclide with a protonbound ground state. There is a prediction ${ }^{3}$ that several nuclides in the mass 80 region could also have high-spin isomers which would be unbound to proton emission.

One such nuclide, ${ }^{77} \mathrm{Rb}$, is predicted to have an isomer that is unbound by $2.93 \mathrm{MeV}$. This isomer would have a spin of $19 / 2^{-}$and would be formed by coupling a single $f_{5 / 2}$ proton hole state with a pair of $g_{9 / 2}$ neutrons. Although proton decays of this energy typically occur in $<10^{-15} \mathrm{~s}$, Bugrov et al., ${ }^{4}$ have predicted a proton-decay partial half life of $240 \mathrm{~ms}$ due to the large centrifugal barrier for this decay $(l=9)$. In order to allow the proton decay to be observed, gamma decay would have to be strongly hindered. Although gamma deexcitation to one of the high-spin rotational-band states known to lie below the isomer in excitation energy could in principle occur, differences in the wavefunctions of these collective-mode states and the manyparticle isomeric state may lead to significant gamma-decay suppression.

${ }^{77} \mathrm{Rb}$ is produced in high yield in the $4^{40} \mathrm{Ca}\left(4^{0} \mathrm{Ca}, 3 \mathrm{p}\right)$ reaction at $150 \mathrm{MeV}$. Unfortunately, competing reactions produce not only the well-known beta-delayed proton emitter ${ }^{77} \mathrm{Sr} 5$, but also weak delayed-proton emitters, observed by our group in previous helium-jet studies. This leads to a weak proton background up to $6 \mathrm{MeV}$. For this reason, we had to employ our on-line mass separator $\mathrm{RAMA}^{6}$ to eliminate this proton background. Previous studies of alkali metals demonstrated typical efficiencies of $1 \%$. However, the large recoil energies present in this reaction significantly lower the helium-jet efficiency from a typical value of $50 \%$ to less than $5 \%$. A recent test measurement demonstrated an efficiency for $\mathrm{Rb}$ commensurate with these expectations.

RAMA $^{7}$ has been fully upgraded in the past several years. To allow the separator to be fed by a capillary that is only $25 \mathrm{~cm}$ long, the ion source has been moved to the bombardment area, just above the target. This change has reduced the He-jet transport time from 200-300 ms to approximately $15 \mathrm{~ms}$. The recently-completed search for the proton decay of $77 \mathrm{~m} R b$ represented the first long experiment to utilize RAMA since the upgrade was completed.

Throughout the experiment, the amount of ${ }^{77} \mathrm{Rb}$ activity collected was monitored via observation of the beta-delayed gamma-ray decays of the ground state. Preliminary analysis indicates that approximately $2 \times 10^{6}{ }^{77} \mathrm{Rb}$ atoms were transported to the detector station during the 5-shift bombardment. To measure proton decays, we employed a single gas $\Delta \mathrm{E}-$ gas $\Delta \mathrm{E}$ silicon $E$ telescope subtending a solid angle of 23 $\pm 1 \%$ of $4 \pi$. Since there could have been unusual events due to high voltage sparking of the RAMA electrostatic components, we continued running the detectors and RAMA at mass 77 for an additional 6 shifts to adequately check the background rate. No adjustments to the mass separator were necessary during the entire 11 shifts.

Preliminary analysis of the data has yielded no evidence of the predicted proton peaks. Our sensitivity is approximately $1 \times 10^{-5}$ relative to feeding of the ground-state (based on the detector solid angle and the number of atoms transported). This indicates that either the isomeric state is not strongly fed by the reaction used, gamma-decay is insufficiently hindered to allow proton emission to compete or a combination of these two effects is present.

${ }^{1}$ K. P. Jackson et al. , Phys. Lett., 33B (1970) 281.

2J. Cerny et al. , Phys. Lett., 33B (1970) 284.

${ }^{3}$ L. K. Peker et al. , Phys. Lett., 36B (1971) 547.

4V. P. Bugrov et al., Yad. Fiz. 42 (1985) 57.

5J. C. Hardy et al. , Phys. Lett., 63B (1976) 27.

${ }^{6}$ F. B. Blonnigen et al. , Nucl. Instr. Meth., B26(1987) 328.

${ }^{7}$ T. J. Ognibene, P.hD. Thesis, Report LBNL-38848, unpublished. 


\title{
Calibration of Low-Energy Particle-Identification Telescopes
}

\author{
M. W. Rowe, D. M. Moltz, T. J. Ognibene, J. Powell and Joseph Cerny
}

In 1993, this group began making proton spectroscopy measurements with a unique detector array incorporating six low-energy particle identification telescopes ${ }^{1}$. These telescopes represent the culmination of a series of designs intended to lower the proton energy threshold from $\sim 700 \mathrm{keV}$ using traditional telescopes to $\sim 200 \mathrm{keV}$, allowing a wide range of novel measurements to be made. The traditional Si $\Delta E$ detector has been replaced in this design by two gas-ionization $\Delta \mathrm{E}$ detectors. The gas is confined by a thin polypropylene window; the electric field is created collinear with the incident radiation between a central grid held at a high positive voltage and two outer signalgrids floating near ground.

Over the following two years, much effort went into understanding the response of these telescopes to protons and alpha particles in a high beta radiation environment. One of the first experiments performed using this array was a measurement of the beta-delayed proton decay of ${ }^{23} \mathrm{Al}$ through its isobaric analog state (IAS) in ${ }^{23} \mathrm{Mg}$. Because this state is unbound to proton emission by only $220 \mathrm{keV}$ (c.m.), calibration of the decay spectrum at low energies required the development of a reliable method of extrapolation from higher-energy calibration points down to the low-energy threshold of the detectors.

Since there are no standard calibration sources of decay protons, proton calibrations are generally accomplished in situ using betadelayed protons emitted from ${ }^{25} \mathrm{Si}$ produced via the ${ }^{3} \mathrm{He}+{ }^{24} \mathrm{Mg}$ reaction at $40 \mathrm{MeV} \cdot{ }^{3}$. Unfortunately, the lowest energy proton group emitted by ${ }^{25} \mathrm{Si}$ has a laboratory energy of 386.1 $\mathrm{keV}$; other than ${ }^{23} \mathrm{Al}$, no emitter of lower energy protons is known. A linear extrapolation of the calibration of the $\mathrm{Si} \mathrm{E}$ detector from the higher energy points would not be accurate due to two factors which increasingly cause the calibration to deviate from linearity at low energies. First, the stopping power of the detector materials increases with decreasing proton energy. Because of this, as the threshold is approached an evergreater proportion of the incident energy is lost in the non-active components of the telescope (the window, part of the gas and the $\mathrm{Si} \mathrm{E}$ dead layer). Secondly, there is a slight non-linearity between the input and output of the slow-signal electronics that is most severe near the detection threshold.
To calibrate the detector telescopes before each experiment, we make ${ }^{25} \mathrm{Si}$ as described above. We calculate the energy which will be lost by its decay protons in the window, gas and Si deadlayer using the stopping powers given in ref. 4; these are subtracted from the incident energy to determine the energy which will be detected in the Si E detector. We measure the non-linearity of the detector electronics with a precision pulser; observed channel numbers are then corrected for this non-linearity. A linear fit is made of calculated energy detected in the $\mathrm{E}$ detector to corrected channel. If new proton groups are observed during the primary experiment, the energy detected in the Si E is calculated from this fit, then the energy loss calculation is reversed to find the incident energy of the new groups.

To test the reliability of this procedure, two experiments were performed in which a series of thin Al foils were used to degrade protons from ${ }^{25} \mathrm{Si}$ (and alpha particles from ${ }^{20} \mathrm{Na}$ ) to energies below the threshold for detection. The figure below shows a linear fit of energy to channel before and after correction for the electronics and energy losses. Using this method, it was determined that the low-energy threshold is $\sim 180 \mathrm{keV}$ for detecting protons and is $\sim 450 \mathrm{keV}$ for detecting alpha particles.

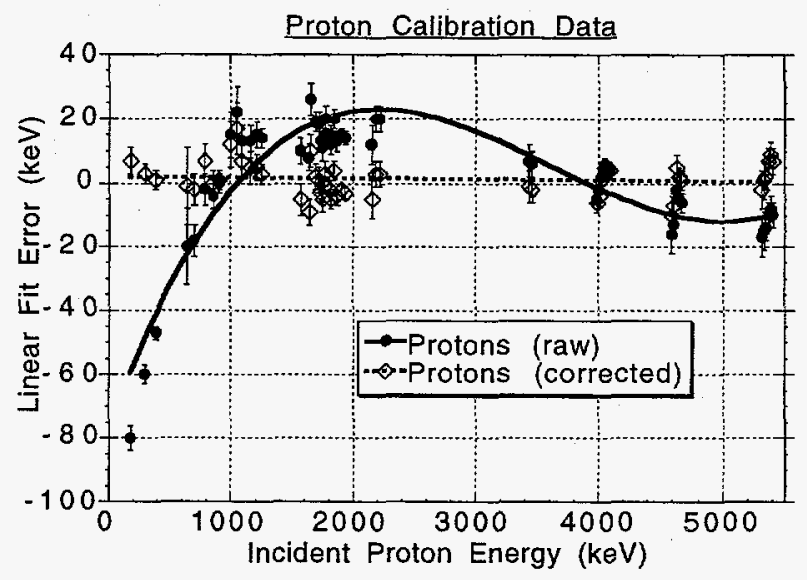

1. D. Moltz et al., Nuc. Instr. Meth A349 (1994) 210.

2. R. J. Tighe et al., Phys. Rev. C52 (1995) R2298.

3. J. D. Robertson et al., Phys. Rev. C42 (1990) 1922. 4. M. J. Berger, ed., Stopping Powers and Ranges for Protons and Alpha Particles (ICRU Report 49, 1993). 


\title{
Gas Phase Chromatography of $\mathrm{FrBr}$ and $\mathrm{BiBr}_{3}$.
}

\author{
E. R. Sylwester, D. C. Hoffman, J. Adams, Y. H. Chung, K. E. Gregorich, M. B. Hendricks, M. R. \\ Lane, C. Laue, D. M. Lee, C. A. McGrath, D. A. Shaughnessy, D. A. Strellis, P. A. Wilk.
}

The Heavy Element Volatility Instrument $(\mathrm{HEVI})^{1}$ was used to investigate the volatility of the $\mathrm{Fr}$ and $\mathrm{Bi}$ bromides. HEVI is an isothermal gas phase chromatography system which separates short lived isotopes based on their volatility. A $\mathrm{He} / \mathrm{KBr}$ gas jet was used to transport the recoil products- the $4.8-\mathrm{min} .{ }^{221} \mathrm{Fr}$ and its granddaughter the $45-\mathrm{min} .{ }^{213} \mathrm{Bi}$ - from a ${ }^{225} \mathrm{Ra} /{ }^{225}$ Ac source.

The recoil products were continuously collected on a quartz wool plug kept at $900^{\circ} \mathrm{C}$ in a quartz chromatography column. $\mathrm{HBr}$ was added at a rate of $100 \mathrm{ml} / \mathrm{min}$ in order to form the volatile bromide species of $\mathrm{Fr}$, which were then swept into the isothermal section of the chromatography column. The separated species were reattached to $\mathrm{KBr}$ aerosols and transported through a capillary system onto a glass wool filter. After a collection time of 5 minutes, the filter was taken out and counted on a PIPS (Passivated Ion implanted Planar Silicon) detector.

The volatility of each species was established by determining yield as a function of the temperature of the column. Due to the long halflife of the Bi granddaughter, the chemistry and collection time used (5 minutes each) was insufficient to provide an accurate measure of volatility. The alpha peak from the ${ }^{217} \mathrm{At}$ daughter was also seen, however due to the extremely short half-life of this species ( $32 \mathrm{~ms}$ ) no information about the chemical behavior could be determined; instead, the Astatine appeared wherever the Fr parent was found. $\mathrm{FrBr}$ was shown to be volatile at $550^{\circ} \mathrm{C}$ while preliminary data on $\mathrm{BiBr}_{3}$ show it to be volatile around $250^{\circ} \mathrm{C}$.

In past experiments the species studied in HEVI have shown an unusually high (non-zero) yield at the low temperatures of $50-150^{\circ} \mathrm{C}$. This yield was observed to drop to $0 \%$ at about 150 $200^{\circ} \mathrm{C}$ before rising in the expected volatility curves for each species. The $4.8-\mathrm{min} .{ }^{221} \mathrm{Fr}$ recoil product was used to investigate this effect by varying the amount of $\mathrm{HBr}$ and quartz wool used in column and again determining the yield as a function of temperature.

The high low-temperature yield effect was seen to appear at temperatures up to $250^{\circ} \mathrm{C}$ even with no halogenating agent $(\mathrm{HBr})$ present and no quartz wool in the column. With the addition of quartz wool, the effect was seen to drop but still appeared at temperatures up to $150^{\circ} \mathrm{C}$. With the addition of more quartz wool, the effect again dropped in yield but was still present at the temperatures of 50 and $100^{\circ} \mathrm{C}$. In none of these experiments did the addition or lack of the halogenating agent make any significant difference in the low-temperature yield.

These results indicate that the lowtemperature yield observed is not a result of the chemical volatility of the compound- which would require a halogenating agent. Instead it is a function of mass transport that allows a percentage of the activity to pass through the column unimpeded, in effect acting as a gas jet instead of a chromatography column. This effect is reduced by the presence of the quartz wool at the beginning $\left(900^{\circ} \mathrm{C}\right)$ section of HEVI, which breaks up the $\mathrm{KBr}$ clusters which are responsible for transport in the gas jet, and reduces the amount of activity entering the isothermal section of the column without the presence of a halogenating agent.

\section{Footnotes and References}

1. B. Kadkhodayan et al., Nucl. Instr. Meth. A317 254 (1992). 


\section{Gas Phase Chromatography of $\mathrm{HfBr}_{4}$}

E. R. Sylwester, D. C. Hoffman, J. Adams, Y. H. Chung, K. E. Gregorich, M. B. Hendricks, M. R. Lane, C. Laue, D. M. Lee, C. A. McGrath,

D. A. Shaughnessy,

D. A. Strellis, P. A. Wilk.

The Heavy Element Volatility Instrument $(\mathrm{HEVI})^{1}$ was used to investigate the volatility of $\mathrm{HfBr}_{4}$. HEVI is an On-line isothermal gas phase chromatography system which separates short lived istopes based on their volatility. The $6.8-\mathrm{min}{ }^{166} \mathrm{Hf}$, the $1.7-\mathrm{min}{ }^{165} \mathrm{Hf}$, and the $2.0-\mathrm{min} 167 \mathrm{~W}$ were produced at the 88 -Inch Cyclotron by the reaction of ${ }^{19} \mathrm{~F}$ with a target of nat $\mathrm{Eu}$. Reaction products were transported by a $\mathrm{He} / \mathrm{KBr}$ gas jet system and continuously collected on a quartz wool plug kept at $900^{\circ} \mathrm{C}$ in a quartz chromatography column. $\mathrm{HBr}$ was added at a rate of $100 \mathrm{ml} / \mathrm{min}$ in order to form the volatile species $\mathrm{HfBr}_{4}$, which was then swept into the isothermal section of the chromatography column, where it was separated from the other reaction products. The separated species was reattached to $\mathrm{KBr}$ aerosols and transported through a capillary system onto a glass wool filter placed in front of an intrinsic Ge gamma detector.

${ }^{165} \mathrm{Hf}$ was identified by gamma spectroscopy of the $180 \mathrm{keV}$ gamma line. ${ }^{166} \mathrm{Hf}$ was identified by gamma spectroscopy of the 78.76 $\mathrm{keV}$ line, and ${ }^{167} \mathrm{Hf}$ was identified by its 315.24 $\mathrm{keV}$ line. Volatility was determined by measuring the yield of each isotope simultaneously as a function of the temperature of the column. All three isotopes showed exactly the same volatility behavior when their different half-lives were taken into account, as expected. The temperature at which $\mathrm{HfBr}_{4}$ was observed to be volatile was $250^{\circ} \mathrm{C}$.

A Monte Carlo simulation program ${ }^{2}$ was used to calculate the adsorption enthalpy of these species based upon their volatilities. The adsorption enthalphy for $\mathrm{HfBr}_{4}$ on a $\mathrm{SiO}_{2}$ surface based upon the volatility data collected was calculated to be $-107 \pm 5 \mathrm{~kJ} / \mathrm{mol}$.
Figure 1 shows the volatity data collected for ${ }^{165} \mathrm{Hf}$ and ${ }^{167} \mathrm{Hf}$. The yield curves shown are the volatility curves calculated by the best fit of the data to an adsorption enthalpy value of $-107 \pm 5 \mathrm{~kJ} / \mathrm{mol}$ using the Monte Carlo simuation program.

\section{Footnotes and References}

1. B. Kadkhodayan, et al., Nucl. Instr. Meth. A317 254 (1992).

2. A. Türler, K. E. Gregorich, D. C. Hoffman, D. M. Lee, H. W. Gäggeler, LBL annual report \#31855, Nuclear Science Division (1991).

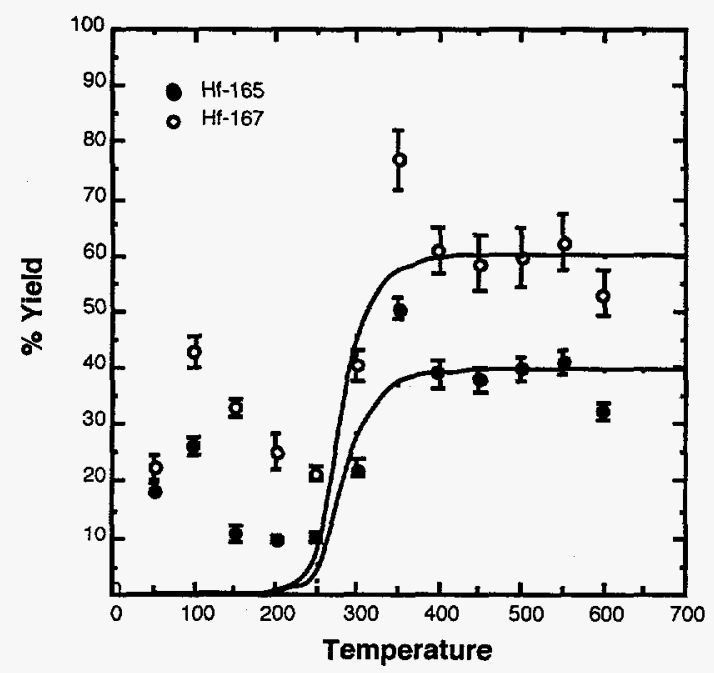

Fig. 1. Observed yields of $\mathrm{HfBr}_{4}$ as a function of temperature. The fitted curves show the expected yield based upon the Monte Carlo calculation of an adsorption enthalpy of $-107 \pm 5$ $\mathrm{kJ} / \mathrm{mol}$, which is the best fit to the data. 


\section{Gas Phase Chromatography of $\mathrm{WBr}_{6}$}

E. R. Sylwester, D. C. Hoffman, J. Adams, Y. H. Chung, K. E. Gregorich, M. B. Hendricks, M. R. Lane, C. Laue, D. M. Lee, C. A. McGrath, D. A. Shaughnessy, D. A. Strellis, P. A. Wilk.

The Heavy Element Volatility Instrument $(\mathrm{HEVI})^{1}$ was used to investigate the volatility of the $W$ bromides. HEVI is an On-line isothermal gas phase chromatography system which separates short lived isotopes based on their volatility. The $2.4-\mathrm{min}$. ${ }^{171} \mathrm{~W}$ and the 8.0 min. ${ }^{172} \mathrm{~W}$ were produced at the 88 -Inch Cyclotron by the reaction of $20 \mathrm{Ne}$ with a target of nat $\mathrm{Gd}$. Reaction products were transported by a $\mathrm{He} / \mathrm{KBr}$ gas jet system and continuously collected on a quartz wool plug kept at $900^{\circ} \mathrm{C}$ in a quartz chromatography column. $\mathrm{HBr}$ was added at a rate of $100 \mathrm{ml} / \mathrm{min}$ in order to form the volatile bromide species of $W$, which were then swept into the isothermal section of the chromatography column. The separated species were reattached to $\mathrm{KBr}$ aerosols and transported through a capillary system onto a glass wool filter placed in front of an intrinsic Ge gamma detector. $171 \mathrm{~W}$ was identified by gamma spectroscopy of the 184, 294, and $479 \mathrm{keV}$ lines and it's volatility determined. $172 \mathrm{~W}$ was identified by gamma spectroscopy of the 424 and $548 \mathrm{keV}$ lines and it's volatility was determined. As expected, both isotopes showed exactly the same volatility behavior when their different half-lives were taken into account. The temperature at which $\mathrm{WBr}_{6}$ was observed to be volatile was determined to be $130^{\circ} \mathrm{C}$.

A Monte Carlo simulation program ${ }^{2}$ was used to calculate the adsorption enthalpy of these species based upon their volatilities. The adsorption enthalpy was calculated to be $-82 \pm 5$ $\mathrm{kJ} / \mathrm{mol}$ for $\mathrm{WBr}_{6}$ on a $\mathrm{SiO}_{2}$ surface based upon the volatility data collected.

Figure 1 shows the data for the most prominent gamma lines of each of the three isotopes, and the volatility curve calculated using an adsorption enthalpy of $-82 \pm 5 \mathrm{~kJ} / \mathrm{mol}$ for ${ }^{172} \mathrm{~W}$. The variation in yield with isotope is a function of the differences in their half-lives, but this variation results in no change in adsorption enthalpy when half-life is taken into consideration.

\section{Footnotes and References}

1. B. Kadkhodayan, et al., Nucl. Instr. Meth. A317 254 (1992).

2. A. Türler, K. E. Gregorich, D. C. Hoffman, D. M. Lee, H. W. Gäggeler, LBL annual report \#31855, Nuclear Science Division (1991).

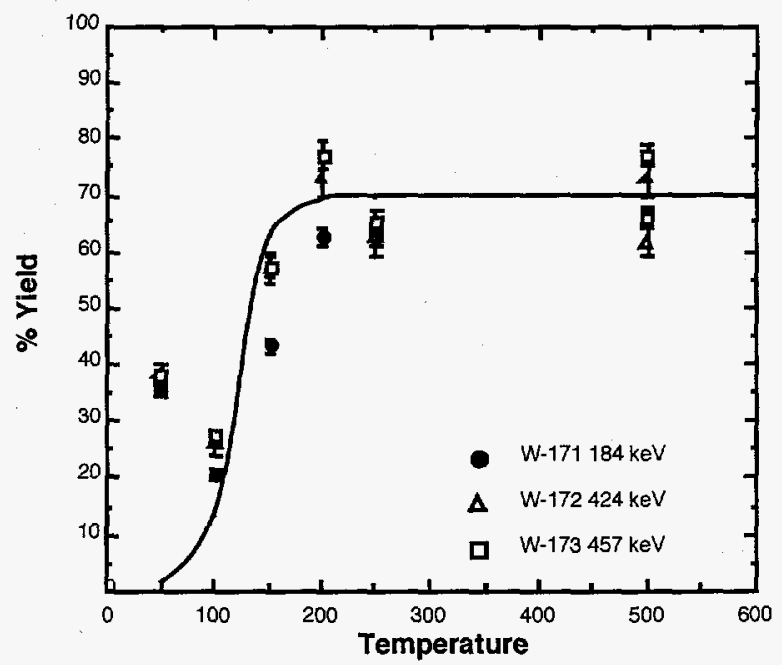

Fig. 1. Observed yields of $\mathrm{WBr}_{6}$ as a function of temperature. Only the data from the most prominent gamma line of each isotope is shown. The fitted curve shows the expected yield based upon the Monte Carlo calculation of an adsorption enthalpy of $-82 \pm 5 \mathrm{~kJ} / \mathrm{mol}$, and is the best fit to the data. 


\title{
Chemical Studies of Rutherfordium (Element 104)*
}

\author{
C.D. Kacher**, K.E. Gregorich, D.M. Lee, B. Kadkhodayant, S.A. Kreek‡, M.R. Lane, M.F. Moharß,
} M.P. Neu\%, N.J. Stoyerf, E.R. Sylwester, Y. Watanabett, B. Wierczinski, A. Bilewicz\#, D.C. Hoffman

Abstracts of three papers on chemical studies of rutherfordium* which have been accepted for publication in Radiochemica Acta are given below:

Part I. Thin Film Ferrocyanide Surfaces for the Study of the Hydrolysis of Rutherfordium.

The chemical properties of element 104, rutherfordium ( $\mathrm{Rf})$, and its group 4 homologs were studied by sorption on glass support surfaces coated with cobalt ferrocyanide. The surface studies showed that the hydrolysis trend in the group 4 elements and the pseudogroup 4 element, $\mathrm{Th}$, decreases in the order $\mathrm{Rf}>\mathrm{Zr} \approx \mathrm{Hf}>\mathrm{Th}$. This unexpected trend was attributed to relativistic effects which predict that $\mathrm{Rf}^{4+}$ would be more prone to having a coordination number $(\mathrm{CN})$ of 6 than 8 in aqueous solutions due to a destabilization of the $6 \mathrm{~d}_{5 / 2}$ shell.

Part II. Solvent Extraction into Tributylphosphate from $\mathrm{HBr}$ Solutions.

The chemical properties of element 104, rutherfordium (Rf), and its group 4 homologs and other tetravalent cations were studied by solvent extraction into tributylphosphate (TBP) from various concentrations of $\mathrm{HBr}$ and $\mathrm{HCl}$. Since bromide is a larger and softer (more polarizable) anion than chloride, the stability of Rf-bromide complexes was expected to differ from the stability of Rf-chloride complexes, and a comparison of the two systems was undertaken. Our studies showed that the extraction trend decreased in the order $\mathrm{Zr}>\mathrm{Hf}>\mathrm{Rf} / \mathrm{Ti}$ for $\mathrm{HBr}$, compared to $\mathrm{Zr}>\mathrm{Hf}>\mathrm{Rf}>\mathrm{Ti}$ for $\mathrm{HCl}$, showing that $\mathrm{Rf}$ and $\mathrm{Ti}$ did not extract as well in either system, probably because they hydrolyzed more easily than $\mathrm{Zr}$ and $\mathrm{Hf}$.

\section{Part III. Solvent Extraction into Triisooctyl-} amine from HF Solutions.

The extraction of element 104, rutherfordium (Rf), and its group 4 homologs into $0.25 \mathrm{M}$ triisooctylamine (TIOA) in $o, m, p$-xylene from various concentrations of hydrofluoric acid was studied. This extraction system was of particular interest because all group 4 cations studied formed complexes with the same coordination number (CN) of 6. Therefore, species extracted were similar and the extractability depends on the radii of the fluoride complexes. The studies showed that the extractability for the group 4 elements in this system decreases in the order $\mathrm{Ti}>\mathrm{Zr} \approx \mathrm{Hf}>\mathrm{Rf}$, in inverse order from the decrease in ionic radii $\mathrm{Rf}>\mathrm{Zr} \approx \mathrm{Hf}>\mathrm{Ti}$. This order is different than the order in the hydrolysis, $\mathrm{TBP}-\mathrm{HCl}$, and TBP- $\mathrm{HBr}$ extraction systems where $\mathrm{Rf}$, due to decreasing $\mathrm{CN}$, behaves more similarly to $\mathrm{Ti}$ than to $\mathrm{Zr}$ and Hf.

Footnotes and References

*This research constituted a major part of Christian D. Kacher's Ph. D. Thesis, December, 1995, Chemistry Department, University of California, Berkeley, CA.

** William O'Neil \& Co., Inc., 12655 Beatrice St., Los Angeles, CA 90066.

† LAM Associates, 4330 Terrabella Way, Oakland, CA 94611 .

‡ Lawrence Livermore National Laboratory, L-231, Livermore, CA 94550.

§ EG\&G Energy Measurements, Washington Aerial Measurements Division, P.O. Box 380, Suitland, MD 20752.

\%Los Alamos National Laboratory, MS G-739, Los Alamos, NM 87544.

t+ \#102 GS-heim, 3-3-8 Asahi-ga-oka, Hino, Tokyo 191 JAPAN

\# Department of Radiochemistry, Institute of Nuclear Chemistry and Technology, 03-195, Warszawa, Poland 


\title{
First Chemical Separations of Seaborgium (Element 106)
}

\author{
K.E. Gregorich, D.C. Hoffman, E.R. Sylwester, D.M. Lee, and the LBNL, GSI, + Mainz, $\ddagger$ Dresden, $€$ \\ Krakow, JAERI * PSI,ø Bern, $\triangle$ Rossendorf, $\infty$.JINR, $\S$ Oslo, $₫$ Chalmers, $\partial$ Collaboration
}

The first chemical separations of seaborgium ( $\mathrm{Sg}$, element 106) have been performed in both the aqueous- and gas-phase.

Experiments to determine the chemical properties of lawrencium ( $\mathrm{Lr}$, element 103), rutherfordium ( $\mathrm{Rf}$, element 105), and hahnium ( $\mathrm{Ha}$, element 105$)$ have clearly shown that the actinide series ends with $\mathrm{Lr}$, and that $\mathrm{Rf}$ and $\mathrm{Ha}$ are the first two members of the $6 \mathrm{~d}$ series of transition metals, with $\mathrm{Lr}^{3+}, \mathrm{Rf}^{4+}$, and $\mathrm{Ha}^{5+}$ being the most stable oxidation states. While the most general chemical properties of $\mathrm{Rf}$ and Ha indicate that they are members of the fourth and fifth groups of the periodic table, respectively, we have found that the detailed chemical properties of these first two transactinide elements cannot be reliably extrapolated from the chemical properties of their lighter homologs. These deviations from the periodicity of the elements have been explained as being due to relativistic effects in the electronic orbitals. In this light, it is of great interest to investigate the the chemical properties of $\mathrm{Sg}$, and compare its chemical properties to the lighter periodic table homologs, W and Mo.

Recently, ${ }^{265} \mathrm{Sg}\left(\mathrm{E}_{\alpha}=8.8 \mathrm{MeV}, \mathrm{t}_{1 / 2} \sim 8 \mathrm{~s}\right)$ and ${ }^{266} \mathrm{Sg}\left(\mathrm{E}_{\alpha}=8.6 \mathrm{MeV}, \mathrm{t}_{1 / 2} \sim 13 \mathrm{~s}\right)$ have been identified in ${ }^{22} \mathrm{Ne}+{ }^{248} \mathrm{Cm}$ bombardments. ${ }^{1}$ The half-lives are long enough to allow chemical separation with the most modern techniques, and the production rates are sufficient for detection of about 1 atom per day (after chamical separation).

In the chemical experients, ${ }^{266} \mathrm{Sg}$ and ${ }^{265} \mathrm{Sg}$ were produced in ${ }^{22} \mathrm{Ne}$ bombardments of ${ }^{248} \mathrm{Cm}$ targets at the GSI UNILAC. Products were transported to the chemical separation apparatus via an aerosol-loaded gas-jet.

Gas-phase chemical separations ${ }^{2}$ were performed with the OLGA III gas-phase isothermal chromatography apparatus. Volatile oxychlorides of Sg were formed, and their retention time in a quartz column was measured as a function of the temperature of the column. We found $\mathrm{Sg}$ oxychlorides to be somewhat less volatile than oxychlorides of Mo and $W$ formed under the same conditions.

Aqueous-phase chemical separations ${ }^{4}$ were carried out with the ARCA ${ }^{5}$ apparatus, a miniaturized, computer-controlled, liquid column chromatography apparatus. ${ }^{265} \mathrm{Sg}$ was eluted from cation exchange-resin (Aminex A6) columns with $0.1 \mathrm{M} \mathrm{HNO}_{3} / 5 \times 10^{-4} \mathrm{MHF}$. Under these conditions, the ${ }^{261} \mathrm{Rf}$ and ${ }^{257}$ No $\alpha$-decay products of ${ }^{265} \mathrm{Sg}$ are strongly retained on the columns. Observation of the decays of ${ }^{265} \mathrm{Sg}$ daughter activities, ${ }^{261} \mathrm{Rf}$ and ${ }^{257}$ No, after the columns indicates that $\mathrm{Sg}$ forms neutral or anionic complexes (presumably $\mathrm{SgO}_{4}{ }^{2-}$ ). This behavior is similar to measured properties of the periodic table homologs, $\mathrm{W}^{6+}$ and $\mathrm{Mo}^{6+}$, and unlike that of $U^{6+}$.

\section{Footnotes and References}

+ Gesellschaft für Schwerionenforschung, Germany

$\ddagger$ Universität Mainz, Germany

$£$ Technische Universität Dresden, Germany

- Niewodniczanski Inst. of Nucl. Phys, Poland

* Japan Atomic Energy Research Institute, Japan

ø Paul Scherrer Institut, Switzerland

$\Delta$ University of Bern, Switzerland

$\infty$ Institut for Radiochemistry, Rossendorf, Germany

$\S$ Joint Institutes for Nuclear Research, Dubna, Russia

\& University of Oslo, Norway

$\partial$ Chalmers University of Technology, Sweden

$¥$ Inst. for Radiochem. Research, Rossendorf, Germany

1. Yu.A. Lazarev, et al., Phys. Rev. Lett. 73, 624 (1994).

2. A Türler et al., NRC4 Conf., St. Malo, France, (1996). 


\section{Production and Decay of ${ }^{265} \mathrm{Sg}$ and ${ }^{266} \mathrm{Sg}$}

K.E. Gregorich, B. Kadkhodayan, B. Wierczinski, D.M. Lee, C.D. Kacher, D.A. Shaughnessy, M.R. Lane, E.R. Sylwester, M.B. Hendricks, N.J. Stoyer, A.C. Veeck, D.C. Hoffman

Predictions of enhanced nuclear stability ${ }^{1}$ due to deformed nuclear shells near $\mathrm{N}=162$ and $Z=108$, have recently been confirmed with the detection ${ }^{2}$ of ${ }^{265} \mathrm{Sg}$ and ${ }^{266} \mathrm{Sg}$ using the Dubna gas-filled separator. In the Dubna experiments, the ${ }^{265} \mathrm{Sg}$ and ${ }^{266} \mathrm{Sg}$ half-lives were not measured (although $\alpha$-decay systematics indicated half-lives between 2 -s and 30-s). In addition, the measured production cross sections for the ${ }^{248} \mathrm{Cm}\left({ }^{22} \mathrm{Ne}, 4-5 \mathrm{n}\right){ }^{266-265} \mathrm{Sg}$ reactions were accurate to only a factor of three.

The estimated half-lives for $265,266 \mathrm{Sg}$, together with the production cross sections of $\sim 100 \mathrm{pb}$, make measurement of the chemical properties of $\mathrm{Sg}$ possible for the first time. Measurement of the half-lives, and refinement of the production cross sections are important in the understanding of the strength and extent of the $\mathrm{N}=162$ deformed shell, and necessary in planning for the chemical experiments.

In experiments at the LBNL 88-Inch Cyclotron, we searched for ${ }^{265} \mathrm{Sg}$ and ${ }^{266} \mathrm{Sg}$ produced in the ${ }^{22} \mathrm{Ne}+{ }^{248} \mathrm{Cm}$ reaction at $22 \mathrm{Ne}$ bombarding energies of 116- and 121-MeV. Activities recoiling out of the target were transported with a $\mathrm{He} / \mathrm{KCl}$ gas-jet to the $\mathrm{MG}$ rotating wheel system where we looked for the following decay chains: ${ }^{265} \mathrm{Sg} \Rightarrow{ }^{261} \mathrm{Rf}+\alpha \Rightarrow{ }^{257} \mathrm{No}+\alpha \Rightarrow{ }^{255} \mathrm{Fm}$ and ${ }^{266} \mathrm{Sg} \Rightarrow{ }^{262} \mathrm{Rf} \Rightarrow$ fission. At the $M G$, the activities were collected on $50-\mu \mathrm{g} / \mathrm{cm}^{2}$ polypropylene foils at the periphery of a $20^{\prime \prime}$ diameter wheel, which was periodically stepped to advance the newly collected sources between pairs of ion-implanted Si detectors.

A parent-daughter stepping mode was used to provide unambiguous identification of the decay of $266,265 \mathrm{Sg}$. In this mode, upon detection of a potential Sg $\alpha$-event in a bottom detector, it is assumed that the corresponding $\mathrm{Rf}$ daughter atom recoils into the top detector. The source is then removed from the detector station to allow detection of the daughter-atom $\alpha$-decay in a background-free environment.

In these experiments, the gas-jet efficiency was found to be $70 \%$ by measuring the detection rates of $254,256 \mathrm{Fm}$ (binary transfer products) and $213,214 \mathrm{Ra}$ (compound nucleus products from $\mathrm{a} \mathrm{Pb}$ impurity in the target). In addition, the efficiency for collecting $\alpha$-daughter recoils in the top detector upon detection of an $\alpha$-particle in the bottom was found to be $\sim 70 \%$ by monitoring the $211,212 \mathrm{Ra} \rightarrow 207,208 \mathrm{Rn}$ decay chains.

At the $116-\mathrm{MeV}$ bombarding energy (where ${ }^{266} \mathrm{Sg}$ was observed in the Dubna experiments), we observed no correlated $\alpha$-fission pairs, indicating an upper limit for the ${ }^{266} \mathrm{Sg}$ of $25 \mathrm{pb}$ ( $95 \%$ confidence limit). This is below the lower limit reported in the Dubna experiment.

At the 121-MeV bombarding energy (where ${ }^{266} \mathrm{Sg}$ and ${ }^{265} \mathrm{Sg}$ were observed in the Dubna experiments), We performed two experiments. In the first, we observed one decay chain from ${ }^{266} \mathrm{Sg}$, and background problems prevented positive assignment of four candidate events to the decay of ${ }^{265} \mathrm{Sg}$. In the second experiment, two decay chains were observed which are unambiguously assigned to the decay of ${ }^{265} \mathrm{Sg}$.

\begin{tabular}{|c|c|c|c|c|c|c|}
\hline $\begin{array}{l}E_{\alpha} S g \\
(\mathrm{keV})\end{array}$ & $\begin{array}{l}\tau \mathrm{Sg} \\
(\mathrm{sec})\end{array}$ & $\begin{array}{l}E_{\alpha} R f \\
(\mathrm{keV})\end{array}$ & $\begin{array}{l}\tau \mathrm{Rf} \\
(\mathrm{sec})\end{array}$ & $\begin{array}{l}E_{\alpha} \text { No } \\
(\mathrm{keV})\end{array}$ & $\begin{array}{l}\tau \text { No } \\
(\mathrm{sec})\end{array}$ & \\
\hline 8690 & 3.809 & $S F$ & 0.875 & \multirow{3}{*}{\multicolumn{2}{|c|}{$8280 \quad 37.68$}} & $266 \mathrm{Sg}$ \\
\hline 8840 & 5.640 & 8250 & 28.51 & & & $265 \mathrm{Sg}$ \\
\hline 8860 & 25.80 & 8185 & 56.5 & & & $265 \mathrm{Sg}$ \\
\hline
\end{tabular}

From these results, we conclude that ${ }^{265} \mathrm{Sg}$ and ${ }^{266} \mathrm{Sg}$ are formed in $121-\mathrm{MeV}{ }^{22} \mathrm{Ne}+{ }^{248} \mathrm{Cm}$ bombardments with cross sections of $\sim 200 \mathrm{pb}$ and $\sim 100 \mathrm{pb}$, respectively.

\section{Footnotes and References}

1. R. Smolanczuk, et al., Phys. Rev. C $\underline{52} 1871$ (1995).

2. Yu. A. Lazarev, et al., Phys. Rev. Lett. 73 , 624 (1994). 


\section{A Search for ${ }^{264} \mathrm{Sg}$}

C. A. McGrath, K. E. Gregorich, D. M. Lee, M. R. Lane, E. R. Sylwester, D. A. Shaughnessy, M. B. Hendricks, D. A. Strellis, P. A. Wilk, D. C. Hoffman

A search was undertaken at the 88-Inch Cyclotron at the Lawrence Berkeley National Laboratory for the new isotope ${ }^{264} \mathrm{Sg}$. This nucleus is predicted to decay with nearly equal probability by spontaneous fission (SF) and by alpha emission with $\mathrm{E}_{\alpha} \approx 9 \mathrm{MeV}$ and with a half life between 0.5 and 2 seconds ${ }^{1-2}$. The ${ }^{260} \mathrm{Rf}$ daughter of this alpha decay undergoes SF with a reported half life of $20 \mathrm{~ms}$. We tried to produce ${ }^{264} \mathrm{Sg}$ via the ${ }^{250} \mathrm{Cf}\left({ }^{18} \mathrm{O}, 4 \mathrm{n}\right){ }^{264} \mathrm{Sg}$ reaction at $111 \mathrm{MeV}$ and to correlate parent and daughter decays using our MG rotating wheel system.

Due to high background activities produced from binary transfer reactions, fairly stringent requirements were needed for an event. An acceptable event was defined as a parent alpha decay of between 8.5 and $9.2 \mathrm{MeV}$ detected in the bottom detector (which would cause the daughter to recoil from the source into the top detector) followed within $40 \mathrm{~ms}$ by detection of a SF single event in the top detector (indicating fission from an atom on the top detector surface). Unfortunately, only 4 of these $\alpha$-SF correlations were detected, while our calculations predict 2 or 3 random correlations of unrelated background activity. This result permits us to set an upper limit on the cross section for this reaction. If the $\alpha$-decay branch is $\geq 25 \%$ and the half life is between 0.5 and 2 seconds, the cross section is $\leq 200 \mathrm{pb}$ at $111 \mathrm{MeV}$. Figure 1 shows a plot of cross section versus half life, when a $25 \% \alpha$-decay branch is assumed. The expected cross section for this reaction is $200 \mathrm{pb}$.
In addition to looking for $\alpha-S F$ correlations, we also searched for $\alpha-\alpha$ and $\alpha-$ $\alpha-\alpha$ correlations. These would result from an alpha decay branch in a possible longer-lived isomer of ${ }^{260} \mathrm{Rf}$. However, we saw no evidence for these correlations .

These negative results have forced us to consider other production methods.

\section{References}

1. Smola 'nczuk, R., Skalski, J., Sobiczewski, A., Phys. Rev. C 52, 1871 (1995).

2. Möller, P., Nix, J. R., Myers, W. D., Swiatecki, Atomic Data Nucl. Data Tables 59, 185-381 (1995)

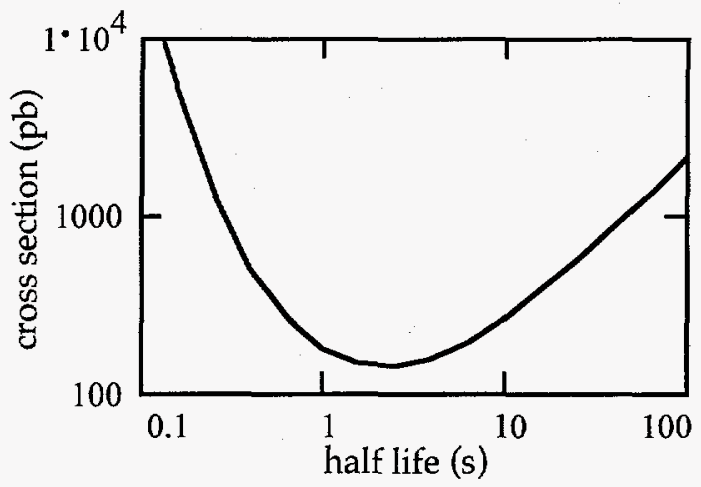

Fig. 1. Cross section upper limit plotted versus assumed $264 \mathrm{Sg}$ half life for the ${ }^{250} \mathrm{Cf}\left({ }^{18} \mathrm{O}, 4 \mathrm{n}\right){ }^{264} \mathrm{Sg}$ reaction at $111 \mathrm{MeV}$. An $\alpha-$ decay branch of $25 \%$ was assumed. 


\title{
Electronics for On-Line Liquid Scintillation $\alpha$-Particle Spectroscopy
}

\author{
K.E.Gregorich
}

Recent developments in liquid scintillation counting now make $\alpha$-particle pulse-height spectroscopy possible with energy resolution as good as $200 \mathrm{KeV} \mathrm{FWHM}{ }^{1,2}$. Since the light output per $\mathrm{MeV}$ of decay energy is much greater for $\beta$ - particles than for $\alpha$-particles, it is necessary to use pulse shape discrimination, based on the different fractions of slow- and fastdecaying components of the $\alpha$ - and $\beta$-light pulses, to produce a clean $\alpha$-energy spectrum.

In recent experiments we used this technique to study the chemical properties of transactinide elements. The $\alpha / \beta$ pulse-shape discrimination was implemented with a standard constant fraction discriminator on the fast (7-ns risetime) pulse from the photomultiplier tube (PMT) to start a time-to amplitude converter (TAC). This PMT signal was then shaped to undershoot by $10 \%$ to $20 \%$ of the full pulse amplitude. A zerocross-discriminator was then used to stop the TAC, providing discrimination between $\alpha$ - and $\beta$ pulses. Using this pulse shape discrimination technique, we found the $\beta$-decay of large amounts $(>1 \mathrm{kHz})$ of background activities caused pileup pulses which passed the pulse shape discrimination circuit.

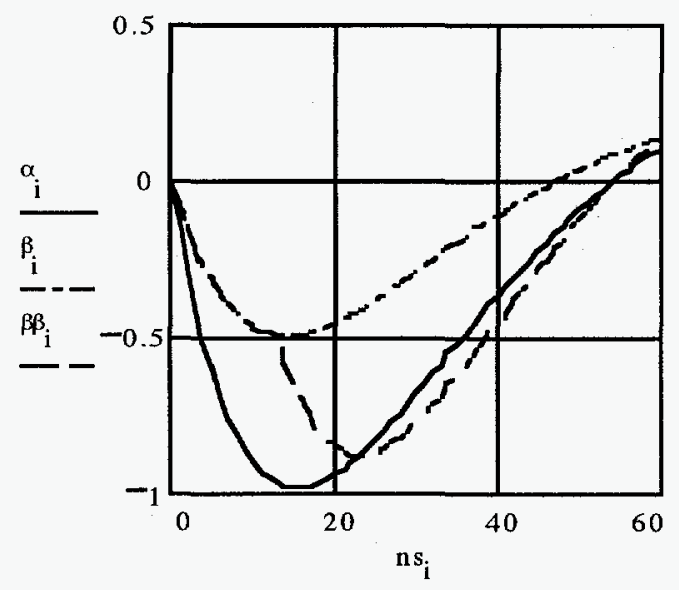

Fig. 1. Shaped pulses for the standard $\alpha / \beta$ pulse shape discrimination. The solid line is the shape for an $\alpha$-pulse. The short dashed line is for a $\beta$ pulse. The long dashes indicate a $\beta-\beta$ pileup with the same zero cross time as the $\alpha$.
In subsequent experiments an additional pulse shape discrimination technique ${ }^{3}$ was used on the shaped pulse mentioned above, which acted as a fast pileup rejector. This pileup rejector was simply a CFD run with a fraction of 1.0 and a constant fraction delay equal to the full risetime of the shaped pulse (15ns), making it sensitive to the width of the pulse. The combination of this pileup rejector with the standard pulse shape discriminator allows the measurement of alpha decay rates as small as a few counts per hour in samples with beta background rates of well over $1 \mathrm{kHz}$.

\section{Footnotes and References}

1. W.J. McDowell, Alpha Counting and Spectrometry using Liquid Scintillation Methods, Technical Information Center, Office of Scientific and Technical Information, U.D. DOE, (1986).

2. B. Wierczinski, Doctoral Thesis, U.Mainz, (1994).

3. B. Wierczinski, et al., Nucl. Instr. Meth. A $\underline{370}, 532$ (1995).

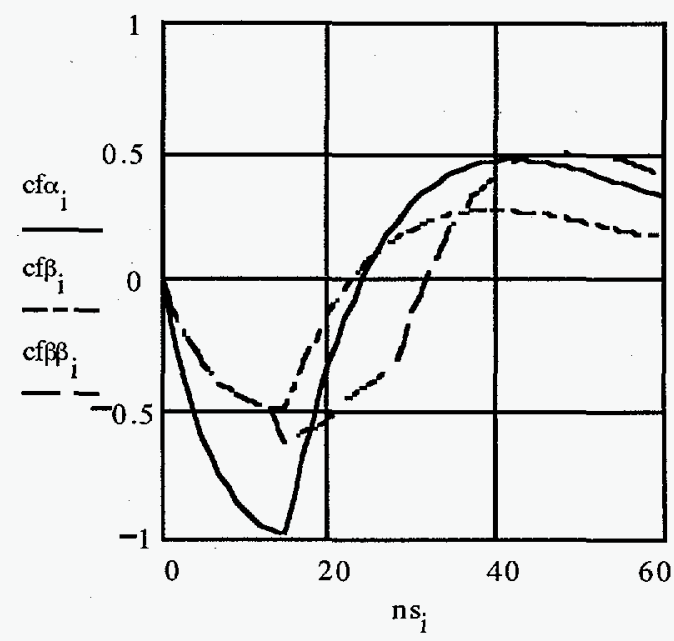

Fig. 2. The same pulses as in Fig.1, after passing through the $f=1 \mathrm{CFD}$. Note that the zero-cross time of the $\beta-\beta$ pileup (long dashes) pulse is well separated from that for the alpha pulse (solid line). 


\section{Improved Half-life Measurement of ${ }^{224} \mathrm{~Pa}$ and its ${ }^{209} \mathrm{Bi}\left({ }^{18} \mathrm{O}, 3 n\right){ }^{224} \mathrm{~Pa}$ Production Cross Section}

Philip A. Wilk, Ken E. Gregorich, Melissa B. Hendricks, Mike R. Lane, Diana M. Lee, Chris A. McGrath, Dawn A. Shaughnessy, Dan A. Strellis, Eric R. Sylwester, and Darleane C. Hoffman

Two half-lives for ${ }^{224} \mathrm{~Pa}$ have been reported previously. However, the error bars of these two measurements, $600 \pm 50 \mathrm{~ms}$ measured for ${ }^{224} \mathrm{~Pa}$ from the ${ }^{232} \mathrm{Th}(p, 9 n)^{224} \mathrm{~Pa}$ reaction ${ }^{1}$ and $950 \pm 150 \mathrm{~ms}$ measured for ${ }^{224} \mathrm{~Pa}$ from the ${ }^{205} \mathrm{Tl}\left({ }^{22} \mathrm{Ne}, 3 n\right){ }^{224} \mathrm{~Pa}$ reaction, $^{2}$ do not overlap. The former measurement's low value could be due to misinterpretation of the very complex alpha spectrum that was obtained. The disparity could lie in the misidentification of a ${ }^{223} \mathrm{Th}\left(t^{1} / 2=650 \mathrm{~ms}\right.$, $\mathrm{a}=7.324 \mathrm{MeV}$ ) decay peak produced by the ${ }^{232} \mathrm{Th}(p, p 9 n){ }^{224} \mathrm{~Pa}$ reaction.

In our experiment ${ }^{224} \mathrm{~Pa}$ was produced via the ${ }^{209} \mathrm{Bi}\left({ }^{18} \mathrm{O}\right.$, $\left.3 n\right){ }^{224} \mathrm{~Pa}$ reaction which has been previously reported ${ }^{3}$, but no production cross section or additional half-life measurement was given. The Lawrence Berkeley National Laboratory 88-Inch Cyclotron provided a 20 particle- $n A$ beam of $111 \mathrm{MeV}$ (laboratory frame) ${ }^{18} \mathrm{O}^{5+}$. The target system has been descibed in detail previously ${ }^{4}$. The energy calibration was performed on-line using known alpha decay energies from the following nuclides: ${ }^{211} \mathrm{Bi},{ }^{214} \mathrm{Ra}$, ${ }^{211} \mathrm{Po},{ }^{212} \mathrm{Ac},{ }^{216} \mathrm{Fr}$,

The odd-odd nucleus ${ }^{224} \mathrm{~Pa}$ is not expected to have a significant beta-decay branch and appears to alpha decay mainly to a single nuclear level in ${ }^{220} \mathrm{Ac}$ with an alpha particle energy of $7.49 \mathrm{MeV}^{2.5}$. Unfortunately, while alpha particles with this energy are identifiable in our alpha-energy spectrum, they are largely obscured by the ${ }^{211} \mathrm{Po}$ transfer product which decays via a $7.45 \mathrm{MeV}$ alpha. Therefore the ${ }^{224} \mathrm{~Pa}$ decay was identified by observing its alpha-decay daughters ${ }^{220} \mathrm{Ac}(\mathrm{t} 1 / 2=26$ $\mathrm{ms} ; \alpha=7.610,7.680,7.790,7.850 \mathrm{MeV})^{5}$ and ${ }^{216} \mathrm{Fr}$ $\left(t^{1 / 2}=0.7 \mathrm{~ms} ; \alpha=9.01 \mathrm{MeV}\right)^{5}$. The ${ }^{224} \mathrm{~Pa}$ daughter events were observed with 6 pairs of opposing detectors $^{6}$ within $2 \mu$ s of each other. By fitting the resultant decay curve with two components, the half-life of ${ }^{224} \mathrm{~Pa}$ was determined to be $850 \pm 20 \mathrm{~ms}$ (see Fig. 1). From these data, we also determined the production cross section to be $0.5 \pm 0.1 \mathrm{mb}$, after taking into account the detector efficiency, capillary transport efficiency, and capillary transport time.

The half-life for ${ }^{224} \mathrm{~Pa}$ as determined by this experiment is consistent with, but much more precise than the value of $950 \pm 150 \mathrm{~ms}$ reported by Borggreen $e t \mathrm{al}^{2}$. Our measured production crosssection is consistent with the value of $0.35 \mathrm{mb}$ calculated with the SPIT code $^{7}$.

\section{Footnotes and References}

1. P. A. Tove, Arkiv Fysik. 13, 549 (1958).

2. J. Borggreen, K. Valli, E. K. Hyde. Phys. Rev. C 2, 1841 (1970).

3. M. Fukuda et al., RIKEN Accel. Prog. Rep. 20, 14 (1986).

4. D. Lee et al., Phys. Rev. C 25, 286 (1982).

5. R. B. Firestone, Table of Isotopes, 8 th ed. (WileyInterscience, New York, 1996).

6. D. C. Hoffman et al., Phys. Rev. C 41, 631 (1990).

7. T. Sikkeland, Ark. Fys. 36, 539 (1967); J. Alonso, Gmelin Handbook of Inorg. Chem., $7 \mathbf{b}, 28$ (1974).

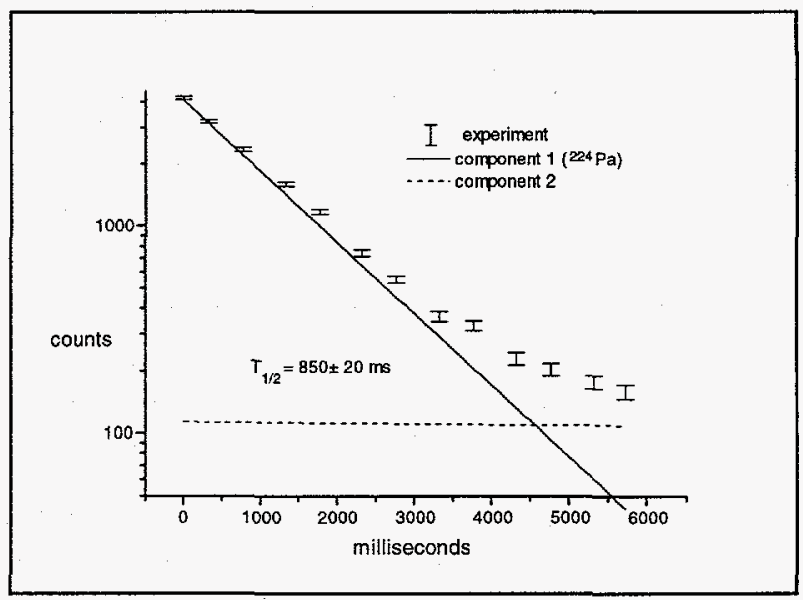

Fig. 1. Decay of ${ }^{224} \mathrm{~Pa}$ 


\section{Off-line Generation of ${ }^{233} \mathrm{~Pa}$ and the ${ }^{227} \mathrm{~Pa}$ Yield Through SISAK Philip A. Wilk and Darleane C. Hoffman}

During the experiment described in the previous article we were able to determine the production cross section for ${ }^{224} \mathrm{~Pa}$ by bombarding a ${ }^{209} \mathrm{Bi}$ target with ${ }^{18} \mathrm{O}$ at $88 \mathrm{MeV}$ and looking for subsequent fusion product alpha decays with our MG system ${ }^{1}$. This is the same reaction and energy that was used to produce $\mathrm{Pa}$ for the SISAK extraction. SISAK is an automated system for doing ultra-fast chemical extractions. By looking at time-correlated alpha decays from the ${ }^{224} \mathrm{~Pa}$ daughters ${ }^{220} \mathrm{Ac}$ and ${ }^{216} \mathrm{Fr}$, the cross section was determined to be $0.5 \pm 0.1 \mathrm{mb}$. Also during this experiment, a more precise half-life $-850 \pm$ $20 \mathrm{~ms}$ - was determined than was previously available. Based on this cross section, the chemical yield of Pa through SISAK 3 was very low. Taking into account the reported cross section ${ }^{2}$ of ${ }^{261} \mathrm{Ha}$, the amount of this element that we should have seen would have been vanishingly small.

Calculating from the cross-section for ${ }^{224} \mathrm{~Pa}$, we should have seen about 26,000 atoms of ${ }^{224} \mathrm{~Pa}$ if we had $100 \%$ chemical yield. Assuming every alpha higher in energy than ${ }^{224} \mathrm{~Pa}$ that correlated with ${ }^{224} \mathrm{~Pa}$ within $26 \mathrm{~ms}$ to be evidence for its alpha decay, resulted in $650{ }^{224} \mathrm{~Pa}$ atoms through the SISAK system during the 40 minute experiment. This gives a yield through SISAK of only about $2.5 \%$.

The lost $\mathrm{Pa}$ could be accounted for by sorption on SISAK/LISSY surfaces. To look at its sorption behavior, ${ }^{233} \mathrm{~Pa}$ was generated off-line (see Fig. 1) from the decay of ${ }^{237} \mathrm{~Np}$ by elution of 27 day ${ }^{233} \mathrm{~Pa}$ from an anion-exchange column. The ${ }^{233} \mathrm{~Pa}$ was eluted from the column with $12 \mathrm{M} \mathrm{HCl} / 0.1 \mathrm{M} \mathrm{HF}$. This $\mathrm{Pa}$ was then applied to samples of the materials used in the SISAK experiment to determine if $\mathrm{Pa}$ would strongly sorb on components of the experimental apparatus. 10 to $50 \mu \mathrm{L}$ aliquots of this solution were evaporated directly on a glass slide, Teflon square, and $\mathrm{Ti}$ foil, respectively (the surface area covered was about $1 \mathrm{~cm}^{2}$ ). These were then dried under a warm heat lamp - so as not to boil the solution and washed five times with $50 \mu \mathrm{L}$ of $1 \mathrm{M} \alpha-\mathrm{HIB}$. After the samples were allowed to dry, the samples and the $250 \mu \mathrm{L}$ of wash solution were counted for $\gamma$ activity with an HPGe spectrometer. Pa was found to sorb very strongly to $\mathrm{Ti}$, and to a lesser extent on Teflon and glass. The following results should be considered only preliminary because each material was tested only once. Similar experiments should be conducted for the organic solution for complete characterization.

\begin{tabular}{cc}
\hline Material & $\%$ Pa per $\mathrm{cm}^{2}$ \\
\hline Teflon & $1 \pm 1$ \\
Glass & $4 \pm 1$ \\
$\mathrm{Ti}$ & $18 \pm 2$ \\
\hline
\end{tabular}

It seems likely that the group 5 elements $\mathrm{Pa}$ and $\mathrm{Ha}$ (element 105) sorb so strongly on $\mathrm{Ti}$ that we were unable to observe them.

\section{Footnotes and References}

1. D. C. Hoffman et al., Phys. Rev. C 41, 631 (1990).

2. A. Ghiorso, M. Nurmia, K. Eskola, P. Eskola, Phys. Rev. C 4, 1850 (1971).

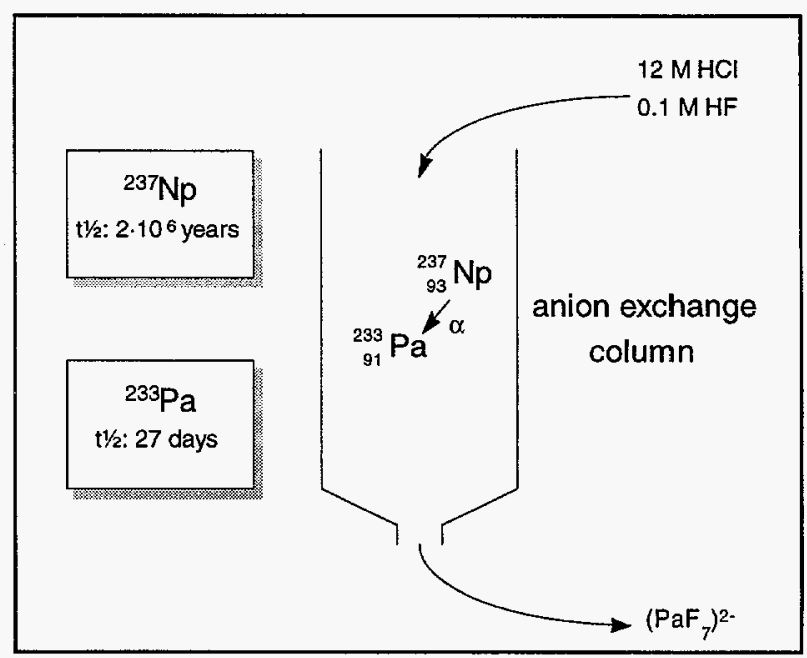

Fig. 1. ${ }^{233} \mathrm{~Pa}$ Generator 


\section{Production of ${ }^{211} \mathrm{Bi}$ from the Reaction of ${ }^{209} \mathrm{Bi}$ with ${ }^{22} \mathrm{Ne}$ Projectiles}

Y. H. Chung, * K. E. Gregorich, D. M. Lee, and D. C. Hoffman

Production of 2.14-min ${ }^{211} \mathrm{Bi}$ was investigated by bombarding $2.5 \mathrm{mg} / \mathrm{cm}^{2}{ }^{209} \mathrm{Bi}$ target with $140-$ $\mathrm{MeV}{ }^{22} \mathrm{Ne}$ ions, whose average energies at the target were $\sim 108 \mathrm{MeV}$ with correction of energy losses in the Be windows, $\mathrm{N}_{2}$ cooling gas, and the target itself, at the Lawrence Berkeley National Laboratory 88-Inch Cyclotron. The experiments were performed using the helium-jet system to transport the activity via a capillary from the production site to the MG rotating wheel ${ }^{1}$ for measurement of alpha activity. Eskola et al. ${ }^{2}$ studied multi-particle transfer reactions using the ${ }^{208} \mathrm{~Pb}\left({ }^{18} \mathrm{O}, \mathrm{pxn}\right){ }^{211-213} \mathrm{Bi}$ reactions in the $5-10$ $\mathrm{MeV} /$ nucleon range. In their study the production cross section of ${ }^{211} \mathrm{Bi}$ via $\mathrm{p} 2 \mathrm{n}$-transfers was $\sim 2 \mathrm{mb}$ in the vicinity of $110 \mathrm{MeV}$, which induced $\sim 55 \mathrm{MeV}$ excitation energy in the compound system. In our study, we deduced the cross section of ${ }^{211} \mathrm{Bi}$ using its two alpha-decay lines: 6.28 and $6.62 \mathrm{MeV}$. Based on their decay intensities, the excess intensity of the $6.62 \mathrm{MeV}$ line was obtained, and it was found to be due to the 6.65 and $6.66 \mathrm{MeV}$ lines from 2.1-min ${ }^{223} \mathrm{Ac}$. The excitation energy of the compound nucleus is about $36 \mathrm{MeV}$, which is lower by $20 \mathrm{MeV}$ than that estimated in Eskola's work. From our data, the preliminary cross section of ${ }^{211} \mathrm{Bi}$ via the $2 \mathrm{n}$ transfer reaction has been estimated to be $\sim 3 \pm 1$ $\mathrm{mb}$, assuming $70 \%$ gas transport efficiency. This result indicates that the $2 \mathrm{n}$ transfer cross section is comparable to the $\mathrm{p} 2 \mathrm{n}$ transfer, which was observed in the interaction of ${ }^{208} \mathrm{~Pb}$ with ${ }^{18} \mathrm{O}$ in this energy regime. Further detailed analysis of data obtained from the interaction of ${ }^{204} \mathrm{Bi}$ with $103-$ and $117-\mathrm{MeV}^{22} \mathrm{~N}$ ions and $128-, 140-$, and $149-\mathrm{MeV}^{26} \mathrm{Mg}$ ions is in progress.

Footnotes and References

*Department of Chemistry, Hallym University, Chunchon, Korea 200-702.

1. D. C. Hoffman et al., Phys. Rev. C41, 631(1990).
2. K. Eskola, P. Eskola, M. M. Fowler, H.Ohm, E. N. Treher, J. B. Wilhelmy, D. Lee and G.T. Seaborg, Phys. Rev. C29, 2160(1984). 


\section{Production Cross Sections for ${ }^{254} \mathrm{Fm}$ and ${ }^{256} \mathrm{Fm}$ in ${ }^{248} \mathrm{Cm}+{ }^{22} \mathrm{Ne}$ Reactions M.B. Hendricks, R.J. Wallsgrove, K.E. Gregorich, M.R. Lane, D.M. Lee, D.A. Shaughnessy, D.A. Strellis, E.R. Sylwester, P.A. Wilk, D.C. Hoffman}

The cross sections for ${ }^{254} \mathrm{Fm}$ and ${ }^{256} \mathrm{Fm}$ produced in the interactions of ${ }^{22} \mathrm{Ne}$ ions with ${ }^{248} \mathrm{Cm}$ were measured. Excitation functions provide information about the reaction mechanism of transfer reactions involving actinides. By studying these reactions, we can design better experiments for production of exotic isotopes, particularly neutron-excess actinides. More immediately, if the production cross sections are known, ${ }^{254} \mathrm{Fm}$ and ${ }^{256} \mathrm{Fm}$ can be used to measure the efficiency of transporting reaction products to the detectors during compound nucleus experiments to produce $\mathrm{Sg}$ isotopes in the ${ }^{248} \mathrm{Cm}$ $+{ }^{22} \mathrm{Ne}$ reaction.

${ }^{254} \mathrm{Fm}$ and ${ }^{256} \mathrm{Fm}$ were chosen for this experiment for three reasons. The ${ }^{22} \mathrm{Ne}+{ }^{248} \mathrm{Cm}$ reaction produces sufficient quantities of both isotopes. Detection of these nuclides is relatively easy as ${ }^{256} \mathrm{Fm}$ decays predominantly by spontaneous fission and ${ }^{254} \mathrm{Fm}$ emits a relatively high energy alpha particle that can be distinguished from the background activity. In addition, these Fm isotopes have half-lives that are suitable for this experiment.

Experiments were performed at two different cyclotron energies. With a lab energy of 139 $\mathrm{MeV}$ out of the cyclotron, the energy range of the $\mathrm{Ne}$ ions in the $1.26 \mathrm{mg} / \mathrm{cm}^{2}$ target was $114.4-118$ $\mathrm{MeV}$. This energy range includes the Coulomb barrier, which is close to $116 \mathrm{MeV}$. The experiment was performed with a second target of $0.96 \mathrm{mg} / \mathrm{cm}^{2}$. The projectile energy out of the cyclotron used with this target was $151 \mathrm{MeV}$ in the lab frame. This is degraded to $119-121.5 \mathrm{MeV}$ inside the target.

During each experiment, a gold foil was placed behind the $\mathrm{Cm}$ target to catch recoiling reaction products. The foil was then dissolved in aqua regia and the lanthanides and actinides were separated from the gold with an ion-exchange column. The eluate was dried on a platinum disk to produce a source for alpha and SF spectroscopy. These sources were monitored at various intervals with surface barrier detectors to discover isotopes that decay by alpha emission or by spontaneous fission.

Cross sections for production of the two isotopes were determined in each experiment. At the energy near the Coulomb barrier, the cross section for production of ${ }^{256} \mathrm{Fm}\left(\sigma_{256}\right)$ was found to be $0.27 \pm 0.11 \mu \mathrm{b}$ and the cross section for production of ${ }^{254} \mathrm{Fm}\left(\sigma_{254}\right)$ was measured as $5.3 \pm 1.1 \mu \mathrm{b}$. The ${ }^{254} \mathrm{Fm}$ value does not agree within $1 \sigma$ error limits with that obtained by Lee et al. at the same energy. ${ }^{1}$ In that study, $\sigma_{256}$ was measured as $0.15 \pm 0.04 \mu \mathrm{b}$ and $\sigma_{254}$ was quoted as $3.1 \pm 0.4 \mu \mathrm{b}$. In two experiments at the higher energy, the production cross sections increased. $\sigma_{256}$ was measured as $0.57 \pm 0.11 \mu \mathrm{b}$ and $0.48 \pm 0.08 \mu \mathrm{b} . \quad \sigma_{254}$ was found to be $9.7 \pm 1.4 \mu \mathrm{b}$ and $7.8 \pm 1.1 \mu \mathrm{b}$. These results indicate that further studies of this system should be undertaken. In particular, excitation functions over a wider energy range for these $\mathrm{Fm}$ isotopes should be produced.

\section{Footnotes and References}

1. D. Lee et al., Phys. Rev. C, 25286 (1982). 


\title{
Production of Neutron Deficient Plutonium Isotopes
}

\author{
M.B.Hendricks, K.E. Gregorich, M.R. Lane, D.M. Lee, D.A. Shaughnessy, D.A. Strellis, E.R. Sylwester, \\ P.A. Wilk, D.C. Hoffman
}

Production of three highly neutron-deficient isotopes of plutonium, ${ }^{229} \mathrm{Pu},{ }^{230} \mathrm{Pu}$ and unknown ${ }^{231} \mathrm{Pu}$, was attempted with the highly assymetric reaction ${ }^{233} \mathrm{U}\left({ }^{3} \mathrm{He}, 5-7 \mathrm{n}\right)$. An attempt was made to measure half-lives, alpha-decay energies and alpha/electron capture branching ratios for the three isotopes. Until Andreyev et al. identified ${ }^{228} \mathrm{Pu},{ }^{229} \mathrm{Pu}$ and ${ }^{230} \mathrm{Pu}$, the lightest plutonium isotope known was ${ }^{232} \mathrm{Pu}^{1,2}$ In these experiments, ${ }^{229} \mathrm{Pu}$ and ${ }^{230} \mathrm{Pu}$ were produced in a ${ }^{208} \mathrm{~Pb}\left({ }^{26} \mathrm{Mg}, 4-5 \mathrm{n}\right)$ reaction. Production of ${ }^{228} \mathrm{Pu}$ was achieved by a similar reaction: ${ }^{208} \mathrm{~Pb}\left({ }^{24} \mathrm{Mg}, 4 \mathrm{n}\right)$. Andreyev et al. were able to measure alphadecay energies of these new isotopes, but could not measure half-lives or branching ratios.

The ${ }^{233} \mathrm{U}\left({ }^{3} \mathrm{He}, 5-7 \mathrm{n}\right)$ experiment was performed to study both decay properties and reaction mechanisms. Knowledge of decay characteristics of highly neutron-deficient plutonium isotopes is valuable to the study of electron capture delayed fission (ECDF) of light americium isotopes, since these plutonium isotopes are members of the americium decay chains. Determination of plutonium masses and decay Q-values aids estimation of electron-capture Q-values for potential ECDF precursors, like ${ }^{230} \mathrm{Am}$. By studying the reaction mechanism involved in different systems, investigators can design better experiments. The two main criteria in designing an experiment to study exotic nuclei are high production rate of the nuclide under investigation and low production rate of interfering background activity. Comparisons between the lead target heavy-ion beam system used by Andreyev et al. and the actinide target light-ion beam system used in this experiment will aid in designing future experiments involving neutron-deficient actinides.

Eleven ${ }^{233} \mathrm{U}$ targets were mounted in the lightion multiple target system. ${ }^{3}$ The reaction products were transported via a helium jet system from the reaction chamber and deposited on polypropylene foils in our MG rotating wheel system. It can be rotated to place the foils successively between six pairs of surface barrier detectors. Identification of a plutonium isotope relies on observation of the alpha decay of members of its decay chain. Measurements were attempted at three cyclotron energies: 40,50 and $60 \mathrm{MeV}$ in the laboratory frame. The helium ions lose less than $1 \mathrm{MeV}$ in interactions with the cyclotron window and the target system itself. The ${ }^{231} \mathrm{Pu},{ }^{230} \mathrm{Pu}$ and ${ }^{229} \mathrm{Pu}$ cross sections were predicted to peak at these energies, respectively.

Many alpha-decay chains were observed. However, large amounts of interfering activity were produced. This activity may have been due to $\left({ }^{3} \mathrm{He}, \mathrm{pxn}\right)$ and $\left({ }^{3} \mathrm{He}, \alpha \times \mathrm{xn}\right)$ reactions that directly yield neptunium and uranium isotopes. These high background levels and relatively long halflives of the plutonium, neptunium, and uranium isotopes at the beginning of the deay chains made determination of half-lives and branching raios of the light plutonium isotopes impossible. These problems will be avoided in the future by chemically separating plutonium, neptunium and uranium reaction products from each other before attempting detection of alpha-decay.

\section{Footnotes and References}

1. A.N. Andreyev et al., Z. Phys. A, 337, 231 (1990).

2. A.N. Andreyev et al., Z. Phys. A, 347,225 (1994).

3. H.L. Hall, M.J. Nurmia and D.C. Hoffman, Nucl. Instrum. Meth., A276, 649 (1989). 


\section{Electron-Capture Delayed Fission of ${ }^{242}$ Es \\ D.A. Shaughnessy, K.E. Gregorich, M.B. Hendricks, M.R. Lane, D.M. Lee, D.A. Strellis, E.R. Sylwester, P.A. Wilk, D.C. Hoffman}

We previously reported evidence that ${ }^{242}$ Es undergoes electron-capture delayed fission $(E C D F .)^{1}$ We have performed additional experiments in order to determine the decay properties of ${ }^{242} \mathrm{Es}$ and its probability of delayed fission $\left(\mathrm{P}_{\mathrm{DF}}\right)$

The ${ }^{242}$ Es was produced at the LBNL 88-Inch Cyclotron via the ${ }^{233} \mathrm{U}\left({ }^{14} \mathrm{~N}, 5 \mathrm{n}\right){ }^{242} \mathrm{Es}$ reaction. To prepare the target ${ }^{233} \mathrm{U}$ was placed on an anion exchange column and washed several times with concentrated $\mathrm{HCl}$ to remove lead impurities. The target material was eluted from the column with dilute $\mathrm{HCl}$ and electroplated on Be foil. Reaction products from the cyclotron were transported via a $\mathrm{He} / \mathrm{KCl}$ aerosol gas-jet through a capillary to our MG rotating wheel detection system. The aerosols were deposited consecutively on 80 thin polypropylene foils located on the periphery of the wheel which was moved every 10 seconds between six pairs of $\mathrm{Si}(\mathrm{Au})$ surface barrier detectors.

${ }^{242} \mathrm{Es}$ is reported to decay with a $7.9 \mathrm{MeV}$ alpha particle and a half-life of $16_{-4}^{+0}$ seconds. ${ }^{2}$ We looked for alpha particles coming from ${ }^{242} \mathrm{Es}$, as well as its EC daughter, ${ }^{242} \mathrm{Cf}$. The ${ }^{242}$ Es branching ratio and initial EC activity could be determined by comparing the production of ${ }^{242} \mathrm{Cf}$ to that of ${ }^{242} \mathrm{Es}$. Once the initial EC activity is measured, the $\mathrm{P}_{\mathrm{DF}}$ is calculated by comparing the number of fission events to the total number of EC decays.

Even though most of the lead was removed from the ${ }^{233} \mathrm{U}$ target, a large amount of ${ }^{213} \mathrm{Fr}$ was made. Fr is produced when lead is bombarded with nitrogen. This huge peak in the spectra obscured the much smaller ${ }^{242} \mathrm{Cf}$ peak at 7.385 $\mathrm{MeV}$ making the $\mathrm{EC}$ branching ratio impossible to determine. This in turn prevented us from measuring the $\mathbf{P}_{\mathrm{DF}}$.

Another problem we encountered was the production of ${ }^{243} \mathrm{Es}$ at $7.895 \mathrm{MeV}$. We ran the ${ }^{14} \mathrm{~N}$ at a higher energy than before ( $91 \mathrm{MeV}$ on target) to try and suppress ${ }^{243}$ Es production, but enough was made to interfere with the ${ }^{242}$ Es peak at 7.9 $\mathrm{MeV}$. When this entire region was integrated, it was found to decay with a half-life of 35 seconds. This is longer than that of ${ }^{243} \mathrm{Es}$ (21 seconds) which indicates that the region was probably a combination of both ${ }^{242} \mathrm{Es}$ and ${ }^{243} \mathrm{Es}$. Unfortunately our system could not resolve the two into separate peaks.

We saw 16 coincident fissions with a half-life of $25 \pm 2$ seconds. Since the fissions decay with the half-life of the EC parent, this would also be the half-life of ${ }^{242} \mathrm{Es}$. This is slightly longer than previously reported. The fission fragments had an asymmetric mass distribution and a preneutron average total kinetic energy (TKE) of 199 $\pm 21 \mathrm{MeV}$. This is 6 to $13 \mathrm{MeV}$ higher than semiempirical fits predict for ${ }^{242} \mathrm{Cf}^{3}{ }^{3}$

Based on the fission half-life, the fission events could not have come from a different Es isotope. Also, the $P_{D F}$ has been shown to increase sharply with increasing electron-capture $Q$-value $\left(Q_{\mathrm{EC}} \cdot\right)^{4}$ The $Q_{E C}$ of ${ }^{242} E s$ is greater than all other $E s$ isotopes by at least $1 \mathrm{MeV}$ making it the most probable delayed fission precursor. The $\mathrm{Q}_{\mathrm{EC}}$ of its neighboring isotopes would correspond to a $P_{D F}$ too small to account for 16 fissions over the whole experiment, implying that the ECDF was from ${ }^{242} \mathrm{Es}$.

\section{Footnotes and References}

1. Nuclear Science Division Annual Report, LBL37384 (1994.)

2. V. Ninov, private communication (1997.)

3. D.C. Hoffman and M.R. Lane, Radiochimica Acta, 70/71, 135 (1995).

4. S.A. Kreek et al., Phys. Rev. C $\underline{50} 2288$ (1994). 


\section{Electron-Capture Delayed Fission Study of ${ }^{246}$ Es \\ D.A. Shaughnessy, K.E. Gregorich, M.B. Hendricks, M.R. Lane, D.M. Lee, C.A. McGrath, D.A. Strellis, E.R. Sylwester, P.A. Wilk, D.C. Hoffman}

We continued our study of electron-capture delayed fission (ECDF) in the neutron-deficient Es isotopes with ${ }^{246} \mathrm{Es}$. The ${ }^{246} \mathrm{Es}$ was produced at the LBNL 88-Inch Cyclotron via the ${ }^{249} \mathrm{Cf}(\mathrm{p}$, $4 n)^{246}$ Es reaction. According to $\mathrm{SPIT}^{1}$, a neutron evaporation code, the production cross section would be $350 \mu \mathrm{b}$ for $37 \mathrm{MeV}$ protons. Based on this cross section, we expected to make $1 \times 10^{8}$ ${ }^{246}$ Es per day.

The ${ }^{249} \mathrm{Cf}$ targets were prepared by first performing an extraction with bis(2ethylhexyl)orthophosphoric acid (HDEHP) to remove $\mathrm{Bk}$, and then electroplating the $\mathrm{Cf}$ onto Be foils. 19 targets between $6 \mu \mathrm{g} / \mathrm{cm}^{2}$ and 20 $\mu \mathrm{g} / \mathrm{cm}^{2}$ were prepared and stacked in our light ion multiple (LIM) target system. ${ }^{2}$ Reaction products were transported with a $\mathrm{He} / \mathrm{KCl}$ aerosol jet via a capillary to our MG rotating wheel detection system. The aerosols were collected consecutively on 80 thin polypropylene foils located on the periphery of the wheel which was moved every two minutes between six pairs of PIPS detectors to look for ${ }^{246} \mathrm{Es}$ alpha particles as well as fissions. Some samples were removed from the foils with a buffered solution of acetic acid and sodium acetate maintained at $\mathrm{pH} 5$ and extracted with $0.5 \mathrm{M}$ thenoyltrifluoroacetone (TTA) in toluene. TTA complexes $3+$ and higher oxidation states from aqueous solutions between $\mathrm{pH} 4$ and $5 .^{3}$ Es and $\mathrm{Cf}$ are complexed by the TTA, removing them from the $\mathrm{KCl}$ and any interfering activities. The TTA was dried on glass cover slips and placed in front of a solid state particle detector which was sandwiched between two $x$-ray detectors. This configuration allowed us to look for $\mathrm{K} x$-rays, which follow $\mathrm{EC}$ decay, in coincidence with fission fragments. Once the initial EC activity is determined, the probability of delayed fission $\left(\mathrm{P}_{\mathrm{DF}}\right)$ is calculated by comparing the number of delayed fissions to the total number of EC decays.
During the experiment we saw 25 times fewer ${ }^{246}$ Es than predicted. The spectra showed multiple components in the Es region indicating that several isotopes were being produced concurrently. The last samples collected were counted for several days after the end of the experiment to look for ${ }^{246} \mathrm{Cf}\left(\mathrm{t}_{1 / 2}=1.5 \mathrm{~d}\right.$.) Based on the amount of ${ }^{246} \mathrm{Cf}$, a production cross section of $13 \pm 5 \mu \mathrm{b}$ was calculated for ${ }^{246} \mathrm{Es}$. This is much lower than the original prediction and accounts for the lower production rate. The other Es isotopes seen in the spectra were predicted to have much lower production rates than ${ }^{246}$ Es. We now believe that the predicted cross sections in this region are not valid for $(p$, $\mathrm{xn})$ reactions.

We plan to run an Es excitation function to determine optimal proton energies and cross sections for each isotope. If the cross section is large enough, the delayed fission experiment will be repeated to produce more ${ }^{246} \mathrm{Es}$ than we did with $37 \mathrm{MeV}$ protons. This in turn should lead to more fission events and a determination of the $P_{D F}$.

\section{Footnotes and References}

1. SPIT is a version of the JORPLE code with a proximity potential in the entrance channel. The JORPLE reference is J. Alonso, Gmelin Handbuch der Anorganischen Chemie, 7b, 28 (1974.)

2. H. L. Hall, LBL Report LBL-27878 (1989.)

3. S. A. Kreek, LBL Report LBL-33766 (1993.) 


\title{
Design of a Sample Changer/Transport System to be used for Gammasphere Studies on Electron-capture Delayed Fission
}

\author{
D.A. Strellis, K.E. Gregorich, Y.H. Chung, M.R. Lane, E.R. Sylwester, M.B. Hendricks, D.A. \\ Shaughnessy, C.A. McGrath, P.A. Wilk, D.M. Lee, D.C. Hoffman
}

A sample changer/transport system has been designed to rapidly transport isotopes from high beam intensity irradiations in Cave 0 to the center of Gammasphere to study electron-capture delayed fission (ECDF).

In the Cave 0 target system, recoiling reaction products are captured by $\mathrm{KCl}$ aerosols in a flowing stream of He gas. The activity-laden aerosols are transported from the irradiation vault to the Gammasphere cave through a polyvinyl chloride capillary tube. The aerosol is then deposited on a thin polypropylene foil at one of four stations on a sample changer wheel. This wheel sits approximately four feet from the center of the Gammasphere array. After a specified collection time, the wheel is rotated $90^{\circ}$. A solenoidoperated piston then moves the foil from the wheel to the center of the array. After a specified counting time, the piston returns the foil to its wheel position. After the wheel rotates another $90^{\circ}$, the counted foil is deposited into a reservoir of used foils. After another $90^{\circ}$ rotation, an unused foil is dropped from a reservoir of new foils to the open wheel position. This four station process is continuous and simultaneous. For example, when one foil is being counted in Gammasphere; one foil is collecting aerosol from the capillary tube, one foil is being dumped into the used reservoir, and another is being dropped into the open position on the wheel.

A motion controller program controls the stepping motor that turns the wheel and the solenoids that activate the piston and vacuum systems. These programs are written on a PC and downloaded to the motion controller before the experiment.

At the counting station, the activitybearing polypropylene foil will be placed between two ion-implanted $\mathrm{Si}$ detectors at the center of Gammasphere. Fission fragments, alpha particles, $\mathrm{K} x$-rays, and gamma rays will be measured while the sample sits inside the array.

Gammasphere is an ideal detection system for studying the gamma spectroscopy associated with the ECDF process for two primary reasons. First, the small solid angle of each detector prevents summing of the ECDF gamma rays with those from the deexcitation of the fission fragments. Second, a high detector efficiency is desired when dealing with relatively low production rates.

The centerpiece of the Gammasphere measurements will be a search for gamma transitions within the second well of the nuclear potential. Based on results from earlier measurements of the $\mathrm{K} x$-rays in coincidence with delayed fission fragments, we have found that in regions of $N$ and $Z$ where a fission isomer is expected, the delayed fission process is slow enough for the K-vacancies left by the electron capture of the precursor to fill. The most likely explanation for this delay is that the ECDF process is proceeding through the fission shape isomer in the EC daughter. The observation of some common gamma transitions in coincidence with various fission fragments and $\mathrm{K} x$-rays would be indicative of transitions within the second well.

The first experiment to utilize this sample changer/transport system will take place in Spring 1997 with the ECDF study ${ }^{1,2}$ of ${ }^{232} \mathrm{Am}$ and ${ }^{234} \mathrm{Am}$ with half-lives: 1.31 \pm 0.04 and $2.32 \pm 0.08$ minutes, respectively. The speed at which the sample changer/transport system moves the sample from the target area to Gammasphere could be as low as 10 seconds- a benefit in studying isotopes with short half-lives.

\section{Footnotes and References}

1. H.L. Hall, et.al., Phys. Rev. C 41 , 618 (1990).

2. H.L. Hall and D.C. Hoffman, Annual Rev. Nucl. Part. Sci. $\underline{42}, 147$ (1992). 


\title{
Confirmation of the Assignment of a 1.5-Second Fission Activity to ${ }^{259} \mathrm{Fm}$
}

\author{
C. A. McGrath, K. E. Gregorich, D. M. Lee, M. R. Lane, E. R. Sylwester, D. A. Shaughnessy, M. B.
} Hendricks, D. A. Strellis, B. Wierczinski, D. C. Hoffman

In 1995, two separate experiments were performed at the 88-Inch Cyclotron at Lawrence Berkeley National Laboratory to study the nuclear properties of ${ }^{266} \mathrm{Sg}$ using the reaction ${ }^{248} \mathrm{Cm}\left({ }^{22} \mathrm{Ne}, 4 \mathrm{n}\right){ }^{266} \mathrm{Sg}$ at $118 \mathrm{MeV}$ and $121 \mathrm{MeV}$. The results of these experiments will be discussed in a separate submission to this annual report. However, the use of this particular reaction provides an important opportunity to study the production of ${ }^{258} \mathrm{Fm}$ and ${ }^{259} \mathrm{Fm}$ in binary transfer reactions.

The assignment of a 1.5-second spontaneous fission(SF) decay to ${ }^{259} \mathrm{Fm}$ results from the tritium bombardment of a ${ }^{257} \mathrm{Fm}$ target ${ }^{1}$. Since ${ }^{258} \mathrm{Fm}$ was already known to decay by. SF with a half life of $0.37 \mathrm{~ms}$, the observed 1.5-second fission activity was assigned to ${ }^{259} \mathrm{Fm}$, produced by the ${ }^{257} \mathrm{Fm}(\mathrm{t}, \mathrm{p})^{259} \mathrm{Fm}$ reaction.

However, the possible discovery ${ }^{2}$ of a SF isomer in ${ }^{262} \mathrm{Rf}$ suggests that the 1.5 second fission activity could be from an isomer of ${ }^{258} \mathrm{Fm}$. Because the target material is very scarce and finding a tritium accelerator is next to impossible, it would be difficult to repeat the original measurement. Even then it might still be difficult to differentiate between the $(t, p)$ and $(t, d)$ reactions.

Therefore, a different reaction was chosen to try to confirm the assignment of the 1.5 -second fission activity. This reaction must selectivity produce either ${ }^{258} \mathrm{Fm}$ or ${ }^{259} \mathrm{Fm}$. Bombarding ${ }^{248} \mathrm{Cm}$ with ${ }^{22} \mathrm{Ne}$ provides just such a reaction.

Several different isotopes of fermium are produced via transfer reactions in the bombardment of. ${ }^{248} \mathrm{Cm}$ with ${ }^{22} \mathrm{Ne}$. The excitation energy $\left(E^{*}\right)$ of the target-like fragments help indicate the relative yields from the binary transfer reaction ${ }^{3}$. If the $E^{*}$ is very negative that isotope will be formed with lower yield. A positive $\mathrm{E}^{*}$ below the threshold for neutron emission and fission is ideal for production of a particular nuclide. The following table shows the $E^{*}$ values for the production of several fermium isotopes.

$\begin{array}{cc}\text { Table 1: } \mathrm{E}^{*} \text { for }{ }^{248} \mathrm{Cm}\left({ }^{22} \mathrm{Ne},{ }_{\mathrm{Be}}{ }^{270-\mathrm{A}} \mathrm{Fm}\right. \\ \frac{\text { Isotope }}{257} & \frac{\mathrm{E}^{*}(\mathrm{MeV})}{}{ }^{\#} \\ { }^{258} \mathrm{Fm} & 5.7 \\ { }^{259} \mathrm{Fm} & 6.5 \\ 260 \mathrm{Fm} & -7.8 \\ & -15.0\end{array}$

This table shows that conditions are optimal for the production of ${ }^{258} \mathrm{Fm}$, but not for ${ }^{259} \mathrm{Fm}$. This is, however, a blessing. While ${ }^{258} \mathrm{Fm}$ should be produced in measurable quantities, production of ${ }^{259} \mathrm{Fm}$ should be very limited. Therefore, the presence or absence of a 1.5-second fission activity should indicate its origin.

After analyzing all the available data, no evidence is seen for a 1.5 -second SF activity. An upper limit on the cross section for production of a 1.5-second activity was found to be $150 \mathrm{pb}$. Comparison of this reaction with similar transfer reactions would indicate a cross section of approximately $10 \mathrm{nb}$ for the production of ${ }^{258} \mathrm{Fm}$. A longer-lived isomer would be expected to receive some significant portion of this cross section. Thus, it is likely that the 1.5-second SF activity arises from ${ }^{259} \mathrm{Fm}$ and not from ${ }^{258} \mathrm{Fm}$.

\section{Footnotes and References}

\# $\mathrm{E}^{*}$ values calculated at the coulomb barrier 1. Hoffman, D. C. , et al, "Discovery of ${ }^{259} \mathrm{Fm}$," Proc. 3rd Intl. Conf. on Nuclei Far from Stability, Cargese, Corsica, France, 19-26 May, 1976, Geneva, CERN 75113, 15 July 1976, p. 558.

2. Lane, M. R. et al, Phys. Rev. C. 53, 2893 (1996).

3 Hoffman, D. C. and Hoffman, M. M. " Calculated

Excitation Energies for Transcurium Products of Binary Heavy Ion Reactions;" LA-UR-82-824 (1982). 


\section{Spontaneous fission properties of ${ }^{262} \mathbf{R f}$}

M.R. Lane, K.E. Gregorich, D.M. Lee, M.F. Mohar, M. Hsu, C.D. Kacher, B. Kadkhodayan, M.P. Neu, N.J. Stoyer, E.R. Sylwester, J.C. Yang, and D.C. Hoffman

In the time since our experiment on the spontaneous fission properties of ${ }^{262} \mathrm{Rf}$ was last reported ${ }^{1}$, we have obtained more data and have published the results. We have measured the mass and kinetic-energy distributions of 200 pairs of coincident fission fragments from the spontaneous fission (SF) of ${ }^{262} \mathrm{Rf}$. The ${ }^{262} \mathrm{Rf}$ was produced via the ${ }^{244} \mathrm{Pu}\left({ }^{22} \mathrm{Ne}, 4 n\right)$ reaction with a production cross section of $\sim 0.7 \mathrm{nb}$ using 114.4$\mathrm{MeV}$ projectiles. The kinetic energies and times of the coincident fission fragments were measured using our rotating wheel system. From these data the half-life, mass, and kineticenergy distributions were derived. The total kinetic-energy (TKE) distribution (Fig. 1) appears to consist of a single component with a most probable pre-neutron-emission TKE of $215 \pm 2$ $\mathrm{MeV}$. The mass distribution (Fig. 2) is symmetric with a full width at half maximum of about 22 mass numbers. These results are consistent with trends observed for other trans-berkelium spontaneously fissioning isotopes. We determined the half-life to be $2.1 \pm 0.2 \mathrm{~s}$ by measuring its spontaneous fission decay. We also attempted to observe the alpha decay of ${ }^{262} \mathrm{Rf}$ by searching for alpha decay correlated in time with SF from the alpha daughter, $1.2-\mathrm{ms}{ }^{258} \mathrm{No}$. We observed no such decays and have set an upper limit of $0.8 \%$ ( $68 \%$ confidence level) on the alpha decay branch of ${ }^{262} \mathrm{Rf}$.

\section{References}

1. D.C. Hoffman et al.; K.E. Gregorich et al., LBL35768, Annual Report (1993).

2. M.R. Lane et al, Phys. Rev. C $\underline{53}, 2893$ (1996).

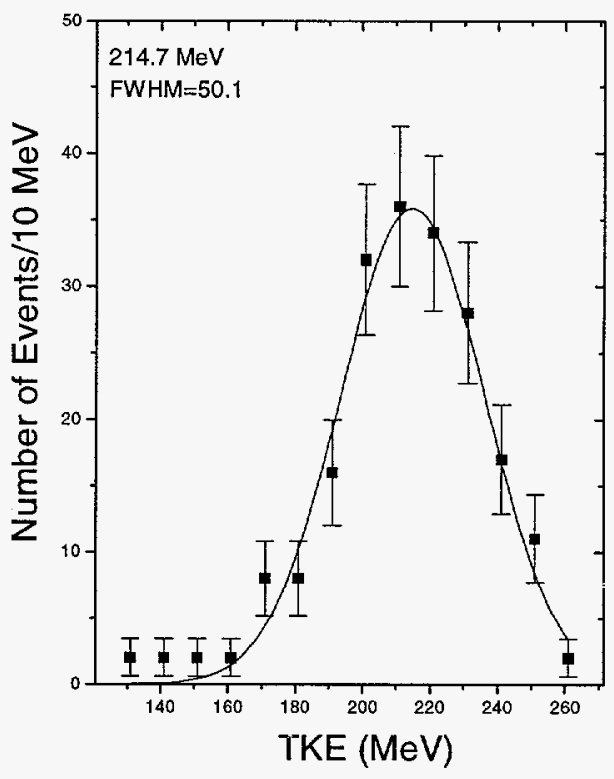

Fig. 1. Gaussian fit to the pre-neutron-emission TKE distribution from the SF of ${ }^{262} R f$.

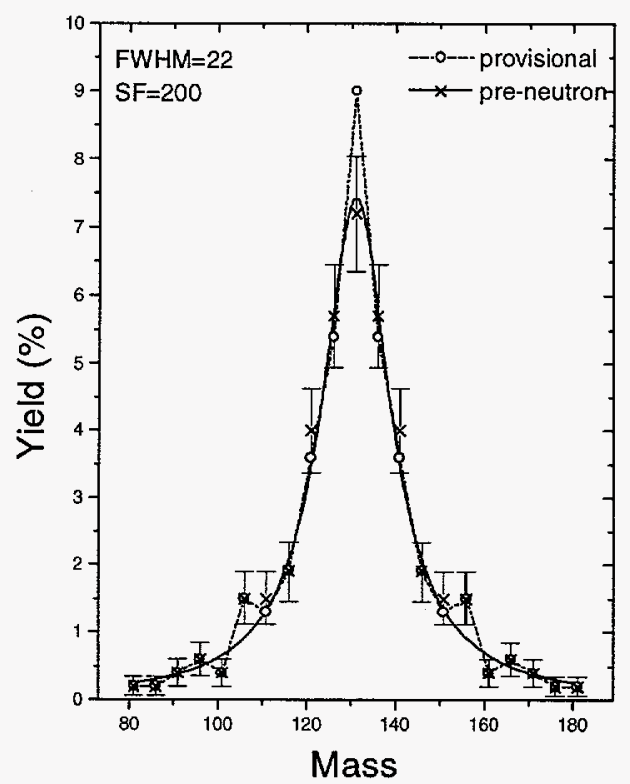

Fig. 2. Lorentzian fit to the pre-neutron-emission mass-yield distribution for ${ }^{262} \mathrm{Rf}$. Also shown is the provisional mass-yield curve to which no neutron correction was applied. The bars indicate $1 \sigma$ error limits. 


\section{Spontaneous fission reviews}

D.C. Hoffman, T.M. Hamilton, ${ }^{\dagger}$ M.R. Lane

Two spontaneous fission review articles have been published. Each presents the known data for spontaneous fission properties. The systematics are discussed and compared to theoretical models.

I. Spontaneous Fission ${ }^{1}$ by Darleane Hoffman, Todd Hamilton and Michael Lane. Since the comprehensive Hoffman and Somerville review ${ }^{2}$ of experimentally determined spontaneous fission properties published in 1989 (literature to mid-1986 considered), major conferences have been held at Berlin and Washington, D.C. in 1989 to commemorate the 50th anniversary of the discovery of nuclear fission. Many review papers and much new information concerning spontaneous fission were presented. The intent of the present review is to update the information of Hoffman and Somerville on spontaneous fission half-lives, fission fragment kinetic energy, mass and charge distributions, neutron and photon emission at scission or from the excited fragments and new theoretical developments. New experimental results on SF phenomena are reviewed. The relevant published literature to mid-1992 has been considered.

II. Spontaneous Fission ${ }^{3}$ by Darleane Hoffman and Michael Lane. Recent experimental results for spontaneous fission half-lives, total kinetic energies, fragment mass and kinetic-energy distributions, and other properties of the fragments are reviewed and compared with recent theoretical models. The experimental data lend support to the existence of the predicted deformed shells near $Z=108$ and $N=162$. Prospects for extending detailed studies of spontaneous fission properties to elements beyond hahnium (element 105) are discussed. The relevant published literature to mid-1995 has been considered. This article updates the information from the last published review by Hoffman, Hamilton, and Lane ${ }^{1}$ (see above).

\section{Footnotes and References}

† Present address: IUCF, Bloomington, IN

1. D.C. Hoffman et al.: Spontaneous Fission, in Nuclear Decay Modes, D.N. Poenaru, Ed., IOP Publishing, Bristol UK, chap. 10 (1996).

2. D.C. Hoffman and L.P. Somerville: Spontaneous Fission, in Charged Particle Emission from Nuclei, Vol. III, D.N. Poenaru and M.S. Ivascu, Eds., CRC Press Inc., Boca Raton, Florida, chap. 1 (1989).

3. D.C. Hoffman and M.R. Lane, Radiochim. Acta 70/71, 135 (1995). 


\title{
Relative Deformations of Superdeformed Bands in ${ }^{131,132} \mathbf{C e}$.
}

\author{
R.M.Clark ${ }^{1}$, I.Y.Lee ${ }^{1}$, P.Fallon ${ }^{1}$, D.T.Joss ${ }^{2}$, S.J.Asztalos ${ }^{1}$, J.A.Becker ${ }^{3}$, L.Bernstein ${ }^{3}$, B.Cederwall ${ }^{1}$, \\ M.A.Deleplanque ${ }^{1}$, R.M.Diamond ${ }^{1}$, L.P.Farris ${ }^{3}$, K.Hauschild ${ }^{4}$, W.H.Kelly ${ }^{5}$, A.O.Macchiavelli ${ }^{1}$, \\ P.J.Nolan ${ }^{2}$, N.O'Brien ${ }^{4}$, A.T.Semple ${ }^{2}$, F.S.Stephens ${ }^{1}$, R.Wadsworth ${ }^{4}$ \\ 1 Nuclear Science Division, Lawrence Berkeley National Laboratory, Berkeley CA 94720, U.S.A. \\ 2 Oliver Lodge Laboratory, University of Liverpool, Liverpool, L69 $3 B X, U K$ \\ ${ }^{3}$ Lawrence Livermore National Laboratory, Livermore, California 94550 \\ ${ }^{4}$ Department of Physics, University of York, Heslington, York, YO1 5DD, UK \\ ${ }^{5}$ Iowa State University, Ames, Iowa 50011, U.S.A
}

The quadrupole deformations of states in some SD bands have been determined through lifetime measurements and confirm their SD nature, but very little is known about the relative deformations of yrast and excited SD bands in the same, and neighbouring, nuclei. These differences should reflect the deformation-driving effects of specific configurations and the stability of the second minimum with respect to various nucleon excitations. This information is crucial in providing a stringent test of current theoretical models. With this in mind, superdeformed states in ${ }^{131,132} \mathrm{Ce}$ were populated via the ${ }^{100} \mathrm{Mo}\left({ }^{36} \mathrm{~S}, \mathrm{xn}\right)$ reactions at a beam energy of $155 \mathrm{MeV}$. The target comprised a $\sim 600 \mu \mathrm{g} / \mathrm{cm}^{2}$ ${ }^{100} \mathrm{Mo}$ foil evaporated on a $12 \mathrm{mg} / \mathrm{cm}^{2}$ Au backing which slows down and stops the recoiling nuclei. The beam was provided by the $88-\mathrm{Inch} \mathrm{Cy}$ clotron at the Lawrence Berkeley National Laboratory, and $\gamma$ rays were detected by the Gammasphere array which, for this experiment, had 55 large-volume HpGe detectors. A total of $9 \times 10^{8}$ events with a fold $\geq 5$ was collected. A Doppler Shift Attenuation Method (DSAM) centroid shift analysis was then performed.

Experimental fractional Doppler shift, $F(\tau)$, curves were extracted and are shown in the figure. Calculated $F(\tau)$ curves, assuming a rotational cascade with a constant quadrupole moment $(6.4,7.4$, and $8.4 \mathrm{eb})$ and using the stopping powers of Ziegler et al., are shown for comparison with the data. Comparing the curves allowed a number of important conclusions to be drawn. We have extracted the relative deforma- tions of all five known SD bands in ${ }^{131,132} \mathrm{Ce}$ to an accuracy of $\simeq 5-7 \%$. We find that the yrast bands in these two nuclei have very similar deformations implying that the shape-driving force of the $N=6$ neutron orbital is less than previously thought. The yrast and excited bands in ${ }^{132} \mathrm{Ce}$ have very similar deformations indicating that the second minimum is stable to the excitations responsible for these excited bands. The excited band in ${ }^{131}$ Ce has a significantly larger deduced Qo than any of the other bands. Information on the effect and nature of side-feeding was also extracted and it was found that there is a significant slow side-feeding component for the yrast band in ${ }^{131} \mathrm{Ce}$.

Further details of the work can be found in [1]

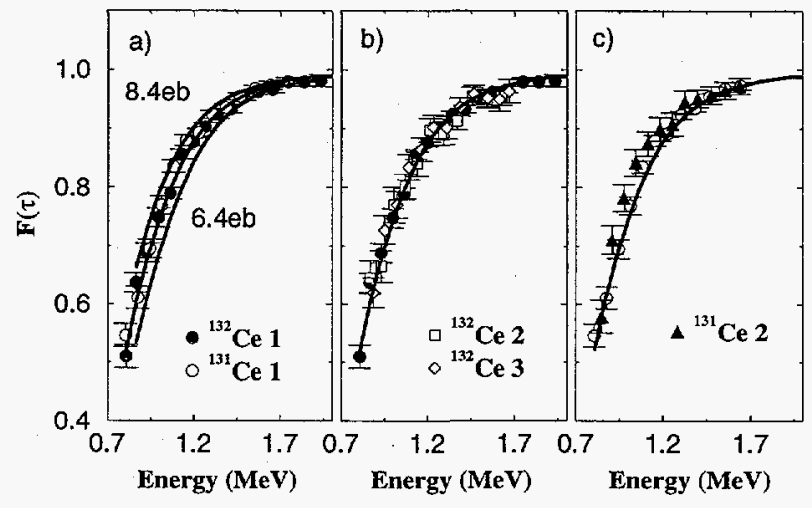

\section{References}

[1] R. M. Clark et al., Phys. Rev. Lett. 76 (1996) 3510 . 


\title{
Low-Spin Termination of the Superdeformed Band in ${ }^{135} \mathrm{Nd}$
}

\author{
M.A.Deleplanque ${ }^{1}$, S. Frauendorf ${ }^{1}$, R.M.Clark ${ }^{1}$, R.M.Diamond ${ }^{1}$, F.S.Stephens ${ }^{1}$, J.A.Becker ${ }^{2}$, \\ M.J.Brinkman ${ }^{2}$, B. Cederwall ${ }^{1}$, P.Fallon ${ }^{1}$, L.P.Farris ${ }^{2}$, E.A.Henry ${ }^{2}$, H.Hübel ${ }^{\beta}$, \\ J.R.Hughes ${ }^{2}$, W.Korten ${ }^{3}$ I.Y.Lee ${ }^{1}$, A.O.Macchiavelli ${ }^{1}$, M.A.Stoyer ${ }^{2}$, \\ P.Willsau ${ }^{3}$, J.E.Draper ${ }^{4}$, C.Duyar ${ }^{4}$, E.Rubel ${ }^{4}$ \\ 1 Nuclear Science Division, Lawrence Berkeley National Laboratory, Berkeley CA 94720, U.S.A. \\ ${ }^{2}$ Lawrence Livermore National Laboratory, Livermore, California 94550 \\ ${ }^{3}$ Institut für Strahlen- und Kernphysik, Universität Bonn, D-53115 Bonn, Germany \\ 4 University of California, Davis, CA 95616
}

In an effort to understand the decay out of superdeformed (SD) bands, the nucleus ${ }^{135} \mathrm{Nd}$ was studied with the early implementation of Gammasphere at the 88-Inch Cyclotron of the Lawrence Berkeley National Laboratory. In two separate experiments, the reaction ${ }^{40} \mathrm{Ar}$ on ${ }^{100} \mathrm{Mo}$ at 182 and $176 \mathrm{MeV}$ produced $1.0 \times 10^{9}$ and $1.8 \times 10^{9}$ three and higher fold suppressed events, respectively. Both angular correlations and triple $\gamma$ coincidences were used to construct the level scheme of Fig. 1. A total of $75 \%$ of the decay of the SD band (and $84 \%$ of the decay of the $3.324 \mathrm{MeV}$ state) has been placed in the level scheme. These results determine the spins of the SD states. Also the proposed positiive signature is consistent with the $i_{13 / 2}$ neutron configuration previously assigned. The intensity of the linking transitions, together with previous RDM lifetime measurements [1] indicate that the observed E2 linking transitions have a reduced transition probability that is a factor about 10 lower than that of a SD band transition. Therefore, we propose that the band ceases to exist below the $3.324 \mathrm{MeV}$ energy level.

Ultimate Cranker calculations with pairing, in which the $i_{13 / 2}$ configuration could be followed, were used to try to understand this behavior. The results show that, as the frequency decreases, a lower-deformation triaxial minimum competes with the SD minimum and the nucleus "slides over" from a high-deformation to the triaxial minimum. The rapid change is a consequence of pair correlations. The pair field will scatter pairs between the neutron levels $\left(h_{9 / 2}\right.$ $\left.\leftrightarrow \mathrm{g}_{7 / 2}\right)$ and proton levels $\left(\mathrm{h}_{11 / 2} \leftrightarrow \mathrm{g}_{7 / 2}\right)$ distinguishing the two minima. The example of ${ }^{135} \mathrm{Nd}$ clearly demonstrates the importance of underlying structural changes in the decay of highly deformed configurations [2]. Such configurations could play the role of doorway states in other decays of SD bands in which a larger number of pairs is rearranged.

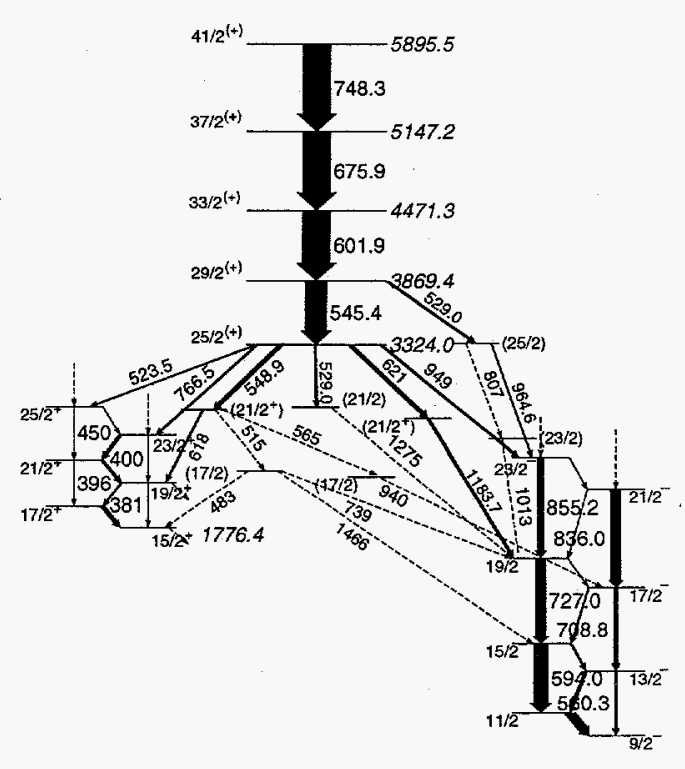

\section{References}

[1] P. Willsau et al., Phys.Rev.C48, R494 (1993).

[2] M.A. Deleplanque et al., Phys.Rev.C52, R2302 (1995). 


\title{
Proton Superdeformed Bands below the $\mathrm{Z}=80$ Gap in ${ }^{191} \mathrm{Au}$ : More Evidence for Pseudo-spin Coupling
}

\author{
C. Schück ${ }^{1}$, E. Gueorguieva ${ }^{1}$, A. Lopez-Martens ${ }^{1}$, F. Hannachi ${ }^{1}$, Ch. Vieu ${ }^{1}$, M. Kaci ${ }^{1}$, J.S. Dionisio ${ }^{1}$, \\ W.H. Kelly ${ }^{2}$, M.A. Deleplanque ${ }^{3}$, F.S. Stephens ${ }^{3}$, B. Kharraja ${ }^{4}$, B.J.P. Gall ${ }^{4}$, A. Korichi ${ }^{5}$, \\ U.J. van Severen ${ }^{6}$ W. Pohler ${ }^{6}$, B. Schulze ${ }^{6}, H$. Hübel $^{6}$, R. Wadsworth ${ }^{7}$,$$
1 \text { C.S.N.S.M. CNRS-IN2P3, F-91405 Orsay (France) }
$$ \\ ${ }^{2}$ Iowa State University, Ames, Iowa 50011, USA \\ ${ }^{3}$ Lawrence Berkeley National Laboratory, Berkeley, California 94720, USA \\ ${ }^{4}$ C.R.N. Strasbourg, F-67037 Strasbourg, France \\ ${ }^{5}$ Institut Physique Nucleaire, IN2P3-CNRS, F-9140 Orsay, France \\ 6 University of Bonn, Nussallae 14-16, D-53115 Bonn, Germany \\ 7 University of York, Dept of Physics, Helsington, York, Y01 5DD, U.K.
}

Three superdeformed bands have been observed in ${ }^{191} \mathrm{Au}$ using the EUROGAM II multidetector array. The yrast superdeformed band has been extended to very high rotational frequencies and two new excited bands have been found. These bands behave as signature partners and display characteristics which suggest they could be based on the [532] $3 / 2$ or [530]1/2 configurations, which become the $[\widetilde{431}] 3 / 2,1 / 2$ doublet in the pseudo-spin formalism. It is important to note that band 3 has energies identical $(\langle\Delta \mathrm{E} \gamma\rangle=1.5 \mathrm{keV})$ to those of band 1 in ${ }^{192} \mathrm{Hg}$ within the observed range of frequencies.

The occurrence of the same transition energies (isospectral) in an even-even and an oddmass nucleus requires an alignment that is exactly half-integer. For several cases in the mass $\sim 150$ region the special proton orbital, [301]1/2, provides the half-integer alignment which comes about naturally [1] in a pseudospin scheme where the above orbital becomes [200]1/2, implying an alignment of exactly one half, i.e. of the pseudo intrinsic spin. All the normal parity orbitals are either such pseudospin-singlets $(\tilde{\Lambda}=0)$ or fall into pairs (pseudospin doublets) corresponding to a $\tilde{\Lambda} \neq 0$ with a small pseudo spin-orbit splitting. It was pointed out [2] that these pairs could also decouple the $1 / 2 \hbar$ pseudo intrinsic spin, providing alignments of one half, but such behavior has so far not been observed.
For the pseudo-spin doublet, $[\widetilde{431}] 1 / 2,3 / 2$, the Coriolis mixing should first decouple the pseudo intrinsic spin and produce two signaturedegenerate bands, having alignments $+1 / 2$ and $-1 / 2$. The properties of bands 2 and 3 fit well those expected for the lower degenerate band with alignment $+1 / 2$. The HFB routhians for quasi-protons in a ${ }^{190} \mathrm{Pt}$ core show that these two orbitals are the lowest quasi-particle excitations at low frequencies. However, both bands develop some signature splitting and the alignment of the lower one increases significantly beyond $1 / 2$ at the higher frequencies. These features indicate deviations of the calculation from the simple decoupling of the pseudo-intrinsic spins; whereas, the data agree well with those expectations.

It is not really clear whether the calculations underestimate the effects of the pseudospin symmetry or whether the better agreement with the simple expectations is just an accident. However, that quantized alignments may be coming from pseudospin symmetry is an exciting possibility.

\section{References}

[1] W. Nazarewicz et al., Phys. Rev.Lett. 64 1654 (1990).

[2] F.S. Stephens et al., Phys. Rev. Lett. 642623 (1990). 


\title{
Confirmation of a superdeformed band in ${ }^{192} \mathbf{P b}$
}

\author{
S.J.Astalos ${ }^{1}$, P.Fallon ${ }^{1}$, J.A.Becker ${ }^{2}$, L.A.Bernstein ${ }^{2}$, R.M. Clark ${ }^{1}$, M.A. Deleplanque ${ }^{1}$, \\ R.M.Diamond ${ }^{1}$, L.P.Farris ${ }^{2}$, E.A.Henry ${ }^{2}$, W.H.Kelly ${ }^{\dagger}$,I.Y.Lee ${ }^{1}$, A.O. Macchiavelli ${ }^{1}$, and F.S.Stephens ${ }^{1}$, \\ ${ }^{1}$ Lawrence Berkeley Laboratory, Berkeley, California, 94720 \\ ${ }^{2}$ Lawrence Livermore National Laboratory, Livermore, California, 94550 \\ ${ }^{\dagger}$ Permanent address: Iowa State University, Ames, Ia, 50011
}

A number of experiments have been performed to map out the region of superdeformed shapes in and around the neutron deficient $\mathrm{Pb}$ isotopes. Of particular interest are the edges of such regions, where theoretical nuclear models are most rigorously tested. For this purpose, during a brief test of the electronics of the GAMMASPHERE array at the 88-Inch Cyclotron we conducted a 7 hour test run using a reaction ${ }^{173} \mathrm{Yb}\left({ }^{24} \mathrm{Mg}, 5 \mathrm{n}\right)$ to populate high spin states in ${ }^{192} \mathrm{~Pb}$ at a beam energy of $140 \mathrm{MeV}$. In spite of the brief duration of the experiment, subsequent analysis allowed us to confirm the original observation [1] of the $\mathrm{SD}$ band assigned to ${ }^{192} \mathrm{~Pb}[2]$, the assignment of which had previously been under dispute[3].

The increased resolution afforded by GAMMASPHERE with its 49 Compton-suppressed detectors permitted the SD band to be seen from only three clean gates at 304,345 and $424 \mathrm{keV}$ (Fig 1a). As previously reported [1] and as is seen in Figure 1b, the entire SD band consists of nine transitions, extending over a range of approximately $300 \mathrm{keV}$. The relatively small number of transitions populating ${ }^{192} \mathrm{~Pb}$ in this experiment as compared with other heavier even-even lead isotopes may reflect greater fission competition as the reduction in the fissility parameter (which scales roughly as $\mathrm{Z}^{2} / \mathrm{A}$ ) for lighter isotopes in $\mathrm{Pb}$ makes it more difficult to populate a superdeformed (SD) band in ${ }^{192} \mathrm{~Pb}$. The $\gamma$-ray energies are also shown in Figure $1 \mathrm{a}$ and agree with previous published values to within 1 $\mathrm{keV}[1]$. The band has a moment of inertia similar to ${ }^{194} \mathrm{~Pb} \mathrm{SD}$ band 1 . The presence of lowlying isomers prevents definitive isotopic assignment to ${ }^{192} \mathrm{~Pb}$, although this has recently been confirmed[4]. The inband relative intensities of the SD band transitions are shown in the inset of Figure 1b. In summary, we confirm the earlier observation of the SD band assigned to ${ }^{192} \mathrm{~Pb}$. To date, no SD bands in lighter $\mathrm{Pb}$ isotopes have been observed.

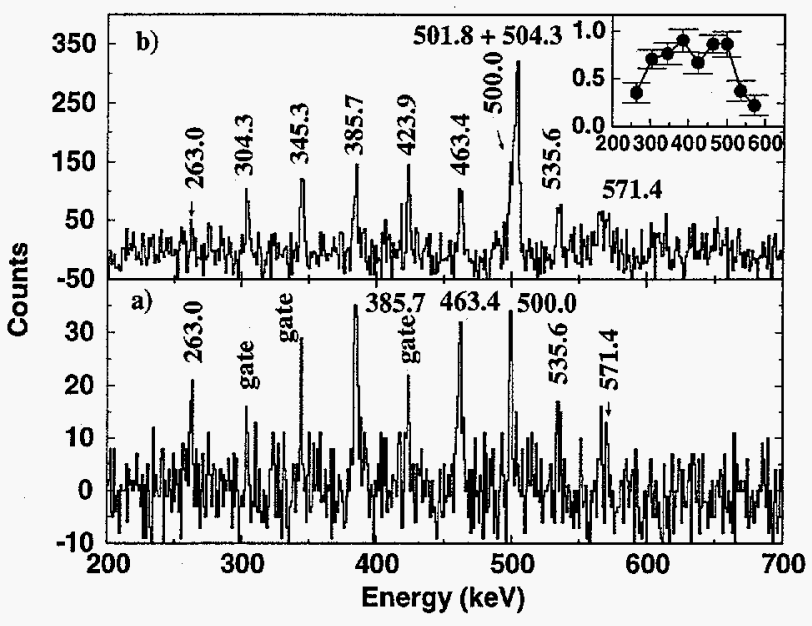

Figure 1: Sum of coincidence spectra of the SD band in ${ }^{192} \mathrm{~Pb}$, obtained from triples data, showing: a) from clean gates at 304,345 and $424 \mathrm{keV}$, and b) a sum of all gates on known in-band transitions. Band members, are labelled with their energies in $\mathrm{keV}$. Also labelled are the prominent $5^{-} \rightarrow 4^{+}$and $4^{+} \rightarrow 2^{+}$transitions at 504.3 and $501.8 \mathrm{keV}$, respectively, in ${ }^{192} \mathrm{~Pb}$.

\section{References}

[1] E.A.Henry et al., Z. Phys. A 338 (1991) 469

[2] S.J. Asztalos et al., Z. Phys. A 352 (1995) 239

[3] A.J.M.Plompen et al., Phys. Rev. C 47 (1993) 2378

[4] L. Ducroux et al., Z. Phys. A 352 (1991) 13 


\title{
Octupole Vibrations and Signature Splitting in Even Mass Hg Superdeformed Bands
}

\author{
P.Fallon ${ }^{1}$, F.S.Stephens ${ }^{1}$, S.Asztalos ${ }^{1}$, B.Busse ${ }^{1}$, R.M.Clark ${ }^{1}$, M.A.Deleplanque ${ }^{1}$, R.M.Diamond ${ }^{1}$, \\ R.Krücken ${ }^{1}$, I.Y.Lee ${ }^{1}$, A.O.Macchiavelli ${ }^{1}$, R.W.MacLeod ${ }^{1}$, G.Schmid ${ }^{1}$, K.Vetter ${ }^{1}$ and T.Nakatsukasa ${ }^{2}$. \\ ${ }^{1}$ Lawrence Berkeley National Laboratory. \\ ${ }^{2}$ Department of Physics, UMIST, P.O. Box 88, Manchester M60 1QD, U.K.
}

The possibility that the lowest excited SD bands in even mass mercury isotopes $\left({ }^{190,192,194} \mathrm{Hg}\right)$ are based on octupole vibrations has been discussed previously $[1,2]$ in terms of the moments of inertia and decay properties. In this present work[3] we have investigated the signature splitting of excited SD bands in ${ }^{194} \mathrm{Hg}$ isotopes. The signature splitting depends on the mixing with other states, e.g. other $K$ components of the octupole vibration. Since the $K=0$ octupole vibration contains only the $\alpha=1$ sequence, there will be an additional Coriolis mixing among $\alpha=1$ states. Odd spin $(\alpha=1)$ bands which lie below the $K=0$ band will therefore be favored with respect to the corresponding even spin $\alpha=0$ band. Thus if the octupole interpretation is valid, and in the absence of other effects (band-crossings etc), one may expect the odd spin band to be favored in a signature-split pair of bands.

The signature splitting (difference in the Routhian energies) for the ${ }^{194} \mathrm{Hg}$ excited SD bands is shown in Fig. 1. The experimental data (circles) exhibits an initial splitting which is consistent with these bands being based on octupole vibrational states. At higher frequencies the $\alpha=0$ band (band 2) gains in energy relative to band 3 at the highest frequencies. In other words, after an initial 'octupole-like' splitting, there appears to be a trend towards 'signature inversion', whereby the $\alpha=0$ band is becoming favored relative to the $\alpha=1$ band. For the three calculated cases in Fig. 1 the $\alpha=1$ structure starts lower in energy but at higher frequencies the $\alpha=0$ band becomes increasingly favored due to the mixing with close lying 2-quasiparticle states, e.g. $\pi[642] 5 / 2 \otimes \pi[514] 9 / 2$. The calculations are able to reproduce, in a quantitative manner, both the initial signature splitting observed in ${ }^{194} \mathrm{Hg}$ and the subsequent 'inversion' lending support to the presence of octupole effects in this nucleus.

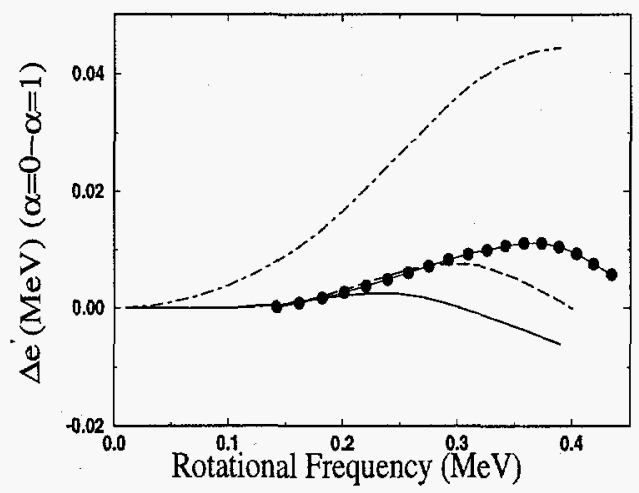

Fig. 1: The difference $\left(e^{\prime}(\alpha=0)-e^{\prime}(\alpha=1)\right)$ for both the experimental (circles) and calculated ${ }^{194} \mathrm{Hg}$ band 2 and 3 Routhians. Three curves are shown for the calculated Routhians, each with the same octupole strength, $f_{3}=1.05$, but with differing pairing; (i) The solid line has the critical frequency for pairing $\hbar \omega_{c}=$ $0.5(0.3) \mathrm{MeV}$ for neutrons(protons). (ii) The dashed line has $\hbar \omega_{c}=0.5(0.5) \mathrm{MeV}$ for neutrons(protons). (iii) The dot-dashed has constant pairing strength as a function of rotational frequency.

\section{References}

[1] B.Crowell et al., Phys. Lett. B333, 320 (1994) and Phys. Rev. C51, R1599 (1995).

[2] T.Nakatsukasa, K.Matsuyanagi, S.Mizutori and Y.R.Shimizu, Phys. Rev. C53, 2213 (1996).

[3] P.Fallon et al., submitted to Phys. Rev. C. 


\section{Relative Quadrupole Moments of ${ }^{192,193} \mathbf{H g}$ Superdeformed Bands.}

B.C.Busse ${ }^{1}$, P.Fallon ${ }^{1}$, R.Krücken ${ }^{1}$, D.Ackermann ${ }^{2}$, I.Ahmad ${ }^{2}$, S.J.Asztalos ${ }^{1}$, D.J.Blumenthal , M.P.Carpenter ${ }^{2}$, R.M.Clark ${ }^{1}$, M.A.Deleplanque ${ }^{1}$, R.M.Diamond ${ }^{1}$, S.M.Fischer ${ }^{2}$, F.Hannachi ${ }^{4}$, R.V.F.Janssens ${ }^{2}$, T.L.Khoo ${ }^{2}$, A.Korichi ${ }^{5}$, T.Lauritsen ${ }^{2}$, I.Y.Lee ${ }^{1}$, C.J.Lister ${ }^{2}$, A.Lopez-Martens ${ }^{4}$, A.O.Macchiavelli ${ }^{1}$, R.W.MacLeod ${ }^{1}$, E.F.Moore ${ }^{3}$, D.Nisius ${ }^{2}$, G.Schmid ${ }^{1}$, D.Seweryniak ${ }^{2}$, F.S.Stephens ${ }^{1}$, K.Vetter ${ }^{1}$

${ }^{1}$ Lawrence Berkeley Laboratory, Berkeley, California 94720

${ }^{2}$ Argonne National Laboratory, Argonne, Illinois 60439

${ }^{3}$ North Carolina State University, Raleigh, North Carolina 27695

4 Centre de Spéctrometrie Nucléaire, IN2P3-CNRS bat 104, F-91405, Orsay, France

${ }^{5}$ Institut de Physique Nucléaire, IN2P3-CNRS bat 104, F-91406, Orsay, France

Relative quadrupole moments ( $\mathrm{Q}_{0}$ 's) of yrast and excited SD bands can give insight into the deformation-driving effects of specific nucleon configurations and aid in the interpretation of "identical" bands. A pair of "identical" bands will have transition energies that are equal to, or are at the quarter points or half points of, each other. A clear understanding of this phenomenon has yet to be developed. With this in mind, $\mathrm{SD}$ states in ${ }^{192,193} \mathrm{Hg}$ were populated simultaneously via the ${ }^{176} \mathrm{Yb}\left({ }^{22} \mathrm{Ne}, \mathrm{xn}\right)$ reaction at a beam energy of $118 \mathrm{MeV}$. The target consisted of a $500 \mu \mathrm{g} / \mathrm{cm}^{2}{ }^{176} \mathrm{Yb}$ foil evaporated on a $6.8 \mathrm{mg} / \mathrm{cm}^{2} \mathrm{Au}$ backing. The beam was accelerated by the 88 -Inch Cyclotron at Lawrence Berkeley National Laboratory, and $\gamma$ rays were detected by the Gammasphere array which, for this experiment, had $85 \mathrm{Ge}$ detectors. A total of $1.9 \times 10^{9}$ events with a fold $\geq 5$ were collected. A Doppler Shift Attenuation Method (DSAM) centroid shift analysis was then performed.

Experimental fractional Doppler shifts, $F(\tau)$, were extracted and are shown in the figure for the ${ }^{192} \mathrm{Hg}$ and ${ }^{193} \mathrm{Hg}$ SD yrast bands. Calculated $\mathrm{F}(\tau)$ curves, that assume a rotational cascade and constant $\mathrm{Q}_{o}\left(\mathrm{Q}_{s f}\right)$ for the inband(sidefeeding) states, are shown for comparison. The stopping powers of Ziegler were used. A lineshape analysis on some transitions in the ${ }^{192,193} \mathrm{Hg}$ SD bands was also performed, leading to results consistent with the $F(\tau)$ analysis. Comparing the $F(\tau)$ curves allowed several im- portant conclusions. The relative $\mathrm{Q}_{0}$ 's of the yrast SD bands of ${ }^{192} \mathrm{Hg}$ and ${ }^{193} \mathrm{Hg}$ are different to a significance $>2 \sigma$, with values of 19.8 $\pm 1.2 \mathrm{eb}$ and $17.2 \pm .7 \mathrm{eb}$ respectively. The uncertainty in the sidefeeding time, obtained by $\chi^{2}$ minimization, has been included in the $Q_{0}$ errors. Nevertheless, radically different sidefeeding in the two SD Hg nuclei could cause the apparent Qo difference, although this solution is less likely. Based on current theoretical work, the difference between ${ }^{192} \mathrm{Hg}$ and ${ }^{193} \mathrm{Hg}$ is unexpected. The experimental Qo's of all six SD bands in ${ }^{193} \mathrm{Hg}$ are the same to $\approx 1 \sigma$, which seems to eleminate a large polarizing effect of the different single particle levels. This similarity in $Q_{0}$ is difficult to reconcile, given the difference in the ${ }^{192} \mathrm{Hg}$ and ${ }^{193} \mathrm{Hg} \mathrm{Q}_{0}$ 's. Since ${ }^{192} \mathrm{Hg}$ and ${ }^{193} \mathrm{Hg}$ have a SD "identical" band relationship, the data may imply that "identical" transition energies don't always lead to identical deformations. This is the first accurate measurement of $Q_{0}$ 's in an oddmass SD nucleus in the mass 190 region.

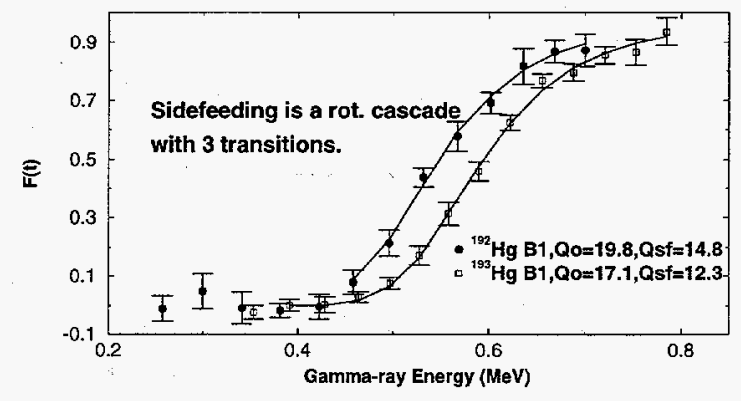




\title{
Test of $\Delta I=2$ Staggering in the superdeformed bands of ${ }^{194} \mathbf{H g}$
}

\author{
R.Krücken ${ }^{1}$, G.Hackman ${ }^{2}$, M.A.Deleplanque ${ }^{1}$, R.V.F.Janssens ${ }^{2}$, D. Ackermann ${ }^{2}$, S. Asztalos ${ }^{1}$, \\ R.M. Clark ${ }^{1}$, R.M. Diamond ${ }^{1}$, P.Fallon ${ }^{1}$, B.Herskind ${ }^{3}$ T.L.Khoo ${ }^{2}$, T. Lauritsen ${ }^{2}$, \\ I.Y.Lee ${ }^{1}$, A.O. Macchiavelli ${ }^{1}$, R.W. MacLeod ${ }^{1}$, G.J.Schmid ${ }^{1}$, F.S.Stephens ${ }^{1}$, and K. Vetter ${ }^{1}$ \\ ${ }^{1}$ Nuclear Science Division, Lawrence Berkeley National Laboratory, Berkeley CA 94700, U.S.A. \\ ${ }^{2}$ Argonne National Laboratory, Argonne IL 60439, U.S.A. \\ 3 The Niels Bohr Institute, University of Copenhagen, Denmark
}

Recently a regular staggering pattern of the transition energies was observed in the yrast superdeformed band in ${ }^{149} \mathrm{Gd}$ [1]. Evidence for similar effects has also been reported for ${ }^{194} \mathrm{Hg}[2]$. The theoretical investigation of these effects has so far not been conclusive. It remains unclear whether to associate the observed staggering with a $Y_{44}$ deformation of the superdeformed shape or with numerous band-crossings, since a single band-crossing cannot explain the extension of a staggering over a larger frequency range.

Since the observed energy shifts are only of the order of $100 \mathrm{eV}$ or less, experimental confirmation of the reported effects is extremely important. Here we report on results from a new experiment on the known SD bands of ${ }^{194} \mathrm{Hg}$, looking for the previously reported [2] staggering in those bands.

Superdeformed states in ${ }^{194} \mathrm{Hg}$ were populated in the reaction ${ }^{150} \mathrm{Nd}\left({ }^{48} \mathrm{Ca}, 4 \mathrm{n}\right)$ using a $201 \mathrm{MeV}$ ${ }^{48} \mathrm{Ca}$ beam. The emitted $\gamma$-rays were detected by the GAMMASPHERE array, which at the time of the experiment consisted of 70 Comptonsuppressed Ge detectors. A total of $1.4 \times 10^{9}$ events with fold four and higher were taken in this thin-target experiment. The transition energies in the three known SD bands of ${ }^{194} \mathrm{Hg}$ have been determined with an accuracy of around $30 \mathrm{eV}$. The resulting staggering values $\Delta E_{\gamma}$, defined as the fourth derivative of the $\gamma$-ray energies, are presented in the figure. No statistically significant staggering was found in band 1 . However, a significant staggering has been observed for band 2 in the frequency range above $\hbar \omega=0.3 \mathrm{MeV}$ that includes one phase inversion. A short regular staggering with an amplitude of
$50 \mathrm{eV}$ has been observed in band 3 in the frequency range $0.25 \mathrm{MeV}<\hbar \omega<0.375 \mathrm{MeV}$. The observed staggering patterns show similarities with patterns that can be induced by the crossing of weakly interacting bands. However, such a scenario cannot account in a simple manner for the observed staggering. Detailed information can be found in Ref. [3].

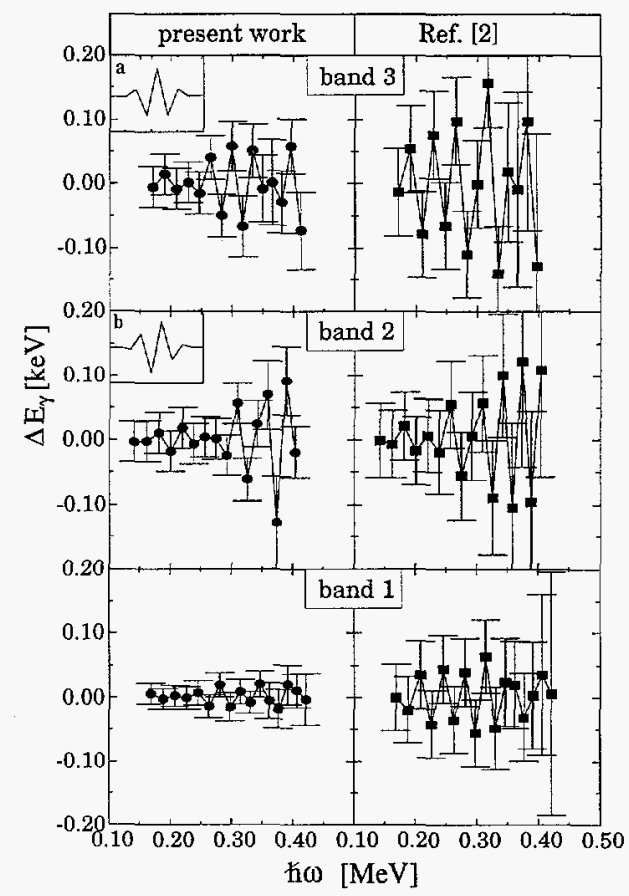

\section{References}

[1] S. Flibotte et al., Phys. Rev. Lett. 71, 4299 (1993)

[2] B. Cederwall et al., Phys. Rev. Lett. 72, 3150 (1994)

[3] R. Krücken et al., Phys. Rev. C 54, R2109 (1996) 


\title{
Discrete SD-ND linking transitions from the yrast superdeformed band in ${ }^{194} \mathrm{~Pb}: J^{\pi}$ and $E_{x}$
}

\author{
K. Hauschild, L.A. Bernstein, J.A. Becker, D.E. Archer, R.W. Bauer: LLNL \\ D.P. McNabb, J.A. Cizewski, K.-Y. Ding, W. Younes: Rutgers University. \\ R. Krücken, R.M. Diamond, R.M. Clark, P. Fallon, I.-Y. Lee, A.O. Macchiavelli, \\ R. MacLeod, G.J. Schmid, M.A. Deleplanque, F.S. Stephens: LBNL \\ W.H. Kelly: Iowa State University
}

Twelve one-step linking transitions between the yrast $\mathrm{SD}$ band and low-lying states in ${ }^{194} \mathrm{~Pb}$ have been identified, shown in Fig. 1. A further 12 high-energy transitions have also been identified to be in coincidence with the yrast SD band. Anisotropy measurements have determined that the linking decays include E1, M1 and mixed M1/E2 transitions. $J^{\pi}=6^{+}, E_{x}=4878.4(3)$ $\mathrm{keV}$ and $J^{\pi}=8^{+}, E_{x}=5047.8(3) \mathrm{keV}$ were unambiguously assigned to the two lowest lying observed superdeformed states without a priori assumptions about the properties of SD bands. These results represent the first experimentally self-consistent $J^{\pi}$ assignments to an SD band.

The experiment was performed at the Lawrence Berkeley Laboratory 88-Inch Cyclotron Facility using the ${ }^{174} \mathrm{Yb}\left({ }^{25} \mathrm{Mg}, 5 \mathrm{n}\right)$ reaction at $\mathrm{E}\left({ }^{25} \mathrm{Mg}\right)=130 \mathrm{MeV}$ and the GAMMASPHERE array [1]. The isotopically enriched $(>98 \%){ }^{174} \mathrm{Yb}$ target was $1.21-\mathrm{mg} / \mathrm{cm}^{2}$ thick,

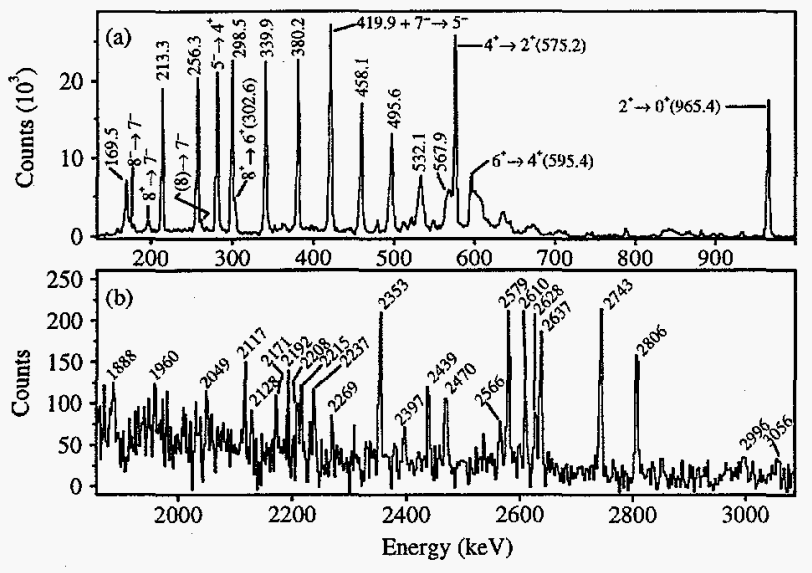

Figure 1: Triple-gated ${ }^{194} \mathrm{~Pb}$ SD spectra. and was evaporated directly onto a $6.13-\mathrm{mg} / \mathrm{cm}^{2}$ Au backing. The signal-to-noise ratio of the SD primary $\gamma$ rays is enhanced when a thick backing is used.

$21(2) \%$ of the ${ }^{194} \mathrm{~Pb}$ yrast SD band intensity has been observed to decay out through the onestep linking transitions. At first glance this is a suprisingly large proportion of the SD flux when compared to the $\sim 5 \%$ observed for the ${ }^{194} \mathrm{Hg}$ yrast SD band [2]. However, arguments based on level density considerations at the point of SD decay, combined with the different low-lying ND structure of ${ }^{194} \mathrm{Hg}$ and ${ }^{194} \mathrm{~Pb}$ indicate a more highly fragmented decay in ${ }^{194} \mathrm{Hg}$ is to be expected.

Greater understanding of these properties is anticipated with the improved resolving power of the "complete" Gammasphere and Euroball arrays, for example, excited bands and their decay properties. Unique determination of the $J^{\pi}$ and $E_{x}$ values of SD bands are needed to place more stringent restrictions upon theoretical calculations which predict the SD states and their properties, in particular, the excitation energy of the SD bandheads. In addition, this knowledge will help to address the phenomena of "identical" bands.

\section{References}

[1] I. Y. Lee, Nucl. Phys. A520, 641c (1990).

[2] T. L. Khoo et al., Phys. Rev. Letts. 76, 1583 (1996). 


\section{The decay of the superdeformed band in ${ }^{194} \mathrm{~Pb}$ : Electromagnetic Properties}

\section{R. Krücken, ${ }^{1}$ S.J. Asztalos, ${ }^{1}$ J.A. Becker ${ }^{3}$ B. Busse, ${ }^{1}$ R.M. Clark, ${ }^{1}$ M.A. Deleplanque, ${ }^{1}$}

A. Dewald, ${ }^{2}$ R.M. Diamond, ${ }^{1}$ P. Fallon, ${ }^{1}$ K. Hauschild, ${ }^{3}$ I.Y. Lee, ${ }^{1}$ A.O. Macchiavelli, ${ }^{1}$ R.W. MacLeod, ${ }^{1}$ R. Peusquens, ${ }^{2}$ G.J. Schmid ${ }^{1}$ F.S. Stephens, ${ }^{1}$ K. Vetter, ${ }^{1}$ and P. von Brentano ${ }^{2}$

${ }^{1}$ Nuclear Science Division, Lawrence Berkeley National Laboratory, Berkeley, CA 94720, U.S.A.

${ }^{2}$ Institut für Kernphysik, Universität zu Köln, 50937 Köln, Germany

${ }^{3}$ Lawrence Livermore National Laboratory, Livermore, CA 94550, U.S.A.

Lifetimes of the $14^{+}, 12^{+}, 10^{+}$states and, for the first time, the $8^{+}$state in the yrast superdeformed (SD) band of ${ }^{194} \mathrm{~Pb}$ were measured at GAMMASPHERE (95 detectors) with the recoil-distance Doppler-shift method utilizing the Cologne Plunger. The reaction ${ }^{164} \mathrm{Dy}\left({ }^{34} \mathrm{~S}, 4 \mathrm{n}\right)$ with a $166-\mathrm{MeV}$ beam from the 88-Inch Cyclotron of the Lawrence Berkeley National Laboratory was used. $2 \times 10^{8}$ threefold and higher coincidence events recorded at 12 target-to-stopper distances between 2.6 and $1200 \mu$. The lifetimes of the $14^{+}, 12^{+}$, $10^{+}$and for the first time the $8^{+} \mathrm{SD}$ states were determined (see Tab.I). Constant transition quadrupole moments with an average of 18.8 (11) e $b$ were found at the bottom of the SD band.

The decay out of the SD band can be viewed as governed by a small admixture $\left(a_{n}^{2}\right)$ of normal deformed (ND) states to the SD wavefunction which is assessed for the $8^{+}$and $10^{+}$SD states based on a simple mixing model (see Ref.[1] and refs. therein). The results $\left(a_{n}^{2} \leq 1 \%\right)$ show that the structure of the SD states is not drastically changed. Spins and parity and the excitation energy of the SD states in ${ }^{194} \mathrm{~Pb}$ were recently established $[2,3]$ by the observation of direct linking transitions between SD and near yrast ND states. The lifetimes of the $8^{+}$and $10^{+} \mathrm{SD}$ states and their branching ratios [3] determine the reduced transition probabilities for these discrete linking transitions. By correcting for the amount of admixture between SD and ND states one can extract the electromagnetic properties of the pure $\mathrm{ND}$ states that mix into the $\mathrm{SD}$ wavefunction. The extracted $\mathrm{B}(\mathrm{E} 1)$ values were found to be of the order of $10^{-6}-10^{-5}$ W.U. Upper limits of $3 \times 10^{-2} \mathrm{~W} . \mathrm{U}$. for the $\mathrm{B}(\mathrm{E} 2)$ values and $5 \times 10^{-4} \mathrm{~W}$.U. for $\mathrm{B}(\mathrm{M} 1)$ values were determined. These values are all consistent with a statistical decay, even when the possibility of an increase by one order of magnitude is considered. They show no enhancement that would point to the presence of structural effects. Despite the large intensity of observed linking transitions, the new data show that the decay out of this SD band is statistical in nature.

Table 1: Mean lifetimes $\tau$ of SD states in the yrast $\mathrm{SD}$ band in ${ }^{194} \mathrm{~Pb}$. Reduced transition probabilities $\mathrm{B}(\mathrm{E} 2)$ and transition quadrupole moments $Q_{t}$ are given for the intra-band transitions.

\begin{tabular}{|cc|ccc|}
\hline$I^{\pi}$ & $\begin{array}{c}E_{\gamma} \\
{[\mathrm{keV}]}\end{array}$ & $\begin{array}{c}\tau \\
{[\mathrm{ps}]}\end{array}$ & $\begin{array}{c}\text { B(E2) } \\
10^{3} \text { W.U. }\end{array}$ & $\begin{array}{c}Q_{t} \\
{[\mathrm{eb}]}\end{array}$ \\
\hline $14^{+}$ & 298 & $2.6 \pm 0.7$ & $1.8_{-0.4}^{+0.8}$ & $18.5_{-2.0}^{+3.2}$ \\
$12^{+}$ & 256 & $5.5 \pm 1.0$ & $1.7_{-0.3}^{+0.3}$ & $18.2_{-1.5}^{+1.9}$ \\
$10^{+}$ & 214 & $8.3 \pm 1.7$ & $2.2_{-0.4}^{+0.6}$ & $20.7_{-1.8}^{+2.5}$ \\
$8^{+}$ & 170 & $20.0 \pm 6.9$ & $1.5_{-0.4}^{+0.7}$ & $17.3_{-2.4}^{+4.0}$ \\
\hline
\end{tabular}

\section{References}

[1] R. Krücken et al., Phys. Rev. C54, 1182 (1996)

[2] A. Lopez-Martens et al., Phys. Lett. B380, 18 (1996)

[3] K. Hauschild et al., to be published 


\section{Evidence for 'Magnetic Rotation': Lifetimes of States in the M1-bands of ${ }^{198,199} \mathbf{P b}$

\author{
R.M.Clark ${ }^{1}$, S.J.Asztalos ${ }^{1}$, G.Baldsiefen ${ }^{2}$, J.A.Becker ${ }^{3}$,L.Bernstein ${ }^{3}$, M.A.Deleplanque ${ }^{1}$, \\ R.M.Diamond ${ }^{1}$, P.Fallon ${ }^{1}$, I.M.Hibbert ${ }^{4}$, H.Hübel ${ }^{2}$, R.Krücken ${ }^{1}$, I.Y.Lee ${ }^{1}$, \\ A.O.Macchiavelli ${ }^{1}$, R.W.MacLeod ${ }^{1}$, G.Schmid ${ }^{1}$, F.S.Stephens ${ }^{1}$, R. Wadsworth ${ }^{4}$ \\ 1 Nuclear Science Division, Lawrence Berkeley National Laboratory, Berkeley CA 94720, U.S.A. \\ ${ }^{2}$ Institut für Strahlen- und Kernphysik, Universität Bonn, D-53115 Bonn, Germany \\ ${ }^{3}$ Lawrence Livermore National Laboratory, Livermore, California 94550 \\ 4 Department of Physics, University of York, Heslington, York, YO1 5DD, UK
}

The observation of long cascades of magnetic dipole transitions in the neutron deficient $\mathrm{Pb}$ nuclei has posed a serious challenge to conventional collective models. They are thought to be based on high-K proton configurations coupled to neutron configurations involving low- $\mathrm{K}_{13 / 2}$ holes. The properties of the bands are extremely unusual: 1) the structures follow the rotational $\mathrm{I}(\mathrm{I}+1)$ rule over many states despite very low deformations; 2) the levels show no signature splitting; 3) the levels are linked by strong M1 transitions with a typical $\mathrm{B}(\mathrm{M} 1)$ of the order of several Weisskopf units; 4) the $\mathrm{B}(\mathrm{M} 1) / \mathrm{B}(\mathrm{E} 2)$ ratios are very large (typically $\geq 20-40 \mu_{N}^{2} / \mathrm{e}^{2} \mathrm{~b}^{2}$ ); 5) the ratio $\Im^{(2)} / \mathrm{B}(\mathrm{E} 2)$ is roughly ten times larger than that for well deformed nuclei. It has been suggested that the bands represent a novel mode of nuclear excitation, namely 'magnetic rotation' which arises as a consequence of breaking the intrinsic rotational symmetry by a large magnetic dipole (as opposed to the more familiar 'electric rotation' which arises when an electric quadrupole (deformation) breaks the symmetry).

An intuitively appealing description of the behaviour of the M1-bands naturally arises from the Tilted Axis Cranking (TAC) model [1]. Close to the band-head the proton and neutron angular momentum vectors are coupled almost perpendicular to each other. The total angular momentum vector, $\underline{J}$, then lies along a tilted axis (see Fig. 1). Angular momentum is generated as the proton and neutron angular momentum vectors slowly tilt towards $\underline{\mathrm{J}}$. This has been called the 'shears mechanism'. Since the magnetic transition probability is determined by the components of the magnetic moments perpendicular to $\underline{\mathrm{J}}$, the $\mathrm{B}(\mathrm{M} 1)$ should drop with increasing spin. This is a crucially important prediction, since the B(M1)'s are a sensitive probe of the underlying mechanism. Previous attempts to deduce B(M1)'s through lifetime measurements have proven inconclusive. A new high-precision measurement of lifetimes of states in the M1bands of ${ }^{198,199} \mathrm{~Pb}$ has been performed using Gammasphere [2]. The deduced $\mathrm{B}(\mathrm{M} 1)$ values show a remarkable agreement with the TAC predictions (see Fig. 2). The results represent the first convincing proof of the phenomenon of nuclear 'magnetic rotation'.

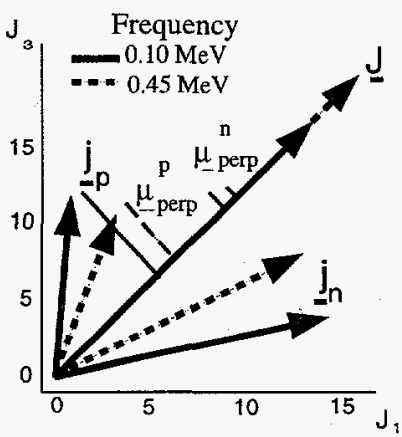

Fig1: The TAC Mechanism

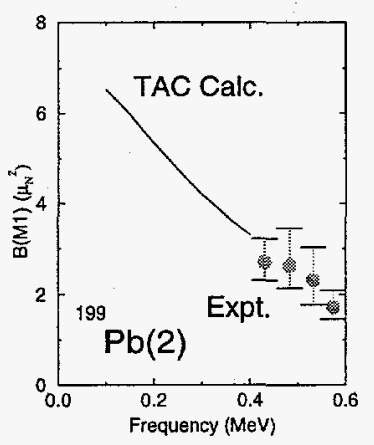

Fig2: $B(M 1)$ 's: TAC vs. Expt

\section{References}

[1] S. Frauendorf, Nucl. Phys. A 557 (1993) 259c

[2] R. M. Clark et al., accepted, Phys. Rev. Lett. 


\title{
Search for two-phonon octupole vibrational states in ${ }^{208} \mathrm{~Pb}$
}

\author{
K. Vetter, A.O. Macchiavelli, S.J. Asztalos, R.M. Clark, M.A.Deleplanque, R.M. Diamond, \\ P.Fallon, R. Krücken, I.Y.Lee, R.W.MacLeod, G.J.Schmid and F.S. Stephens
}

For many years the first excited state of ${ }^{208} \mathrm{~Pb}$ has been interpreted as a one-phonon vibration of octupole character. The identification of the two-phonon vibration is mandatory in order to prove the vibrational picture and to measure the harmonicity of octupole vibrations. Only recently the first promising experimental evidence for the existence of the two-phonon vibration was observed using a $\left(n, n^{\prime} \gamma\right)$ reaction, indicating only a slight deviation of an harmonic oscillation [1]. We have performed an experiment to populate the two-phonon vibrational states in ${ }^{208} \mathrm{~Pb}$ with ${ }^{154} \mathrm{Sm}$ at a bombarding energy of $1000 \mathrm{MeV}$ and measuring $\gamma$-rays in Gammasphere consisting of 60 Ge-detectors at this time. To be independent of the lifetime of the two-phonon state we used a thin target requiring the measurement of the position of the scattered particles to correct for the Doppler-shift of the emitted $\gamma$-rays. Scattered particles were detected by a Silicon strip detector covering the range close the grazing angle. Like all previous heavy-ion induced experiments designed to measure the $\gamma$-decay following the possible excitation of the second octupole phonon in ${ }^{208} \mathrm{~Pb}$ this experiment was performed at a bombarding energy which is about $20 \%$ above the Coulomb Barrier. At this energy the nuclear interaction is expected to increase strongly the population probability of the double phonon members [2]. Coulomb excitation as well as coupled-channel calculations indicate that the $6^{+}$state dominates the population of the multiplet. The decay of the $6^{+}$state is expected to feed the $5^{-}$state at $3198 \mathrm{keV}\left(\mathrm{E}_{\gamma}(E 1) \approx 2 \mathrm{MeV}\right)$ followed by an $\mathrm{E} 2$ transition of $\mathrm{E}_{\gamma}=584 \mathrm{keV}$ to the $3^{-}$state at $2614 \mathrm{keV}$. As can been seen in the partial energy spectrum shown in fig. 1 obtained by gating on the $584 \mathrm{keV}$ transition it is not possible to identify any candidate for the decay of members of the two-phonon octupole vibra- tion. However, based on the efficiency-corrected

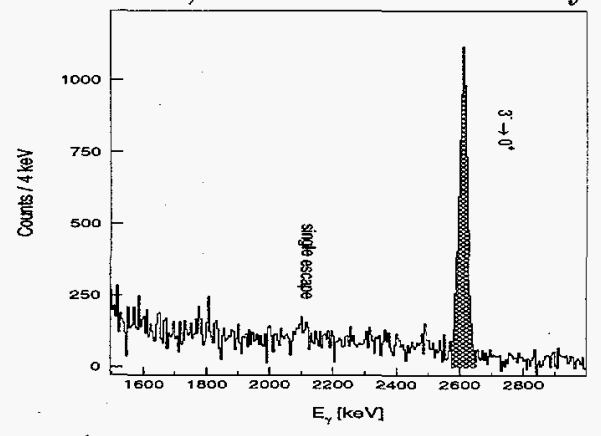

Figure 1: Partial $\gamma$-energy spectrum of ${ }^{208} \mathrm{~Pb}$ for grazing collisions after gating on the $5^{-} \rightarrow 3^{-}$transition at $584 \mathrm{keV}$.

intensities we can establish lifetime independent limits for the observation of a $\gamma$-transition in a region between $2 \mathrm{MeV}$ and $3 \mathrm{MeV}$ relatively to the $3^{-} \rightarrow 0^{+}$or the $5^{-} \rightarrow 3^{-}$transition. Particularly, the limit for the collisions at the grazing angle are about a factor of 10 below the yield predicted by coupled-channel calculations [2]. However, one has to realize that this limit may not be very sensitive to the population of the two-phonon states since the intensity of the $5^{-} \rightarrow 3^{-}$transition is primarily determined by the decay of energetically higher-lying states, bypassing the higher order vibrational states. Having not been able to observe the decay of any second phonon state it is suggested that we need to perform an experiment at a "safe" bombarding energy well below the Coulomb barrier where only Coulomb excitation takes place as a process which can be described by theory accurately.

\section{References}

[1] M. Yeh et al., Phys. Rev. Lett. 76, 1208 (1996).

[2] S. Landowne, C.H. Dasso, R.A. Broglia and A. Winther, Phys. Lett. 70B, 294 (1977). 


\title{
Study of Neutron-rich Nuclei Using Deep-inelastic Reactions
}

\author{
I.Y.Lee ${ }^{1}$, S. Asztalos ${ }^{1}$, M.A.Deleplanque ${ }^{1}$, B. Cederwall ${ }^{1}$, R.M. Diamond ${ }^{1}$, P. Fallon ${ }^{1}$ \\ A.O. Macchiavelli ${ }^{1}$, L. Phair ${ }^{1}$, F.S.Stephens ${ }^{1}$, G.J. Wozniak ${ }^{1}$, S.G. Frauendorf ${ }^{2}$ \\ J.A. Becker ${ }^{3}$, E.A. Henry ${ }^{3}$, P.F. Hua ${ }^{4}, D . G$. Sarantites ${ }^{4}$, J.X. Saladin ${ }^{5}$, and C.H. Yu \\ ${ }^{1}$ Nuclear Science Division, Lawrence Berkeley National Laboratory, Berkeley CA 94720, U.S.A. \\ ${ }^{2}$ Research Center Rossendorf, Dresden, Germany \\ ${ }^{3}$ Lawrence Livermore National Laboratory, Livermore, CA 94550, U.S.A. \\ 4 Washington University, St. Louis, MO 63130 U.S.A. \\ ${ }^{5}$ University of Pittsburge, Pittsburge, PA 15260 U.S.A. \\ ${ }^{6}$ University of Rochester, Rochester, NY 14627 U.S.A.
}

Neutron-rich nuclei are of particular interest since they might reveal new aspects of nuclear structure associated with an excess of neutrons, such as a neutron skin, a modified shell structure and new modes of excitation. These nuclei are difficult to produce, particularly in high-spin states. However, using deep-inelastic reactions together with the new gamma-ray detector arrays, one expects to have enough sensitivity, in spite of the low cross sections, to reach these nuclei in high-spin states.

We have carried out the reaction ${ }^{48} \mathrm{Ca}+{ }^{176} \mathrm{Yb}$ at a beam energy of $250 \mathrm{MeV}$. A thin target was used to allow both the projectile- and target-like fragments to decay outside the target so that gamma rays from short-lived high-spin states can be observed as sharp lines after Dopplershift correction. An annular silicon-strip detector was used to detect the scattered fragments. The early implementation of Gammasphere with 36 detectors was used to detect the gamma-rays. Coincidence events with at least one fragment and two Compton-suppressed gamma rays detected were taken at a rate of $1000 / \mathrm{sec}$.

Two- and three-fold gamma-ray-coincidence data were analyzed for the gamma rays from the target-like fragments, and about 10 different nuclei were observed. In this data set, we were able to study nuclei produced with a cross section as low as $0.1 \mathrm{mb} / \mathrm{sr}$. The gamma-ray yield of $\mathrm{Yb}$ nuclei as a function of spin is shown in Fig. 1. The sensitivity of the current setup allowed states with spin as high as 20 to be observed.
Before this study, only three levels in the yrast band were known in ${ }^{175,177} \mathrm{Yb}$ and ${ }^{178} \mathrm{Yb}$. This work extends the yrast band of ${ }^{178} \mathrm{Yb}$ to spin 12 and the yrast bands in ${ }^{175,177} \mathrm{Yb}$ to spin $37 / 2$ and $33 / 2$, respectively. These new transitions allow us to study the systematics of back bending in neutron rich $\mathrm{Yb}$ nuclei. The $\mathrm{i}_{13 / 2}$ band in the odd-mass nuclei provided an experimental reference for the extraction of the Routhians in the neighboring even-mass nuclei. The experimental Routhians compare well with cranked shell model calculations[1].

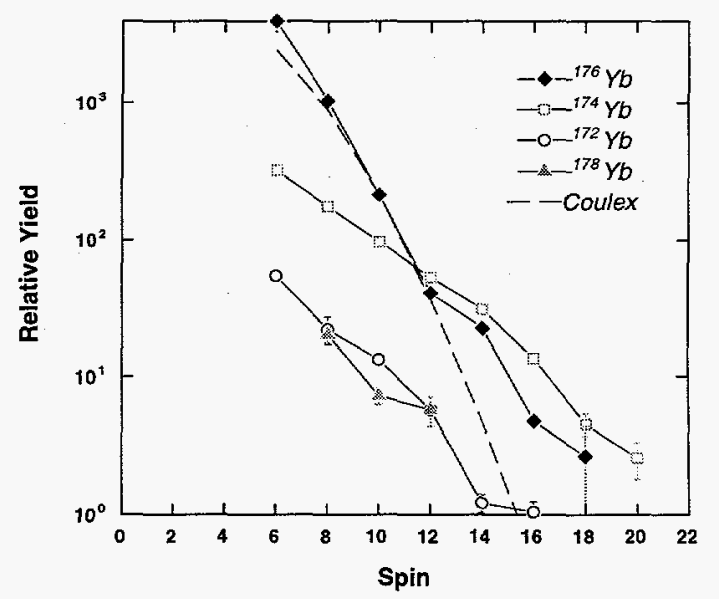

Figure 1: Gamma-ray yield of $\mathrm{Yb}$ nuclei as a function of spin

\section{References}

[1] R. Bengtsson, S. Frauendorf and F.-R. May, Atomic Data and Nuclear Data Tables, 35 (1986)15. 


\title{
A Measurement of the Gamma Ray Polarization Sensitivity of Gammasphere
}

\author{
G.J.Schmid, A.O.Macchiavelli, M.A.Deleplanque, S. Asztalos, R.M.Clark, R.M. Diamond, \\ P. Fallon, R. Krücken, I.Y. Lee, R.W. MacLeod, F.S. Stephens, and K. Vetter \\ Nuclear Science Division, Lawrence Berkeley National Laboratory, Berkeley CA 94720, U.S.A.
}

The Gammasphere array currently in operation at Berkeley has the potential to operate as a $\gamma$-ray polarimeter (for linear polarization) by taking advantage of the segmented Ge detectors that will eventually comprise $65 \%$ of the completed array.

The polarization sensitivity for this type of segmented detector can be related to the polarization, $\mathrm{P}$, of the radiation through the usual formula $\mathrm{Q}\left(\mathrm{E}_{\gamma}\right)=\mathrm{A}\left(\mathrm{E}_{\gamma}\right) / \mathrm{P}$, if the experimental asymmetry, $\mathrm{A}\left(\mathrm{E}_{\gamma}\right)$, is defined (for example) as follows:

$$
A\left(E_{\gamma}\right)=\left(\frac{1}{\sqrt{\eta\left(E_{\gamma}\right)}}\right)\left(\frac{\eta\left(E_{\gamma}\right) C\left(E_{\gamma}\right)-S\left(E_{\gamma}\right)}{C\left(E_{\gamma}\right)+S\left(E_{\gamma}\right)}\right)
$$

In this relation, $\mathrm{C}\left(\mathrm{E}_{\gamma}\right)$ and $\mathrm{S}\left(\mathrm{E}_{\gamma}\right)$ represent the number of confined and shared events in the segmented detectors (i.e. the full absorption events which deposit their energy either confined to one side, or shared between the two sides $)$, and $\eta\left(E_{\gamma}\right)$ is a parameter whose value is determined so as to give zero asymmetry for unpolarized $\gamma$-rays.

To determine $\mathrm{Q}\left(\mathrm{E}_{\gamma}\right)$ we measured asymmetries associated with $\gamma$-rays of known polarization [1]. In particular, we looked at $\gamma$-rays from excited states in ${ }^{24} \mathrm{Mg},{ }^{56} \mathrm{Fe}$, and ${ }^{109} \mathrm{Ag}$ which were populated following inelastic scattering of protons at $\mathrm{E}_{p}=2.46,3.0$ and $2.54 \mathrm{MeV}$ respectively. This allowed determination of $\mathrm{Q}\left(\mathrm{E}_{\gamma}\right)$ at $1.368 \mathrm{MeV}, 0.847 \mathrm{MeV}$, and $0.415 \mathrm{MeV}$ respectively. The $\eta\left(\mathrm{E}_{\gamma}\right)$ parameter for these experiments was determined as a function of $\mathrm{E}_{\gamma}$ by using a ${ }^{152} \mathrm{Eu}$ source placed at the target location. Figure 1 shows (by the solid data points)the results for $\mathrm{Q}\left(\mathrm{E}_{\gamma}\right)$. A computer Monte Carlo simulation was also performed to compare with these experimental values. In this simulation, the $\gamma$ ray polarization vector was rigorously tracked so as to give realistic azimuthal Compton scattering for all the multiple interactions of each $\gamma$-ray. The results of these calculations (along with the associated errors) are shown by the open data points in Figure 1. A solid line connects these points. The agreement between experiment and theory is seen to be good. The dashed line in Figure 1 is the energy dependence of the KleinNishina formula in a point detector approximation [1]. This energy dependence is the one which is traditionally used to fit $\mathrm{Q}\left(\mathrm{E}_{\gamma}\right)$ data from 4-fold segmented polarimeters. However, due to the 2 -fold segmented nature of the current geometry, this energy dependence is seen to be inadequate. In particular, the side-photoabsorption at low energies is seen to reduce $Q$.

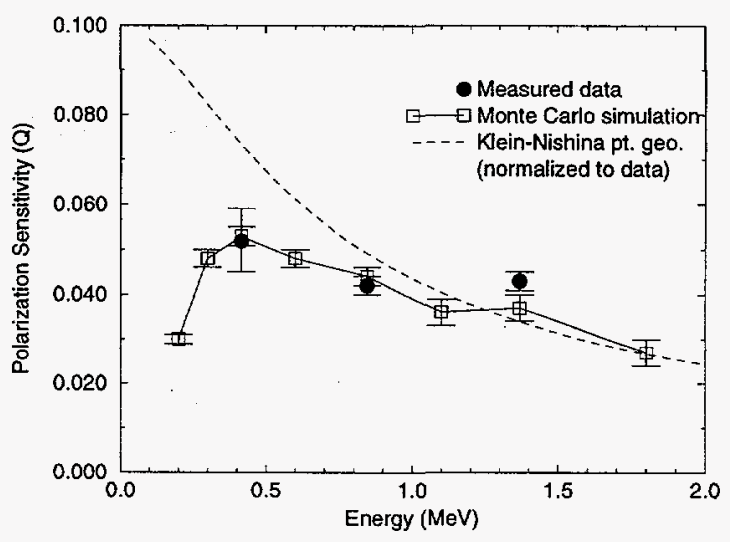

Figure 1: The experimental results for $\mathrm{Q}$ are compared to theoretical energy dependences.

\section{References}

[1] P.M. Jones et al., NIM A362,556 (1995) 


\section{Multiparticle-rotor model for rotational band structure of ${ }^{154} \mathrm{Gd}$}

S.Y. Chu, J.O. Rasmussen, and M.A. Stoyer

Nuclear Science Division, Lawrence Berkeley National Laboratory, Berkeley, CA 94720, USA P. Ring

Physik Department Technische Universität München, D-85747 Garching, Germany

L.F. Canto

Instituto de Fisica, Universidade Federal do Rio de Janeiro, 21945 Rio de Janeiro, Brasil

Band-crossing phenomena in the high-spin region of deformed nuclei are studied in this paper. The power of modern computers for large-matrix diagonalization (up to $17527^{*} 17527$ ) is exploited in a multinucleon-plus-rotor model, the interesting test case of ${ }^{154} \mathrm{Gd}$ being treated in this paper. Though a small basis set of 9-18 Nilsson orbitals is used, the strict angular momentum and particle number conservation guarantees superior orthogonal microscopic wave functions for nucleon transfer calculations. This method takes account of pairing correlations among the valence particles and shows the effects of quadrupole pairing and the usually neglected $\mathbf{j} . \mathbf{j}$ recoil terms.

\section{References}

[1] Published in Phys. Rev. C52 (1995) 1407 


\title{
Models for Rotational Spin Distributions of Primary Fission Products
}

\author{
J.O. Rasmussen, S.Y. Chu, D. Strellis and S. Asztalos, \\ Nuclear Science Division, Lawrence Berkeley National Laboratory, Berkeley, CA 94720, USA \\ R. Donangelo and L.F. Canto \\ Instituto de Fisica, Universidade Federal do Rio de Janeiro, C.P. 68528 \\ 21945-970 Rio de Janeiro, Brazil \\ J.D. Cole \\ Idaho National Engineering Laboratory, Idaho Falls, ID 83415 USA \\ M.A. Stoyer and Y.X. Dardenne \\ Lawrence Livermore National Laboratory, Livermore, CA 94550 USA \\ J.H. Hamilton \\ Dept. of Physics and Astronomy, Vanderbilt University, Nashville, TN 37235 USA
}

Data from the 1993 Gammasphere measurements of the GANDS93 collaboration [1] were analyzed for rotational band spin feeding intensities by Dubna members of the collaboration and presented [2] at the DANF96 Conference in the Slovak Republic. They found in the zero-neutron, so-called "cold fission," of ${ }^{104} \mathrm{Mo}$ (with ${ }^{148} \mathrm{Ba}$ partner) relative feeding intensities of 0.21:0.74:0.05 to $2+, 4+$, and $6+$ levels (with feeding to $0+$ indeterminate, for an average spin of 3.7. This average is below measured average spin values of fission fragments for fission with more neutrons evaporated, which averages around 5 for the light fragments. The ${ }^{106} \mathrm{Mo}$ "cold fission" has an even lower (2.4) average spin.

We have in our paper [3] at the DANF96 conference presented results from a careful theoretical study of the Coulomb excitation following scission, using a modified semiclassical theory with finite moment-of-inertia. Coulex starting from rest at scission gives too much spin, even when the initial spin is unrealistically set at zero. We must assume Coulex is reduced by substantial Coulomb-nuclear interference and fragment kinetic energy present at scission. Data analysis and theoretical studies are continuing.

\section{References}

[1] S.J. Zhu, Q.H. Lu, J.H. Hamilton, A.V. Ramayya, L.K. Peker, M.G. Wang, W.-C. Ma, B.R.S. Babu, J. Kormicki, D. Shi, J.K. Deng, J.O. Rasmussen, M.A. Stoyer, S.Y. Chu, K.E. Gregorich, M.F. Mohar, S. Asztalos, S.G. Prussin, J.D. Cole, R. Aryaenejad, Y.X. Dardenne, M. Drigert, K.J. Moody, R.W. Lougheed, J.F. Wild, N.R. Johnson, I.Y. Lee, F.K. McGowan, G.M. Ter-Akopian, and Yu.Ts. Oganessian, from Idaho National Engineering Laboratory; Vanderbilt University; Lawrence Berkeley National Laboratory; Lawrence Livermore National Laboratory; Joint Institute for Heavy Ion Research, Oak Ridge; Flerov Laboratory of Nuclear Reactions, JINR, Dubna, Russia; Qinghua University, Beijing

[2] G. Popeko, A. Daniel, and GANDS93 authors of preceding reference; Poster at Conf. on Dynamical Aspects of Fission (DANF96), CastaPapiernicka, Slovakia, Aug30-Sep 4, 1996, Proceedings to be published.

[3] Authors of this Annual Report, Invited paper by J.O. Rasmussen at DANF96 Conference of preceding reference. 


\title{
Multiband theory for heavy-ion neutron-pair transfer among deformed Gd nuclei.
}

\author{
S.Y. Chu, J.O. Rasmussen, and M.A. Stoyer \\ Nuclear Science Division, Lawrence Berkeley National Laboratory, Berkeley, CA 94720, USA \\ P. Ring \\ Physik Department Technische Universität München, D-85747 Garching, Germany \\ L.F. Canto and R. Donangelo \\ Instituto de Fisica, Universidade Federal do Rio de Janeiro, 21945 Rio de Janeiro, Brasil
}

In this paper our microscopic wave functions for $\mathrm{Gd}$ isotopes are applied to calculating neutron-pair transfer probabilities in heavy-ion collisions. The approximately 2600 -term wave functions come from Hamiltonian matrix diagonalization of systems of 12 Nilsson neutron orbitals, nearly half-filled. We use the lowest five bands in initial and final nuclei and calculate transfer for all even spins from 0 through 30 . Results for the sudden approximation (infinite moment-of-inertia) for neutron-pair extraction from ${ }^{156} \mathrm{Gd}$ by ${ }^{58} \mathrm{Ni}$ at near coulomb barrier energy, and extraction by ${ }^{206} \mathrm{~Pb}$ are shown. Next neutron-pair deposition by the $\mathrm{Ni}$ and $\mathrm{Pb}$ projectiles is calculated. Finally, a finite momentof-inertia semiclassical calculation is formulated and performed on the above collision system for n-pair transfer in both directions. The results are compared with experimental results. It is clear that the inclusion of the additional bands above the lowest two is important. Theory and experiment agree qualitatively on the rise of population above the yrast line at higher spins.

\section{References}

[1] Published in Phys. Rev. C52 (1995) 685 


\title{
Form factors for two-nucleon transfer in the diabolical region of rotating nuclei
}

\author{
A.R. Farhan \\ Physics Department, Kuwait University, Kuwait \\ L.F. Canto \\ Instituto de Fisica, Universidade Federal do Rio de Janeiro, C.P. 68528 \\ J.O. Rasmussen \\ Nuclear Science Division, Lawrence Berkeley National Laboratory, Berkeley, CA 94720, USA \\ P. Ring \\ Physik Department Technische Universität München, D-85747 Garching, Germany
}

The theoretical investigation of rotational bands in strongly deformed nuclei by Coulomb excitation and the subsequent transfer of nucleon pairs requires the knowledge of two-nucleon transfer amplitudes between rotating eigenstates of the $A$ and the A+or- 2 systems. In a semiclassical approximation these amplitudes are obtained by integrating over transfer form factors depending on the orientation angle of the deformed target nucleus. We use the cranking approximation based on a rotating mean field in order to calculate these transfer form factors. Their behavior is studied as a function of angular momentum, deformation and pairing correlations. In the region of diabolical points these form factors show considerable oscillations and phase changes reflecting the microscopic singleparticle structure involved in these matrix elements. In this way we are able to understand the underlying microscopic structure causing the diabolical behavior of pair transfer in this region.

\section{References}

[1] Published in Nucl. Phys. A 597 (1996) 387 


\section{The 8th Edition of the Table of Isotopes}

\section{R.B. Firestone, V.S. Shirley, C.M. Baglin, S.Y.F. Chu, and J. Zipkin}

A new edition of the Table of Isotopes has been published by John Wiley \& Sons, Inc. This edition is the eighth in a series started by Glenn T. Seaborg in 1940. The two-volume, 3168 page, cloth-bound edition contains nuclear structure and decay data for over 3100 isotopes and isomers. Approximately 24,000 references are cited and the appendices have been extended and completely rewritten. The book is packaged with an interactive $\mathrm{CD}$ ROM that contains the Table of Isotopes in Adobe Acrobat Portable Document Format for convenient viewing on PC and Macintosh personal computers and Unix workstations. The CD-ROM version contains a chart of the nuclides graphical index and separate indices organized for applied radioisotope users and nuclear structure physicists. Over 100,000 hypertext links are provided to move the user quickly through related information free from the limitations of page size. The data are presented more completely in a form that is easy to read on any monitor. Complete references with keyword abstracts are provided. The CD-ROM also contains the Table of Superdeformed Nuclear Bands and Fission Isomers; Tables of Atoms, Atomic Nuclei, and Subatomic Particles by Ivan P. Selinov the Evaluated Nuclear Structure Data File (ENSDF) and the ENSDF Manual; the Nuclear Science Reference file (NSR); and Adobe Acrobat Reader software. The databases on the CD-ROM can be viewed with VuENSDF software provided separately. More information about the Table of Isotopes and a faxable order form can be obtained through the Table of Isotopes Internet site at http://isotopes.lbl.gov/isotopes/toi.html.

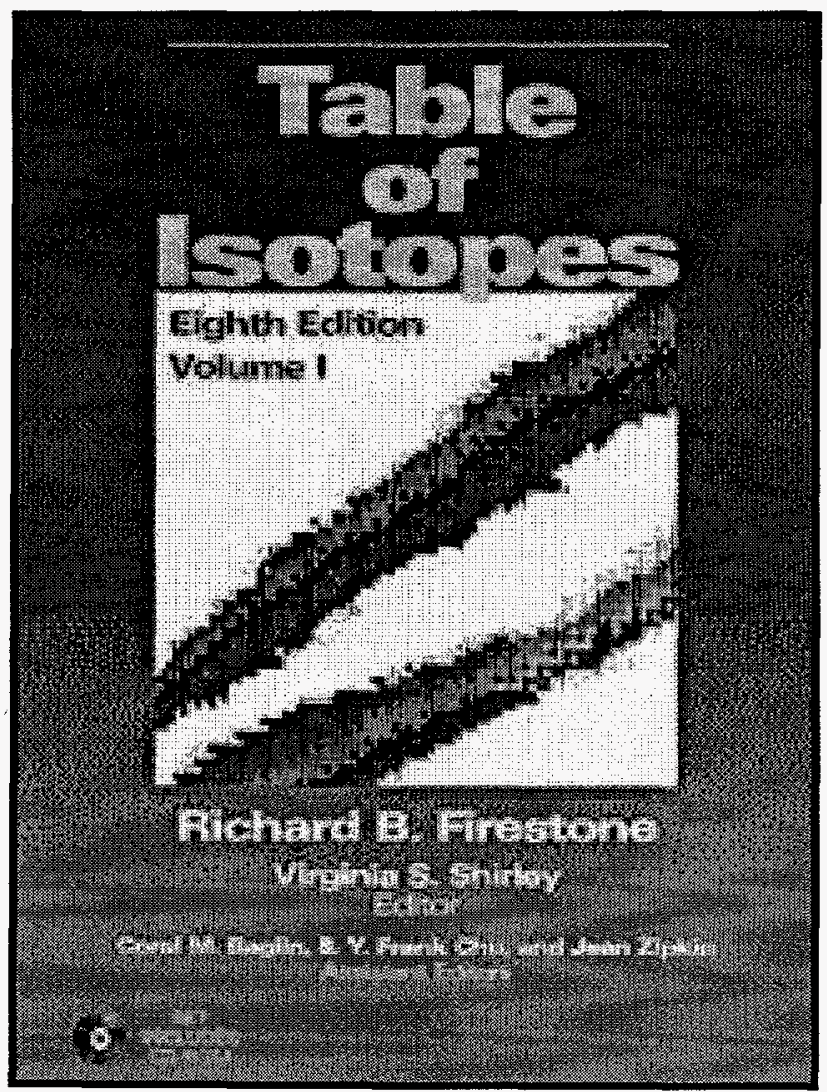




\section{The Isotope Explorer}

\section{S.Y.F. Chu ${ }^{I}$, H. Nordberg ${ }^{1,2}$, L.P. Ekstrom ${ }^{2}$, and R.B. Firestone ${ }^{I}$}

The Isotope Explorer (formerly VuENSDF) is a Windows $\mathrm{C}++$ application for searching and displaying the Evaluated Nuclear Structure Data File (ENSDF) and the Nuclear Science Reference file (NSR). The data can be downloaded by the Isotope Explorer from the Internet or from the Table of Isotopes CDROM. Data can be selected by level or transition properties, nuclear structure information, and transition coincidence relationships. The nuclear chart can be used to display selected nuclear properties, generate functional relationships between data, and display data from the nuclear databases. The database can be searched by restricting the range of nuclear properties, and the results can be displayed on the chart or in tables, level scheme drawings, and plots. Keyword abstracts for over 140,000 references can be retrieved and displayed.

Version 1.0 of VuENSDF was released in 1996, and Version 2.0 of the Isotope Explorer is available for beta testing. Both versions are freely distributed and can be downloaded from the WWW home page at http://isotopes.lbl.gov/isotopes/vuensdf.html. Sample windows for the isotope explorer are shown in figure 1 below.

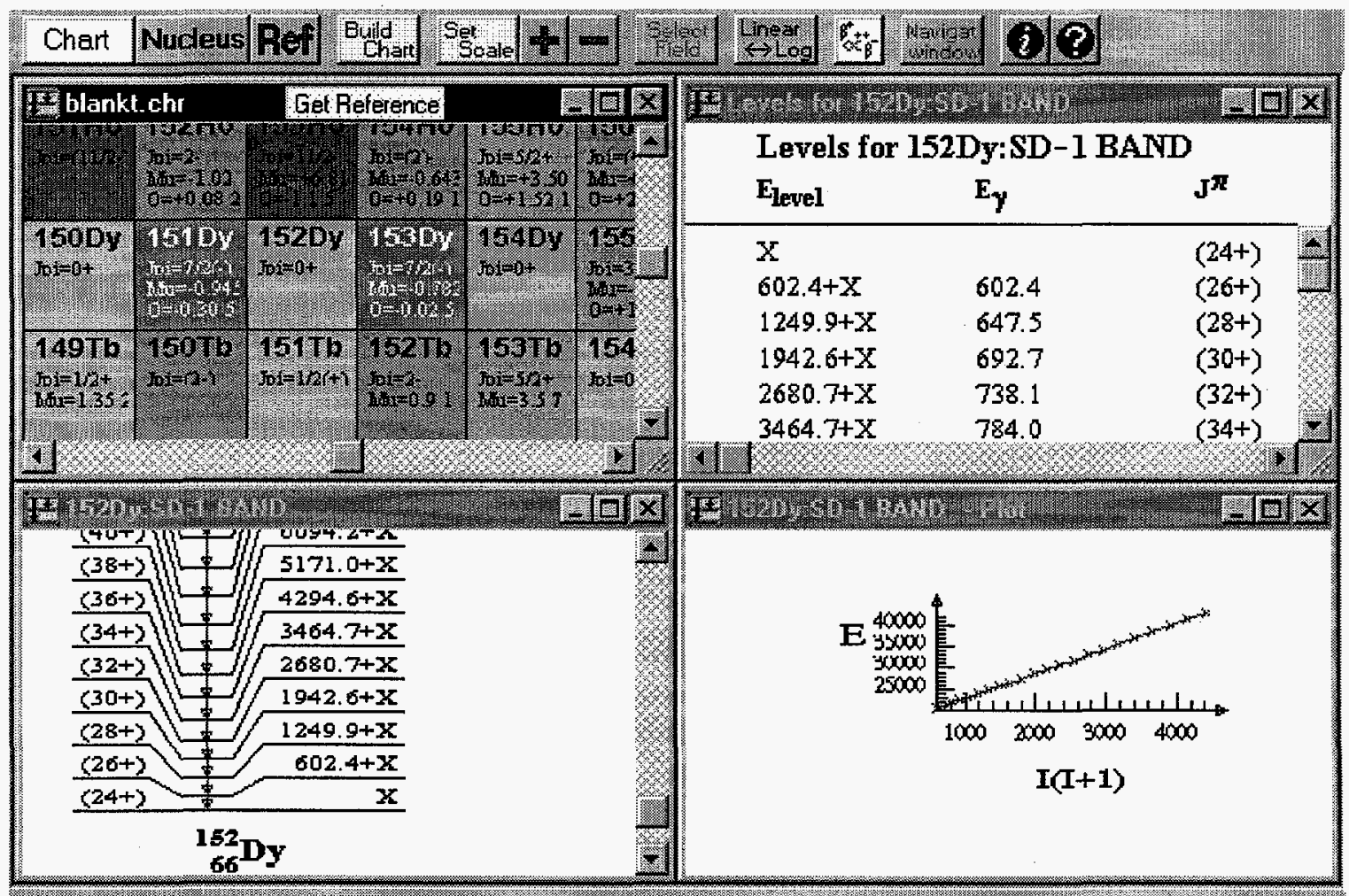

Nueler scrence References lokk:

Figure 1. Representative chart, table, level scheme, and plot for a ${ }^{152}$ Dy superdeformed band.

${ }^{1}$ Lawrence Berkeley National Laboratory

${ }^{2}$ Lund University, Sweden 


\title{
Nuclear Data on the Internet
}

\author{
R.B. Firestone ${ }^{1}$, S.C. Wu ${ }^{1,2}$, and S.Y.F. Chu ${ }^{1}$
}

The LBNL Isotopes Project has been providing nuclear data over the Internet since April, 1995. Eight primary home pages are supported for the Table of Isotopes, radioactive decay, Isotopes Explorer (VuENSDF) software and ENSDF data, nuclear astrophysics, nuclear structure and high-spin, atomic masses, thermal neutron capture, and Isotopes Project information. About $800 \mathrm{MB}$ of information is currently available through these websites.
The following table summarizes how often each of the Isotopes Project websites was accessed each month. The number of individual users, determined by counting connections from separate computer IP addresses, has risen to over 2500 during the peak months of 1996. Total link requests, indicating how many files or pages were accessed, have run as high as 38,400 in the busiest month.

Access Frequency for the Isotopes Project Nuclear Data WWW Home Pages

\begin{tabular}{lrrrrrrrrrrr}
\hline \multicolumn{1}{c}{ Date } & \multicolumn{1}{c}{ TOI } & DECAY & VUENSDF & ASTRO & HSPIN & TOIMASS & NG & \multicolumn{1}{c}{ IP } & $\begin{array}{r}\text { Total Link } \\
\text { Requests }\end{array}$ & Total \\
Users
\end{tabular}

${ }^{1}$ Lawrence Berkeley National Laboratory

${ }^{2}$ Tsing-Hua University, Taiwan 


\title{
Superdeformed and High-Spin Nuclear Structure Data on the Internet
}

\author{
B. Singh ${ }^{1,2}$, R.B. Firestone ${ }^{2}$ and S.Y.F. $\mathrm{Chu}^{2}$
}

With the advent of large detector arrays, a wealth of new information about the properties of nuclei at high-spin has become available. Rapid progress in this field has created a demand for up-to-date sources of information. We have published two editions of the Table of Superdeformed Nuclear Bands and Fission Isomers $^{1,2}$ to meet this need. This publication provides Table of Isotopes style summary tables, superdeformed band drawings, and dynamic moment of inertia plots for for all nuclei with superdeformed bands or fission

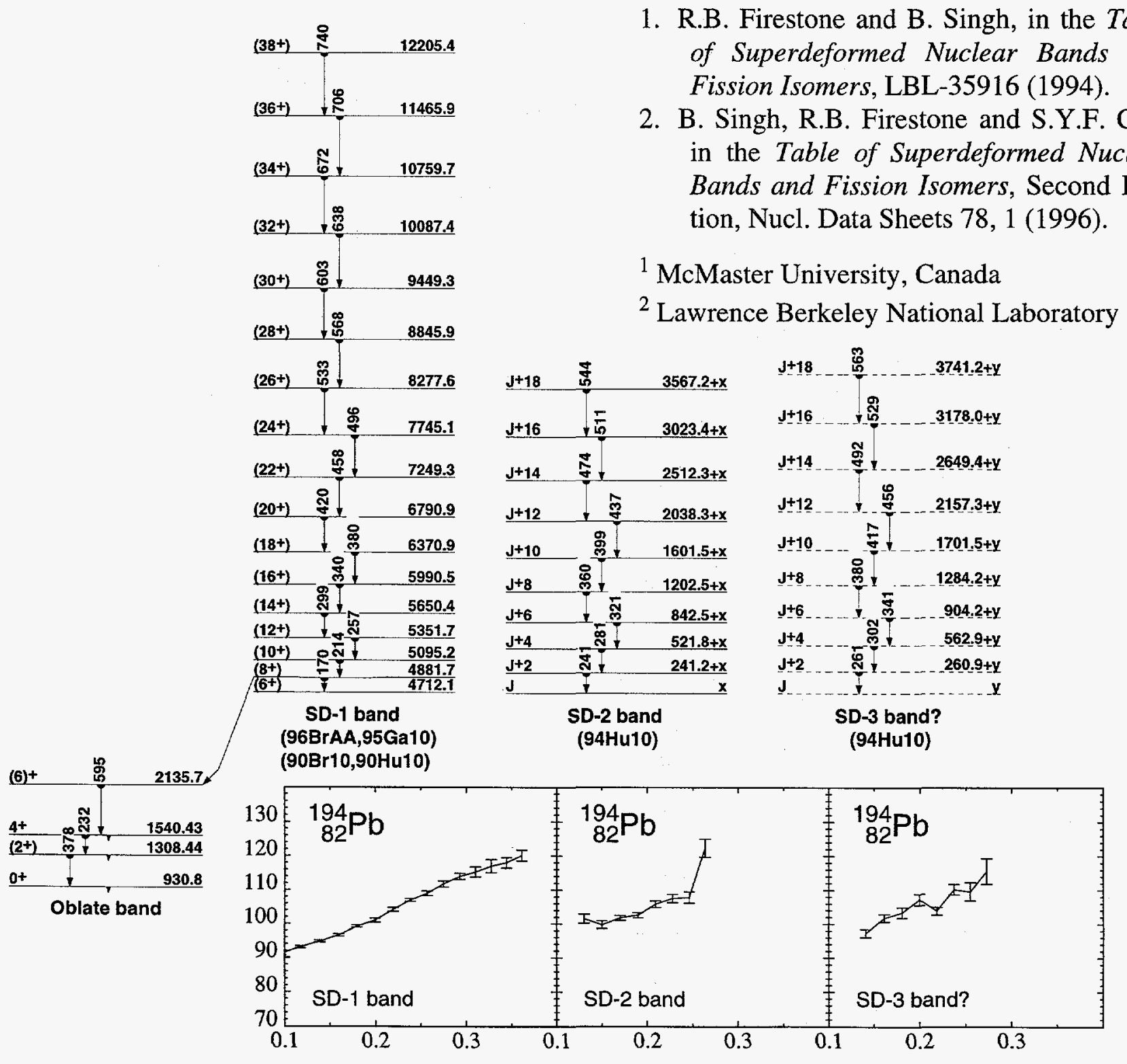

isomers. The Table of Superdeformed Nuclear Bands and Fission Isomers is being updated continuously and provided on our High-Spin and Nuclear Structure home page at http://isotopes.lbl.gov/isotopes/hspin.html on the Internet. In addition, recent high-spin references are being scanned continuously and new lists are posted on the WWW approximately every three months. Nilsson diagrams, links to the large detector array home pages, and other nuclear data are also available on this home page.

1. R.B. Firestone and B. Singh, in the Table of Superdeformed Nuclear Bands and

. Sin. Chu, in the Table of Superdeformed Nuclear Bands and Fission Isomers, Second Edition, Nucl. Data Sheets 78, 1 (1996).

${ }^{1}$ McMaster University, Canada

${ }^{2}$ Lawrence Berkeley National Laboratory

(90Br10,90Hu10)

100

${ }_{82}^{194} \mathrm{~Pb}$ superdeformed band drawings and dynamic moment of inertia plots 


\title{
Reducibility and Thermal Scaling in Nuclear Multifragmentation
}

\author{
L.G. Moretto, R. Ghetti, L. Phair, K. Tso, and G.J. Wozniak \\ Nuclear Science Division, Lawrence Berkeley National Laboratory
}

Recent studies have revealed the existence of a number of reducibility and thermal scaling properties in nuclear multifragmentation. The probability of emitting $n$ intermediate-mass fragments is found to be reducible to the probability of emitting a single fragment through the binomial expression $[1,2]$. The resulting one fragment probability shows thermal scaling by producing linear Arrhenius plots [1-3].

Similarly, the charge distributions associated with $n$ fragment emission are reducible to the one-fragment charge distribution [4]. Thermal scaling is also observed [4]. The reducibility equation contains a constant whose value, zero or positive, can be related to a univariant (two phases) or bivariant (one phase) regime [5].

The light fragment particle-particle angular correlations also show reducibility to the single-particle angular distributions as well as thermal scaling [6]. A mass scaling associated with the angular correlations suggests emission from several small sources $(A \approx 20)$.

The limits of applicability of scaling and reducibility are discussed in ref. [7] as well as their implications for the mechanism of multifragmentation.

The picture of multifragmentation, as it appears in ref. [7], is still sketchy and incomplete. However, we believe we have succeeded in unveiling important features which may be the key to deeper understanding.

The pervasive aspect of reducibility indicates that, whatever. the mechanism, the fragments are emitted essentially independent of one another. Thus we have shown that the probability $P_{n}$ of emitting $n$ fragments can be reduced to the probability of emitting a single fragment through the binomial equation. Similarly the $n$ fragment charge distributions can be reduced to the one fragment charge distribution. Furthermore, the particleparticle angular correlation can be reduced to the individual particle angular distributions.

In all the above quantities, reducibility is somehow restricted by what we may call "dynamical constraints". For the emission probabilities, the constraint is the binomial parameter $m$ (the number of "throws"), indicative either of a dynamical time window, or of the finite source size.

For the charge distributions, reducibility is restricted by the parameter $c$ (see refs. [4,5]), which seems to indicate some special way of enforcing charge conservation. We speculate that its transition from near zero to a finite value with increasing energy could be an indication of a transition from phase coexistence (liquid-vapor) to a single phase (vapor).

Finally, the angular correlations violate reducibility at small relative angle where particle-particle interactions become manifest [6].

These broad features of reducibility speak to the near independence of fragment emission but not to its mechanism.

Thermal scaling instead makes a clear statement about the fact that the elementary probabilities entering in the $n$ fragment emission probabilities, the $n$ fragment charge distributions, and the two fragment angular correlations are thermal. In other words, these probabilities have the form of a Boltzmann factor and clearly portray its characteristic energy dependence (Arrhenius plots).

Thus the resulting picture is tantalizingly close, but not quite that of a compound nucleus emission. Apparently sources are dynamically generated which, within dynamical constraints of time and size, emit fragments in a thermal manner. Among the potential fruits that can be reaped from the pursuit of the analysis outlined so far are dynamical features of source formation, size and lifetime, as well as static features like barriers, source sizes and densities.

[1] L.G. Moretto et al., Phys. Rev. Lett. 74, 1530 (1995).

[2] K. Tso et al., Phys. Lett. B 361, 25 (1995).

[3] L.G. Moretto et al., Phys. Rev. Lett. 71, 3935 (1993).

[4] L. Phair et al., Phys. Rev. Lett. 75, 213 (1995).

[5] L.G. Moretto et al., Phys. Rev. Lett. 76, 372 (1996).

[6] L. Phair et al., Phys. Rev. Lett. 77, 822 (1996).

[7] L.G. Moretto et al., Phys. Rep. (in press). 


\title{
Aspects of Reducibility and Thermal Scaling in Multifragmentation
}

\author{
R. Ghetti, L.G. Moretto, L. Phair, K. Tso, G.J. Wozniak \\ Nuclear Science Division, Lawrence Berkeley National Laboratory
}

Recent experimental evidence of binomial reducibility and thermal scaling in multifragmentation, has raised a number of physical and technical issues that need to be addressed. To this end, two simulations of multifragment decay have been implemented, a binomial simulation and a Poisson one [1]. By means of the binomial simulation, the reducibility of the $n$-fold intermediate-mass-fragment multiplicity and charge distributions to the one-fragment emission values has been investigated. With both simulations, the effects of using the transverse energy as a measure of the system excitation energy have been evaluated. Possible experimental biases in the results have been evaluated as well.

We have implemented binomial and Poisson decay simulations to address a number of issues connected to the experimental multifragmentation studies presented in Refs. [2-7]. For the binomial simulation, we have assumed that the $n$-fragment emission probabilities $P_{n}$ are distributed according to the binomial distribution, and that multifragmentation is empirically reducible to a combination of nearly independent fragment emissions. Moreover, we have assumed a thermal dependence of the elementary emission probability $p$ on the nuclear temperature.

By processing simulated events, we have tested the standard procedures utilized in the experimental data analysis. We have verified that the simulation input is recovered without significant loss of information and how the final results reflect different input conditions.

The simulation results strengthen the validity of the reducibility approach used in the experimental work and the physical meaning of quantities such as the elementary probability $p$. Reducibility is a valid approach also when multiple sources of fragment emission are created in the collision, if one of the sources is dominant. The Arrhenius plot is a powerful tool to explore the thermal features of the elementary probability $p$, even though the determination of the actual fragment emission barrier $B$, proportional to the slope of the plot, can be hampered by the presence of source residues and by small size effects.

From both binomial and Poisson simulations it appears that the total transverse kinetic energy $E_{t}$ is a good observable that can be reliably used for the measurement of the excitation energy in multifragmentation studies at intermediate energies. In our simulations, the transverse energy is linearly correlated with the system excitation energy and weakly correlated with the IMF multiplicity. We have demonstrated that neither event-to-event fluctuations nor $E_{t}$ auto-correlation effects are large enough to distort a Poisson distribution into a binomial distri- bution. In particular the mean and variance of the $N_{\mathrm{IMF}}$ distribution are reasonably well preserved, even when the measurement of $E_{t}$ is limited by the detection efficiency.

We have tested the effects of a reduced geometric efficiency as well as the effects of a software replica of the detection device. We have shown that a reduced geometric efficiency preserves binomiality and that efficiency corrections can be applied through the binomial parameters $p$ and $m$. We have explored the effects of a finite detection acceptance on the Arrhenius plot and have shown that when the geometric acceptance is reduced, it is still possible to recover the binomial input without significant loss of information. The introduction of an energy dependent efficiency, instead, can compromise this possibility, especially when the transverse energy is used as a measure of the system excitation energy.

We have also shown that, in a binomial decay scenario, the $n$-fold IMF charge distributions are reducible to the 1-fold, consistent with the experimental findings. In the binomial simulation, the $n$-fold charge distributions are independent of the fold number $n$ as long as a sizeable remnant serves as a reservoir of mass, charge and excitation energy. A dependence on $n$ arises when the entire system is consumed.

The results of the decay simulations have made us more confident that the experimental procedures utilized in the data analysis of Refs. [2-7] are correct, and that the empirical findings of reducibility and thermal scaling in multifragmentation are not an artifact of incomplete detection efficiency nor are they generated by the autocorrelation of the variable used to estimate the excitation energy.

[1] R. Ghetti et al., LBNL-39196.

[2] L.G. Moretto et al., Phys, Rev. Lett. 74 (1995) 1530.

[3] K. Tso et al., Phys. Lett. B 361 (1995) 25.

[4] L. Phair et al., Phys. Rev. Lett. 75 (1995) 213.

[5] L. Phair et al., Phys. Rev. Lett. 77 (1996) 822.

[6] L.G. Moretto et al., Phys. Reports (1996), accepted, LBNL-39388.

[7] A. Ferrero et al., Phys. Rev. C 53 (1996) R5. 


\title{
Reducibility, Thermal and Mass Scaling in Angular Correlations from Multifragmentation Reactions
}

\author{
L. Phair, L.G. Moretto, G.J. Wozniak, \\ Nuclear Science Division, Lawrence Berkeley National Laboratory
}

R.T. de Souza, D. R. Bowman, N. Carlin, C. K. Gelbke, W.G. Gong, Y.D. Kim, M.A. Lisa, W.G. Lynch, G.F. Peaslee, M.B. Tsang, and F. Zhu NSCL and Department of Physics and Astronomy, Michigan State University, East Lansing, MI 48824

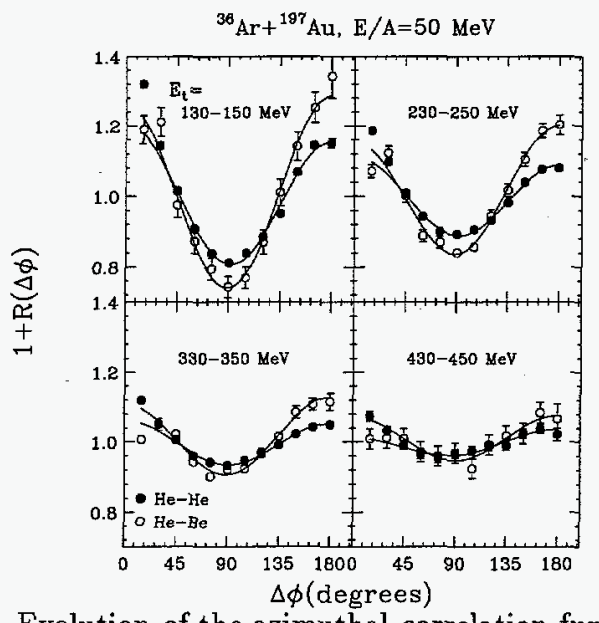

FIG. 1. Evolution of the azimuthal correlation functions of two He particles (solid circles) and $\mathrm{He}$ and Be particles (open circles) emitted at $\theta_{l a b}=31^{\circ}-50^{\circ}$ for four different cuts on the transverse energy $E_{t}$. The solid lines are fits described in ref. [1].

We have explored the azimuthal correlations between emitted particles to search for thermal scaling of the correlation amplitudes and reduciblity of the two-fold emission probability to that of the one-fold [1]. Fig. 1 shows azimuthal correlation functions of different particle pairs for different values of the transverse energy $E_{t}$. Consistent with previous observations, the azimuthal correlation functions exhibit a slightly distorted V-shape pattern. At larger excitation energies (assumed proportional to $E_{t}$ ) the correlations become progressively damped.

To understand the evolution of the correlation functions of Fig. 1, we have considered the exactly solvable problem of thermal particle emission from a rotating source. The classical probability of emitting a particle with reduced mass $\mu$ from the surface of a rotating system (of angular momentum $I$, moment of inertia $\Im$, temperature $T$ and distance $R$ between centers of the "daughter" and emitted nuclei) in a direction given by polar angle $\theta$ (in the center of mass frame) and azimuthal angle $\phi$ (measured with respect to the reaction plane) is:

$$
P(\theta, \phi) \propto \exp \left[-\beta \sin ^{2} \theta \sin ^{2} \phi\right]
$$

where

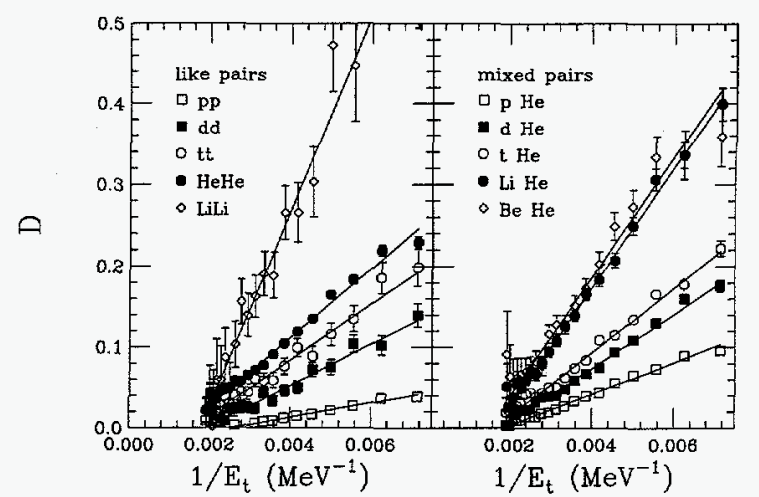

FIG. 2. Left panel: The fit parameter $D$ as a function of $1 / E_{t}\left(\propto 1 / T^{2}\right)$ for the indicated identical particle pairs. Solid lines are linear fits to the data. Right panel: Same as left panel but for particle pairs of different masses.

$$
\beta=\frac{\hbar^{2} I^{2}}{2 \Im T} \frac{\mu R^{2}}{\left(\Im+\mu R^{2}\right)}=\frac{E_{r o t}}{T} \frac{\mu R^{2}}{\left(\Im+\mu R^{2}\right)}
$$

and $E_{\text {rot }}$ is the rotational energy of the source.

If the fragments are emitted independently of one another, the joint probability of observing two particles at a given polar angle $\theta$ and different azimuthal angles $\phi$ and $\phi+\Delta \phi$ is $P(\theta, \phi, \Delta \phi)=P(\theta, \phi) P(\theta, \phi+\Delta \phi)$. The resulting probability distribution must be averaged over the different directions of $\vec{I}$ arising from different orientations of the impact vector and one obtains proportionality to a modified Bessel function of zeroth order. Expanding this function, the joint probability is approximately:

$$
P(\theta, \Delta \phi) \propto 1+\frac{D}{1+D / 2} \cos 2 \Delta \phi+\frac{D^{2}}{(D+2)^{2}} \cos ^{2} 2 \Delta \phi
$$

where $D=\left(\beta^{2} \sin ^{4} \theta\right) / 8 \propto 1 / T^{2} \propto 1 / E_{t}$.

A plot of $D$ (extracted from fits to the correlation data, see Fig. 1) as a function of $1 / E_{t}$ is given in Fig. 2. The simplest explanation for the observed linear behavior (thermal scaling) is that the fragmenting system attains an average rotational energy which is largely independent of $E_{t}$.

[1] L. Phair et al., Phys. Rev. Lett. 77, 822 (1996). 


\title{
Comment on "Circumstantial Evidence for Critical Behavior in Peripheral Au+Au Collisions at $35 \mathrm{MeV} /$ nucleon."
}

\author{
L. Phair, Th. Rubehn L.G. Moretto, G.J. Wozniak, \\ Nuclear Science Division, Lawrence Berkeley National Laboratory
}

Mastinu et al. recently reported the observation of several positive signals possibly indicating critical behavior in peripheral collisions of $\mathrm{Au}+\mathrm{Au}$ at $E / A=35 \mathrm{MeV}$ [1]. In our comment [2], we examine the choice of variables used to determine the presence (or absence) of critical behavior. We do this by repeating the analysis of ref. [1] on "data" from a simulation with no critical behavior.

The simulation samples a charge distribution and conserves charge (breaking up a source of size $Z_{0}$ ). The charge particle multiplicity $N_{C}$ is specified at the outset. Within an event, at multiplicity $n$ (where $1 \leq n \leq$ $N_{C}-1$ ) the probability to emit a particle of a given $\mathrm{Z}$ is

$$
P_{n}(Z) \propto e^{-\alpha Z}
$$

under the constraint that at each "emission step" $n$, the $Z$ of the emitted particle be sufficiently small so that the event will satisfy the requirement of containing $N_{C}$ particles. We chose $\alpha=0.3$ and $Z_{0}=79$. The choice of an exponential charge distribution (and $\alpha=0.3$ ) is arbitrary as is the specific implementation of charge conservation.

Using this simulation we constructed "events" and examined the proposed observables for critical behavior. In Fig.1a is shown the Campi scatter plot of $Z_{\max }$ versus $M_{2} / Z_{0}$. We observe the two-branch feature commonly interpreted as indicating "sub-critical" and "over-critical" events.

By applying cuts similar to those in ref. [1], we have plotted the resulting multiplicity distribution (Fig.1b). Qualitative agreement with the experimental data [1] is achieved with this simple event selection. We question whether these cuts "select" events that can be associated with critical behavior.

Using the same cut (2) in the Campi plot for "potentially critical" events as in ref. [1], we have constructed the horizontally scaled factorial moments. These moments are shown in Fig.1c. The linear rise with decreasing bin size is quite apparent. It has already been pointed out $[3,4]$ that spurious intermittency signals can be observed by mixing events of different multiplicity, which is clearly the case for ref. [1] and for which the authors appropriately express concern.

Finally, we show a plot of $M_{2}$ versus $N_{c}$ (Fig.1d). A peak in such a plot is often mistakenly taken as an indication of critical behavior. While we observe a peak, our simulation is one that assuredly contains no critical behavior.

We have repeated the analysis shown in Fig. 1 for power law charge distributions and different implemen-
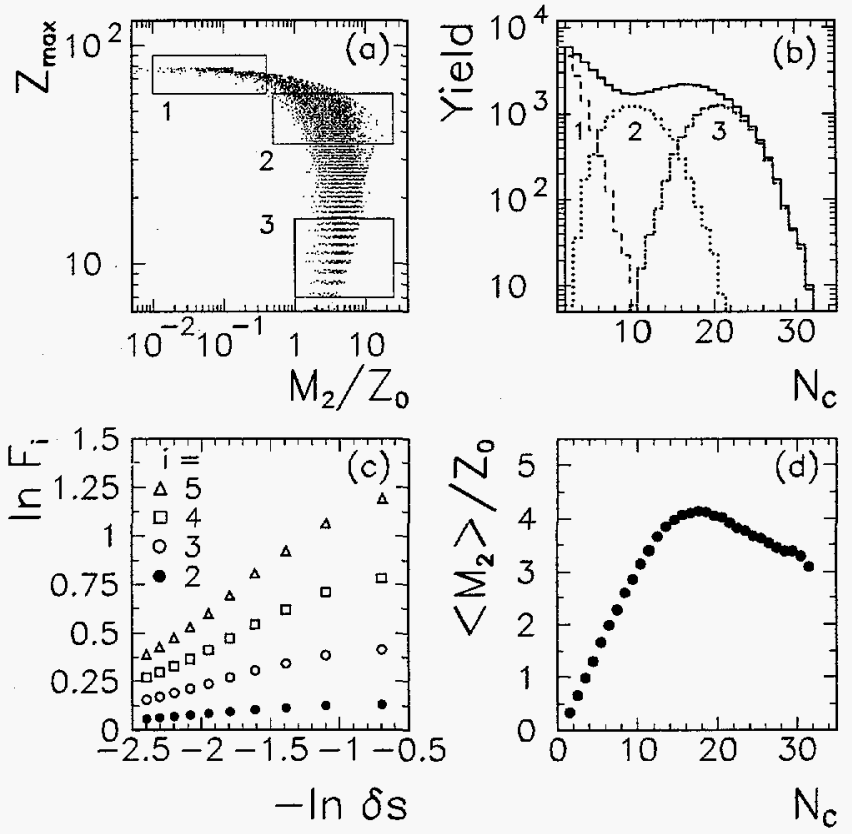

FIG. 1. a) $Z_{\max }$ vs $M_{2} / Z_{0}$ with cuts similar to those used in ref. [1]. b) Input multiplicity distribution for the simulation (solid line) along with output distributions for cuts 1 (dashed), 2 (dotted) and 3 (dotted-dashed). c) Log of the scaled factorial moments $(i=2,3,4,5)$ as a function of the negative $\log$ of the bin size $\delta s$ for cut 2. d) $\left\langle M_{2}\right\rangle / Z_{0}$ versus $N_{C}$.

tations of charge conservation but the qualitative results remain the same.

Before doing this analysis, we were under the mistaken impression that the simple observables listed above give an indication of the presence or absence of critical behavior. Part of our confusion came from the vast commentary in the literature that points to these observables as indicators of critical phenomena. In fact, positive signals in all of these observables are probably found in any simulation that conserves charge and where light particle emission is preferred over heavier fragments. And so we caution that the positive signals observed in ref. [1] are insufficient to establish critical behavior since they appear even in simple models which contain neither a phase transition nor critical behavior.

[1] P.F. Mastinu et al., Phys. Rev. Lett. 76, 2646 (1996).

[2] L. Phair et al., Phys. Rev. Lett. (in press).

[3] L. Phair et al., Phys. Lett. B 291, 7 (1992).

[4] X. Campi and H. Krivine, Nuc. Phys. A 589, 505 (1995). 


\title{
Comment on "Probing the Nuclear Liquid-Gas Phase Transition"
}

\author{
L.G. Moretto, R. Ghetti, L. Phair, K. Tso, and G.J. Wozniak
}

Nuclear Science Division, Lawrence Berkeley National Laboratory

In a recent paper Pochodzalla et al. [1] report a tantalizing dependence of nuclear temperature upon excitation energy. This dependence is characterized by an initial rise, followed by a wide plateau and a subsequent rise. The authors tentatively ascribe the initial rise to "ordinary" evaporation, the middle plateau to a liquid-vapor phase transition, and the final rise to a nearly ideal gas dependence for the overheated vapor.

The question arises whether the entire experimental curve can be interpreted in terms of equilibrium thermodynamics. If so, several problems arise. For instance, the claimed distinction between the initial rise (interpreted as the fusion-evaporation regime) and the plateau (hinted at as liquid-vapor phase transition) is not tenable, since evaporation is the liquid-vapor phase transition and no thermodynamic difference exists between evaporation and boiling.

Furthermore, the "caloric curve" requires for its interpretation an additional relationship between the variables $T, P$, and $V$. More to the point, the plateau is a very specific feature of the constant pressure condition rather than being a general indicator of a phase transition. For instance, a constant-volume liquid-vapor phase transition is not characterized by a plateau but by a monotonic rise in temperature. This can be easily proven by means of the Clapeyron equation, which gives $d P / d T$ along the univariance line (liquid-vapor transition) together with the ideal-gas equation for the vapor [2].

As an example, Fig. 1 shows a standard temperature $T$ vs entropy $S$ diagram for water vapor. The region under the bell is the phase coexistence region. For the constant pressure curves $(\Delta S=\Delta H / T)$, the initial rise along the "liquid" curve is associated with pure liquid, the plateau with the liquid-vapor phases, and the final rise with overheated vapor. The constant volume curves $(\Delta S=\Delta E / T)$ cut across the coexistence region at an angle, without evidence for a plateau.

Thus one concludes that the alleged reminiscence of the observed "caloric curve" with "the paradigm of a phase transition" may be more pictorial than substantive, and that indicators other than the plateau may be needed to substantiate a possible transition from one to two phases. More specifically, an additional relationship between the three variables $P, T, V$ (like $P=$ const, or $V=$ const, etc.) is needed to interpret a $T$-E diagram unequivocally.

[1] J. Pochodzalla et al., Phys. Rev. Lett. 75, 1040 (1995).

[2] L.G. Moretto et al., Phys. Rev. Lett. 76, 2822 (1996).

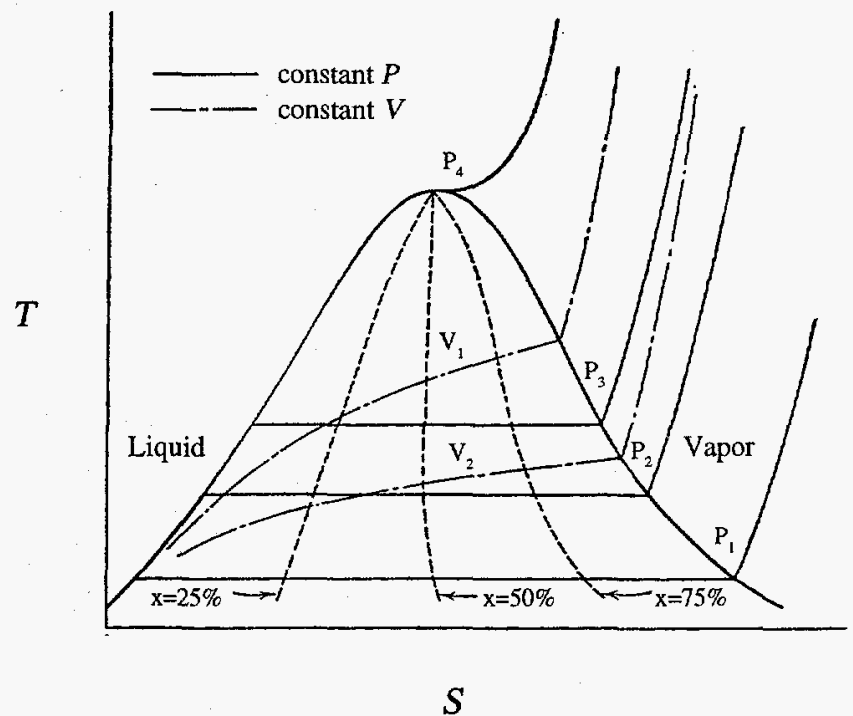

FIG. 1. Temperature-entropy diagram for steam. Curves are shown for constant pressure $\left(P_{4}>P_{3}>P_{2}>P_{1}\right)$, constant volume $\left(V_{1}<V_{2}\right)$ and constant percentage in the gas phase (dashed lines). 


\title{
Stable Coulomb bubbles?
}

\author{
L.G. Moretto, K. Tso and G.J. Wozniak \\ Nuclear Science Division, Lawrence Berkeley Laboratory, Berkeley, California 94720
}

Within the framework of the liquid drop model, the energy of a bubble in units of twice the surface energy of the equivalent sphere (constant volume) can be expressed as a function of the bubble monopole coordinate [1]. At zero pressure and angular momentum, the surface energy increases as a bubble develops from a sphere, but the Coulomb energy decreases as the charges are brought farther apart due to the bubble expansion. Therefore, an interplay between the Coulomb and surface energies may generate a minimum energy point along the monopole coordinate. The bubble minimum appears first at a value of the fissility parameter $\mathrm{X}=2.022$, and becomes the absolute minimum at $\mathrm{X}=2.204$.

A Coulomb bubble that is stable against monopole oscillations, may be subjected to higher order perturbations. The higher deformation modes of the bubble can be divided into two classes: the radial modes and the crispation modes. The deformations on the two surfaces are in phase with each other for a radial mode, and they are out of phase for a crispation mode.

The monopole oscillation obviously belongs to the class of radial modes. The lowest order crispation mode is the dipole mode which corresponds to a rigid displacement of the two spheres, one with respect to the other.

Unlike the dipole oscillation, higher multipole perturbations tend to increase the surface energy, and thus stabilize the unperturbed bubbles. This surface effect is the same for the radial and crispation modes, since the two modes differ only in the relative orientation of their surfaces. However, the Coulomb effect is drastically different for the two modes. The Coulomb perturbation energy is always negative for the radial mode, since the average distance between charges is increased slightly due to the perturbation. A similar effect of Coulomb destabilization is observed for the crispation mode in case of thick bubbles. In fact, the two modes are indistinguishable for a solid sphere. However, this destabilization effect becomes progressively weaker as the bubble expands. When a bubble is sufficiently thin, the Coulomb perturbation energy becomes positive, and stabilizes the crispation modes. This is because the Coulomb force tends to resist the attempt to concentrate the charge in "clumps" distributed on the surface of the thin bubble, as required by the higher order crispation modes. At the threshold fissility of $X=2.022$, the value of $X_{\text {eff }}$ lies just about at the $n=4$ line, indicating that the bubble is unstable up to the $n=4$ mode. As more charge is brought into the bubble with increasing values of $\mathrm{X}$, the Coulomb bubble expands and it becomes stable with respect to the $n=4$ and even to the octupole mode $(n=3)$ at $\mathrm{X}=2.5$. How- ever, the Coulomb bubble is still unstable with respect to the quadrupole mode $(n=2)$. In fact, a further increase of $\mathrm{X}$ does not stabilize the quadrupole mode.

Yet, it may be possible to have a stable nuclear bubble. If the bubble is warm, it fills up with vapor arising from the fluid itself. The resulting pressure acts only upon the monopole mode, by displacing outwards the Coulomb minimum. The effect on the other radial modes is nil, since only changes in volume are relevant to pressure. Thus the bubble has become secularly stable with respect to all the modes.

However, when a bubble becomes rather thin, a possible demise of the bubble may be associated with the sheet instability. The sheet instability is a new kind of Rayleigh-like surface instability associated with the crispation modes. A nuclear sheet of any thickness tends to escape from the high surface energy by breaking up into a number of spherical fragments with less overall surface. However, any perturbation of finite wavelength increases the surface area, and consequently the energy of the sheet, independent of the sheet thickness. Clearly, this barrier prevents the sheet from reaching the more stable configurations. However, when a nuclear sheet becomes sufficiently thin, the two nuclear surfaces interact with each other. This proximity interaction may become sufficiently strong to overcome the sharp barrier and causes the sheet to puncture into numerous fragments.

Since a bubble, like a sheet, must rely on the proximity interaction to become unstable, it will retain its surface stability until the range of the surface-surface interaction is of the order of its thickness.

In conclusion, the depletion of charge in the central cavity of nuclear bubbles reduces the Coulomb energy significantly and thus stabilizes "Coulomb" bubbles against monopole oscillations. These Coulomb bubbles, however, are at least unstable to perturbation of the quadrupole radial mode. On the other hand, a sufficiently high temperature generates a vapor pressure in the central cavity which drives the bubble to a thinner configuration that is stable against all the radial modes. Finally, a thin Coulomb bubble behaves like a sheet, and becomes susceptible to a proximity surface instability via the crispation modes when its thickness is comparable to the range of the proximity interaction.

[1] I.G. Moretto, K. Tso and G.J. Wozniak, Phys. Rev. Lett. (accepted), LBNL-39678. 


\title{
Charge correlations and dynamical instabilities in multifragmentation*
}

\author{
L.G. Moretto, Th. Rubehn, L. Phair, N. Colonna, and G.J. Wozniak \\ Nuclear Science Division, Lawrence Berkeley National Laboratory, Berkeley, California 94720
}

\begin{abstract}
D.R. Bowman, G.F. Peaslee, N. Carlin, R.T. de Souza, C.K. Gelbke, W.G. Gong, Y.D. Kim, M.A. Lisa, W.G. Lynch, and C. Williams

National Superconducting Cyclotron Laboratory and Department of Physics and Astronomy, Michigan State University, East Lansing, MI 48824
\end{abstract}

In recent years, it has been suggested that multifragment production can be related to the occurrence of instabilities in the intermediate system produced by heavy ion collisions. Although the scenarios and the models vary, breakup into several nearly equal-sized fragments is a common result of instabilities. We report the examination of model independent signatures that would indicate decay into a number of nearly equal-sized fragments by investigating charge correlations from both experimental data and simulations [1].

For comparison with experimental data, and to determine the sensitivity of our analysis, Monte Carlo calculations have been performed. The created events obey two conditions: the sum charge of all fragments is conserved within an adjustable accuracy, and a fragment is produced according to a probability consistent with the experimental finding, that the charge distributions are nearly exponential functions. Furthermore, the simulation allows one to smear out the charge distributions of the individual fragments of an event according to a Gaussian distribution.
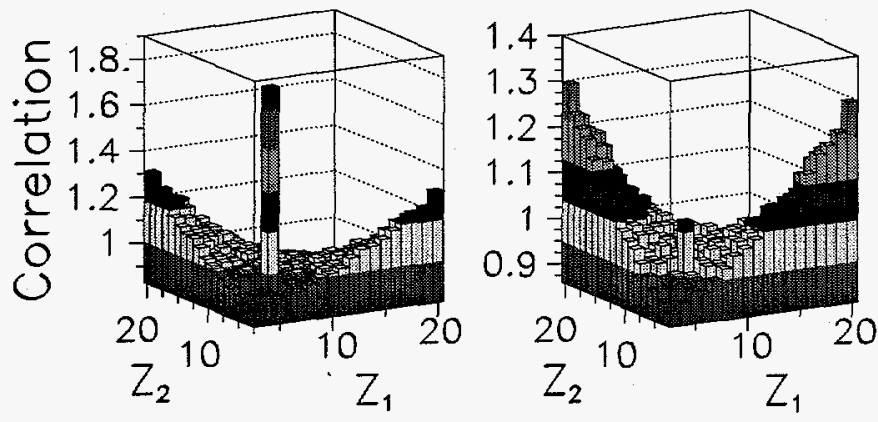

FIG. 1. Two particle charge correlations of IMF's from simulations for $N_{\text {IMF }}=6$ plus $1 \%$ (left) and $0.1 \%$ (right panel) of $Z_{\text {art }}=6$.

To demonstrate the sensitivity of our method to breakup configurations producing equal-sized fragments, Fig. 1 shows the results of simulations for the case $N_{\text {IMF }}=6$. Here, a "contamination" of $1 \%$ of the events consisting of fragments which all have the size $Z_{\text {art }}=6$ has been added to the data set. The peak produced by these fragments is clearly visible, even if we decrease the yield of equal-sized fragments to only $0.1 \%$. We note that the "background" seen in Fig. 1 is only due to charge con- servation. The same analysis used for the simulation has been applied to experimental data of the reaction $\mathrm{Xe}+\mathrm{Cu}$ at $E / A=50 \mathrm{MeV}$ for different $N_{\text {IMF }}$ cuts. An enhanced signal for breakup into nearly equal-sized fragments was not observed in any of the $N_{\text {IMF }}$ bins.
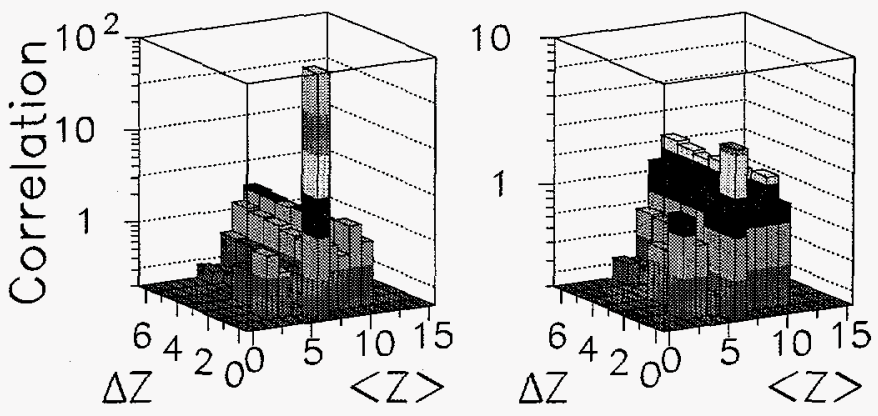

FIG. 2. Higher order charge correlations from the simulations for a system size of $83, N_{\mathrm{IMF}}=6$ and widths of $\omega=0$ and $\omega=2$.

To increase the sensitivity of the method, we have also investigated higher order charge correlations [1]. In Fig. 2, we show the results of such an analysis. Here, $\langle Z\rangle$ denotes the average fragment charge of the event, and $\Delta Z$ is the standard deviation. The same simulation which has already been shown in Fig. 1 was used. Here, only $0.1 \%$ of the events were chosen to have fragments with equal size. We show two cases with a "smearing" width of $\omega=0$ and $\omega=2$, respectively. The comparison between the two particle and the higher order charge correlation functions for the same simulations using $\omega=0$ shows an enhancement of $\sim 20 \%$ for the first case (right panel of Fig. 1) while the signal in the second case exceeds the "background" by roughly a factor of 100 (left panel of Fig. 2).

We have also analysed our experimental data for higher order charge correlation. No signals are observed that can be attributed to an enhanced production of nearly equal-sized fragments. This results in an upper limit of breakup events with nearly equal-sized fragments of less than $0.05 \%$ if we assume a width $\omega<3$. Similar results have been obtained for other systems albeit with significantly poorer statistics.

[1] , ${ }^{*}$ L.G. Moretto et al., Phys. Rev. Lett. 77, 2634 (1996). 


\title{
Scaling Laws, Shell Effects, and Transient Times in Fission Probabilities*
}

\author{
L. G. Moretto, K. X. Jing, R. Gatti, and G. J. Wozniak \\ Nuclear Science Division, Lawrence Berkeley National Laboratory \\ R. P. Schmitt \\ Cyclotron Institute, Texas ABM University
}

In a recent paper [1] we analyzed the intermediate mass fragment excitation functions for an extensive range of fragment atomic numbers, obtained for four different compound nuclei. A special way of plotting these data permits the ready observation of deviations from the transition state rates as a departure from a straight line. For over 70 excitation functions, the lack of deviations from the transition state null hypothesis both as a function of fragment $Z$ and excitation energy led to the conclusion that the transition state rates were closely obeyed, and that no substantial transient time effects were present in these systems over the covered experimental energy and lifetime ranges.

In this work we extend the method to the fission of systems in the lead region, where lies the dramatic onset of the shell effects, and for which transient time effects have been claimed. Specifically we show the following: a) fission excitation functions for nuclei ranging from $\mathrm{A}=186$ to 213 are rigorously scalable in terms of the transition state rates once the shell effects are accounted for; $b$ ) the shell corrections $\Delta_{\text {shell }}$ obtained from the data are in excellent agreement with those obtained from the ground state masses.

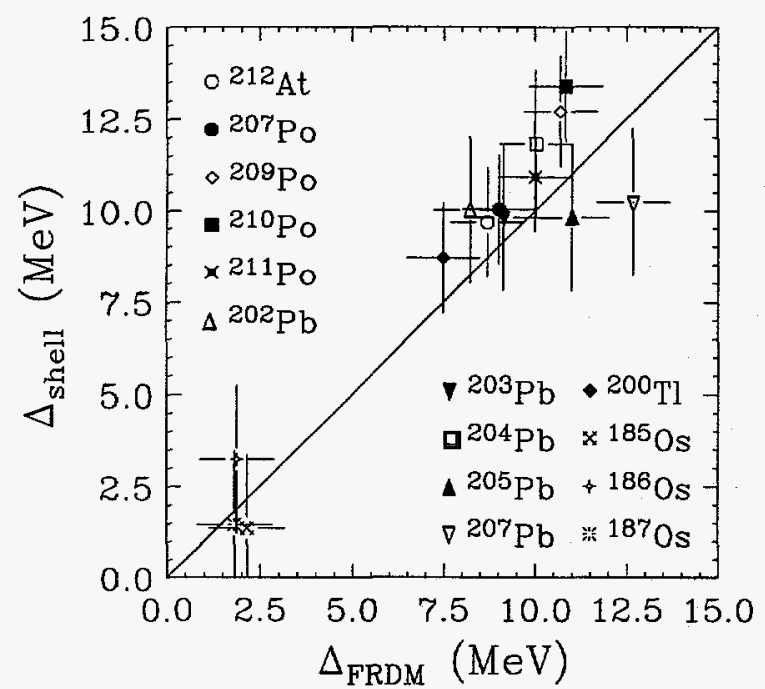

FIG. 1. Shell corrections $\Delta_{\text {shell }}$, for the daughter nuclei $\left(A_{C N}-\mathbf{n}\right)$, extracted from fits to the fission excitation functions, plotted against the values determined from the ground state masses [2]. The diagonal line is to guide the eye.

In fig. 1 are shown the values of the ground state shell effect $\Delta_{\text {shell }}$ of the daughter nuclei after neutron emission, obtained by fitting the fission excitation func- tions with a transition state formalism in which $\Delta_{\text {shell }}$ is included in the expression of the level density of the residual nucleus. In this figure we plot these values of $\Delta_{\text {shell }}$ versus the corresponding values obtained as the difference of the ground state mass and the corresponding liquid drop value. The observed correlation is excellent. Note that the present shell effects are obtained in a totally independent way, which, in contrast to the standard procedure [2] is completely local, namely it depends only on the properties of the nucleus under consideration.

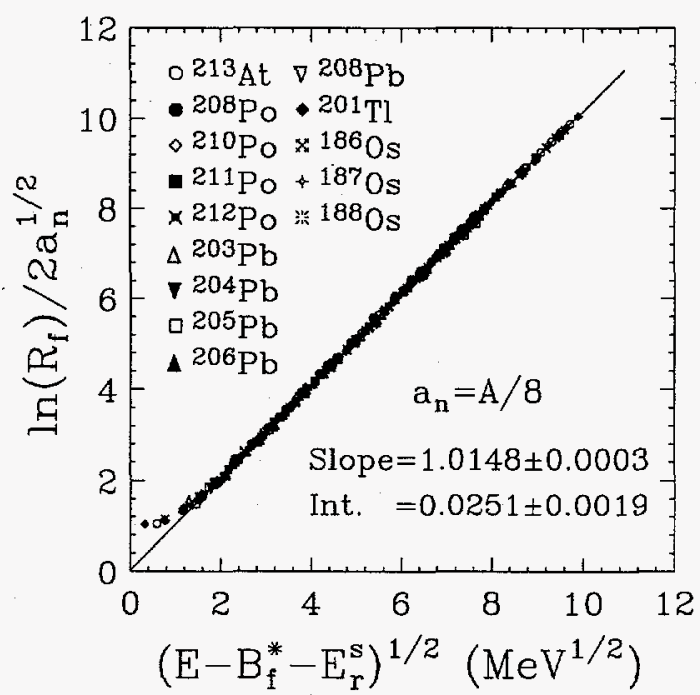

FIG. 2. The quantity $\ln \left(R_{f}\right)$ divided by $2 a_{n}{ }^{1 / 2}$ vs the square root of the intrinsic excitation energy over the saddle for fission of the fourteen compound nuclei indicated in the figure. The straight line is a linear fit to all but the lowest two or three data points.

As shown in fig. 2, all of the excitation functions for fourteen different compound nuclei reduce beautifully to a single straight line, according to the transition state predictions. This scaling extends well over seven orders of magnitude in the fission probability and is even better than that observed in ref.[1] for complex fragment emission, despite the fact that the systems cover a region in $A$ and $Z$ where shell effects vary dramatically. This indicates that the transition state fission rates hold extremely well. No evidence for the effects of transient times longer than $3 \times 10^{-20}$ seconds is found.

* Excerpted from Phys. Rev. Lett. 75, 4186 (1995).

[1] L.G. Moretto et al.,Phys. Rev. Lett. 74, 3557 (1995).

[2] P. Möller et al., LA-UR-3083 (1994). 


\title{
Scaling laws and transient times in ${ }^{3} \mathrm{He}$ induced nuclear fission ${ }^{*}$
}

\author{
Th. Rubehn, K.X. Jing, L.G. Moretto, L. Phair, K. Tso, and G.J. Wozniak \\ Nuclear Science Division, Lawrence Berkeley National Laboratory
}

It is well known from early studies that fission excitation functions vary dramatically from nucleus to nucleus over the periodic table [1-3]. Some differences can be understood in terms of a changing liquid-drop fission barrier with the fissility parameter, others are due to strong shell effects which occur e.g. in the neighborhood of the double magic numbers $Z=82$ and $N=126$. Further effects may be associated with pairing and the angular momentum dependence of the fission barrier.

Fission rates have been successfully calculated most often with the transition state method introduced by Wigner [4] which has then been applied to fission by Bohr and Wheeler [5]. However, recent measurements of an enhancement of prescission emission of neutrons and $\gamma$-rays in relatively heavy fissioning systems claim the failure of the transition state method. The alleged failure has been attributed to the transient time necessary for the so-called slow fission mode to attain its stationary decay rate. Since these methods are indirect and are fraught with difficulties, we have experimentally investigated the fission probability to search for possible deviations from the predicted transition state rates. Therefore, we have measured ${ }^{3} \mathrm{He}$ induced fission cross sections of three compound nuclei using two large area PPACs which allowed for the detection of both fission fragments in coincidence; the results are shown in Fig. 1 [6].

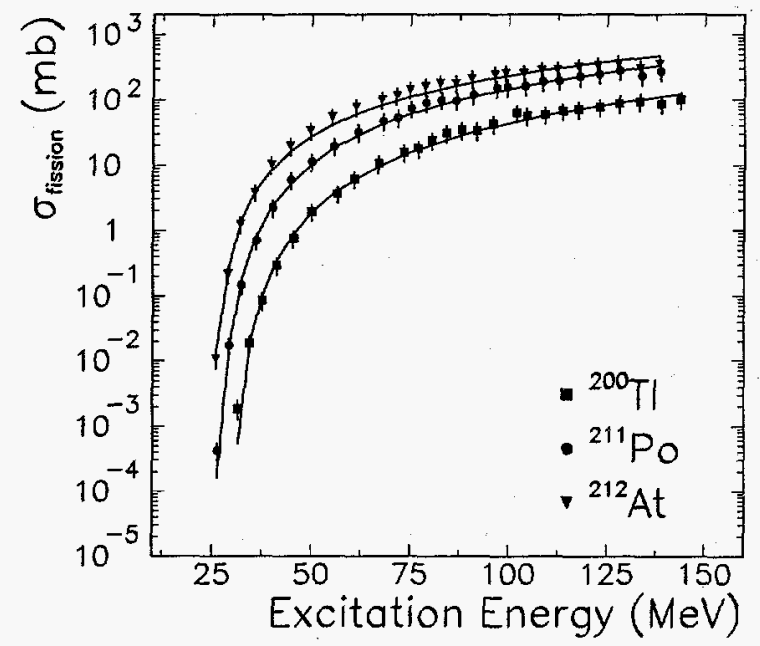

FIG. 1. Excitation function for fission of several compound nuclei formed in ${ }^{3} \mathrm{He}$ induced reactions. The different symbols correspond to the experimental data points. The solid line shows the results of a fit to the data.

Recently, we have introduced a method to analyze fission excitation functions $[6,7]$ : it has been shown that this analysis allows the scaling of different excitation func- tions according to the transition state predictions, once the shell effects are taken into account. As we show in Fig. 2, no deviations from the straight line, which would indicate a failure of the transition state method, are observable. Furthermore, this method allows one to determine the effective fission barrier, the shell effect, and $a_{f} / a_{n}$.

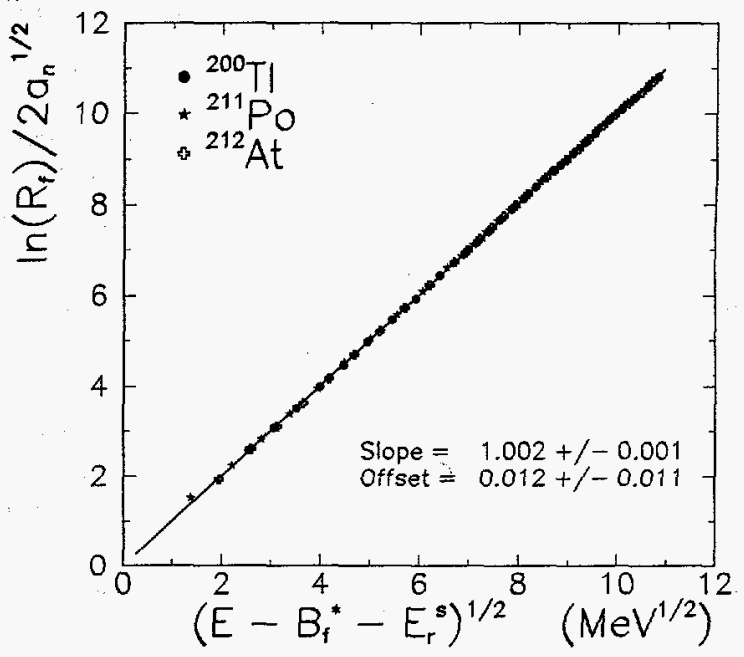

FIG. 2. The scaled fission excitation functions vs the square root of the intrinsic excitation energy over the saddle for fission of several compound nuclei. The straight line represents a fit to the whole data set.

The excitation energy covered by our experiment (25$145 \mathrm{MeV}$ ) corresponds to compound nucleus life times between $10^{-18}$ and $10^{-22}$ seconds, and is therefore sensitive to delay times in the first chance fission probability. We have assumed a step function for the transient time effect and calculated accordingly the resulting fission width. Modest fission transient times would show up as significant deviations from the straight line presented in Fig. 2. This allows us to determine an upper limit for the transient time of no longer than $10^{-20}$ seconds. Since the experimental fission rates are well described by the transition state method, it seems likely that most prescission emission occurs during the descent from saddle to scission.

[1] G. M. Raisbeck, J.W. Cobble, Phys. Rev, 153, 1270 (1967).

[2] L.G. Moretto et al., Phys. Lett. 38B, 471 (1972).

[3] A. Khodai-Joopari, Ph.D. thesis, UC Berkeley, 1966.

[4] E. Wigner, Trans. Faraday Soc. 34, 29 (1938).

[5] N. Bohr and J.A. Wheeler, Phys. Rev. 56, 426 (1939).

[6] , * Th. Rubehn et al., Phys. Rev. C 54, 3062 (1996).

[7] L.G. Moretto et al., Phys. Rev. Lett. 75, 4186 (1995). 


\title{
First chance fission probability of neighboring Po isotopes*
}

\author{
Th. Rubehn, K. X. Jing, L. G. Moretto, L. Phair, K. Tso, and G. J. Wozniak \\ Nuclear Science Division, Lawrence Berkeley National Laboratory
}

Fission excitation functions of four neighboring polonium isotopes, ${ }^{209,210,211,212} \mathrm{Po}$, have been precisely measured in ${ }^{3} \mathrm{He}$ and ${ }^{4} \mathrm{He}$ induced reactions with isotopic lead targets at the 88-Inch Cyclotron to experimentally determine first chance fission probabilities. An estimate on the latter is essential to test the validity of the recently observed transition state scaling and to extract fission transient times. In the formalism described in Refs. [1,2], we have only accounted for 1 st chance fission while for the experimental data we have the measured total fission probability. This approximation is certainly correct at low excitation energies where 1st chance fission is the dominant contribution. However, at higher energies, multi-chance fission is expected to become more important. Thus, the percentage of 1 st chance fission will decrease.

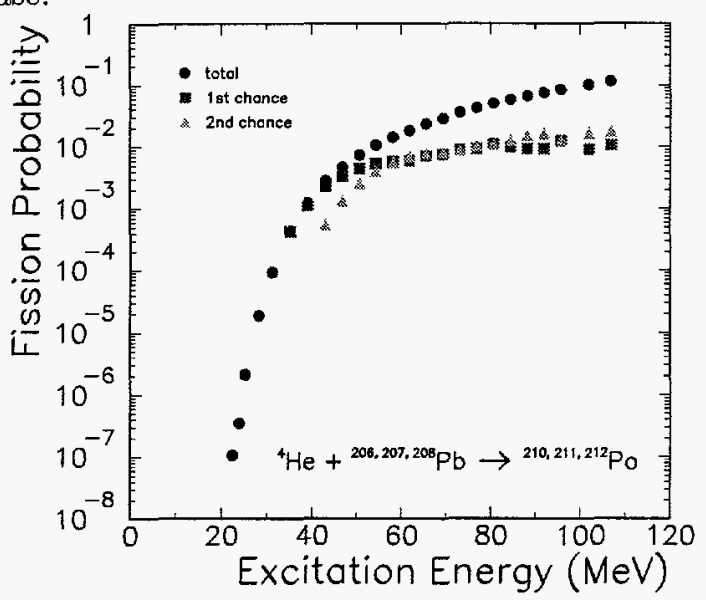

FIG. 1. First and second chance fission probabilities for the reaction ${ }^{4} \mathrm{He}+{ }^{206,207,208} \mathrm{~Pb}$. (Preliminary.)

The difference in the cumulative fission probability of neighboring isotopes can be used to determine the 1st chance fission probability. Since the energy dependence of the first chance fission probability is determined by subtracting similar cross sections, it is essential to measure the cumulative cross sections with high precision; see Ref. [3]. First chance fission at a given excitation energy can be determined by subtracting the fission probabilities of two neighboring isotopes by using the separation energy of the last neutron and the temperature of the daughter nucleus given by $T=\sqrt{E^{*} / a_{n}}$. We note that the angular momentum dependence is neglected in this simple ansatz.

In Fig. 1, we show the preliminary results of this analysis for ${ }^{4} \mathrm{He}$ induced reactions. At excitation energies smaller than $\sim 45 \mathrm{MeV}$, 1st chance fission accounts for practically all the fission yield. However, at higher excita-

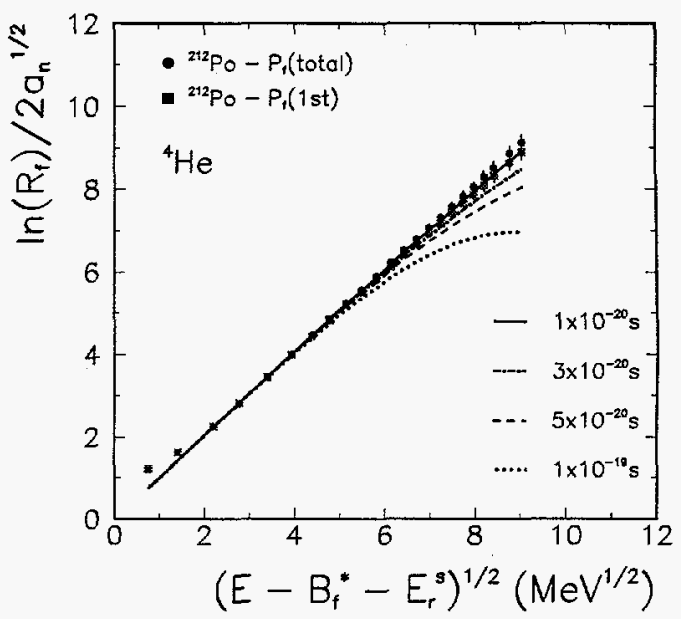

FIG. 2. The quantity $\frac{\ln R_{f}}{2 \sqrt{a_{n}}}$ vs the square root of the intrinsic excitation energy over the saddle for fission of the compound nucleus ${ }^{212}$ Po investigating both the total fission probability and the 1st chance fission probability only. The lines represent calculations assuming that no fission occurs during a given transient time which is indicated in the figure.

tion energies, multi-chance fission sets in and 1st chance fission only accounts for $\sim 10 \%$ of the total fission probability at the highest excitation energies investigated. It is interesting that 2 nd chance fission becomes somewhat stronger than 1st chance fission around $100 \mathrm{MeV}$.

As pointed out before, the formalism used in Ref. [2] has been established for first chance fission only. In Fig. 2, we show the scaled fission excitation functions for both 1st chance and the total fission probability. Although there is a small difference between the two cases at high excitation energies, no significant deviations from the straight line are visible. Similar results have been obtained for the other ${ }^{3} \mathrm{He}$ and ${ }^{4} \mathrm{He}$ induced fission reactions. We thus conclude that the 1st chance fission probability scales as the transition state method predicts and that fission transient times must be shorter than 30 zs. It seems likely that that any excess prescission emission occurs during the descent from saddle to scission. If this is the case, then the present fission results are not in contradiction with recent measurements of prescission neutron and $\gamma$ rays.

[1] ,* Th. Rubehn et al., LBNL-39895 (1997).

[2] Th. Rubehn et al., Phys. Rev. C 54, 3062 (1996) and this report.

[3] Th. Rubehn et al., Nucl. Instr. Meth. A (in print); LBNL39398; Los Alamos e-print nucl-ex/9609004; and this report. 


\title{
Transition State Rates and Mass Asymmetric Fission Barriers of Compound Nuclei ${ }^{90,94,98} \mathrm{Mo}$
}

\author{
K. X. Jing, L. G. Moretto, A. C. Veeck, N. Colonna, I. Lhenry, K. Tso, K. Hanold, \\ W. Skulski, Q. Sui and G. J. Wozniak \\ Nuclear Science Division, Lawrence Berkeley National Laboratory
}

The emission of complex fragments (or massasymmetric fission) can be treated in an analogous fashion to mass-symmetric fission by introducing the ridge line of conditional saddle points. Each mass or charge emission can be associated with a conditional saddle (or barrier) with the constraint of a fixed mass asymmetry. The locus of all such conditional saddles define the ridge line in the potential energy surface of a nucleus. This ridge line controls the emission of complex fragments, and can be measured with techniques similar to those used to determine fission barriers. In this work, we measured the excitation functions for complex fragments with atomic number $Z=5$ - 25 emitted from the compound nuclei ${ }^{90,94,98}$ Mo produced in the reactions ${ }^{78,82,86} \mathrm{Kr}+{ }^{12} \mathrm{C}$. Mass-asymmetric fission barriers (or the ridge line) for the compound nuclei ${ }^{90} \mathrm{Mo},{ }^{94} \mathrm{Mo}$, and ${ }^{98} \mathrm{Mo}$ (see fig. 1) were extracted by fitting the measured excitation functions with a transition state formalism.

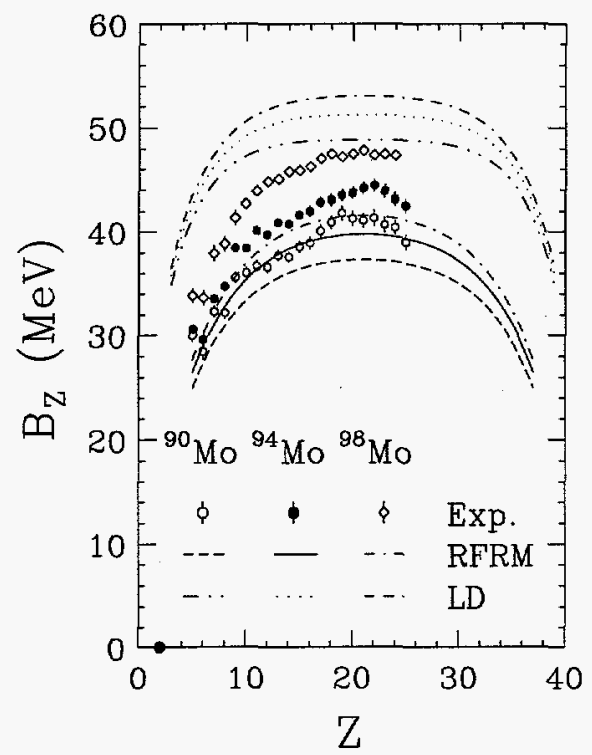

FIG. 1. The shell corrected mass-asymmetric fission barriers $\left(B_{Z}\right)$ for compound nuclei ${ }^{90} \mathrm{Mo},{ }^{94} \mathrm{Mo}$, and ${ }^{98} \mathrm{Mo}$ are compared with the Rotating Finite Range Model and the Rotating Liquid Drop Model calculations.

With the shell correction, the measured barriers are several $\mathrm{MeV}$ higher on average than the predictions of the Rotating Finite Range Model, and substantially lower than that predicted by the Rotating Liquid Drop Model. A stronger $N / Z$ dependence of the experimental conditional barriers is observed than predicted by either model. These results suggest the need for refinements of the parameters in both models, if not modifications of the models themselves.

As for the symmetric fission, the rate for massasymmetric fission has been calculated successfully by the means of the transition-state method. However, the claims for the failure of the transition state method to account for the observed prescission particle emission $(n$, $p, \alpha, \gamma)$ prompted an attempt to justify the validity of the transition-state method and/or to identify regimes in which deviations might be expected. In this work we compared the experimental emission rates of complex fragments with the transition state predictions, and searched for $E$ and $Z$ dependent deviations that would indicate the existence of a transient effect which has been advocated as an explanation for the alleged large number of prescission particle emission observed in the fission of many systems.

In fig. 2, we show that, over ninety excitation functions measured for complex fragments emission from five different nuclei $\left({ }^{75} \mathrm{Br},{ }^{90} \mathrm{Mo},{ }^{94} \mathrm{Mo},{ }^{94} \mathrm{Mo}\right.$, and $\left.{ }^{110,112} \mathrm{In}\right)$, can be scaled, as expected from the transition state theory, onto a single universal straight line which has a slope near unity and passes closely through zero. This is strong evidence for the validity of the transition state formalism and allows us to assign an upper limit for the transient time of $1 \times 10^{-20}$ seconds.

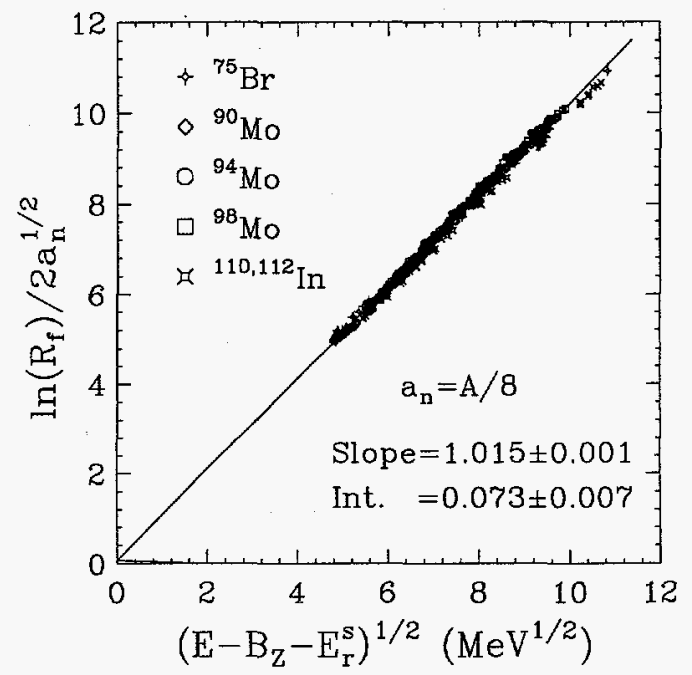

FIG. 2. The logarithm of the reduced mass-asymmetric fission rate $R_{f}$ divided by $2 a_{n}{ }^{1 / 2}$ versus the square root of the intrinsic excitation energy for five different compound nuclei. The solid lines are the linear fits to all of the data. The error bars are smaller than the size of symbols. 


\title{
Characterization of nuclear physics targets using Rutherford backscattering*
}

\author{
Th. Rubehn, G.J. Wozniak, L. Phair, L.G. Moretto, and K. M. Yu \\ Nuclear Science Division and Materials Science Division, Lawrence Berkeley National Laboratory
}

Many experiments in nuclear physics require targets with a precise characterization. In particular, informations on quantities like the target thickness, the homogeneity, and the amount and kind of impurities are essential [1].

In a recent experiment performed at the 88-Inch $\mathrm{Cy}-$ clotron of LBNL, we have investigated first chance fission of various polonium isotopes [2]. To do so, we have precisely measured fission excitation functions of the neighboring isotopes ${ }^{209} \mathrm{Po},{ }^{210} \mathrm{Po},{ }^{211} \mathrm{Po}$, and ${ }^{212} \mathrm{Po}$ produced in the reactions ${ }^{3} \mathrm{He}$ and ${ }^{4} \mathrm{He}+{ }^{206,207,208} \mathrm{~Pb}$. To study the excitation energy dependence of the first chance fission probability, which is determined by subtracting similar cross sections of two neighboring isotopes, it is essential to measure the cumulative fission cross sections with high precision. While statistical errors can be minimized by measuring a sufficiently large number of fission events, systematic errors, as for example caused by uncertainties in the target thickness, are of particular concern.

We have used a Rutherford backscattering (RBS) spectrometer and particle induced $\mathrm{x}$-ray emission for a precise off-line characterization of targets used in our experiments. Monoenergetic ${ }^{4} \mathrm{He}^{+}$particles of $1.95 \mathrm{MeV}$ energy generated by a $2.5 \mathrm{MeV}$ van der Graaf accelerator have been utilized. Combining the measured energy and angle of the backscattered particles, the mass and energy of the projectile allows for the identification of the elementary constituents of the sample. The thickness of the sample can be derived from the energy loss, i.e., by determining the energy of the backscattered particles at both edges of the sample.

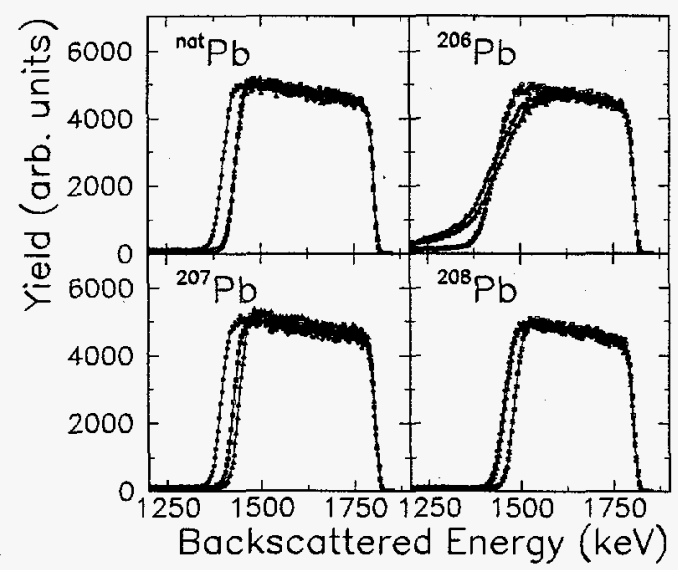

FIG. 1. RBS energy spectra for four lead targets. The different symbols correspond to different positions on the target (center, upper and lower edge).

In Fig. 1, we show the measured energy spectra from

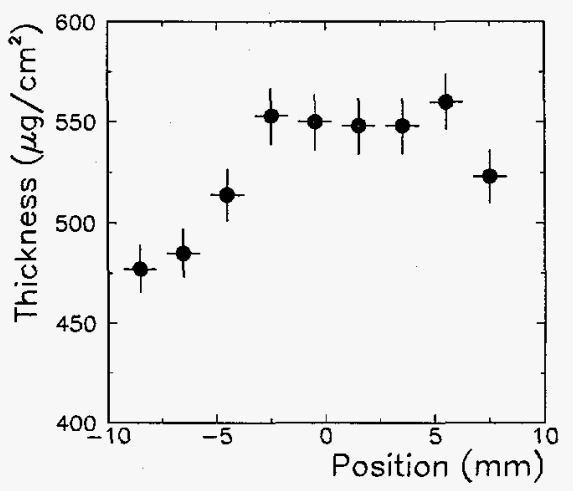

FIG. 2. Target thickness determined by RBS as a function of position on the target surface for ${ }^{207} \mathrm{~Pb}$.

the RBS analysis for four lead targets. The thickness of the foils are deduced from the widths of the RBS spectra using the energy loss data of the ions in lead. The high energy edge reflects the front and the low energy edge the back of the sample. Small inhomogeneities in the target thickness can clearly be seen in the figure. In general, the spectral edges are sharply defined indicating well defined surfaces. A comparison with the thicknesses determined by direct weighing shows good agreement. In contrast to the RBS method, however, weighing only allows for an average thickness determination.

In Fig. 2, we show the thickness as a function of the distance from the center on the surface of the target. Measurements were made in $2 \mathrm{~mm}$ steps to determine the homogeneity. Within the central $8-10 \mathrm{~mm}$, the thickness fluctuation is small. However, the sides are not symmetric. These features have been assigned to the production process. Since the beamspot in our experiment is smaller than $5 \mathrm{~mm}$, the uncertainty in the target thickness is very small.

In order to determine whether any significant target impurities were present, we have furthermore measured particle induced $\mathrm{x}$-ray emission simultaneously during the RBS experiments. This method relies on the spectrometry of characteristic $\mathrm{x}$-rays emitted by the target atoms due to the irradiation with high energy ion beams. Various constituents can be identified via their characteristic $\mathrm{x}$-rays. For the lead targets investigated here, no sizable contribution of contaminations other than a carbon backing, were seen.

[1] , Th. Rubehn et al., Nucl. Instr. Meth. A (in print); LBNL-39398; Los Alamos e-print nucl-ex/9609004.

[2] Th. Rubehn et al., this report. 


\title{
LBNL 88-Inch Cyclotron Improvements*
}

\author{
C.M. Lyneis, Z.Q. Xie, D.J. Clark
}

The 88-Inch Cyclotron is now operating with beams of higher energy, greater intensity and variety, with more hours of beam on target and supporting a larger number of users than any time since it began operation in 1962. There are two major reasons for this.

First, the development of the ECR ion source by Geller et $\mathrm{al}^{1}$ and its subsequent development have made it possible to produce a wide range of beams with intensities and energies well matched to the study of nuclear structure, nuclear reaction mechanisms and heavy element research. In addition the 88-Inch Cyclotron which began its operation as a lightion cyclotron has maintained this capability, which provides research opportunities in new areas such a laser atom trapping for the study of weak interactions and fundamental symmetries.

The second reason is the development of a new generation of high energy resolution detectors such as Gammasphere and Eurogam which has opened up a new area of research in the study of highly deformed nuclei. Much of the research with these new detectors involves the formation of rapidly rotating compound nuclei. Production of the compound nuclei is typically done by using projectiles with $9 \leq \mathrm{A}<80$ with 4 to $6 \mathrm{MeV} /$ nucleon. This research puts strong demands of the ion source capabilities both with respect to making beams from almost every element up to mass 80 and to produce beams economically from separated isotopes. Efficient use of separated isotopic feed material in the ECR sources has been developed. For example in FY94 more than 300 hours of research utilized ${ }^{48} \mathrm{Ca}$ beams with typical usage rate of about $25 \mu \mathrm{g} / \mathrm{hr}$ of $40 \%$ enriched ${ }^{48} \mathrm{Ca}$.

The 88-Inch Cyclotron is nominally a k140 cyclotron with three sectors and three external ion sources. The cyclotron RF frequency can be varied from $5.5 \mathrm{MHz}$ to $16.2 \mathrm{MHz}$ and both first and 3rd harmonic beams are routinely accelerated. Minimum and maximum energies in first harmonic are 6.1 and $55 \mathrm{MeV} /$ nucleon and in third harmonic 0.7 to $6 \mathrm{MeV} /$ nucleon. Currently all beams being used by the research programs are produced by the two high charge state ECR ion sources located on the vault roof of the cyclotron, the LBL ECR ion source and the Advanced ECR

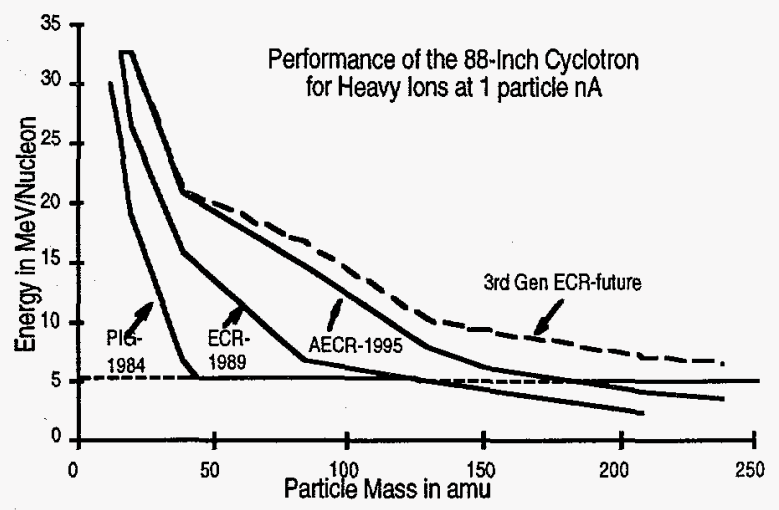

Fig. 1. This shows the energy mass curves for the cyclotron at an intensity of 1 particle nA. The dotted curve shows the projected performance with a new superconducting ECR ion source under development.

The evolution of the heavy-ion performance of the 88-Inch Cyclotron is illustrated in Fig. 1. These curves are drawn for beams of at least 1 particle nA extracted current. Higher intensity beams can be produced at energies less than those indicated by the curves. To date, the 88Inch Cyclotron has produced beams of 41 elements, from hydrogen through zinc (the first 30 elements) as well as $\mathrm{Ge}, \mathrm{Kr}, \mathrm{Ag}, \mathrm{Sn}, \mathrm{Xe}, \mathrm{La}$, $\mathrm{Sm}, \mathrm{Tb}, \mathrm{Au}, \mathrm{Bi}$ and $\mathrm{U}$.

\section{Footnotes and References}

*Condensed from a paper in Proceedings of the 14th International Conference on Cyclotrons and their Applications, Cape Town, S.A, 1995, p173.

1. Geller, R., Appl. Phys. Lett. 16, No 10, 401 (1970). 


\section{Development of Exotic Nuclear Beams at the 88-Inch Cyclotron \\ D. M. Moltz, P. E. Haustein\#,J. Powell, E.B. Norman, M. W. Rowe, and Joseph Cerny}

There has been great interest in recent years in the potential use of radioactive nuclear beams for the study of nuclear science. Experiments have been proposed in nuclear astrophysics, nuclear reactions, nuclear spectroscopy and properties of nuclei near the drip lines. Initial measurements in these areas have required the construction of many prototype first generation RNB facilities. Many second generation facilities are in the planning and construction phases. In principle, a high intensity radioactive beam facility is the highest nuclear physics priority in the U.S. behind the timely completion of RHIC and the Jefferson Laboratory. With the exception of MSU and RIKEN, all other major facilities under construction in the world are using the ISOL approach; this necessitates that most of the technical development in this field has concentrated on target-ion source issues. Although many laboratories have studied post accelerators, most construction projects to date have been limited to fairly low energies $(<5$ $\mathrm{MeV} /$ nucleon ). Unfortunately, almost all experimental ideas involving low-energy beams also require extremely high RNB intensities; this possibility is a long term goal, but hardly realistic for the earliest experiments. Many nuclear reaction experiments can be performed with very small beam currents, but they must have much higher energies ( $>15 \mathrm{MeV} /$ nucleon).

We are proposing to build a modest demonstration project whereby we can produce a few beams with energies up to $20 \mathrm{MeV} /$ nucleon utilizing the 88-Inch Cyclotron as the secondary accelerator. The primary machine is a small medical cyclotron located about $300 \mathrm{~m}$ away from the 88". This small cyclotron produces proton beams at $10 \mathrm{MeV}$ and 30 microamps intensity. The basic idea is to transport these radionuclides via a gas-jet system down to the 88 " for injection into an ECR source. Once injected into the ECR source, a fraction of the injected radioactivity will be ionized directly into an appropriate charge state, extracted, and injected into the 88-Inch Cyclotron for final acceleration. For example, ${ }^{11} \mathrm{C}$ is made in copious yield in the ${ }^{14} \mathrm{~N}(\mathrm{p}, \mathrm{a})$ reaction. It would be transported on organic based aerosols (heavy elements effectively poison the ECR ion source output of lighter species), extracted as $11 \mathrm{C}^{4+}$, and accelerated to a maximum energy of 18.5 $\mathrm{MeV} /$ nucleon. In order to perform nuclear reaction studies with this beam, routine operation will strip this beam to +6 to permit its magnetic separation from ${ }^{11} B$ beam impurities; the cyclotron cannot separate ${ }^{11} \mathrm{C}$ and ${ }^{11} \mathrm{~B}$.

To date we have performed several tests to ascertain the viability of this approach. These tests have included measuring the ${ }^{11} \mathrm{C}$ yields transported through a $25 \mathrm{~m}$ capillary, comparisons of these results with transport through a $300 \mathrm{~m}$ capillary, and yield measurements on the simultaneous production of ${ }^{14} \mathrm{O}$ for possible use as a second radioactive beam. Results of the yield tests along with some sample efficiencies are given in Table 1 . The transit time through the $300 \mathrm{~m}$ capillary was measured to be $69 \mathrm{~s}$; there were no measurable losses of ${ }^{11} \mathrm{C}$ activity. We are currently constructing a system to couple a gas-jet to the ECR to measure the efficiency for a beam extracted as ${ }^{11} \mathrm{C}^{4+}$. This system should be operational in mid 1997.

\#Visitor from Brookhaven National Lab.

Table 1. Yields and Efficiencies

\begin{tabular}{ll}
$\begin{array}{l}\text { Measured }{ }^{11} \mathrm{C} \text { Yield } \\
\text { (extrapolated from } 1 \mathrm{~mA})\end{array}$ & $9.0 \mathrm{E} 08 / \mathrm{s}$ \\
$\begin{array}{l}\text { Measured }{ }^{14} \mathrm{O} \text { Yield } \\
\text { (extrapolated from } 1 \mathrm{~mA})\end{array}$ & $4.8 \mathrm{E} 07 / \mathrm{s}$ \\
$\begin{array}{l}\text { Target Efficiency } \\
\text { Transit Time }\end{array}$ & $50 \%$ \\
\hline Skimmer Efficiency & $69 \mathrm{~s}$ \\
\hline ECR Efficiency & $50 \%$ \\
Cyclotron and Transport Eff. & $30 \%$ \\
Proposed Initial ${ }^{11} \mathrm{C}$ Intensity & $1.0 \mathrm{E} 06 / \mathrm{s}$
\end{tabular}




\title{
Performance of the upgraded LBL AECR Source *
}

\author{
Z.Q. Xie and C.M. Lyneis
}

The LBL AECR source has been upgraded (AECR-U, shown in Figure 1) in July 1996 by increasing its magnetic fields to improve the plasma confinement therefore to enhance the source performance. The new solenoid magnets have pancakes with larger hollow core copper conductor and thicker magnet iron return yokes. Iron plugs are also used to concentrate the field flux in the plasma chamber. With these improvements, the maximum peak axial magnetic field strengths increase from 1.0 to 1.7 Tesla at the injection and from 0.7 to 1.1 Tesla at the extraction region, respectively, with no increase in ac power. A new set of $\mathrm{NdFeB}$ permanent sextupole magnet with a nominal magnetic remanence $\mathrm{Br}$ of 1.3 Tesla was installed in the aluminum plasma chamber to raise the maximum radial field strength. To ensure sufficient cooling, the permanent sextupole magnet bars are enclosed in $0.25 \mathrm{~mm}$ thick watertight stainless steel cans and are directly water cooled. The maximum sextupole field strength at the chamber inner surface is 0.85 Tesla.

After a few months of tailoring the magnetic field configuration to match the incoming microwaves of 14 and $10 \mathrm{GHz}$ (two-frequency plasma heating), the AECR-U source with its higher magnetic fields and increased magnetic mirror ratios has demonstrated significantly enhanced performance. So far the AECR source has produced many record beam intensities and high charge state heavy ions from an ECR source. For heavy ions at intensity of about 1 $\mathrm{e} \mu \mathrm{A}$, the charge state was shifted from $42+$ to $48+$ for the heaviest natural element--uranium and from $41+$ to $46+$ for bismuth. An order of magnitude enhancement for fully stripped argon ions ( $I \geq 60$ en $A$ ) also has been achieved. For elements of mass from 40 to 86 and intermediate charge state, i.e., mass to charge ratios of 4 to 5 , intensities of 100 to $300 \mathrm{e} \mu \mathrm{A}$ were also produced.
These ion beams will be used for the BGS detector under construction at the 88-Inch Cyclotron. High charge state ion beams of xenon136 and uranium-238 produced by the source were injected into the 88-Inch Cyclotron. After acceleration to energies greater than 10 $\mathrm{MeV} /$ nucleon for xenon-136 and $6 \mathrm{MeV} /$ nucleon for uranium, the extracted beam intensities from the cyclotron are $1 \times 10^{7}$ pps for xenon $41+$ and a few hundreds pps of xenon $46+$. In the identification of the high charge state uranium ions, the transmission was less than $0.01 \%$ for these highly-charged ions due to the poor vacuum in the cyclotron. Nevertheless, charge states up to $60+$ uranium ions were confirmed with a crystal detector. Uranium $60+$ at a few pps with a total energy of $1.935 \mathrm{GeV}$, the highest beam energy ever produced by the 88 inch cyclotron, was measured.

\section{Footnotes and References}

${ }^{*}$ Condensed from a paper to be presented at the 13th International Workshop on ECR Ion Sources, College Station, Texas, February 1997.

*

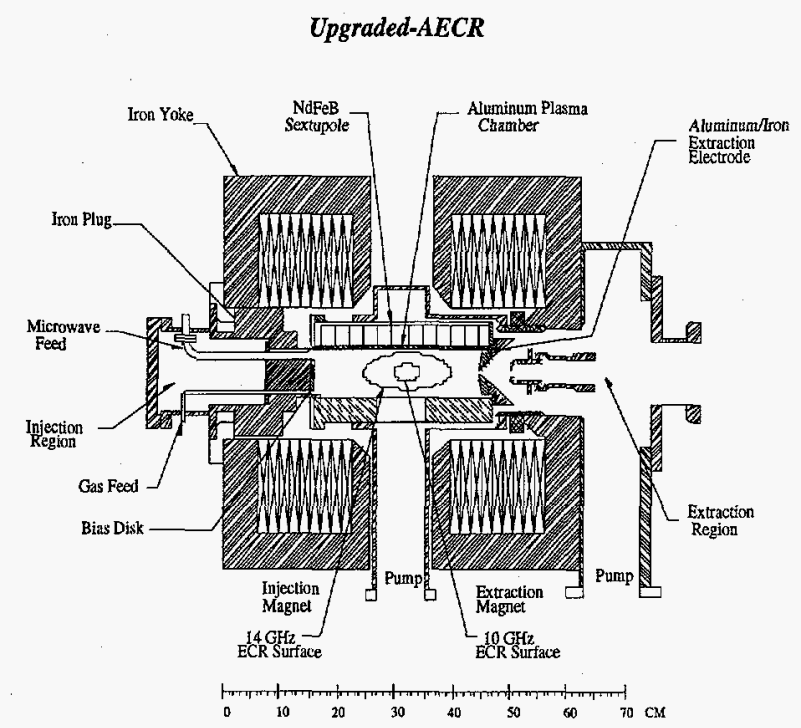

Fig. 1. A cross-section view of the upgraded AECR (AECR-U) ion source. 


\title{
Production of intense high charge state ions with an aluminum liner in the Advanced Electron Cyclotron Resonance Ion Source *
}

\author{
Z.Q. Xie and C.M. Lyneis
}

The basic physics of an electron cyclotron resonance (ECR) ion source involves coupling microwave energy to plasma electrons confined by a magnetic bottle which then produce singly or multiply charged ions by electron impact ionization. The neutrals and ions are mainly stepwise ionized by electron impact ionization and the ionization process provides the primary electrons to compensate the escaped electrons to maintain a dynamic equilibrium plasma in which the plasma loss is electrically neutral. Early experiments on ECR sources demonstrated that adding extra electrons to the ECR plasma with a microwave-driven first stage or by using electrons emitted from the plasma chamber walls coated with materials of high secondary electron emission can substantially enhance the production of high charge state ions. Since the AECR source was built, we have explored various techniques to provide more electrons for the plasma. The early methods used, such as coating the plasma chamber walls with $\mathrm{SiO}_{2}$ or $\mathrm{Al}_{2} \mathrm{O}_{3}$ and using an electron gun to axially injecting electrons to the plasma, substantially enhanced the performance of the AECR source..$^{1,2,3}$

Enhanced production of high charge state ions due to the aluminum oxide wall coating has been demonstrated in various ECR sources. Empirically speaking, a good chamber surface should have high secondary electron emission, long lifetime in against plasma etching and low material sticking coefficients to minimize the surface memory. Aluminum oxide not only has high secondary emission but it is also very resistant to plasma etching. The previous aluminum oxide coating, done by running an aluminum plasma, did not entirely cover all the chamber copper surfaces in the AECR source and only had about one month lifetime. In this test, a $0.38 \mathrm{~mm}$ thick aluminum liner was installed in the copper plasma chamber. The tests with this aluminum liner and two-frequency heating produced an increase of up to $60 \%$ in beam intensity of the high charge state ions was achieved. Shown in Table I are the measured beam intensities for a few example elements.

\section{Footnotes and References}

*Condensed from Proc. 6th International Conference on Ion Sources, Whistler, BC, Canada, 1995.

${ }^{1}$ Z. Q. Xie and C. M. Lyneis, Rev. Sci. Instrum. 65, 2947 (1994).

2Z. Q. Xie, C. M. Lyneis, R. S. Lam, and S. A. Lundgren, Rev. Sci. Instrum. 62, 775 (1991).

${ }^{3}$ T. Nakagawa, T. Kageyama, M. Kase, A. Goto and Y. Yano, Jpn. J. Appl. Phys. 32, 1335 (1993).

Table I. Ion beams produced from the AECR source with an aluminum chamber liner

\begin{tabular}{|c|c|c|c|}
\hline $\mathbf{Q}$ & $I(\mathrm{e} \mu \mathrm{A})$ & $\mathbf{Q}$ & $I(e \mu A)$ \\
\hline${ }^{16} \mathrm{O}^{6+}$ & 510 & ${ }^{197} \mathrm{Au}^{24+}$ & 41.7 \\
\hline $16 \mathrm{O}^{7+}$ & 210 & ${ }^{197} \mathrm{Au}^{26+}$ & 34 \\
\hline${ }^{40} \mathrm{Ar}^{11+}$ & 238 & ${ }^{197} \mathrm{Au}^{29+}$ & 20 \\
\hline${ }^{40} \mathrm{Ar}^{12+}$ & 158 & ${ }^{197} \mathrm{Au}^{30+}$ & 14.3 \\
\hline${ }^{40} \mathrm{Ar}^{13+}$ & 84 & ${ }^{197} \mathrm{Au}^{31+}$ & 10 \\
\hline${ }^{40} \mathrm{Ar}^{14+}$ & 47.5 & ${ }^{197} \mathrm{Au}^{32+}$ & 6.6 \\
\hline${ }^{40} \mathrm{Ar}{ }^{16+}$ & 4.7 & ${ }^{197} \mathrm{Au}^{34+}$ & 3.5 \\
\hline${ }^{209} \mathrm{Bi}^{28+}$ & 33 & ${ }^{197} \mathrm{Au}^{35+}$ & 2.4 \\
\hline${ }^{209} \mathrm{Bi}^{31+}$ & 26 & ${ }^{197} \mathrm{Au}^{36+}$ & 1.3 \\
\hline${ }^{209} \mathrm{Bi}^{34+}$ & 14 & ${ }^{197} \mathrm{Au}^{38+}$ & 0.4 \\
\hline${ }^{209} \mathrm{Bi}^{36+}$ & 6.6 & & \\
\hline
\end{tabular}

All ion beams are extracted at 10 or $15 \mathrm{kV}$ extraction voltage and through an $8 \mathrm{~mm}$ aperture. 


\title{
A Microtron Cyclotron - the Slipatron*
}

\author{
D. J. Clark**
}

The motivation for this "microtron cyclotron" or "slipatron" design arises from the idea that a more compact cyclotron type magnet might be built, using the usual sector cyclotron fields for focusing but having a strongly increasing radial gradient in the field, as in FFAG design, to compact the orbits. This deviation from the isochronous field causes the ion frequency to increase with energy. To obtain high intensity we must use constant rf frequency and an integral number of rf cycles per turn, which is the microtron mode of acceleration. The harmonic number (rf freq/ion freq) then must decrease with energy gain. To allow a sufficient number of turns a high starting harmonic number of 50-100 is used, so that it can be decreased each turn during acceleration. A high energy gain per turn is required and can be accomplished with multiple dees. Unlike a microtron cavity, the dees have to extend over the radial aperture. The change of period during acceleration can be matched by changing the angular width of the dees with radius. Phase stability exists because the ion frequency depends upon radius.

A layout of an optimized design is shown in Figure 1. A four sector structure is chosen to give two valleys for $\mathrm{rf}$ and space for injection and extraction. The magnet and $\mathrm{rf}$ systems are drawn to scale. This design is for a proton accelerator with injection at 2 $\mathrm{MeV}$ and extraction at $30 \mathrm{MeV}$. The harmonic is 100 at injection and 54 at extraction. Average radius at extraction is .84 meter. The field gradient is obtained by tapering the hill gap, with constant hill angular width. The peak field on the hills at the edge is 19 kG. The axial focusing was calculated using the simple hard edge approximation with valley field assumed zero. The high flutter is adequate to compensate the rising average field and give Nuz of .3 or more, using some spiral at the edge. The rf system has 10 gaps in each of 2 valleys. The rf frequency is $770 \mathrm{MHz}$. The energy gain per turn is programmed from the center to the edge by dee shaping to give .2 $\mathrm{MeV} /$ turn at the center and $3.2 \mathrm{MeV} /$ turn, or 160
$\mathrm{keV} / \mathrm{gap}$, at the edge. The injection and extraction are shown schematically. The turn separation is $1.1 \mathrm{~cm}$ at injection and $1.7 \mathrm{~cm}$ at extraction. A figure of merit, $F$, for this type of accelerator is its radial aperture compared to that of a standard cyclotron of the same energy. $F=.7$ for this optimized design.

An advantage of this design over that of a cyclotron with the same magnetic fields at extraction is that the magnet can be more compact. But the disadvantages are the greater complication of the dee system and the need for an injector. Perhaps other variations of this design can be developed.

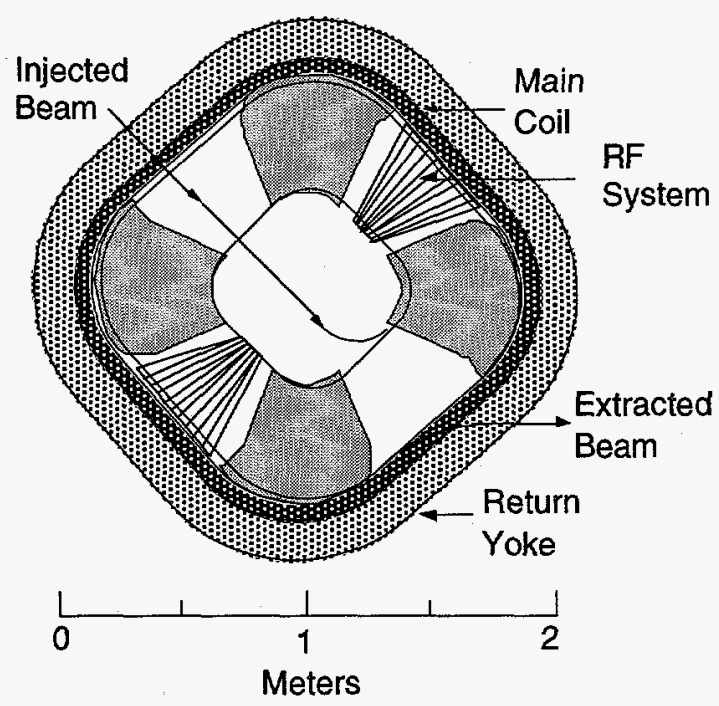

Figure 1: Optimized design layout

\section{Footnotes and References}

*Condensed from Proc. 14th International Conference on Cyclotrons and their Applications, Capetown, South Africa, Oct. 8-13, 1995, p. 618.

**Lawrence Berkeley National Laboratory 


\section{Non-Nuclear Science Programs at the 88-Inch Cyclotron: Part A. Space Applications* M.A. McMahan}

The 88-Inch Cyclotron runs about $20 \%$ of the time for non-nuclear science fields, including high-energy physics, radiobiology, materials, and space applications. The largest part, space applications, consists of two parts: radiationinduced effects in microelectronics and calibration of detectors for NASA missions.

\section{Radiation Induced Effects}

Recent demands by both the Defense Department and the communications industry for cost, flexibility, and speed of getting new satellites into space, have led to an increased use of commercial off-the-shelf (COTS) parts. These parts must be ground tested before committing them to an expensive space mission. Groundbased testing is done at accelerators and with radioactive sources. Accelerators in the USA, Europe, and Japan now provide radiation effects test facilities for heavy ions, protons, or electrons. At the 88-Inch Cyclotron, the bulk of non-nuclear science time $(\approx 15 \%)$ goes to space effects studies for a wide variety of companies and laboratories, on a cost-recovery basis.

Several types of "single event effects (SEEs)" have been identified, ranging from a simple flip of a bit, as in an upset, to more catastrophic effects such as latch-ups or burn-outs, which destroy the part.

Models of these effects begin with an initial charge distribution or current source positioned along the track of an energetic ion. The sensitivity of a device to a particular type of SEE is expressed in terms of the cross section as a function of LET, which gives a characteristic curve with two key parameters: the threshold, $\mathrm{L}_{t h}$ for onset of the SEE, and the cross section at saturation, $\sigma_{\text {sat }}$. The saturation cross-section is related to the total area of the sensitive regions of the component, and the threshold LET is a measure of the critical, or minimum, charge required to trigger the event.

The 88-Inch Cyclotron has features which make it ideal for SEE studies. The Cyclotron runs both heavy-ions and protons, enabling both kinds of measurements to be done at the same facility. Also, the combination of ECR (Electron Cyclotron Resonance) source and cyclotron makes possible the development of "cocktail" beams", which allow the ion species, and thus LET, to be changed very quickly. In addition, with bismuth beams, LETs as high as $100 \mathrm{MeV} / \mathrm{mg} / \mathrm{cm}^{2}$ can be reached.

These studies require accurate measurements of energy, fluence, and spatial uniformity. Energy measurements are performed with silicon detectors, and dose and uniformity obtained with various types of scintillators or ion chambers.

\section{Detector Calibrations}

Another use of the $88^{\prime \prime}$ Cyclotron is for groundbased calibrations and tests of detector packages being prepared for future space launches. NASA's Advanced Composition Explorer (ACE) satellite, scheduled for launch in 1997, will investigate a wide range of fundamental problems in i) the elemental and isotopic composition of matter, ii) origin of the elements and subsequent evolutionary processing, iii) formation of the solar corona and acceleration of the solar wind, and iv) particle acceleration and transport in nature. Two of the nine instruments being designed for this mission have been tested and calibrated at the 88-Inch Cyclotron.

\section{Footnotes and References}

*Condensed from Proceedings of the International Symposium on Large-Scale Collective Motion of Atomic Nuclei, Brolo, IT, October 1996, to be published. ‡ M.A. McMahan, et al., Nucl. Instr. Meth. $\underline{\mathbf{A 2 5 3}}$ 1 (1986). 


\title{
Non-Nuclear Science Programs at the 88-Inch Cyclotron: Part B. Biology, Physics, and New Technologies *
}

\author{
M.A. McMahan
}

Small amounts of beam time at the 88-Inch Cyclotron are dedicated to non-nuclear science programs in radiation biology, high-energy physics, and materials.

\section{Radiobiology}

With the closure of the Bevalac in 1993, part of the Life Science Division's work in radiobiology moved to the Cyclotron. A beamline was developed for light ions tuned with uniformities of $\pm 10 \%$ over diameters of 10 cm. Dose, flux, and uniformity are measured with a segmented ion chamber.

Radiation biologists study the effect of radiation on living tissue from microscopic systems (DNA strands) through macroscopic ones (cataract studies).

One of several radiation biology studies being pursued at the 88-Inch Cyclotron has already made discoveries which may change future textbooks on structural biology. By studying the size distribution of DNA fragments after passage of heavy ions through a cell ${ }^{\dagger}$, shown in Figure 1, and comparing these with Monte Carlo calculations ${ }^{\ddagger}$, one can differentiate between various models for the structure of the DNA inside the nucleus, These studies show that the generally accepted model is most likely not correct.

\section{High Energy Physics}

The irradiation station and diagnostics developed for the biology program have proven to be of direct use to members of the Physics Division involved with the Atlas Detector being designed for the LHC at CERN. Protons from the 88-Inch Cyclotron are used to perform radiation damage studies on silicon diodes being considered for use in Atlas.

\section{Materials Studies}

The discovery of "nuclear tracking" in the late 1950 's led to a new class of nuclear physics detectors as well as a wide range of practical applications. Variations of the nuclear tracking technique have been pursued at the Cyclotron, for example, in the i) calibration of nuclear track detectors for physics experiments, ii) exploration of new techniques to make micropore filters, and iii) development of flat screen displays for lap-top computers. These kinds of studies require the ability to accelerator a variety of heavy, low-energy beams with good uniformity. A scintillating fiber/CCD detector is under development for tuning and dosimetry of these beams.

\section{Footnotes and References}

* Condensed from Proceedings of the International Symposium on Large-Scale Collective Motion of Atomic Nuclei, Brolo, IT, October 1996, to be published.

tBjorn Rydberg, Rad. Res. 145, 200 (1996).

$\ddagger$ W.R. Holley and A. Chatterjee, Rad. Res. 145, 188 (1996).

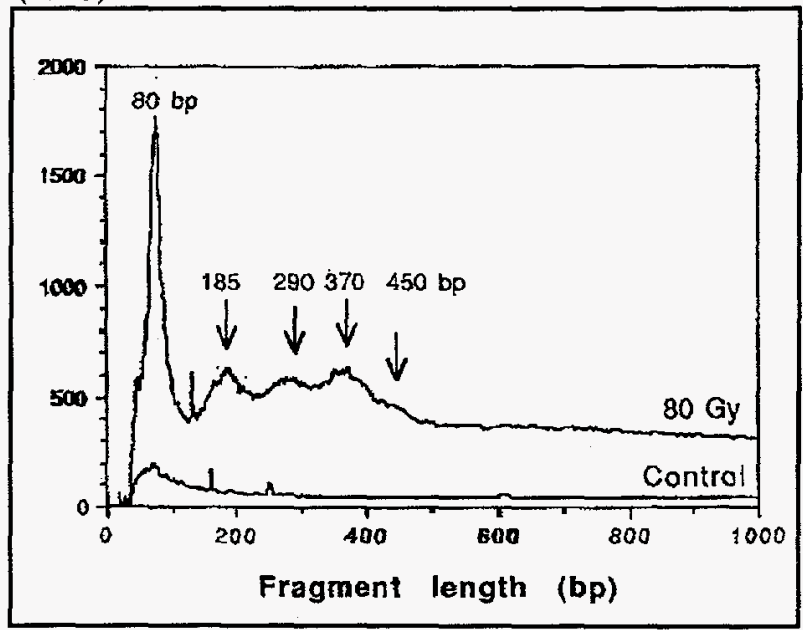

Fig. 1. Experimental distribution of DNA fragments after bombardment by $32.5 \mathrm{MeV} / \mathrm{u} \mathrm{N}$ ions. 



$$
-
$$




\title{
Sudbury Neutrino Observatory - PMT Support Structure \& Panel Arrays
}

K.T. Lesko, Y.D. Chan, M. Dragowsky, M. Isaac, M.E. Moorheadt, E.B. Norman, A. Schuelkeł, and

\author{
R.G. Stokstad
}

The Sudbury Neutrino Observatory detector is. a large heavy water $\check{C}$ erenkov detector designed to detect neutrinos in a 1000 tonne $\mathrm{D}_{2} \mathrm{O}$ target[1]. The detector has sensitivity to the total neutrino flux, $v_{x}$, independent of neutrino family $(x=e, \mu$, $\tau)$ and to the $v_{\mathrm{e}}$ flux, separately, by measuring charged and neutral current reactions and neutrino-electron elastic scattering. The detector is located $2 \mathrm{~km}$ below ground near Sudbury, Ontario Canada in an active nickel mine operated by INCO, Ltd. The $\mathrm{D}_{2} \mathrm{O}$ target is contained in a thin-wall acrylic vessel (AV) of 6 $m$ radius, which is suspended in a $11 m$ radius cavity filled with $\sim 7800$ tons of ultrapure water. The $\mathrm{D}_{2} \mathrm{O}$ and the surrounding $1 m$ shell of $\mathrm{H}_{2} \mathrm{O}$ are viewed by an array of $94568^{\prime \prime}$ Hamamatsu PMTs. Each PMT is coupled to a light concentrator[2] within a plastic hexagonal structure. The hexagons are assembled into 751 flat panel arrays each holding from 7 to 21 PMTs. The panel arrays are suspended on the $9 \mathrm{~m}$ radius stainless steel geodesic sphere (PSUP). This sphere is concentric with the acrylic vessel and is also suspended from the deck structure near the top of the cavity. The PSUP and the panel arrays were designed and fabricated by our group at LBNL.

The PSUP is based on a three-frequency iscosahedron sphere. The $\sim 14,000 \mathrm{~kg}$ structure is capable of supporting a load of nearly $90,000 \mathrm{~kg}$. All materials used in the fabrication of the geodesic sphere were carefully selected to contain $<15 \mathrm{ppb} U$ or Th. All parts were cleaned and double bagged for installation in $\mathrm{SNO}^{\prime} \mathrm{s}$ Class 2000 environment. In addition to the low level of permissible $U$ and Th contamination, mine access and construction concerns heavily influenced our design. The final design consists of 270 struts, 91 nodes, a chimney ring and guide ring to permit the AV's chimney and suspension ropes to penetrate the PSUP and six ports for calibration devices. The upper half of the geodesic structure was installed underground in January and February, 1995.

The PMT panel arrays are fixed to the geodesic structure struts with adjustable and responsive mounts. These permit the panels to adjust to the flexing of the geodesic sphere the cavity if filled with water. The entire array changes from a net weight of $90,000 \mathrm{~kg}$ to becoming slightly buoyant $(-2500 \mathrm{~kg})$ due to the large displacement of the PMTs which necessitates the deployment of anchors at the bottom of the cavity. The PSUP struts will flex by $.64 \mathrm{~cm}$ due to this change in weight. The panel mounts permit an accurate $\left(<0.3^{\circ}\right)$ alignment of the panels so that each PMT-reflector views the $\mathrm{D}_{2} \mathrm{O}$. The reflector-PMT coupling in the hexagons creates a nearly water-tight and lighttight seal with only small engineered leakages to permit water and air to escape during filling and water purification. Each panel is sealed to neighboring panels using thin opaque plastic membranes. All materials used in the fabrication of the panels, except the PMTs, were selected to contain $<15 \mathrm{ppb} U$ and $T h$ and were packaged under cleanroom conditions. The upper half of the geodesic sphere was instrumented with PMTs (390 panels, 5000 PMTs) between April and August, 1995. The final installation will be completed in 1997.

\section{Footnotes and References}

† Present address, Oxford University, Oxford, UK OX13RH

$\ddagger$ Die Arbeit wurde mit Unterstützung eines Stipendiums im Rahmen des Gemeinsamen Hochschulsonderprogramms III von Bund and Ländern über den DAAD ermöglicht.

1. G. Ewan, et al. Sudbury Neutrino Observatory Proposal, SNO 87-12 (1987).

2. G. Doucas, et al. NIM A370, (1996) 579-96. 


\section{${ }^{6} \mathrm{Li}$ Neutron Poison for SNO}

M. E. Moorhead*, Y. D. Chan, M. Dragowsky, M. C. Isaac, R. M. Larimer, K. T. Lesko, E. B. Norman, A. Schuelkeł, R. G. Stokstad

There are two independent possible signatures of neutrino oscillations in SNO: i) a NC/CC (neutral current/charged current) ratio greater than unity, and ii) a distortion of the ${ }^{8} \mathrm{~B} C \mathrm{CC}$ spectrum. If oscillations are occurring, it is most probable that both of these signatures will manifest themselves. However, there are some scenarios where only one of these signatures is present, e.g. in the case of oscillations into "sterile" neutrinos, the NC/CC ratio will be unity, whereas the $\mathrm{CC}$ distortions should still occur. In any case, it is always preferable to investigate a new phenomenon through as many channels as possible.

The motivation for introducing ${ }^{6} \mathrm{Li}$ into the heavy water of SNO is to enhance the detector's ability to measure $\mathrm{CC}$ distortions. For the experimentally favored "non-adiabatic" MSW solution $^{1}$ these distortions correspond to a suppression in the 5-7 MeV part of the spectrum, where complications arise from neutron capture on deuterium ( $Q=6.25 \mathrm{MeV}$ ). To remove this complication and measure a clean $\mathrm{CC}$ spectrum, it is proposed to introduce $300 \mathrm{~kg}$ of $\mathrm{LiNO}_{3}$ salt (enriched to $95 \%{ }^{6} \mathrm{Li}$ ) into the $\mathrm{D}_{2} \mathrm{O}$. The ${ }^{6} \mathrm{Li}$ will absorb neutrons without emitting $\gamma$ rays and reduce the deuterium neutron capture efficiency down to $1 \%$ as compared to $27 \%$ in pure $\mathrm{D}_{2} \mathrm{O}$.

It has previously been shown ${ }^{2}$ that the complication arising from the deuterium neutroncapture gamma peak in pure $\mathrm{D}_{2} \mathrm{O}$ reduces the significance of the non-adiabatic MSW CC distortion to a negligible level (a $1 \sigma$ effect in one year's data). In contrast, by adding a poison such as ${ }^{6} \mathrm{Li}$, the statistical significance of the $\mathrm{CC}$ distortion is greatly enhanced and one can exclude the null hypothesis (no distortion) at the $99.9 \%$ confidence level (one year's data).

Enriched lithium nitrate or sulfate ( 0.3 tonnes) have been chosen as the most suitable chemical forms for introducing a neutron poison into the heavy water because they are very similar to the magnesium chloride salt (2 tonnes), which has already been developed as a neutral current additive. Thus, the reverse osmosis technology already installed by SNO for removing the magnesium chloride from the $\mathrm{D}_{2} \mathrm{O}$ can be used for the lithium nitrate or sulfate. This has been demonstrated, in small scale laboratory tests, by A. Kumar and K. Lamb ${ }^{3}$ of NRC Canada at our request. In addition, small scale tests carried out at LBNL have shown that the technique of seeded ultrafiltration for purifying magnesium chloride worked equally well for lithium nitrate solutions ${ }^{4}$.

The ${ }^{6} \mathrm{Li}$, in the chemical form of $\mathrm{LiOH} \cdot \mathrm{H}_{2} \mathrm{O}$, is supplied by Oak Ridge National laboratory and a $1 \mathrm{~kg}$ sample was purchased and analyzed for chemical and radioactive contamination. A chemical procedure has been established for converting the $\mathrm{LiOH} \cdot \mathrm{H}_{2} \mathrm{O}$ into $\mathrm{LiNO}_{3}$ and, at the same time, removing the $T h$ and $U$ chain contaminants. This procedure has been successfully carried out at the $1 \mathrm{~kg}$ scale using the material from Oak Ridge. Our objective is to produce the 300 kilograms of purified $\mathrm{LiNO}_{3}$ or $\mathrm{Li}_{2} \mathrm{SO}_{4}$ by the end of 1997 so that it is ready soon after the detector is filled with $\mathrm{D}_{2} \mathrm{O}$.

\section{Footnotes and References}

*Present address: Oxford University

$\ddagger D i e$ Arbeit wurde mit Unterstützung eines Stipendiums im Rahmen des Gemeinsamen Hochschulsonderprogramms III von Bund and Ländern über den DAAD ermöglicht.

1. N. Hata, S. Bludman, P. Langaker, Phys. Rev. D 49, 3622 (1994).

2. B. Frati, SNO internal report.

3. A. Kumar and K. Lamb, NRC No. ER-1356-95S.

4. A. R. Smith, D. L. Hurley, R. J. McDonald, LBL internal report. 


\section{A $4 \pi \beta$-tagged Decay Chamber for Gaseous SNO Calibration Sources \\ Y.D. Chan, M. Dragowsky, M.C. Isaac, K.T. Lesko, M.E. Moorhead*, E.B. Norman, A. Schuelke and R.G. Stokstad}

In the Sudbury Neutrino Observatory detector, Cerenkov photons, generated by the fast moving electrons in the final states of the charged current $\left(v_{e}+d-p+p+e\right)$ and elastic scattering $(v+e->v+e)$ interactions, are detected by about 10,000 inward-looking phototubes mounted on a geodesic sphere. The energy of the incoming neutrino is related to the number of fired phototubes (NHIT) in an event.

One important calibration is to establish an absolute energy scale for the NHIT spectrum. This is achieved by deploying calibration devices into the $\mathrm{D}_{2} \mathrm{O}$ volume. Among the planned calibration schemes is a gaseous calibration system, comprised of a shielded underground $d-t$ neutron generator, a capillary transport line, and an active decay chamber. The decay chamber consists of a cylindrical gas-holding volume made of $3 \mathrm{~mm}$ thick plastic scintillator sheets, enclosed in a water-tight stainless pressure vessel. Short-lived gamma $\left({ }^{16} \mathrm{~N}\right)$ and neutron $\left({ }^{17} \mathrm{~N}\right)$ sources are made by neutron-activating ${ }^{16} \mathrm{O}$ and ${ }^{17} \mathrm{O}$ gases. The beta particle accompanying the decay is detected by the scintillator to provide an effective tagging signal.

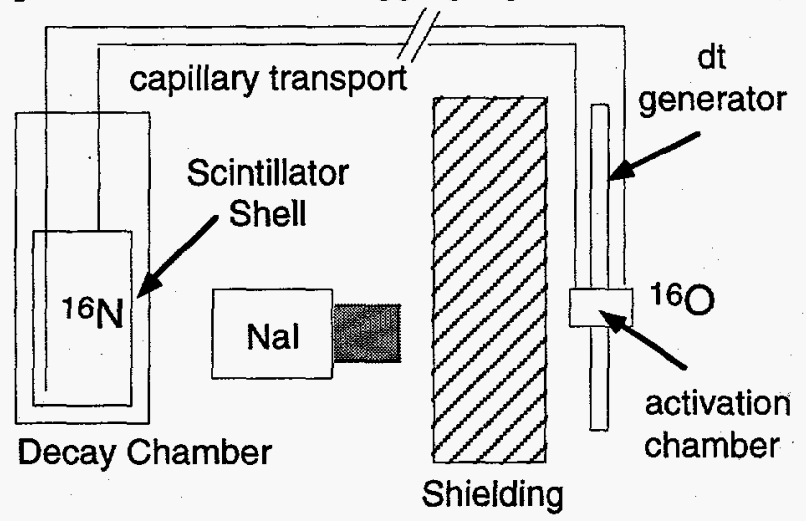

Fig. 1. Schematics of the experimental set up.

A highly successful test of the SNO d-t neutron generator, gas transport system, and decay chamber was carried out in the summer of 1996 at Chalk River Laboratory (Fig.1). The neutron generator produced approximately $10^{8} 14-\mathrm{MeV}$ neutrons per second which irradiated a target cell through which oxygen gas flowed. Through the ${ }^{16} \mathrm{O}(\mathrm{n}, \mathrm{p})$ reaction, ${ }^{16} \mathrm{~N}$ was produced and transported through a thin capillary to the decay chamber. There the betas and $6.13 \mathrm{MeV}$ gamma rays, produced by the decay of ${ }^{16} \mathrm{~N}$, were detected in the plastic scintillator walls of the decay chamber and in a large $\mathrm{NaI}$ detector, respectively (Fig. 2). By comparing the singles counting rate of $6.1 \mathrm{MeV}$ gamma rays in the $\mathrm{NaI}$ detector to the rate of decay chamber-NaI coincidences, we determined that the efficiency of the decay chamber for detecting the betas from ${ }^{16} \mathrm{~N}$ decay is approximately $95 \%$. Thus this system provides a clean source of tagged $6.1 \mathrm{MeV}$ gamma rays that will be used to provide an energy calibration for the SNO detector.

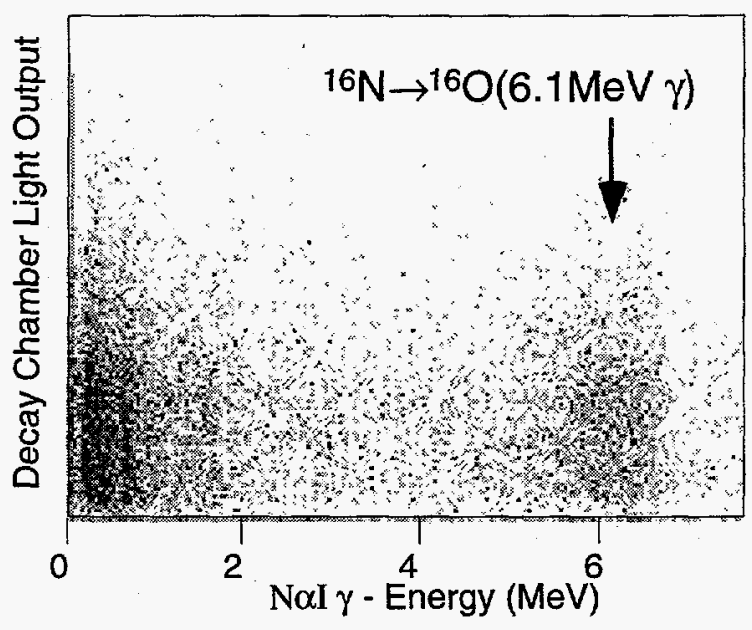

Fig. 2. Raw ${ }^{16} \mathrm{~N}$ decay spectrum.

\section{Footnotes and References}

* Present address : Oxford University, Oxford, UK.

† Die Arbeit wurde mit Unterstützung eines Stipendiums im Rahmen des Gemeinsamen Hochschulsonderprogramms III von Bund and Ländern über den DAAD ermöglicht. 


\title{
Sudbury Neutrino Observatory, \\ Neutral Current Detectors
}

\author{
K.T. Lesko, Y.D. Chan, M. Dragowsky, M. Isaac, M.E. Moorheadt, E.B. Norman, A. Schuelkeł, and \\ R.G. Stokstad
}

With SNO's ability to observe both the Neutral Current (NC) and Charged Current (CC) reaction rates we are able to deduce the existence of neutrino oscillations with two distinct signatures. The first signature is obtained by a measurement of the $\mathrm{CC} / \mathrm{NC}$ ratio. The second method is based on observing deviations in the predicted shape of the $\mathrm{CC}$ spectrum.

SNO is developing two techniques to observe the neutrons liberated by the NC reaction: $v_{x}+D$ $\Rightarrow \mathrm{p}+\mathrm{n}+\mathrm{v}_{\mathrm{x}}, \mathrm{Q}=-2.2 \mathrm{MeV}$. The first technique uses the addition of a salt to the $\mathrm{D}_{2} \mathrm{O}$, which captures the neutrons and generates $\gamma$-rays that are subsequently observed through their Compton scattering and associated Cerenkov light. The salt selected by $\mathrm{SNO}$ is $\mathrm{MgCl}$. The second technique uses an array of $\sim 100{ }^{3} \mathrm{He}$ proportional detectors[1]. The neutrons are detected via the ${ }^{3} \mathrm{He}(\mathrm{n}, \mathrm{p}+\mathrm{t})$ reaction. SNO anticipates using both techniques to reduce the affects of the different systematic errors associated with each technique.

The groups at the University of Washington, Los Alamos National Laboratory, and Lawrence Berkeley National Laboratory have received funds from the Department of Energy to develop the array of ${ }^{3} \mathrm{He}$ proportional counters, or "NCDs". The past year much work as gone into developing and producing the nickel tube detector bodies with low levels of uranium and thorium contamination ( 1 ppt $U$ has been achieved). The technology required for the production of the counters has advanced to the point where prototype counters were produced at the end of 1996.

Our group has applied our experience in engineering, contamination control, and low level counting to assist with the production of the counters. In particular we have drafted cleanliness procedures for the production of the counters, assisted in the selection and production of various NCD components, drafted quality assurance plans for the NCDs, and coordinated much of the low level counting performed at the Oroville facility. We designed, fabricated, and installed the underground facility in the SNO control room for the long-term storage of the detectors while the ${ }^{56} \mathrm{Co}$ decays and the detectors are operated to determine their contamination levels and operational characteristics.

Nickel in the array is activated by cosmic rays and ${ }^{56} \mathrm{Co}$ is produced. This activity adds a significant contribution to the NC backgrounds $(\gamma$-rays $>2.2 \mathrm{MeV})$ unless i) steps are taken to minimize the exposure of the components to cosmic rays and ii) the finished counters have a "cool-down" period ( $\sim 6$ months) during which ${ }^{56} \mathrm{Co}$ will decay $\left(\tau_{1 / 2}=77.27\right.$ day).

We have begun simulations of the NCDs in SNO and are pursuing studies of in situ measurements of the NCD backgrounds using a neutron poison.

\section{Footnotes and References}

† Present address, Oxford University, Oxford, UK OX13RH

$\ddagger$ Die Arbeit wurde mit Unterstützung eines Stipendiums im Rahmen des Gemeinsamen Hochschulsonderprogramms III von Bund and Ländern über den DAAD ermöglicht.

1. Neutral-Current Detection in the Sudbury Neutrino Observatory, Proposal to the Department of Energy FIN-94-ER-E-324, January 31, 1992, T. Bowles, et al. 


\title{
Sudbury Neutrino Observatory - Contamination Control
}

\author{
R.G. Stokstad, K.T. Lesko, Y.D. Chan, M. Dragowsky, M. Isaac, E. Norman and A Schuelket
}

Twelve thousand square meters of surface area will be in the SNO cavity when construction is complete. Dust left on these surfaces may emanate radon and therefore contaminate regions of the detector well removed from the source material. Mine dust (6 ppm Th) is the main problem, but any granular material containing $\mathrm{U}$ and Th are problematic. Our goal is to reduce surface contamination to acceptable levels, which are derived by requiring that they be less than the unavoidable contributions from the bulk materials comprising the detector. Earlier articles ${ }^{1}$ have described the cleanliness infrastructure in the SNO laboratory. During the last two years, experience has been gained through cleanliness monitoring and further measures have been implemented.

The rate at which mine dust settles on surfaces is inferred from measurements of iron, a $7 \%$ constituent of mine dust. These measurements are made using X-Ray Fluorescence Analysis (XRF). A monitoring program extending over several years has shown that the rate of mine dust deposition is typically between 1 and 3 $\mu \mathrm{g} / \mathrm{cm}^{2} / \mathrm{mo}$. Since the construction period is longer, and the above deposition rates are higher than anticipated years ago, additional cleanliness measures are warranted. The solution to this problem has been the introduction of dust covers. Critical components such as the surfaces of the acrylic vessel (AV) are covered with tarpaulins just after being cleaned for the last time. The covers are designed for removal much later in the construction period. Ropes and fastening mechanisms are installed such that the covers can be pulled off from a remote location. (Tests have been made to verify that airborne dust deposited on the covers sticks.) Light/dust covers for the photomultiplier tubes (PMT) were designed, fabricated, and installed before the
PMT panels were put in place on their support structure. Since the PSUP was installed, covers have been placed on top of the upper PSUP (i.e., on the back sides of the PMTs), which will be removed just before the water fill commences.

Procedures for the cleaning of the acrylic surfaces and for measuring the level of cleanliness were developed and recently implemented. The complete procedure involved many steps, but the basic cleaning agent was a dilute solution of Alconox. The level of contamination before and after cleaning was measured by applying a special (low in Fe) adhesive tape to the surface and then analyzing the tape in the XRF spectrometer. By applying the same piece of tape repeatedly to adjacent areas on the surface, the sensitivity of the technique could be increased by a factor of five. It was clear that the cleaning procedure was effective in removing surface contamination containing the elements $\mathrm{Fe}$ and $\mathrm{Zn}$. (The $\mathrm{Zn}$ is believed to come from the galvanized scaffolding used in construction.) After cleaning, the residual mine dust (again, inferred from the amount of $\mathrm{Fe}$ ) was well below the $0.1 \mu \mathrm{g} / \mathrm{cm}^{2}$ value set as a tolerable upper limit for the $A V$. The sensitivity of the method is $0.025 \mu \mathrm{g} / \mathrm{cm}^{2}$ of mine dust $(1 \sigma)$.

The upper hemisphere of the AV is now cleaned and covered.

Footnotes and References

†Die Arbeit wurde mit Unterstützung eines Stipendiums im Rahmen des Gemeinsamen Hochschulsonder-programms III von Bund and Ländern über den DAAD ermöglicht.

1. NSD Annual Reports: 1991, p.81; 1992, p.87; 1994, p. 101. 


\section{Sudbury Neutrino Observatory, Monte-Carlo Simulations and Data Analysis}

M.E. Moorheadt, Y.D. Chan, M. Dragowsky, M. Isaac, K.T. Lesko, E.B. Norman, A. Schuelkeł, and R.G. Stokstad

SNOMAN is the principal the Monte-Carlo simulation and data analysis program for the Sudbury Neutrino Observatory (SNO). The code is being developed by the many of the institutions participating in SNO. The LBNL group plays a major role in the development of this 110,000 line code and this effort has increased as our hardware responsibilities have been met.

One significant LBNL contribution to SNOMAN has been the graphical display package. During the past year we developed a user interface for SNOMAN using the Windows Motif package. The user interface will also help in the comparisons between different SNOMAN runs at different institutions, since it records all the run-dependent input parameters in a standard format.

Other LBNL contributions to SNOMAN have been made in photon transport, neutron code verification, detector geometry, input parameters for radioactive decay schemes and reaction cross-sections, and the generation, propagation and detection of high energy $\gamma$ rays. The goals of these contributions have been to provide accurate and detailed treatments of $i$ ) backgrounds from radioactivity in the photomultiplier support structure and other components, ii) calibration sources, in particular the $16,17 \mathrm{~N}$ calibration sources which are being developed at LBNL, and iii) backgrounds generated from the cavity walls.

On the data analysis side several event fitters [1] are being developed using neural network, maximum likelihood and other techniques. The aim of these fitters is to reconstruct event position and direction as well as to provide some particle identification capabilities.

An analysis of MSW distortions of the charge current spectrum was made. It is clear that the experimentally favored 'small-angle MSW, or nonadiabatic' solution to the solar neutrino problem produces a measurable distortion of the charge current spectrum[2]. However, this distortion occurs mostly at low energies where it is obscured by $n$-capture events on deuterium. To remedy this problem we propose to introduce 30 $\mathrm{Kg}$ of ${ }^{6} \mathrm{Li}$ (in the form of lithium carbonate) into the $\mathrm{D}_{2} \mathrm{O}$ in order to capture $>99 \%$ of the free neutrons on ${ }^{6} \mathrm{Li}$ and reduce the rate of capture on deuterium by a factor $>25$ [3].

A $2 \mathrm{~m}$ diameter test detector was built in Seattle with 128 PMTs. The mini-SNO detector is used to test the electronics and DAQ system and also to exercise the $\mathrm{MC}$ simulation and analysis chain with real events from calibration sources which will be used in SNO[4].

Footnotes and References

† Present address, Oxford University, Oxford, UK OX13RH

fDie Arbeit wurde mit Unterstützung eines Stipendiums im Rahmen des Gemeinsamen Hochschulsonderprogramms III von Bund and Ländern über den DAAD ermöglicht.

1. SNO-STR-95-042 Grid fitter for reducing tails in spatial distributions M.E. Moorhead and LBL-33634 Neural Network for Recognizing Cerenkov Radiation Patterns Dawei W. Dong, Y.D. Chan, M.T.F. da Cruz, A. Garcia, R.M. Larimer, K.T. Lesko, E.B. Norman, R.G. Stokstad, F.E. Wietfeldt, and I. Zlimen.

2. See also Bahcall, Krastev, Lisi IASSNS-AST 96/43

3. SNO-STR-95-056 Li-6 neutron poison for SNO M.E. Moorhead

4. SNO-STR-95-041 Mini-SNO geometry M.E. Moorhead, M.Isaac and K.T. Lesko 


\section{The Sudbury Neutrino Observatory: Monitoring the Experiment}

Y.D. Chan, M.C. Isaac, M. Dragowsky, K.T. Lesko, E.B. Norman, A. Schuelke and R.G. Stokstad

Unlike most accelerator-based experiments, the Sudbury Neutrino Observatory (SNO) will be operated 24-hours a day for a planned duration of 5 - 10 years. It is, therefore, essential to develop a flexible and reliable data monitoring program to provide up-to-date information about the status of the experiment, for SNO participants both on-site or off-site.

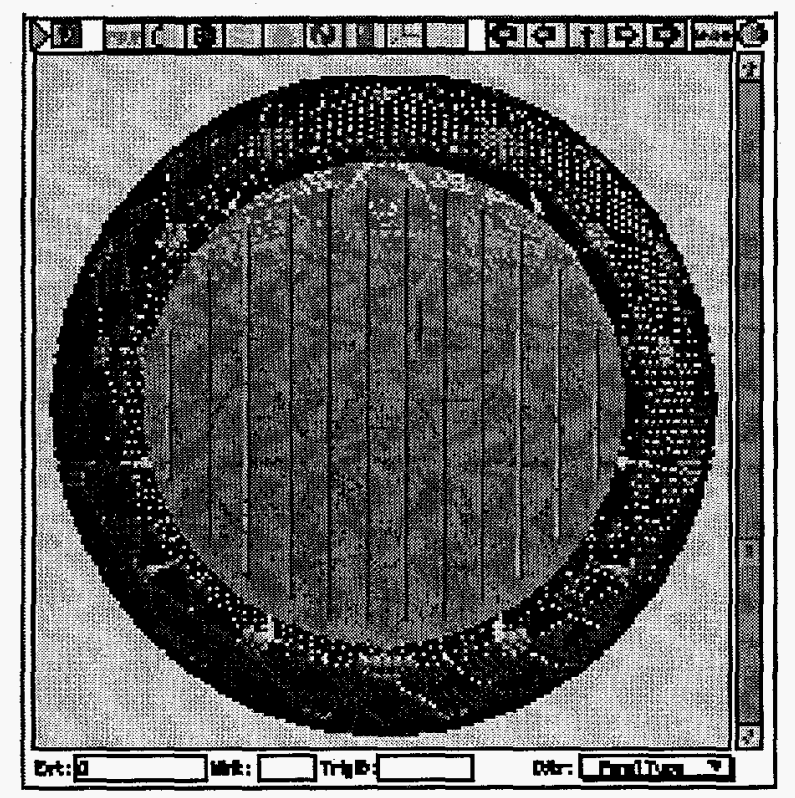

Fig. 1. SNO monitoring object CM_PSUP.

The scope of SNO monitoring covers two areas : (1) the past and present status of the detector/cavity hardware, and (2) the status of data acquisition (DAQ). There are two classes of tasks for DAQ monitoring. The Low-Level tasks are performed by PowerPC CPUs located underground inside the SNO cavity. These desktop computers control the DAQ task and initialize the operation of the $\sim 20$ embedded CPU's in the SNOCrates. The main tasks are component monitoring, diagnostics, conditional alert, and supernovae watch. An object-oriented SNO DAQ C++ Framework has been developed over the years by the SNO DAQ group. In particular, a SNOObject class forms the basis of all monitoring objects. All modules can be pre- configured or set up dynamically during run time. Fig.1 shows an example of a detector geometry access object connected to a data source.

The High-Level monitoring tasks are performed on UNIX workstations situated in the SNO surface building. The main tasks performed here include acquisition statistics, long term stability evaluation, as well as first-pass event fitting and histogram analysis. In compliance with coding standards within the SNO collaboration, a stand-alone monitoring program, SNOMON, has been developed to include features from both the official SNO Monte Carlo code, SNOMAN, and tools from the versatile CERNLIB application, PAW++. Streams of native $D A Q$ data are filtered into CERNLIB/ZEBRA format before they being processed by SNOMON. Fig. 2 shows the familiar PAW++-like user environment for SNOMON.

The surface workstations also serve as hosts for off-site monitoring. Techniques and tools from the WWW community, including Java-based dynamic content update and event browsers, are employed.

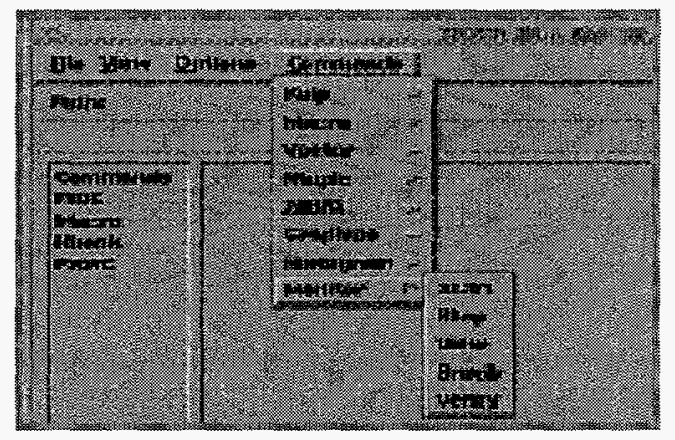

Fig. 2. SNO monitoring program SNOMON.

\section{Footnotes and References}

††Die Arbeit wurde mit Unterstützung eines Stipendiums im Rahmen des Gemeinsamen Hochschulsonder-programms III von Bund and Ländern über den DAAD ermöglicht. 


\title{
Response of the SNO Detector to High Energy Gamma-Rays
}

\author{
M. C. Perillo Isaac, Y.D. Chan, M. Dragowsky, D. Hurley, K. T. Lesko, R. J. McDonald, \\ M. E. Moorhead, E. B. Norman, A. Schuelke, A. R. Smith and R. G. Stokstad
}

A two step Monte-Carlo procedure was done in order to quantify the response of the SNO detector to high energy gamma rays produced by $(\alpha, \gamma),(\alpha, n \gamma)$ and $(\alpha, p \gamma)$ reactions induced by natural radioactivity in the rock and coating of the SNO cavity. In a first step, we used GEANT $3.21^{1}$ to model the SNO cavity and to track gamma rays and the subsequent electromagnetic radiation from the surface of the cavity up to a spherical surface of $18 \mathrm{~m}$ of diameter. The particle type, position and momentum are then recorded and used as input for SNOMAN ${ }^{2}$, the dedicated SNO Monte-Carlo code.

The GEANT 3.21 simulation generates gamma-rays uniformly in the cavity walls in all directions in $1 \mathrm{MeV}$ bins from 3 to $12 \mathrm{MeV}$. Two models were studied: cavity filled with water and cavity empty (air).

As a visual aid, figure 1 shows the projection in the ZX plane of events generated in the cavity walls superimposed with the projection of the events which survive the water shield, i.e., penetrate the region of the photomultiplier support structure.

For the purpose of this study we set standard conditions to the event trigger and data filter, requiring that at least 10 photomultipliers fire and that the events pass the standard SNO event reconstruction program, the FTT fitter.

The results are exemplified in figure 2 where we plot the response of the SNO detector, when filled with water, to gamma-rays originated in the cavity walls with energy between 7 and

Footnotes and References

*present address Oxford University, Oxford, UK

${ }^{\dagger}$ Die Arbeit wurde mit einem Stipendium des Gemeinsamen Hochschulsonderprogramms III von Bund und Landern uber den DAAD ermoglicht

${ }^{1}$ GEANT, CERN Program Library, Long Writeup W5013

${ }^{2}$ SNOMAN, SNO dedicate Monte Carlo code
$8 \mathrm{MeV}$. This response considers the events which were reconstructed inside the heavy water volume only.

The results of this study are combined with the experimental values obtained from the measurement of the high energy gamma-ray flux in the SNO cavity in order to evaluate the contribution of this background to the experiment.

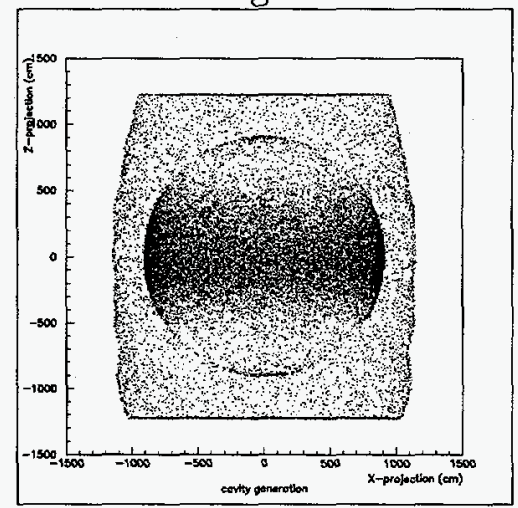

Figure 1: Projection of the GEANT generation in a water filled geometry. In this plot are represented the ZX projection of the position at which events are generated in the cavity walls superimposed to the position where particles intercept a 9 meter sphere. From this sphere on particles are tracked by SNOMAN.

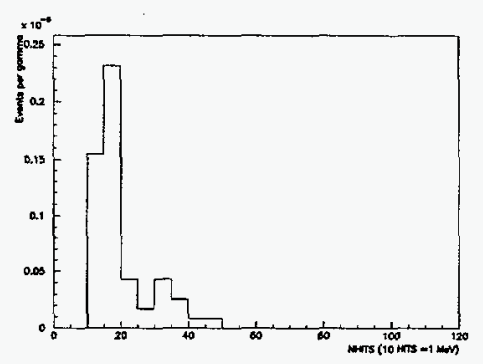

Figure 2: Example of the response of the SNO detector, as a function of the event energy, to gamma-rays of energies between 7 and $8 \mathrm{MeV}$ originated in the cavity walls. 


\title{
Measurements of the High Energy Gamma-Ray Flux at SNO
}

\author{
M. C. Perillo Isaac, Y.D. Chan, M. Dragowsky, D. Hurley, K. T. Lesko, R. J. McDonald,
} M. E. Moorhead, E. B. Norman, A. Schuelke $\dagger$ A. R. Smith and R. G. Stokstad

High energy gamma rays are an important background for both reactions of neutrino detection in SNO, neutral and charged current reactions. Compton scattering and/or pair production of high energy gamma-rays can mimic the electron produced in the charged current reaction. The photodisintegration of the deuteron can produce background neutrons to the neutral current reaction signal.

Our goal with this work is to evaluate the high energy gamma-ray flux induced from $\mathrm{U}$ and $\mathrm{Th}$ long lived isotopes, from the $(\alpha, \gamma),(\alpha, n \gamma)$ and $(\alpha, p \gamma)$ reactions in the rock. This new measurement of the gamma-ray flux was performed after the cavity was completed and coated with low activity concrete and the final sealing plastic liner, Urylon.

The detector used to measure the gamma-ray flux inside the SNO cavity was a $19 \mathrm{~cm}$ diameter by $15 \mathrm{~cm}$ thick NaI crystal to which three 3-inch photomultiplier tubes (PMT) were attached. The gains of the PMT were matched and the signals all of the PMTs were summed and fed into a ORTEC-NOMAD, a portable data acquisition system. We acquired data in this configuration for 1490 hours in the bottom of the SNO cavity. Shielded background runs were performed at the $4600 \mathrm{ft}$ level low background facility of the Creighton mine.

Figure 1 shows the comparison between spectra obtained with the same detector at different sites: at the LBNL Low Background Facility on Bldg. 72 (sea level), at the Oroville Low Background Facility (700 meter-waterequivalent) and at the SNO cavity (6000 meterwater-equivalent).

Footnotes and References

*Present Address Oxford University, Oxford UK

${ }^{\dagger}$ Die Arbeit wurde mit einem Stipendium des Gemeinsamen Hochschulsonderprogramms III von Bund und Landern uber den DAAD ermoglicht

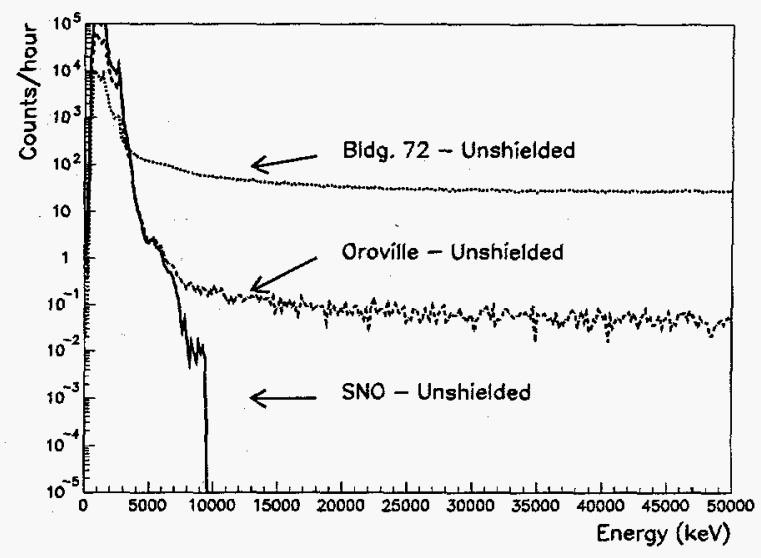

Figure 1: Comparison between spectra obtained at sea level (dotted line) at the Oroville Low Background Facilty (dashed line) and at the SNO cavity (full line).

In table 1 we present the comparison of the fluxes of the gamma-ray lines, $1460 \mathrm{keV}$ from ${ }^{40} \mathrm{~K}$ decay and $2614 \mathrm{keV}$ from ${ }^{208} \mathrm{Tl}$ decay obtained in 1994 and recently in 1996.

Table 1: Measured Fluxes - $6800 \mathrm{ft}$ Level

\begin{tabular}{ccc}
\hline $\begin{array}{c}E_{\gamma} \\
(\mathrm{keV})\end{array}$ & $\begin{array}{c}\text { Flux 3/94 } \\
\mathrm{cm}^{-2} \mathrm{~s}^{-1}\end{array}$ & $\begin{array}{c}\text { Flux 5/96 } \\
\mathrm{cm}^{-2} \mathrm{~s}^{-1}\end{array}$ \\
\hline 1460 & $0.059 \pm 0.003$ & $0.061 \pm 0.006$ \\
2614 & $0.016 \pm 0.001$ & $0.0075 \pm 0.0007$ \\
\hline
\end{tabular}

Based on the measured spectra, on the measured detector's intrinsic background and on the measured detector's intrinsic efficiencies the expected flux of gamma-rays from the cavity walls above $5 \mathrm{MeV}$ in the SNO cavity is $3.4 \times$ $10^{-3}$ gammas $/ \mathrm{cm}^{2} /$ hour, while previous estimatives based on Monte-Carlo and the rock contaminants quote $1.4 \times 10^{-3}$ gammas $/ \mathrm{cm}^{2} /$ hour $^{1}$

Footnotes and References

${ }^{1}$ P. Skensved and B.C. Robertson, SNO internal report. 


\title{
Sudbury Neutrino Observatory: Simulation of the Calibration Decay Chamber for ${ }^{16} \mathrm{~N}$ and ${ }^{17} \mathrm{~N}$
}

\author{
M. Dragowsky, Y.D. Chan, M.C. Perillo Isaac, K.T. Lesko, M.E. Moorheadt, E.B. Norman, \\ A. Schuelke $\ddagger$ and R.G. Stokstad
}

The energy calibration of the SNO detector will be accomplished, in part, through the use of a calibration decay chamber for ${ }^{16} \mathrm{~N}$ and ${ }^{17} \mathrm{~N}$ gas. A SNOMAN Monte-Carlo simulation provided a preliminary estimate of the decay chamber response. We are extending the geometry within the SNOMAN code to improve the accuracy of these simulations. Additionally, we have begun to investigate the effect of the decay chamber an the propagation of Cerenkov light in the $\mathrm{D}_{2} \mathrm{O}$.

Presently, the decay chamber geometry is modeled as three concentric cylinders: an outer canister, a thin shell of plastic scintillator and an interior gas volume. The actual detector has a more detailed internal structure, the most important issue being the nonconcentric location of the gas volume relative to the outer canister. The geometry is also being modified to include a photomultiplier tube (PMT) used to monitor the scintillator, the high voltage generator for the PMT, an optical coupling between the scintillator and PMT and the utility lines which provide power and supply the ${ }^{16,17} \mathrm{~N}$ gas.

The decay chamber is optically opaque and potentially can screen Cerenkov light produced by ${ }^{16} \mathrm{~N}$ decays in the decay chamber from reaching the main PMT array. Using SNOMAN with our extended geometry we can estimate these screening effects by comparing simulations with the stainless steel canister replaced with a transparent media $\left(D_{2} O\right)$. A comparison of the mean number of PMT hits determine the influence of the decay chamber. We will also account for gamma-ray attenuation in the stainless steel decay chamber. A first assessment will be made by reducing the thickness of the outer canister wall in the simulation so that attenuation is less than $1 \%$. We plan further studies of the decay chamber to assess the light blocking of the decay chamber using simulated external sources of
Cerenkov light, as well as using SNOMAN to track specific photon propagation paths.

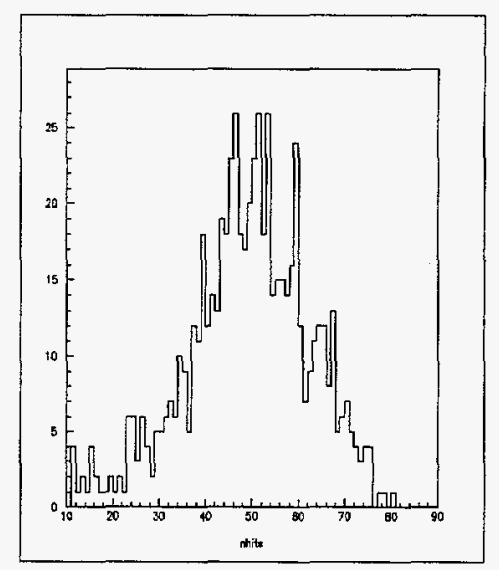

Fig. 1. Plot of PMT hits for decay chamber with stainless steel outer canister.

Footnotes and References

† Present address, Oxford University, Oxford, UK OX13RH

$\ddagger$ Die Arbeit wurde mit Unterstützung eines Stipendiums im Rahmen des Gemeinsamen Hochschulsonderprogramms III von Bund and Ländern über den DAAD ermöglicht. 


\title{
Sudbury Neutrino Observatory, Monte-Carlo Simulation and Background Studies of the PSUP
}

\author{
A. Schuelke $\dagger$, K.T. Lesko, M. Dragowsky, Y.D. Chan, M. Isaac, E. Norman and R. Stokstad
}

The anticipated solar neutrino signal rates of a few tens of events per day require a low background environment for SNO. There are two principal sources of background: cosmic rays and radiation produced by naturally occuring radionuclides. It is expected that the most significant sources of background lie within the detector components themselves. One part is due to the $\gamma$ 's and $\beta$ 's from the Thorium and Uranium chains. The second component is due to the high-energy $\gamma$ 's from the PMT's, their support structure (PSUP) and the SNO cavity. These $\gamma$-rays can have energies exceeding $10 \mathrm{MeV}$ and are produced by $(\alpha, p \gamma)$ or by $(n, \gamma)$ reactions [1].

Since the LBNL group designed and supplied the PSUP[2], we developed here a much improved version of a Monte-Carlo simulation for the PSUP geodesic frame with the SNOMAN code [2]. The exact geometry has not been included into SNOMAN until now.

Two cases were developed to simulate highenergy $\gamma$ 's coming from the PSUP. The first used a shell of water at radius $900 \ldots 910 \mathrm{~cm}$ as the source of $9 \mathrm{MeV} \gamma$ 's. Care was taken to avoid an overlap with other components in this region. The second case placed the shell more correctly at a radius of $883.9 \ldots 894.1 \mathrm{~cm}$. The monoenergetic $\gamma$ 's are distributed isotropically within these shells. We then investigated how many events with an energy greater than $3 \mathrm{MeV}$ were reconstructed as originating inside the $\mathrm{D}_{2} \mathrm{O}$.

In fig. 1 the reconstructed position versus the generated point of the Cerenkov light is shown for the second case. Mostly the Cerenkov light creation is outside of the $D_{2} O$. In the first case with the source shell behind the panel zone $8.7 \%$ of all generated photons resulted in a detected

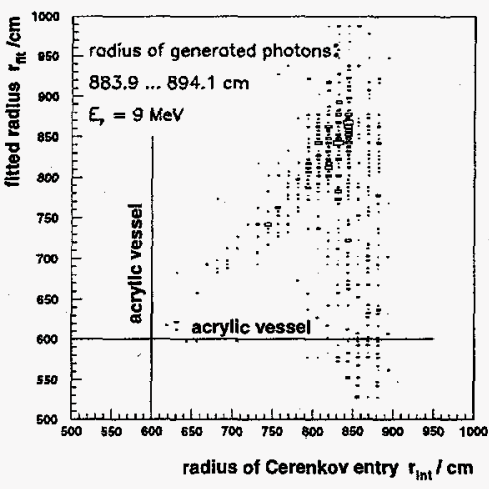

Figure 1: Fitted position of Cerenkov light vs. production point for case 2

signal. From these entries $9.6 \%$ are fitted inside the $\mathrm{D}_{2} \mathrm{O}$ with the standard time fitter and $2 \%$ had a fitted energy $E \geq 3 \mathrm{MeV}$. Therefore of all high-energy $\gamma$-rays originating in the PSUP $0.2 \%$ are fitted in the $D_{2} O$ with energy greater than $3 \mathrm{MeV}$. Tab.1 summarizes and compares the results of both cases. We are continuing our

\begin{tabular}{||l|c|c||}
\hline \hline shell range & case 1 & case 2 \\
\hline hits & $8.7 \%$ & $13.1 \%$ \\
fitted in $\mathrm{D}_{2} \mathrm{O}$ & $9.6 \%$ & $8.9 \%$ \\
$E_{\text {fit,hits } \geq 3 \mathrm{MeV}} \geq 2.0 \%$ & $2.8 \%$ \\
$E_{f i t, g e n} \geq 3 \mathrm{MeV}$ & $0.2 \%$ & $0.4 \%$ \\
\hline \hline
\end{tabular}

Table 1: Results from PSUP shell simulation

Monte-Carlo simulations for background studies for the $\mathrm{CC}$ and $\mathrm{NC}$ spectra with the improved fitters and PSUP geometry.

\section{References}

[1] NIM A364 (1995) 317-327

[2] NSD Annual Report, 1995, p.99ff

$\dagger$ Die Arbeit wurde mit Unterstützung eines Stipendiums im Rahmen des Gemeinsamen Hochschulsonderprogramms III von Bund und Ländern über den DAAD ermöglicht. 


\title{
Sudbury Neutrino Observatory, Extending the Detector Geometry in SNOMAN for Background Investigations
}

\author{
A. Schuelke $\dagger^{\dagger}$ K.T. Lesko, Y.D. Chan, M. Dragowsky, M. Isaac, E. Norman and R. Stokstad
}

Our background study for the PMT support structure and PMT panel area using special water shells was improved by including the PSUP steel frame geometry into the SNOMAN code.

The geometry is an embedding geometry. There are regions with different priorities. The regions with higher priority are embedded in regions with lower. The handling between the various regions is done by a boundary manager.

The geometry software package, GEO, has four tiers: interface, detector element routines geometry primitives and boundary manager. The main task in extending the geometry is the development of the detector element routines. Progressing through the different generations shown in fig. 1 requires using different coordinate systems.

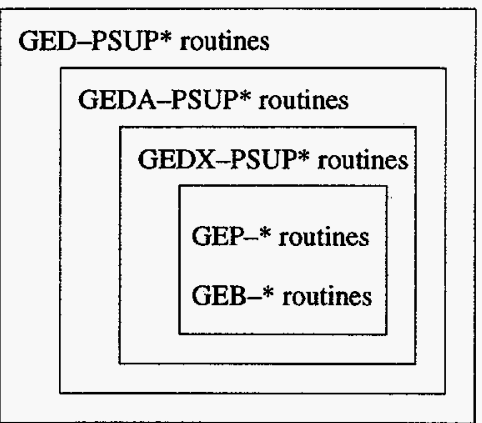

Figure 1: General scheme of extending the geometry in SNOMAN

The $G E D *$ routines call their own element and internal element routines, and these routines know about their relationships to their neighbouring regions. These routines work with the overall detector frame. Next generation, the $G E D A *$ routines, handle all requests estimating the nearest or the next element and picking particular parts of the detector. They are linked to the primitives in this detector element. Therefore, a special detector element coordinate system is used. In case of the PSUP, it is the same as the overall detector coordinate system. The $G E D A *$ routines are only necessary for complex detector elements such as the PSUP steel frame. Modelling the PSUP steel frame in a geometry useable by SNOMAN requires that all components have to be described with the available primitive types. By using the high degree of symmetry in the steel frame a special field including a fifth of all struts, hubs, ropes and acrylic vessel guide cans is generated. This section of the PSUP is then simply rotated five times about $\phi$ to produce the complete frame. Additionally, the PSUP frame model includes a south pole hub, the acrylic vessel guide ring and the chimney ring. The struts, hubs, ropes and acrylic vessel guide ring cans are described as cylinders with radius, thickness and length. Some of them are hollow, which requires creating an internal PSUP region filled with water. The AV guide ring and the chimney ring are described as two half tori. They have an internal water volume, too. The $G E D X *$ routines are called by the $G E D A *$ routines. They handle the external and internal boundaries ( $G E B *$ routines) depending on the tracking request and call the special primitive routines ( $G E P *$ routines). The $G E P *$ and $G E B *$ routines are standard routines of the SNOMAN code. The shapes and the locations of all elements of the steel frame are stored in the GEDS and GEDP banks.

†'Die Arbeit wurde mit Unterstützung eines Stipendiums im Rahmen des Gemeinsamen Hochschulsonderprogramms III von Bund und Ländern über den DAAD ermöglicht. 


\section{R\&D for a Km3 Neutrino Detector}

W. Chinowsky ${ }^{\dagger}$, H. Crawford ${ }^{\ddagger}$, R. Fusezy ${ }^{\dagger}$, YuDong He ${ }^{\ddagger t}$, S. Jackson*, K. Lesko ${ }^{\S}$, D. Lowder ${ }^{\dagger,+t}$, J. Ling*,

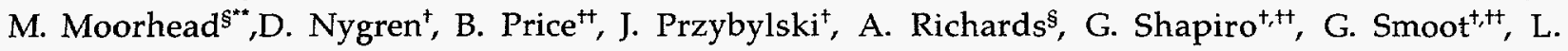
Stevenson ${ }^{\dagger}$, R. Stokstad ${ }^{\S}$, and R. Tripp ${ }^{\dagger}$

The exploration of the neutrino sky is a frontier area of nuclear and particle astrophysics: to date only the sun, one supernova, and the neutrinos generated in the earth's atmosphere have been studied. There is certainly more to be discovered. For example, the most energetic spots in the universe, Active Galactic Nuclei, are very likely sources of highenergy neutrinos. The discovery of these objects in the neutrino spectrum would open a new window for their study and for the investigation of other cosmological sources as well. The detectors currently in operation - AMANDA and BAIKAL - use strings of phototubes arranged in an array, and are the precursors of a future detector with characteristic dimensions of a kilometer. It will enable the measurement of the neutrino spectrum at high energies, a property of the universe that is as fundamental as the cosmic ray spectrum. The collaborations NESTOR (Greece) and ANTARES (France) are two examples of the strong international interest in such a detector, which has acquired the generic name "Km3."

A $\mathrm{Km} 3$ able to identify the relatively rare signals of interest and extract the maximum information from them will not be a straightforward scale-up of the existing detectors. Before designing and constructing a $\mathrm{Km} 3$, an intensive program of $R \& D$ is needed to establish a technical base matched to the scientific goals. An inter-divisional effort at LBNL has been underway and, together with JPL, has produced a key technical advance that, we believe, is ideal for the scientific program of the $\mathrm{Km} 3$. Prototype digital optical modules, which incorporate an advanced microchip enabling full wave form reconstruction, have been built and are undergoing testing here and at the South Pole. Concurrently, a modular computer simulation code is being developed to evaluate detector configurations and performance. Also, we are addressing the challenge of a costeffective deployment of the photomultiplier tubes in deep ocean water. These activities so far have been supported by Director's Development funds at LBNL and JPL.

It is essential to proceed toward an eventual $\mathrm{Km} 3$ in a step-wise manner that can accommodate changes and new developments. Four stages are envisioned:

$$
\begin{aligned}
& \text { Stage I: Systems Design, Simulations, } \\
& \text { and Deployment (one string, } 3 \text { years) } \\
& \text { Stage II: Demonstration (6 to } 12 \text { strings, } \\
& \left.\quad 2 \text { years, } 0.03 \mathrm{~km}^{2}\right) \\
& \text { Stage III: Exploratory Science }\left(0.2 \mathrm{~km}^{2}\right. \text {, } \\
& \quad 3 \text { years) } \\
& \text { Stage IV: Full Scope Science }\left(1 \mathrm{~km}^{2}\right)
\end{aligned}
$$

Stage I is the scope of the $R \& D$ envisioned here. The $R \& D$ activities factor naturally into these areas: electronic systems design, computer simulations, and deployment methodologies. The three R\&D efforts are fully coordinated, however, and each effort includes a phased approach in reaching its goals.

\section{Footnotes and References}

+ Physics Division

₹ Space Sciences Laboratory

$\S$ Nuclear Science Division

${ }^{*}$ Jet Propulsion Laboratory

** Now at Oxford University

t+ Dept. Physics, UC Berkeley 


\section{Half-Life of ${ }^{44} \mathrm{Ti}$}

\section{E. B. Norman, E. Browne, Y. D. Chan, I. D. Goldman*, R.-M. Larimer, K. T. Lesko, M. Nelson ${ }^{\dagger}$, F. E. Wietfeldt $t^{*}$, I. Zlimen}

One of the few long-lived gamma-ray emitting radioisotopes expected to be produced in substantial quantities during a supernova explosion is ${ }^{44} \mathrm{Ti}$. The characteristic $1157-\mathrm{keV} \quad \gamma$ ray from the decay of ${ }^{44} \mathrm{Ti}$ has recently been observed from the supernova remnant Cas A [1]. In order to compare the theoretically predicted gamma-ray flux to that actually observed from this remnant, one must know the half-life of ${ }^{44} \mathrm{Ti}$. However, published values for this quantity range from 39 to 66.6 years $[2,3]$. Given that the Cas A supernova is believed to have occurred approximately 300 years ago, this translates to an uncertainity by a factor of 6 in the amount of ${ }^{44} \mathrm{Ti}$ ejected by this supernova. Thus, in order to provide an accurate and reliable value for this important quantity, we have performed a new experiment to determine the half-life of ${ }^{44} \mathrm{Ti}$.

We produced ${ }^{44} \mathrm{Ti}$ via the ${ }^{45} \mathrm{Sc}(\mathrm{p}, 2 \mathrm{n})$ reaction using $40 \mathrm{MeV}$ protons from the 88 -Inch Cyclotron. This energy was chosen to be just above the maximum of the excitation function for this reaction as reported by McGee et al. [4]. However, in the course of preparing this source, we produced far less ${ }^{44} \mathrm{Ti}$ than expected. We therefore remeasured the excitation function for this reaction and found that it peaks at much lower energy and has a significantly smaller maximum cross section than previously reported [5]. To produce the source for the present halflife measurement, a $99.9 \%$ pure metallic scandium target $37.8 \mathrm{mg} / \mathrm{cm}^{2}$ thick was irradiated for approximately 24 hours with 1 microampere of beam. After allowing the shortlived activities to decay away, approximately $0.01 \mu$ Curie of ${ }^{44} \mathrm{Ti}$ was chemically separated from the target, mixed together with $0.04 \mu$ Curie of ${ }^{22} \mathrm{Na}$ and $0.05 \mu \mathrm{Curie}$ of ${ }^{137} \mathrm{Cs}$, and then dried to make the source for this experiment. This mixed source and a separate $1-\mu$ Curie source of ${ }^{241} \mathrm{Am}$ were then rigidly mounted to a shielded $110-\mathrm{cm}^{3}$ high-purity germanium detector for $\gamma$ ray counting. Data were collected in 1-day time bins for approximately 2 years.

In the present experiment, we attempted to use all three ${ }^{44} \mathrm{Ti} \gamma$-ray lines to determine its half life. However, analysis of the ${ }^{241} \mathrm{Am}$ and ${ }^{137} \mathrm{Cs}$ lines produced an incorrect value for the half life of each of these isotopes. On the other hand, the analysis of the ${ }^{22} \mathrm{Na}$ line produced a result that agreed to within $0.5 \%$ of the known value of 2.603 years. Also, a small ${ }^{54} \mathrm{Mn}$ contaminant line at $835 \mathrm{keV}$ yielded a half life that agreed to within $1 \%$ of its known value. Thus, we decided to concentrate our effort on the analysis of the $1157-\mathrm{keV}$ line. In order to reduce systematic effects such as the possibility of a change in the source-detector distance or a change in the detection efficiency, we compared the area of the $1157-\mathrm{keV}^{44} \mathrm{Ti}$ line to that of the nearby $1275-\mathrm{keV}$ line from the ${ }^{22} \mathrm{Na}$ standard. The ratio of these peak areas was plotted versus time and then fitted to an exponential whose argument was the difference in decay rates between ${ }^{44} \mathrm{Ti}$ and ${ }^{22} \mathrm{Na}$. The chi-squared per degree of freedom for the fit is 1.1. The half life of ${ }^{44} \mathrm{Ti}$ that we deduce from this experiment is $62 \pm 2$ years.

\section{Footnotes and References}

*Permanent address: Univ. of SaoPaulo, Sao Paulo, Brasil

† Present address: Santa Rita High School, Tucson, AZ \#Present address: NIST, Gaithersburg, MD

1. A. F. Iyudin et al., Astron. \& Astrophys. 284 (1994) L1.

2. J. Meissner, Ph.D. thesis, Univ. of Notre Dame, 1996

3. D. Alburger \& G. Harbottle, Phys. Rev. C 41 (1990) 2320.

4. T. McGee, et al., Nucl. Phys. A 150 (1970) 11.

5. R. Ejnisman et al., Phys. Rev. C 54 (1996) 2047. 


\section{Survival of ${ }^{180}$ Ta During the s-Process}

R.-M. Larimer, Y. D. Chan, M. P. C. Isaac, K. T. Lesko, A. O. Macchiavelli, R. W. Macleod, M. E. Moorhead, E. B. Norman, K. S. Krane*, K. Zaerpoor*, J. Beckert, L. A. Bernsteint, L. P. Ferrist

Over the years, many attempts have been made to to photodeexcite ${ }^{180} \mathrm{Ta}$ to ${ }^{180} \mathrm{Tag}$ using intense sources find a plausible production mechanism for Nature's of ${ }^{137} \mathrm{Cs}$ and ${ }^{60} \mathrm{Co}$ have failed [4]. This suggests that rarest isotope and only naturally-occurring isomer, the mediating levels may lie above $1.33 \mathrm{MeV}$ excitation the odd-odd nucleus ${ }^{180} \mathrm{Ta}$. The long-lived ${ }^{180} \mathrm{Ta}$ m energy. Recent Coulomb deexcitation experiments have $\left(0^{\pi}=9^{-}\right)$is located approximately $75 \mathrm{keV}$ above the observed the production of ${ }^{180} \mathrm{Tag}$ from ${ }^{180} \mathrm{Ta}$ and $\mathrm{t}_{1 / 2}=8.1$ hour $\left(\mathrm{J}^{\pi}=1^{+}\right.$) ground state [1]. The suggest that there may in fact be lower lying mediating standard s- , r-, and p-processes all fail to levels [5].

quantitatively account for the observed abundance of ${ }^{180} \mathrm{Ta}\left(\left[{ }^{180} \mathrm{Ta}\right] /\left[{ }^{181} \mathrm{Ta}\right]=10^{-4}\right.$.) Several detours off the standard s- and r-process paths that could lead to ${ }^{180} \mathrm{Ta}$ have been investigated. The s-process path through the stable Hf isotopes weakly populates a $\mathrm{J} \pi=8^{-}$isomer in ${ }^{180} \mathrm{Hf}$ which can then beta decay to ${ }^{180} \mathrm{Ta} \mathrm{m}$. However, this route was shown to be too small to account for ${ }^{180} \mathrm{Ta}$ abundance [1]. In principle 60

In order to answer the question of whether or not ${ }^{180} \mathrm{Ta}$ can survive in stellar environments, we have populated levels in ${ }^{180} \mathrm{Ta}$ using the ${ }^{176} \mathrm{Yb}\left({ }^{7} \mathrm{Li}, 3 \mathrm{n}\right)$ reaction and have used GAMMASPHERE to studying the gamma decays of levels up to approximately 2 $\mathrm{MeV}$ excitation energy. Thus far we have been able 60 different levels. We observe a family of the r-process could also reach ${ }^{180} \mathrm{Ta}$. ${ }^{180} \mathrm{Lu}$ transitions that ultimately lead to the ${ }^{180} \mathrm{Tag}$ and produced in the r-process could feed $\mathrm{J}^{\pi}=8^{-}$isomer in another family that leads to the long-lived ${ }^{180} 0_{\mathrm{Ta}} \mathrm{m}$.

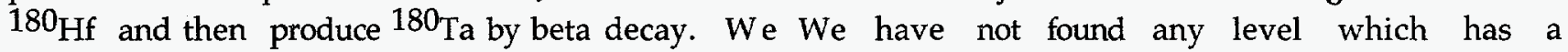
have shown with experiments at the Univ. of measurable decay branch to both levels. Thus it may Washington and at $88^{\prime \prime}$ Cyclotron that this be that ${ }^{180} \mathrm{Ta}$ m can survive in an s-process branching ratio is too small to account for a environment.

significant amount of ${ }^{180} \mathrm{Ta}[2,3]$. Thus, the production mechanism responsible for ${ }^{180} \mathrm{Ta}$ remains an open question.

Regardless of how ${ }^{180} \mathrm{Ta}$ is actually produced in stars, the question remains as to whether it can survive in the hot dense stellar environment? At the temperatures appropriate to the s- and r-processes, there is an enormous bath of high energy photons present. Such photons could excite ${ }^{180} \mathrm{Ta}^{\mathrm{m}}$ up to a higher-lying level of intermediate spin which subsequently decays though a $\gamma$-cascade to the ${ }^{180} \mathrm{Ta}$ ground state. Such photoexcitation could lead to thermal equilibration between the ground state and Footnotes and References

*Oregon State Univ., Corvallis, OR

† Lawrence Livermore National Laboratory

1.S. E. Kellogg and E. B. Norman, Phys. Rev. C 46, 1115

(1992).

2. S. E. Kellogg and E. B. Norman, Phys. Rev. C 34, 2248

(1986).

3. K. T. Lesko et al., Phys. Rev. C 34, 2256 (1986).

4. E. B. Norman et al., Astrophys. J. 281, 360 (1984).

5. C. Schlegel et al., Phys. Rev. C 50, 2198 (1994). isomer, with the result that the effective ${ }^{180} \mathrm{Ta}$ half life becomes on the order of days. This would drastically reduce the amount of $180 \mathrm{Ta}$ m that would actually emerge from a stellar environment.

The question of whether or not this process actually happens in nature depends critically on the excitation energy and $\gamma$-decay modes of levels in ${ }^{180} \mathrm{Ta}$. Attempts 


\title{
Search for the $\beta^{+}$Decay of ${ }^{144} \mathrm{Pm}$
}

\author{
K. Zaerpoor*, M. R. Dragowsky*, K. S. Krane ${ }^{*}$, M. M. Hindit, S. J. Robinsont, D. E. DiGregorioł, E. B. \\ Norman, Y. D. Chan, M. C. P. Isaac, R.-M. Larimer, A. O: Macchiavelli, R. MacCleod
}

The mean confinement time of cosmic rays within our Galaxy can be determined by comparing the cosmic-ray abundances of suitably long-lived radioactive isotopes to those of their stable neighbors. For heavy elements $(Z>28)$ the current state of the art in cosmic-ray mass measurements is not sufficient to allow isotopic abundance measurements. This difficulty prompted Drach and Salamon [1] to consider the possibility of using elemental abundances of Tc and $\mathrm{Pm}$, which have no stable isotopes, as cosmic ray clocks. In the case of $\mathrm{Pm}$, only three isotopes, ${ }^{143,144,145} \mathrm{Pm}$, have sufficiently long half-lives and suitable decay schemes to be present in the cosmic rays. Due to their interactions with the interstellar medium, cosmic rays nuclei are fully stripped of their atomic electrons. This turns a nucleus such as ${ }^{145} \mathrm{Pm}$, which decays in the laboratory via electron capture, into a stable nucleus. Because of their larger EC decay energies, it is possible for ${ }^{143} \mathrm{Pm}$ and ${ }^{144} \mathrm{Pm}$ to also decay by $\beta^{+}$emission. In the laboratory, these forbidden decays have to compete with allowed EC decays and so have not yet been observed. As fully stripped nuclei, however, these $\beta^{+}$decays will determine the cosmic ray half lives of these isotopes.

In a previous experiment [2], we used two $28 \%$ efficient $\mathrm{Ge}$ detectors surrounded by a $4 \pi$ $\mathrm{NaI}$ annular detector to search for the $\beta^{+}$decay branches of ${ }^{143,144} \mathrm{Pm}$. For the case of ${ }^{144} \mathrm{Pm}$ we searched for the $\beta^{+}$decay to the $J^{\pi}=2^{+}$level at $697 \mathrm{keV}$ through the observation of 511-511-697 $\mathrm{keV}$ triple coincident events. While we did not succeed in observing this decay, we were able to establish a limit of $>1.2 \times 10^{6}$ years for the $\beta^{+}$ decay half life of ${ }^{144} \mathrm{Pm}$. This is close to the lower limit for this decay mode estimated by Drach and Salamon. In order to maximize our detection efficiency, we had to sandwich the source right in between the two Ge detectors. Because of this close geometry, a major limitation in this experiment was background produced by $\gamma$ rays Compton scattering from one Ge detector into the other.

In order to improve upon this measurement, we have performed a new experiment by placing a $1.4 \mu \mathrm{Ci}$ source of ${ }^{144} \mathrm{Pm}$ into GAMMASPHERE to search once again for 511-511-697 keV coincidences. This source was produced at the 88-Inch Cyclotron approximately 9 months ago and has been used in one study of the decay scheme of ${ }^{144} \mathrm{Pm}$ [3]. Because of the larger size but smaller solid angle covered by each detector element in GAMMASPHERE and because of the BGO Compton suppressers, this provides a much higher detection efficiency and a substantially lower background environment in which to search for this $\beta^{+}$decay branch. This ${ }^{144} \mathrm{Pm}$ source was counted in GAMMASPHERE for approximately 4 days. Analysis of the data obtained in this experiment is now in progress. We estimate that we will improve our sensitivity by approximately a factor of 10 over our previous results.

Footnotes and References

*Oregon State Univ. Corvallis, OR

+ Tennessee Technological Univ., Cookeville, TN

¥TANDAR Laboratory, Buenos Aires, Argentina

1. J. Drach, M. Salamon, Astrophys. J. 319 (1987) 237.

2. M. M. Hindi et al., Phys. Rev. C 50 (1994) 728.

3. S. J. Robinson et al., Phys. Rev. C 54 (1996) 1478. 


\title{
Preliminary Results from a Study of the Double Beta Decay of ${ }^{150} \mathrm{Nd}$
}

\author{
M. C. Perillo Isaac, Y.D. Chan, D. Hurley, R. J. McDonald, \\ E. B. Norman and A. R. Smith
}

The main contribution to double beta decay (with or without neutrinos) is the ground state to ground state (GS) transition. Transitions to the excited states of the daughter isotope are also possible, if kinematically allowed. The double beta transition to $0^{+}$excited states is analogous to the ground state to ground state transitions, while a double beta decay from a $0^{+}$state to a $2^{+}$excited state is only possible through right handed currents. In the first approximation, the transition rate of the double beta decay with the emission of two neutrinos is proportional to a function of the available energy for the decay ${ }^{1}$. In this scenario the transition rates to excited states are substantially lower than the transition rate to the GS of the daughter isotope.

Here we report the first phase of an experiment where we look for the de-excitation $\gamma$-rays of ${ }^{150} \mathrm{Sm}$ populated by the double beta decay of ${ }^{150} \mathrm{Nd}$ using direct counting techniques.

The half life of the $2 \nu$ double beta transition of ${ }^{150} \mathrm{Nd}$ to the ground state of ${ }^{150} \mathrm{Sm}$ was recently measured to be $\left(1.7_{-0.5}^{+1.0} \pm 0.35\right) \times 10^{19} \mathrm{y}^{2}$ Taking into account the expected dependence on the phase space factor, one can predict a half life for the double beta transition of ${ }^{150} \mathrm{Nd}$ to the first $0^{+}$excited state of ${ }^{150} \mathrm{Sm}$ to be of the order of $10^{20} \mathrm{y}$. The de-excitation from this level gives rise to two coincident gamma-ray lines, 334 and $406 \mathrm{keV}$.

We used a $500 \mathrm{~cm}^{3}$ n-type HPGe detector to look for the $\gamma$-rays following the double beta decay of ${ }^{150} \mathrm{Nd}$ to the excited states of ${ }^{150} \mathrm{Sm}$. A sample of $5 \mathrm{~kg}$ of natural $\mathrm{Nd}_{2} \mathrm{O}_{3}$ was placed in a Marinelli beaker geometry around the de-

\footnotetext{
Footnotes and References

${ }^{1}$ See "Physics of Massive Neutrinos", F. Boehm and P. Vogel, Cambrige Unversity

${ }^{2}$ V. A. Artem et al, JETP Lett. 58, 262,(1993)
}

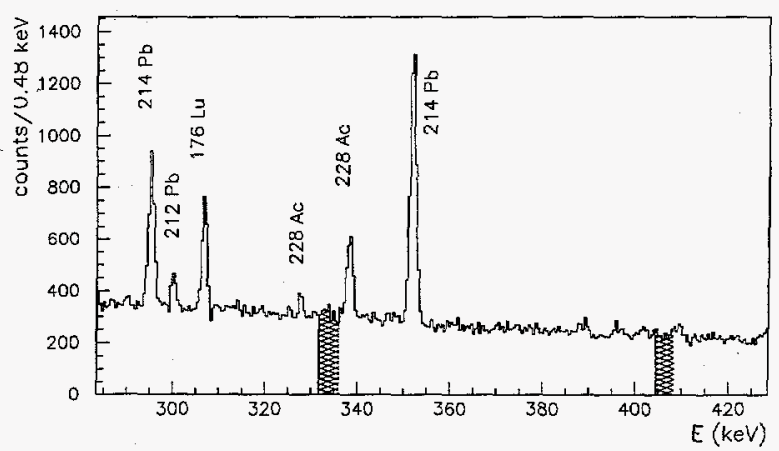

Figure 1: The energy regions of the two gamma-ray lines from the de-excitation of the first $0^{+}$state are hatched. The peaks from natural radioactive contamination are marked

tector. The passive shielding consists of $15 \mathrm{~cm}$ of lead and $2 \mathrm{~cm}$ of copper and the whole setup is located at the LBNL - Oroville low background facility ( $700 \mathrm{mwe}$ ). We accumulated data for $1268.67 \mathrm{~h}$ in this configuration.

We did not observe peaks in the energy region where the gamma-ray lines from the deexcitation of ${ }^{150} \mathrm{Sm}$ levels are expected. The lower limit for the half life can be obtained through the upper limit peak area of the gamma lines 334 and $406 \mathrm{keV}$.

Since the two gamma lines we are looking for have the same intensity ( $100 \%$ branching ratio each), one can add the two energy regions of the spectrum in order to obtain a combined upper limit peak area and hence a better limit for the half life. Our limit for the half life of the double beta decay of ${ }^{150} \mathrm{Nd}$ to the first $0^{+}$excited state of ${ }^{150} \mathrm{Sm}$ is $4.9 \times 10^{19}$ years $\left(2.9 \times 10^{19}\right.$ years $)$, with $68 \%$ (90\%) confidence level. 


\section{Does The Lifetime of ${ }^{40} \mathrm{~K}$ Depend Upon its Chemical State?}

E. B. Norman, M. C. Perillo-Isaac, M. E. Moorhead*, R.-M. Larimer, R. J. McDonald, A. R. Smith, E. Browne

The influence of the chemical state on nuclear decay rates has been observed for a number of isotopes that decay either by electron capture or by internal conversion [1,2]. This is believed to be due to the fact that the density of electrons at the nucleus, upon which such decay rates depend, varies as the chemical state of the radioactive species is altered. The electron-capture decay branch of ${ }^{40} \mathrm{~K}$ is the basis of the potassiumargon dating scheme. This decay leads to the $1461-\mathrm{keV}$ first excited state of ${ }^{40} \mathrm{Ar}$ and has a QEC value of only $44 \mathrm{keV}$. Because of its low decay energy and its first-forbidden-unique character, it is possible that the lifetime of ${ }^{40} \mathrm{~K}$ could depend on its chemical form. In fact, there have been some reports in the literature in which ages of materials derived from $\mathrm{K} / \mathrm{Ar}$ dating disagree with those obtained from $\mathrm{U} / \mathrm{Pb}$ techniques [3]. Such discrepancies in apparent ages could be due to effects of the chemical environments in which the potassium is found.

To search for influences of the chemical state on the ${ }^{40} \mathrm{~K}$ decay rate, we compared the specific gamma activities of ${ }^{40} \mathrm{~K}$ in $\mathrm{KCl}, \mathrm{K}_{2} \mathrm{SO}_{4}, \mathrm{KNbO}_{3}$, and $\mathrm{KTaO}_{3}$. This was done by combining known masses of each of these compounds with known amounts of $\mathrm{La}_{2} \mathrm{O}_{3}$ and gamma counting these mixtures. By comparing the measured yields of the $1461-\mathrm{keV}$ line from the decay of ${ }^{40} \mathrm{~K}$ to that of the $1436-\mathrm{keV}$ line from the decay of ${ }^{138} \mathrm{La}$, we were able to determine the specific activity of each potassium compound. We also compared the specific activity of $\mathrm{KCl}$ in powder form to that of $\mathrm{KCl}$ dissolved in $\mathrm{H}_{2} \mathrm{O}$. Finally, we measured the specific activity of $\mathrm{K}_{2} \mathrm{CO}_{3}$, converted it to $\mathrm{KCl}$, and then measured the specific activity again.

The results obtained in all of these studies were consistent with the $\mathrm{EC}$ decay rate of ${ }^{40} \mathrm{~K}$ being independent of the chemical state. We were able to set a limit on the fractional change in the ${ }^{40} \mathrm{~K}$ decay rate to be $\leq 1 \%$. Thus, the reason for the discrepancies in apparent $\mathrm{K} / \mathrm{Ar}$ and $\mathrm{U} / \mathrm{Pb}$ ages of materials from the Oklo natural reactor [3] must be something other than a chemical dependence of the decay rate of ${ }^{40} \mathrm{~K}$.

\section{Footnotes and References}

*Present address: Oxford University, Oxford, UK

1. G. T. Emery, Ann. Rev. Nucl. Sci. 22 (1972) 165.

2. H. P. Hahn, H. J. Born, J. I. Kim, Radiochim. Acta. 23 (1976) 23.

3. F. Gautier-Lafaye, P. Stille, R. Bros, R. Taieb, Commission of the European Communities Report EUR14877 (1993) p. 57. 


\title{
Weak Gamma Rays in the Electron-Capture Decay of ${ }^{194} \mathrm{Au}^{*}$
}

\author{
R. R. P. Teixeira*, I. D. Goldman*, M. T. F. da Cruz*, E. Browne, I. Y. Lee, A. O. Macchiavelli, E. B. \\ Norman
}

The electron capture decay of ${ }^{194} \mathrm{Au}$ has been extensively studied before, and the spectroscopic and nuclear data have been evaluated and published in Nuclear Data Sheets [1]. However, recently available large Ge detectors and highefficiency Ge detector arrays have provided the opportunity for detecting weaker gamma rays in singles and coincidence experiments, and thus, to verify and extend results from previous measurements.

We produced two sources of ${ }^{194} \mathrm{Au}$. A source for measuring gamma rays in a singles experiment was made by irradiating a foil of natural platinum with $10-\mathrm{MeV}$ protons in the cyclotron of the Instituto de Pesquisas Energeticas e Nucleares (IPEN) at the University of Sao Paulo, Brazil. Another source for gamma-gamma coincidence experiments using the (LBNL) HighEnergy-Resolution-Array (HERA) was made by bombarding a gold foil with $40-\mathrm{Mev}$ protons from the 88-Inch Cyclotron.

Our measurements produced a wealth of gamma-ray data (167 transitions between singles, coincidence, and angular correlation measurements). The complete list of gamma rays measured in this work has been published [2], and some preliminary results have already been included in the most recent data evaluation of ${ }^{194} \mathrm{Au}[3]$. We found thirty three gamma rays that had not been previously observed in the electron-capture decay of ${ }^{194} \mathrm{Au}$, and several coincidence results that have significantly affected its decay scheme. These data have confirmed levels in ${ }^{194} \mathrm{Pt}$ at $1373-$ and $1737 \mathrm{keV}$, and established new placements in the decay scheme for the 59-, 223-, 304, and 562-keV transitions. A paper describing these results have recently been submitted for publication [4].
Footnotes and References

*Permanent address: Univ. of Sao Paulo, Sao Paulo, Brasil

1. B. Singh, Nuclear Data Sheets 56, 75 (1989).

2. R.R. Plaza Teixeira, Espectroscopía Gama e Correlação Angular Gama Gama em ${ }^{194} \mathrm{Pt}$; Ph. D. Thesis, University of Sao Paulo, Brazil, 1996.

3. E. Browne and B. Singh, Nuclear Data Sheets 79, 277 (1996).

4. R. R. P. Teixeira et al., Phys. Rev. C (submitted). 


\section{Nuclear Astrophysics Data Home Page \\ J. Dairiki, R. Firestone, R. Stokstad, S. Woosley ${ }^{\dagger}$, and S.C. Wu ${ }^{\ddagger}$}

A variety of data is needed in nuclear astrophysics; charged particle, neutron, and neutrino cross sections; atomic masses; decay modes and nuclear lifetimes from the valley of stability to the nucleon drip lines; beta decay strength functions; elemental and isotopic abundances; and theoretical values for all of these quantities when experimental values are unavailable. These data are found in many different places and forms including printed publications, computer files, journals and review articles, and private databases. Some data are available through electronic means, but at a variety of locations and in widely varying formats. The disbursed nature of these data often makes it difficult for theorists to perform their calculations with the best available input data.

In the past, much of the compiled data used for nuclear astrophysics was the result of work by relatively few people, in particular Willy Fowler and his collaborators. That era has ended, and astrophysicists recognize that a concerted and coordinated community effort must be initiated for the compilation, evaluation, and stewardship of the nuclear data they both generate and use. The expertise for evaluation of data is spread over many institutions, worldwide, and it seems inevitable that the data will be published in various formats in the foreseeable future. We can, however, bring these data to a central location where they can be (if not already) compiled, and formats standardized. The data can then be made conveniently available electronically through the WWW and file transfer protocols on the Internet.

We have made a start toward this goal by developing a home page for nuclear astrophysics data that can serve as a prototype for a more complete and elaborate effort. Even as a prototype, however, this home page contains a variety of useful data, much of which has not been generally available before, or which appears on the WWW for the first time.

The data are arranged as follows:

\section{Nuclear Astrophysics Data}

1. Nuclear Data Reference List - a comprehensive bibliography for nuclear astrophysics, hypertext linked to available data.

2. Reaction Rates, Charged-Particle Induced Reactions, Neutron-Induced Reactions, EM Interactions, and Weak Interactions.

3. Nuclear Structure and Decay Data, Isotope Explorer (VuENSDF), Super Chart of the Nuclides, Table of Isotopes, Table of Radioactive Isotopes.

4. Atomic Masses, experimental and theoretical.

5. Nuclear and Particle Astrophysics Data from the Particle Data Group, Phys. Rev. D54 (1996)

6. Chemical and Isotopic Data, Elemental Abundance, Isotopic Abundance, Physical Constants, Periodic Table, and Chemical Properties.

\section{Nuclear Astrophysics Meetings}

\section{Web Sites of Interests to Nuclear Astrophysics}

We note that section 2 above contains the comprehensive data bases of Hoffman and Woosley, and of Thielemann, et al.

The Nuclear Astrophysics Data Home Page can be visited at: http:/ / isotopes.lbl.gov/ isotopes/astro.html

\section{Footnotes and References}

† U.C. Santa Cruz

¥ On sabbatical from Tsing-Hua University, Taiwan 


\section{Laser Trapping Radioactive Atoms for Precise Weak Interaction Tests \\ M.A. Rowe, S.J. Freedman, B.K. Fujikawa, G. Gwinner, S.-Q. Shang, P.A. Vetter, E.G. Wasserman}

Radioactive isotopes confined in neutral atom traps will play an important role in future precision measurements of weak interactions such as the parity violating asymmetry in the $\beta$ decay of spin-polarized nuclei, electron-neutrino correlations, and parity violation in atoms. Traps provide a localized, isotopically pure sample in which source scattering is eliminated, and neutral atoms are easily manipulated by optical pumping techniques to achieve high nuclear spin polarization.

Recent improvements to our apparatus have provided us with 50,000 trapped ${ }^{21} \mathrm{Na}$ atoms. In the past, our efforts have been directed towards increasing the size of the trapped sample from the first demonstration of the technique towards a useful level. Our advances during the last year have produced a ${ }^{21} \mathrm{Na}$ trap large enough to begin experiments with radioactive atoms. A precise determination of the ground state hyperfine splitting is now in progress. We will then begin a measurement of the $\boldsymbol{\beta}$-asymmetry parameter in the mirror decay of ${ }^{21} \mathrm{Na} \rightarrow{ }^{21} \mathrm{Ne}$ to search for deviations from the V-A structure of the charged current weak interaction, e. g. from right handed currents.

${ }^{21} \mathrm{Na}\left(\mathrm{t}_{1 / 2}=22 \mathrm{sec}\right)$ is produced on-line at the 88 " Cyclotron at LBNL with the ${ }^{24} \mathrm{Mg}(\mathrm{p}, \alpha)^{21} \mathrm{Na}$ reaction. Efficient extraction of ${ }^{21} \mathrm{Na}$ from our MgO target is crucial for achieving large traps. During 1996, we installed a new, more reliable target oven. In target development runs with this oven we determined that sintering and slow diffusion in the $\mathrm{MgO}$ powder kept the yield low from a powder target. Now we use a series of thin, free-standing, pressed $\mathrm{MgO}$ disks. Another improvement has been better collimation of the ${ }^{21} \mathrm{Na}$ atomic beam into the laser trap region. Since the vapor pressure inside our target oven is much lower than standard atomic beam sources, we can use long narrow exit channels to focus the outgoing beam. We now use channels with a length-to-diameter ratio of 25:1. These refinements provide more ${ }^{21} \mathrm{Na}$ in the forward direction, yielding more trapped atoms.

We have made a preliminary measurement of the atomic ground state hyperfine splitting of ${ }^{21} \mathrm{Na}$ using our trapped atoms (Fig. 1). We induce transitions between the two ground state hyperfine levels with microwaves and then probe the atoms with a laser. A measurement of the atomic fluorescence tells us how many atoms make the transition as a function of microwave frequency. With an improved magnetic field configuration and a new microwave antenna, a final frequency measurement will be made with a precision of 1 part in $10^{7}$. We have already achieved this accuracy with the stable isotope, ${ }^{23} \mathrm{Na}$. This microwave work can also be applied to characterize the nuclear polarization of our trapped atoms, which will be necessary to extract weak interaction parameters from our measurements on the $\beta$-decay. In our last run of 1996 at the 88" Cyclotron, we observed $\beta$ decays of our trapped sample with an in-vacuum detector. This data provides us with signal and noise levels which will guide design changes for the $\beta$ asymmetry measurement.

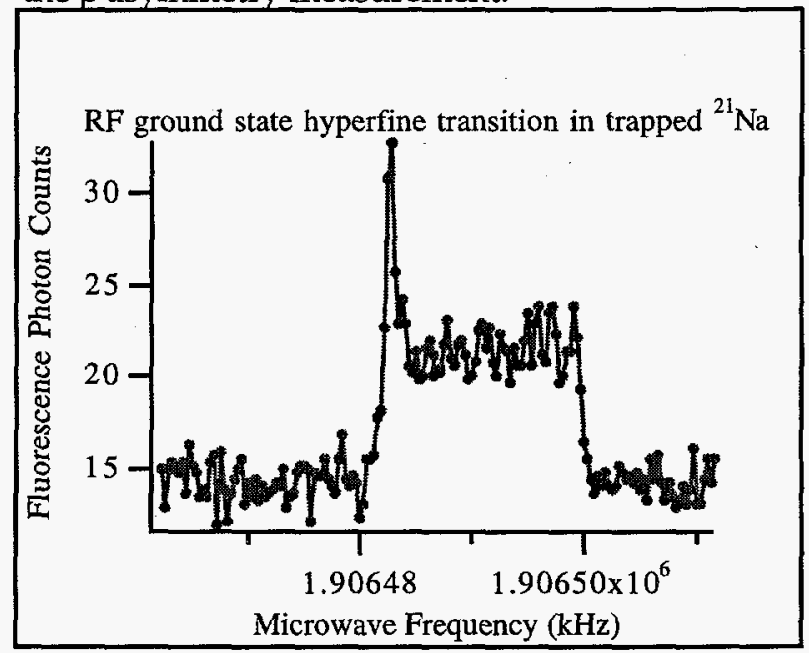

Fig. 1.Microwave absorption by trapped ${ }^{21} \mathrm{Na}$. 


\title{
Measurement of the Partial $0^{+} \rightarrow 0^{+}$Half Life of ${ }^{10} \mathrm{C}$ with GAMMASPHERE
}

\author{
B.K. Fujikawa, S.J. Asztalos, ${ }^{*+}$ C.J. Bowers, ${ }^{*}+$ R.M. Clark, ${ }^{*}$ M.-A. Deleplanque-Stephens, ${ }^{*}$ P. \\ Fallon, ${ }^{*}$ S.J. Freedman, ${ }^{*}+$ J.P. Greene, $\neq$ I. - Y. Lee, ${ }^{*}$ L.J. Lising, ${ }^{*}+$ A.O. Macchiavelli, ${ }^{*}$ R.W. MacLeod, \\ J.C. Reich, ${ }^{*}+$ M.A. Rowe, ${ }^{*}+$ S.-Q. Shang, ${ }^{*}$ F.S. Stephens, ${ }^{*}$ and E.G. Wasserman $\left.{ }^{*}\right\}$
}

The $\mathrm{u}$-d element $\left(\mathrm{V}_{\mathrm{ud}}\right)$ of the CabibboKobayashi-Maskawa (CKM) quark mixing matrix is a fundamental parameter of the Standard Model of Electroweak Interactions. Its most precise determination comes from nuclear physics experiments, in particular, from measurements of superallowed Fermi beta decays. These measurements, requiring both precision nuclear physics experiments and state of the art theoretical nuclear physics calculations, have been made for a variety of nuclei ranging from ${ }^{14} \mathrm{O}$ to ${ }^{54} \mathrm{Co}$. The $\mathrm{u}$-d element obtained from these measurements are in statistical agreement and the average value obtained implies a non-unitary CKM matrix, which if correct, would require exotic extensions to the Standard Model. Unfortunately the theoretical calculations of the isospin breaking corrections, necessary for extracting Vud, are controversial. For example, Wilkinson ${ }^{* *}$ has suggested that these calculations are incomplete and that the isospin breaking corrections must be extracted empirically. In order to resolve this controversy, much effort has recently been invested in measuring $\mathrm{V}_{\mathrm{ud}}$ from the superallowed Fermi decay of ${ }^{10} \mathrm{C}$, where the isospin breaking corrections are expected to be small and any residual charge dependent corrections will be apparent. This is a very challenging measurement, since the beta decay of ${ }^{10} \mathrm{C}$ has a small superallowed Fermi branching ratio which has to be precisely determined in a high background environment. We are currently engaged in series of experiments to measure the superallowed Fermi branching ratio of ${ }^{10} \mathrm{C}$ beta decay using the GAMMASPHERE facility at the Lawrence Berkeley National Laboratory 88-inch cyclotron. Analysis of the first data run is complete and the preparation of publication is in progress. The results are summarized in Fig. 1.

\section{Footnotes and References}

*Lawrence Berkeley National Laboratory

tUniversity of California, Berkeley

$\ddagger$ Argonne National Laboratory

§Present address: Abacus Concepts Incorporated

${ }^{*}$ D.H. Wilkinson, Zeit. Phys. A348, 129, (1994).

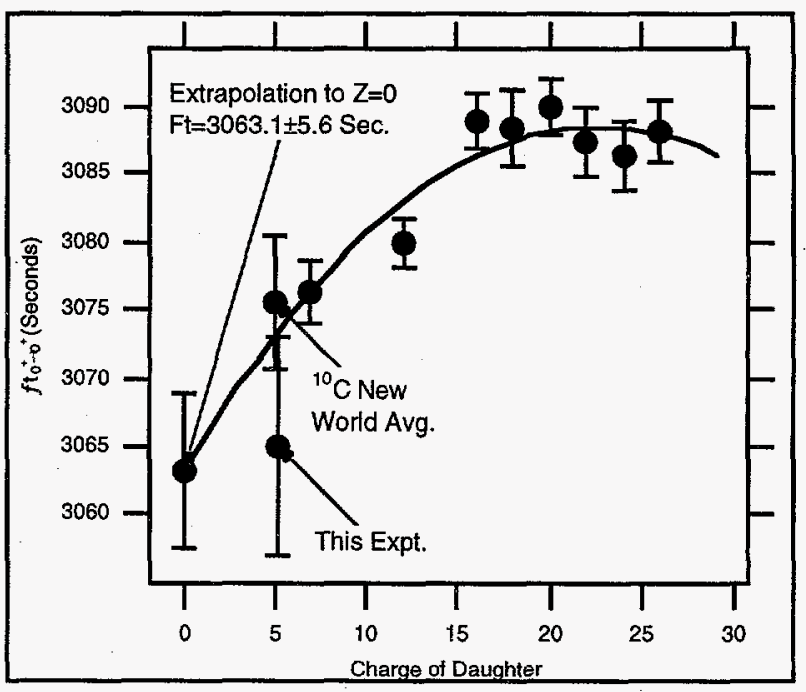

Fig. 1. The radiatively-corrected $f t$ values for superallowed Fermi beta decays plotted against the charge of the daughter nucleus. The solid curve is a fit to the data assuming a hypothetical quadratic $\mathrm{Z}$ dependent isospin breaking correction proposed by Wilkinson.** Recent results from this experiment on ${ }^{10} \mathrm{C}$, taken alone and with previous experiments, tend to support such a dependence. 


\section{Test of the conserved vector current hypothesis in the beta-decay of ${ }^{14} \mathrm{C}$}

J. L. Mortara*t, I. Ahmad**, S. J. Freedmantł, B. K. Fujikawaf, J. P. Greene**, J. P. Schiffer**, A. R. Zeuli**

In 1957, Feynman and Gell-Mann proposed the conserved vector current (CVC) hypothesis. ${ }^{1}$ In the classic case of the mass-12 isospin triplet (B$\mathrm{C}-\mathrm{N}), \quad \mathrm{CVC}$ relates the width of the electromagnetic transition $\left({ }^{12} \mathrm{C}^{*} \rightarrow{ }^{12} \mathrm{C}\right)$ to the shape factors of the beta decay spectra $\left({ }^{12} \mathrm{~B} \rightarrow\right.$ $\left.{ }^{12} \mathrm{C}\right)$ and $\left({ }^{12} \mathrm{~N} \rightarrow{ }^{12} \mathrm{C}\right)$. The shape factor represents a deviation of the beta spectrum from the simple allowed shape by the additional factor $S(E)=1$ $+a E$, where $E$ is the total electron energy Several experiments have measured $a$ in the mass- 12 system, but the agreement with CVC is weak at best. ${ }^{2}$

The mass-14 isospin triplet ( $\mathrm{C}-\mathrm{N}-\mathrm{O})$ represents another viable system for testing CVC (Fig. 1).

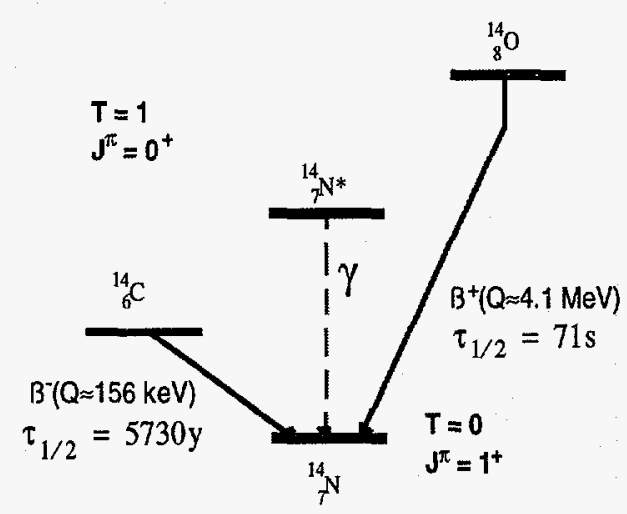

Fig. 1. Mass-14 isospin triplet.

We have performed a measurement of the beta decay spectrum of ${ }^{14} \mathrm{C} \rightarrow{ }^{14} \mathrm{~N}(Q \approx 156 \mathrm{keV})$ toward this end. The apparatus used for this measurement consists of a superconducting solenoid and $\mathrm{Si}(\mathrm{Li})$ solid state detector. ${ }^{3}$ The magnetic field transports the electrons in helical orbits $(r<3 \mathrm{~mm})$ to the $\mathrm{Si}(\mathrm{Li})$ detector without the possibility of scattering on material collimators. The detector response function was determined by measurement of internal conversion spectra from ${ }^{139} \mathrm{Ce}$ and ${ }^{109} \mathrm{Cd}$. The ${ }^{14} \mathrm{C}$ data consists of four separate runs with a total of about $7 \times 10^{9}$ total decays accumulated over a period of 515 livetime hours. The data is fitted from 65 to $250 \mathrm{keV}$. The results are quoted for this energy interval, but the shape factor was observed to be independent of the chosen interval. In Fig. 2 we show the fit from nun 1 which yielded $a=-39.1 \pm 0.4$ (stat.) $\% / \mathrm{MeV}$ for the shape factor. Including the results from the other three data

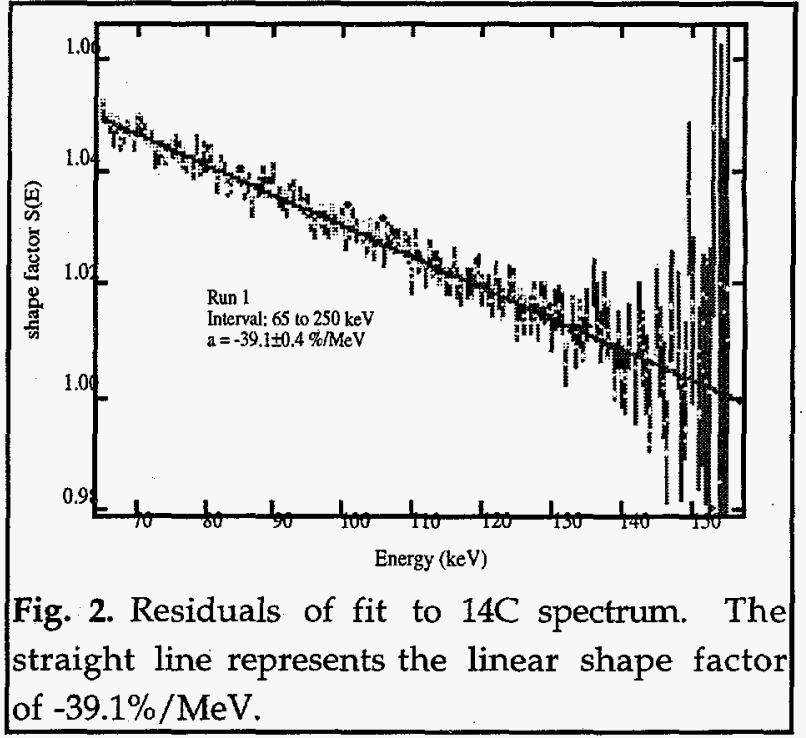

sets and we have $a=-39.2 \pm 0.2$ (stat.) \pm 0.6 (sys.) $\% / \mathrm{MeV}$. This represents the most precise determination of the shape factor in ${ }^{14} \mathrm{C}$ and appears to be in good agreement with the value predicted by CVC of $-38.0 \pm 1.2 \% / \mathrm{MeV}^{2}$ where the error reflects the uncertianty in the radiative width of ${ }^{14} \mathrm{C} \rightarrow{ }^{14} \mathrm{~N}^{*}$.

\section{Footnotes and References}

*P-23 Neutron Science and Technology, Los Alamos National Laboratory

+ Department of Physics, University of California at Berkeley

$\ddagger$ Nuclear Science Division, Lawrence Berkeley National Laboratory

**Physics Division, Argonne National Laboratory

1. Feynman and Gell-Mann, Phys. Rev. 109, 193 (1957).

2. Calaprice and Holstein, Nucl. Phys. A273, 301 (1976).

3. J.L. Mortara et. al., Phys. Rev. Lett. 70, 394 (1993). 


\title{
Test of the conserved vector current hypothesis in the beta-decay of ${ }^{14} \mathrm{O}$
}

\author{
S. J. Freedmantł, B. K. Fujikawał, J. L. Mortarat§, D. W. Rossł, D. Wutte
}

In 1957, Feynman and Gell-Mann proposed the conserved vector current (CVC) hypothesis. ${ }^{1}$ In the classic case of the mass-12 isospin triplet (B$\mathrm{C}-\mathrm{N}), \quad \mathrm{CVC}$ relates the width of the electromagnetic transition $\left({ }^{12} \mathrm{C}^{*} \rightarrow{ }^{12} \mathrm{C}\right)$ to the shape factors of the beta decay spectra $\left({ }^{12} \mathrm{~B} \rightarrow\right.$ $\left.{ }^{12} \mathrm{C}\right)$ and $\left({ }^{12} \mathrm{~N} \rightarrow{ }^{12} \mathrm{C}\right)$. The shape factor represents a deviation of the beta spectrum from the simple allowed shape by the additional factor $S(E)=1$ $+a E$, where $E$ is the total electron energy Several experiments have measured $a$ in the mass- 12 system, but the agreement with $\mathrm{CVC}$ is weak at best. ${ }^{2}$

The mass-14 isospin triplet (C-N-O) represents another viable system for testing CVC (Fig. 1).

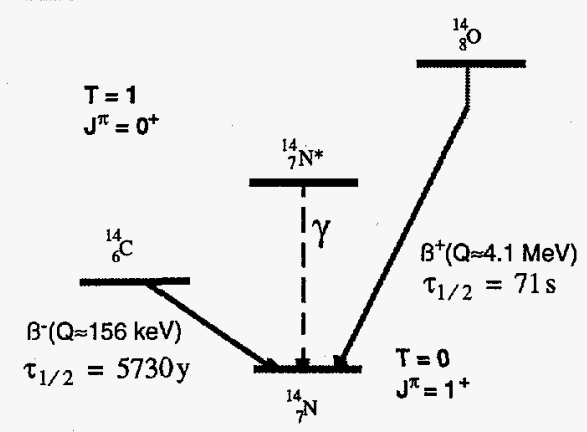

Fig. 1. Mass-14 isospin triplet.

We are preparing to measure the ground state to ground state decay of ${ }^{14} \mathrm{O}$. Since the half-life of this decay is only slightly more than a minute, the activity must be produced on-line at the $88^{\prime \prime}$ cyclotron. In order to avoid spectral distortion associated with thick sources, we have decided to pursue a new approach. Using a cusp source devloped by the AFRD at Lawrence Berkeley National Laboratory, we will produce a radioactive beam of ${ }^{14} \mathrm{O}$. A cross-sectional view of the cups source is shown in Fig. 2. The ${ }^{14} \mathrm{O}$ will be produced by the reeaction ${ }^{12} \mathrm{C}\left({ }^{3} \mathrm{He}, \mathrm{n}\right){ }^{14} \mathrm{O}$ in a heated graphite target. The evolving $\mathrm{C}_{-}{ }^{14} \mathrm{O}$ will be transported to the cusp source where a it will be ionized to produce a beam of ${ }^{14} \mathrm{O}$ to impant in a thin carbon foil.

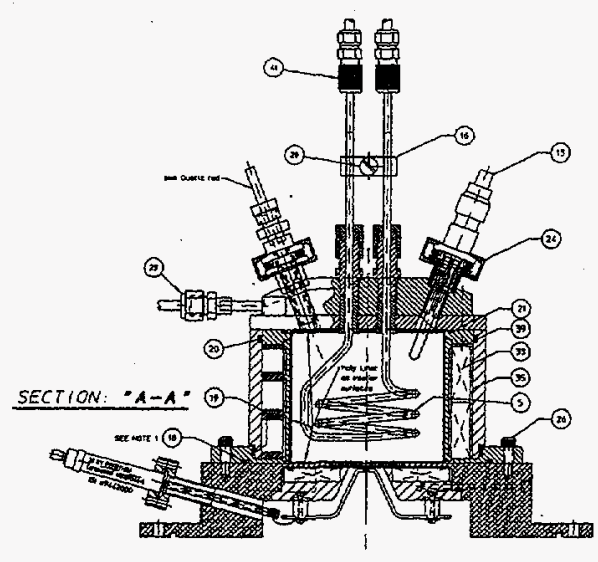

Fig. 2. Cross-sectional view of cusp source.

To measure the beta-spectrum we have a $180^{\circ}$ spectrometer with an approximately $50 \%$ momentum bite. We will detect the positrons with a multi-wire proportional chamber. We expect prelimanry tests of the entire system by late 1997. In addition to measuring the ground state to ground state decay, we also plan to measure the superallowed $0+$ to $0+$ decay. This branching ratio has been measured only once ${ }^{3}$ and is directly related to the value of $V_{u d}$ in the CKM matrix.

\section{Footnotes and References}

† Department of Physics, University of California at Berkeley

$\ddagger$ Nuclear Science Division, Lawrence Berkeley National Laboratory

$\S$ P-23 Neutron Science and Technology, Los Alamos National Laboratory

**Accelerator and Fusion Research Division, Lawrence Berkeley National Laboratory

1. Feynman and Gell-Mann, Phys. Rev. 109, 193 (1957).

2. Calaprice and Holstein, Nucl. Phys. A273, 301 (1976).

3. G.S. Sidhu and J.B. Gerhart, Phys. Rev. 148, 1024 (1966). 


\section{$\beta-\gamma$ Directional Correlation in ${ }^{22} \mathrm{Na}$ \\ C.J. Bowers, S.J. Freedman, B.K. Fujikawa, J.C. Reich, S.-Q. Shang, P.A. Vetter, E.G. Wasserman, and the Gammasphere Collaboration}

\section{Motivation}

An experiment by the Weak Interactions Group in collaboration with Gammasphere to measure nuclear form factors in the $\beta+$ decay of ${ }^{22} \mathrm{Na}$ made good progress in 1996. It has been known for roughly 40 years that for ${ }^{22} \mathrm{Na}$, the usual allowed approximation for $\beta+$ decay does not yield a good estimate of the $\log \mathrm{ft}$ value, suggesting that higher order corrections from nuclear recoil play a significant role. These higher order terms offer an opportunity to test Standard Model predictions and to search for socalled second class currents. This experiment should also clear up a $2.5 \sigma$ discrepancy between two past measurements of the $\beta-\gamma$ correlation.

Experiment

An effort at the $88^{\prime \prime}$ Cyclotron is proceeding to improve the measurement of the directional correlation between the emitted $\beta$ and the subsequent $1.274 \mathrm{MeV} \gamma$ from the $2^{+}$excited state of the ${ }^{22} \mathrm{Ne}$ daughter. The Gammasphere at the 88" Cyclotron is an ideal instrument. Symbiotically, the required precision (about $10^{-4}$ ) of this simple experiment constitutes a stringent test of the Gammasphere array, while using what would otherwise be down time for the array. Our experiment is performed in an offline configuration, using a $10 \mu \mathrm{Ci}{ }^{22} \mathrm{Na}$ source located at the center of the detector array. A plastic scintillator $\beta$ detector is inserted in the array for $8 \%$ detector solid angle. Events when a Ge detector in Gammasphere fires in coincidence with a $\beta$ observation are recorded, and we seek a correlation between the $\beta$ and $\gamma$ directions of the form $\left(1+A \cos ^{2} \theta\right)$ where $\theta$ is the angle between the $\beta$ detector and the coincident $G e$ detector, and $A$ is the directional correlation amplitude. In principle, we could count the $\gamma$ hits in each detector and correlate the Ge detector angular direction to the $\beta$ detector. However, we address the problem of varying Ge detector efficiency by also counting events when only a $\gamma$ is detected independently of a $\beta$. The number of $\gamma$ hits in each detector is proportional to the efficiency, so we take a ratio of coincident $\beta-\gamma$ events to single $\gamma$ counts to cancel the efficiencies. This ratio as a function of $\theta$ yields the directional correlation.

We took data during the winter holiday shutdowns at the 88" Cyclotron in January of 1996 and in January 1997 with the goal of a statistical uncertainty of $5 \times 10^{-4}$ in the correlation coefficient A. The earlier run revealed an unexpected systematic difference in count ratios for the two hemispheres of the array at roughly $1.2 \%$, probably due to staggered detector timing delays. This led to an improvement in the cable routing of the array, which has reduced the effect in our raw data to roughly $0.4 \%$. Software timing cuts on the coincident $1.274 \mathrm{MeV} \gamma$ seem to eliminate this effect to less than $0.1 \%$, and our current analysis in progress seems set to achieve the desired precision (Fig. 1).

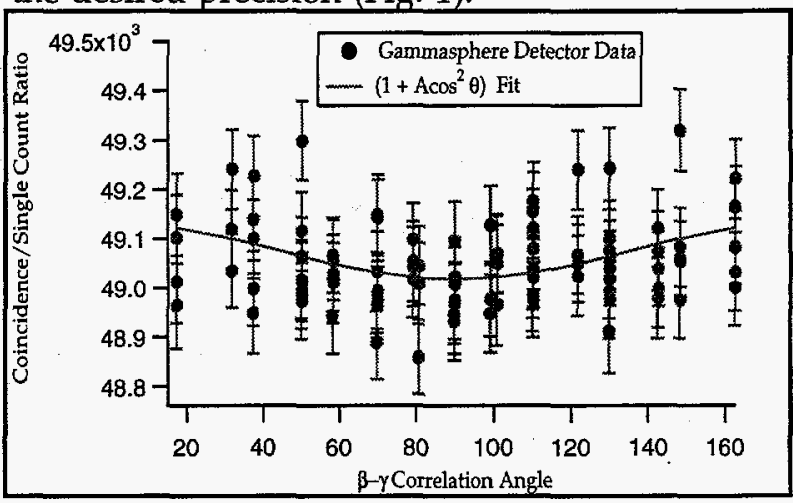

Fig. 1. Jan. 1997 Data, Early Analysis

Recent achievements summarized:

- First $\beta-\gamma$ correlation measured with Gammasphere: Nov. 1995, Jan. 1996.

- Cabling upgrade on Gammasphere to improve pretrigger timing staggering

- Jürgen Reich completed Diplomarbeit in summer, 1996 for TU München.

- New 1997 data should complete directional correlation measurement 


\section{Search for the beta-decay of ${ }^{54} \mathrm{Mn}$}

\section{Ahmad**, S. Austin§, S. J. Freedmant‡, K. T. Leskoł, J. L. Mortara*t, A. Wuosmaa**}

The decay of ${ }^{54} \mathrm{Mn}$ is well known to proceed by electron capture with a half-life of 312 days (Fig. 1). Although it is energetically possible for ${ }^{54} \mathrm{Mn}$ to decay to the ground state of ${ }^{54} \mathrm{Fe}$ by $\beta$ decay or to the ground state of ${ }^{54} \mathrm{Cr}$ by $\beta+$, only upper limits have been set for these second forbidden decay modes. ${ }^{1}$ The current limit on these branches is $\approx 10^{-6}$ for the $\beta$ - decay and $\approx 10^{-8}$ for the for the $\beta+$ decay. The difference in the branching fractions arises from the greater available phase space for the $\beta$ - decay. These hindered decay modes are the primary means by which a fully ionized ${ }^{54} \mathrm{Mn}$ nucleus would decay. Such fully stripped ions are found in cosmic rays and abundances of ${ }^{54} \mathrm{Mn}$ have been reported. ${ }^{2}$ These relative abundances, combined with measured partial half-lives, provide a cosmic ray chronometer by which one can infer the cosmic-ray confinement time.

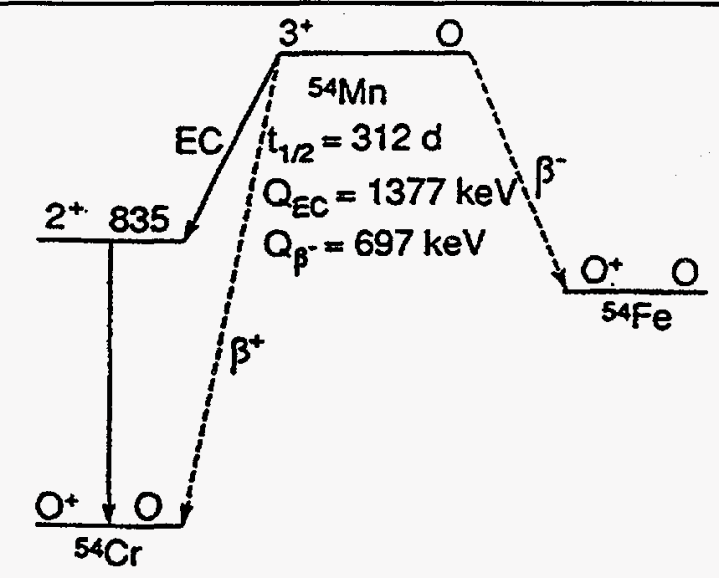

Fig. 1. The decay scheme of ${ }^{54} \mathrm{Mn}$.

Previous efforts ${ }^{1}$ have used the characteristic annihilation radiation from positrons as a signature for the $\beta+$ decay. We intend to use annihilation radiation as a trigger with an apparatus designed specifically for such detection. The APEX spectrometer ${ }^{3}$ was designed to detect the annihilation radiation of positrons created following heavy ion collisions. APEX is a 3 meter long solenoid which will be used to transport positrons to a charge particle detector surrounded by a segmented annihilation radiation detector. The method has high efficiency and allows us to use an extremely intense radioactive source without suffer from the potentially large $835 \mathrm{keV}$ gamma-ray background.

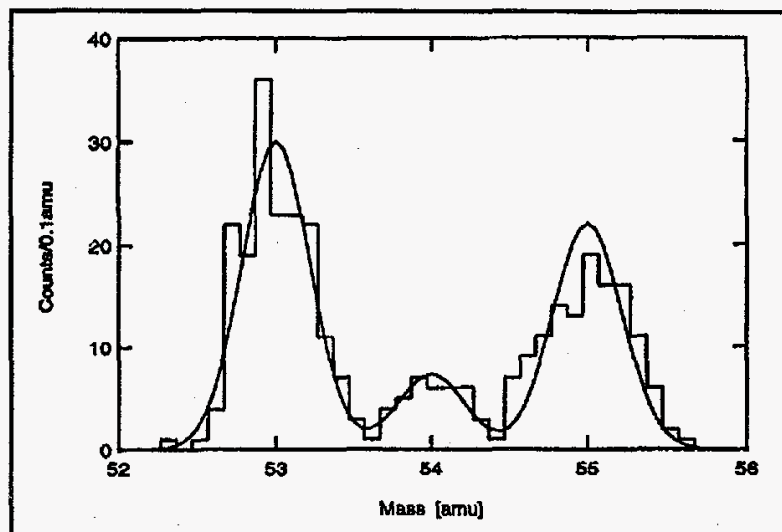

Fig. 1. Recent measurement of $\mathrm{Mn}$ abundances ${ }^{4}$

\section{Footnotes and References}

*P-23 Neutron Science and Technology, Los Alamos National Laboratory

tDepartment of Physics, University of California at Berkeley

$\ddagger$ Nuclear Science Division, Lawrence Berkeley National Laboratory

** Physics Division, Argonne National Laboratory $\S$ Michigan State University

1. M. T. F. da Cruz et. al, Physical Review C, 48, 3110 (1993).

2. R. A. Leske, Astrophys. J. 405, 567 (1993).

3. I. Ahmad et. al., Nuclear Instruments and Methods A 370, 539 (1996).

4. M. DuVernois, private communication. 


\title{
Time Reversal Invariance Violation in Polarized Neutron Beta Decay
}

\author{
L.J. Lising, S.J. Freedman, B.K. Fujikawa, E.G. Wasserman,* and the emiT Collaborationt
}

The existence of $\mathrm{CP}$-symmetry violation in the weak interaction is established in kaon decay, but the implied consequence, time-reversalsymmetry violation, is yet to be observed. The emiT collaboration is mounting an experiment to search for time-reversal-symmetry violation in free neutron beta decay, using cold, polarized neutrons at the National Institute of Standards and Technology's Cold Neutron Research Facility. This experiment, which was put online at the reactor this December, utilizes an octagonal array of detectors to observe neutron decay electron and recoil proton in coincidence. The experiment is designed to detect an angular correlation of the form,

$$
\hat{\sigma}_{n} \cdot\left(\boldsymbol{p}_{e} \times \boldsymbol{p}_{v}\right),
$$

which is odd under time reversal. The coefficient of this term in the neutron decay correlation has been previously shown to be less than 0.0011. The present experiment aims at a factor of three improvement in sensitivity from the present run.

The neutrons in the cold ( $T=40$ Kelvin) beam are polarized to $96 \%$ with a supermirror polarizer. Electrons are detected with four $50 \mathrm{~cm}$ long plastic scintillators. The recoil protons, whose maximum energy is only $750 \mathrm{eV}$, drift in a field free region until they near one of the four proton arrays, where they are accelerated through 30 kilovolts onto thin-window PIN diodes. The characteristic delay time between the decay proton and electron is used to distinguish signal from background. The proton drift time is greater than $0.5 \mu \mathrm{s}$ and most backgrounds are prompt. Figure 1 shows time versus detected proton energy, illustrating the separation of the proton signal from the large prompt background. Anticipated sources of systematic uncertainty were reduced in the detector design and extensive cross checks and tests of the apparatus are planned.
The experiment is scheduled to run during three six week reactor cycles this year. Set up and shakedown of the experiment was completed during the first cycle. Production data taking is scheduled to begin in February during the second reactor cycle.

\section{Footnotes and References}

*Present address: Abacus Concepts Incorporated TThe emiT collaboration institutions are: Lawrence Berkeley National Laboratory, University of California at Berkeley, University of Michigan, National Institute of Standards and Technology, Notre Dame University, Los Alamos National Laboratory, and University of Washington.

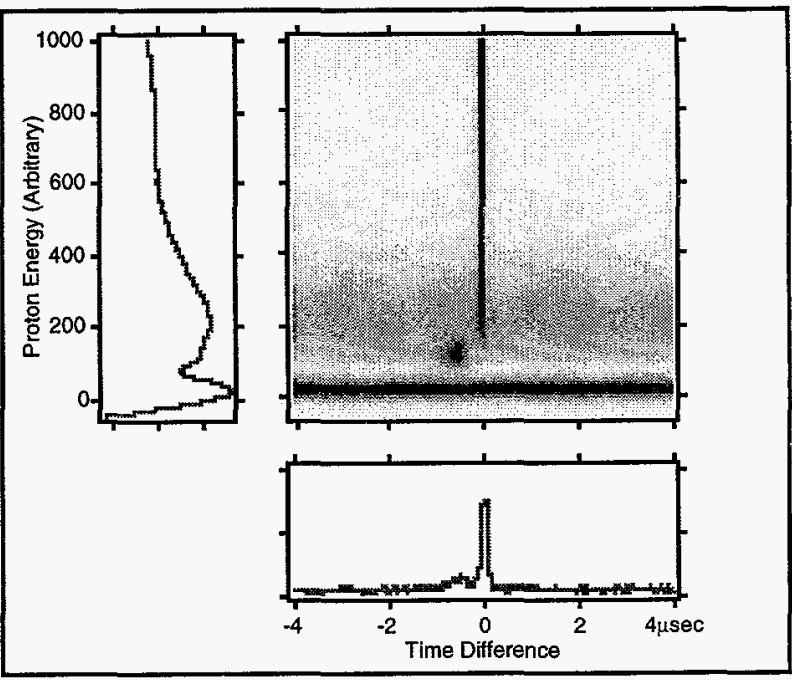

Fig. 1. A plot of proton detector energy versus coincidence time shows clearly the large prompt events, mostly from background gamma rays. Delayed proton coincidences show up to the left of the prompt events in this figure. The enhancement at 0.5 microseconds delay is from neutron decay. 


\title{
Test of time reversal invariance violation in the beta-decay of ${ }^{56} \mathrm{Co}$
}

\author{
J. L. Mortara*t, J. C. Davist, S. J. Freedmant $\neq$
}

Since the detection of $C P$ violation in the neutral kaons, ${ }^{1}$ there have been many attempts to detect the implied $T$ violation in low energy phenomena. To date these searches have yielded no positive results, and for tests involving the beta-decay of the neutron the limits on $T$ violation are quite small and are approaching limits of $10^{-4} .2$ Of course, without a good idea of the mechanism for $T$ violation, present results do not rule out the existence of large effects in other systems.

The present work on ${ }^{56} \mathrm{Co}$ is motivated by the idea that $T$ violation might appear through a mechamism that experiments involving allowed nuclear beta-decay are not sensitive to. One possibility is the existence of $T$ violating second class currents. For the allowed decays of the neutron and ${ }^{19} \mathrm{Ne}$, the dominant terms must be first class and hence any second class term would be kinematically surpressed.

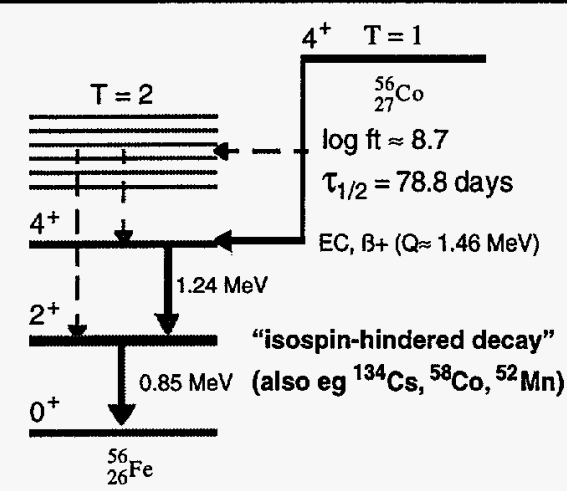

Fig. 1. The decay scheme of ${ }^{56} \mathrm{Co}$.

In the decay of ${ }^{56} \mathrm{Co}$ this is not the case. Since it involves the decay between two different isospin multiplets (Fig. 1), the dominant terms need not necessarily be first class. The current limit on $\mathrm{T}$ violation in ${ }^{56} \mathrm{Co}$ is at the level of $10^{-2}$. We intend to improve this by at least an order of magnitude.

The relevant correlation for the detection of TRIV is of the form $E 1(J \cdot k)(J \bullet p \times k)$, where $J$ is the nuclear spin, $k$ is the gamma-ray momentum and $p$ is the beta-momentum. The required alignment will be achieved by low temperature nuclear orientation with an Oxford Kelvinox 400 that has been assembled and reaches temperatures of $5 \mathrm{mK}$. Unlike the previos effort, ${ }^{3}$ this dilution refrigerator will be capable of maintaining a stable temperature for weeks in a single run. The gamma-rays from the excited state of ${ }^{56} \mathrm{Fe}$ will be detected by conventional $\mathrm{NaI}(\mathrm{Tl})$ detectors. Besides stable refrigeration, the experiment has been improved by the development of a $\mathrm{Si}$ detector that operates at $\mathrm{mK}$ temperatures. ${ }^{4}$ This allows the detector to be placed very near the ${ }^{56} \mathrm{Co}$ source. A spectrum from ${ }^{207} \mathrm{Bi}$ is shown in Fig. 2. Results will be obtained by late 1997.

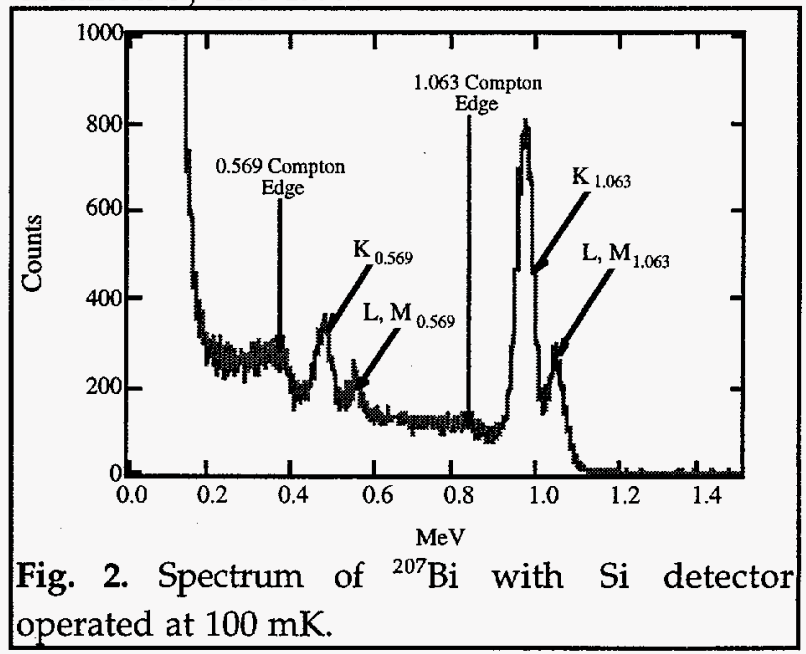

Footnotes and References

${ }^{*}$ P-23 Neutron Science and Techonolgy, Los Alamos National Laboratory

tDepartment of Physics, University of California at Berkeley

$\ddagger$ Nuclear Science Division, Lawrence Berkeley National Laboratory

1. Christenson et. al., Phys. Rev. Lett. 13, 138 (1964).

2. E. G. Wasserman, thesis LA-12735-T.

3. Calaprice et. al., Phys. Rev. C 15, 381 (1977).

4. We gratefully acknowledge the support of B. Young for the fabrication of this device. 


\title{
Atomic Parity Nonconservation in Stable $\mathbf{Y b}$ Isotopes
}

\author{
C.J. Bowers, D. Budker, E.D. Commins, D. DeMille, S.J. Freedman, \\ G. Gwinner, S.-Q. Shang, and J.E. Stalnaker
}

The weak interaction, best known for its role in nuclear beta decay, also plays a minor role in the electronic structure of atoms. Atomic structure is dominated by the electromagnetic interaction, which conserves parity (mirror reflection symmetry), whereas the weak interaction does not. Measurement of parity nonconserving (PNC) effects in atoms allows one to observe the weak interaction in a system dominated by the electromagnetic interaction. The study of atomic PNC, which is due to the weak neutral current (Z-exchange), complements the study of nuclear beta decay, which results from the weak charged current (W-exchange).

The $\left(6 s^{2}\right)^{1} \mathrm{~S}_{0} \rightarrow(6 \mathrm{~s} 5 \mathrm{~d})^{3} \mathrm{D}_{1}$ transition in atomic $\mathrm{Yb}$ is a promising system for the study of $\mathrm{PNC}^{1}$. The E1 transition amplitude is strictly forbidden by the parity selection rule, while the M1 amplitude is highly suppressed.The application of an external electric field mixes even and odd parity states, giving rise to a Stark-induced amplitude $\left(E 1_{\text {st }}\right)$. The weak interaction also mixes even and odd parity states, giving rise to a parity nonconserving amplitude (E1PNC). In order to measure a very small E1PNC, one observes the interference between the much larger $E 1_{\text {st }}$ and $E 1_{P N C}$ as one excites this forbidden transition with intense laser light. The parity violating effect in $\mathrm{Yb}$ is expected to be very large, due to the near degeneracy of two states of nominal opposite parity .

Precise measurements of PNC in single isotopes of $\mathrm{Cs}^{2}$ and $\mathrm{Tl}^{3}$, when combined with atomic structure calculations, have led to a determination of the weak mixing angle $\left(\sin ^{2} \theta_{w}\right)$, rivaling those obtained from high energy physics experiments. Measurement of $\mathrm{PNC}$ in a single isotope of $\mathrm{Yb}$ will not be competitive with $\mathrm{Cs}$ or $\mathrm{Tl}$, because the uncertainty in the calculation of the atomic structure of $\mathrm{Yb}$ is large. However, $\mathrm{Yb}$ has seven stable isotopes. The dominant contribution to atomic PNC comes from the electron-nucleon interaction, and one expects the effect to be roughly proportional to the neutron number, $\mathrm{N}$. Comparing PNC effects in several isotopes of $\mathrm{Yb}$ may allow us to extract information about the weak interaction independent of the atomic structure. The PNC effect for a given isotope also depends on the distribution of neutrons within the nucleus, a nuclear property not readily accessible by other means. In addition, comparison of PNC effects in the different hyperfine components of the two odd $\mathrm{A}$ isotopes of $\mathrm{Yb}$ allows a determination of the nuclear anapole moment.

The past year has seen the publication of $\mathrm{Yb}$ lifetime measurements ${ }^{4}$ and the first observation of the forbidden transition used in the study of $\mathrm{PNC}$ in $\mathrm{Yb}$, as shown in Fig. 1.

Footnotes and References

1. D. DeMille, Phys. Rev. Lett. 74, 4165(1995).

2. M.S. Noecker, B.P. Masterson, and C.E. Wieman, Phys. Rev. Lett. 61, 310 (1988).

3. P.A. Vetter et al.,Phys. Rev. Lett. 74, 2658 (1995).

4. C. J. Bowers et al. , PRA 53, 3103(1996).

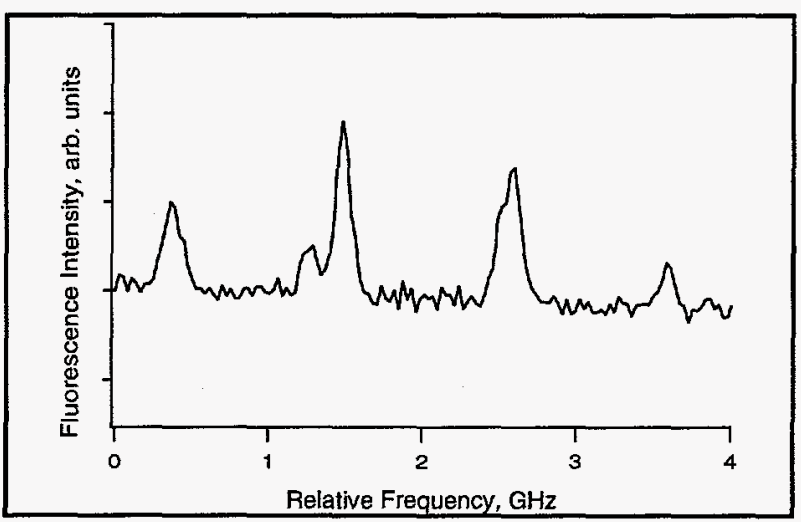

Fig. 1. Fluorescence signal from the forbidden M1 transition in ytterbium in a $45 \mathrm{kV} / \mathrm{cm}$ electric field. The peaks correspond to individual isotopes and hyperfine components. 


\title{
Experimental Study of the ${ }^{8} B$ Neutrino Spectrum
}

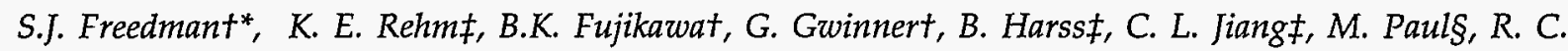 \\ Pardo $\neq$, J. P. Schiffer $\neq$, S. Q. Shangt, and P. Vettert
}

The principal source of high energy neutrinos form the Sun is the beta decay of ${ }^{8} \mathrm{~B}$ produced by nucleosynthesis deep in the solar interior. The exact shape of the emitted neutrinos from ${ }^{8} \mathrm{~B}$ is of current interest because of plans to measure the solar-neutrino energy spectrum directly in the $\mathrm{SNO}$ and SuperK detectors. Distortions from the primitive spectrum may provide evidence of "matter enhanced neutrino oscillations" in the solar interior.

The beta decay of ${ }^{8} \mathrm{~B}$ is allowed but both the beta and neutrino energy spectrum deviate significantly from an ordinary allowed decay. The decay proceeds primarily to the umbound first excited state of ${ }^{8} \mathrm{Be}$ at $2.9 \mathrm{MeV}$ which decays to two alpha particles. The distortion is due to the broad and complicated energy profile of the final state.

In the past the neutrino spectrum has been calculated from measurements of the delayed alpha spectrum and in one case from constraints imposed from measurements of the beta spectrum $^{1}$. Recently, Bahcall et. $\mathrm{al}^{2}$ reviewed all the available experimental data attempting to predict the $8 \mathrm{~B}$ neutrino spectrum and establish the uncertainty with which it is know. It is pointed out in that work that the five existing measurements of the alpha spectrum are in poor agreement. While the basic shape of the spectra are similar there are discrepancies of about 80 $\mathrm{keV}$ in the energy scales, making it difficult to asses the overall systematic uncertainty.

We proposed to measure the alpha spectrum directly using the novel technique of implanting $8 \mathrm{~B}$ in a silicon detector. In previous experiments $8 \mathrm{~B}$ was implanted in thin metallic foils. We suspect that the discrepancies in the energy scales of the various experiments are due to the difficulties of correcting for energy losses of the alpha particles and uncertainties in the distribution of the $8 \mathrm{~B}$ in the foil. Our technique will avoid these uncertainties. The experiment makes use of the Enge Spectrograph at ATLAS and a new technique of producing secondary beams with a gas cell target. In this case we use the ${ }^{6} \mathrm{Li}\left({ }^{3} \mathrm{He}, \mathrm{n}\right)^{8} \mathrm{~B}$ reaction. The experimental plan was developed this year and the proposal was recently approved by the ATLAS PAC to run this Spring at Argonne.

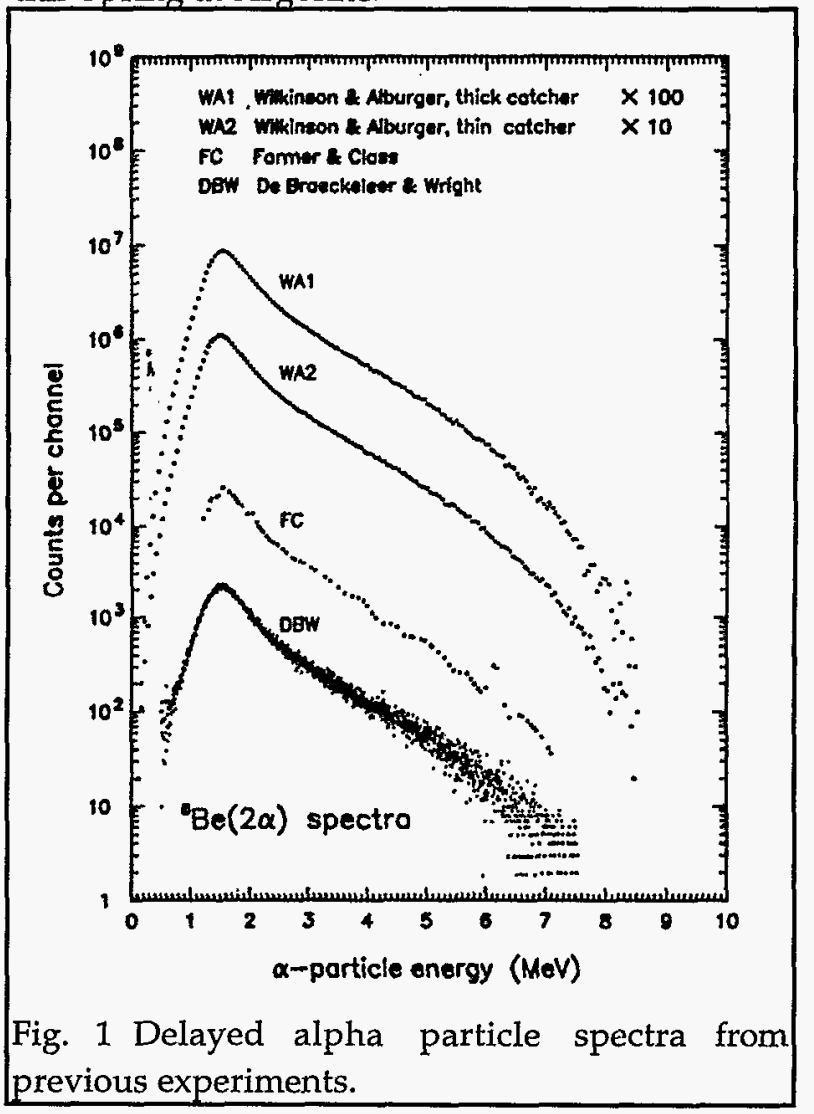

\section{Footnotes and References}

tLawrence Berkeley National Laboratory

*University of California at Berkeley

$\ddagger$ Argonne National Laboratory

§ Hebrew University, Jerusalem

1. J. Napolitano, S. J. Freedman, and J. Camp, Phys. Rev. C36, 298 (1987)

2. J. N. Bahcall, et al, Phys. Rev. C58, 411, (1996). 


\section{Search for Narrow Positron Emission Lines in Heavy Ion Collisions with APEX}

Stuart Freedman, Michael Maier, as part of the Apex collaboration

\section{Motivation}

The apparent discovery of narrow positron emission lines in two separate experiments at the GSI, Darmshtad has been an object of intense investigation for nearly two decades. The signal reported in both singles and in other experiments with electrons detected in coincidence were taken as potential evidence for the decay of a new light neutral particle or other exotic phenomena. The APEX collaboration built a sensitve spectrometer at the ATLAS facility at Argonne to help discover the explanation for the surprising effect. ${ }^{1}$

\section{Experiment}

During experimental runs in 1995 we searched for the previously reported effect in the reaction ${ }^{238} \mathrm{U}+{ }^{232} \mathrm{Th}$ at $5.95 \mathrm{MeV} / \mathrm{u}$ and the reaction ${ }^{238} \mathrm{U}+$ ${ }^{181} \mathrm{Ta}$ at $5.95,6.10$ and $6.60 \mathrm{MeV} / \mathrm{u}$. We searched the data for evidence of narrow structures in the positron singles spectrum and the positronelectron coincidence energy spectrum. Using analysis techniques motivated by the previous experiments we failed to find any effects consistent with the previous positive reports. ${ }^{2}$ Figure 1 shows the electron-positron sum energy spectrum from the ${ }^{238} \mathrm{U}+{ }^{232} \mathrm{Th}$ reaction with APEX and the resulting limits. Since the publication of this work, the EPOS experiment at GSI has reported that they have been unable to reproduce their previous findings with a more sensitive apparatus.

There are no plans to continue these searches at the present time. Other uses for the APEX spectrometer are under consideration.

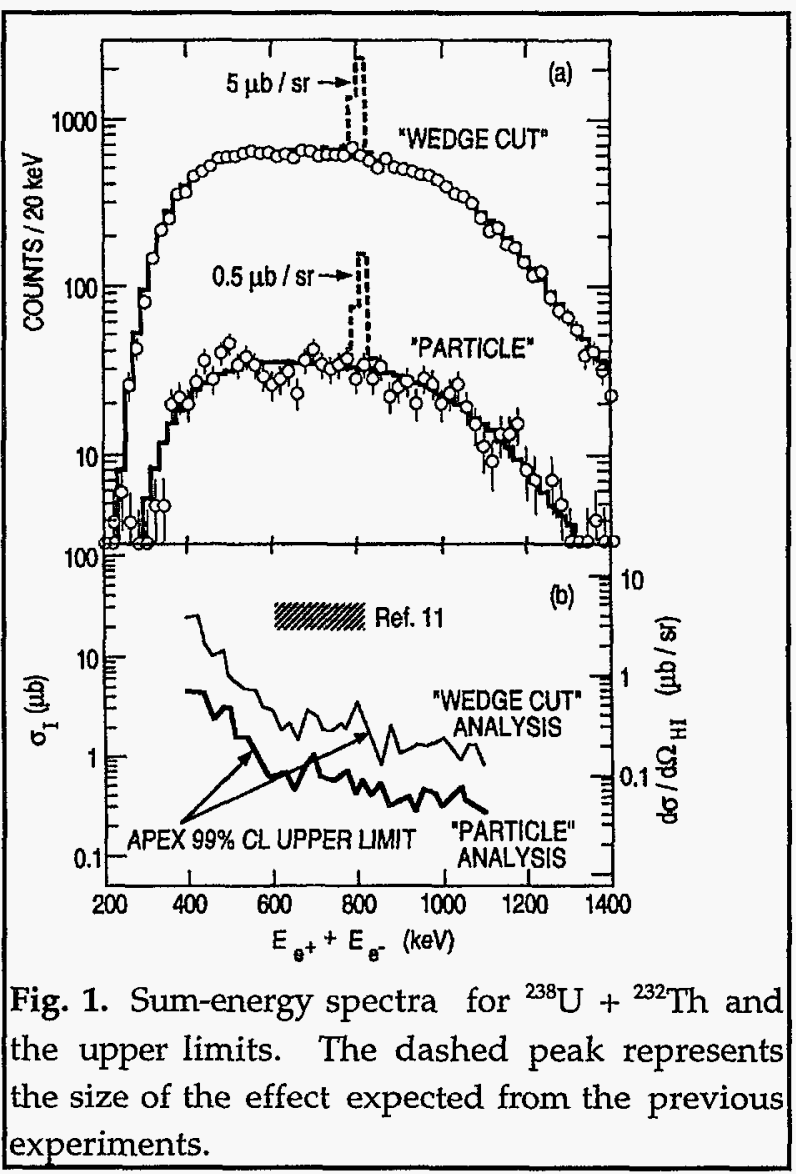

Footnotes and References

The APEX collaborating institutions consist of:

Argonne National Laboratory, Florida State University, Lawrence Berkeley National Laboratory, Michicagn State University, Princeton University, Rochester University, University of Washington, and Yale University.

${ }^{1} \mathrm{I}$. Ahmad, et. al. Nuclear Instruments and Methods A 370, 539 (1996)

${ }^{2}$ I. Ahmad, et. al. Phys. Rev. Lett. 75, 2658 (1995) 


\title{
Studying New Physics Using Ultrahigh-Energy Cosmic-Ray $\gamma$-Family Data ${ }^{*}$
}

\author{
Y. D. He
}

This paper discusses some recent developments in the study of ultrahigh-energy interactions using cosmic-ray $\gamma$-family data obtained with emulsion chambers at mountain altitudes. The apparent excess of the event rate at large transverse momenta with respect to the prediction based on perturbative QCD indicates new interaction mechanisms at $E_{\mathrm{lab}} \simeq 10^{4}-10^{5} \mathrm{TeV}$, an energy regime beyond the reach of existing colliders. To reconcile the data with a compositeness model of quarks, the characteristic energy scale would have to be as low as $\Lambda_{c} \leq 0.1$ $\mathrm{TeV}$, in conflict with the lower limit of $\Lambda_{\mathrm{c}}>1.4$ $\mathrm{TeV}$ or a possible value of $\Lambda_{\mathrm{c}} \simeq 1.6 \mathrm{TeV}$ obtained by the CDF experiment at Tevatron. Possible new physics beyond the standard model, together with future experiments at the LHC, are also discussed.

*Abstract of an invited review paper in Modern Physics Letter A 11 (1996) 2013-2026. 


\title{
Search for Dirac Magnetic Monopole Production in High Energy Heavy Ion Collisions*
}

\author{
Y. D. He and P. B. Price
}

Extensive searches for monopole pair production in $e^{+} e^{-}, p p$, and $\bar{p} p$ collisions have been performed at various high energy colliders. In each of these, an upper limit on its production cross section has been placed. In all these collisions, the monopole pairs are expected to be produced via Drell-Yan mechanism. In $A A$ collisions, the thermal production of monopole pairs has also been predicted in addition to Drell-Yan.

We exploit a useful feature of the BP-1 trackrecording glass: its sensitivity can be tuned by a suitable choice of chemical etchants. Our detector consisted of 17 sheets of $\mathrm{BP}-1$ glass and $\mathrm{a} \mathrm{Pb}$ target. Two of the BP-1 plates were placed in front of the target and 15 downstream. For particles with $Z / \beta>84$, we measured their instantaneous values of $Z / \beta$ along their paths. With measurements of $Z / \beta$ as a function of penetrating depth, we were able to determine $Z$ and $\beta$.

In a search for Lee-Wick matter, we found nine events with $Z / \beta>82$. All of them were slowing particles $(\beta<0.79)$ from interactions of beam particles with pipe or air. None of the candidates has $d E / d x$ that matches that predicted for a magnetic monopole with $n=2$.

Based on a null result in $\sim 10^{9}$ interactions of $11 \mathrm{~A} \mathrm{GeV} \mathrm{Au}$ with a $\mathrm{Pb}$ target, we can place an upper limit on its production cross section: $2 \times 10^{-32} \mathrm{~cm}^{2}$. No one has realistically calculated the cross section for monopole production. The
Drell-Yan cross section serves as a rough point of reference for production of monopole pairs via an intermediate massive virtual photon, multiple virtual photons, or gluon-gluon fusion.

At BNL AGS, we are currently analyzing a new search in $\sim 10^{11} \mathrm{Au}$ on $\mathrm{Pb}$ interactions at $\sim 11 \mathrm{~A} \mathrm{GeV}$. With this new experiment, we expect to achieve an improvement of $\sim 2$ orders of magnitude in sensitivity. We have also proposed a new search at CERN SPS using the high intensity $\mathrm{Pb}$ beam at $160 \mathrm{~A} \mathrm{GeV}$.

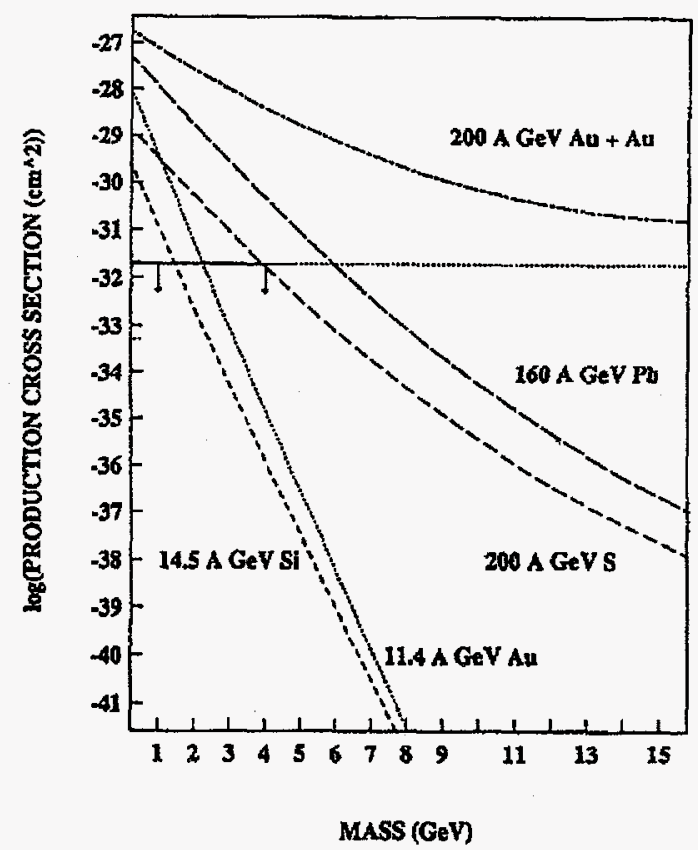

Figure - Upper limit to the production cross section for Dirac monopole pair in heavy ion collisions.

* Condensed from a paper in the Proceedings of $24^{\text {th }} \mathrm{In}$ ternational Conference on Cosmic Rays, Rome, Italy 1 (1995) 845-848. 


\title{
Measurement of Low Energy Ion Tracks in CR-39 Plastic with An Atomic Force Microscope*
}

\author{
Y. D. He and C. I. Hancox
}

We demonstrate that an atomic force microscope allows one to measure the detailed geometry of etch pits due to heavy ions with kinetic energies of $1-10 \mathrm{keV} / \mathrm{amu}$ at small distance scales registered in the nuclear track detector CR-39 plastic. With a typical range of a few hundred $\mathrm{nm}$ and an etch pit depth on the orders of a few tens of $\mathrm{nm}$, these ion tracks cannot be measured using conventional methods. With the technique developed in this work one is able to study the response of CR-39 plastic to particles at low ionization rates by precise measurements of low energy ion tracks. Among other applications, of particular interest is to explore the response of CR-39 in the regime in which nuclear stopping is comparable to electronic stopping.

\footnotetext{
*Abstract of a paper in Review of Scientific Instruments 66 (1995) 4575-4582.
} 


\title{
Low-background Counting Facilities
}

\author{
A.R. Smith, R.J. McDonald, D.L. Hurley, and E.B. Norman
}

The LBNL Low Background Facilities (LBF) consist of a Berkeley site and an Oroville site specially configured for low-background gammaray spectroscopy. The Berkeley site was established in 1963 and consists of a $3 \mathrm{~m}$ by $7 \mathrm{~m} \mathrm{x}$ $3 \mathrm{~m}$ room surrounded by $1.6 \mathrm{~m}$ of specially-selected low-background concrete shielding. The aggregate in this concrete is from serpentine gravel which is low in $U, T h$, and $K$. This barrier was made to shield against accelerator-produced neutrons and natural gamma radiation as well as some cosmic rays. Also, the low-activity concrete emits little radon, and a HEPA-filtered air system constantly purges the room to reduce airborne radon.

Detectors at this site include a $20 \mathrm{~cm}$ diameter by $10 \mathrm{~cm}$ thick $\mathrm{NaI}$ crystal, two $30 \%$ p-type $\mathrm{Ge}$ spectrometers (one of these with an external active cosmic ray suppresser), an $80 \%$ p-type Ge spectrometer and a 55\% n-type spectrometer suitable for low-energy gamma-ray and X-ray measurements. These detectors each have small local shields consisting of $10 \mathrm{~cm}$ of $\mathrm{Pb}$. The overall shielding reduces background to the point where internal activity in the detectors and cosmic-rays are the dominant source of background.

The LBF Oroville site is located in the powerhouse of the Oroville Dam, under $180 \mathrm{~m}$ of rock cover. This site now has a $115 \%$ n-type and a $30 \%$ p-type Ge spectrometer and is used for our most sensitive counting. Sensitivities of 50 partsper-trillion (PPT) for U and daughters, 200 PPT for Th and daughters, and 100 parts-per-billion for $\mathrm{K}$ are realized at the Oroville site.

The LBF has been involved in a wide variety of experiments supporting programs from LBNL and a variety of other institutions. There are five main types of work the facility supports: low-activity materials certification, cosmic ray activation, neutron activation, nuclear science experiments and environmental health and safety activities. The ongoing materials certification work is for two large detector projects: SNO and the UCB Dark Matter Search. A variety of stock materials and components were surveyed for these projects. Cosmic-ray activation work, begun with the LDEF and HST, continued with a $\mathrm{Pb}$-glass sample from $\mathrm{Mir}$ and a stainless steel sample from another Russian satellite. The latter study involved looking for excess Be-7 on the earth-facing side of the sample. Neutron Activation Analysis work continued on semiconductor-grade silicon, and verified parts-per-trillion sensitivity to 29 elements in wafer-sized samples (Ref. 1). A new initiative was begun with biological samples. Selenium-laden bacteria and sludges were studied as proof-of-principle for a program in bioremediation. Uranium uptake was measured in another bacterial sample. The facility supported research aspects of the Nuclear Astrophysics Group including looking for electron density effects on the half-life of ${ }^{40} \mathrm{~K}$, a halflife determination of ${ }^{108} \mathrm{Ag}$, and measurements of a large sample of ${ }^{150} \mathrm{Nd}$ separated isotope proposed to be used in a double beta decay experiment. An additional program involved developing mixtures of natural $\mathrm{La}, \mathrm{Lu}$, and $\mathrm{K}$ as a "non-radioactive" calibration source. A substantial amount of the facility's time was involved with Environmental Health and Safety work. This included a major radiological survey of the building and site of the new waste handling facility, Building 85, measurements of sub-surface soils under the BEVALAC, characterization of concrete shielding blocks for disposal, and measurements of a variety of samples in support of the ES\&H analytical lab.

During the coming year, new projects screening materials for semiconductor manufacturing are proposed, including both direct counting and neutron activation. Work in support of bioremediation is expected to increase. Cosmicray activated parts are expected from the second HST servicing mission in February 1997. Characterization work on LBNL radioactive and mixed waste will increase.

\section{Footnotes and References}

1. A.R. Smith, R.J. McDonald, H. Manini, D.L. Hurley, E.B. Norman, M.C. Vella, and R.W. Odom: J. Electrochem Soc. 143 (1996) 339-346 


\title{
Low-background Gamma-ray Spectroscopy Applied to Measuring the Uptake of Metals by Bacteria
}

\author{
A.R. Smith, R.J. McDonald, D.L. Hurley, and E.B. Norman
}

Low-background gamma-ray spectroscopy offers a sensitivity to $U$ of 10 micro-grams of depleted $U$ and 100 nano-grams of $235 \mathrm{U}$ by direct counting. Following neutron activation, sensitivities of 0.01 nano-grams have been observed for 29 elements in semiconductor-grade silicon [1], and should be similar for pure lifeelements $(\mathrm{H}, \mathrm{C}, \mathrm{N}, \mathrm{O})$. In real organisms, this sensitivity will be reduced due to trace elements such as $\mathrm{Na}$ and $\mathrm{Fe}$. However, the sensitivity to per-cent level uptakes in micro-gram sized samples or PPB sensitivities in gram-sized samples should have many applications in studying the uptake of metals by bacteria. Two proof-of-principle experiments follow:

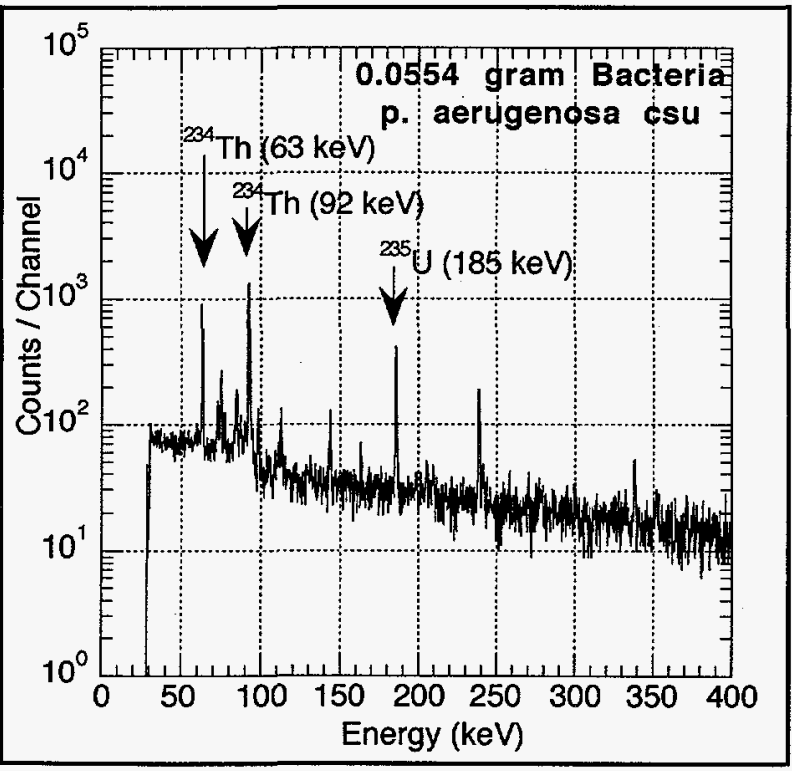

Figure 1 shows a gamma-ray spectrum observed from a $0.055 \mathrm{~g}$ sample of $\mathrm{p}$. aerugenosa bacteria which absorbed $1.55 \mathrm{mg}$ (2.8\%) of (depleted) U. Characteristic gamma-ray lines at 63 and $92 \mathrm{keV}$ are observed from the decay of the 24.1 day half-life ${ }^{234} \mathrm{Th}$ (the first daughter of ${ }^{238} \mathrm{U}$ ) and at $185 \mathrm{keV}$ from ${ }^{235} \mathrm{U}$. Observed over 30 days, the Th activity "grows in" to a level of $2.8 \%$ of the sample mass by weight. Not knowing the relative abundance of ${ }^{235} \mathrm{U}$ compared to ${ }^{238} \mathrm{U}$ in the depleted solution, there was no way to correlate this measurement with that from ${ }^{235} \mathrm{U}$ as a check. This technique can be used, for example, to screen large numbers of bacteria and characterize their ability to take up $U$ as a function of variables such as $\mathrm{pH}$ and concentrations of other elements in the growth medium.

Figure 2 shows a gamma-ray spectrum observed following neutron activation of $0.84 \mathrm{mg}$ Se absorbed by $20 \mathrm{mg}$ of bacteria $(4.2 \%)$. Since most of the bacteria is composed of $\mathrm{H}, \mathrm{C}, \mathrm{N}, \mathrm{O}$, the background is favorably low. $\mathrm{Fe}$, at the $0.2 \%$ level is observed, and Compton-scattered gamma rays reduce somewhat the sensitivity to $\mathrm{Se}$ compared to pure $\mathrm{H}, \mathrm{C}, \mathrm{N}, \mathrm{O}$ or $\mathrm{Si}$. Again, because of the accuracy and the small sample size, this technique can be used to determine uptake in large numbers of samples as functions of a number of variables, including other contaminants such as $\mathrm{Cr}$, in the growth medium.

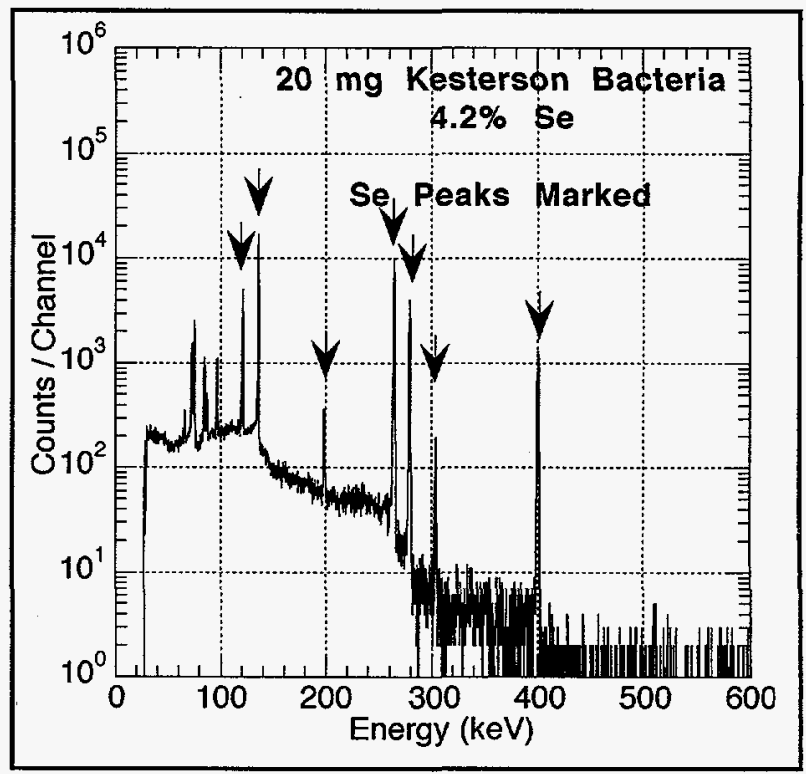

Footnotes and References

1. A.R. Smith, R.J. McDonald, H. Manini, D.L. Hurley, E.B. Norman, M.C. Vella, and R.W. Odom: J. Electrochem Soc. 143 (1996) 339-346 


\title{
Low-Level Measurement of Alpha-Particle Emitters in Ceramics and Metals
}

\author{
A.R. Smith, R.J. McDonald, D.L. Hurley, and E.B. Norman
}

Nearly all natural materials contain trace quantities, typically in the PPM range, of $U$ and Th. Even processed materials often contain these elements in the PPB range. These radioactive elements decay via complex alpha and beta decay chains, forming 11-15 daughter nuclides each, many of which emit alpha particles in their own decay. When close enough to sensitive electronic components, such as transistors in an IC chip, they can disrupt the normal operation of prcessors or memory.

Direct alpha counting techniques using solid state or gas-filled detectors can be used to measure activity, but this method is only sensitive to a $0.01-0.03 \mathrm{~mm}$ surface layer and, unless even much thinner films are used, provides only a count rate and not spectrographic information. Besides examining only a small part of the material, alpha detecection rates are low and can be distorted by contamination, i.e. from radon in the air.

For the assay of $U$ and Th in materials, at the PPB level, low-background gamma-ray spectroscopy is a very sensitive and isotopespecific method for determining the bulk concentrations of these radionuclides in essentially all solid and liquid materials, including ceramics, metals, plastics, and etchants. While some of the U- and Th-series alpha-emitters are not detected directly, the gamma-ray evidence correctly indicates the present levels of alpha-activity, and also provides accurate predictions for how the alpha-activity may change with time. $5 \mathrm{~kg}$ samples provide adequate counting rates at the PPB level for 1 day counting times.

For the analysis of ${ }^{210} \mathrm{~Pb}$ in $\mathrm{Pb}$, thinner samples are required to measure the characteristic $46 \mathrm{keV}$ gamma ray, but counting rates are similar to those for alpha counters, spectrographic information is obtained, and no wait is needed for the ingrowth of $210 \mathrm{Po}$.
The low-background facity is presently seeking an industrial partner to work on these problems. The $115 \%$ n-type detector at the LBF underground Oroville site has the sensitivity to measure $10 \mathrm{~Bq} / \mathrm{kg}$ levels in $100 \mathrm{~g}$ samples of $\mathrm{Pb}$ and 1 PPB levels of $U$ and Th in $5 \mathrm{~kg}$ samples of metals or ceramics. 




\section{$\Delta$ Resonance Production in ${ }^{58} \mathrm{Ni}+\mathrm{Cu}$ Collisions at $\mathrm{E}=1.97 \mathrm{~A} \mathrm{GeV}$}

\section{E.L. Hjort and the EOS Collaboration}

In heavy-ion collisions above $1 \mathrm{~A} \mathrm{GeV}$ a significant fraction of the baryonic content is in the form of resonances, primarily the $\Delta$ resonance. Using data taken with the EOS TPC we have performed invariant mass analyses of $\left(\mathrm{p}, \pi^{ \pm}\right)$pairs which show correlations resulting from the decays of the $\Delta$ resonance, the $\Lambda$ baryon and the $N^{*}(1440)$ resonance. A reduction in the $\Delta$ mass which is maximized in central collisions is seen, and this feature is related to the size of the reaction volume and the production mechanisms of the $\Delta$ resonance.

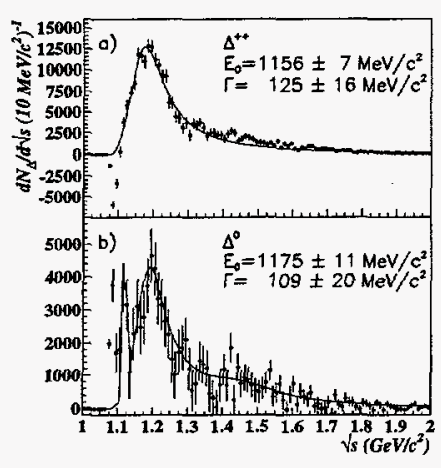

Figure 1:

The $\Delta^{++}$resonance [a]; the $\Delta^{0}$ and $\mathrm{N}^{*}$ resonances and $\Lambda$ peak [b].

Figures $1 \mathrm{a}$ and $1 \mathrm{~b}$ show the $\Delta^{++}$and $\Delta^{0}$ resonance content of the data along with the best-fit Breit-Wigner functions. In the case of the $\Delta^{0}$ a second Breit-Wigner function has been included to account for $N^{*}(1440)$ decays. Decays of the $\Lambda$ hadron into a $\left(\mathrm{p}, \pi^{-}\right)$pair produce the narrow peak in two channels at $\sqrt{s} \approx 1.116 \mathrm{GeV} / \mathrm{c}^{2}$. The fact that the $\Lambda$ mass is correct confirms the absolute momentum calibration of the TPC.
The negative(positive) values in Fig. $1 \mathrm{a}(\mathrm{b})$ at $\sqrt{s}<1.12 \mathrm{GeV} / \mathrm{c}^{2}$ result from the Coulomb repulsion(attraction) of the $\left(p, \pi^{+}\left(\pi^{-}\right)\right)$pair.

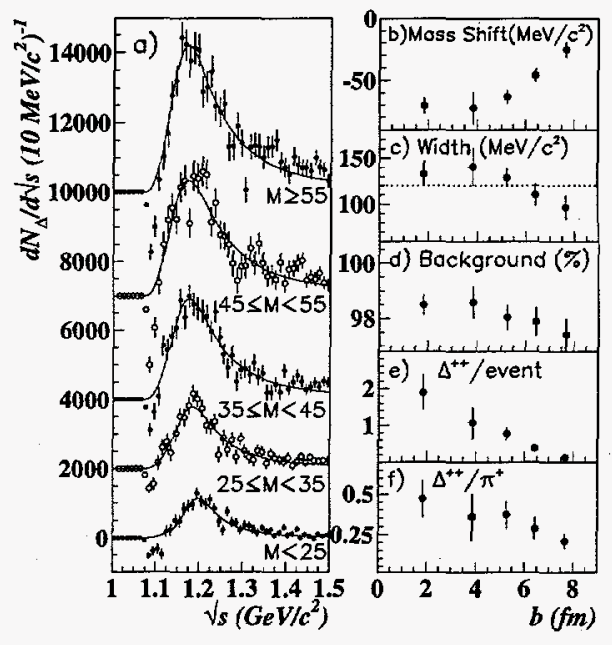

Figure 2: The $\Delta^{++}$resonance for 5 multiplicity (M) bins [a]; and the mass [b], width [c], combinatoric background [d], yield [e] and the $\Delta^{++}$to $\pi^{+}$ratio [f] versus impact parameter.

The $\Delta^{++}$has also been analyzed according to the charged particle multiplicity of each event. Fig. 2a shows the resulting fits for each of $5 \mathrm{mul}$ tiplicity bins, and Figs. 2b-e show related quantities as a function of impact parameter. A mass reduction is observed in all bins and the reduction increases by about $50 \mathrm{MeV} / \mathrm{c}^{2}$ from the most peripheral collisions to the most central collisions. A larger reaction volume in central collisions increases the proportion of $\Delta$ 's which are produced by pion scattering $(\pi N \rightarrow \Delta)$, a process which has less available energy and populates a lower mass region of the resonance relative to initial nucleon-nucleon collisions $(N N \rightarrow \Delta N)$, resulting in the observed mass shift. 


\title{
Dielectron Production in A-A Reactions at $1.0 \mathrm{~A} \cdot \mathrm{GeV}$
}

\author{
R. Jeff Porter and the DLS Collaboration
}

Measurements of dielectron production in nucleus-nucleus collisions offer a unique probe into the dynamics of heavy-ion reactions. Dielectrons are not subject to the same rescattering rates within the collision as hadronic participants and can yield direct information about internal properties of the reacting system. Important features such as baryon resonance formation, sub-threshold meson production $(\eta, \rho$, and $\omega$ ), and $\pi^{+} \pi^{-}$annihilation have been predicted to contribute to dielectron production at Bevalac energies[1]. Dielectron production measurements have yielded interesting results at both Bevalac[2] and SPS[3] energies where unresolved differences between the data from $\mathrm{p}$-A and A-A collisions have been found.

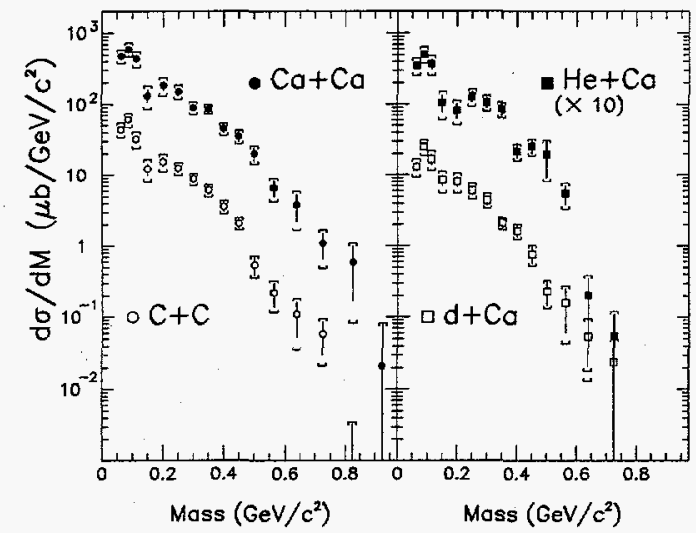

Figure 1: Dielectron cross sections in A-A systems at $1.0 \mathrm{~A} \cdot \mathrm{GeV}$.

The Dilepton Spectrometer (DLS) Collaboration has performed dielectron cross section measurements in $\mathrm{Ca}+\mathrm{Ca}, \mathrm{C}+\mathrm{C}, \mathrm{He}+\mathrm{Ca}$, and $\mathrm{d}+\mathrm{Ca}$ reactions at $1.0 \mathrm{~A} \cdot \mathrm{GeV}$. The invariant mass spectra, $\mathrm{d} \sigma / \mathrm{d} M$, from the four measurements are shown in Fig. 1. A common shape to the four spectra at low mass includes a large signal below the pion mass suggesting $\pi^{0}$ Dalitz decays and a distribution in the $0.2-0.4 \mathrm{GeV} / \mathrm{c}^{2}$ mass region resembling that predicted from $\eta$ Dalitz decays[1], The cross sections in this low mass region are found to scale in proportion to the product of the projectile and target numbers, $d \sigma / d M \sim\left(\mathrm{A}_{p} \cdot \mathrm{A}_{t}\right)^{1.0}$.

A direct comparison of the cross sections from $\mathrm{Ca}+\mathrm{Ca}$ and $\mathrm{C}+\mathrm{C}$ reactions is shown in Fig. 2 as the ratio in the measurements as a function of the pair mass. The low-mass scaling with $\left(A_{p} \cdot A_{t}\right)^{1.0}$ is illustrated by the dashed line in the figure marking the $\mathrm{A}_{p} \cdot \mathrm{A}_{t}$ ratio value. For $M \geq$ $0.5 \mathrm{GeV} / \mathrm{c}^{2}$, the ratio increases significantly indicating that a density dependent mechanism(s) is exhibited in the high-mass cross section.

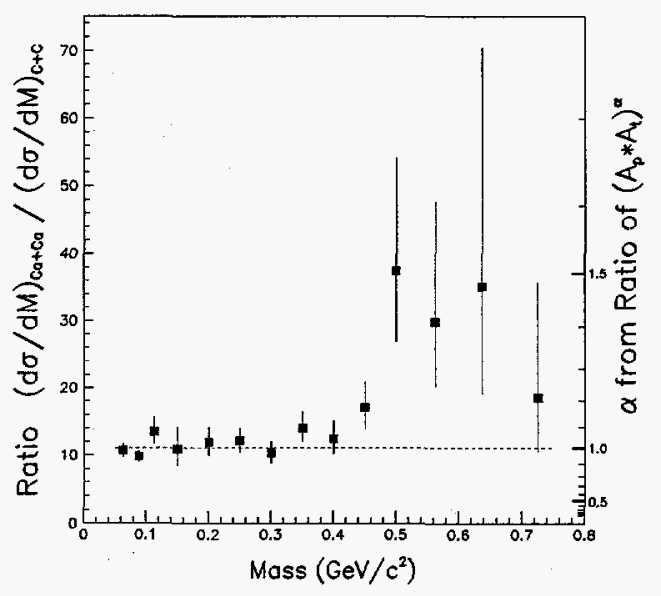

Figure 2: Cross section ratio from $\mathrm{Ca}+\mathrm{Ca}$ and $\mathrm{C}+\mathrm{C}$ reactions.

\section{References}

[1] G. Wolf et al., Prog. Part. Nucl. Phys. 30, 273 (1993); K.K. Gudima, Sov. Jour. of Nucl. Phys. 55, 1715 (1992); E.L. Bratkovskaya et al., Phys. Lett. B376 12 (1996).

[2] G. Roche et al., Phys. Lett. B226, 228 (1989).

[3] G. Agakichiev et al., Phys. Rev. Lett. 75, 1272 (1995). 


\section{The E895 experiment at the AGS}

\section{G. Rai and the E895 Collaboration}

The acceleration of $\mathrm{Au}$ beams at the Brookhaven National Laboratory's AGS accelerator facility has made it feasible to investigate the bulk characteristics of nuclear matter and its Equation of State (EOS) at high energy and baryon density. Latest experimental data and theoretical calculations on collective phenomena at low beam energies suggest that highest compression of nuclear matter might occur in the 2-10 A GeV energy range. Moreover, the potential for interesting new physics exist in the form of resonance matter and signals of phase transitions such as QGP[2] and chiral symmetry restoration[3].

The E895 experiment has, for the first time at the AGS, carried out an excitation study of $\mathrm{Au}$ induced reactions in fine detail. Specifically, E895 is currently studying particle production yields and cross-sections, global characteristics and energy spectra, multiparticle correlations, directed flow in symmetric and asymmetric systems, radial flow and squeeze-out of light fragments, pion production and shadowing (antiflow) effects, and strangeness production.

E895 has the unique ability to measure the four-momentum of light mass particles, projectile fragments from $Z=6$ to $Z=79$, and antiparticle production. All of the data was collected on an event by event basis from a Time Projection Chamber (EOS TPC). The EOS TPC provides continuous tracking, almost $4 \pi$ acceptance and particle identification for the light mass particles. Projectile fragments are identified in a multiple ionization sampling device called MUSIC. In this way full event reconstruction allowed simultaneous measurement of many experimental observables.

The E895 experiment[1] was made operational at BNL and, in December 1995, had a sucessful first run using low energy Au beam from the AGS. High statistics data was recorded using the
2 and $4 \mathrm{~A} \mathrm{GeV}$ Au beams impinging on a $\mathrm{Au}$ target, and with less statistics on $\mathrm{Ag}, \mathrm{Cu}$ and Be targets. During the second run in 1996 data was recorded at 6 and $8 \mathrm{~A} \mathrm{GeV}$ using the same combination of targets.

Preliminary online analysis showed good $\mathrm{dE} / \mathrm{dx}$ particle identification in the TPC. The p, d, ${ }^{3} \mathrm{H},{ }^{4} \mathrm{He}, \mathrm{K}^{+}$and pion bands were visible over a wide rigidity range. Multiplicity and rapidity distributions along with transverse momentum spectra were also extracted from the uncalibrated data. Figure 1 is representive of the quality of pion data and highlights the very low $P_{t}$ acceptance of the experiment. A complete offline data analysis effort is now underway at LBNL utilizing the Parallel Distributed Scientific Farm (PDSF) at NERSC.

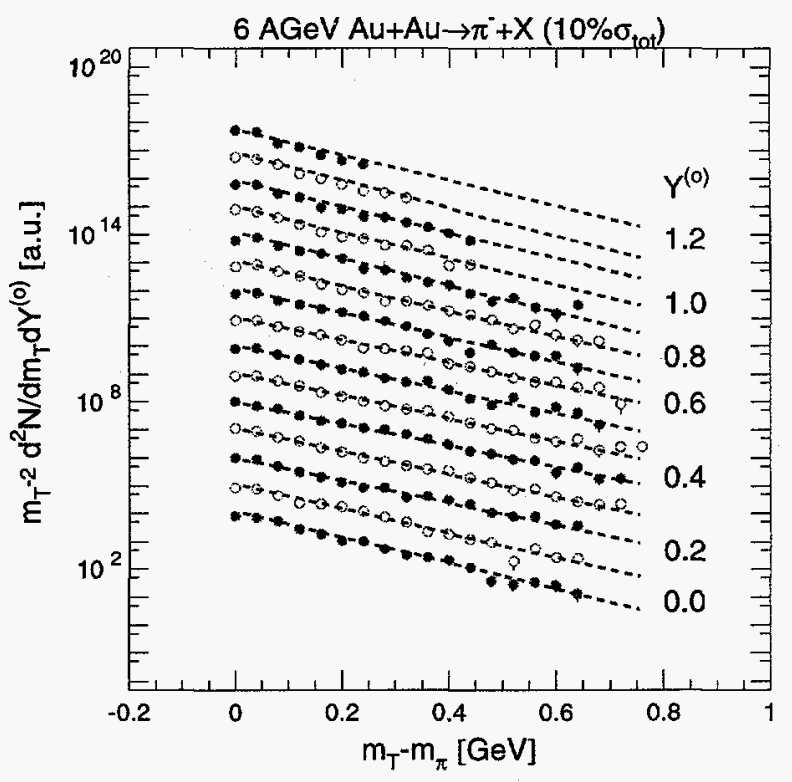

Figure 1: Pion $P_{t}$ spectra at $6 \mathrm{~A} \mathrm{GeV}$ for $\mathrm{Au}+\mathrm{Au}$ collisions plotted for various rapidity intervals

\section{References}

[1] G. Rai et al., AGS Proposal, LBL-PUB-5399, 1993

[2] D. Rischke, Proc. HIPAGS,(1996),138

[3] B.A. Li and C.M. Ko, Nucl. Phys. A601,(1996),447 


\title{
Rare Processes in Relativistic Heavy Ion Collisions--Results from BNL E878
}

\author{
M.J. Bennett, J.B.Carroll, H. Crawford, M. Cronqvist, J. Engelage, I. Flores, L. Greiner, T.J. \\ Hallman, H.H. Heckman, C. Kuo, P.J. Lindstrom
}

E878 is a high sensitivity search experiment, designed to investigate rare proceses in relativistic heavy ion collisions, e.g. antibaryon production and the formation of new, previously undiscovered particles. The experiment was conducted at the Alternating Gradient Synchrotron (AGS) at Brookhaven National Laboratory, and is a followon to experiment E858. Data were recorded using $\mathrm{Au}$ beams on various nuclear targets during the spring of 1992 and the fall of 1993. Over the course of the last two years, analysis of this data has been completed, resulting in several publications and one Ph D thesis.

The primary goal of E878 was a search for strangelets, which are multiquark hadrons made of roughly equal number of $u, d$ and $s$ quarks which have been predicted to be stable. E878 was sensitive to strangelets up to $A=30$ (90 quarks) with lifetimes of $\sim 100 \mathrm{~ns}$ or longer. Our search yielded no strangelet candidates. Sensitivites for positively charged strangelets are shown in figure 1; similar sensitivities were reached for negatively charged strangelets.

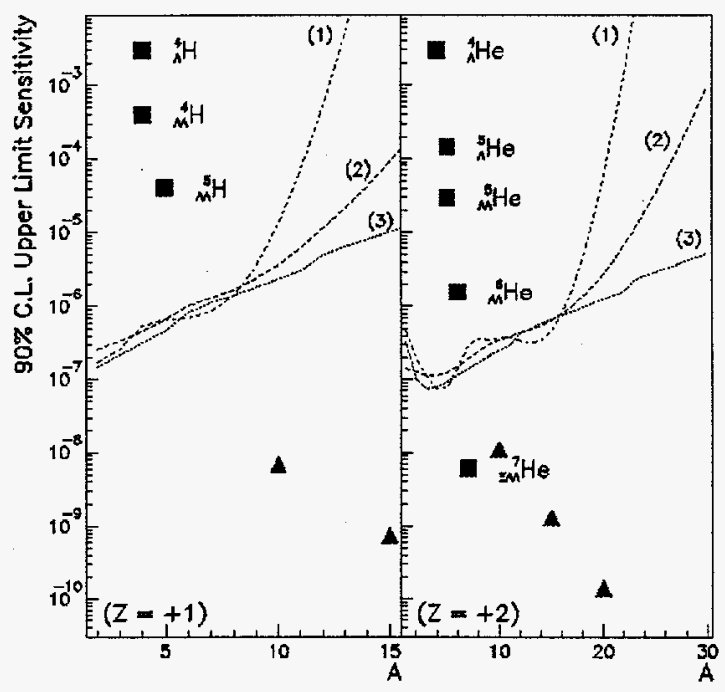

Figure 1. The sensitivity of E878 for the detection of charge $+1,+2$ strangelets. The three curves are $90 \%$ sensitivity upper limits based on specific models of strangelet production. Strangelet predictions of Crawford et al are shown as triangles and hypernuclei predictions of Baltz et al are shown as squares. [ 1 and references therein]
The biggest improvement in E878 over its forerunner E858 is the ability to measure the centrality of the nucleus-nucleus collision producing the detected particle. This information has been used to investigate pion and kaon production cross sections, as well as a study of the formation of light nuclei as a function of centrality. Manuscripts of these studies are in the final stages of preparation.

The most interesting use of centrality information in E878 is in the study of antiproton production. As shown in Figure 2, the E878 data differed widely from theoretical predictions for models which heretofore had been quite successful in matching particle spectra. This result has spurred new thinking about how the antiproton annihilation process proceeds in the hot, dense environment of central $\mathrm{Au}+\mathrm{Au}$ collisions.

Footnotes and References

1 D. Beavis et al, Phys. Rev. Lett. 75, 3078 (1995)

2 D. Beavis et al, Phys. Rev. Lett. 75, 3633.(1995)

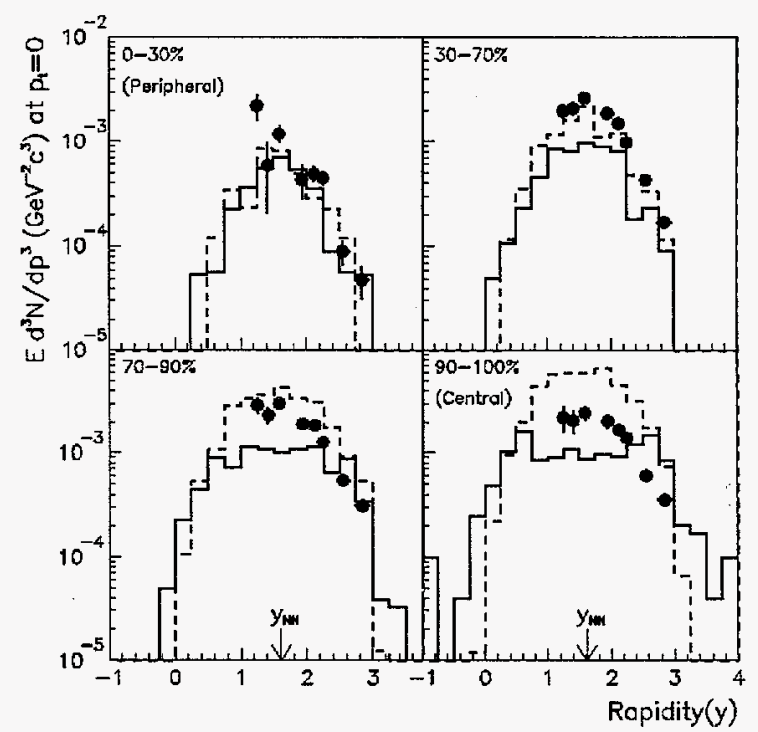

Figure 2. The invariant multiplicities of antiprotons measured in $\mathrm{Au}+\mathrm{Au}$ collisions plotted as functions of rapidity for four non-overlapping centrality bins. The data are shown as symbols, RQMD calculations as solid lines and ARC calculations as dashed lines. [2 and references therein] 


\title{
Development of a Distributed Drift Chamber for BNL E896
}

\author{
J. Engelage, M.J. Bennett, J.B.Carroll, H. Crawford, M. Cronqvist, I. Flores, L. Greiner, E. Judd, \\ P.J. Lindstrom, J.Marx, I.Sakrejda, and G.Visser
}

Experiment 896 (E896) is a search for the $\mathrm{H}_{\mathrm{o}}$ (a six quark, neutral charged, short-lived strange dibaryon state) in nucleus-nucleus (AA) collisions at the AGS. The primary detector in this experiment is a large volume tracking detector located in a $1.5 \mathrm{~T}$ field. A superconducting magnet will be utilized to sweep the beam and charged secondaries away from the area occupied by neutral particles. The tracking detector has been designed to unambiguously identify the topological signature of particle decays and provide enough track information to reconstruct the rigidity of each charged particle produced. A Distributed Drift Chamber (DDC) was determined to be the optimum tracking detector for this application, capable of higher event rates than a TPC and better spatial resolution than an MWPC.

The design of the 144 plane DDC relies heavily on GEANT-based simulations ${ }^{1}$. A $2 \mathrm{~mm}$ wire spacing was determined to be sufficiently large to avoid electrostatic breakdown and still achieve the double track resolution required to resolve the $\Sigma+p$ decay channel of the $\mathrm{H}_{\mathrm{o}}$. A granularity of $0.6 \mathrm{~cm}$ along the beam direction allows for multiple measurements along the short lived sigma track while maintaining a reasonable total depth of the detector to increase momentum resolution. Conductive kapton foil High Voltage planes were used because the low $\mathrm{Z}$ material reduces the delta ray background and foils facilitate transitions between different sense wire orientations. A $3 \mathrm{~cm}$ diameter hole was cut in the foils to reduce background from passage of beam through a non-active region of the chamber. A Helium-based gas, $\mathrm{He}: \mathrm{C}_{2} \mathrm{H}_{6}$ (50:50), was chosen to reduce the amount of $\delta$-rays and secondary interactions produced in the gas by the uninteracted beam and projectile fragments. It is expected that the effect of the magnetic field on the drift velocity of this "cooler" helium-based gas will be less than that experienced in the "hotter" Argon-based gases. However, the effect of the magnetic field will reduce the pulse height and distort the drift paths which has been a concern ${ }^{2}$.

In June 1996 a Prototype Drift Chamber (PDC) using $\mathrm{He}: \mathrm{C} 2 \mathrm{H} 6$ was tested in a $1.65 \mathrm{~T}$ field at the AGS. The PDC consisted of 3 full size DDC planes. The PDC resolution was measured to be $2.8 \mathrm{~ns}$, cor- responding to a spatial resolution of $\sim 100 \mu \mathrm{m}$ (the effective drift velocity being $\sim 35 \mu \mathrm{m} / \mathrm{ns}$ ). The efficiency for most of the volume of the drift cell was determined to be $\sim 99 \%$. The distortion of the drift region by the magnetic field caused the efficiency at the edges of the cell to be reduced to $\sim 75 \%$.

It is important for the analysis to be able to simulate these effects. Using the CERN packages GEANT and GARFIELD, along with a time to TDC interpolation $\operatorname{code}^{3}$, we are able to reproduce the data quite well, as shown below. The simulations clearly reproduce all the major features of the measured data.

The results of the testbeam show 1) that the response of the $\mathrm{He}: \mathrm{C}_{2} \mathrm{H}_{6}$ gas in the presence of the magnetic field is more that sufficient for our application 2) the distortion of the drift lines is consistent with the GEANT/GARFIELD simulations 3) the resolution is within the tolerances set by the GEANT simulations and track reconstruction algorithms developed for this experiment.
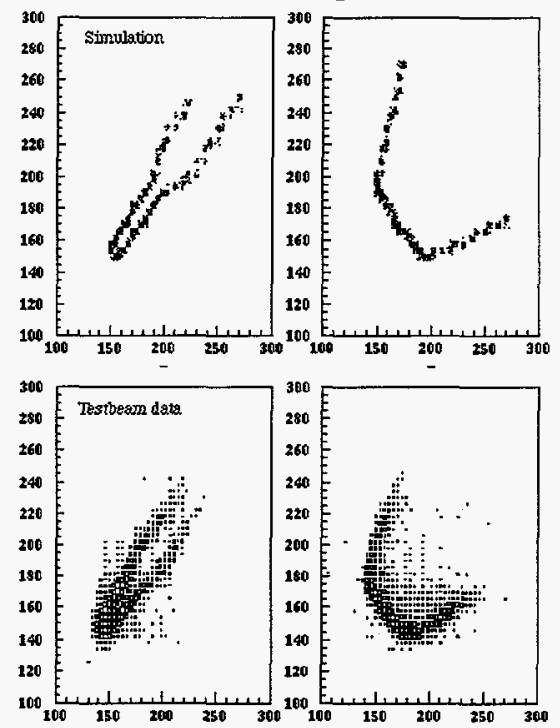

Figure 1. TDC distributions of neighboring wires from $X$ and $X^{\prime}$ planes for simulated and testbeam data. Testbeam data was produced by $10 \mathrm{GeV} / \mathrm{c}$, $\mathrm{Z}=+1$ particles at normal incidence to the PDC.

Footnotes and References

1 I.Sakrejda, LBL-37384 UC-413, 125 (1995).

2 Becker, NIM A335, 439 (1993).

3 http:/ / centauri.lbl.gov/ mats/e896/digit.html 


\title{
Spectroscopy of Scalar Mesons with the Crystal Barrel Detector
}

\author{
K.M. Crowe, T. Case, F.H. Heinsius, P. Kammel, M. Lakata \\ and the Crystal Barrel Collaboration
}

Three new scalar mesons have been observed by the Crystal Barrel experiment at LEAR(CERN) in the analysis of high statistics/ high precision data on $\bar{p} p$ annihilation into three neutral pseudoscalars ${ }^{1}$. The Crystal Barrel detector allows the complete reconstruction of $\bar{p} p$ annihilations into charged as well as neutral final states. Its main components are a multiplicity trigger, a Jet Drift Chamber (furnished by the Berkeley group) and a $\mathrm{CsI}(\mathrm{Tl})$ calorimeter.

These new results led to a major revision of our understanding of the $J^{P C}=0^{++}$scalar meson sector ${ }^{2}$. An alternative model for the classification of scalar states is discussed where the new scalars $f_{0}(1370), a_{0}(1450)$ replace the well established states $f_{0}(980)$ and $a_{0}(980)$ as members of the $J^{P C}=0^{++}$ground state nonet. The $f_{0}(1500)$ is supernumerary in all classification schemes and difficult to interpret as a $q \bar{q}$ state.

This experimental progress is of particular relevance in the search for exotic meson resonances containing constituent gluons $g$ (in particular $g g$ states called glueballs) which are suggested by the strong gluon self coupling in QCD. The discovery of gluonic mesons would directly demonstrate the unique features of strong QCD. During the last years large scale lattice QCD calculations succeeded in quantitative predictions for glueball masses and even provide some guidance on their properties. They firmly predict the ground state glueball as an isoscalar, scalar object in the $1.5-1.8 \mathrm{GeV}$ mass region.

Several papers and arguments appeared recently which identify the new state $f_{0}(1500)$ as a promising candidate for this long-sought scalar glueball. However, another contender, the

$\overline{\text { Footnotes and References }}$

${ }^{1}$ C. Amsler et al., (CBAR-Coll.), Phys. Lett. B355

(1995) 425, and references given there.

${ }^{2}$ c.f. Review of Particle Physics, R.M. Barnett et al., Phys. Rev. D 54 (1996) 1.
$f_{J}(1710)$, is hotly debated as well. More information about decay couplings and production reactions of these states are needed for definite conclusions.

Our recent work focussed on establishing the properties of the $f_{0}(1500)$ using both new data (higher statistics and new channels) as well as coupled channel analyses of several related data sets. We obtain a mass and width of $m=$ $(1505 \pm 10) \mathrm{MeV}$ and $\Gamma=(135 \pm 15) \mathrm{MeV}$ and the following invariant couplings to the different pseudoscalar states relative to $\pi \pi$ :

\begin{tabular}{ccc}
\hline$\eta \eta$ & $K \bar{K}$ & $\eta \eta^{\prime}$ \\
\hline $0.27 \pm 0.11$ & $0.24 \pm 0.10_{-0.05}^{+0.13}$ & $0.19 \pm 0.08$ \\
\hline
\end{tabular}

The decay into $4 \pi$ is found to be the dominant decay mode of the $f_{0}(1500)$, being $(3.4 \pm 0.8)$ times more frequent than $2 \pi$ decays $^{3}$.

In order to further clarify the quark-gluon content of these new scalar mesons, it is essential to systematically study their $K \bar{K}$ coupling with similar precision as their coupling to $\pi^{0}$ and $\eta$. For that purpose the Crystal Barrel detector was upgraded with a new silicon vertex detector with allows a highly selective trigger on $K_{S}$ decays.

With this method we were able to increase the world statistics on $K_{S}$ channels in liquid/gaseous hydrogen and in liquid deuterium by $1-2$ orders of magnitude ${ }^{4}$. The key information for $K \bar{K}$ decays of intermediate scalar resonances is contained in the $K \bar{K} \pi$ final states, where we expect $(2-5) \times 10^{4}$ events (depending on reaction) in the final Dalitz Plots. These data are currently being analyzed in Berkeley.

\footnotetext{
Footnotes and References

${ }^{3}$ A. Abele et al. (CBAR-Coll.), Phys. Lett. B380,(1996) 453.

${ }^{4}$ P. Kammel et al., (CBAR-Coll.), PANIC 96, Williamsburg, 1996.
} 


\section{Measurement of Rare $\omega \rightarrow \eta \gamma$ Radiative Decay* \\ Mark Lakata, Ken Crowe, Tom Case, Peter Kammel, Fritz-Herbert Heinsius}

The radiative decays of vector and pseudoscalar mesons are useful tests of the simple quark model and its extensions. In the family of the eleven vector meson to pseudoscalar meson decays, the decay of $\omega$ into to $\eta \gamma$ is one of the least well measured because of its very small branching ratio into neutral final states.

The Crystal Barrel Experiment (PS197) at Cern, Geneva, Switzerland, was used to measure the annihilations of an antiproton beam on a proton target. The nearly $4 \pi$ solid angle coverage of the decay allows full event reconstruction from all of the final $\gamma$ rays. The annihilation channel of $\eta \omega$ has used to produce decays of $\omega$ into the reference channel $\pi^{0} \gamma$ and into the rare channel $\eta \gamma$ (where $\pi^{0} \rightarrow \gamma \gamma$ and $\eta \rightarrow \gamma)$. Thus the intermediate states were $\eta \pi^{0} \gamma$ and $\eta \eta \gamma$ respectively, which end up finally as five $\gamma$ states. Nearly 20 million neutral annihilations, triggered by an incoming antiproton and a veto from the tracking chamber, were used the analysis.

The dominant source of background was feedthrough from six $\gamma$ final states, where a soft $\gamma$ 's is lost in the detection or reconstruction process, resulting in a five $\gamma$ final state. The respective backgrounds of $\eta \pi^{0} \gamma$ and $\eta \eta \gamma$ were therefore $\eta \pi^{0} \pi^{0}$ and $\eta \eta \pi^{0}$, where the soft $\gamma$ is due to the last $\pi^{0}$. The $\eta \eta \pi^{0}$ channel's production rate is several orders of magnitude higher than that of $\eta(\omega \rightarrow \eta \gamma)$, so even a small percentage of feed-through causes a large background.

Extensive Monte Carlo simulations of all of the signal and background event channels were produced using the GEANT package.

The $\gamma$ reconstruction for each event was repeated twice. The first reconstruction used a high energy threshold to discard low energy noisy signals to enhance the desired channels. The second reconstruction used a low energy threshold to look for the soft $\gamma$ present in the background channels.

The first cuts on the data and simulated data selected $5 \gamma$ states, with each $\gamma$ having a minimum energy of $20 \mathrm{MeV}$, which passed a $4 \mathrm{C}$ fit to total momentum and energy conservation. This selected data was reprocessed from the original measured signals, but this time with a $4 \mathrm{MeV}$ minimum $\gamma$ energy. The $\gamma$ 's were paired to form $\pi^{0}$ and $\eta$; any event with more than $1 \pi^{0}$ or with having $\eta \eta \pi^{0}$ were discarded. The $\gamma$ s from the $20 \mathrm{MeV}$ reconstruction were used in the further analysis.

The $5 \gamma$ events were kinematically fitted to $\eta \pi^{0} \gamma$ and $\eta \eta \gamma$ hypotheses and separated into two groups, where ambiguous or bad events were discarded. From each group, a Dalitz plot of $\eta \gamma$ vs. $\pi^{0} \gamma$ and $\eta \gamma$ vs. $\eta \gamma$, respectively, was made. The real data and simulated data were treated to the same analysis chain, and in the end the real data was fitted bin by bin to a sum of the appropriate simulated data. $65 \mathrm{~K}$ events of $\omega \rightarrow \pi^{0} \gamma$ and 150 events of $\omega \rightarrow \eta \gamma$ were identified in the fit.

Using tabulated values for all branching ratios except the rare channel, the branching ratio for the rare channel was measured,

$$
\mathrm{BR}(\omega \rightarrow \eta \gamma)=(6.2 \pm 0.7 \pm 1.0) \times 10^{-4} .
$$

where the first error is statistical and the second is systematic. This improves the errors of the previous tabulated value by $20 \%$.

\section{Footnotes and References}

*Ph.D. Thesis of Mark Lakata, to be published. 


\title{
A Precision Measurement of Nuclear Muon Capture on ${ }^{3} \mathrm{He}$
}

\author{
K.M. Crowe, T. Case, P. Kammel *
}

In an experiment performed at PSI Switzerland our collaboration has measured the muon capture rate in the reaction $\mu^{-}+{ }^{3} \mathrm{He} \rightarrow \nu_{\mu}+$ ${ }^{3} \mathrm{H}(1.9 \mathrm{MeV}, \mathrm{BR} \approx 0.33 \%)$ with unprecedented accuracy. Our results allow new tests of PCAC and chiral perturbation theory ${ }^{123}$.

At first glance it might seem that one could more easily compare the simpler system $\mu^{-}+\mathrm{p} \rightarrow \nu_{\mu}+\mathrm{n}$ to the more accurate theoretical predictions associated with it. However, the experiment is difficult to carry out with precision as both reaction products are neutral and also the initial state of the muon is uncertain due to the formation of $\mathrm{p} \mu \mathrm{p}$ mesonic molecules. The $\mu^{3} \mathrm{He}$ system turns out to be much more accessible; the muon is quickly bound in the 1s state and there is a charged particle (triton) which can be detected with extreme efficiency.

We used a high-pressure ionization chamber as an active target to count both the tritons and the incoming muons with $100 \%$ efficiency for the time region of our measurement. The monoenergetic tritons form a peak at $1.9 \mathrm{MeV}$ in figure 1. The only remaining background is from the "breakup" reactions $\mu^{-}+{ }^{3} \mathrm{He} \rightarrow \nu_{\mu}+\mathrm{d}+\mathrm{n}$ and $\mu^{-}+{ }^{3} \mathrm{He} \rightarrow \nu_{\mu}+\mathrm{p}+\mathrm{n}+\mathrm{n}$, which occur at rates of $27 \%$ and $15 \%$ relative to the main reaction.

The theory of the $A=3$ system is well known

Footnotes and References

${ }^{*}$ With a Collaboration from: The Austrian Academy of Sciences Institute for Medium Energy Physics / St. Petersburg Nuclear Physics Institute / Université Catholique de Louvain / Paul Scherrer Institute / Technical University of Munich / University of California at Berkeley and Lawrence Berkeley Laboratory / Kurchatov Institute / and University of Victoria.

${ }^{1}$ C.W. Kim and H. Primakoff, Phys. Rev: 140B (1965) 566.

${ }^{2}$ J.G. Congleton and H.W. Fearing, Nucl. Phys. A552 (1993) 534; J.G. Congleton, Nucl. Phys. A570 (1994) 511.

${ }^{3}$ H. Kameyama, M. Kamimura and Y. Fukushima, Phys. Rev. C40 (1989) 974. from the related system ${ }^{3} \mathrm{H} \rightarrow{ }^{3} \mathrm{He}+\bar{\nu}_{\mathrm{e}}+\mathrm{e}^{-}$. Recent improved calculations ${ }^{4}$ predicted a capture rate $\lambda_{\text {stat }}=1502 \pm 27 \mathrm{~s}^{-1}$ (as compared with $1305 \mathrm{~s}^{-1}$ for no meson exchange currents). Our final result $\lambda_{\text {stat }}=1496 \pm 3 \pm 3 \mathrm{~s}^{-1}$ confirms this calculation and even calls for more precise predictions (stat refers to the statistical population of the muonic hyperfine levels).

Our result confirms PCAC for the nuclear pseudoscalar ${ }^{3} \mathrm{H}-{ }^{3} \mathrm{He}$ form factor $F_{P}\left(q_{0}^{2}\right)$ to within $15 \%$ :

$$
\frac{F_{P}\left(q_{0}^{2}\right)}{F_{P}^{\mathrm{PCAC}}\left(q_{0}^{2}\right)}=1.005 \pm 0.145
$$

where most of the error now resides in the theoretical calculations ( $q_{0}^{2}$ is the momentum relevant to the capture reaction).

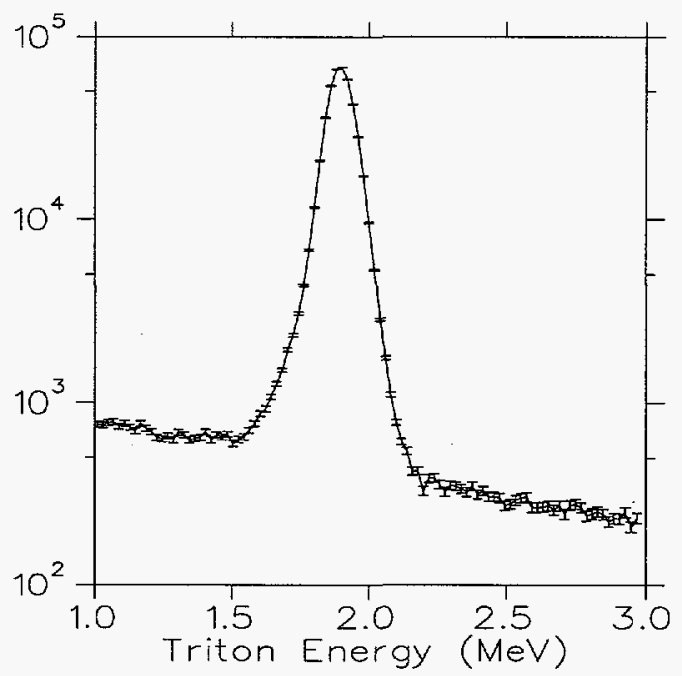

Figure 1: Energy distribution of the tritons from $\mu^{3} \mathrm{He}$ capture. The background is from the associated breakup reactions with energies from 0 to $50 \mathrm{MeV}$.

Footnotes and References

${ }^{4}$ J.G. Congleton and E. Truhlík, Phys. Rev. C53 (1996) 956 . 


\section{Elliptic Event Shapes at the CERN SPS}

\section{T. Wienold, I. Huang, and the NA49 Collaboration}

The azimuthal asymmetry of hadron emission and transverse energy production in non-central $\mathrm{Pb}+\mathrm{Pb}$ collisions at SPS energies was studied. The transverse energy was measured by the NA49 Ring Calorimeter in a special run without magnetic field to ensure azimuthally symmetric acceptance in momentum space. This calorimeter is segmented into 240 modules, configured in 10 radial rings and 24 azimuthal sectors. To search for azimuthal correlations we construct a two dimensional tensor as suggested in [1]. We define:

$$
\vec{E}_{T, k}=\left(E_{T, k} * \cos \phi_{k}, E_{T, k} * \sin \phi_{k}\right)
$$

and the tensor

$$
F_{x y}=\sum_{k} E_{T, k}(x) * E_{T, k}(y)
$$

with $E_{T, k}(x), E_{T, k}(y)$ being the components of the hadronic part of the transverse energy vector $\vec{E}_{T, k}$ for a given cell $\mathrm{k}$ centered at $\phi_{k}$.

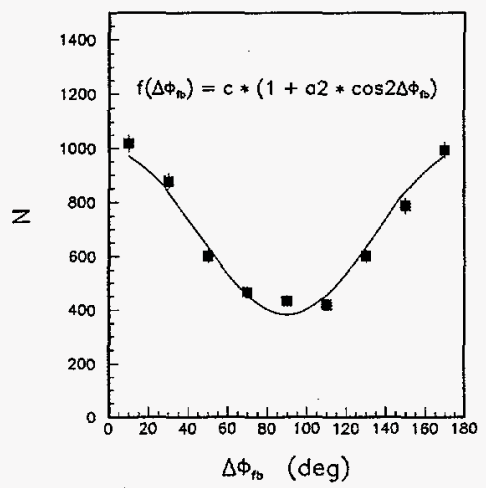

Figure 1: Forward-backward angular correlation of the major transverse energy flow axes.

This tensor is then evaluated to give the oval shape of the event separately in regions forward $(2.9<\eta<3.8)$ and backward $(2.1<\eta<2.9)$ of mid-pseudo rapidity for each event as a function of $E_{V E T O}$, which is directly related to the impact parameter. Fig. 1 demonstrates a strong angular correlation of the major axes between the two. hemispheres in an event sample, corresponding to an impact parameter range of approximately $7-8 \mathrm{fm}$. The orientations of the forward and backward ellipses describing the azimuthal $E_{T}$ distribution are shown to be aligned. This observation cannot be explained as a detector bias since the individual angular distributions of the major axes are uniform. To quantify the correlation strength of the orientation of the shape we fit the angular distribution in Fig. 1 with the function shown in the figure where $\mathrm{c}$ is a normalization constant. The centrality dependence is

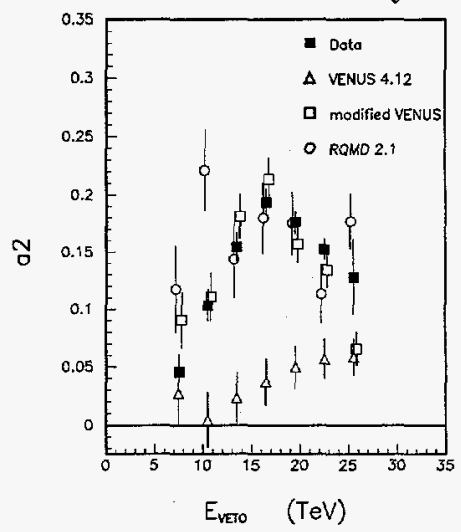

Figure 2: Centrality dependence of the a2 correlation strength.

displayed in Fig. 2. We used the pseudo rapidity intervals $2.1<\eta<2.6$ and $3.3<\eta<3.8$ to reduce the influence of shower leakage to our analysis. The correlation strength a 2 exhibits a strong impact parameter dependence and is largest at roughly half overlap collisions. Filtered VENUS events fail to reproduce the data whereas RQMD (in mean field mode) agrees roughly within the statistical errors.

\section{References}

[1] J.-Y. Ollitrault, Phys. Rev. D48 (1993) 1132 


\title{
Proton and Negative Hadron Spectra from NA49
}

\author{
Milton Toy, Peter Jacobs, and the NA49 Collaboration
}

The proton and negative hadron spectra from central $\mathrm{Pb}-\mathrm{Pb}$ collisions at $158 \mathrm{GeV} /$ nucleon have been measured by the NA49 experiment at the CERN SPS. These two distributions show two facets of the collision process: the rapidity shift (and thus energy loss) of the beam nucleons, which are represented by the protons, and the subsequent production of particles. The data shown here were taken by the NA49 Main TPCs and are fully corrected for acceptance, tracking efficiency, and non-target vertex track backgrounds.

Figure 1 shows the proton distribution in rapidity determined by the 'plus-minus-minus' method. The transverse momentum inverse slope parameter at mid-rapidity is $291 \pm 22 \mathrm{GeV}$. This technique uses the fact that charge and baryon number are conserved to form the basis of the net proton yield on the charge difference of all detected particles. Corrections are then made for the residual non-proton content composed of $\mathrm{K}^{+}-\mathrm{K}^{-}$from associated production, $\pi^{+}{ }_{-} \pi^{-}$from the isospin carried by the participating neutrons, and decay products from $\Lambda$ baryons that are misidentified as coming from the target vertex. The VENUS ${ }^{1}$ model was used to generate the particle distributions used for these corrections. Preliminary strange particle yields from NA49 agree with the predictions from VENUS.

Also shown are data from central S-S collisions at $200 \mathrm{GeV} /$ nucleon by the NA35 experiment ${ }^{2}$. The data are scaled for the difference in beam energy and number of participating nucleons. The $\mathrm{Pb}$ results show a flatter spread in rapidity which suggest a greater rapidity loss than the S data.

Because the beam projectile and target are composed of only nucleons, the negatively charged particles from the collision are a mea-

Footnotes and References

${ }^{1}$ K. Werner, Phys Rep 232 (1993) 87

${ }^{2}$ J. Baechler et. al., Phys. Rev. Lett. 72 (1994) 1419 sure of the particle production. Figure 2 shows the negative hadron rapidity spectrum that is the sum of the $\pi^{-}, \mathrm{K}^{-}$, and $\bar{p}$ yields. The scaled NA35 S-S data shows good agreement with the $\mathrm{Pb}-\mathrm{Pb}$ data and indicates that multiparticle production scales simply with the number of participating nucleons.

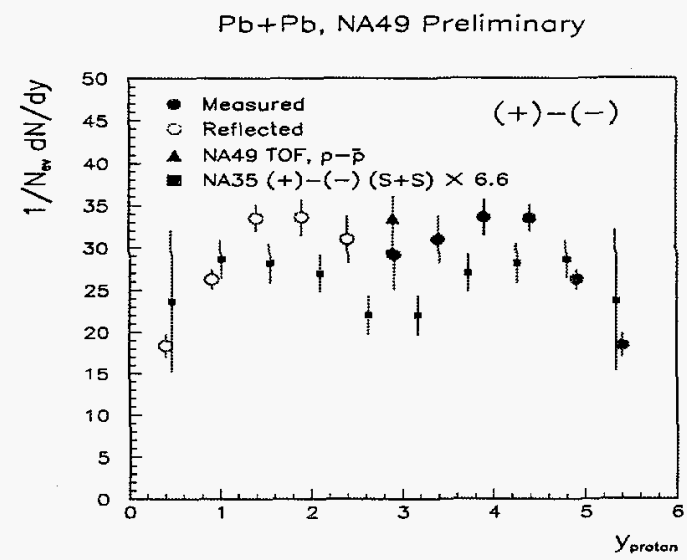

Figure 1: Rapidity distribution of protons

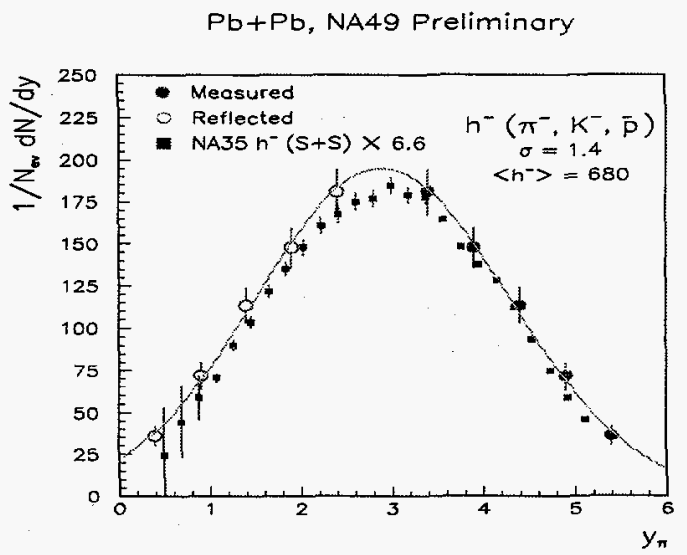

Figure 2: Rapidity distribution of $\mathrm{h}^{-}$ 


\section{A new method for V0 reconstruction in NA49 CERN experiment}

\section{S. Margetis}

The NA49 CERN experiment uses four large Time Projection Chambers (TPC) for tracking. Two (VT1/2) are placed inside magnets, and two (MTPCs) are placed further downstream and on both sides of the beam. $\mathrm{A}$ set of central $\mathrm{Pb}+\mathrm{Pb}$ events was taken with the target placed about $30 \mathrm{~cm}$ upstream of the entrance of the MTPCs. In this configuration (without magnetic field) the reconstruction of $\mathrm{V} 0$-type vertices is done the conventional way (by combining track pairs), but by using straight instead of curved tracks [1]. The momentum of the parent and daughter particles can be inferred from the decay angles if one assumes their masses. This is done by solving the energy/momentum conservation equations. The advantages of this method include

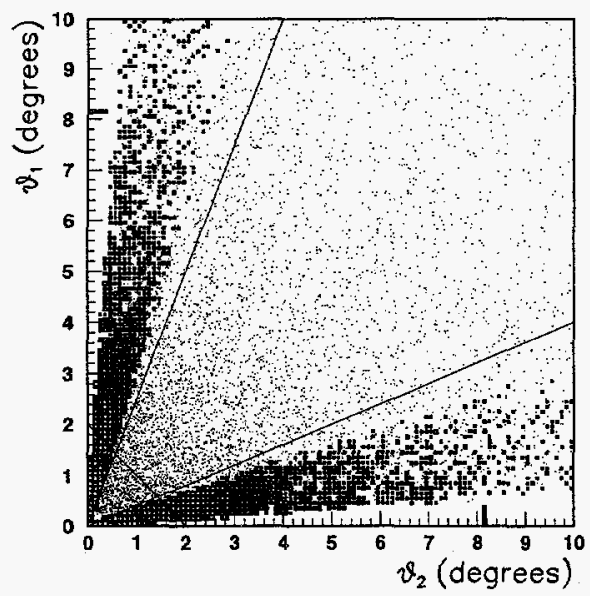

Figure 1: Daughter particle decay angle distribution (relative to the parent direction) for $\mathrm{K}_{S}^{0}$ (dots) and Lambdas (squares).

the easier/high-accuracy (straight line) tracking in the large MTPC volumes, the absence of $\mathrm{ExB}$ distortions, and the target proximity to the active volume. Also, this method provides complementary information to the standard V0 analysis done in the two vertex TPCs [2].

The separation of $\mathrm{K}_{S}^{0}$ from Lambdas is done by placing cuts in the angles between the parent and daughter particles. In Lambda decays the opening angle of the proton cannot exceed a certain value whereas the $K_{S}^{0}$ decay is symmetric (see Fig.1). The background was found to be combinatoric and, depending on the set of cuts, in the range $20-30 \%$. It was estimated using simulated events (see Fig.2) and subtracted from the reconstructed signal. The reconstruction efficiency was estimated by either, using simulated events, or by embedding in raw events simulated decays.

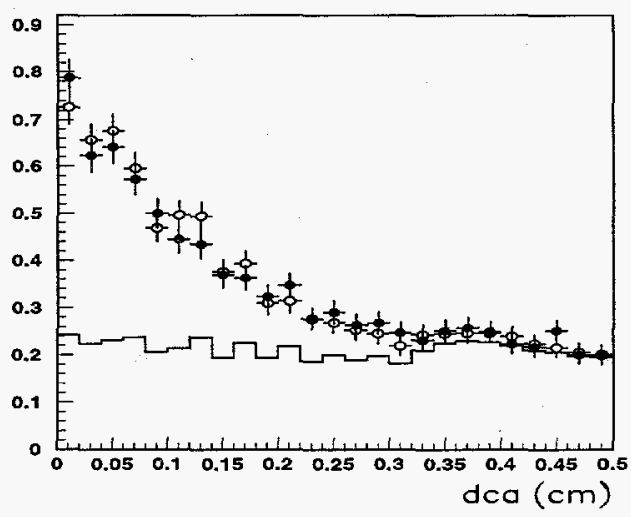

Figure 2: Normalized distribution of the distance of two daughter particles at the decay vertex (dca). Filled symbols are data, open symbols are simulated data and the solid line is the background only in the simulated data.

\section{References}

[1] K. Alpgard et al., Phys.Lett. 115B(82)65 G.J. Alner et al., Nucl.Phys B 258(85)505

[2] S. Margetis, Heavy Ion Phys. 4 (96) 63 


\section{$\mathbf{K}_{S}^{0}$ production in $\mathbf{P b}+\mathbf{P b}$ collisions at CERN SPS}

\section{S. Margetis and the NA 49 Collaboration}

Neutral kaons were reconstructed in NA49 Time Projection Chambers (TPC) using a method described in [1], via their V0 decay topology. A sample of $20000 \mathrm{~Pb}+\mathrm{Pb}$ events at $158 \mathrm{GeV} / \mathrm{c}$ was analyzed and 15000 simulated events were used for efficiency and background estimations. The interaction cross-section is $5 \%$ of $\sigma_{\text {inel }}^{\text {tot }}$ which corresponds to $\mathrm{b}_{\max }=3 \mathrm{fm}$. Results were obtained in the rapidity region $2.0<$ $y<2.8$ and $p_{T}>0.4 \mathrm{GeV} / \mathrm{c}$.

The resulting transverse mass distribution is shown in Fig. 1. An exponential fit $\left(1 / m_{T} d N / d m_{T}=e^{-m_{T} / T}\right)$ gives an inverse slope parameter $(T)$ of $220 \pm 15 \mathrm{MeV}$.

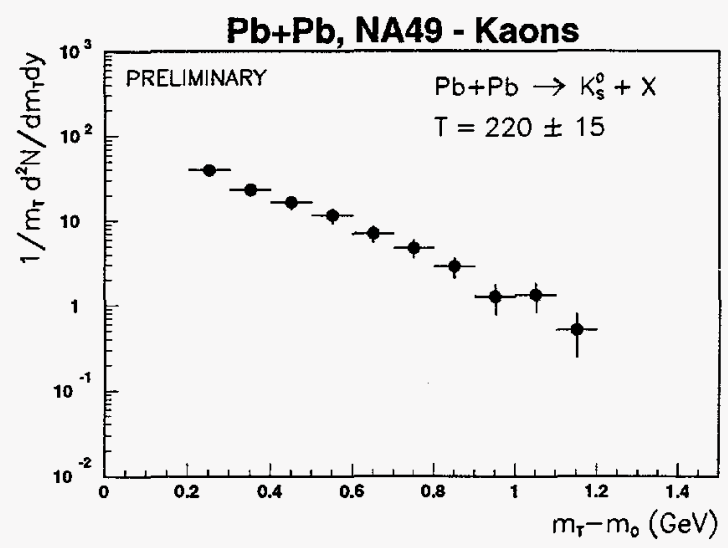

Figure 1: Transverse mass distributions of $\mathrm{K}_{S}^{0}$ in the rapidity region $2.2<y<2.6$.

By integrating over all $p_{T}$ values one obtains the rapidity density $(d N / d y)$ which is shown in Fig. 2. The filled symbols are the measured data whereas the open ones are the same values but reflected around mid-rapidity $\left(y_{m i d}^{P b+P b}=\right.$ 2.92). This is because $\mathrm{Pb}+\mathrm{Pb}$ is a symmetric system and assuming the trigger biases are negligible. The solid line is the fitted $\mathrm{S}+\mathrm{S}$ distribution scaled with the ratio of number of participants in the two systems $(390 / 58=6.7)$ which is also the ratio of produced negative hadrons (mostly pions). The two distributions are compatible within errors. This leads to the conclusion that the $\mathrm{K}_{S}^{0} / \pi$ ratio is about the same in $\mathrm{S}+\mathrm{S}$ and $\mathrm{Pb}+\mathrm{Pb}$ collisions [2]. This ratio in $\mathrm{S}+\mathrm{S}$ was found to be twice as large than in nucleonnucleon collisions at the same energy [3]. It is therefore intriguing that in a much larger system, like $\mathrm{Pb}+\mathrm{Pb}$, there is no further enhancement of this ratio.

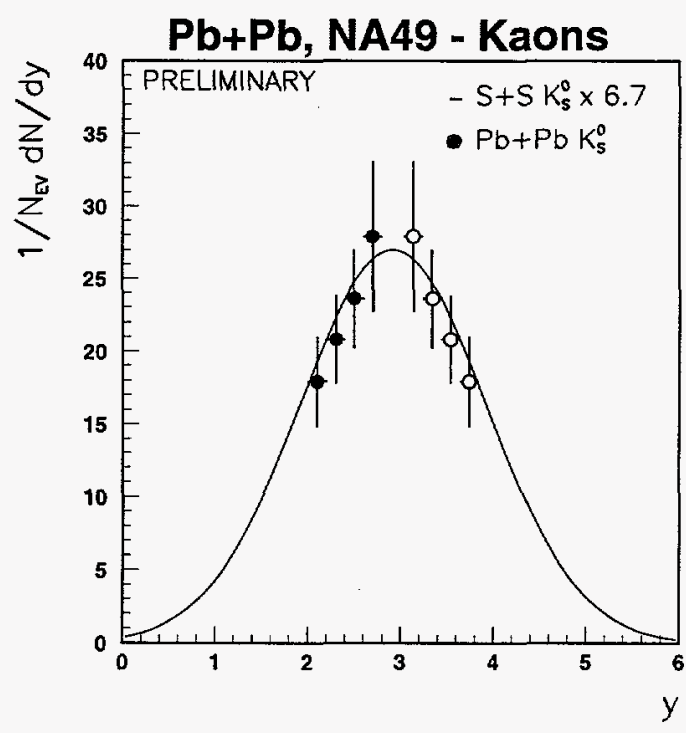

Figure 2: Rapidity distribution of $\mathrm{K}_{S}^{0}$ in $\mathrm{Pb}+\mathrm{Pb}$ collisions (symbols). See text for an explanation of the symbols.

\section{References}

[1] S. Margetis, Elsewhere in this report

[2] S. Margetis, Heavy Ion Phys. 4 (96) 63

[3] T. Alber et al, NA35 Collaboration, Z.Phys.C $64(94) 195$ 


\title{
Projectile Fragmentation in $\mathrm{Pb}+\mathrm{Pb}$ Collisions at $158 \mathrm{~A} \cdot \mathrm{GeV}$
}

\author{
I. Huang, S. Margetis, G. Odyniec and the NA49 Collaboration
}

The fragmentation of the projectiles in $\mathrm{Pb}+\mathrm{Pb}$ collisions at $158 A \cdot \mathrm{GeV}$ at the CERN SPS is studied in order to determine how many spectator nucleons are bound in fragments as a function of centrality. This is accomplished by measuring the forward-going energy in the NA49 zerodegree calorimeter (called Veto calorimeter) in conjunction with the adjustable iron collimator, which defines the acceptance of the calorimeter. NA49 uses two superconducting dipole magnets with a total bending power of $7.8 \mathrm{~T} \cdot \mathrm{m}$. Since the spectator nucleons in the projectile fragmentation region have approximately the beam energy and small $p_{T}$, the deflections of nucleons and fragments in the bending plane depend mostly on their charge-to-mass ratios. (The amount of deflection of spectator protons is $30 \mathrm{~cm}$ at the collimator.) Thus, neutrons, protons, and fragments of the spectator matter can be disentangled and measured separately in the Veto calorimeter.

The centrality trigger is provided by the NA49 Ring calorimeter (positioned upstream of the collimator and the Veto calorimeter) which measures energy deposited near mid-rapidity in each event. By comparing the data with the prediction of VENUS, the impact parameter was deduced. Forward energy data were taken in 1995 and '96 with appropriate configurations of the collimator in four separate runs to measure 1) neutrons only, 2) protons only, 3) fragments only, and 4) all three simultaneously. To estimate the amount of background contamination, a target-out run was also performed.

The magnitude of the contamination in the Veto calorimeter signal from showers that originate in the collimator and reach the Veto calorimeter, as well as from produced particles, is estimated using GEANT simulation. A fraction of the spectator nucleons in VENUS are assigned to fragments such that the full-physics GEANT simulation for the neutron configura- tion resembles the neutron data. This procedure was reiterated until the results of all configurations roughly fitted the respective data sets. The corrections for these effects were applied to the data.

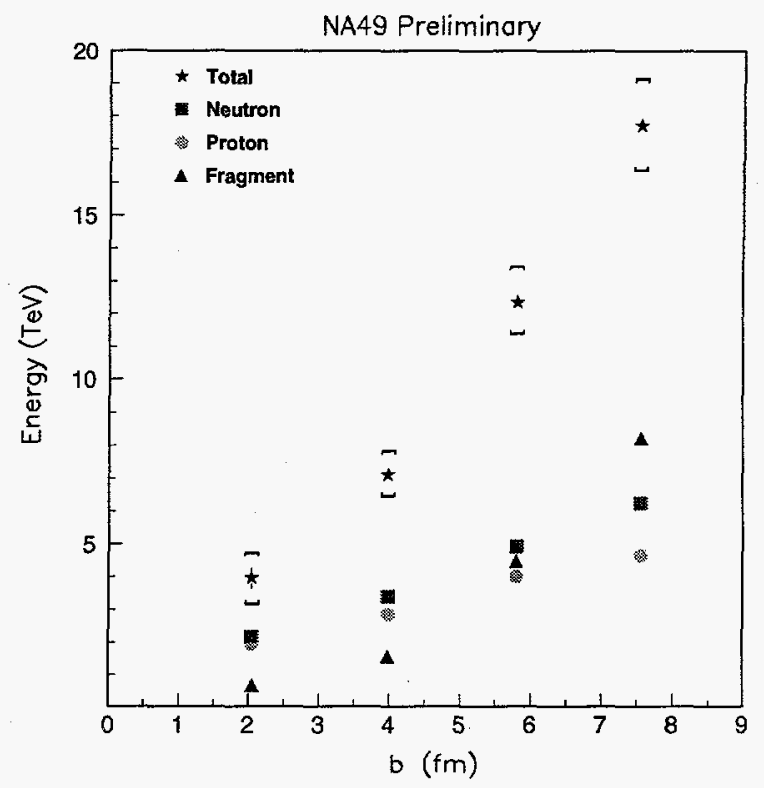

Figure 1: Projectile spectator energy as a function of the impact parameter. The square brackets are the estimated systematic errors.

Fig. 1 shows the energy carried by spectator neutrons, protons and fragments as a function of the impact parameter. The sum of energies carried by neutrons, protons and fragments agrees with the total energy to within the systematic uncertainties. These data, however, have not been corrected for the small nonuniformity of the Veto calorimeter. This correction will slightly affect the relative energy calibration of protons and neutrons. We observe that the energy carried by fragments is small for central collisions but increases dramatically for larger values of $b$. The final analysis of these data is in progress. 


\title{
Fragmentation of $160 \mathrm{~A} \mathrm{GeV} \mathrm{Pb}$ in Various Targets*
}

\author{
$Y$. D. He and P. B. Price
}

A substantial fraction of ultrarelativistic jectile fragments obtained in 5 sheets of glass heavy ion collisions results in fragmentation of in the figure. The charge resolution is found projectile nuclei in peripheral collisions involving both nuclear and electromagnetic interactions. These reactions are intrinsically of interest, as an aspect of ultrarelativistic nucleusnucleus interactions which is not thoroughly understood. Moreover, measurements of cross sections for fragmentation are needed in studies of cosmic ray propagation. Measurements of some of these cross sections have been previously made using beams of $14.5 \mathrm{~A} \mathrm{GeV}{ }^{28} \mathrm{Si}$ and $11.4 \mathrm{~A} \mathrm{GeV}$ ${ }^{197} \mathrm{Au}$ at BNL AGS, and 60 and $200 \mathrm{~A} \mathrm{GeV}{ }^{18} \mathrm{O}$ and $200 \mathrm{~A} \mathrm{GeV}{ }^{32} \mathrm{~S}$ at CERN SPS. In this paper, we shall report new measurements using the beam of $160 \mathrm{~A} \mathrm{GeV}{ }^{208} \mathrm{~Pb}$ at CERN SPS.

We exposed 10 stacks of BP-1 glass stacks that were sandwiched with various targets to the beam of $160 \mathrm{~A} \mathrm{GeV} \mathrm{Pb}$ in December 1994. The exposure was carried out at normal incidence at a density of $\sim 800 \mathrm{~cm}^{-2}$. The dimension of each plate of BP-1 glass was $50 \mathrm{~mm} \times 50 \mathrm{~mm} \times 1$ $\mathrm{mm}$. We etched the glass in $70 \% \mathrm{CH}_{3} \mathrm{SO}_{3} \mathrm{H}$ at $50{ }^{\circ} \mathrm{C}$ for 120 hours. The charge threshold is $Z_{\text {th }} \sim 68$.

We typically obtain $\sim 1.5 \times 10^{4}$ events in each stack. The charge resolution is $\sim 0.14$ charge unit. The charge resolution can even be improved by multiple measurements of etch pits in several sheets of BP-1 detectors. We present the charge distribution of beam particles and proto be $\sim 0.06$ charge unit. This charge resolution is more than adequate to identify fragments and hence to measure cross sections for chargechanging interactions. The measurements of fragmentation cross section of $160 \mathrm{~A} \mathrm{GeV} \mathrm{Pb}$ in various targets are presented.

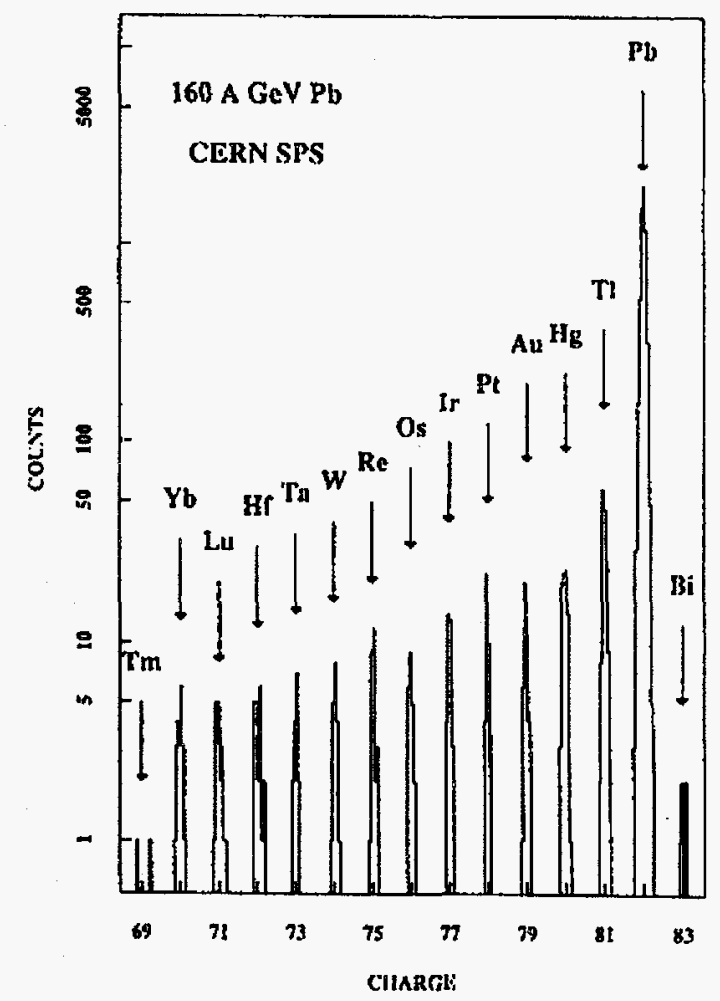

Figure - The distribution of averaged charges from 5 sheets of BP-1 detectors for beam particles and projectile fragments created in collisions of $160 \mathrm{~A}$ $\mathrm{GeV} \mathrm{Pb}$ with target.

"Condensed from a paper in the Proceedings of $24^{\text {th }} \mathrm{In}$ ternational Conference on Cosmic Rays, Rome, Italy 1 (1995) 119-122. 


\section{STAR TPC System Test}

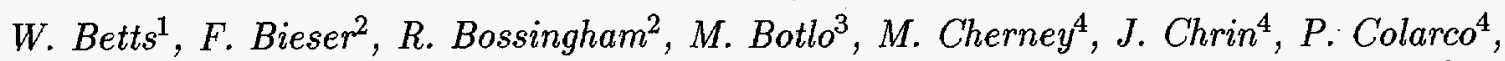

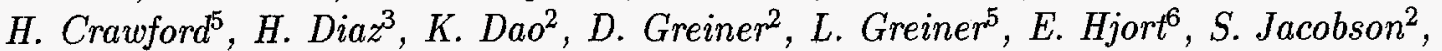
R.C. Jared ${ }^{2}$, E. Judd ${ }^{5}$, S.R. Klein ${ }^{2}$, A.N. Lebedev ${ }^{7}$, M.J. Levine ${ }^{3}$, V. Lindenstruth ${ }^{2}$, M.A. Lisa ${ }^{2}$, K. Marks ${ }^{2}$, C. McParland ${ }^{2}$, T.S. McShane ${ }^{4}$, J. Meier ${ }^{4}$, M.T. Nguyen ${ }^{3}$, D.L. Olson ${ }^{2}$, I. Sakrejda ${ }^{2}$, J. Schambach ${ }^{1}$ R.A.Scheetz ${ }^{3}$, N.T.B. Stone ${ }^{2}$, C.E. Tuld ${ }^{2}$, G. Visser ${ }^{2}, C$. Vu ${ }^{2}, H$. Wieman ${ }^{2}, E$. Yee and the STAR Collaboration

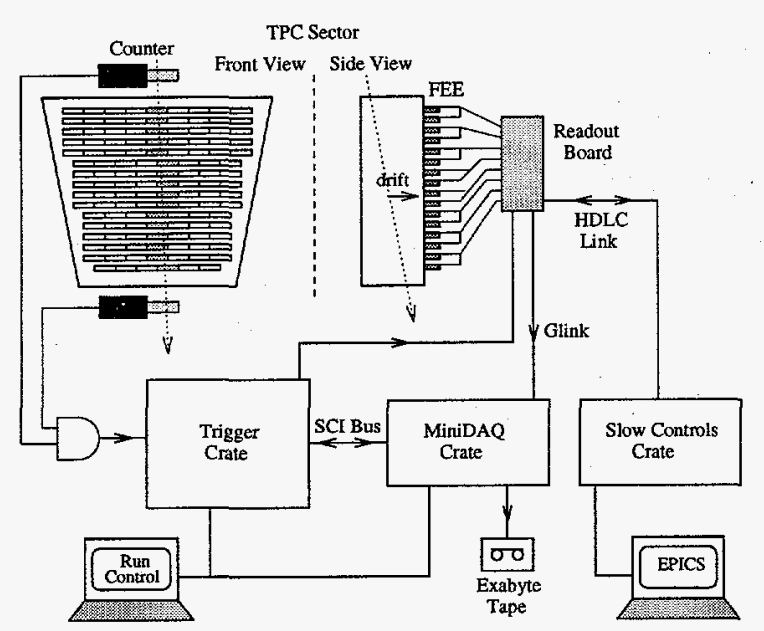

Figure 1: TPC system test cosmic-ray setup.

The STAR TPC system test assembles components at their current stage of development, testing functionality and interactions in a semirealistic environment. Preliminary results have been reported at conferences[1],[2].

A typical setup is shown in Fig. 1. A TPC subsector is mounted in a field cage with a $10-\mathrm{cm}$ drift region; 20 front-end cards and a prototype readout board instrument up to 640 pads. After a trigger, data are transmitted to "MiniDAQ" to be stored on tape and/or analyzed on line. System parameters are monitored by an EPICSbased slow controls system, while run control and a state manager coordinate the systems.

The front-end electronics gain, noise and stability have been studied, while cosmic-ray and laser tracks are used to debug and tune the analysis codes, as well as to test the hardware. A reconstructed track is shown in Fig. 2; rectangles mark the instrumented areas. A fully instru- mented sector will soon be available for testing, leading to TPC field-cage tests in spring, 1997.

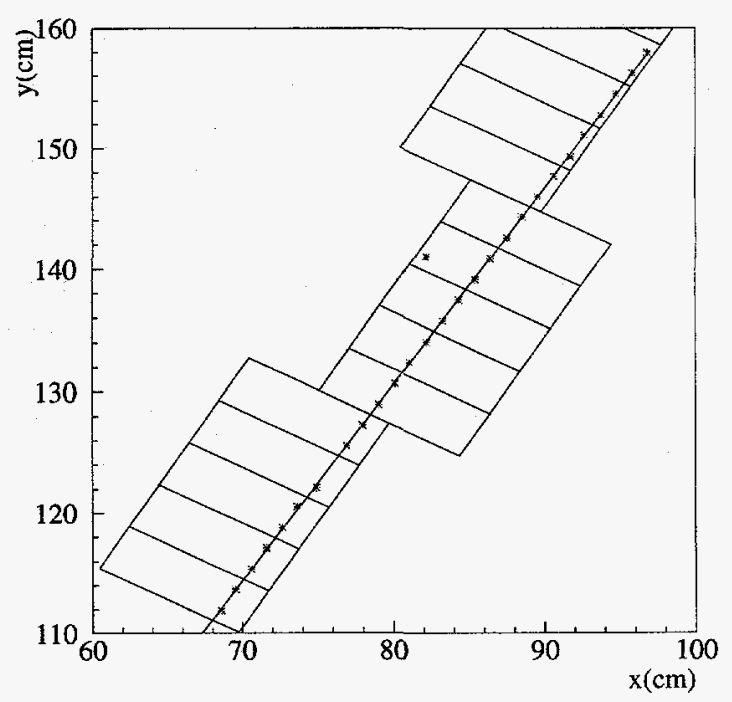

Figure 2: Reconstructed cosmic-ray track.

${ }^{1}$ University of Texas at Austin

${ }^{2}$ Lawrence Berkeley National Laboratory

${ }^{3}$ Brookhaven National Laboratory

${ }^{4}$ Creighton University

${ }^{5}$ Univ. Calif. Space Sciences Laboratory

${ }^{6}$ Purdue University

${ }^{7}$ Moscow Engineering Physics Institute

\section{References}

[1] Presented by I. Sakrejda, APS DNP Meeting, Indianapolis, IN., Oct. 2-5, 1996.

[2] Presented by J. Chrin, IEEE Nuclear Science Symposium, Anaheim, CA., Nov. 3-9, 1996. 


\section{Front End Electronics for the STAR TPC \\ F. S. Bieser, J. Hunter, S. R. Klein, C. Vu, and the STAR Collaboration}

The front end electronics (FEE) for the STAR TPC receives signals from the 136,600 pads on the TPC, amplifies them, shapes them, and digitizes them with a 512 time sample, $6 / 12 \mathrm{MHz}$, 10 bit waveform digitization system[1]. The high channel count requires a highly integrated design to minimize size, power, and costs, and to maximize reliability[2].

The analog functionality is contained in two custom $1.2 \mu$ CMOS chips, the STAR Amplifier/Shaper (SAS), which contains a low noise preamplifier, shaper (with tail correction), and output buffer[3], and the SCA, which contains a 512 time bucket switched capacitor array, plus a 12 bit ADC. Both chips are 16 channels wide, with 32 channels ( 2 chip sets) packaged onto 2.9 " by 7" FEE cards. The FEE cards contain all of the analog circuitry, and plug onto the TPC sectors, eliminating cabling and associated ground loops. A water cooled aluminum bar maintains FEE temperature within $0.5^{\circ} \mathrm{C}$, necessary for accurate $\mathrm{dE} / \mathrm{dx}$ measurement in the TPC.

The FEE cards are read out by readout boards, which control 36 FEE cards (1152 channels) of FEE. Data is sent from the readont boards to STAR DAQ over a fiber optic link at $1.2 \mathrm{Gbit} / \mathrm{sec}$. A trigger bus provides trigger, abort and calibration event functionality, while a slow controls link provides for monitoring and control, such as turning groups of FEE cards on and off. The readout board sequencer, readout buffers, and control are implemented in.FPGA's; the system can read out events at $200 \mathrm{~Hz}$.

$20 \mathrm{FEE}$ cards and a prototype readout board are now in use in the STAR system test[4]. These tests have shown that the system meets it's requirements, and that the on-detector noise levels match the bench levels. Output stability with temperature, humidity and time have all been monitored, and grounding and cooling addressed.
The FEE cards went into low rate production ( $4 \%$ build, or 240 cards) at the end of 1996; full production is slated to begin in 1997, to be complete at STAR turn on in 1999. Readout board prototyping is almost complete, and production should follow a similar schedule.

Roughly 25,000 channels of this electronics will also be used for the STAR forward TPC's. This application takes advantage of the flexibility of the design; the SAS shaping time is adjusted to 400 nsec FWHM, and the SCA sampling clock and number of samples are reduced. Because of the reduced noise and output slew rate, SAS power consumption can be reduced to about $1 / 3$ of that in the main TPC. The chips and circuitry will be repackaged to fit the allowable space. STAR will also provide 3840 channels of this electronics (plus spares) to the BRAHMS collaboration at RHIC, for use with their TPC's.

\section{References}

[1] S. Klein et al., "Front End Electronics for the STAR TPC", IEEE Transactions on Nuclear Science 43, 1768 (1996).

[2] S. Klein, et al., "The STAR TPC Front End Electronics", presented at the First Workshop for LHC Electronics", Lisbon, Portugal, September 11-15, 1995, and published in the proceedings.

[3] E. Beuville et al., "A Low Noise AmplifierShaper with Tail Correction for the STAR Detector", IEEE Transactions on Nuclear Science 43, 1619 (1996).

[4] W. Betts et al., Results from the STAR TPC System Test., preprint CU (Creighton U.) -PHY-NP 96/03, Nov. 1996, submitted to IEEE Transactions on Nuclear Science. 


\section{Triggering in the STAR Experiment at RHIC}

Eleanor Judd and the STAR trigger group

The STAR collaboration was formed to investigate the behavior of matter under conditions of extremely high temperature and density at RHIC. One particular goal is to search for the existence of a deconfined state of quarks and gluons, the quark-gluon plasma. The UCB contingent in STAR is part of the group responsible for triggering the experiment. The major goals of the trigger group are:

- Analyze data from every RHIC crossing in the trigger detectors.

- Issue triggers based on the total charged multiplicity, the energy deposited in the calorimeter and the interaction position.

- Abort events based on a more detailed analysis of the trigger detector data and on data from slower detectors.

- Issue triggers to fast detectors (EMC) while the slow detectors (TPC and SVT) are busy.

- Inform DAQ when an event is ready to be built and taped.

- Implement enough scalars to enable crosssections to be calculated.

The UCB STAR collaborators have concentrated on designing and building the custom hardware for the early levels of the trigger, and the control software for the complete trigger system.

Over the past year a prototype Data Storage and Manipulation board (DSM) and Trigger Control Unit (TCU) have been built and tested. In the final system a tree of 16-channel DSM boards will be used to store the data from the trigger detectors every RHIC crossing. The tree will also calculate triggerable quantities; total multiplicity, total energy, etc.... The output from each branch of the tree will then be collected in the last DSM board, which feeds the TCU. The prototype DSM board had just two input channels, but the rest of its functionality was the same as for the production board. A fast, high precision, TDC, used as input to one branch of the DSM tree has also been prototyped.

The prototype TCU board was very close to the final production model. The pre-scalars and trigger word look-up tables were fully implemented, and so were most of the output FIFOs. The board could also drive the trigger word on the VME backplane to be distributed to the main detector front-end electronics.

A prototype SCI-PCI (SPSB) card was designed and built for use as the internal trigger network. This should allow the trigger group to use a Scalable Coherent Interface (SCI) network to communicate between processors in many different VME crates. The prototype has been successfully used to connect the two processors that make up the trigger-daq interface.

In software, the Level 1 controller, which reads out the TCU and feeds tokens back to the TCU, and the trigger-daq interface (TDI), which informs minidaq of an event, have been written and used extensively in testing the hardware.

All the trigger pieces have now been integrated into an on-going system test at LBL, along with a TPC sector, minidaq, slow controls and experiment controls. The trigger can reliably detect a cosmic-ray induced coincidence between two scintillators placed around the TPC sector. The TCU can issue a trigger to the TPC front-end cards. Level 1 can read out the TCU FIFOs and inform minidaq that an event has occurred.

Finally, the whole STAR trigger group has just been through a successful, internal STAR review. The review committee was very impressed with the power and flexibility of the trigger system and the project can now move to the production phase. 


\title{
STAR Analysis Framework*
}

\author{
D. L. Olson, C. E. Tull,, , D. Prindle§
}

The analysis framework in STAR (called STAF) generally provides the interface between the physicists doing data analysis and the off-line computing facilities. STAF provides an encapsulation for data analysis algorithms permitting the integration of components provided by many physicists with those components which provide the rest of the computing environment, i.e., data access, user interface, control logic, etc.

This framework, developed in C++ and following an interface standard for component software (CORBA) $\neq$ was released to the collaboration during the summer and process of moving the pre-existing simulation and analysis software into the new framework is nearly complete.

There are many components to this framework as shown in figure 1 and some of the major ones are:

- DUI - unix-like user interface to the hierarchical data structures

- DIO - interface to disk, network and tape data input \& output

- AMI - interface to user-written analysis code

- TBR - table browser for viewing data

A new user interface based on Tcl/Tk is being developed for STAF (and the whole STAR offline environment) at UW, called the STAR Offline Notebook (figure 2). This will provide a graphic interface to the available set of programs and off-line activities. This interface should permit more reliable usage of the off-line software as well as enabling non-experts to play a significant role in the simulations and data analysis activities.

Besides its current use for STAR simulations software, STAF is being used for analysis of actual cosmic-ray test data in the STAR System Test environment**.
Footnotes and References

* http://www.rhic.bnl.gov/STAR/star.html

+ LBNL ICSD

§ UW NPL

thttp://www.omg.org/orb.htm

** See separate report on STAR system test.

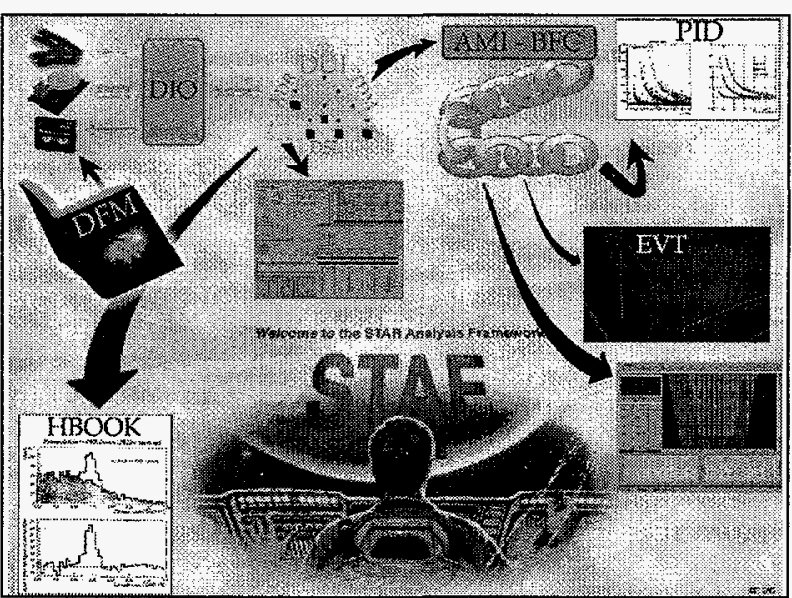

Fig. 1. Artistic rendition of a scientist controlling the components of the STAR analysis framework.

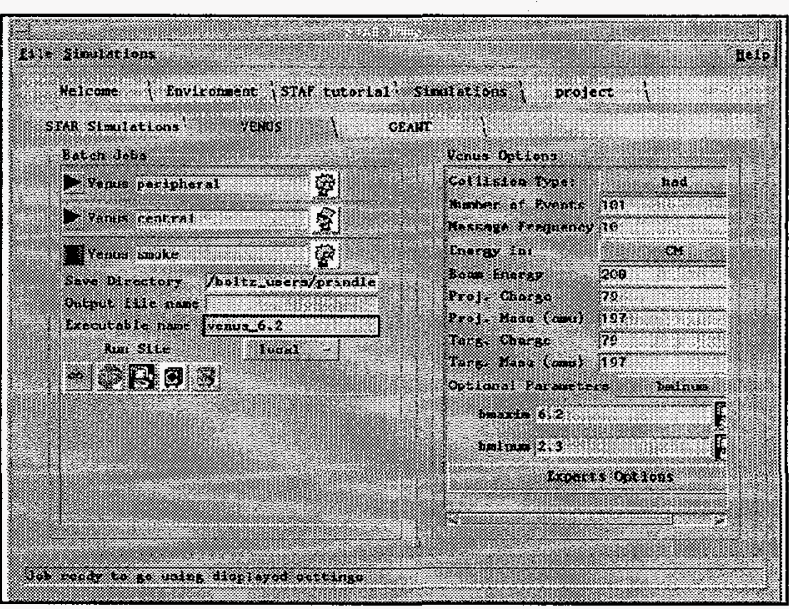

Fig. 2. Screen view of STAR Offline Notebook. Programs or activities are selected by clicking on the notebook tabs. 


\title{
Simulations and Analysis Software for the STAR TPC
}

\author{
P.M. Jacobs, M.A. Lisa, I.M. Sakrejda, N.T.B. Stone and the STAR Collaboration
}

A set of programs to simulate and analyze data from the STAR Time Projection Chamber has been developed. This package includes the detector simulations, reconstruction of the particle tracks in the detector, and the particle identification software. The simulations and reconstruction modules are accompanied by the calibrations and geometry packages.

The first step in the simulation chain is to track particles produced in a $100 \mathrm{GeV} /$ nucleon $\mathrm{Au}+\mathrm{Au}$ collision through the STAR detector. This task is performed by GSTAR [1], a module based on the CERN simulations package GEANT. It accounts for the hadronic interactions of the particles produced during the collision with the detector material, multiple scattering, decays in flight, and many other effects. The next step is designed to study impact of the detector performance on the ionization deposited in the volume of the TPC by charged products of the collision. Two packages were provided to perform this task. First, the TPC Slow Simulator (tss) [2] includes effects of the charge drifting in the electric and magnetic field and the signal processing by the Front-End Electronics (FEE). The Slow Simulator writes output in the raw data format. This simulated data then serves as an input to the cluster finder and hit reconstruction software [3]. The other option is to forgo detailed simulations of the detector and use a parametrization of the reconstructed position resolution and the hit finding efficiency to directly create reconstructed hits. The second option is implemented in the form of the TPC Fast Simulator, (tfs) [4]. After the hits are reconstructed or simulated, the next step in data analysis is the track reconstruction. This task is performed by the TPC tracking module - tpt [5]. High tracking efficiency (>95\%) and good momentum resolution provide input for the particle identification based on the $\mathrm{dE} / \mathrm{dx}$ analysis.

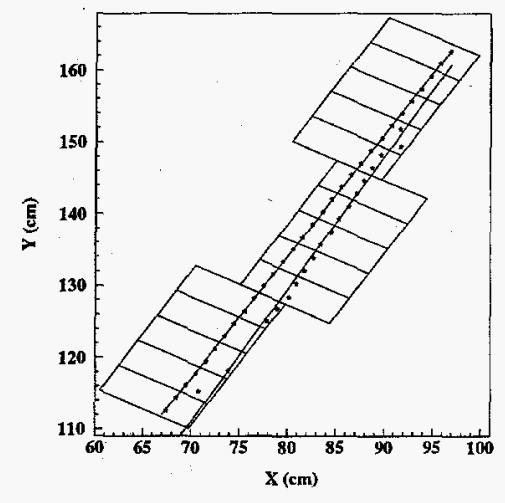

Figure 1: Reconstructed laser tracks in a test module. The rectangles outline parts of the detector that have been instrumented so far.

Recently parts of the TPC software chain were tested (Fig. 1) during the STAR TPC System Test. Data taken in the fall of 1996 agreed with the simulated detector parameters. The simulation and reconstruction software chain is used to develope physics analysis software so that, when the experiment starts taking data in the year 1999, a prompt and reliable analysis will be possible.

\section{References}

[1] P.M. Jacobs GSTAR: A GEANT-based Detector Simulation Chain for STAR, STAR Note SN0235.

[2] Wen Gong; The STAR-TPC Slow Simulator, Star Note SN0197

[3] M.A. Lisa; The STAR TPC Cluster finder Hit finder, Star Note SN0238.

[4] P.G. Jones; The STAR Fast TPC Simulation (A User's Guide), STAR Note SN0056

[5] I.M. Sakrejda; Tracking for the STAR TPC, STAR Note SN0190 


\title{
The PDSF Computing Facility at LBNL
}

\author{
R. Jeff Porter
}

During the past year, the computing facility known as PDSF was relocated from it original home at the SSC laboratory in Texas to LBNL. PDSF is a multi-platform workstation farm designed to support the computing needs of detector development projects at the SSC laboratory. It was moved to LBNL to take advantage of both the scientific computing environment provided by the National Energy Research Scientific Computing Center (NERSC) and the strong presence in Nuclear Science (NS) and High Energy Physics (HEP) research found at LBNL. The PDSF facility is being supported jointly by NERSC, the Nuclear Science Division, and the Physics Division. PDSF will be used to meet the computing requirements of large-scale data analysis and detector simulation projects in HEP and NS investigations.

PDSF at LBNL was initially brought on-line in a temporary form during the summer of this past year before moving to its final home in October. The Phase I hardware configuration, shown in Fig. 1, was designed using equipment obtained from the SSC laboratory. The current status of the facility can be found on the PDSF WWW pages noted in Fig. 1. At present, the PDSF systems include,

- 32 HP 9000/735(25) workstations

- 32 SUN Sparc10 workstations

- 2 80-Gbyte Data-Vault disk storage

- 2 SGI Challenge-L data servers

- 100BaseT external ethernet connection

- FDDI internal PDSF network

The nuclear science research community has been very active in making use of PDSF. A listing of NS groups currently using or preparing to use the facility is given below.
STAR is running event and detector simulation projects on PDSF.

NA49 is preparing to use PDSF for data analysis and physics simulations.

PHENIX is preparing to run event and detector simulation projects on PDSF.

E895 is preparing to use PDSF as a data analysis facility.

EOS-Bevalac is analyzing data on PDSF

SNO will use PDSF in developing data analysis systems and plans to analyze data on PDSF.

Gammasphere is preparing to extend its data analysis program to PDSF.

PDSF Configuration at Phase I http://www.nersc.gov/hardware/PDSF

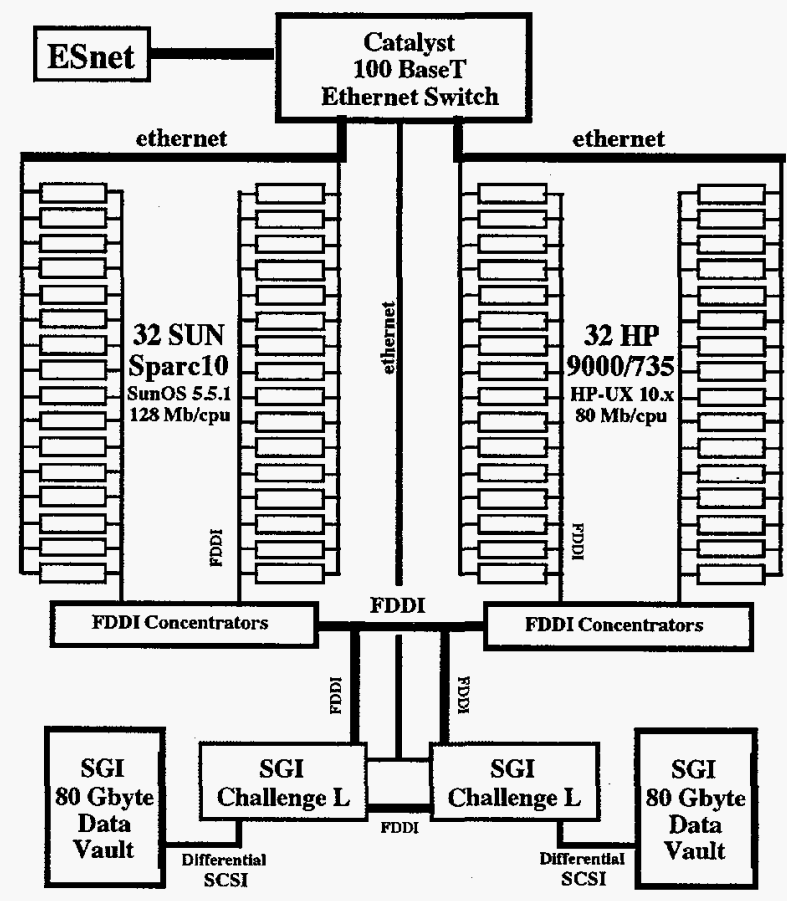

Figure 1: PDSF hardware configuration 


\section{Development of a Micro TPC Vertex Detector}

H. Wieman, E. Beuville, $†$ M. Burks, $\ddagger$ D. Fritz,, S. Margetis, F. Retiere, S. Trentalange, $\S$ and R. Wells $†$

We are developing a new Micro TPC vertex detector that will be capable of tracking in the high track density environments which will be encountered in the STAR experiment at RHIC and in the ALICE experiment at LHC. This detector can handle track densities of 10 tracks $/ \mathrm{cm}^{2}$, approaching the capabilities of silicon devices.[1] It has, however, the important advantage that it is essentially massless in comparison. This greatly reduces problems due to multiple scattering and secondary interactions, which is of utmost importance for vertex detectors. This technology could provide a significant improvement over silicon in these experiments particularly at low pt where exciting results have been predicted for heavy ion collisions. In STAR the combined radiation length of the beam pipe plus silicon vertex detector averages $4.8 \%$. Replacing the silicon detector with a Micro TPC will reduce the combined radiation length to $0.85 \%$, an improvement factor of over 5 .

The Micro TPC will use micro-strip gas chambers (MSGCs) to read out the signal. The fine pitch of these devices is well matched to the low diffusion that can be achieved with a short drift distance in dimethyl-ether (DME). This combination makes it possible to achieve much better two-track resolution than has been possible with other TPCs. The main thrusts of the program have been the demonstration that electrons can be drifted in DME over the planned drift distance of $15 \mathrm{~cm}$ without significant attenuation and the development of a MSGC system that can constructed with the required channel density. We have built a drift cell, shown in fig. 1, in which we measured electron attenuations of less than $0.1 \%$ loss per $\mathrm{cm}$.[2] We have also produced a MSGC on a silicon amplifier chip and demonstrated suitable operation.

The MSGC was fabricated with the amplifier using a standard HP CMOS process. A postprocess layer of partially conductive SiCarbide was added to correctly shape the electric field at the readout anode. Integrating the detector with the amplifier on a single chip reduces noise permitting low gas gain operation. We have successfully tested the detectoramplifier chip using an $\mathrm{Fe}^{55} \mathrm{X}$-ray source. The amplifier noise was 110 electrons rms which allowed excellent signal to noise operation with a gas gain of only 20 .

\section{Footnotes and References}

+ Engineering Division LBNL

$\ddagger$ Univ. of Arkansas

$\S$ Univ. Calif. Los Angeles

1. S. Margetis, H. Wieman, W. Gong and the STAR Collaboration, "VTX: A compact TPC with Microstrip readout for STAR", LBL-37384, NSD Ann. Rep. 1994

2. M. Burks, S. Trentalange, S. Margetis and $\mathrm{H}$. Wieman, "Electron drift parameters in dimethyl ether", NIM to be published

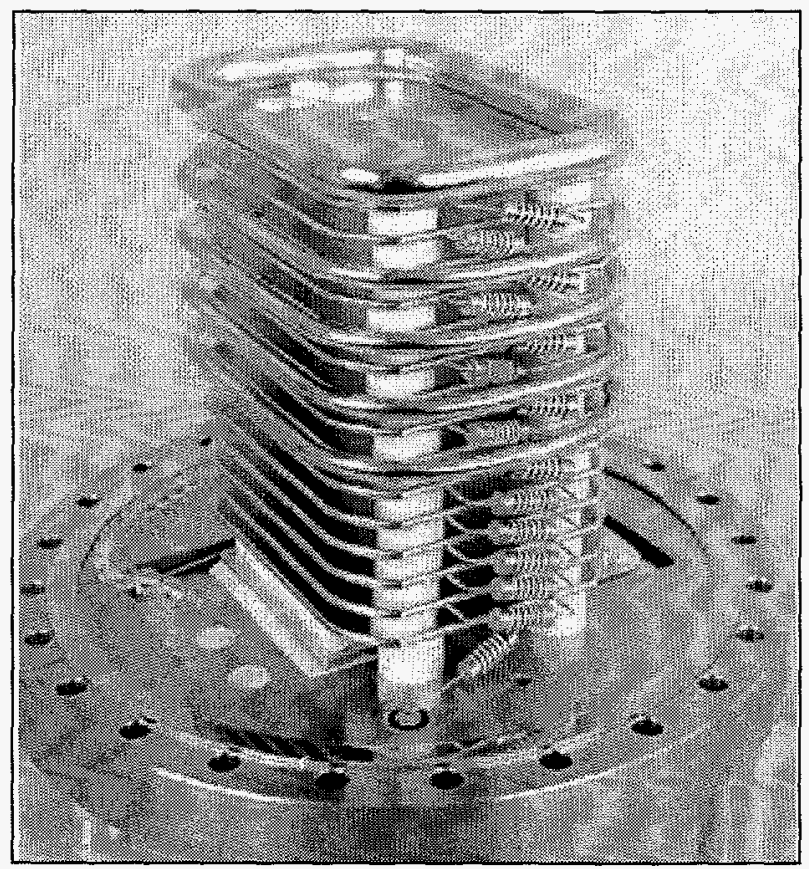

Fig. 1. Drift cell for testing electron attenuation while drifting through DME gas 


\title{
Production and Detection of Hyperfragments in High Energy Nucleus-Nucleus Collisions*
}

\author{
$Y$. D. He and P. B. Price
}

We discuss electromagnetic production of hyperfragments induced by the extremely intense flux of photons generated by heavy target nuclei and seen by rapidly moving projectile nuclei in high energy distant nucleus-nucleus collisions. The cross section estimated using WilliamsWeizsäcker method is found to be accessible to future experiments at the Relativistic Heavy Ion Collider. We also discuss its signature and detection.

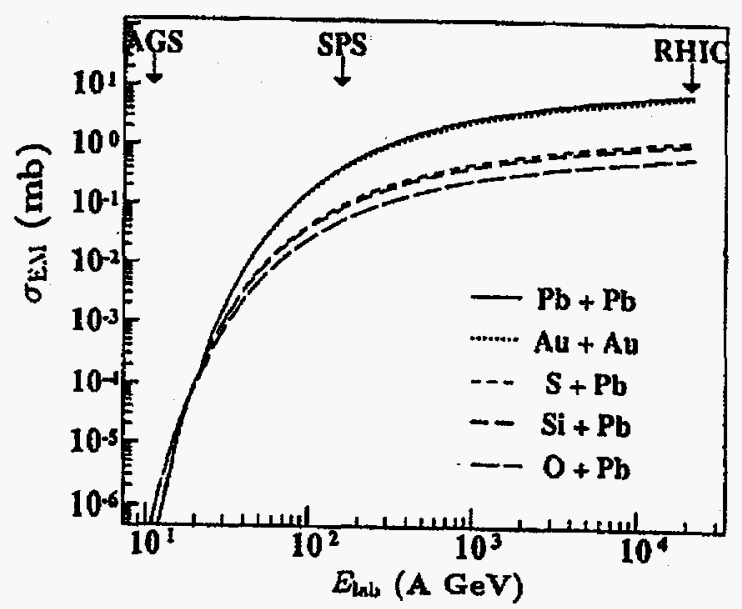

Fig. 2 - Estimated cross sections for electromagnetic production of hyperfragments in nucleus-nucleus collisions.

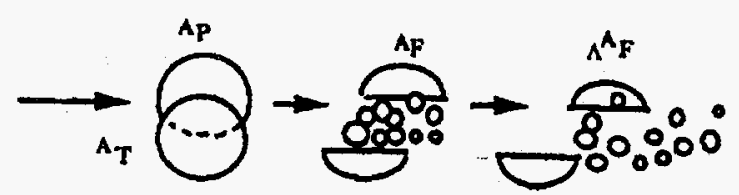

(a) NUCI.EAR PRODUCTION

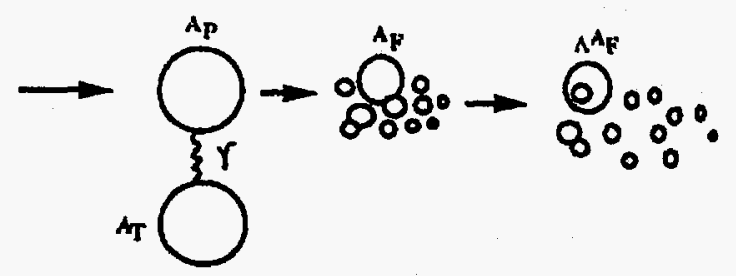

(b) BLECTROMAGNETC PRODUCTION

Fig. 1 - Hyperfragment production in high energy nucleus-nucleus collisions by nuclear and electromagnetic modes.
*Abstract of a paper in the Proceedings of $24^{\text {th }}$ International Conference on Cosmic Rays, Rome, Italy 1 (1995) 64-67. 


\title{
Coherent Nuclear Interactions at RHIC
}

\author{
Spencer Klein and Evan Scannapieco
}

When ultrarelativistic nuclei undergo interactions at large impact parameters, the entire nucleus can interact as a single particle, via a coherent electromagnetic (photon) or Pomeronic fields. Collisions between these fields have a broad physics interest.

Two-photon collisions result from the interactions of the electromagnetic fields of the two nuclei. This process can be modelled by the Weizsacker-Williams method as the collision of two almost-real photons; any spin 0 or spin 2 state with internal or external charge is accessible[1]. We have calculated the photon fluxes, collision rates and final state production rates for a variety of final states at RHIC[2]. For final state energies below $1.5 \mathrm{GeV}$, RHIC will have a higher $\gamma \gamma$ luminosity than $e^{+} e^{-}$colliders such as CESR and LEP II.

Photon-Pomeron and double-Pomeron interactions are also possible. Pomerons are a representation of colorless interactions involving the strong force, such as $p p$ elastic scattering, and having the same quantum numbers as the vacuum. They represent the absorptive part of the interaction cross section. At RHIC, interactions such as $\gamma+P \rightarrow V$, where $V$ is a vector meson will produce large numbers of $\rho, \phi$ and $\mathrm{J} / \psi$. Double Pomeron interactions can lead to spin 0 or 2 final states, at higher energies than can be produced in $\gamma \gamma$ collisions. These interactions measure the photon-Pomeron and nucleonPomeron coupling, and shed light on the nature of the Pomeron.

We have investigated the feasibility of studying these interactions, using two-photon collisions as our model[2]. These interactions have signatures that can be distinguished from backgrounds such as grazing nuclear collisions, beam gas interactions, cosmic rays, and the like. Since the nuclei interact as a single particle, the perpendicular momentum scale $p_{\perp}$ of the prod- ucts is determined by the nuclear size $R$, with $p_{\perp}=\hbar c / R$, or about $30 \mathrm{MeV} / \mathrm{c}$ for gold. In contrast, incoherent background has a $p_{\perp}$ scale given by QCD, typically $300 \mathrm{MeV} / \mathrm{c}$. For the reactions of interest studied here, there are typically 2 or 4 charged and 0 neutrals, allowing for complete reconstruction of the event, and hence accurate $p_{\perp}$ determination. Because the photons and Pomerons are both color singlets, the final state should not be accompanied by additional particles, and should be well separated in rapidity space from both beams.

We have simulated $\gamma \gamma$ collisions to a number of final states: $\mu^{+} \mu^{-}, \tau^{+} \tau^{-} \rightarrow l^{+} l^{-}, \eta_{c} \rightarrow$ $K^{* 0} K^{+} \pi^{-}, \eta^{\prime}, f_{0}(975), f_{2}(1270)$, and $\rho^{0} \rho^{0} \rightarrow$ $\pi^{+} \pi^{-} \pi^{+} \pi^{-}$, and found the rates and acceptances in the STAR detector. Rates are high, and, except for the $\eta_{c}$, all states may be seen and studied with good statistics; the $\eta_{c}$ is marginal. We have also simulated the backgrounds due to grazing nuclear collisions and beam gas events. Except for the $\eta_{c}$, signal to noise ratios are good.

We have also developed a scheme for STAR to trigger on these events at level $0,1,2$, and 3 . This trigger selects events based on multiplicity and topology in levels 0 through 2 , adding tracking information to find the total charge, vertex position and momentum balance at level 3. At each stage, we have demonstrated good acceptance, while using a small fraction of the available bandwidth.

\section{References}

[1] S. Klein, "Two-Photon Physics at RHIC", in Photon '95, ed. D. J. Miller, S. L. Cartwright and V. Khoze, World Scientific, 1995.

[2] Spencer Klein and Evan Scannapieco, "TwoPhoton Physics with STAR, STAR Note 243, March 22, 1996. 


\title{
NSD Pre-College Educational Activities
}

\author{
Howard S. Matis, Chairman NSD Educational Committee
}

The Nuclear Science Division has established an active education outreach program which has developed several strategies to introduce nuclear physics into the high school and middle school classroom. Our goals are to prepare educational literature that teachers can introduce into their curriculum and have experimental equipment that they can borrow.

To disseminate this material, we have developed a teaching center in cooperation with Oakland Public School's Chabot Science Center. Using a modest grant (the Meggers Award) from the American Institute of Physics, sufficient equipment was purchased to provide classroom hands-on activities centered on the general topic of "The ABCs of Radioactivity." This unit covers general properties of radioactivity and common radioactive materials found in the home and environment. Division scientists have given several workshops at Chabot Science Center for teachers. After attending the workshop, teachers have the opportunity to borrow the detectors for classroom use.

A teacher working with several members of the Nuclear Science Division created a laboratory manual that describes several experiments and activities on radioactivity. These experiments can be done with either equipment borrowed from the Chabot Science Center or from items bought from a science supply house. We have been distributing this manual to teachers. Last summer, we put these experiments on our educational web site.

To continue our work on the $A B C^{\prime} s$ : of Radioactivity, we have created a low cost cosmic ray detector that has been used in several schools and a 25 minute video on Cosmic Rays and Radiation. We have recently received another Meggers Foundation grant to add this equipment to the Chabot Science Center.

Another educational activity for the Division in the past few years has been participation in the annual California Chemathon which is attended by more than 2000 high school students. During the Chemathon, NSD scientists provide several "hands-on" demonstrations of nuclear science research. More than 400 students visit this popular station; it provides students from many diverse backgrounds an opportunity to talk to "real scientists" and see what they do. In 1995, we presented many of our demonstrations at the LBL Open House.

The major activity of 1996 has been the creation of a "Nuclear Science Wall Chart." This chart will illustrate the basics of Nuclear Physics coupled with the most exciting research that is being done in the world. We intend to make this chart widely available to schools. In August of 1996, we joined the organization called Contemporary Physics Education Project (CPEP). CPEP is the creator of the popular Particle Physics Wall chart and the recently produced Fusion chart. NSD is working with scientists and teachers from throughout the nation to produce this chart.

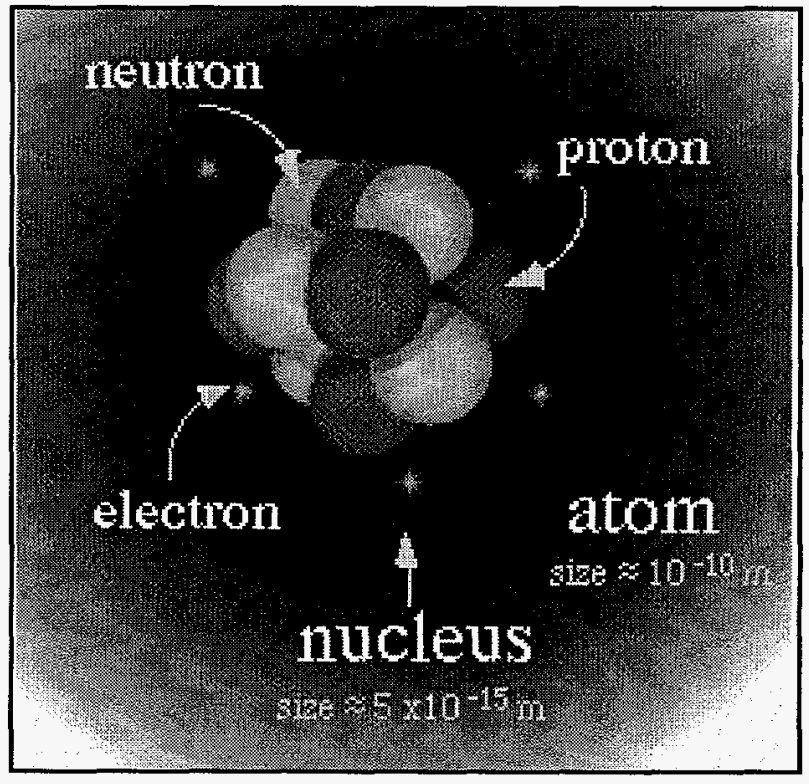

Fig. 1. One of the graphics that can be found in the Nuclear Science Educational Web site -http://user88.lbl.gov/NSD_docs/ed.html. 


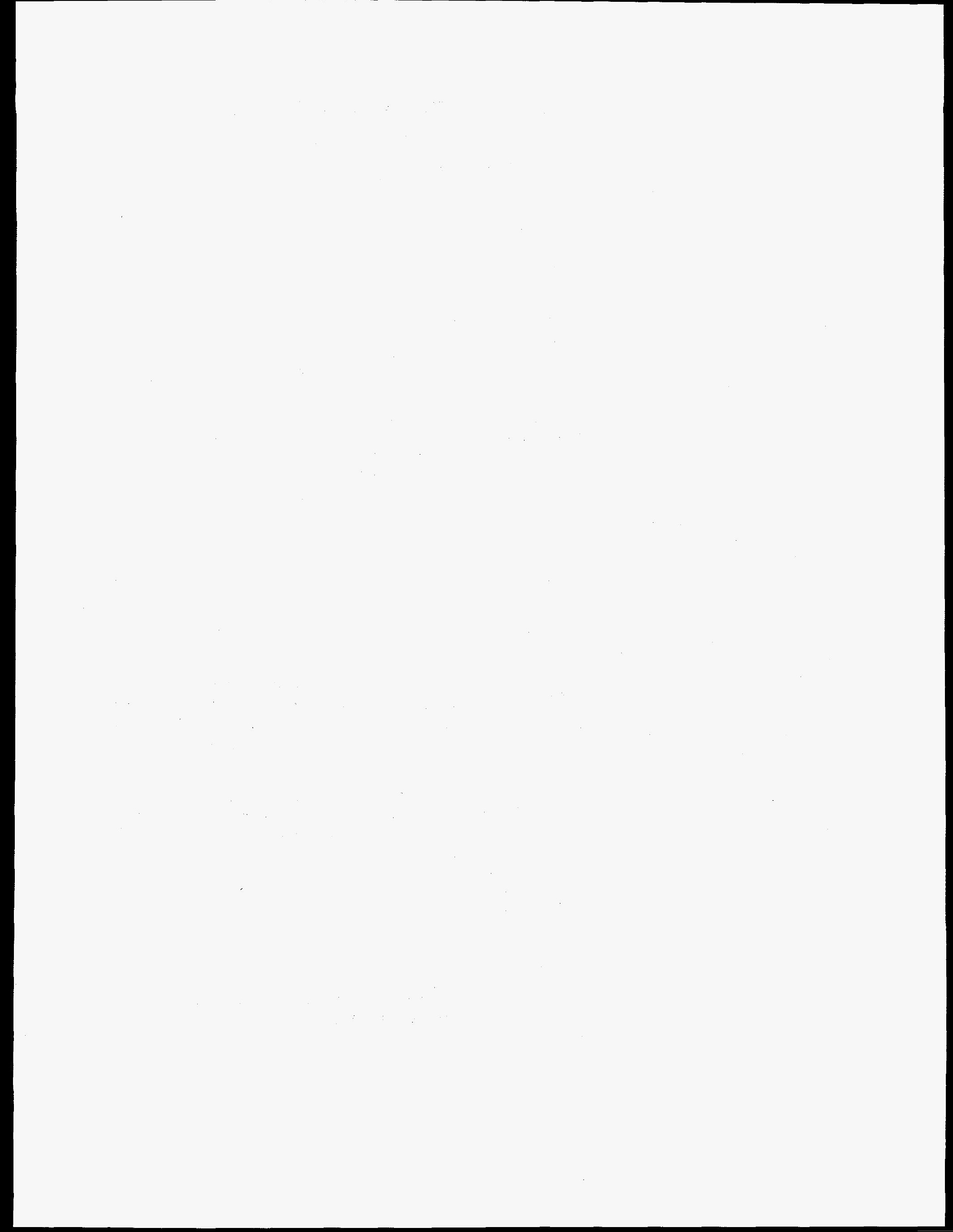




\title{
Study of Medium-induced Parton Energy Loss in $\gamma+$ jet Events of High-Energy Heavy-Ion Collisions *
}

\author{
$X$. N. Wang and Z. Huang ${ }^{\dagger}$
}

Among the proposed hard probes of dense matter in high-energy heavy-ion collisions, large transverse momentum jets or partons are especially useful because they interact strongly with the medium. Jet quenching due to the mediuminduced radiative energy loss of a high-energy parton propagating through a dense medium can provide important information on the properties of the medium and interaction processes that may lead to partial thermalization of the produced parton system.

In QCD, one can measure the radiative energy loss indirectly via the modification of the jet fragmentation function. In order to measure the fragmentation function one has to first determine the initial energy of the fragmenting parton. We propose to measure the particle $p_{T}$ distribution in the opposite transverse direction of a tagged direct photon. Since a direct photon in the central rapidity region $(y=0)$ is always accompanied by a jet in the opposite transverse direction with roughly equal transverse energy, the $p_{T}$ distribution of particles in that direction is directly related to the jet fragmentation function with known initial energy, $E_{T}^{\text {jet }} \approx E_{T}^{\gamma}$. In such $\gamma+$ jet events, one can easily extract the fragmentation function from the experimental data. By comparing the extracted jet fragmentation function in $A A$ to that in $p p$ collisions, one can then measure the modification of the fragmentation function and determine the parton energy loss.

In this paper, we have studied in detail the effect of parton energy loss on the jet fragmentation function as extracted from the $p_{T}$ spectrum in the opposite direction of a triggered direct photon. In particular, we have taken into account the $E_{T}$ smearing of the jet due to initial state radiations associated with the $\gamma+$ jet processes. We have shown that the particle spectrum from jet fragmentation at $p_{T} \sim E_{T}^{\gamma}$ is very sensitive to the $E_{T}$ broadening from initial and final state scatterings with beam partons (see Fig. 1). One can then use our proposed measurement to determine the $E_{T}$ broadening in

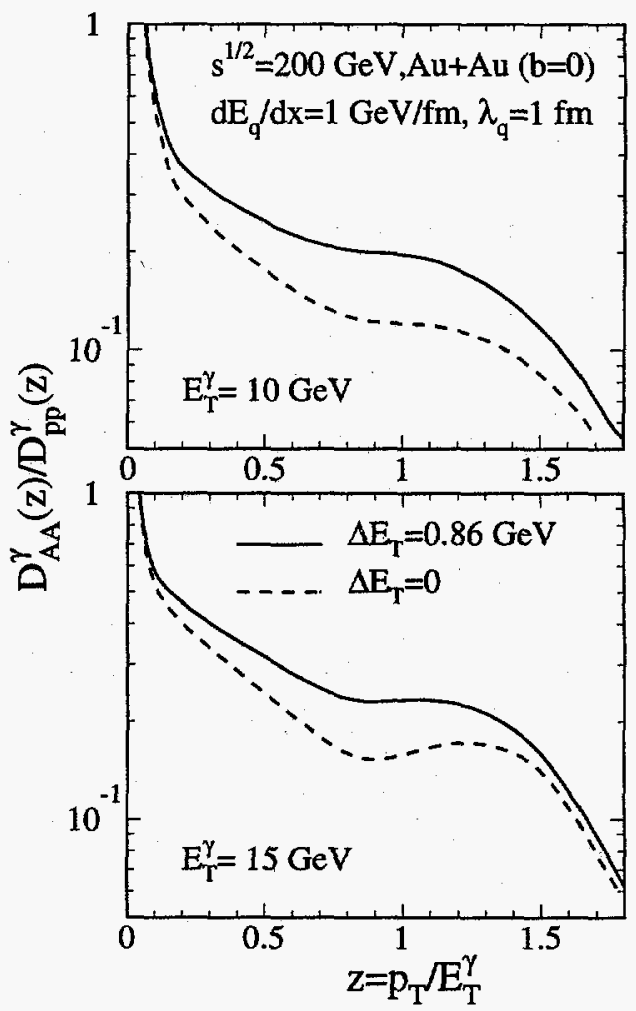

Figure 1: The modification factor for the inclusive fragmentation function of photon-tagged jets with (solid) and without (dashed) $E_{T}$ broadening due to initial parton scatterings.

$p A$ collisions. This small but finite effect must then be subtracted out when one determines the medium-induced parton energy loss in $A A$ collisions.

\footnotetext{
*LBNL-39742, 1997, submitted to Phys. Rev. C.

${ }^{\dagger}$ Department of Physics, University of Arizona, Tucson, AZ 85721
} 


\section{Jet Quenching in the Direction Opposite to a Tagged Photon in High-Energy Heavy-Ion Collisions *

\author{
X. N. Wang Z. Huang ${ }^{\dagger}$ and Ina Sarcevic ${ }^{\dagger}$
}

The study of large $p_{T}$ jets can probe their energy loss due to inelastic scatterings inside dense matter or a quark-gluon plasma. Because of the enormous background in high-energy heavy-ion collisions, the conventional calorimetric study cannot measure the jet energy to the accuracy required to determine the energy loss.

In this work, we proposed thestudy of jet quenching in high-energy heavy-ion collisions by measuring the $p_{T}$ distribution of charged hadrons in the opposite direction of a tagged direct photon. A direct photon is produced by quark-antiquark annihilation or quark(antiquark)-gluon Compton scatterings in which a gluon or quark(antiquark) jet is also produced in the opposite direction of the photon. By tagging a direct photon with a given transverse energy $E_{T}^{\gamma}$, one can avoid the uncertainties associated with the jet production cross section. One can also determine the initial transverse energy of the produced jet, $E_{T} \approx E_{T}^{\gamma}$, from momentum conservation, modulo calculable corrections from initial state radiations.

In such events, the $p_{T}$ spectrum of charged hadrons from jet fragmentation in the opposite direction of the tagged photon is a very good approximation of the jet fragmentation function and is estimated to be well above the background which can be reliably subtracted at moderately large $p_{T}$. We demonstrate that comparison between the extracted fragmentation function in $A A$ and $p p$ collisions can be used to determine the jet energy loss and the interaction meanfree-path in the dense matter produced in highenergy heavy-ion collisions.

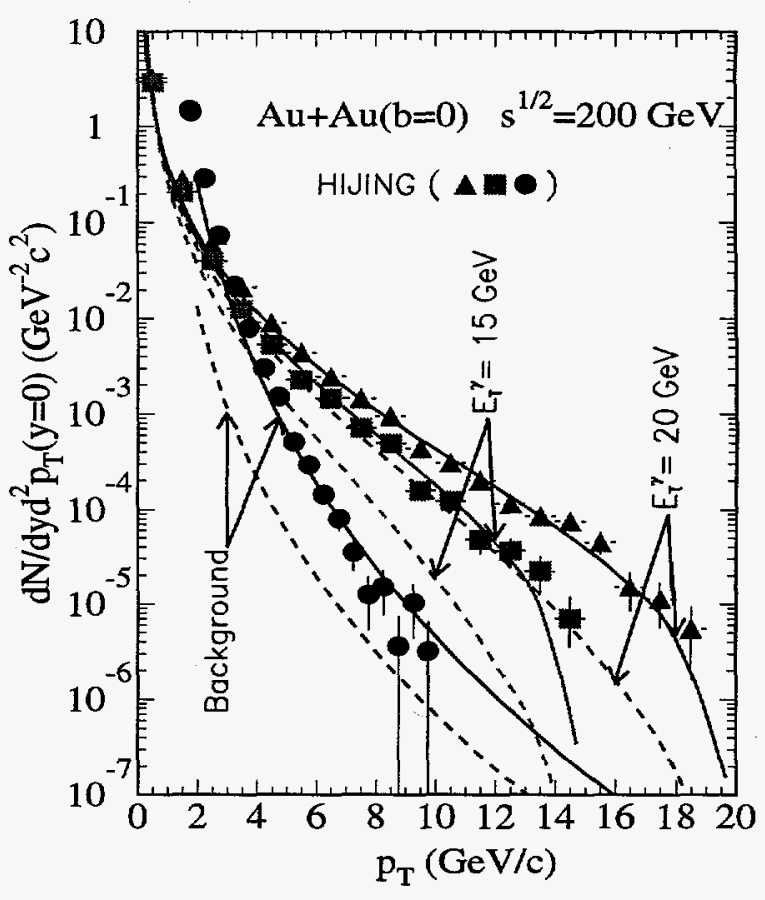

Figure 1: The differential $p_{T}$ spectrum of charged particles from the fragmentation of a photon-tagged jet with $E_{T}^{\gamma}=15,20 \mathrm{GeV}$ and the underlying background in central $A u+A u$ collisions at $\sqrt{s}=200 \mathrm{GeV}$. The direct photon is restricted to $|y| \leq \Delta y / 2=0.5$. Charged particles are limited to the same rapidity range and in the opposite direction of the photon, $\left|\phi-\phi_{\gamma}-\pi\right| \leq \Delta \phi / 2=1.0$. Solid lines are perturbative calculations and points are HIJING simulations of $10 \mathrm{~K}$ events. The dashed lines are calculations with jet energy loss, $d E_{q} / d x=1$ $\mathrm{GeV} / \mathrm{fm}$ and the mean-free-path $\lambda_{q}=1 \mathrm{fm}$.

\footnotetext{
*Phys. Rev. Lett. 77, 231 (1996).

${ }^{\dagger}$ Department of Physics, University of Arizona, Tucson, AZ 85721
} 


\title{
Dilepton and Photon Production from a Coherent Pion Oscillation *
}

\author{
Z. Huang ${ }^{\dagger}$ and $X$. N. Wang
}

Electromagnetic probes are known to be ideal probes of dense hadronic matter owing to the fact that they escape the strong interaction region once produced without further final state interactions and thus carry the information on the early dynamical evolution. In this work, we studied the dilepton and direct photon production from the classical pion field in the context of a disoriented chiral condensate (DCC), or most generally, a nonequilibrium pion cloud. We developed a general formalism for the dilepton and photon emissions in the presence of a classical electromagnetic current. Since the electromagnetic current coincides with the third component of the isovector current, the isospin angular oscillation of the condensate field can be a significant source of electromagnetic emission in the low mass (transverse momentum) region.

The dilepton differential distribution with respect to the lepton pair 4-momentum $q$ can be derived as

$$
\frac{d N_{\ell+\ell^{-}}}{d^{4} q}=\frac{\alpha^{2}}{6 \pi^{3}} \frac{B}{q^{4}}\left[q^{\mu} q^{\nu}-q^{2} g^{\mu \nu}\right] J_{\mu}^{\mathrm{cl}}(q) J_{\nu}^{\mathrm{cl} *}(q)
$$

where $J_{\mu}^{\mathrm{cl}}(q)$ is the electromagnetic current carried - by the classical pion fields, $B=[1+$ $\left.2 m_{\ell}^{2} / q^{2}\right]\left[1-4 m_{\ell}^{2} / q^{2}\right]^{1 / 2}$ and $m_{\ell}$ is the lepton mass.

Given an analytical solvable model where a general class of solutions for the pion fields can be obtained, we calculate the dilepton spectrum. as shown in Fig. 1.

Both the dilepton and the photon spectra fall off exponentially for large transverse momentum which is characteristic of coherent production from a finite domain. The electromagnetic emission from a DCC domain is thus only important in the low momentum region where the spectra increase as some inverse power law of the momentum.

The result depends on a constant $a_{3}$ in the third component of the isospin which is conserved, $J_{\mu}^{(3)}=a_{3} f_{\pi}^{2} x_{\mu} / \tau^{2}$. The constant $a_{3}$ can

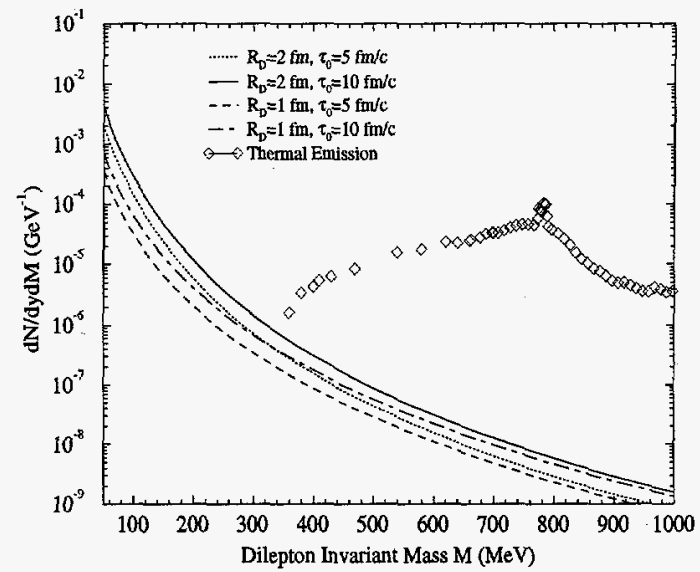

Figure 1: The dilepton invariant mass spectrum for different choices of initial time scale $\tau_{0}$ and the coherent field domain size $R_{D}$. The initial energy density is assumed to be $\epsilon_{0}=0.1$ $\mathrm{GeV} / \mathrm{fm}^{3}$. The typical thermal spectrum due to the $\pi-\pi$ annihilation is also plotted for a comparison

be related to the initial energy density using the symmetry argument,

$$
\left\langle a_{3}^{2}\right\rangle=\frac{1}{6} \frac{\epsilon_{0} \tau_{0}^{2}}{2 f_{\pi}^{2}} \quad(i=1,2,3) .
$$

Most notably, there does not exist a pion mass threshold for dilepton production from a coherent field: the spectrum rises even below $M=$ $2 m_{\pi}$. The finite pion mass plays no roles in the conserved isovector current whose third component directly couples to the photon. Therefore, the coherent field is most effective in producing lepton pairs in the low mass region $M<2 m_{\pi}$.

\footnotetext{
${ }^{*}$ Phys. Lett B383, 457 (1996).

'Department of Physics, University of Arizona, Tucson, AZ 85721
} 


\title{
Thermal Equilibration in an Expanding Parton Plasma *
}

\author{
H. Heiselberg ${ }^{\dagger}$ and X. N. Wang
}

The approach to thermal equilibrium in relativistic heavy ion collisions is dictated by the competition between expansion and parton interactions. If the expansion is much more rapid than the typical collision time among partons, the expansion is closer to free-streaming than hydrodynamic expansion. Only at times on the order of the collision time may the parton gas reach local thermal equilibrium and expand hydrodynamically. Furthermore, the time dependence of the collision time (or the relaxation time) will determine whether the system can eventually reach local thermal equilibrium because of the competition between expansion and parton interactions. If the collision time increases rapidly with time, the parton system may never thermalize, leading only to a free-streaming limit. The collision time, therefore, is a very important quantity which in turn depends sensitively on the infrared behavior of parton interactions.

What determine the thermalization processes are the transport rates which are free of logarithmic divergences after the resummation of thermal loops. This is because thermalization is achieved to leading order mainly through momentum changes by elastic scatterings. The resultant transport times for a system near thermal equilibrium behave like

$$
\frac{1}{\tau_{\mathrm{tr}}} \sim T \alpha_{s}^{2} \ln \left(1 / \alpha_{s}\right)
$$

to leading order in $\alpha_{s}$.

For a system near local thermal equilibrium, the time dependence of the transport times is through the temperature according to Eq. (1). This dependence is in general slower than $1 / \tau$ and thus can lead to local thermal equilibrium according to arguments based on the relaxation time approximation. We can solve the Boltzmann equation in the relaxation time approximation and demonstrate how the time dependence of the relaxation time will affect the approach to thermal equilibrium.

From Fig. 1, one can see that if the time dependence of the relaxation time is weaker than linear, the thermal equilibrium limit will

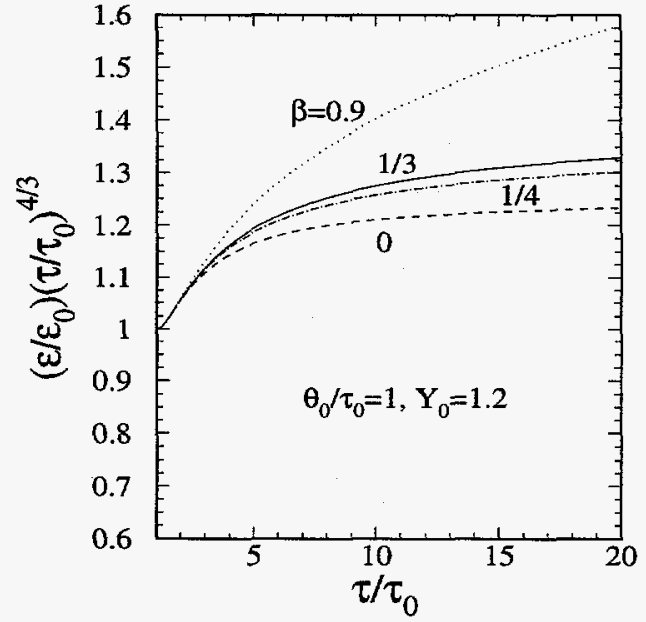

Figure 1: Time evolution of $G\left(\tau / \tau_{0}\right)\left(\tau / \tau_{0}\right)^{1 / 3}=\left(\epsilon / \epsilon_{0}\right)\left(\tau / \tau_{0}\right)^{4 / 3}$ according to the solution to Boltzmann equation, for different time dependence of the relaxation time $\theta=\theta_{0}\left(\tau / \tau_{0}\right)^{\beta}$, with $\theta_{0} / \tau_{0}=1, \beta=0.9$ (dotted), $1 / 3$ (solid), $1 / 4$ (dot-dashed)and 0 (dashed line). $Y_{0}=1.2$ is the width of the initial rapidity distribution.

eventually be reached. For a time dependence stronger than the linear one, the system will never thermalize, only leading to a free-stream limit. For an exact linear time dependence, the system will reach an asymptotic state between free-streaming and thermal equilibrium. We find that the thermalization process also depends on the initial condition of the system. The deviation of the initial momentum distribution from an isotropic one in the longitudinal direction determines the initial approach to thermal equilibrium. This initial approach will then carry its inertia throughout the whole thermalization process. This "memory effect" can be seen from the dependence of the final total entropy production on the initial momentum distributions.

\footnotetext{
"Nucl. Phys. B462, 389 (1996).

${ }^{\dagger}$ NORDITA, Blegdamsvej 17, DK-2100 Copenhagen $\emptyset$., Denmark
} 


\title{
Domain Structure of a Disoriented Chiral Condensate from a Wavelet Perspective *
}

\author{
Z. Huang I. Sarcevic $^{\dagger}$, R. Thews ${ }^{\dagger}$ and X. N. Wang
}

Theoretical investigations of nonequilibrium dynamics using the classical linear $\sigma$-model have found some evidence for the formation of Disoriented Chiral Condensate (DCC) or the growth of long wavelength pion modes. Although the precise dimension of a typical domain or cluster is still under debate, it seems likely, especially in heavy-ion collisions, that many domains or clusters could be formed in the large interaction volume. If there are many uncorrelated small domains, the integrated probability distribution of the neutral pion fraction $f$ emitted from a disoriented region, predicted to be $P(f)=1 / 2 \sqrt{f}$, would become Gaussian, following the Central Limit Theorem.

Normally DCC domains are localized in coordinate space. If they develop collective motion in the course of their time evolution, they should also appear localized in momentum space. In order to disentangle the DCC domain structure in high energy heavy-ion collisions, we propose a new method which emphasizes not only the behavior of the probability distribution in the full phase space region but also its fluctuation in rapidity $\eta$ or azimuthal angle $\phi$. It is a multiresolution analysis performed by a discrete wavelet transformation (DWT) which has been found effective in systematically detecting structures on various scales in turbulence, astrophysics, and multiparticle production. We demonstrate that the DWT proves to be very useful in identifying and measuring the DCC domain structures simultaneously in terms of their size (in scale) and location (in space). Since it is likely that there are other physical scales accompanying the typical DCC domain scale in a physical process, the multiresolution feature of the DWT is essential for identification of the structures of interest. It acts like a mathematical microscope which can zoom in or out to various scales at each location. Due to the completeness and orthogonality of the DWT basis, there will be no information loss.

Shown in Fig. 1 are the wavelet power spectrum in rapidity of different samples. The ran-

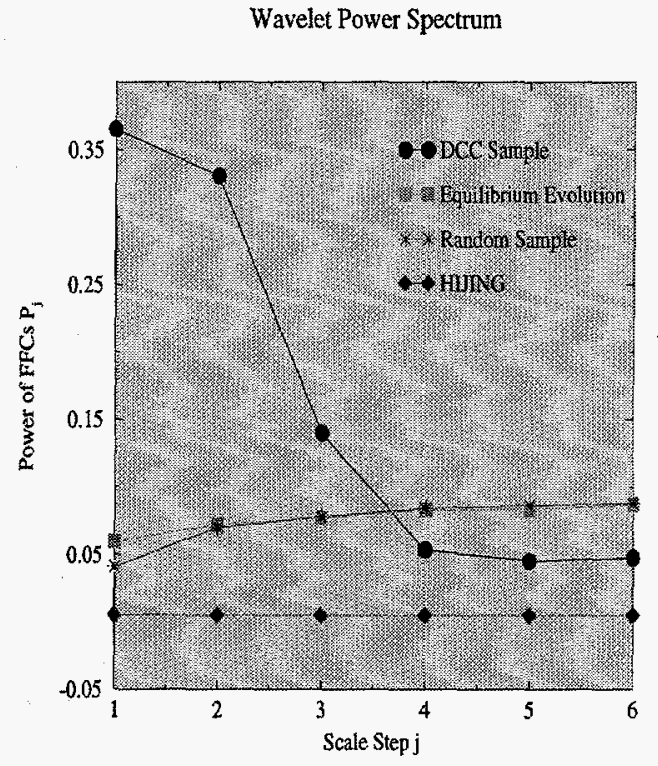

Figure 1: The wavelet power spectra for different dynamical scenarios.

dom noise sample features a flat power spectrum, i.e. the power of fluctuations is the same at any scale. For the DCC sample there should be a flat spectrum when the scale is small and some power build-up should show up when the scale becomes larger than the DCC scale. The crossing point in Fig. 1 is found to be at $j_{d}=3.6$ which unambiguously suggests the existence of the DCC clustering with a typical size of $\Delta \eta_{d}=2 \eta_{\max } / 2^{j_{d}} \simeq 0.8$ units in rapidity. Also plotted is the power spectrum from HIJING Monte Carlo data which is also flat, consistent with the random noise case.

The existence of a plateau structure in the "wavelet power spectrum is important in that one may attempt to define an effective "temperature" inside a domain structure where the fluctuations are relatively "stable" against the scale change.

*Phys. Rev. D 54,750 (1996).

${ }^{\dagger}$ Department of Physics, University of Arizona, Tucson, AZ 85721 


\section{$J / \psi$ Suppression in an Equilibrating Parton Plasma * \\ Xiao-Ming Xu ${ }^{\dagger}$ D. Kharzeev ${ }^{\ddagger}$ H. Satz and X. N. Wang}

$J / \psi$ suppression due to color screening has been proposed to probe deconfinement. This requires that the interactions of $J / \psi$ with hadrons and deconfined partons are different. A partonbased calculation of $J / \psi$-hadron cross section via an operator product expansion gives a very small break-up cross section at low energies. Only at very high energies, this cross section will reach its asymptotic value of a few $\mathrm{mb}$. Therefore, a slow $J / \psi$ is very unlikely to be absorbed inside a hadron gas of reasonable temperature.

On the other hand, a deconfined partonic system contains much harder gluons which can easily break up a $J / \psi$. A study of the energy dependence of the gluon- $J / \psi$ inelastic cross section shows a strong peak just above the break-up threshold of the gluon energy, $\epsilon_{0}=2 M_{D}-M_{J / \psi}$, where $M_{J / \psi}$ and $M_{D}$ are the $J / \psi$ and $\mathrm{D}$ meson masses, respectively. In the pre-equilibrium stage, i.e., before the partons have reached equilibrium, the average parton transverse momentum is sufficiently large to break up a $J / \psi$, provided the partons are deconfined. The dissociation of the $J / \psi$ will continue during the whole equilibration process until the effective temperature drops below a certain value or the beginning of hadronization, whichever takes place first. Therefore measurements of $J / \psi$ suppression can probe the deconfinement of the early partonic system and shed light on the subsequent equilibration process, provided that possible nuclear effects on the production of $Q \bar{Q}$ pairs and on pre-resonance charmonium states are understood and taken into account.

In this paper we first calculated the thermal gluon- $J / \psi$ dissociation cross section at different temperatures and for different $J / \psi$ transverse momentum. We then follow the evolution of an initially produced parton gas toward equilibrium and calculate the resulting total survival probability of a $J / \psi$ and its $p_{T}$-dependence.

Shown in Fig. 1 are the $J / \psi$ survival probabilities in the deconfined and equilibrating parton plasma at RHIC and LHC energies with initial conditions given by HIJING Monte Carlo simulations. We find that there is stronger $J / \psi$ sup-

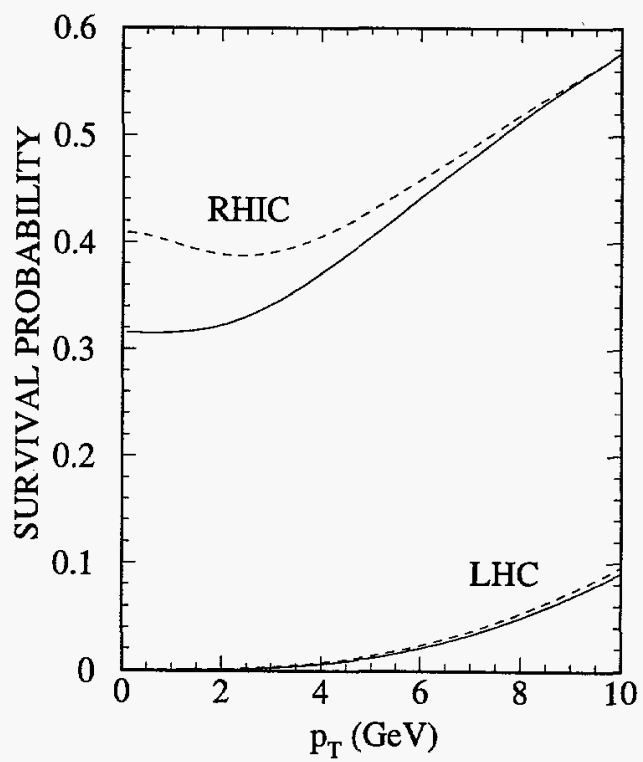

Figure 1: The survival probability of $J / \psi$ in an equilibrating parton plasma at RHIC and LHC energies with initial conditions given by HIJING.

pression at LHC than at RHIC energy, due both to the higher initial parton densities and longer lifetime of the parton plasma. The increase of the survival probabilities with the $J / \psi$ transverse momentum is a consequence of the decrease of the thermal cross section with increasing $P_{T}$ at high temperatures and the shorter time spent by a higher- $p_{T} J / \psi$ inside the parton plasma.

For a parton system with a low initial temperature (below $300 \mathrm{MeV}$ ), the $p_{T}$ dependence of the survival probability should be flatter. One can therefore use the $p_{T}$ dependence to shed light on the initial temperature and the evolution of the system.

\footnotetext{
${ }^{*}$ Phys. Rev, C 53, 3051 (1996).

${ }^{\dagger}$ Theory Division, Shanghai Institute of Nuclear Research, Chinese Academy of Sciences, P.O. Box 800204, Shanghai 201800, China

${ }^{\ddagger}$ Fakultät für Physik, Universität Bielefeld

D-33501 Bielefeld, Germany
} 


\title{
Screening of Initial Parton Production in Ultrarelativistic Heavy-ion Collisions *
}

\author{
K. J. Eskola $\dagger^{\dagger} B$. Müller ${ }^{\ddagger} a b d X$. N. Wang
}

Because the dominant QCD parton cross sections are singular in the soft scattering limit, most model calculations of minijet production via perturbative $\mathrm{QCD}$ ( $\mathrm{pQCD}$ ) have introduced an infrared cut-off, $p_{0}$, corresponding to the smallest permissible transverse momentum transfer in a $2 \rightarrow 2$ parton scattering, to which perturbative QCD can still be applied. This cutoff is used in most of the models to separate perturbative hard processes from nonperturbative soft interactions. Since there is no distinct boundary between soft and hard physics, both the hard and soft part of the interaction in this scheme are very sensitive to the cut-off $p_{0}$.

Heavy ion collisions differ from $p p$ collisions in that minijets are produced in large number, so that a medium of minijets is formed. In the space-time evolution of a heavy-ion collision soft particle production will be completed after semihard and hard processes. Therefore, the soft interactions are expected to be screened by interactions with the semihard quanta (minijets). We here take a step further by proposing that the perturbative semihard particle production should also be screened by the processes which have happened even earlier, i.e., by the harder processes.

We considered color screening of initial semihard parton production in a phenomenological but self-consistent manner. We first compute the static electric screening mass of the parton system by using the number distributions of produced minijets with transverse momentum larger than $p_{T}$ and by taking into account the formation time of the minijets. We then use the obtained electric screening mass as a regulator in divergent $t$ and $u$-channel sub-processes for the production of partons with transverse momentum smaller than $p_{T}$. We iteratively compute the lowest order minijet cross section with a feedback from the screening mass towards the region $p_{T} \sim \mu_{D} \lesssim 2 \mathrm{GeV}$.

In Fig. 1 we show the screening mass $\mu_{D}$ and the screened one-jet cross section as functions of $p_{T}$. We can see that the medium of produced

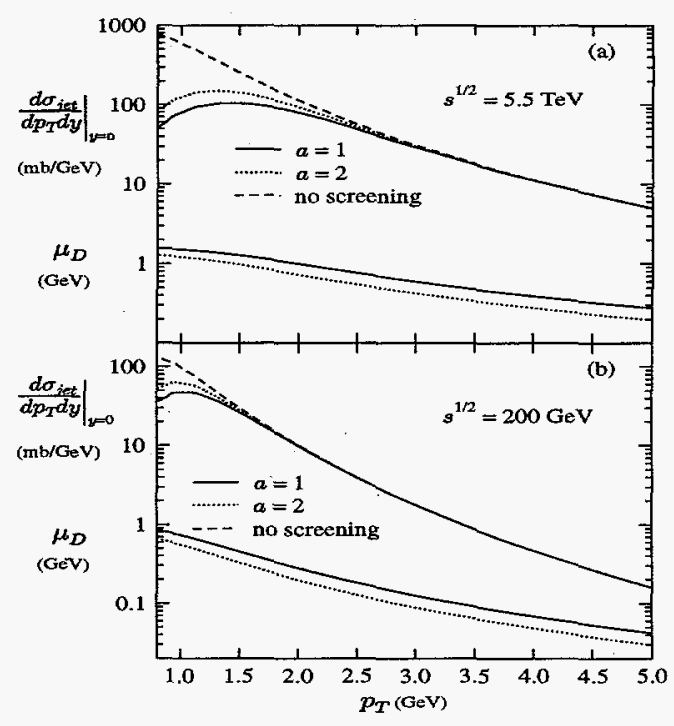

Fig. 2

Figure 1: (a) Differential minijet cross section $d \sigma_{\text {jet }} / d p_{T} d y$ at $y=0$ and screening mass $\mu_{D}$ as functions of transverse momentum $p_{T}$ in a $p p$ sub-process of a central $A A$ collision at $\sqrt{s}=5.5 A \mathrm{TeV}$ with $A=200$. The dashed line is for the unscreened cross section, the solid and dotted curves for the screened case with different parameters a. Nuclear shadowing is not taken into account. (b) The same as in panel (a) but for $\sqrt{s}=200 \mathrm{AGeV}$.

minijets regulates the rapid growth of the jet cross section. Finally, at $\mu_{D} \sim p_{T}$, the cross section saturates. For sufficiently large nuclei and for high collision energies this happens in the perturbative region $p_{T} \gg \Lambda_{Q C D}$.

\footnotetext{
${ }^{*}$ Phys. Lett. B374, 20 (1996).

${ }^{\dagger}$ TH Division, CERN, CH-1211 Geneve 23, Switzerland ${ }^{\dagger}$ Department of Physics, Duke University, Durham, NC 27708-0305
} 


\title{
Partial $U(1)_{A}$ Restoration and $\eta$ Enhancement in High-Energy Heavy-Ion Collisions *
}

\author{
Z. Huang ${ }^{\dagger}$ and $X$. N. Wang
}

It is well known that the $U(1)_{A}$ symmetry in QCD is violated by the axial anomaly present at the quantum level and thus cannot give rise to the Goldstone boson which would occur when $U\left(N_{f}\right) \times U\left(N_{f}\right)$ chiral symmetry is spontaneously broken. The $U(1)_{A}$ particle, known as $\eta^{\prime}(958)$ in the $N_{f}=3$ case, acquires an additional mass through the quantum tunneling effects mediated by instantons, breaking up the mass degeneracy with pions, kaons and $\eta$ 's. The $\eta(547)$ particle also acquires an additional mass through the mixing with the $\eta^{\prime}$. It is believed that at high temperatures the instanton effects are suppressed due to the Debye-type screening. Then one expects a practical restoration of $U(1)_{A}$ at high temperatures.

One of the consequences of $U(1)_{A}$ restoration is the enhancement of $\eta$ particle production at small and intermediate transverse momenta due to the softening of its mass at high temperatures. However, the final yield of the $\eta$ particles and their $p_{t}$ distributions both depend crucially on the chemical and thermal equilibrating processes involving the $\eta$.

In this paper, we examine the rates of various processes relevant for the thermal $\eta$ particle production, in particular, whether or not the $\eta$ can decouple early enough from the thermal system expected to be produced in relativistic heavy-ion collisions. We present a theoretical calculation of thermal cross sections for the processes $\eta \eta \leftrightarrow \eta \eta$, $\pi \eta \leftrightarrow \pi \eta$ and $\eta \eta \leftrightarrow \pi \pi$, essential to the thermal and chemical equilibration. Our calculations are based on models which explicitly incorporate the $U(1)_{A}$ anomaly. We also assume an exponential suppression of the $U(1)_{A}$ anomaly due to the Debye-type screening of the instanton effect, which leads to the temperature dependence of the $\eta$ and $\eta^{\prime}$ masses. Our results, as shown in Fig. 1 and 2, suggest that the $\eta$ particles are out of chemical equilibrium long before the thermal freeze-out.

*Phys. Rev. D 53, 5034 (1996).

${ }^{\dagger}$ Department of Physics, University of Arizona, Tucson, AZ 85721

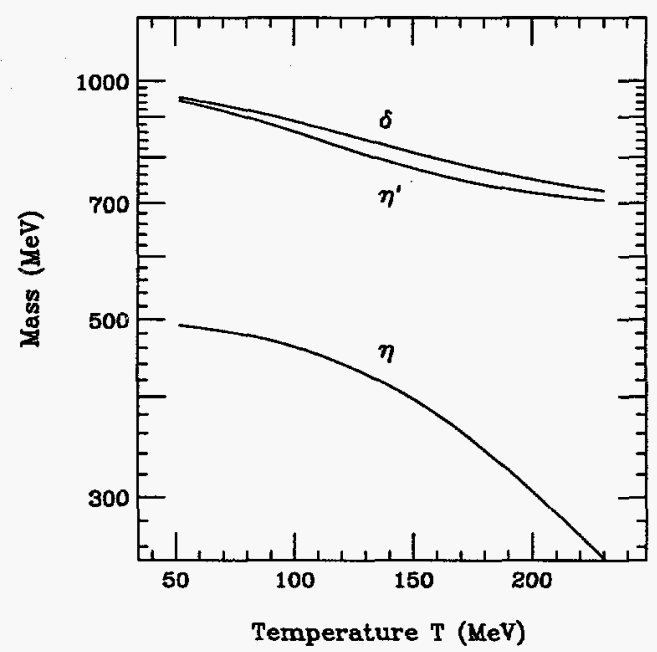

Figure 1: The temperature dependence of $m_{\eta}$, $m_{\eta^{\prime}}, m_{\delta}$.

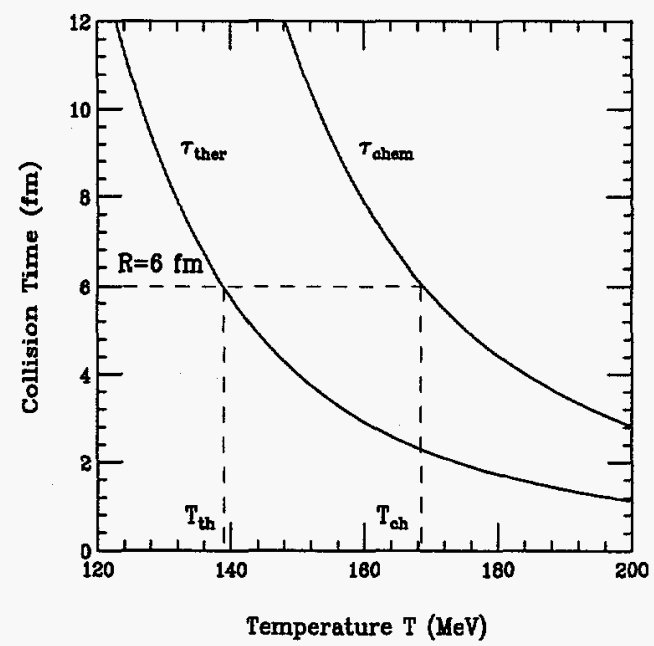

Figure 2: The characteristic time scales of the thermal and chemical equilibration for the $\eta$ particle. 


\title{
Study of Parton Energy Loss in High-energy Heavy-ion Collisions *
}

\author{
X. N. Wang
}

Medium-induced radiative energy loss of a high-energy parton traversing a dense QCD medium is interesting not only because it illustrates the importance of quantum interference effects in QCD, but also because it depends sensitively on the density of the medium and thus can be used as a probe of the dense matter formed in ultrarelativistic heavy-ion collisions. Unlike in the QED case, where one can measure directly the radiative energy loss of a fast electron, one cannot measure directly the energy loss of a fast leading parton in QCD. One can only measure parton energy loss indirectly via the modification of the jet fragmentation function and jet profile. Here, the effects of energy loss on single-particle distributions both in the normal central $A+A$ collisions and in events with a tagged direct photon with known transverse energy are reviewed.

The $p_{T}$ distribution of particles from jet fragmentation in a normal central heavy-ion collision can be calculated via the convolution of the fragmentation functions with the jet cross sections. In $A A$ collisions, one has to take into account the modification of the jet fragmentation functions due to parton energy loss inside the medium. Then, jet energy loss will result in the suppression of high $p_{T}$ particles as compared to $p p$ collisions. Therefore, the ratio,

$$
R_{A A}\left(p_{T}\right)=\frac{d N_{A A} / d y / d^{2} p_{T}}{\sigma_{p p} T_{A A}(0) d N_{p p} / d y / d^{2} p_{T}},
$$

at large $p_{T}$ in Fig. 1 is smaller than one due to the energy loss suffered by the jet partons. It, however, increases with $p_{T}$ because of the constant energy loss. The ratio is normalized to the effective total number of binary $p p$ collisions in a central $A A$ collision.

In order to study the modification of the fragmentation function due to energy loss, one might in principle measure the inclusive $p_{T}$ spectrum in the direction of a triggered jet. However, with the large background and its fluctuation due to hadrons from many other minijets and soft processes, the determination of the jet en-

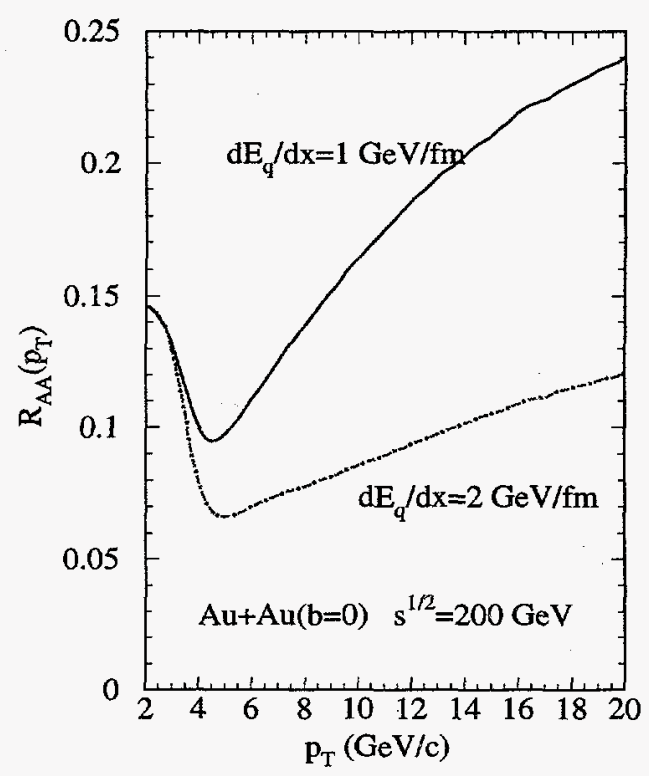

Figure 1: The ratio of charged particle $p_{T}$ spectrum in central $A u+A u$ collisions at $\sqrt{s}=200$ $\mathrm{GeV}$ over that of $p p$ collisions, normalized by the total binary nucleon-nucleon collisions in central $A u+A u$ collisions. The mean-free-path of a quark inside the medium is assumed to be $1 \mathrm{fm}$.

ergy is almost impossible. To overcome this difficulty, we proposed the study of the high $p_{T}$ particle spectrum in the opposite direction of a tagged direct photon. Direct photons are always accompanied by a jet in the opposite transverse direction. Even taking the initial state radiation into account, the average energy of the jet is approximately that of the tagged photon. One can therefore relate the $p_{T}$ distributions of hadrons in the opposite direction of a tagged photon to the fragmentation function of a jet with known initial energy and study the modification of the fragmentation function due to parton energy loss.

${ }^{*}$ LBNL-39408, in the proceedings of RHIC Summer Study 196. 


\title{
A pQCD-based Approach to Parton Production and Equilibration in High-Energy Nuclear Collisions *
}

\author{
X. N. Wang
}

In this report, I presented a perturbative QCD (pQCD)-based picture of ultrarelativistic heavyion collisions. In this framework, a nucleus in the infinite momentum frame consists of many partons (quarks and gluons). The interactions among these partons can be divided into perturbative, which can be described by pQCD calculations, and nonperturbative, which can only be modeled phenomenologically. I demonstrate that $\mathrm{pQCD}$ processes dominate the underlying dynamics of heavy-ion collisions at extremely high energies. It is then reasonable to assume that the evolution of the initially produced partons can be described by PQCD processes. Using the initial conditions estimated by the HIJING Monte Carlo model, the following picture emerges:

(1) During the early stages of ultrarelativistic heavy-ion collisions, hard or semihard parton scatterings, which happen in a time scale of about $0.2 \mathrm{fm} / c$, produce a hot and undersaturated parton gas. This parton gas is dominated by gluons and is far from chemical equilibrium. Multiple hard scatterings suffered by a single parton during this short period of time when the beam partons pass through each other are suppressed due to the interference embedded in the Glauber formula for multiple scatterings. Interference and parton fusion also lead to the depletion of small $x$ partons in the effective parton distributions inside a nucleus. This nuclear shadowing of parton distributions reduces the initial parton production.

(2) After the two beams of partons pass through each other, the produced parton gas in the central rapidity region starts its evolution toward (kinetic) thermalization and (chemical) equilibration through elastic scatterings and induced radiations. Further evolution of the parton gas toward a fully equilibrated parton plasma is dictated by the parton proliferation through induced radiation and gluon fusion. Though the gluon equilibration rate is reduced by the inclusion of the Landau-Pomeranchuk-Migdal effect, the gluon fugacity still increases rapidly toward its equilibrium value. Due to the consumption of energy by the additional parton production, the effective temperature of the parton plasma cools down considerably faster than the ideal Bjorken scaling solution. Therefore, the life time of the plasma is reduced to $4-6 \mathrm{fm} / c$ before the temperature drops below the QCD phase transition temperature.

(3) The evolution of the quark distribution always lags behind that of gluons due to a smaller equilibration rate and the initial density. For heavy quarks, the equilibration rate is even smaller. Take charm quarks for example. The thermal production during the equilibration period is much smaller than the initial direct production, due to the small initial gluon fugacity and the short lifetime during which the temperature remains high enough to produce charm quarks. For the same reason, dilepton and photon production during the evolution of the parton plasma is also small because of small quark number density. Therefore, observation of large charm and dilepton enhancement would imply high initial gluon and quark density and thus a longer life time of the parton plasma.

(4) Even though the initial parton system is not in full equilibrium, a study of color screening shows that the system is already in a deconfined state with large average momentum (or effective temperature). Such a deconfined parton system, though not in equilibrium, will dissociate hadronic states such as the $J / \psi$. It was shown that the $J / \psi$ can be substantially suppressed during the evolution of the parton plasma toward equilibrium. The measurements of this suppression can reveal the initial conditions and the evolution history of the parton plasma in highenergy heavy-ion collisions.

${ }^{*}$ LBNL-38145, 1997, Physics Report in press. 


\title{
Phenomenology of Charm and Bottom Production*
}

\author{
R. Vogt
}

Previous measurements of the $c \bar{c}$ production cross section at $\sqrt{S} \leq 63 \mathrm{GeV}$ suggested that the Born cross section underpredicted the data by a factor of two to three, called the $K$ factor after a similar situation in Drell-Yan production,

$$
K_{\text {exp }}=\frac{\sigma_{\text {data }}(A B \rightarrow Q \bar{Q})}{\sigma_{\text {theory }}(A B \rightarrow Q \bar{Q})},
$$

where $Q$ is the produced heavy quark. An analogous theoretical $K$ factor can be defined from the ratio of the next-to-leading order (NLO) to the Born cross sections,

$$
K_{\mathrm{th}}=\frac{\sigma_{\mathrm{NLO}}(A B \rightarrow Q \bar{Q})}{\sigma_{\mathrm{Born}}(A B \rightarrow Q \bar{Q})},
$$

where $\sigma_{\mathrm{NLO}}$ is the sum of the Born and $\mathcal{O}\left(\alpha_{s}\right)$ corrections. The NLO cross section is strongly dependent on the choice of the renormalization and factorization scales, $\mu_{R}$ and $\mu_{F}$, which determine both $K_{\exp }$ and $K_{\text {th }}$.

We discuss the scale dependence of $c$ and $b$ quark production and its influence on the $K$ factors. We adjust the scales and the heavy quark mass, $m_{Q}$, to achieve $K_{\exp }^{\mathrm{NLO}} \approx 1$ [1] keeping in mind that further corrections to the cross section could also be large. Our calculations are done with a Monte Carlo program developed by Nason and collaborators [2].

The physical cross section should be independent of the scale. If the perturbative expansion is convergent, i.e. if further higher-order corrections are small, at some scale the $\mathcal{O}\left(\alpha_{s}^{n+1}\right)$ contribution to the cross section should be smaller than the $\mathcal{O}\left(\alpha_{s}^{n}\right)$ contribution. If the scale dependence is strong, the perturbative expansion is untrustworthy. Since $K_{\text {th }}-1>1$, further higher-order corrections are needed, particularly for charm and bottom quarks which are rather "light" when $\sqrt{S}$ is large. Although the scales are, in principle, independent, we take $\mu_{F}=$
$\mu_{R}=\mu$ because this assumption is inherent in global analyses of parton densities.

In calculations of the total cross section, $\mu \propto$ $m_{Q}$. However, for single inclusive or double differential distributions, it may be more appropriate to choose a scale proportional to the transverse momentum of the heavy quark, $p_{T}$, or its transverse mass, $m_{T}$. A constant scale would be appropriate if $m_{Q} \simeq p_{T}$, but generally $m_{c} \ll p_{T}$ and $m_{b} \ll p_{T}$ at collider energies. Therefore the $p_{T}$ dependent scale absorbs (resums) large logarithmic terms such as $\ln \left(p_{T} / \mu\right)$ appearing when $p_{T} \gg m_{Q}$ and producing collinear divergences which are unregulated if $\mu=m_{Q}$.

Since the $Q \bar{Q}$ pair distributions are essential to determine their contribution to lepton pair production, it would be convenient if the nontrivial distributions could be modeled by the Born distributions to within a constant $K_{\text {th }}$. With $\mu \propto m_{T}, K_{\text {th }}$ is nearly constant, even in a regime where $m_{Q} \ll \sqrt{S}$ although some variations occur near the boundaries of phase space. Thus event generators for heavy quark production can, with relative confidence, scale all non-trivial Born results. Thus for heavy quark and quark pair distributions calculable at the Born level, $K_{\text {th }}$ is nearly constant provided that $\mu \propto m_{T}$. The actual value of $K_{\text {th }}$ can be determined by a comparison of the NLO and Born total cross sections.

[1] P.L. McGaughey et al., Int. J. Mod. Phys. A10 (1995) 2999.

[2] M.L. Mangano, P. Nason, and G. Ridolfi, Nucl. Phys. B373 (1992) 295; M.L. Mangano, P. Nason, and G. Ridolfi, Nucl. Phys. B405 (1993) 507.

*LBL-37105; Z. Phys. C71 (1996) 475. 


\section{Lepton Production from Charm Decay in Nuclear Collisions at $\sqrt{s}=200 \mathrm{GeV}$ and 5.5 TeV per Nucleon* \\ S. Gavin, P. L. McGaughey $y^{\ddagger}$ P. V. Ruuskanen ${ }^{\S}$ and R. Vogt}

Dilepton production provides an important tool for measuring the temperature of the high density matter produced in the early stages of a relativistic heavy-ion collision [1]. In high energy $p p$ interactions, the continuum in the dilepton mass range above $M \sim 2 \mathrm{GeV}$ is dominated by the Drell-Yan process and by semileptonic decays of charm mesons. This production can be addressed using perturbative QCD. In contrast, the lower mass region arises from soft processes, for which theory offers little guidance. We therefore hope to find a signal of thermal dilepton production at masses $M \sim 2-3 \mathrm{GeV}$ where $i$ ) the thermal contribution can still be sizable and $i i$ ) the background is calculable.

We compare the predictions of dilepton production from a simple thermal model with the hard 'background' from heavy quark pair, $Q \bar{Q}$, decays and Drell-Yan production at RHIC and LHC nucleus-nucleus collision energies, $\sqrt{s}=$ $200 \mathrm{GeV}$ and $5.5 \mathrm{TeV}$ in the nucleon-nucleon center of mass. This background is now calculated to next-to-leading order (NLO), shown to agree with $p p$ and $p \bar{p}$ data [2], and is no longer dependent on arbitrary phenomenological ' $\mathrm{K}$ factors'. The remaining uncertainties in the NLO perturbative approach are well defined and likely rather small [2]. We also calculate the invariant mass and transverse momentum distributions of the lepton pairs and use these distributions to illustrate how thermal and hard dileptons can be distinguished in an experiment. In addition, we introduce the dilepton contribution from $B$ meson decays, and discuss the effects of nuclear shadowing on the initial production.

We find that hard charm quark production and decay dominates the continuum below the $\Upsilon$ mass. In particular, the charm signal is more than an order of magnitude above the optimistic thermal dilepton and thermal charm rates for $M>2 \mathrm{GeV}$. Thus the isolation of thermal signals will not be straightforward.

To experimentally enhance the thermal signal, one can count only those lepton pairs with a small separation in rapidity. Lepton pairs from charm decays typically occur with a large rapidity gap, absent in Drell-Yan and thermal pairs. Together with like-sign subtraction, rapidity gap cuts can essentially remove the uncorrelated charm and greatly suppress the correlated charm background. The finite acceptance of a real detector can serve this purpose. Then at least one of the $D \bar{D}$ decay leptons may be outside the finite detector acceptance. In particular, relatively few high mass lepton pairs from uncorrelated $D \bar{D}$ decays will be detected, significantly reducing the uncorrelated yield even before like-sign subtraction. We show that the acceptance window of PHENIX/RHIC and ALICE/LHC can enhance the signal from thermal charm decays to the point of measurability by rejecting pairs with large gaps. However, charm production remains the dominant source of dileptons in heavy-ion collisions, even with acceptance cuts, for $M<6-8 \mathrm{GeV}$. Since charm cannot be removed simply by the finite detector acceptance, charm measurements are crucial.

[1] R. Vogt, B.V. Jacak, P.L. McGaughey and P.V. Ruuskanen, Phys. Rev. D49 (1994) 3345. [2] P.L. McGaughey et al., Int. J. Mod. Phys. A10 (1995) 2999; S. Gavin et al., Int. J. Mod. Phys. A10 (1995) 2961.

\footnotetext{
${ }^{*}$ LBL-37981; Phys. Rev. C54 (1996) 2606.

${ }^{\dagger}$ Physics Department, Brookhaven National Laboratory

${ }^{\ddagger}$ Los Alamos National Laboratory

${ }^{\S}$ Department of Physics, University of Jyväskylä, Jyväskylä, Finland
} 


\title{
Charm and Bottom Quark Production Cross Sections Near Threshold*
}

\author{
J. Smith ${ }^{\dagger}$ and R. Vogt
}

Next-to-leading order calculations of charm production are large compared to the Born cross section, suggesting that further higher order corrections are substantial. Near threshold large logarithms in the perturbative expansion can arise from an imperfect cancellation of the softplus-virtual $(S+V)$ terms, which must be resummed. An approximation of these terms was used to resum the leading logarithms to all orders in perturbation theory [1]. The method relies on the proportionality of the higher order terms to the Born cross section. A cutoff parameter, $\mu_{0}$, is introduced to keep the result finite. A strong dependence on $\mu_{0}$, implies that the cross section requires nonperturbative contributions.

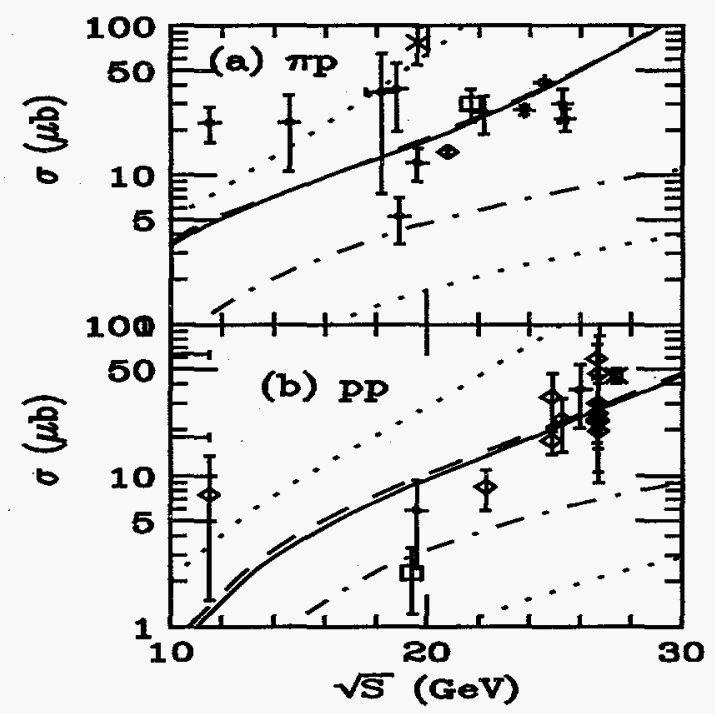

Figure 1: We show the $c \bar{c}$ cross sections, $\sigma^{\text {res }}$ (solid), $\sigma^{\text {imp }}=\sigma^{\text {res }}+\left.\sigma^{\text {NLO }}\right|_{\text {exact }}-\left.\sigma^{\text {NLO }}\right|_{\text {app }}$ (dashed), and $\left.\sigma^{\text {NLO }}\right|_{\text {exact }}$ (dot-dashed), in (a) $\pi^{-} p$ and (b) $p p$ interactions. Extreme values of $\sigma^{\text {res }}$ are shown in the dotted lines.

Because $m_{c}$ is relatively small, $1.2 \leq m_{c} \leq 1.8$ $\mathrm{GeV} / c^{2}$, charm production must be treated with some care. The only consistent NLO evaluation of the pion and proton parton densities is GRV HO [2], in the $\overline{\mathrm{MS}}$ scheme. This set also has a small initial scale so that $\mu=m_{c}=1.5$ $\mathrm{GeV} / c^{2}$ is used. We find that resummed cross section, $\sigma^{\text {res }}$, in the $q \bar{q}$ channel in the $\overline{\mathrm{MS}}$ scheme converges for $\mu_{0} \approx 0.15 m_{c}$ while the $g g$ channel, with its larger color factor, converges for $\mu_{0} \approx 0.35 m_{c}$. The ratios $\mu_{0} / m_{c}$ agree with the convergence ratios for bottom and top production.

Figure 1 shows that the perturbative expansion no longer converges at higher energies, since

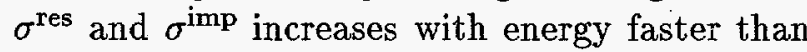

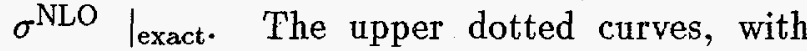
$\mu=m_{c}=1.3 \mathrm{GeV} / c^{2}$, increase faster than $\sigma^{\text {res }}$, implying that the resummation breaks down at lower energies for lighter masses. The lower dotted curves are calculated with $\mu / 2=m_{c}=1.8$ $\mathrm{GeV} / c^{2}$ and the MRS $\mathrm{D}-{ }^{\prime}$ proton and SMRS P2 pion distributions [3]. The larger quark mass requires a larger $\mu_{0}$ for convergence.

[1] E. Laenen, J. Smith, and W.L. van Neerven, Nucl. Phys. B369 (1992) 543.

[2] M. Glück, E. Reya and A. Vogt, Z. Phys. C53 (1992) 127; Z. Phys. C53 (1992) 651.

[3] A.D. Martin, W.J. Stirling and R.G. Roberts, Phys. Lett. B306, 145 (1993); P.J. Sutton, A.D. Martin, R.G. Roberts and W.J. Stirling, Phys. Rev. D45 (1992) 2349.

${ }^{*}$ LBL-38282 and ITP-SB-95-60; Z. Phys. $\mathbf{C}$ in press.

'Institute for Theoretical Physics, State University of New York at Stony Brook 


\title{
Resummed Heavy Quark Production Cross Sections to Next-to-Leading Logarithm*
}

\author{
N. Kidonakis, J. Smith ${ }^{\ddagger}$ and R. Vogt
}

The first resummation of leading logarithmic, LL, terms in heavy quark production was discussed in [1]. The analysis was based on the fact that the LL terms are identical to those in the Drell-Yan process. The importance of incorporating resummation effects in threshold production of heavy quarks in QCD was apparent. The order $\alpha_{s}^{2}$ corrections to the Drell-Yan process allow a check of the NLL terms in the resummation formulae. However, this information cannot be used in heavy quark resummation since the color structure is more complicated. A resummation formalism which correctly incorporates the NLL heavy quark resummation near threshold has recently been presented [2].

We apply this new NLL resummation formalism to calculate the top quark production cross section at the Fermilab Tevatron and the bottom quark production cross section at fixed target energies. We are particularly interested in the size and therefore the phenomenological importance of the NLL terms with respect to the LL terms.

Our calculations are based on the factorization of soft gluons from high-energy partons in perturbative QCD. In [2] it was shown that to NLL it is possible to pick a color basis in which moments of the short distance cross section, $\hat{\sigma}_{a b}^{(I J)}$, exponentiate so that

$$
\begin{aligned}
\tilde{\sigma}_{a b}^{(I J)} & =\int_{0}^{1} d z z^{n-1} \hat{\sigma}_{a b}^{(I J)}\left(z, \theta^{*}, \alpha_{s}\left(Q^{2}\right)\right) \\
& =h_{I}\left(\theta^{*}, Q^{2}\right) h_{J}^{*}\left(\theta^{*}, Q^{2}\right) e^{E_{I J}^{(a b)}} .
\end{aligned}
$$

The hard scattering prefactor is a product of contributions from the amplitude $h_{I}$ and its complex conjugate $h_{J}^{*}$. The functions $h_{I}$ and $h_{J}^{*}$ have no collinear or soft divergences at the partonic threshold since these terms have been factored into the exponent $E_{I J}$ in eq. (1), given by

$$
\begin{aligned}
& E_{I J}^{(a b)}=-\int_{0}^{1} d z \frac{z^{n-1}-1}{1-z} \\
& \times\left\{(2-r) \int_{0}^{z} \frac{d y}{1-y} g_{1}^{(a b)}\left(y, z, Q^{2}\right)+g_{2}^{(a b)}\left(z, Q^{2}\right)\right. \\
& \left.+g_{3}^{(I)}\left(z, Q^{2}, \theta^{*}\right)+g_{3}^{(J) *}\left(z, Q^{2}, \theta^{*}\right)\right\}
\end{aligned}
$$

where $g_{1}, g_{2}$ and $g_{3}$ are functions of the running coupling constant $\alpha_{s}$. The scheme dependent parameter $r$ changes the lower limit of the $y$-integral in the $\overline{\mathrm{MS}}$ scheme.

The NLL functions $g_{3}^{(I)}$, depend on the color structure in the hard scattering so that $g_{3}^{(I)}$ is a function of both $\alpha_{s}$ and the angle $\theta^{*}$ between the incoming and outgoing partons. Although the eigenvalues are complex, the total $g_{3}$ contribution to $E_{I J}^{(a b)}$ in eq. (2) is real.

We have investigated the numerical importance of the NLL terms in the resummation of subleading soft gluon contributions near threshold for heavy quark production cross sections. We have shown that these contributions are generally quite small for top production either because they are numerically small or due to cancellations between them. This result has only been demonstrated for the resummation method of [1] and at $\theta^{*}=90^{\circ}$.

[1] E. Laenen, J. Smith, and W.L. van Neerven, Nucl. Phys. B369, 543 (1992).

[2] N. Kidonakis and G. Sterman, Phys. Lett. B387,867 (1996); N. Kidonakis, SUNY at Stony Brook Ph.D. Thesis (1996), hep-ph/9606474.

\footnotetext{
*LBNL-39025, ITP-SB-96-32 and Edinburgh 96/30; submitted to Phys. Rev. D.

tDepartment of Physics and Astronomy, University of Edinburgh, Scotland

${ }^{\ddagger}$ DESY, Hamburg, Germany and Institute for Theoretical Physics, State University of New York at Stony Brook
} 


\title{
Reanalysis of the EMC Charm Production Data With Extrinsic and Intrinsic Charm at NLO* $^{*}$
}

\author{
B. W. Harris, J. Smith ${ }^{\ddagger}$ and R. Vogt
}

The European Muon Collaboration (EMC) [1] established that photon-gluon fusion (PGF)in QCD, the analogue of the Bethe-Heitler reaction in QED, explains most of the charm deep inelastic structure function $F_{2}^{c}$. However, the EMC data was not in complete agreement with PGF at all $x$ and $Q^{2}$. The disagreement at large $x$ and $Q^{2}$ suggested that a higher-twist component of charm production was necessary, called intrinsic charm (IC) [2] to distinguish it from PGF, referred to as extrinsic charm (EC).

In the analysis of the EMC data [1], a relatively simple model was used for both the EC and IC components. At the time, only the LO contributions to $F_{2}^{c}$ from both models were available. Afterwards, Hoffman and Moore [3] calculated the next-to-leading order (NLO) corrections to the IC component and discussed their effects on the EMC analysis. Based on LO photongluon fusion, they found evidence for an $0.3 \%$ IC component in the proton. Since the complete NLO results are now available for both EC and IC production, a more detailed QCD analysis of the EMC results is finally possible, allowing us to make a more reliable determination of the IC content of the proton.

Up to NLO, the IC component of the structure function is [3]

$F_{2}^{c}=\frac{8}{9} \xi\left[c(\xi, \gamma)+\int_{\xi / \gamma}^{1} \frac{d z}{z} c(\xi / z, \gamma) \sigma_{2}^{(1)}(z, \lambda)\right]$.

Likewise the NLO EC structure function is

$$
\begin{aligned}
& F_{2}=\frac{Q^{2} \alpha_{s}\left(\mu^{2}\right)}{4 \pi^{2} m_{c}^{2}} \int_{\xi_{0}}^{1} \frac{d \xi}{\xi}\left[e_{c}^{2} f_{\frac{g}{P}}\left(\xi, \mu^{2}\right) c_{2, g}^{(0)}\right] \\
& +\frac{Q^{2} \alpha_{s}^{2}\left(\mu^{2}\right)}{\pi m_{c}^{2}} \int_{\xi_{0}}^{1} \frac{d \xi}{\xi}\left\{e_{c}^{2} f_{\frac{g}{P}}\left(\xi, \mu^{2}\right)\left(c_{2, g}^{(1)}+\bar{c}_{2, g}^{(1)} \ln \frac{\mu^{2}}{m_{c}^{2}}\right)\right. \\
& +\sum_{i=q, \bar{q}} f_{\frac{i}{P}}\left(\xi, \mu^{2}\right)\left[e_{c}^{2}\left(c_{2, i}^{(1)}+\bar{c}_{2, i}^{(1)} \ln \frac{\mu^{2}}{m_{c}^{2}}\right)\right.
\end{aligned}
$$

$$
\left.\left.+e_{i}^{2} d_{2, i}^{(1)}+e_{c} e_{i} o_{2, i}^{(1)}\right]\right\} \text {. }
$$

The coefficient functions are of different origin: $c_{2, i}^{(1)}$ and $\bar{c}_{2, i}^{(1)}$ originate from the virtual photoncharmed quark coupling and appear for both charged and neutral parton-induced reactions; $d_{2, i}^{(1)}$ arise from the virtual photon-light quark coupling; $o_{2, i}^{(1)}$ come from their interference and do not contribute to $F_{2}^{c}$. Both the IC and EC components hold only in the DIS regime where $Q^{2}>0$.

We fit the EMC data with the sum of the EC and IC component of $F_{2}^{c}$. The normalization of both the IC and EC components are taken as free parameters,

$$
F_{2}^{c}=\alpha F_{2}^{c, \mathrm{EC}}+\beta F_{2}^{c, \mathrm{IC}},
$$

with $\mu=\mu_{0}$. The shift in the normalization of the EC component may be considered as an estimate of the size of the NNLO contribution, equivalent to a shift in the scale $\mu$. Since we have assumed a $1 \%$ normalization of the IC component, the fitted $\beta$ is the fraction of this normalization. Our fitted value of $\beta$ confirms the Hoffman-Moore result of an $\approx 0.3 \%$ IC contribution to $F_{2}^{c}$.

[1] J.J. Aubert et al., (EMC), Nucl. Phys. B 213 (1983) 31; Phys. Lett. B 94 (1980) 96; ibid. B110 (1982) 73 .

[2] S.J. Brodsky, P. Hoyer, C. Peterson and N. Sakai, Phys. Lett. B93 (1980) 451; S.J. Brodsky, C. Peterson and N. Sakai, Phys. Rev. D23 (1981) 2745.

[3] E. Hoffmann and R. Moore, Z. Phys. C20 (1983) 71 .

${ }^{*}$ LBL-37266, ITP-SB-95-15, and FSU-HEP-951030; Nucl. Phys: B461 (1996) 181.

${ }^{\dagger}$ Physics Department, Florida State University

Institute for Theoretical Physics, State University of New York at Stony Brook 


\title{
Charmed Hadron Asymmetries in the Intrinsic Charm Coalescence Model ${ }^{*}$
}

\author{
R. Vogt and S.J. Brodsky ${ }^{\dagger}$
}

In leading-twist charm production, there is no connection between the spectator and participant partons. However, in higher-twist processes, the interaction between spectators and participants can be strong. These higher-twist processes, such as intrinsic charm, are usually suppressed by $\alpha_{s}^{2}\left(M_{c \bar{c}}\right) / M_{c \bar{c}}^{2}$ relative to leadingtwist production. However, if the transverse distance between the partons is small, they may interact during the time they are near each other, allowing the higher-twist processes to become dominant.

The intrinsic $c \bar{c}$ production cross section, $\sigma_{\text {ic }}(h N)=P_{\text {ic }} \sigma_{h p}^{\text {in }} \mu^{2} /\left(4 \widehat{m}_{\mathrm{c}}^{2}\right)$, extracted from 200 $\mathrm{GeV}$ proton- and pion-induced interactions, is $\sigma_{\mathrm{ic}}\left(\pi^{-} N\right) \approx 0.5 \mu \mathrm{b}$ and $\sigma_{\mathrm{ic}}(p N) \approx 0.7 \mu \mathrm{b}[1]$. The probability of finding a $c \bar{c}$ pair in the proton wavefunction was determined from a fit to the EMC charm structure function [2]. Then the total charm production cross section is the sum of leading-twist fusion and higher-twist intrinsic charm, $d \sigma(h N) / d x_{F}=d \sigma_{1 \mathrm{t}} / d x_{F}+d \sigma_{\mathrm{ic}} / d x_{F}$.

There are two ways of producing $D$ mesons from intrinsic $c \bar{c}$ pairs. The first is through standard fragmentation processes. The $c$ quarks can also coalesce with projectile valence spectators to produce leading charmed mesons. Coalescence introduces flavor correlations between the projectile and the final-state hadrons. A critical test of flavor correlations in charm production is the asymmetry between leading and nonleading charm. For example, in $\pi^{-}(\bar{u} d)$ interactions, the $D^{-}(\bar{c} d)$ is leading since it has a valence quark in common with the projectile while the $D^{+}(c \bar{d})$ with no valence quark in common, is nonleading. This observed leading behavior suggests that hadronization at large $x_{F}$ involves the coalescence of the charmed quarks with projectile spectator quarks.
Here we extended this model to other charmed hadrons. As expected, the asymmetries predicted by the intrinsic charm coalescence model are a strong function of $x_{F}$. We find that $\Lambda_{c}$ production in the proton fragmentation region ( $x_{F}<0$ in $\pi^{-} p$ collisions) is dominated by the coalescence of the intrinsic charm quark with the $u d$ valence quarks of the proton. The production of $D_{s} / \bar{D}_{s}$ and, at $x_{F}>0, \Lambda_{c} / \bar{\Lambda}_{c}$ by coalescence must occur within still higher Fock states such as $\left|n_{V} c \bar{c} d \bar{d}\right\rangle$ and $\left|n_{V} c \bar{c} s \bar{s}\right\rangle$. These states are normalized from a calculation of $\psi \psi$ production from $\left|n_{V} c \bar{c} c \bar{c}\right\rangle$ configurations which allows us to obtain the probability of additional light quark pairs in the Fock states by mass scaling. As more partons are included in the Fock state, the coalescence distributions soften and approach the standard fragmentation distributions. Thus we do not consider $\bar{\Lambda}_{c}$ production by coalescence at $x_{F}<0$ since a minimal nine-parton Fock state is required. Therefore there is no asymmetry in the model for these cases.

[1] R. Vogt and S.J. Brodsky, Nucl. Phys. B438 (1995) 261; R. Vogt and S.J. Brodsky, Phys. Lett. B349 (1995) 569; R. Vogt, Nucl. Phys. B446 (1995) 149.

[2] B.W. Harris, J. Smith, and R. Vogt, Nucl. Phys. B461 (1996) 181.

\footnotetext{
${ }^{*}$ LBL-37666 and SLAC-PUB-95-7068; Nucl. Phys. B478 (1996) 311.

'Stanford Linear Accelerator Center
} 


\title{
Charmonium Suppression by Comover Scattering in $\mathbf{P b}+\mathbf{P b}$ Collisions*
}

\author{
S. Gavin ${ }^{\dagger}$ and R. Vogt
}

Experiment NA50 has reported an abrupt decrease in $\psi$ production in $\mathrm{Pb}+\mathrm{Pb}$ collisions at $158 \mathrm{GeV}$ per nucleon [1]. Specifically, the collaboration presented a striking 'threshold effect' in the $\psi$-to-continuum ratio by plotting it as a function of a calculated quantity, the mean path length of the $\psi$ through the nuclear medium, $L$. This apparent threshold has sparked considerable excitement as it may signal the formation of quark-gluon plasma in the heavy system.

We study the $\mathrm{Pb}$ results in the context of a hadronic model of charmonium suppression [2]. We point out that the behavior as a function of $L$ is not a threshold effect but, rather, reflects the approach to the geometrical limit of $L$ as the collisions become increasingly central. When plotted as a function of the measured neutral transverse energy $E_{T}$, the data varies smoothly as in $S+U$ measurements [1]. The difference between $\mathrm{S}+\mathrm{U}$ and $\mathrm{Pb}+\mathrm{Pb}$ data lies strictly in the relative magnitude. To assess this magnitude, we compare $\psi$ and $\psi^{\prime}$ data to expectations based on the hadronic comover model [2].

The hadronic contribution to the suppression arises from scattering of the $\psi$ with produced particles, comovers, and nucleons [2]. The standard nucleon absorption survival probability is

$$
S_{A}=\exp \left\{-\int_{z}^{\infty} d z \rho_{A}(b, z) \sigma_{\psi N}\right\}
$$

where $\rho_{A}$ is the nuclear density, $b$ the impact parameter and $\sigma_{\psi N}$ the absorption cross section for $\psi$-nucleon interactions. One can estimate $S_{A} \sim \exp \left\{-\sigma_{\psi N} \rho_{0} L_{A}\right\}$, where $L_{A}$ is the path length traversed by the $c \bar{c}$ pair.

Suppression can also be caused by scattering with produced particles traveling with the $c \bar{c}$ pair. This comover density scales $\propto E_{T}$. The corresponding survival probability is

$$
S_{\mathrm{co}}=\exp \left\{-\sigma_{\mathrm{co}} v n_{0} \check{\tau}_{0} \ln \left(\frac{\tau_{F}}{\tau_{0}}\right)\right\} .
$$

Thus $S_{\text {co }}$ depends on the scattering frequency, the formation time of the comovers and the transverse size of the central region. The comover density, $n_{0}$, scales roughly with $E_{T}$.

The path length $L$ and transverse size $R_{T}$ depend on the collision geometry. The path length grows with $E_{T}$, asymptotically approaching the geometric limit $R_{A}+R_{B}$. Nucleon absorption saturates for $b<R_{A}$, where $R_{A}$ is the smaller of the two nuclei. On the other hand, $E_{T}$ continues to grow for $b<R_{A}$ due, e.g., to fluctuations in the number of $N N$ collisions.

Our $\mathrm{Pb}+\mathrm{Pb}$ predictions account for the NA50 data. Nucleon absorption indeed saturates for small values of $b$. Comover scattering accounts for the remaining suppression. To compare our predictions with the NA50 analysis [1], we calculate the continuum dimuon yield for $2.9<$ $M<4.5 \mathrm{GeV}$ and adjust the $E_{T}$ scale to the NA50 calorimeter, $1.1<\eta<2.3$. The agreement depends on these updates. The saturation with $L$ but not $E_{T}$ suggests an additional density-dependent suppression mechanism. Comover scattering explains the additional suppression. Nevertheless, it is unlikely that this explanation is unique.

[1] M. Gonin et al. (NA50), Proc. Quark Matter '96, Heidelberg, Germany, P. Braun-Munzinger et al., eds. (1996).

[2] S. Gavin and R. Vogt, Nucl. Phys. B345 (1990) 104; S. Gavin, H. Satz, R. L. Thews, and R. Vogt, Z. Phys. C61 (1994) 351; S. Gavin, Nucl. Phys. A566 (1994) 383c.

${ }^{*}$ LBL-37980; to be published in Phys. Rev. Lett.

${ }^{\dagger}$ Physics Department, Brookhaven National Laboratory 


\title{
Transverse Momentum of $\psi$ and Dimuon Production in $\mathbf{P b}+\mathbf{P b}$ Collisions*
}

\author{
S. Gavin ${ }^{\dagger}$ and R. Vogt
}

The NA50 collaboration at the SPS has reported a suppression of $\psi$ production in $\mathrm{Pb}+\mathrm{Pb}$ collisions relative to Drell-Yan dimuon production as the neutral transverse energy of hadrons, $E_{T}$, is increased [1]. They further presented a striking 'threshold effect' by comparing the data to $\mathrm{S}+\mathrm{U}$ results as a function of the mean path length of the $\psi$ through nuclear matter, $L$, calculated assuming a sharp-edged nucleus. We point out that the centrality dependence of the transverse momenta of Drell-Yan dimuons essentially provide an experimental determination of $L$. Such a determination can provide vital evidence for or against the threshold behavior.

Important information can be extracted from the nuclear dependence of the $\psi$ 's transverse momentum, $p_{T}$. Hadronic suppression [2] does not modify the transverse momentum dependence of $\psi$ production appreciably. To account for the nuclear modification measured in $p \mathrm{~A}$ and $\mathrm{S}+\mathrm{U}$ collisions, initial-state parton scattering was introduced [3]. This elastic parton scattering broadens the $p_{T}$ distributions of charmonium and Drell-Yan production without affecting the $p_{T}{ }^{-}$ integrated yields. Partons essentially undergo a random walk in momentum space, so that $\left\langle p_{T}^{2}\right\rangle$ grows linearly with $L$.

In a hadron-nucleus collision, a parton from the projectile can suffer soft quasielastic interactions as it crosses the nuclear target [3]. The $\left\langle p_{T}^{2}\right\rangle$ of the dimuon or $\psi$ is increased by:

$$
\Delta p_{T}^{2} \equiv\left\langle p_{T}^{2}\right\rangle-\left\langle p_{T}^{2}\right\rangle_{N N}=\lambda^{2}\left(\bar{n}_{A}-1\right),
$$

where $\bar{n}_{A}$ is the number of $N N$ subcollisions that the projectile suffers in the target, $\lambda^{2}$ determines the $p_{T}^{2}$ increment from each subcollision and $\left\langle p_{T}^{2}\right\rangle_{N N}$ is $A$-independent contribution from the hard scattering.

In a nucleus-nucleus collision, both projectile and target partons scatter,

$$
\Delta p_{T}^{2} \equiv\left\langle p_{T}^{2}\right\rangle-\left\langle p_{T}^{2}\right\rangle_{N N}=\lambda^{2}\left(\bar{n}_{A}+\bar{n}_{B}-2\right),
$$

where the path length is

$$
L \equiv\left(\bar{n}_{A}+\bar{n}_{B}\right) / 2 \sigma_{N N} \rho_{0} \equiv \bar{n} / 2 \sigma_{N N} \rho_{0} .
$$

The relation between $\bar{n}$ and the impact parameter $\vec{b}$ depends on the collision geometry.

We expect $\left\langle p_{T}^{2}\right\rangle$ to increase by $12.3 \%$ for $\psi$ as $E_{T}$ increases from 50 to $150 \mathrm{GeV}$. This represents a flattening of $\left\langle p_{T}^{2}\right\rangle\left(E_{T}\right)$ in comparison to the $\mathrm{S}+\mathrm{U} \rightarrow \psi+X$ data, which show an $18.5 \%$ increase as $E_{T}$ varies from 30 to $90 \mathrm{GeV}$. However, we stress that the most direct extraction of $L$ comes not from $\psi$ but from Drell-Yan production since this process is unaffected by final-state interactions. To check that the NA50 extraction of $L$ is correct, Drell-Yan data in $\mathrm{Pb}+\mathrm{Pb}$ would have to establish a $2.7 \%$ increase in $\left\langle p_{T}^{2}\right\rangle$. This requires dimuon data far more precise than that for $\mathrm{S}+\mathrm{U}$ collisions.

The model parameters were revised to describe the latest E772 $p \mathrm{~A} \rightarrow \mu^{+} \mu^{-}+X$ data. Our revision implies a ratio $\left(\lambda_{\psi} / \lambda_{\mu^{+} \mu^{-}}\right)^{2} \sim 3.9$, larger than that extracted earlier, indicating that finalstate scattering of the octet $c \bar{c}$ may occur. More precise $p \mathrm{~A} \rightarrow \psi+X$ measurements are needed to explore this possibility.

[1] M. Gonin et al. (NA50), Proc. Quark Matter '96, Heidelberg, Germany, P. Braun-Munzinger et al., eds. (1996).

[2] S. Gavin and R. Vogt, LBL-37980, to be published in Phys. Rev. Lett.

[3] S. Gavin and M. Gyulassy, Phys. Lett. B214 (1988) 241.

*CU-TP-791, DOE/ER/40561-292-INT96-21-01; submitted to Phys. Rev. C.

${ }^{\dagger}$ Physics Department, Brookhaven National Laboratory and Physics Department, Columbia University

${ }^{\ddagger}$ Institute for Nuclear Theory, University of Washington 


\title{
Systematics of quarkonium production*
}

\author{
G. A. Schuler ${ }^{\dagger}$ and R. Vogt
}

Some time ago we [1] showed that existing quarkonium production data at fixed-target energies are, in fact, compatible with the assumption that the non-perturbative transition of the $Q \bar{Q}$ pair to quarkonium is more universal than expected from the velocity-scaling rules. Lowenergy data are well reproduced if this infinite sum is truncated to a single term

$$
\sigma\left[H\left(n J^{P C}\right)\right]=F\left[n J^{P C}\right] \tilde{\sigma}[Q \bar{Q}] .
$$

We demonstrate that the colour-evaporation model (CEM) of eq. (1) also accounts for quarkonium production at the Tevatron. The number of non-perturbative parameters is minimal, giving the model great predictive power.

The short-distance part of eq. (1) is the perturbative subthreshold cross section expanded in powers of $\alpha_{s}(\mu)$ where $\mu \propto m_{Q}$. Specifying to charm, the cross section is

$$
\tilde{\sigma}[c \bar{c}]=\int_{2 m_{c}}^{2 m_{D}} d M_{c \bar{c}} \frac{d \sigma[c \bar{c}]}{\mathrm{M}_{c \bar{c}}}
$$

where $\sigma[c \bar{c}]$ is the spin-and colour-averaged open heavy-quark pair production cross section. The CEM is hence based on two ingredients. First, the quarkonium dynamics are assumed to be identical to those of low mass open $Q \bar{Q}$ pairs. All perturbative QCD corrections apply to the short-distance cross section and hence are identical for open and bound heavy-quark production. Second, although the $Q \bar{Q}$ pair is produced at short distances in different states, distinguished by colour, angular momentum, relative momentum, and their relative production rates may be different for different high-energy collisions, the average over many long-distance matrix elements, combined in the long-distance factor $F\left[n J^{P C}\right]$, determines the probability to form a specific bound state. Necessarily, the factor $F$ needs to be universal, i.e. process- and kinematics-independent.
The long-distance factors determined from the low-energy total cross sections are

$$
\begin{aligned}
F_{t o t}[J / \psi] & =2.5 \% \\
\sum_{n=1}^{3} \mathrm{~B}[\Upsilon(n S) \rightarrow \mu \mu] F_{t o t}[\Upsilon(n S)] & =1.6 \times 10^{-3}
\end{aligned}
$$

Here the subthreshold cross sections were calculated in next-to-leading order (NLO) using the MRS D-' parametrization [2] of the partondistribution functions (PDF) with renormalization and factorization scales $\mu_{R}$ and $\mu_{F}$ chosen to be $\mu_{R}=\mu_{F}=2 m_{c}=2.4 \mathrm{GeV}$ and $\mu_{R}=\mu_{F}=m_{b}=4.75 \mathrm{GeV}$, respectively. The results in eq. (4) are rather insensitive to variations of the parameters in the open heavy-quark cross section, if they are tuned to the open heavyflavour total cross section data.

The transverse momentum distributions of prompt charmonium production (i.e. not originating from $B$ decays) and bottomonium production at the Tevatron energy $\sqrt{s}=1.8 \mathrm{TeV}$, with normalizations extracted from eq. (4), are in good agreement with the data, typically better than $50 \%$. The CEM prediction is based on the subthreshold cross section calculated to $O\left(\alpha_{s}^{3}\right)$, NLO for the $p_{T}$ integrated cross section but LO only for the $p_{T}$ distribution. In the absence of the NLO corrections to the $p_{T}$ spectrum one might apply a $K$ factor to account for the unknown higher-order corrections.

[1] P.L. McGaughey et al., Int. J. Mod. Phys. A10 (1995) 2999.

[2] A.D. Martin, R.G. Roberts and W.J. Stirling, Phys. Lett. B306 (1993) 145.

${ }^{*}$ LBL-39012 and CERN-TH/96-157; Phys. Lett. B387 (1996) 181.

${ }^{\dagger}$ Theory Division, CERN 


\title{
Upsilon Suppression in $\mathbf{P b}+\mathbf{P b}$ Collisions at the LHC?*
}

\author{
R. Vogt
}

One of the proposed signatures of the QCD phase transition is the suppression of quarkonium production, particularly of the $J / \psi$ [1]. In a quark-gluon plasma, the suppression occurs due to the shielding of the $c \bar{c}$ binding potential by color screening, leading to the breakup of the resonance. Because the $\Upsilon$ is much smaller than the $c \bar{c}$ and other $b \bar{b}$ resonances, a much higher initial temperature, $T_{i}$, than expected from past estimates is needed to dissociate the $\Upsilon$ [1]. Therefore it was previously assumed that the $\Upsilon$ would not be suppressed by QGP production. However, in view of the high $T_{i}$ of a gluon plasma [2], $T_{i} \sim 1$ $\mathrm{GeV}$, a reexamination of this assumption is in order. The relative suppression of the members of the $\Upsilon$ family should be testable at the LHC.

The potential for nonrelativistically bound quarkonium at finite temperature is [2]

$$
V(r, T)=\frac{\sigma}{\mu(T)}\left(1-e^{-\mu(T) r}\right)-\frac{\alpha}{r} e^{-\mu(T) r},
$$

where $r$ is the separation between the $Q$ and $\bar{Q}$ and $\mu(T)$ is the screening mass. The parameters are $\sigma=0.192 \mathrm{GeV}^{2}, \alpha=0.471, m_{c}=1.32 \mathrm{GeV}$ and $m_{b}=4.746 \mathrm{GeV}$. Minimizing the quarkonium energy gives the radius of the bound state at each $T$. Where there is no longer a minimum and $\mu\left(T=T_{D}\right)=\mu_{D}$ the resonance no longer forms in the plasma.

We concentrate on the $\Upsilon$ states since they can be cleanly measured by CMS. It is necessary to know the dependence of the screening mass on temperature before $T_{D}$ can be determined. Since the behavior of $\mu(T)$ is unknown for $T \gg T_{c}$, we show two rather extreme scenarios which may be expected to bracket the realistic situation. The first is a parameterization based on $\mathrm{SU}(N)$ lattice simulations,

$$
\frac{\mu(T)}{T_{c}} \simeq 4 \frac{T}{T_{c}}
$$

which results in the lowest values of $T_{D}$. Athough $\mu(T)$ is independent of $T_{c}$, since we present our results as a function of the ratio $T / T_{c}$, for definiteness we take $T_{c}=260 \mathrm{MeV}$, consistent with $\mathrm{SU}(3)$ lattice calculations. For this value of $T_{c}$, the $\Upsilon$ breaks up at $T_{D} / T_{c} \approx 1.5$. In [2], this parameterization was used with $T_{c}=$ $150 \mathrm{MeV}$, leading to the estimate $T_{D} / T_{c} \sim 2.6$ for the $\Upsilon$. With initial temperatures of $260 \mathrm{MeV}$ predicted for the LHC, $\Upsilon$ suppression appeared unlikely. The second is a perturbative estimate,

$$
\frac{\mu(T)}{T_{c}}=\sqrt{1+\frac{n_{f}}{6}} g\left(\frac{T}{T_{c}}\right) \frac{T}{T_{c}},
$$

with a temperature-dependent running coupling constant. In this case, $T_{D}$ can be quite high and depends strongly on $n_{f}$ and $T_{c}$. We present results use $n_{f}=3$ and $T_{c}=150 \mathrm{MeV}$.

When the parameterization of eq. (3) is used, the $\Upsilon$ can easily be suppressed in the minijet gluon plasma. However, the perturbative estimate leads to much higher breakup temperatures, indicating that while the $\Upsilon^{\prime}$ and $\chi_{b}$ resonances may break up, directly produced $\Upsilon$ 's will probably not. The initial energy density and temperature have been shown to be much larger at the LHC than previously predicted. One advantage of these higher densities is that $\Upsilon$ production may be suppressed, depending on the temperature dependence of the screening mass. The rates for bottomonium production are large enough for such effects to be observable in the dilepton spectra.

[1] T. Matsui and H. Satz, Phys. Lett. B178 (1986) 416; F. Karsch, M.T. Mehr, and H. Satz, Z. Phys. C37 (1988) 617.

[2] K.J. Eskola, K. Kajantie and P.V. Ruuskanen, Phys. Lett. B332 (1994) 191; K.J. Eskola, Nucl. Phys. A590 (1995) 383c.

${ }^{*}$ CMS TN/96-041 


\title{
Determining the Existence and Nature of the Quark-Gluon Plasma by Upsilon Suppression at the LHC*
}

\author{
J. F. Gunion ${ }^{\dagger}$ and R. Vogt
}

Following up on the work presented in Ref. [1], we found that expectations for $\Upsilon$ production in $\mathrm{Pb}+\mathrm{Pb}$ collisions at the LHC are very dependent upon the nature and details of the quark-gluon plasma (QGP). We demonstrated that this dependence may allow us to determine the fundamental nature of the QGP, including: the energy density, the initial temperature, the plasma radius and the screening mass. We chose the $\Upsilon$ family to examine in detail because while the $\Upsilon$ rate is high enough for statistically significant measurements to be made, particularly at the LHC, the $\Upsilon$ is not strongly affected by interactions with hadronic matter. Strikingly different expectations for the $p_{T}$-dependence of the $\Upsilon^{\prime} / \Upsilon$ ratio, are found depending upon whether or not the QGP is formed and on the QGP properties. While our calculations of the survival probability are rather schematic, the general trend is correct.

The much higher energy of the LHC could be crucial for $\Upsilon$ suppression by a QGP. The high minijet density at the LHC implies that the $p_{T}$ spectra of the $\Upsilon$ resonances are likely to be highly sensitive to details of QGP formation. In contrast, at RHIC, the minijet density will be a factor of 10 to 30 smaller, and the maximum initial temperature predicted is $T_{0} \sim 0.5 \mathrm{GeV}$. Thus no suppression is expected. Thus even a very high $T_{0}$ does not guarantee plasma suppression. Models assuming a slow equilibration result in greater suppression because of the longer time the system spends in the screening region with $T>T_{D}$ where $T_{D}$ is the resonance dissociation temperature.

Even if suppression is absent at RHIC, it is only at RHIC that $p A$ measurements will be possible. Despite the lower energy, these $p A$ measurements will greatly aid in constraining and checking our understanding of nuclear effects.
Thus, RHIC measurements will provide important benchmarks.

We demonstrated that statistics should be adequate to detect the differences in the $\Upsilon^{\prime} / \Upsilon$ ratio that would discriminate between different QGP models. Although such ratios have the advantage that systematic effects will cancel, further vital information can be extracted if the $p_{T}$ spectra for the individual resonances can be measured. We have pointed to a comparison with $Z$ production as a potentially useful benchmark. The ideal situation arises if HERA nuclear shadowing data is available for the $x$ and $Q^{2}$ values appropriate for both $\Upsilon$ and $Z$ production at the LHC so that the observed $p_{T}$ spectrum for the $Z$ could be compared to predictions using the experimentally measured nuclear parton densities. If there is agreement, then we can unfold the shadowing effects on the $\Upsilon p_{T}$ spectra.

Certain detector features will play a key role in carrying out the analysis as envisioned here. The resolution for the CMS detector is such that the $\Upsilon, \Upsilon^{\prime}$ and $\Upsilon^{\prime \prime}$ resonances can be cleanly separated. Less certain is the extent to which the $\chi_{b} \rightarrow \gamma \Upsilon$ process can be separated from direct $\Upsilon$ production. The statistics necessary to discriminate between different QGP models is much greater if the direct 'prompt' $\Upsilon^{\prime} / \Upsilon$ ratio cannot be isolated. One also would lose the ability to observe the $\chi_{b} / \Upsilon$ ratio. It will be important for the LHC detector groups to give further attention to both experimental issues.

[1] R. Vogt, CMS-TN/96-041.

*LBNL-39474 and UCD-96-31; submitted to Nucl. Phys. B.

${ }^{\dagger}$ Davis Institute for High Energy Physics, Physics Department, University of California at Davis 


\title{
Aspects of chiral symmetry* \\ Volker Koch
}

In this review the basic concepts of chiral symmetry in nuclear physics are reviewed. Effective chiral models such as the linear and nonlinear sigma model will be discussed as well as the essential ideas of chiral perturbation theory. Based on these models heuristic derivations of important current algebra relations, such as the GellMann Oakes Renner relation, are provided. Emphasis is given to the applications for the physics of ultrarelativistic heavy ion collisions. In particular questions such as chiral

* LBNL-39463: Jou. Mod. Phys. E, in press. restoration, temperature and density dependencies of the scalar quark condensate are discussed. We also give a critical review concerning changes of vector meson masses and their relation to chiral symmetry. The basic principles behind the formation of disoriented chiral condensates are also discussed. The purpose of this review is to serve as an introduction to the chiral symmetry aspects of the physics of ultrarelativistic heavy ion collisions.

\section{Properties of hadrons in the nuclear medium *}

\author{
C.M. $K o^{\dagger}$, V. Koch and G.Q. $L i^{\$}$
}

This review is devoted to the discussion of hadron properties in the nuclear medium and its relation to the partial restoration of chiral symmetry. Special attention is given to disentangling in-medium effects due to conventional many-body interactions from those due to the change of the chiral condensate. In particular, we medium effects on the Goldstone bosons (pion, kaon and eta), the vector mesons (rho, omega, phi),

* LBNL-39866: To appear in Rev. Nucl. Part. Sci., Volume 47.

$\dagger$ Cyclotron Institute and Physics Department

Texas A\&M University, College Station, Texas 77843

Department of Physics State University of New York at

Stony Brook, Stony Brook, N.Y. 11794 and baryons (nucleon, delta, hyperons) are discussed. Also, for each proposed in-medium effect the experimental consequences are reviewed. These include subthreshold kaon production, kaon flow and pion spectra for the Goldstone bosons. Dilepton production is the central observable for the vector mesons and the relevance of the most recent measurements for possible inmedium modifications is thoroughly discussed. 


\title{
Cold Kaons from Hot Fireballs*
}

\author{
Volker Koch
}

We have studied the effect of an attractive mean field potential on the low $p_{t}$ behavior of particle spectra. Specifically, we concentrate on kaons in order to compare with preliminary data of the E-814 collaboration [1]. An attractive kaon potential would be conceivable in the framework of chiral perturbation theory if one allows for a considerable drop in the vector meson coupling above $T_{c}$, as suggested by lattice gauge calculations. We have used a relativistic transport model in order to simulate the expansion dynamics, since this approach also allow to include the effect of rescattering of the kaons. For the kaons, we take a potential of the form

$$
U_{K}(r)=U_{0} \frac{\rho_{s}}{\rho_{0}}
$$

where the density $\rho_{s}$ is the scalar density $<\bar{\psi} \psi>$. Using a kaon potential of $U_{0} \simeq$ $-50 \mathrm{MeV}$ and a reduced vector coupling for the nuclear mean field of $g_{v}=5.5$, which leads to an expansion velocity of the fireball of $V_{\text {exp }} \simeq 0.1 c$ we obtain a slope parameter as low as $15 \mathrm{MeV}$ for the low momentum kaons. Slow expansion is expected if the system is close to the chiral phase transition, where the expansion parameter $p / \epsilon$ is close to zero. If we, on the other hand, do not reduce the vector mean field coupling, the expansion velocity is $v_{e x p} \simeq 0.3 c$ and no soft component in the spectrum is seen.

A similar sensitivity is seen if one considers the coulomb interaction of the positively charge kaons (see. figure 1) with the charged fireball. If slow expansion is assumed the low $p_{t}$ kaons are pushed away leading to an observable dip in the spectrum (dashed curve). For fast expansion, on the other hand, the potential has disappeared before the kaons have been shifted to

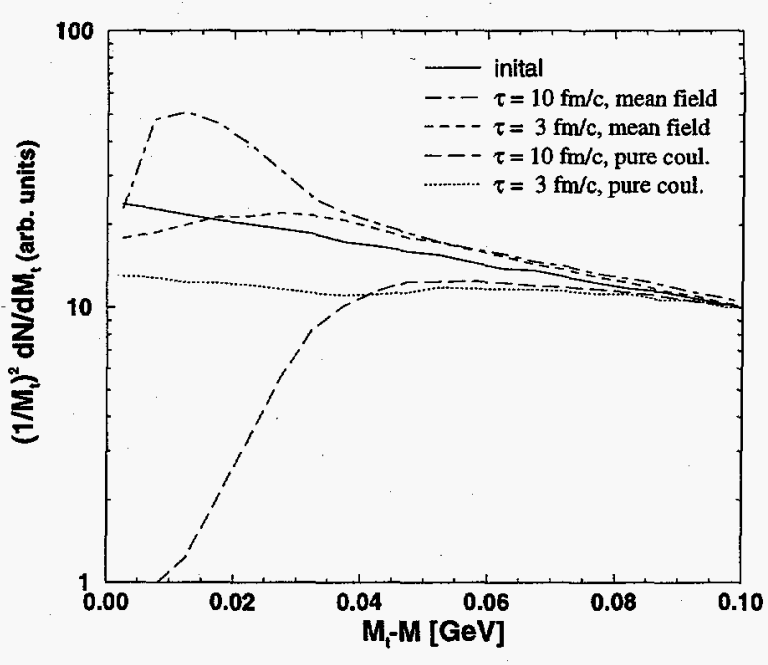

Figure 1: Initial and final kaon spectra for different expansion times $\tau$ including the coulomb interaction. The initial value for the kaon mean field has been taken to be $U_{0}=-100 \mathrm{MeV}$. All spectra are normalized at $m_{t}-m=100 \mathrm{MeV}$.

higher momenta resulting in no change of the slope (dotted curve). Similarly the low $p_{t}$ spectrum of negative kaons will be enhanced. Therefore, a careful measurement of the ratio of positive to negative charge particles may reveal at low transverse momenta may reveal important information about the expansion velocity of the fireball.

[1] J. Stachel, Nucl. Phys. A566 (1994) 183c. 


\section{Thermal effects on dilepton production from pion-annihilation * \\ C. Song, V. Koch, S.H. Lee ${ }^{\dagger}$ and C.M. Ko}

Recently the CERES-collaboration has reported [1] a considerable enhancement in the dilepton spectrum over a hadronic cocktail in the invariant mass range $300 \mathrm{MeV} \leq \mathrm{M}_{\text {inv }} \leq$ $500 \mathrm{MeV}$. Part of this enhancement is certainly due to pion annihilation which has not been taken into account in the CERES-cocktail. In a pion gas the pion-annihilation rate is modified by interactions with the environment in two ways. First, the pion form factor is reduced close to the mass of the $\rho$-meson [2]. This is a direct consequence of chiral restoration, where some of the strength of the current-current correlation function is moved to higher masses, around that of the $a_{1}$ meson. Secondly, the pion dispersion relation is modified in a pion gas due to the attractive p-wave interaction. As a result the pion annihilation cross section is enhanced for low invariant masses, just above the two pion threshold. A similar enhancement has been proposed by Gale and Kapusta in the context of BEVALAC energy heavy ion collisions, where the pion dispersion relation is modified due to the interaction with nucleons forming deltas. The combined effect of both in-medium modifications is to flatten the invariant mass spectrum due to pion annihilation in qualitative agreement with the CERES measurement (see figure 1). A quantitative comparison, however, requires to take into account the experimental acceptance cuts as well as the proper expansion dysnamics of the system created in the heavy ion reaction [4].

\footnotetext{
* LBNL-37388: Phys. Lett. B 366 (1996) 379.

$\dagger$ Department of Physics, Yonsei University, Seoul 120749 , South Korea

Cyclotron Institut, Texas A \& M University, College Station, TX 77843
}

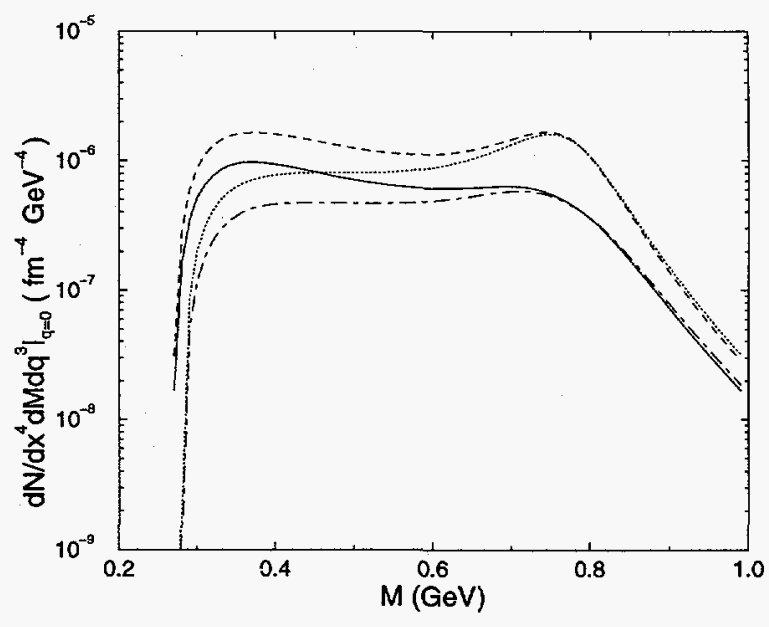

Figure 1: Dilepton production rate from pion-annihilation at $T=160 \mathrm{MeV}$. The dotted line is the result obtained without medium effects, the dashed line is that including modifications of the pion dispersion relation. The dashed-dotted line is the production rate obtained by only taking into account the in medium pion form-factor and the solid line is the result when both effects are taken into account.

[1] G. Agakichiev et al., Phys. Rev. Lett. 75 (1995) 1272.

[2] C. Song, S.H. Lee and C.M. Ko, Phys. Rev. C52 (1995) R476.

[3] C. Gale and J. Kapusta, Phys. Rev. C35 (1987) 2107.

[4] V. Koch and C. Song, Phys. Rev. C54 (1996) 1903. 


\title{
Dilepton production in relativistic heavy-ion collisions*
}

\author{
Volker Koch and Chungsik Song
}

Recently the CERES-collaboration has reported [1] a considerable enhancement in the dilepton spectrum over a hadronic cocktail in the invariant mass range $300 \mathrm{MeV} \leq \mathrm{M}_{\mathrm{inv}} \leq$ $500 \mathrm{MeV}$. Using a hadronic transport model we have calculated dilepton spectra using a wide range of initial conditions for the hadronic phase which are only constrained by the hadronic observables. The purpose of this exercise was twofold. First of all, we wanted to see to which extent the CERES data can be explained in a purely hadronic scenario including pion annihilation and other channels not included in the CERES cocktail. Secondly, we wanted to investigate to which extent a dilepton measurement puts additional constraints on the initial conditions of the hadronic phase. We found a very weak sensitivity of the dilepton invariant mass spectrum on the initial hadronic configurations (figure 1). As far as the CERES data are concerned we could reach the lower end of the sum of statistical and systematic error. It appears impossible, however, to explain the central points if they turn out to be correct after an improved measurement.

We also included the in-medium corrected pion annihilation cross section [2] into our calculation. While the pion-annihilation contribution is enhanced by a factor of about 2 in the low mass range, the total dilepton yield is only increased by $30 \%$ and, thus our medium effect does not explain the CERES enhancement (figure 2).

We predicted no additional enhancement when going from the small system $\mathrm{S}+\mathrm{Au}$ to the large system $\mathrm{Pb}+\mathrm{Pb}$. This is in agreement with preliminary data from the CERES collaboration [3].

* LBNL-38619: Phys. Rev. C54 (1996) 1903.
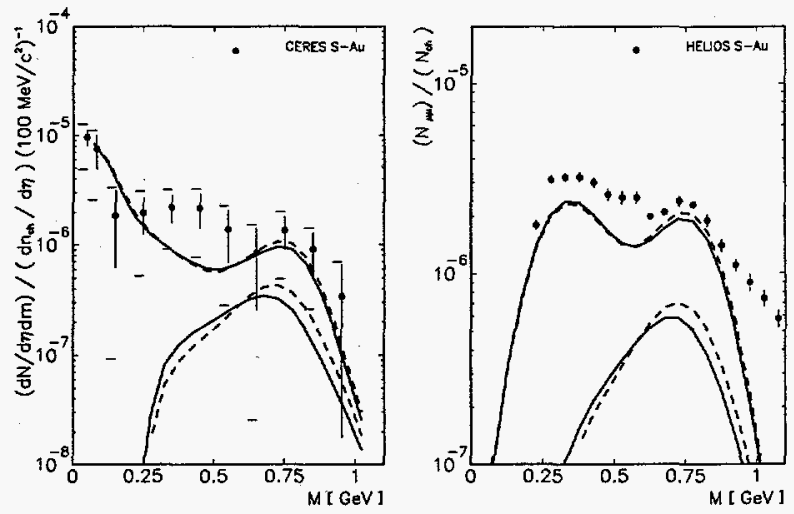

Figure 2: Dilepton invariant mass spectrum with (full line) and without (dashed line) in-medium corrected pion annihilation in comparison with CERES (left) and HELIOS [4] (right) data.

[1] G. Agakichiev et al., Phys. Rev. Lett. 75 (1995) 1272.

[2] C. Song, V. Koch, S.H. Lee and C.M. Ko, Phys. Lett. B366 (1996) 379.

[3] A. Drees, Proceedings Quark Matter '96, to appear in Nucl. Phys. A.

[4] M. Masera, Nucl. Phys. A590 (1995) 93c. 


\section{Pion electromagnetic formfactor at finite temperature * Chungsik Song and Volker Koch}

In [1] we have proposed that a combined effect of an in medium modified pion dispersion relation together with a change of the pion formfactor due to chiral symmetry could provide a qualitative explanation for the enhancement of the dilepton production reported by the CERES collaboration [2]. As previous studies in the context of BEVALAC energy heavy ion collisions have shown $[3,4]$, special care needs to be taken to ensure gauge invariance of the medium modified production amplitude. Using the soft pion limit, we explicitly could prove the gauge invariance of our result by showing that the relevant Ward-Takahashi identities are all satisfied.

An important result is that for back-to-back dilepton pairs the pion form factor in the timelike region does not have to go to one in the limit $k^{2} \rightarrow 0$. Charge conservation constrains only the limit of the zero-component of the vertexfunction

$$
\Gamma_{0}\left(k_{0} \rightarrow 0, \vec{k}=0\right)=-\partial \Pi_{\pi}(p) / \partial p_{0}
$$

If, on the other hand, one approaches $k^{2} \rightarrow 0$ from the space-like region the Ward-Takahashi identities constrain the spatial components of the vertex function

$\Gamma_{i}\left(k_{0}=0, k_{j}=0, j \neq i ; k_{i} \rightarrow 0\right)=-\frac{\partial \Pi_{\pi}(p)}{\partial p_{i}}$.

This of course is due to the fact that the heat bath breaks Lorentz invariance. In other words, there may very well a discontinuity in either $\Gamma_{0}$ or $\Gamma_{i}$ when going from space-like to time like photons. The actual pion formfactor we obtain is shown in figure 1.
As far as a quantitative comparison with the CERES data is concerned, we found [5] that the change in the pion form-factor around the mass of the $\rho$-meson, which is directly related with the onset of chiral restoration, can not be extracted from present data. To achieve that on needs to have good enough a mass resolution to identify the $\omega$-meson.

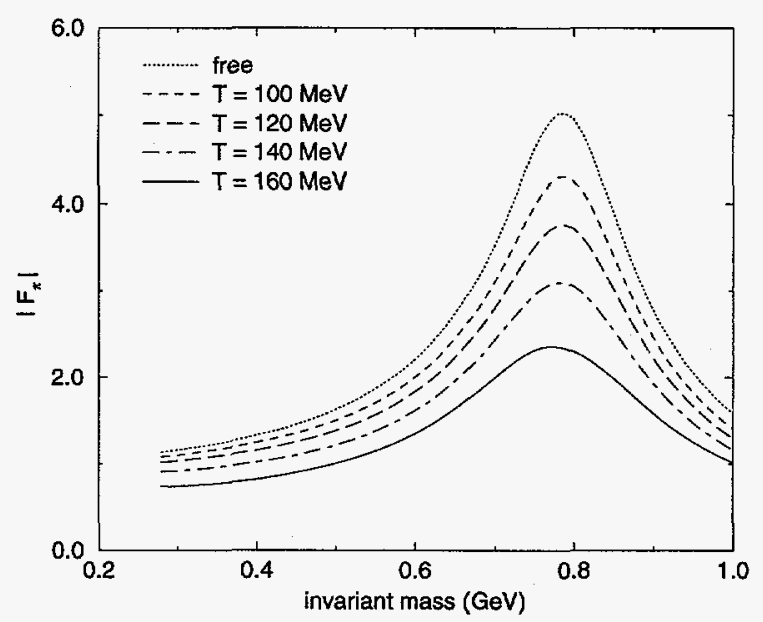

Figure 1: Pion form-factor at finite temperatures

[1] C. Song, V. Koch, S.H. Lee and C.M. Ko, Phys. Lett. B 366 (1996) 379.

[2] G. Agakichiev et al., Phys. Rev. Lett. 75 (1995) 1272.

[3] C. Gale and J. Kapusta, Phys. Rev. C35 (1987) 2107.

[4] C. Korpa and S. Pratt, Phys. Rev. Lett. 64 (1990) 1502.

[5] V. Koch and C. Song, Phys. Rev. C54 (1996) 1903.

* LBNL-38362: Phys. Rev. C54 (1996) 3218. 


\title{
Chemical relaxation time of pions in hot hadronic matter*
}

\author{
Chungsik Song and Volker Koch
}

We have studied the thermal and chemical relaxation time scales of pions in hot hadronic matter with an effective chiral Lagrangian. From the explicit calculation we show that pions in hot hadronic matter are in a phase where elastic collisions rates are very fast compared to typical expansion rates of the system. For chemical equilibration the dominant contribution comes from the inelastic collision involving $a_{1}$ mesons. Comparing with previous calculations [1] based on chiral perturbation theory, the inclusion of the resonances has reduced the chemical relaxation time by about a factor of 10 . When we neglect the formation time of these resonances, the resulting chemical relaxation time of pions is $10 \mathrm{fm} / \mathrm{c}$ at $T=150 \mathrm{MeV}$. This value is comparable to the size of the hot system produced the collision of large nuclei.

Given a system size of $5 \sim 10 \mathrm{fm}$ we obtain a thermal freeze-out temperature which is small compared to those extracted from experiments $[2,3]$. This might be due to flow effects which lead to smaller effective system sizes. If we take the thermal freeze-out temperature be about $150 \mathrm{MeV}$, then the freeze-out size of the system would be $2 \sim 3 \mathrm{fm}$. On the other hand the chemical relaxation time for a system of this size would be $T=170 \mathrm{MeV}$. This implies that chemical freeze-out of pions happens at considerably higher temperatures than thermal freezeout.

We also have studied the effect of baryons on the chemical relaxation time of pions. Since the effect of baryons is suppressed by their large mass, we consider only low laying baryons, $N(938)$ and $\Delta(1236)$. To estimate the relaxation time we use the phenomenological cross section for $N N \rightleftharpoons N \Delta$. The effect of baryons is very small and can be neglected in SPS experiments.

* LBNL-38363: submitted to Phys. Rev. C

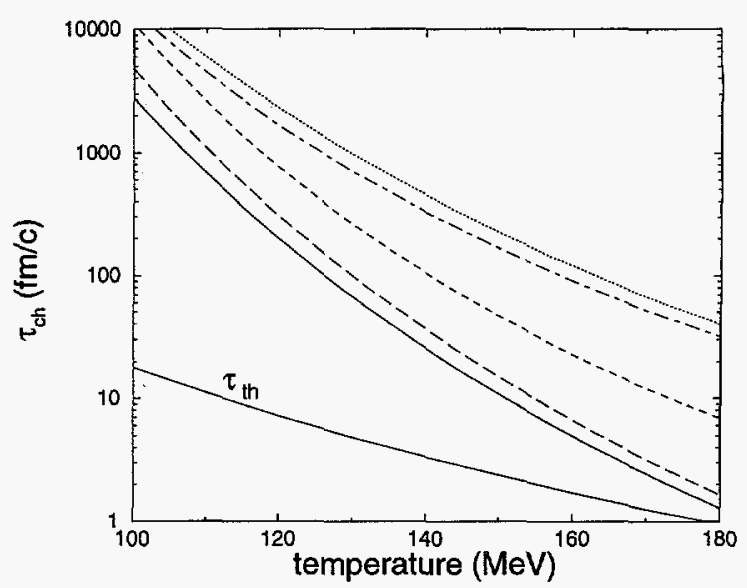

Figure 1: Chemical relaxation time of pions in hot hadronic matter. The reactions $\rho \rho \leftrightarrow \pi \pi$ (long dashed curve), $\pi \omega \leftrightarrow \pi \pi$ (dashed-dot curve), $\pi a_{1} \leftrightarrow \pi \pi$ (dashed curve) and $\pi \pi \pi \pi \leftrightarrow \pi \pi$ (dotted curve) are considered. The solid curve represents the total relaxation time. These results are compared with the thermal relaxation time, $\tau_{t h}$.

However, it becomes important in AGS experiments where the baryon chemical potential is much larger than that in the SPS experiments.

We have extend the definition for the chemical relaxation time to a system of pions out of equilibrium with $\mu_{\pi} \neq 0$. In this case it is possible that the overpopulated pions will be reduced by the inelastic reactions involving vector and axial vector mesons. At $T=150$ with $\mu_{\pi}=100 \mathrm{MeV}$ the relaxation time is about $2 \mathrm{fm} / \mathrm{c}$ which is certainly comparable to the system size at freezeout.

[1] J. L. Goity, Phys. Lett. B 319 (1993) 401.

[2] J. Stachel and P. Braun-Munzinger, Phys.

Lett. B 216 (1989) 1.

[3] U. Heinz, Nucl Phys. A 566 (1994) 205c. 


\title{
Excess of pions with chiral symmetry restoration*
}

\author{
Chungsik Song and Volker Koch
}

Chiral symmetry, a symmetry of quantum chromodynamics (QCD) in the limit of massless quarks, is spontaneously broken in the ground state of QCD as indicated by the small mass of the pion. At high temperatures chiral symmetry is expected to be restored. However, some intriguing questions still remain on how chiral symmetry is actually restored in hot hadronic matter and what are the signatures of the restored phase.

One of the interesting features of the symmetry restored phase is the appearance of the scalar $\sigma$-meson which forms a chiral multiplet with the pions. At low temperatures where chiral symmetry is spontaneously broken, the $\sigma$-meson has very large width due to the strong decay channel into two pions. On the other hand, as the quark condensate drops with increasing temperature, the mass difference of the $\sigma$-meson and pion becomes small. As a result, the decay width of scalar meson decreases because the phase space available for the outgoing pions is reduced. Close to the phase transition temperature $T_{\chi}$, the $\sigma$ becomes an elementary excitation.

The observation of a narrow width scalar meson has been suggested as a direct signature of chiral symmetry restoration [1], but since the $\sigma$ does not couple to any penetrating probes such as photons and dileptons this is difficult to observe in experiment. The purpose of this paper is to study the effect of the $\sigma$-meson on the pion production in high energy nucleus-nucleus collisions.

The pion production will depend on the thermodynamic conditions of a system during the evolution from hadronization to freeze-out. In the symmetric phase a relative chemical equilibrium among the particles will be established rapidly, leading to $\mu_{\sigma}=\mu_{\pi}$ and $\mu_{\rho}=2 \mu_{\pi}=\mu_{a_{1}}$.

* LBNL-39391: submitted to Phys. Lett. B
The number of scalar mesons is then given by one third of the total number of pions at the given temperature. This is quite different from the situation encountered in the broken phase, where $\mu_{\sigma}=2 \mu_{\pi}$ and $\mu_{a_{1}}=3 \mu_{\pi}$. This is mainly due to the appearance of the light $\sigma$ meson and the drop of the chiral condensate.

This difference in chemical equilibration conditions of pions might lead to the excess of pions at freeze-out. As temperature decreases and the symmetry is broken scalar mesons become heavier and decay into two pions. Also $a_{1}$ mesons decay into three pions. The number of observed pions will be given by the number of pions plus contributions from the resonance decay. When we include the scalar and $a_{1}$ meson contributions to observed pions, we have $1.4 \sim 1.6$ times more pions compared to the case when there is no chiral phase transition. This, however, can only be observed, if the chiral transition temperature is not too high $T_{\chi} \leq 180 \mathrm{MeV}$. Otherwise, pion number changing processes in the broken phase will absorb the excess obtained from the chiral phase transition.

Finally, let us conclude by pointing out that an analysis of the particle abundances measured at SPS-energy heavy ion collisions found an excess of pions by a factor of 1.6 over the expected thermal value [2]. However, a different analysis based on the same data, does not find such an excess of pions [3].

[1] T. Hatsuda and T. Kunihiro, Prog. Theor. Phys. Suppl. 91 (1987) 284.

H. A. Weldon, Phys. Lett. B 274(1992) 133.

R. Pisarski, Phys. Rev. Lett. 76 (1996) 76.

[2] M. Gazdzicki for the NA35 Collaboration, Nucl. Phys. A590, (1995) 215c.

[3] P. Braun-Munzinger, J. Stachel, J. Wessels and N. Xu, Phys. Lett. B 365 (1996) 1. 


\title{
Effective mass of phi mesons at finite temperature*
}

\author{
Chungsik Song
}

For phi mesons, changes in their properties are interesting because they would affect the production rate in high energy nucleus-nucleus collisions, which probes the enhancement of strangeness in quark-gluon plasma phase [1]. Recently, it also has been suggested that a change of phi mass in hot hadronic matter might be a possible probe for the chiral phase transition in hot matter [2].

The effective mass of phi meson at non-zero temperature is examined with an effective chiral Lagrangian. We find that $\phi$ effective mass decreases with temperature and is reduced by about $20 \mathrm{MeV}$ at $T=200 \mathrm{MeV}$. The dominant contributions come from kaon loops due to $\mathcal{L}_{\phi K K}$ and the $S U(3)_{V}$ symmetry breaking terms. As temperature increases effects from $K^{*}$ mesons become important and increase the effective mass of phi meson.

Compared with the calculation done by $\mathrm{K}$. Haglin and C. Gale [3], we do not have the large effect from kaon tadpole loops which increases phi mass. In our calculation we also have kaon tadpole loop corrections. However, these contributions come from $S U(3)_{V}$ symmetry breaking terms and show different effect. In the massive Yang-Mills approach which also includes vector mesons in chirally symmetric way, these tadpole loop contributions are exactly canceled by those from axial-vector-pseudoscalar loops [4]. This implies that chiral symmetry plays an important role in vector meson mass at finite temperature and shows different result. Calculations using QCD sum rules also show that the effective mass of phi mesons decreases with temperature. However, we have a small effect compared to the result obtained from in Ref. [2] and have an opposite result for $K^{*}$ contributions. This difference requires more study in future.

* LBNL-38475: Phys. Lett. B 388 (1996) 141.

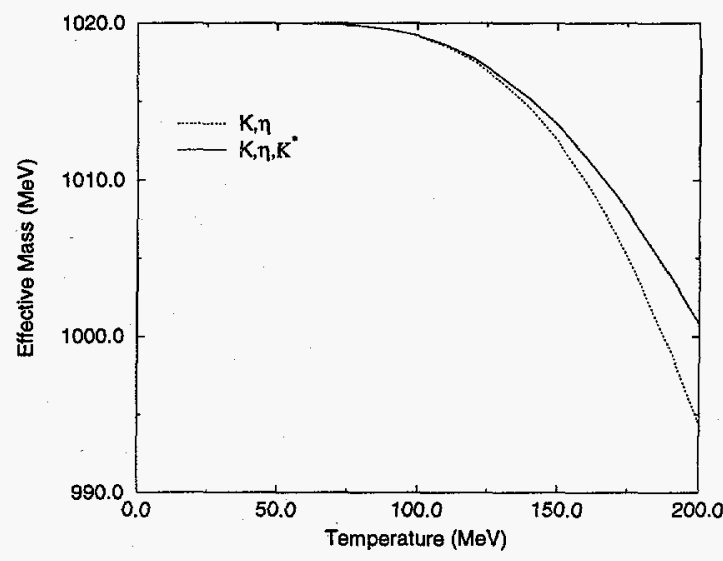

Figure 1: Effective mass of phi meson at finite temperature

A small change in $\phi$ effective mass makes it hard to observe the double phi peak in dilepton spectrum which has been suggested as a possible probes of chiral phase transition in hot hadronic matter. Since it has been estimated that the effective width of phi mesons in hot hadronic matter becomes about $20 \sim 30 \mathrm{MeV}$, the mass shift obtained from the calculation will be within the effective width. With very accurate mass resolution, we might have a chance to see these small changes of the effective mass in dilepton spectrum. Details will also depend on the coupling of phi meson to photon which will be modified in hot hadronic matter as for $\rho$ mesons.

[1] Pin-Zhen Bi and J. Rafelski, Phys. Lett. B 262 (1991) 485.

[2] M. Asakawa and C. M. Ko, Phys. Lett. B

322 (1994) 33, Nucl. Phys. A 572 (1994) 732.

[3] K. Haglin and C. Gale, Nucl. Phys. B 421 (1994) 613.

[4] Chungsik Song, Phys. Rev. D 53 (1996) 3962. 


\title{
Dissipation and Decoherence in Mean Field Theory*
}

\author{
S. Habib ${ }^{\dagger}$, Y. Kluger, E. Mottola ${ }^{\dagger}$, and J.P. Paz ${ }^{\dagger}$
}

The time evolution of a closed system of mean fields and fluctuations is Hamiltonian, with the canonical variables parameterizing the general time-dependent Gaussian density matrix of the system. Yet, the evolution exhibits both quantum decoherence and apparent irreversibility of energy flow from the coherent mean fields to fluctuating quantum modes. Using scalar QED as an example, we show how this collisionless damping and decoherence may be understood as the result of dephasing of the rapidly varying fluctuations and particle production in the time varying mean field.

Mean field methods have a long history in such diverse areas as atomic physics (BornOppenheimer), nuclear physics (Hartree-Fock), condensed matter (BCS) and statistical physics (Landau-Ginzburg), quantum optics (coherent/squeezed states), and semiclassical gravity. Because no higher than second moments of the fluctuations are incorporated, the mean field approximation is related to a Gaussian variational ansatz for the wave function of the system.

The broad applicability of the approximation, as well as the variety of different approaches to it in the literature makes it worthwhile to exhibit its general features unobscured by the particulars of specific applications. Accordingly, our first purpose in this work is to demonstrate the equivalence of the time-dependent mean field approximation to the general Gaussian ansatz for the mixed state density matrix $\rho$, and to underline its Hamiltonian structure.
The Hamiltonian nature of the evolution makes it clear from the outset that the mean field approximation does not introduce dissipation or time irreversibility at a fundamental level. Nevertheless, typical evolutions seemingly manifest an arrow of time, in the sense that energy flows from the mean field to the fluctuations without returning over times of physical interest.

Closely connected to this effective dissipation is the phenomenon of quantum decoherence, i.e. the suppression with time of the overlap between wave functions corresponding to two different mean field evolutions. This decoherence is the reason why quantum superpositions of different mean field states are difficult to observe in nature, and is crucial to understanding the quantum to classical transition in macroscopic systems.

Our second aim in this investigation is to present an explicit example of a quantum field theory (scalar QED) treated in mean field approximation where these effects are observed, and to provide a clear physical explanation of the behavior in terms of dephasing of the fluctuations, i.e., the averaging to zero of their rapidly varying phases on time scales short compared to the collective motion of the mean field(s).

\footnotetext{
* LBNL-37786; Phys. Rev. Lett. 76 (1996) 4660.

† Theory Division, Los Alamos National Laboratory, Los Alamos, New Mexico.
} 


\title{
Anomalous transverse distribution of pions as a signal for disoriented chiral condensates*
}

\author{
F. Cooper ${ }^{\dagger}, Y$. Kluger, and E. Mottola ${ }^{\dagger}$
}

We give evidence that the production of DCC's during a non-equilibrium phase transition can lead to an anomalous transverse distribution of secondary pions when compared to a more conventional boost invariant hydrodynamic flow in local thermal equilibrium. Our results pertain to the linear $\sigma$ model, treated in leading order in large- $N$, in a boost invariant approximation

In this work we see that a non-equilibrium phase transition taking place during a time evolving quark-gluon or hadronic plasma can lead to an enhancement of the low transverse momentum distribution. In particular, if a Centauro type event is not accompanied by such an enhancement one would be suspicious of ascribing this event to the production of disoriented chiral condensates as a result of a rapid quench.
Transverse expansion and direct pion-pion collisions are two competing effects that might change the degree of enhancement. The inclusion of transverse expansion might lead to amplification of the low momenta modes. On the other hand in the mean field approximation the pions can interact with the mean fields (DCC) via the auxiliary field $\chi$ but do not interact directly with each other. Direct pion-pion collisions will tend to thermalize the system, and reduce the amplification of the unstable low momentum modes. This effect is included only in the next to leading order term of the $1 / N$ expansion, and we plan to incorporate it in a future work.

* LBL-38585; hep-ph/9604284; Phys. Rev. C (in press).
† Theory Division, LANL, Los Alamos, New Mexico.

\section{Symmetry breaking in $\lambda \phi^{4}$ theory* \\ F. Cooper ${ }^{\dagger}, S . H a b i b^{\dagger}, Y$. Kluger, and E. Mottola ${ }^{\dagger}$}

The time evolution of $O(N)$ symmetric lambda $\Phi^{4}$ scalar field theory is studied in the large $N$ limit. In this limit the $\langle\Phi\rangle$ mean field and two-point correlation function $\langle\Phi \Phi\rangle$ evolve together as a self-consistent closed Hamiltonian system, characterized by a Gaussian density matrix. The static part of the effective Hamiltonian defines the True Effective Potential $U_{\text {eff }}$ for configurations far from thermal equilibrium. Numerically solving the time evolution equations for energy densities corresponding to a quench in the unstable spinodal region, we find results quite different from what might be inferred from the equilibrium free energy "effective" potential $F$. Typical time evolutions show effectively irreversible energy flow from the coherent mean fields to the quantum fluctuating modes, due to the creation of massless Goldstone bosons near threshold. The plasma frequency and collisionless damping rate of the mean fields are calculated in terms of the particle number density by a linear response analysis and compared with the numerical results. Dephasing of the fluctuations leads also to the growth of an effective entropy and the transition from quantum to classical behavior of the ensemble. In addition to casting some light on fundamental issues of nonequilibrium quantum statistical mechanics, the general framework presented in this work may be applied to a study of the dynamics of second order phase transitions in a wide variety of Landau-Ginsburg systems described by a scalar order parameter.

\footnotetext{
* hep-ph/9610345; Phys. Rev. D. (in press)

$\dagger$ Theory Division, LANL, Los Alamos, New Mexico.
} 


\title{
Statistical Properties of the Linear Sigma Model ${ }^{*}$
}

\author{
Jørgen Randrup
}

The statistical equilibrium properties of the linear sigma model are studied, with a view towards characterizing the field configurations employed as initial conditions for numerical simulations of the formation of disoriented chiral condensates in high-energy nuclear collisions.

The $O(4)$ chiral field $\phi=(\sigma, \pi)$ is subject to a non-linear interaction, $V=(\lambda / 4)\left(\phi^{2}-v^{2}\right)^{2}-H \sigma$. The field can be decomposed into its spatial average, the order parameter, and the fluctuations, the quasi-particles, and enclosed in a box.

The quantized quasi-particle modes are described approximately by Klein-Gordon dispersion relations with an effective mass that depends on both the temperature and the magnitude of the order parameter:

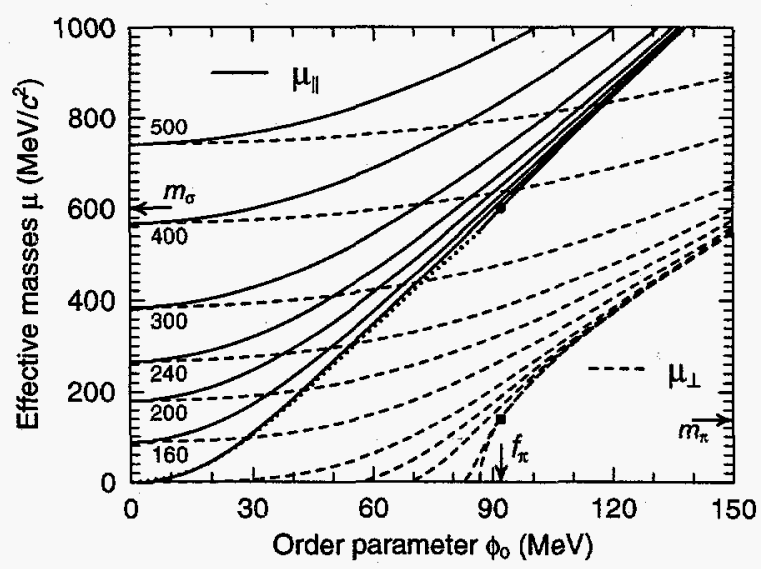

Figure 1: The effective masses $\mu_{\|}$and $\mu_{\perp}$ as functions of $\phi_{0}$ for a range of temperatures, calculated in the thermodynamic limit, $L \rightarrow \infty$. For temperatures above $T_{c}$, the curves start at $\phi_{0}=0$ with degenerate values, whereas below $T_{c}$ they only exist if $\phi_{0}$ is sufficiently large. The corresponding starting points for $\mu_{\|}$are connected by the dotted curve (only $T=0$ is shown). Also shown are the free masses $m_{\sigma}$ and $m_{\pi}$.

* LBNL-38125 (1996); Physical Review D (in press).
The thermal fluctuations are instrumental in shaping the effective potential for the order parameter. As the system is cooled the field fluctuations subside, causing a smooth change from the hot phase having approximate chiral symmetry towards the broken phase:

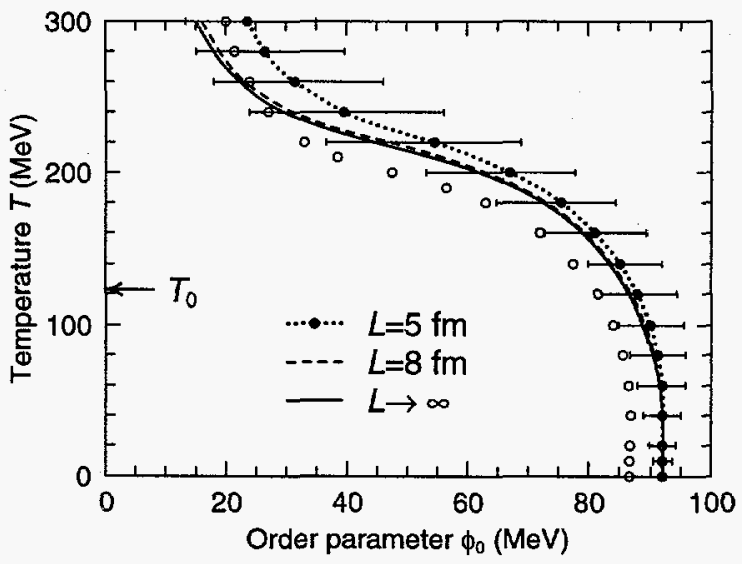

Figure 2: The magnitude of the order parameter, $\phi_{0}$. For a box with $L=5 \mathrm{fm}$, the bars show the full width at half maximum of the thermal distribution of $\phi_{0}$ and the open dots show the centroids for the idealized case having $H=0$; the behavior is qualitatively similar even though the nature of the phase transition changes.

Of practical interest is the fact that the equilibrium field configurations can be sampled in a simple manner, thus providing a convenient means for specifying the initial conditions in $d y$ namical simulations of the non-equilibrium relaxation of the chiral field.

The corresponding correlation function is briefly considered and used to calculate the spectral strength of radiated pions.

Finally, by propagating samples of initial configurations by the exact equation of motion, it has been ascertained that the treatment is sufficiently accurate to be of practical utility. 


\section{Amplification of pionic instabilities in high-energy collisions?* \\ Jørgen Randrup}

The possibility of forming disoriented chiral condensates in high-energy collisions has generated considerable research activity in recent years. We have developed a simple and instructive framework for analyzing the conditions for the occurrence of the $D C C$ phenomenon.

Enclosing the system in a box, we decompose the $O(4)$ chiral field $\phi=(\sigma, \pi)$ into its spatial average $\phi(t)$, the order parameter, and the fluctuating remainder $\delta \phi(x, t)$ representing the quasiparticle excitations. A Hartree factorization [1] then yields Klein-Gordon equations,

$$
\left[\square+\mu^{2}\right] \delta \phi(x, t)=0
$$

The associated effective masses are given by

$$
\begin{aligned}
& \mu_{\sigma}^{2}=\lambda\left[3 \sigma_{0}^{2}+<\delta \phi^{2}>+2<\delta \sigma^{2}>-v^{2}\right], \\
& \mu_{\pi}^{2}=\lambda\left[\sigma_{0}^{2}+<\delta \phi^{2}>+2<\delta \pi_{i}^{2}>-v^{2}\right],
\end{aligned}
$$

where the usual non-linear interaction hass been employed, $V=(\lambda / 4)\left(\phi^{2}-v^{2}\right)^{2}-H \sigma$.

Imagine that the system is formed at a high temperature. The field fluctuations are then sufficiently large to ensure $\mu^{2}>0$. The combined effect of expansion and radiation cools the system and the fluctuations thus decrease in the course of time. This reduces $\mu^{2}$ which allows the order parameter to grow larger, thus counteracting the decrease of the effective masses. The resulting behavior of $\mu^{2}$ is then a delicate balance: for slow cooling the induced growth of $\sigma_{0}$ is approximately adiabatic and the system relaxes towards the vacuum through metastable configurations. On the other hand, for a sufficiently rapid cooling a compensating growth of the order parameter cannot occur quickly enough and the effective pion mass may turn imaginary, $\mu_{\pi}^{2}<0$, indicating that the system has entered a regime exhibiting exponential growth of some modes.

* LBNL-38379: Physical Review Letters 77 (1996) 1226

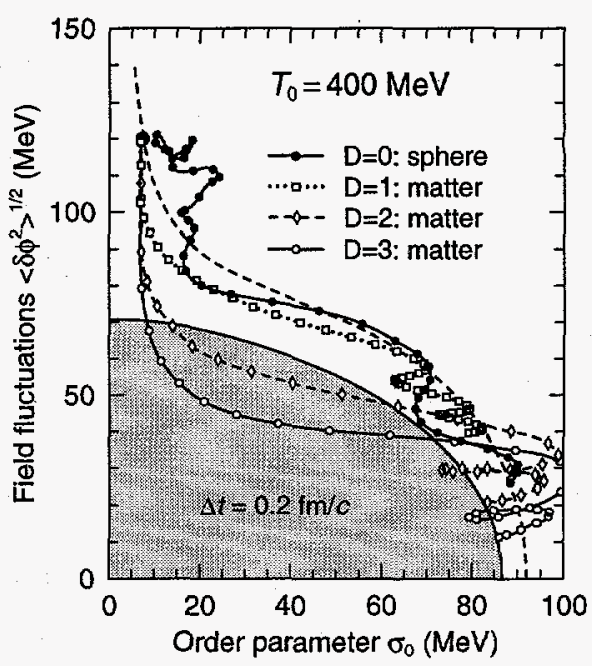

Figure 1: The combined dynamical evolution of the order parameter and the field fluctuations. The dashed curve connects the equilibria and the shaded region is the unstable region within which $\mu_{\pi}^{2}<0$. The systems start in equilibrium at $T_{0}=400 \mathrm{MeV}$. The irregular solid trajectory $(D=0)$ has been obtained by solving the standard equations of motion for a spherical source with $R=5 \mathrm{fm}$. The other three trajectories have been obtained by adding a cooling term emulating expansion in $D=1,2,3$ dimensions.

The resulting distortion of the pion spectrum can be estimated by a simple WKB method in which the integral of the imaginary frequency yields an amplification factor given by $\exp \left(G_{k}\right)$ :

Table 1: Amplification coefficients $\dot{G}_{k=0}^{\pi}$.

\begin{tabular}{|c|llc|}
\hline$T_{0}(\mathrm{MeV})$ & $D=1$ & $D=2$ & $D=3$ \\
\hline 200 & 0.00 & 0.02 & 0.11 \\
220 & 0.00 & 0.50 & 0.55 \\
240 & 0.01 & 1.20 & 1.19 \\
300 & 0.00 & 1.84 & 2.06 \\
400 & 0.00 & 1.67 & 2.49 \\
500 & 0.00 & 1.31 & 2.61 \\
\hline
\end{tabular}

[1] J. Randrup, Physical Review D (in press). 


\title{
Mean-field treatment of the linear sigma model in dynamical calculations of DCC observables*
}

\author{
Jørgen Randrup
}

Approximate mean-field equations of motion for the classical chiral field are developed within the linear sigma model by means of a Hartree factorization. Both the approximate and the unapproximated equations of motion are augmented with a Rayleigh cooling term to emulate a uniform expansion, thereby allowing the extraction of observables relevant to the detection of disoriented chiral condensates. While the mean-field dynamics appears to be sufficiently accurate to be practically useful, the results also illustrate some of the difficulties associated with identifying the phenomenon experimentally.

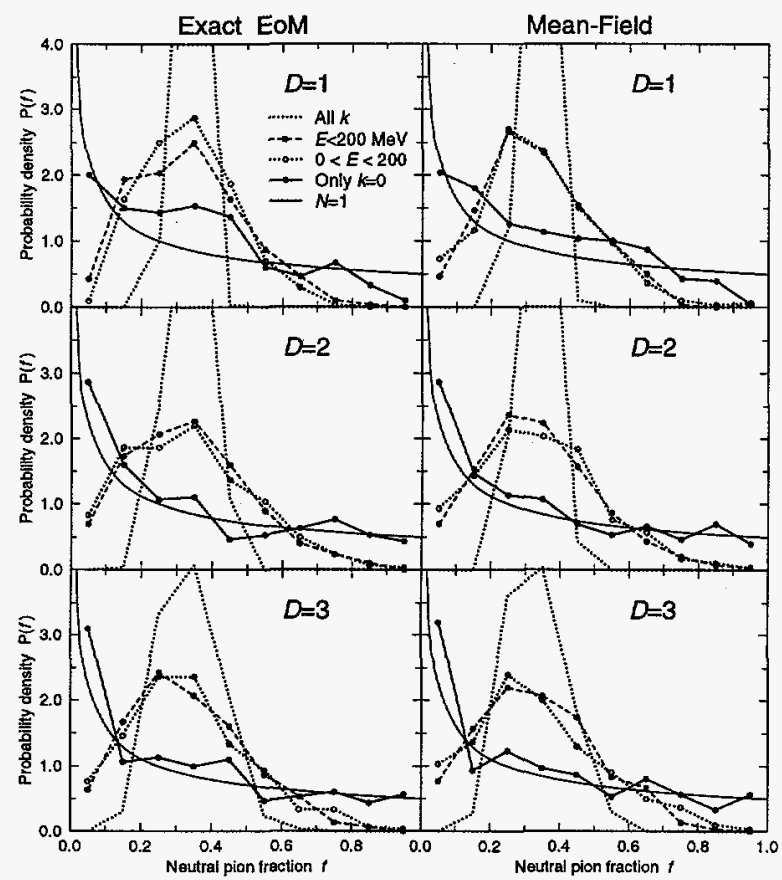

Figure 1: Distribution of the neutral fraction $=n_{0} / n$ after pseudo-expansion in $D$ dimensions, using either the exact equation of motion (left) or the proposed mean-field approximation (right).

* LBNL-39328 (1996): Nuclear Physics A (in press)

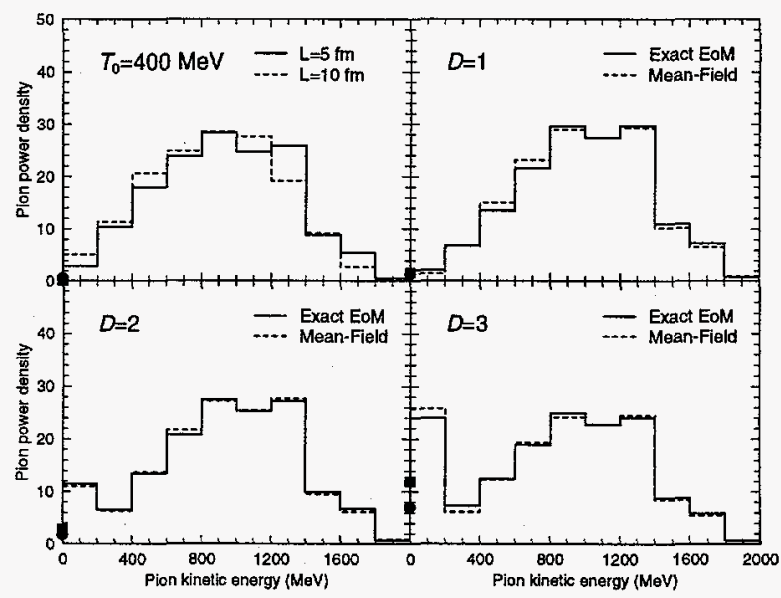

Figure 2: Pion power spectrum either in thermal equilibrium (upper-left) [1] or after a pseudo-expansion in $D$ dimensions [2], with the exact equation of motion (solid) as well as the developed mean-field approximation (dashed).

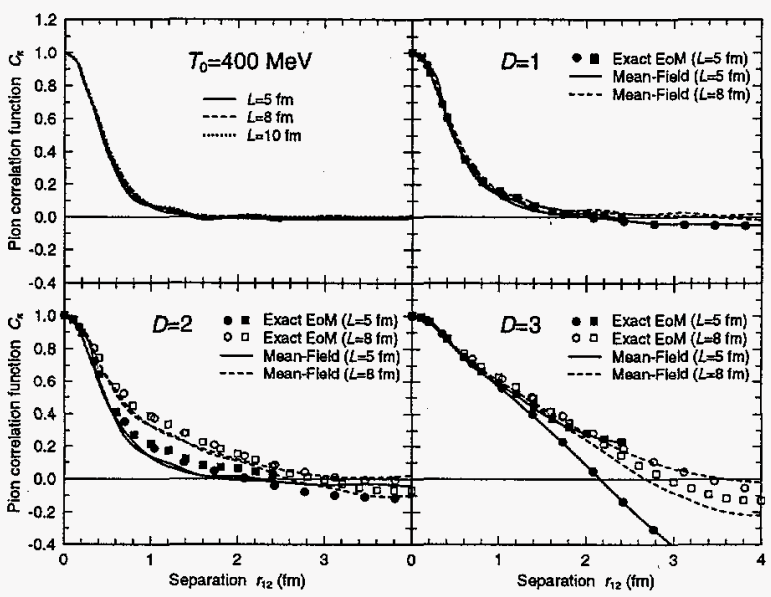

Figure 3: Pion correlation function for the same scenarios as in fig. 2.

[1] J. Randrup, Physical Review D (in press).

[2] J. Randrup, Physical Review Letters 77 (1996) 1226 


\title{
Treatment of Pionic Modes at the Nuclear Surface for Transport Descriptions*
}

\author{
Johan Helsgesson ${ }^{\dagger}$ and Jørgen Randrup
}

Using a recently developed $\pi+N N^{-1}+\Delta N^{-1}$ model $[1,2]$, we derive dispersion relations and amplitudes for collective pionic modes for use in transport descriptions.

In-medium properties obtained in an infinite stationary system consisting of interacting nucleons, nucleon resonances and mesons, can be incorporated into transport descriptions by a local density approximation. We have obtained density dependent dispersion relations of the pionic modes, as well as density dependent amplitudes of the components constituting the pionic mode. These quantities are conveniently parametrized in terms of a few density dependent coefficients.

While such a prescription is rather straightforward to implement in the interior regions of the nuclear system, conceptual problems exist at the nuclear surface. When the nuclear density approaches zero, collective mesonic modes formed in the medium have to be converted to real particles in vacuum. The problems arise since some collective modes (e.g. $\Delta N^{-1}$-like) may exists in the infinite stationary system at arbitrary low (but non-vanishing) density, but no corresponding real particle exists in vacuum. It is discussed how pionic modes can be converted to real particles when penetrating the nuclear surface and how earlier treatments can be improved. When the surface is stationary only free pions emerge.

For the transport process it is not necessary to determine the character of the pionic modes until they penetrate the surface and emerge as free particles. This is automatically determined within our formalism from the amplitudes at zero density. The time-dependent situation has also been addressed.

ECT $^{*}$, European Centre for Studies in Theoretical Nuclear Physics and Related Areas, Trento, Italy.

* LBL-37623: Nucl. Phys. A597 (1996) 672.

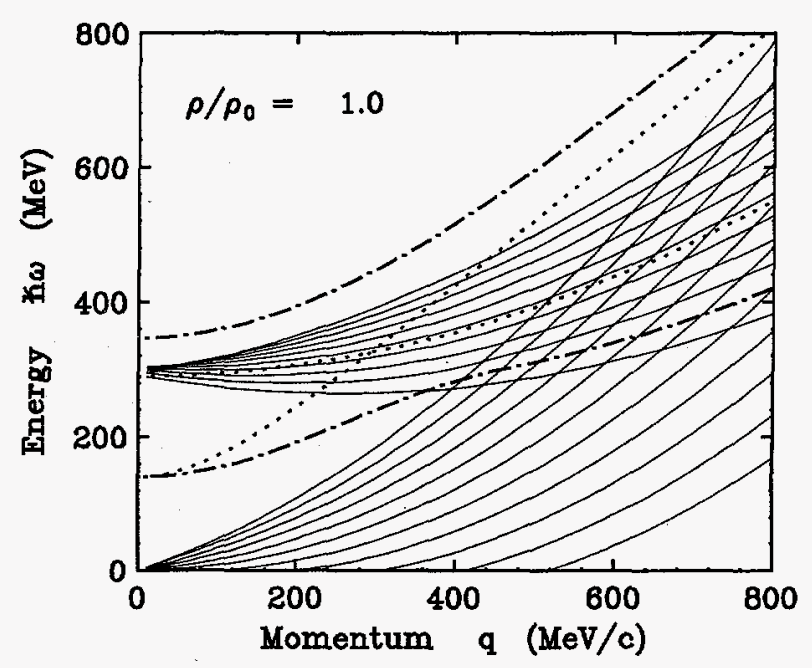

Figure 1: The dispersion relations for the spin-isospin modes in the spin-longitudinal channel, in infinite nuclear matter at normal nuclear density and for zero temperature. The non-collective modes are shown by solid curves, while the collective modes $\tilde{\pi}_{1}$ and $\tilde{\pi}_{2}$ are represented by dot-dashed curves.

We have further shown that for a stationary density profile, the conservation of the energy of the pionic mode and the partial $\Delta$ decay width combine to gurantee that only real pions are realized as free particles when the pionic mode penetrates the surface.

A simplified one-dimensional scenario is used to investigate the reflection and transmission of pionic modes at the nuclear surface. It is found that reflection of pionic modes is rather unlikely, but the process can be incorporated into transport descriptions by the use of approximate local transmission coefficients.

[1] J. Helgesson and J. Randrup, Ann. Phys. 244 (1996) 12

[2] J. Helgesson and J. Randrup, Phys. Rev. C52 (1995) 427 


\title{
Simplified Treatment of Collective Instabilities in Matter*
}

\author{
Jørgen Randrup
}

A formal analysis was made of the onset of instabilities in dilute nuclear matter in which collective modes are agitated by stochastic nucleonnecleon collisions and then exponentially amplified by the self-consistent field. Moreover, employing approximate expressions for the transport coefficients [1], simple expressions were derived for the key quantities, so that survey calculations are facilitated.

It is convenient to discuss instabilities in uniform matter in terms of the Landau parameter $F_{0}=\left(3 \rho / 2 \epsilon_{F}\right)(\partial h / \partial \rho)$. When harmonic modes in matter are considered, this quantity generalizes to $F_{0}(k) \equiv\left(3 \rho / 2 \epsilon_{F}\right)\left(\partial h_{k} / \partial \rho\right)$, involving the appropriate Fourier component of the selfconsistent response. Further generalization is useful at finite $T$,

$$
F_{T}(k) \equiv \phi_{0} \frac{\rho}{T} \frac{\partial h_{k}}{\partial \rho} \approx F_{0}(k)\left[1-\frac{\pi^{2}}{12}\left(\frac{T}{\epsilon_{F}}\right)^{2}\right],
$$

employing the Sommerfeld expansion of the Fermi-surface moment $\phi_{0}$ [2]. The dispersion relation for the collective growth time $t_{k} \equiv$ $m / k P_{F} \gamma_{k}$ is then to a good approximation given by $1=F_{T}(k)\left(\gamma_{k} \arctan \left(1 / \gamma_{k}\right)-1\right)$, which can readily be solved by iteration:

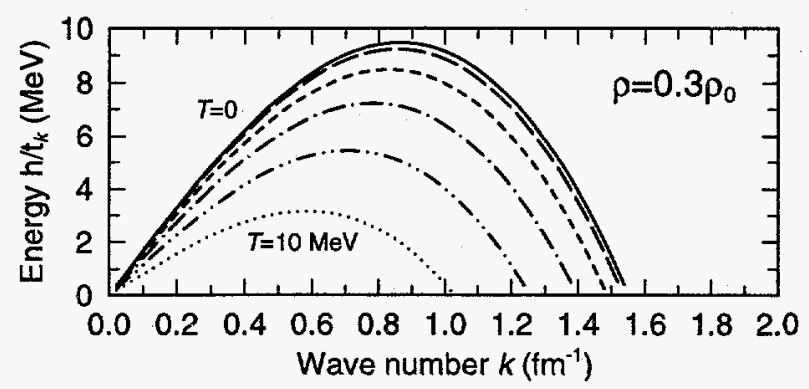

Figure 1: Characteristic energies for the collective modes in unstable matter, $E_{k}=\hbar / t_{k}$, with the generalized Seyler-Blanchard model [3].

* LBL-35847: Nucl. Phys. A583 (1995) 329
The random collisions provide a source term generating density irregularities which are then amplified by the unstable mean field $h$ [4], leading quickly from a linear growth to an explosive development, $\sigma_{k} \approx \mathcal{D}_{k} \exp \left(2 t / t_{k}\right)$ :

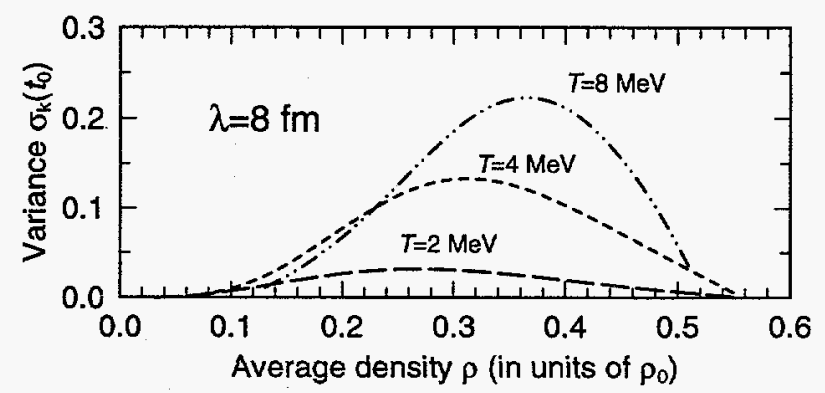

Figure 2: The magnitude of the variance $\sigma_{k}$ of density fluctuations having the wave length $\lambda=2 \pi / k=8 \mathrm{fm}$ (near which the most rapid growth occurs), after a given time $t_{0}=10^{-22} \mathrm{~s}$, as a function of the average density $\rho$ and for specified temperatures $T$.

The derived analytical approximations facilitate the analysis of the onset of fragmentation in the spinodal zone of the phase diagram. Both the source terms for the fluctuations and the amplification times can be easily obtained. Several additional results are also useful, including the expansion of the angular quantities on complex Legendre polynomials, which is helpful for understanding the multipolarity properties.

[1] J. Randrup and S. Ayik, Nucl. Phys. A572 (1994) 489

[2] J. Randrup, Statistical Description of Transport in Plasma, Astro, and Nuclear Physics, Les Houches, France (1992), Nova Science, p. 353

[3] E. de Lima Medeiros and J. Randrup, Phys. Rev. C45 (1991) 372

[4] M. Colonna, Ph. Chomaz, and J. Randrup, Nucl. Phys. A567 (1994) 637 


\title{
Multifragmentation with Brownian One-Body Dynamics*
}

\author{
A. Guarnera ${ }^{1,2}, P h$. Chomaz ${ }^{1}, M$. Colonna ${ }^{1,3}$, and Jørgen Randrup
}

A first application to nuclear multifragmentation has been made of Brownian One-Body Dynamics [1], a recently suggested simplified model for the dynamics of the one-body phasespace density that approximates the nuclear Boltzmann-Langevin description.

A gold nucleus is compressed to double density and then let free to evolve under the combined influence of the effective one-body field and the residual two-body collision processes, with the effects of the fluctuations included whenever local spinodal instability occurs.

The system quickly expands into a hollow and unstable configuration which transforms into several intermediate-mass fragments. The analysis of the resulting fragment pattern suggests that the model provides a physically reasonable description of nuclear multifragmentation.

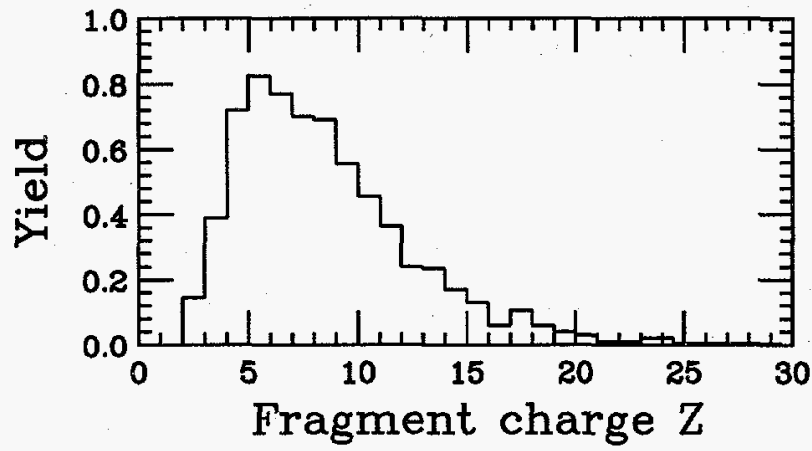

Figure 1: Fragment charge distribution. The fragment charge distribution as obtained by analyzing the matter density at time $t=200 \mathrm{fm} / \mathrm{c}$ using a density cut equal to $\rho_{\text {cut }}=0.05 \mathrm{fm}^{-3}$.

1 GANIL, B.P. 5027, F-14021 Caen Cedex, France
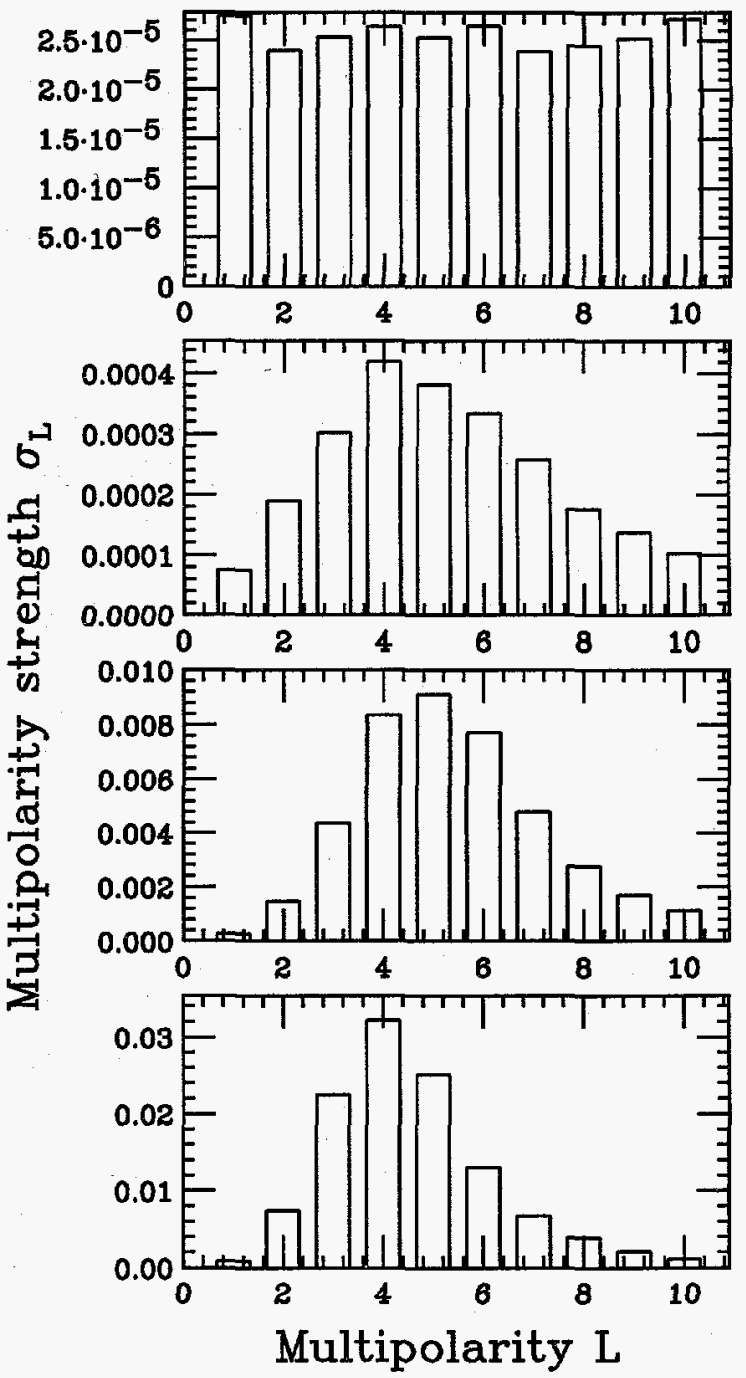

Figure 2: Multipole strength distribution. The multipole strength coefficients $\sigma_{L}$ [2] are shown at various points in time, $t=0,60,120,180 \mathrm{fm} / c$, as the initially compressed gold nucleus expands towards a fragmenting hollow shell. The results are obtained by averaging over 100 events.

${ }^{2}$ Laboratorio Nazionale del Sud, Viale Andrea Doria, I95129 Catania, Italy

3 CEA DAPNIA, CE Saclay, F-91191 Gif-sur-Yvette Cedex, France

* LBL-39544 (1996): Physics Letters B (submitted).

[1] Ph. Chomaz, M. Colonna, A. Guarnera, and

J. Randrup, Phys. Rev. Lett. 73 (1994) 3512

[2] G. Batko and J. Randrup, Nucl. Phys. A563 (1993) 97 


\title{
Inclusion of Quantum Fluctuations in Wave Packet Dynamics*
}

\author{
Akira Ohnishi ${ }^{\dagger}$ and Jørgen Randrup
}

We have addressed the treatment of quantum fluctuations in microscopic descriptions based on wave packets, a problem encountered in a broad range of fields involving quantum physics. Since the wave packets are not energy eigenstates, the statistical operator $\exp (-\beta \hat{H})$ cannot be treated as a $c$-number. In order to take account of the associated spectral distribution and ensure that the statistical properties are quantal, it is necessary to introduce suitable modifications relative to the ordinary equations of motion for the wave packet parameters which are basically classical in character, having been derived from the timedependent variational principle.

We have formulated a simple but apparently successful treatment by including the first correction term in the cumulant expansion of the statistical weight. The associated small parameter is $\sigma_{E}^{2} / T \mathcal{H}$, where $\mathcal{H} \equiv\langle Z|\hat{H}| \boldsymbol{Z}\rangle$ is the mean energy of a wave packet and $\sigma_{E}^{2}$ is the corresponding energy variance. This approach is exact when the spectral distribution is of Poisson form, and the corresponding effective level spacing is $D \equiv-\partial \log \mathcal{H}_{\beta} / \partial \beta=\sigma_{E}^{2} / \mathcal{H}$. It is then straightforward to write down the improved expression for the statistical weight. Moreover, the associated thermal distortion of the internal structure of the wave packet can be determined.

Since our initial suggestion [1] that this treatment might be useful has led to some debate $[2,3]$, we have discussed and illustrated the various possible approaches to determining the statistical behavior of one-body observables, such as the occupation number. The key to resolving the issue lies in the inevitable distortion of the many-body wave packet caused by the canonical operator $\exp (-\beta \hat{H})$.

* LBNL-38596 (1996); Annals of Physics 253 (1997) 279.

† Department of Physics, Faculty of Science, Hokkaido University, Sapporo 060, Japan.
The practical utility was exemplified by ${ }^{12} \mathrm{C}$ and ${ }^{40} \mathrm{Ca}$ which exhibit the desired evolution from a quantum fluid to a fragment gas, as the temperature is raised.

The quantum fluctuations can be incorporated into the dynamics by allowing stochastic transitions between the wave packets [4]. This can conveniently be done by introducing a quantum Langevin force in the equations of motion for the wave packet parameters. We derived the general form of the associated transport coefficients and verified that the proper microcanonical equilibrium distribution is indeed achieved. Simple approximate expressions for the specific values of the transport coefficients were then obtained, leading to a practically useful treatment.

The proposed extension of the standard treatment represents a formally well based approach. Moreover, it leads to the desired statistical properties in static scenarios and can be included in the dynamics in a conceptually simple and tractable manner. The method may, therefore, find useful application in the context of microscopic simulations of actual many-body processes, such as the production of nuclear fragments [5] and atomic clusters [6].

[1] A. Ohnishi and J. Randrup, LBL-33703, Nucl. Phys. A565 (1993) 474

[2] J. Schnack and H. Feldmeier, Nucl. Phys. A601 (1996) 181

[3] A. Ono and H. Horiuchi, Phys. Rev. C53 (1996) 2341

[4] A. Ohnishi and J. Randrup, LBL-36358, Phys. Rev. Lett. 75 (1995) 596

[5] A. Ohnishi and J. Randrup, LBNL-39473, Phys. Lett. B (in press).

[6] A. Ohnishi and J. Randrup, LBNL-39754, Phys. Rev. A (submitted). 


\title{
Quantum Fluctuation Effects on Nuclear Fragment Formation*
}

\author{
Akira Ohnishi ${ }^{\dagger}$ and Jørgen Randrup
}

Many-body systems described by wave packets contain quantum fluctuations which may significantly affect the statistical properties [1]. The effect can be included in dynamical treatments by means of a quantal Langevin force $[2,3]$. We have adapted the treatment to QMD simulations of nuclear collisions where the individual nucleons are described by gaussian wave packets. This augmentation endows the system with larger fluctuations and the excitation of the emerging fragments is smaller. These two features conspire to enhance the production of intermediate-mass fragments. The effect on the mass discribution of atomic clusters has also been studied [4].

The usual QMD treatment leads to primary fragments that are typically sufficiently excited to emit nucleons and it is therefore essential to consider the statistical decay chain. This process causes a strong suppression of the IMFs, and a corresponding enhancement of lighter fragments. By contrast, the quantum Langevin treatment leads to fragments having a relatively small degree of excitation and, consequently, a larger proportion of them survive the statistical decay.

Our results for $\mathrm{Au}+\mathrm{Au}$ collisions (illustrated in fig. 1) indicate that the inclusion of the quantum fluctuations in the wavepacket dynamics leads to a significant increase in the production of massive fragments with low excitation. Although the experimental data are not yet reproduced quantitatively, the improvement is significant over the results obtained with the usual treatments in which the quantum fluctuations are ignored. This general qualitative result suggests that the underlying quantum nature of the nuclear many-body system may indeed play a significant role in fragmentation reactions.

* LBNL-39473 (1996); Physics Letters B (in press).

$t$ Department of Physics, Faculty of Science, Hokkaido University, Sapporo 060, Japan.

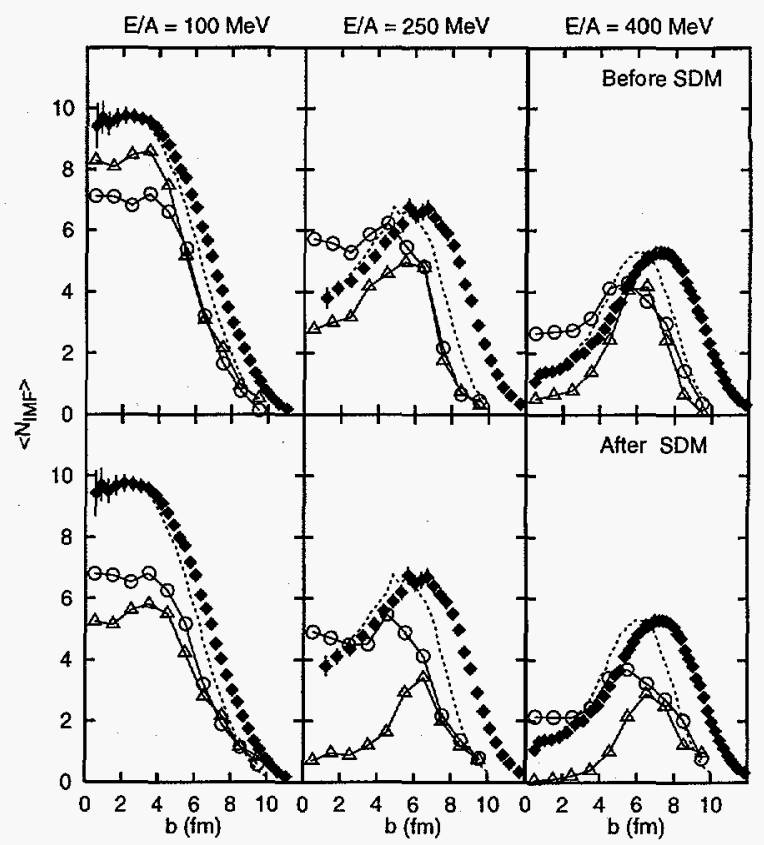

Figure 1: IMF multiplicity before and after statistical decay for central Au+Au collisions. Circles and triangles indicate $\mathrm{QMD}$ results at given energies with and without the quantal Langevin force, respectively. The upper and lower parts show the distributions before and after the statistical decay [5], respectively. The experimental data [6] are shown by solid diamonds.

[1] A. Ohnishi and J. Randrup, LBL-33703, Nucl. Phys. A565 (1993) 474

[2] A. Ohnishi and J. Randrup, LBL-36358, Phys. Rev. Lett. 75 (1995) 596

[3] A. Ohnishi and J. Randrup, LBL-38596, Ann. Phys. (in press).

[4] A. Ohnishi and J. Randrup, LBNL-39754, Phys. Rev. A (submitted)

[5] F. Pühlhofer, Nucl. Phys. A280 (1977) 267

[6] M.B. Tsang et al., Phys. Rev. Lett. 71 (1993) 1502 


\section{Quantum Fluctuations Affect Critical Properties of Noble Gases*}

Akira Ohnishi ${ }^{\dagger}$ and Jørgen Randrup

Molecular dynamics presents a powerful tool for elucidating the features of mesoscopic systems. The present communication draws attention to the possible importance of quantum fluctuations in such treatments. To illustrate this issue, we focus on the cluster mass distribution for argon atoms in thermal equilibrium.

We have adapted a recently developed quantal Langevin treatment $[1,2]$ to a system of argon atoms in thermal equilibrium. The method was developed in the context of nuclear dynamics and takes approximate account of the energy fluctuations that are necessarily present when wave packets are used to describe the system. The presence of these quantum fluctuations changes the character of the specific heat from classical to quantal and their inclusion by the developed method leads to a significant improvement of the statistical properties in a number of simple test cases that can be subjected to exact analysis $[2,3]$ as well as for finite nuclei $[1,2]$. When incorporated into microscopic dynamical simulations of nuclear collisions, it leads to a significant improvement of the calculated fragment mass distribution [4].

Our studies suggest that quantum fluctuations inherent in wave packet dynamics may also play a role in atomic physics. As illustrated in fig. 1, the cluster mass distribution for noble gases can be affected significantly. In particular, the critical temperature for argon is reduced by around $20 \%$, which is the value of the factor $\alpha$ entering into the modified Einstein relation. The quantum fluctuations may therefore also affect the formation process and might affect the outcome of dynamical simulations aimed at understanding the observed mass distributions.

\footnotetext{
* LBL-39754 (1996); Physical Review A (submitted).

† Department of Physics, Faculty of Science, Hokkaido University, Sapporo 060, Japan.
}

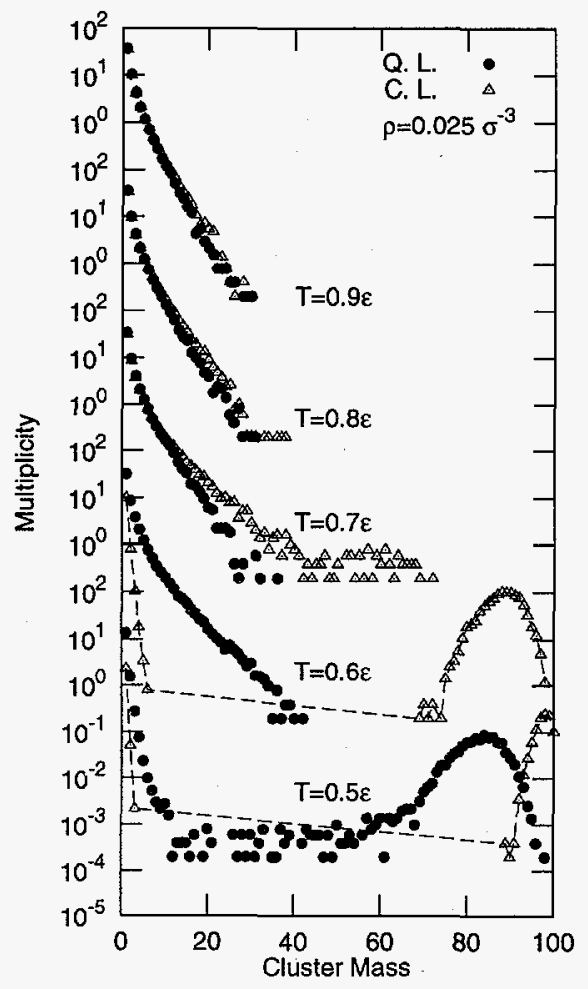

Figure 1: The mass distribution of argon clusters calculated either with (solid circle) or without (open triangle) the quantum Langevin force emulating the quantum fluctuations inherent in wave-packet dynamics. The argon atoms interact with a Lennard-Jones potential, $V(r)=\epsilon\left((\sigma / r)^{12}-(\sigma / r)^{6}\right)$; the temperatures are indicated in units of $\epsilon$ and the atomic density is $\rho=0.025 \sigma^{-3}$.

[1] A. Ohnishi and J. Randrup, LBL-36358, Phys. Rev. Lett. 75 (1995) 596

[2] A. Ohnishi and J. Randrup, LBL-38596, Ann. Phys. (in press)

[3] A. Ohnishi and J. Randrup, LBNL-39473, Phys. Lett. B (in press)

[4] A. Ohnishi and J. Randrup, LBL-33703, Nucl. Phys. A565 (1993) 474 


\title{
$K^{+}$Momentum Spectrum from $\left(K^{-}, K^{+}\right)$Reactions in the Intranuclear Cascade Model ${ }^{*}$
}

\author{
Yasushi Nara $^{a}$, Akira Ohnishi ${ }^{a}$, Toru Harada ${ }^{b}$, Andreas Engel ${ }^{c}$
}

Multi-strange systems have recently attracted much attention in nuclear, particle, and astrophysics, as they may yield information on the generalized nuclear interaction between octet baryons, may reveal the inter-quark forces through the study of " $H$ " particle, and may be realized as strange matter in neutron stars. Among them, nuclear systems with $S=-2$ form the starting point of these studies.

Recently, it has become possible to measure double strangeness exchange reactions $\left(K^{-}, K^{+}\right)$ on nuclear targets. For example, small angle $\left(K^{-}, K^{+}\right)$cross sections at $p_{K^{-}}=1.65 \mathrm{GeV} / \mathrm{c}$ were measured on several targets at KEK-PS [1]. The measured $K^{+}$momentum spectrum shows a striking structure: in addition to quasifree peaks reflecting the elementary process $\mathrm{K}^{-} p \rightarrow$ $K^{+} \Xi^{-}$, there appears a broad bump extending from $p_{K^{+}}=0.35 \mathrm{GeV} / \mathrm{c}$ to $1.0 \mathrm{GeV} / \mathrm{c}$.

We have studied $K^{+}$momentum spectra in $\left(K^{-}, K^{+}\right)$reactions and double-hyperfragment $(S=-2)$ formation by using a intranuclear cascade model calculation. We have found that this bump is well explained by taking account of direct reactions $p\left(K^{-}, K^{+}\right) \Xi^{(*)}$, heavy meson subthreshold production and decay [2], and various two-step processes (Fig. 1); in the first step, the strange quark in the $K^{-}$particle is transferred to other baryons, and $\bar{s} s$ pair creation occurs in the second step. These two-step processes are effective in producing $K^{+}$mesons in this energy region for the following two reasons:

* nucl-th/9608017 (1996): Nuclear Physics A (in press).

$a$ Department of Physics, Faculty of Science, Hokkaido University, Sapporo 060, Japan.

$b$ Department of Social Information, Sapporo Gakuin University, Ebetsu 069, Japan.

c Department of Physics, Kyoto University, Kyoto 606-01, Japan. (a) The incident energy corresponds to baryon resonance region, where the cross section becomes maximum. (b) When the intermediate mesons are heavy $(\rho, \eta, \omega, \ldots)$, the second step reaction becomes exoergic $(Q>0)$, then the strangenes production cross sections with these mesons are much larger than those with pions.

This two-step mechanism not only explains the $K^{+}$spectra for various nuclear targets, but also suggests that more double-hyperfragments would be formed at this momentum region. More detailed study of double-hyperfragment formation in $\left(K^{-}, K^{+}\right)$reaction and $\Xi^{-}$absorption at rest, which is the other clean reaction to make double-hyperfragments, is in progress.

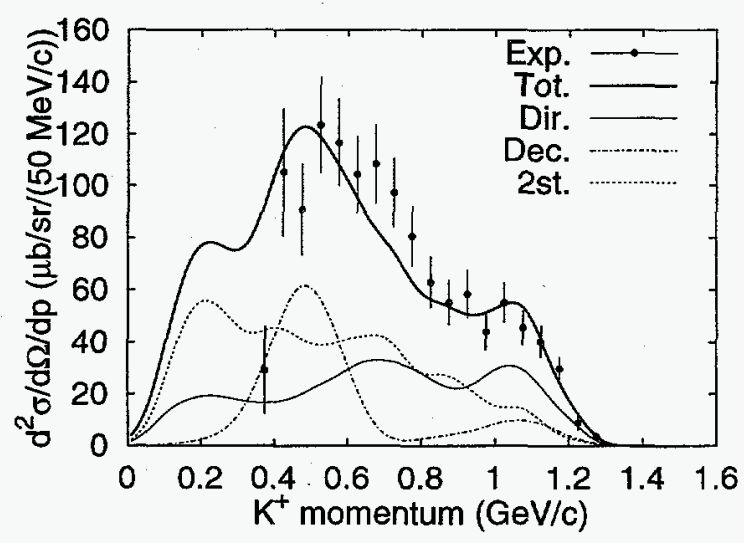

Figure 1: $K^{+}$momentum spectrum from the ${ }^{107} \mathrm{Ag}\left(K^{-}, K^{+}\right)$reaction. The thin solid, dot-dashed, and dotted lines show the contribution from direct processes, meson decays, and two-step processes, respectively. The thick solid line represents the calculated total spectrum. The experimental data are taken from [1].

[1] T. Tijima et al., Nuc. Phys. A546 (1992) 588. [2] C. Gobbi, C. B. Dover and A. Gal, Phys. Rev. C50 (1994) 1594. 


\section{Four-Alpha Linear-Chain States in ${ }^{16} \mathbf{O}^{*}$ \\ Naoyuki Itagaki ${ }^{\dagger}$, Akira Ohnishi ${ }^{\dagger}$, and Kiyoshi Katō ${ }^{\dagger}$}

Nuclei with large deformations are at the frontier in nuclear spectroscopy. It is expected that deformations above 1:3 may be possible in light nuclei due to the $\alpha$-cluster structure. For example, the possibility of multi- $\alpha$ linear-chain configuration in light nuclei, such as the $4 \alpha$ linearchain band starting around the $4 \alpha$ threshold energy region in ${ }^{16} \mathrm{O}$, has been suggested experimentally [1].

Here we study $4 \alpha$ linear-chain states in ${ }^{16} \mathrm{O}$ in comparison with $3 \alpha$ states in ${ }^{12} \mathrm{C}$ by using the Generator Coordinate Method within a microscopic $N \alpha$-cluster model. In contrast to the previous theoretical works, we discuss the stability of the $N \alpha$ linear-chain structure by solving dynamics of $N \alpha$ systems. Also it is shown to be very important to take into account the orthogonality between the linear-chain states and other low-lying states including the ground state. This is achieved by diagonalizing the Hamiltonian matrix in a wide space which also covers low-lying levels including the ground state. The framework used here is based on the combination of the Generator Coordinate Method (GCM) and the Constraint Cooling Method [2] proposed in the framework of AMD in order to generate GCM basis which describes low-lying levels.

The linear-chain state of ${ }^{12} \mathrm{C}$ is hardly considered to be a stable state as we increase the number of the GCM basis states. Their components fragment to the levels over broad range of excitation energy, especially to the $0_{2}^{+}$state, and these couplings make a pure linear-chain state unstable.

* nucl-th/9606056.

$\dagger$ Department of Physics, Faculty of Science, Hokkaido University, Sapporo 060, Japan.

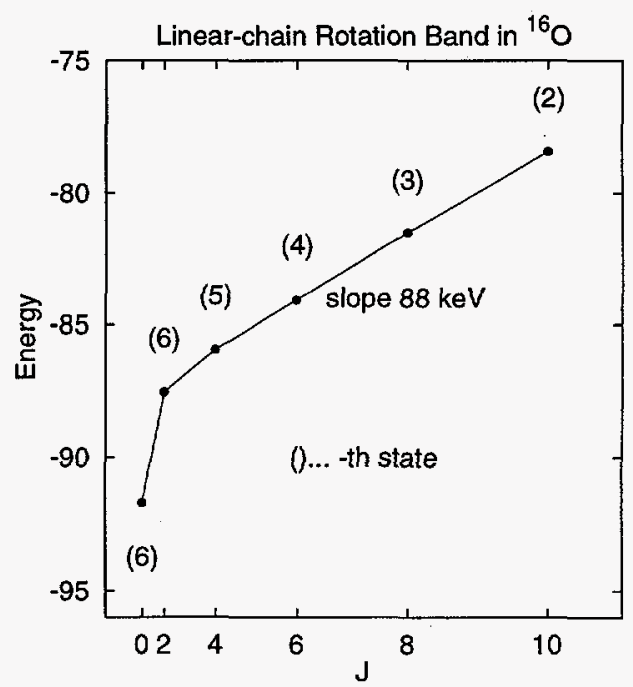

Figure 1: The rotational band of ${ }^{16} \mathrm{O}$ linear-chain state. The numbers show the order of excited state.

On the other hand, the $4 \alpha$ chain state in ${ }^{16} \mathrm{O}$ is hardly affected by low-lying states and persists to remain above the $4 \alpha$ threshold. The calculated moment of inertia of the $4 \alpha$ linear-chain rotational band ( $88 \mathrm{keV}$ ) reproduces the experimentally suggested value qualitatively. These conclusions are consistent with the previous work by Ikeda [3] where the stability against small vibrations around the equilibrium configuration is considered. Moreover, we have shown that the linear-chain state of ${ }^{16} \mathrm{O}$ may survive even after the couplings to the lower states are taken into account.

[1] P. Chevallier and F. Scheibling, Phys. Rev. 160 (1967) 160.

[2] Y. Kanada-En'yo and H. Horiuchi, Prog. Theor. Phys. 93 (1995) 115.

[3] K. Ikeda, N. Takigawa and H. Horiuchi, Prog. Theor. Phys. Suppl. Extra Number (1968) 464. 


\title{
Detectability of Strange Matter in Heavy Ion Experiments*
}

\author{
J. Schaffner-Bielich ${ }^{\dagger, \ddagger}$, C. Greiner $\$$, A. Diener ${ }^{\dagger}$ and H. Stöcker ${ }^{\dagger}$
}

Strangeness and antistrangeness is abundantly produced in heavy ion collisions. Baryonic objects with a high strangeness fraction may be formed, like small droplets of strange quark matter (strangelets) or metastable exotic multihypernuclear objects (MEMOs) consisting of nucleons and multiple hyperons [1]. Both are expected to possess properties quite different form ordinary nuclei, like a negative charge while carrying a positive baryon number. We examined the properties of both forms of strange matter for low masses, its decay properties and its detectability for heavy ion experiments.

The MEMOs were treated within the framework of an extended relativistic mean-field theory. We extended the calculation of [1] to small mass numbers which are most interesting for heavy ion physics. We found that the two smallest bound systems for $A=4$ are ${ }^{4} \mathrm{He}$ and the corresponding $\Xi$-system, i.e. two $\Xi^{-}$and two $\Xi^{0}$. The next heavier ones $(A=6)$ are the combinations ${ }_{\Lambda \Lambda}^{6} \mathrm{He},\left\{2 \mathrm{n}, 2 \Lambda, 2 \Xi^{-}\right\},\left\{2 \mathrm{p}, 2 \Lambda, 2 \Xi^{0}\right\}$, and $\left\{2 \Lambda, 2 \Xi^{0}, 2 \Xi^{-}\right\}$. Note that the double $\Lambda$ hypernucleus $\wedge{ }^{6} \mathrm{He}$ has already been seen experimentally. These objects live, if formed, on the time scale of the weak hyperon decay, i.e. $\tau \approx 10^{-10}$ $\mathrm{s}$. They can not be seen with the present experimental setups, as they are only sensitive to much larger lifetimes.

We calculated the binding energy of strangelets for an arbitrary number of up, down, and strange quarks using the MIT bag model. Afterwards we look for possible strong decays, i.e. nucleon, hyperon and pion emission. We checked also for fission of a strangelet into another strangelet and an arbitrary number of hadrons. This allows for example for a decay where the a strangelet emits a neutron and a pion. A strangelet stable against weak hadronic decays can then only decay via weak leptonic de- cay or via weak radiative decays and lives on the time scale of $\tau_{l}=10^{-4}-10^{-5} \mathrm{~s}$ which is well in reach of present heavy ion experiments.

The long-lived strangelets found are mainly lying on a chain which starts from the triple magic strangelet $(6 u 6 d 6 s)$. The 'valley of stability' continues then towards negative charges by adding one unit of negative charge when going to a higher mass number. The reason for this stability line is a pronounced shell effect as e.g. the first candidate is also triple magic ( $6 u 6 d 18 s$ or $6 u 18 d 6 s)$. Note that these candidates are all highly negative charged contrary to common belief, while most experiments are, however, designed to detect only particles with small chargeto-mass ratio.

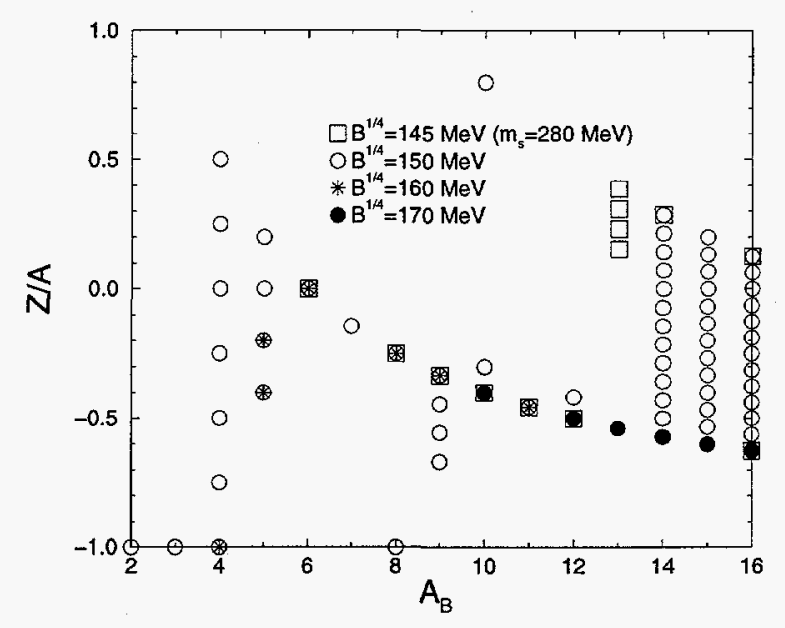

${ }^{\dagger}$ Niels Bohr Institute, Copenhagen, Denmark

‡ Institut für Theoretische Physik, University of Frankfurt, Germany

$\S$ Institut für Theoretische Physik, University of Giessen, Germany

* LBL-39652; Physical Review C (submitted)

[1] J. Schaffner, C.B. Dover, A. Gal, C. Greiner, H. Stöcker, Phys. Rev. Lett. 71, 1328 (1993) 


\title{
Quark Phase Transition \& Time-structure of Pulsar Spin-down*
}

\author{
N. K. Glendenning, S.Pei ${ }^{\dagger}$ and F. Weber ${ }^{\dagger \dagger}$
}

Pulsars-highly magnetized rotating neutron stars-emit magnetic dipole radiation and a wind of electron-positron pairs. Through the energy and angular momentum loss the angular velocity decreases. As a pulsar spins down it becomes less deformed and the central density rises. For some pulsars the mass and initial angular velocity $\Omega$ may be such that the central density rises from below to above the critical density for dissolution of baryons into their quark constituents. Because the compressibility of quark matter is greater than that of the confined phase, the phase change will be mirrored in structural changes in the star such as its size and moment of inertia: the star has entered an era in which it shrinks anomalously as it spins down over time because an increasing proportion of the stellar mass is converted to the more compressible deconfined phase. The star's mass becomes ever more concentrated near its center-more so than would be the case for a star composed of a simple fluid on which a weakening centrifugal force was acting. The concentration arising from the greater compressibility of quark matter is amplified by its greater gravitational attraction on the outer parts of the star.

At the stage described, the tendency of the star to shrink as the region occupied by quark matter grows in radius, counteracts (by angular momentum conservation) the spin-down rate $(\dot{\Omega})$ caused by radiation. The development of a growing central region of deconfined quark matter acts, so to speak, as a governor. Therefore the rates at which the mass becomes concentrated near the center and the star shrinks are large as functions of decreasing angular velocity but small as functions of time. A strong anomalous time-structure, which will endure for a long time, is introduced into the spin-down of a pulsar by conversion of the core to quark matter.

The anomaly in time-structure will appear in the value and behavior of the so-called braking index, a dimensionless measurable quantity,

$$
n(\Omega) \equiv \frac{\Omega \ddot{\Omega}}{\dot{\Omega}^{2}}=n-\frac{3 I^{\prime} \Omega+I^{\prime \prime} \Omega^{2}}{2 I+I^{\prime} \Omega},
$$

where $I^{\prime} \equiv d I / d \Omega$ reflects the response of the moment of inertia to centrifugal and structural changes and $n=3$ for magnetic dipole radiation. The behavior of $n(\Omega)$ for a model star is shown in Fig. 1 where the anomaly is clearly visible in the departure from the smooth curve.

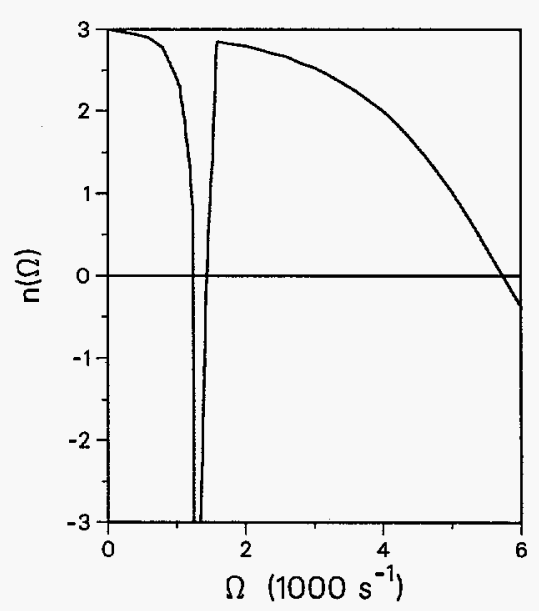

Figure 1: Braking index anomaly.

We estimate the plausibility of observing the phase transitions in the pulsar population. The duration over which the observable index is anomalous is $\Delta T=-\Delta \Omega / \dot{\Omega}$ where $\Delta \Omega$ is the frequency interval of the anomaly. For a typical period derivative, $\dot{P} \sim 10^{-16}$, we find $\Delta T \sim 10^{5}$ years. During a typical pulsar's active lifetime, about $10^{7} \mathrm{yr}$, the signal (negative index) would endure for $1 / 100$ of the lifetime. Given that $\sim 10^{3}$ pulsars are known about 10 of them may be signaling the phase transition.

* LBL-39746.

$\dagger$ Beijing Normal University.

${ }^{\dagger \dagger}$ Ludwig-Maximilians University of Munich. 


\title{
Response of Pulsar Braking to Rotation and Internal Structure*
}

\author{
N. K. Glendenning, S. Pei ${ }^{\dagger}$ and F. Weber ${ }^{\dagger \dagger}$
}

Pulsar spin-down occurs because of energy and angular momentum loss due to radiative processes. The energy loss equation representing processes of multipolarity $n$ is of the form

$$
\frac{d E}{d t}=\frac{d}{d t}\left(\frac{1}{2} I \Omega^{2}\right)=-C \Omega^{n+1}
$$

where, for magnetic dipole radiation, $C=$ $\frac{2}{3} m^{2} \sin ^{2} \alpha, n=3, m$ is the magnetic dipole moment and $\alpha$ is the angle of inclination between magnetic moment and rotation axis. Other multipoles may participate or be important over certain eras of a pulsar's lifetime (for example gravitational radiation in an early era before pulsations are damped). For all such processes the response of the moment of inertia $I$ to the changing angular velocity $\Omega$ will produce its effect upon the behavior of $\dot{\Omega}$. While such other processes may be uncertain or speculative, the response of the internal constitution and moment of inertia of a neutron star to changing rotational frequency are not. Taking account of the response, the rate of change of frequency is governed by

$$
\dot{\Omega}=-\frac{C}{I(\Omega)}\left[1+\frac{I^{\prime}(\Omega) \Omega}{2 I(\Omega)}\right]^{-1} \Omega^{n}
$$

where $I^{\prime} \equiv d I / d \Omega$. This reduces to the usual form quoted in the literature [1] for low frequency or if changes in $I$ are ignored, namely

$$
\dot{\Omega}=-K \Omega^{n} \quad(K=C / I) .
$$

The usual expression for the moment of inertia in General Relativity [2] is not adequate for our purpose. It ignores the alteration of the metric of spacetime by rotation and the dragging of local inertial frames, and even the centrifugal flattening and changes in internal constitution resulting from the changing density profile of the star with changing rotational frequency. Instead we must use an expression that incorporates these effects as derived by Glendenning and Weber [3].
The dimensionless quantity $\Omega \ddot{\Omega} / \dot{\Omega}^{2}$ would be equal to the intrinsic index $n$ if the frequency were small or if the moment of inertia were a constant, as can be obtained from (3). However these conditions are not usually fulfilled and the measurable quantity is not the constant index of the energy-loss mechanism (1) but a variable 'braking' index as can be calculated from (2). The measurable quantity is

$$
n(\Omega) \equiv \frac{\Omega \ddot{\Omega}}{\dot{\Omega}^{2}}=n-\frac{3 I^{\prime} \Omega+I^{\prime \prime} \Omega^{2}}{2 I+I^{\prime} \Omega} .
$$

It approaches the index $n$ that characterizes the energy loss mechanism only in the limits $\Omega \rightarrow 0$ or $I \rightarrow$ constant.

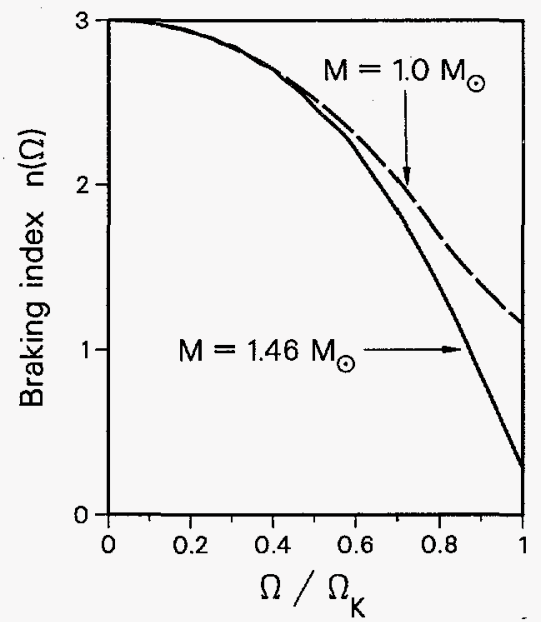

Figure 1: Braking index for two stellar models of different mass showing the strong departure from $n=3$ for higher frequencies.

* LBL-37592.

$\dagger$ Beijing Normal University.

${ }^{\dagger \dagger}$ Ludwig-Maximilians University of Munich.

[1] R. N. Manchester and J. H. Taylor, Pulsars, (W. H. Freeman and Co., San Francisco, 1977). [2] J. B. Hartle, Astrophys. J. 150 (1967) 1005.

[3] N. K. Glendenning and F. Weber, Astrophys. J. 400 (1992) 647; Phys. Rev. D 50 (1994) 3836 . 


\title{
Crystalline Structure Size in Neutron Stars*
}

\author{
N. K. Glendenning and S. $P_{e i}^{\dagger}$
}

We investigate the size of the objects forming a crystal lattice in the mixed phase of confined and deconfined matter in neutron stars whose presence was predicted in [1]. The objects take various forms according to the pressure and therefore the location in the star. We idealize the geometry of the objects as droplets, rods and slabs. As the spacing between droplets decreases, they merge to from rods, and the rods to form slabs.

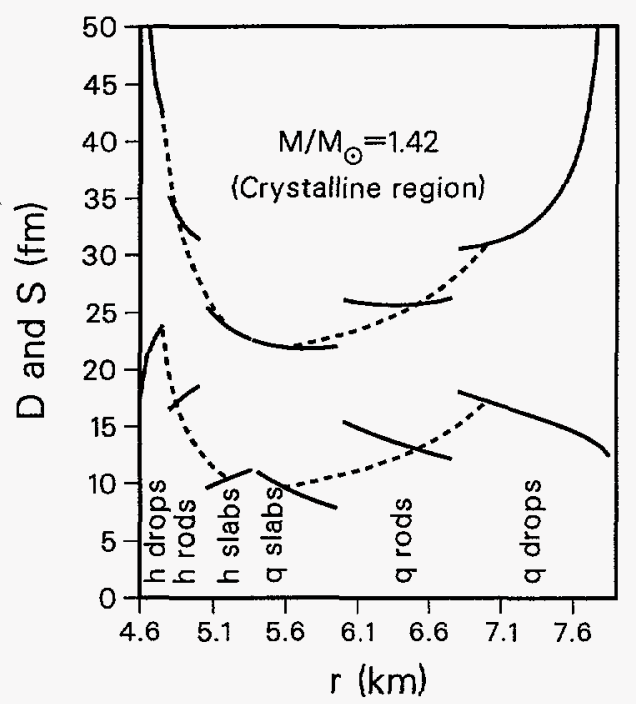

Figure 1: Crystalline mixed region in a neutron star showing the diameter D (lower curves) and spacing $S$ (upper curves) of indicated geometric structures as a function of radial Schwarzschild coordinate. Region internal to $4.6 \mathrm{~km}$ is pure quark matter. Notation ' $h$ drops' denotes hadronic drops immersed in quark matter, and so on. Stellar radius is $10.7 \mathrm{~km}$. Dashed line is continuous dimensionality interpolation

In Fig. 1 we show the diameter of the geometrical objects as a function of radial location in a star, identify their forms and plot the spacing between centers of the rare phase objects immersed in the other phase. The pressure at the center and extending to $4.6 \mathrm{~km}$ is sufficiently high that this region is occupied by pure quark mat- ter. The region between 4.6 and $7.8 \mathrm{~km}$ is in the crystalline confined-deconfined phase. Since the phase transition is completed before the center of the star is reached, the geometrical structures span the full range of forms. At the outer edge of the mixed phase region, quark drops of finite diameter are spaced, in the limit of no quark matter, at infinite distance. At greater depth and therefore proportion of quark matter the drops are more closely spaced and slightly larger in size. Deeper in the star, drops are no longer the favored configuration and merge to from rods of varying diameter

It is almost certain that solid regions in a pulsar will play a role in the period glitch phenomenon, whether associated with cracking of the solid as in star quakes, or with unpinning of superfluid vortices from solid regions. Glitch behavior is highly individualistic from one pulsar to another. We tentatively suggest that this may be due to the extreme sensitivity on stellar mass of the radial extent of the solid crystal region and the forms that occupy it as well as the moments of inertia associated with different gaseous (quark), liquid and solid regions. The interior solid region of the mixed phase and the crustal solid, separated by a nuclear liquid, offer interesting possibilities for interaction or stimulated response at the time of a glitch originating in one of them and in post-glitch recovery.

* N. K. Glendenning and S. Pei, Phys. Rev. C 52 (1995) 2250.

$\dagger$ Beijing Normal University.

[1] N. K. Glendenning, Phys. Rev. D, 46 (1992) 1274. 


\title{
Crystalline Cores of Neutron Stars*
}

\author{
N. K. Glendenning
}

First order phase transitions have been much studied in nuclear physics and cosmology. A remarkable aspect of the coexistence phase in substances having more than one independent component (or conserved charge) has been overlooked until recently [1]. If the substance is allowed to reach equilibrium and one of the conserved charges is the electric charge, then the rarer phase in the coexistence regime will form a crystalline lattice immersed in the dominant phase. The form and spacing of the rare phase objects will vary as its proportion of the mixed phase varies. This is true in general.

In particular, the quark phase transition has been discussed for many years. In the original and succeeding discussions between 1976 and 1990 , the transition was treated in analogy to the liquid-vapor transition in water-a singlecomponent substance. However neutron stars are characterized by two independent conserved charges, the baryon and electric charges. A first order phase transition in multi-component substances is profoundly different from that in a single-component one. There are four main aspects that impinge on the structure of neutron stars: (1) The two phases in coexistence adjust their internal structure at each proportion of the phases so as to minimize the total energya degree of freedom unavailable to a singlecomponent substance. (2) One consequence of the readjustment-actually a rearrangement in the concentrations of the conserved charges - is that the pressure varies as a function of the proportion. For this reason the coexistence region of the two phases can extend over a non-zero radial range in the monotonically varying pressure environment of a star (unlike the coexistence of the two phases of a single-component substance for which the pressure is constant, causing the mixed phase to be squeezed out by the gravitational field of a star). (3) The readjustment of the two phases in each others presence lowers the value of the critical pressure (and density). (4) The degree of freedom afforded by the possibility of reapportioning the conserved charges is exploited by the isospin symmetry energy of nuclear matter with the twin consequence that (a) the charge densities of the two equilibrium phases are unequal and non-zero so that neutrality is achieved not by vanishing charge density but by cancelling charges (neutrality of stars is a global, not a local constraint on their constitution); (b) the spatial arrangement of the two phases in equilibrium that minimizes the Coulomb and surface energies is a crystal lattice (of typically nuclear dimensions).

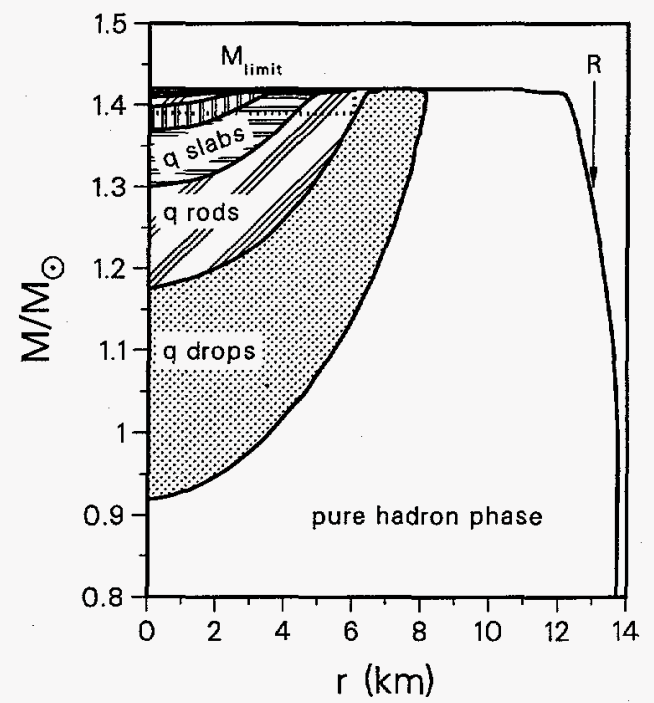

Figure 1: Radial boundaries of the various phases, pure quark, mixed (and the geometrical phases, for which ' $q$ drops' means quark drops immersed in nuclear matter) and pure hadronic fluid for stars of various masses and stellar radius $R$ for stars.

* LBL-39808

[1] N. K. Glendenning, Phys. Rev. D, 46 (1992) 1274. 


\section{Internal Constitution of Neutron and Strange Stars*}

\section{N. K. Glendenning}

The weakest force-gravity-binds a nucleon in a neutron star 10 times more strongly than the strong force binds a nucleon in a nucleus. In doing so it works against the strong shortrange repulsion of the nuclear force and against the Fermi pressure. Gravity therefore brings the Pauli principle into play in distributing the conserved baryon number of the star over many baryon species so as to reach the ground state of charge neutral matter. The name "neutron star" therefore has to be understood as a generic name for a star populated by many baryon species, also by quarks and also a mixed phase of confined and deconfined matter, that as I will discuss, arranges itself in a very intricate pattern in the deep interior of the star.

I will discuss several consequences of the rich constitution of compact stars: (1) a mechanism for the formation of low-mass black holes $(M \sim$ $\left.1.5-2 M_{\odot}\right),(2)$ a multi-layered crystalline structure of confined and deconfined quark phases and (3) the effect of a quark core on the braking index of pulsars as well as the general effect of rotational distortion on the inferred magnetic fields and spin-down times of millisecond pulsars.

In this paper we explore possibilities - not certainties. The properties of matter at densities higher than nuclear are essentially unknown, although they are the subjects of investigation at several ultra high energy accelerators. Essentially all we can be fairly confident of are: (1) the equation of state of dense matter obeys the condition of causality, (2) the equation of state also obeys the condition of microscopic stability $(d p / d \rho \geq 0)$ known as Le Chatelier's principle and (3) at sufficiently high density, asymptotic freedom of quarks is achieved. Beyond this, a theory of dense matter ought to be firmly anchored to what is known at nuclear density. Within these constraints we explore what is allowed by the laws of physics, in the belief that the laws of nature are likely to be realized in many if not all possible ways in the universe. The vehicle for the exploration is a covariant nuclear field theory that embraces the above constraints [1].

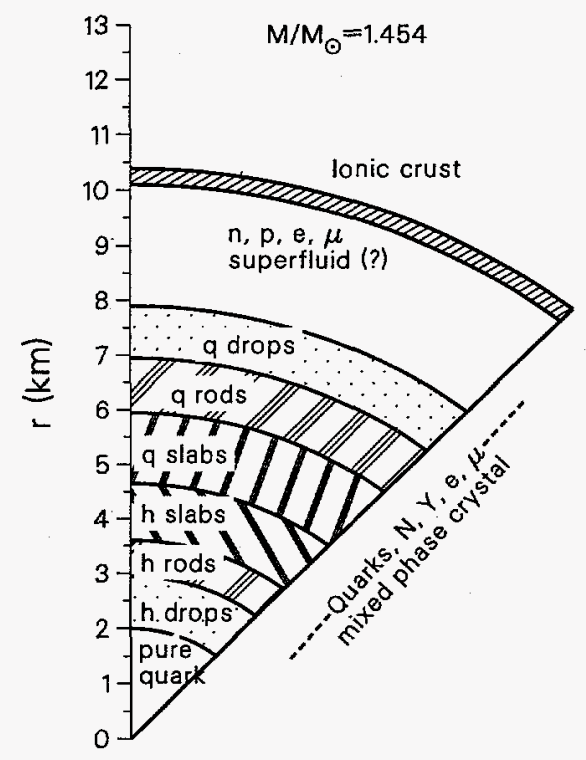

Figure 1: Pie section of a hybrid star (nuclear matter outer region enclosing a solid mixed phase and pure phase of quark matter) showing regions of quantum liquid (white areas) and solid regions of various geometric phases [2]. Notation 'q drops' means quark droplets immersed in nuclear matter, etc.

* Abstract of two lectures delivered at "The Many Faces of Neutron Stars" ,Lipari, Sicily, October 1996, (Organizers: M. A. Alpar, R. Buccheri, H. Ogelman and J. van Paradijs), LBL39638

[1] N. K. Glendenning, Astrophys. J. 293 (1985) 470 .

[2] N. K. Glendenning, COMPACT STARS, Nuclear Physics, Particle Physics, and General Relativity (Springer-Verlag New York, 1997). 


\title{
Thermal Evolution of Compact Stars*
}

\author{
Christoph Schaab ${ }^{\dagger}$, Fridolin Weber ${ }^{\dagger}$, Manfred K. Weigel ${ }^{\dagger}$ and Norman K. Glendenning
}

A broad collection of modern, field-theoretical equations of state (EOSs) is applied to studying the cooling behavior of both neutron stars and strange matter stars. The collection was derived under numerous assumptions about the behavior of superdense stellar matter. To mention several are: (1) the many-body technique used to determine the equation of state; (2) the model for the nucleon-nucleon interaction; (3) description of electrically charge neutral neutron star matter in terms of either only neutrons, (4) neutrons and protons in generalized chemical equilibrium ( $\beta$ equilibrium) with electrons and muons, or (5) nucleons, hyperons and more massive baryon states in $\beta$ equilibrium with leptons; (6) behavior of the hyperon coupling strengths in matter, (7) inclusion of meson $(\pi, K)$ condensation; (8) treatment of the transition of confined hadronic matter into quark matter; and (9) assumptions about the true ground state of strongly interacting matter (i.e., absolute stability of strange quark matter relative to baryon matter).

We find that standard cooling gives agreement between the theoretical cooling curves and the observed data for some but not all pulsars (cf. upper band in Fig. 1). On the other hand, the enhanced cooling mechanisms, which are connected to higher neutrino-emission rates coming from the direct Urca process, $\pi$ - and $K$-meson condensates, or up, down and strange quarks in the cores of neutron stars, lead to too rapid a cooling. Therefore one is left to examine processes which somewhat delay enhanced cooling. A plausible candidate is superfluidity, of which there occur probably two different types in the cores of neutron stars, namely ${ }^{1} \mathrm{~S}_{0}$ and ${ }^{3} \mathrm{P}_{2}$. We find that already small changes in the theoretical value for the ${ }^{1} \mathrm{~S}_{0}$ gap shift the cooling curves into the region of the observed data, giving an overall good agreement (band labeled "intermediate" in Fig. 1). An accurate determination of the superfluid gaps in neutron star matter therefore attains its particular interest.

With respect to the cooling behavior of strange stars, whose luminosities lie in the lower part of the band labeled "enhanced", we find that pulsars like Geminga, Monogem, and PSR 105552 can probably ruled out to be strange pulsar candidates. There is however one pulsar, PSR $1929+10$, which could be interpreted as a strange star. Of course, the QCD related uncertainties in the properties of strange matter as well as observational uncertainties do not permit us to draw stringent conclusions yet.

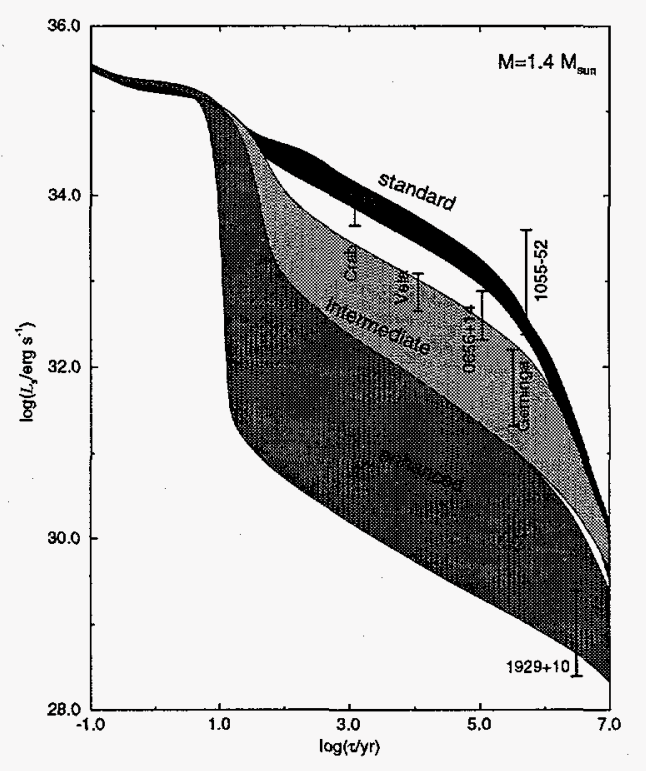

Figure 1: Comparison of the luminosity of observed pulsars with theoretical model calculations. The bands represent uncertainties inherent in the EOS (see text).

${ }^{*}$ Ch.Schaab, F. Weber, M. K. Weigel and N. K. Glendenning, Nucl. Phys. A605 (1996) 531.

$\dagger$ Institut für Theoretische Physik, LudwigMaximilians Universität München, Theresienstr. 37, D-80333 München. 


\title{
The Nuclear Thomas-Fermi Model* \\ W.D. Myers and W.J. Swiatecki
}

The statistical Thomas Fermi model is applied to a comprehensive survey of macroscopic nuclear properties. The adjustable parameters of the effective nucleon-nucleon interaction were fitted to shell-corrected masses of 1654 nuclei, to the diffuseness of the nuclear surface and to the measured depths of the optical model potential. With these parameters nuclear sizes are well reproduced, and only relatively minor deviations between measured and calculated fission barriers of 36 nuclei are found. The model determines the principal bulk and surface properties of nuclear matter and provides estimates for the more subtle, Droplet Model, properties. The predicted energy vs. density relation for neutron matter is in striking correspondence with the 1981 theoretical estimate of Friedman and Phandaripande [1]. Other extreme situations to which the model is applied are a study of $\mathrm{Sn}$ isotopes from ${ }^{82} \mathrm{Sn}$ to ${ }^{170} \mathrm{Sn}$, and the rupture into a bubble configuration of a nucleus (constrained to spherical symmetry) which takes place when $Z^{2} / A$ exceeds about 100 .

*Condensed from Acta Physica Polonica B26 (1995) 111 [1] B. Friedman and V.R. Pandharipande, Nucl. Phys. A361 (1981) 502

\section{The Rotating Nuclear Thomas-Fermi Model ${ }^{*}$}

W.D. Myers and W.J. Swiatecki

The Thomas Fermi model of Ref. [1] is generalized by the addition of a rotational energy. For heavy elements the calculated fission barriers of rotating nuclei agree with those of Ref. [2]. For lighter elements the barriers are lowered appreciably by the inclusion of the shape dependence of the congruence energy, but even then they exceed those of Ref. [2] by up to a few MeV. The shapes, density distributions, deformation energies and saddle-point properties of the superdeformed nuclei ${ }^{152} \mathrm{Dy}$ and ${ }^{83} \mathrm{Sr}$ are calculated.

${ }^{*}$ Condensed from Acta Physica Plonica B27 (1996) 99

[1] W.D. Myers and W.J. Swiatecki, Nucl. Phys. A601 (1996) 141

[2] A. J. Sierk, Phys. Rev. C33 (1986) 2039

\section{The Nuclear Thomas-Fermi Model with Angular Momentum: Fission Barriers, Superdeformations, Moments of Inertia*}

\author{
W.D. Myers and W.J. Swiatecki
}

When rotational energy is added to the Thomas-Fermi nuclear model of Ref. [1] a number of new predictions are possible. In this paper we present a survey of fission barriers, deformation energies, gamma-ray rotational cascades and moments of inertia. We also explore a hypothesis according to which the moment of inertia of a deformed nucleus can be estimated by subtracting from the rigid body value the moment of inertia of an inscribed sphere.

${ }^{*}$ Condensed from LBNL-39224

[1] W.D. Myers and W.J. Swiatecki, Nucl. Phys. A601 (1996) 141 


\title{
The Congruence Energy: A Contribution to Nuclear Masses, Deformation Energies and Fission Barriers*
}

\author{
W.D. Myers and W.J. Swiatecki
}

It has been know for a long time that nuclear binding energies, when plotted along isobaric sequences that cross the $N=Z$ locus, exhibit (after correction for even-odd effects) a slope discontinuity roughly proportional to $|I|$, where $I=(N-Z) / A$.

Our interpretation of a negative contribution with a kink at $N=Z$ is related to the quantal granularity of nucleonic densities. Thus the density distribution of a quantized particle in a potential well consists of cushion-like bumps boxed in by a latticework of the wave function nodal surfaces. A pair of nucleons with congruent nodal surfaces, say a neutron and a proton, will interact more strongly (in the case of short range forces) than a pair with uncorrelated density modulations. Since the number of neutron-proton pairs is the lesser of $\mathrm{N}$ and $\mathrm{Z}$, i.e., $\frac{1}{2}(N+Z)-\frac{1}{2}|N-Z|$, and since each pair interacts with a strength proportional to the reciprocal of the nuclear volume, i.e., to $A^{-1}$, the congruence energy should contain a negative term independent of $A$, modified by a positive term proportional to $|N-Z| / A$. A correction of this type should thus be added to a Thomas-Fermi model, whose smooth, structureless density distributions ignore this rather obvious consequence of quantization in a finite potential well.

A remarkable feature of the congruence energy, in addition to its telltale dependence on $|I|$, is that, being independent of $\mathrm{A}$, it has the same value for a fissioning nucleus as for each of the resulting fission fragments. Hence the total congruence energy must somehow double as the fissioning nucleus deforms into a necked-in scission shape. There is empirical evidence for such doubling.
Figure 2 in Ref. [3] shows the fission barriers of 36 nuclei as a function of a fissility parameter. Two curves in this figure connect points calculated using the above-mentioned Thomas-Fermi model. The upper one assumed that the congruence energy is the same at the saddle point as in the ground state, the lower that it has doubled. There is a fascinating hint in the fact that the almost perfect agreement with the upper curve for Radium and heavier elements, gives place to an approach to the lower curve for lighter nuclei. The Radium region is precisely where saddlepoint shapes develop (rather suddenly) a pronounced neck!

We have developed a model for the shape dependence of the congruence energy according to which the congruence energy is linear in the neck radius, and doubles its original value as the neck tends to zero. We have incorporated this shape dependence into our Thomas-Fermi calculations of nuclear deformabilities and fission barriers. The method we use is to solve the Thomas-Fermi equations first for a spherical nucleus and then for a sequence of elongating configurations for which the separation between the centers of mass of the two reflection symmetric halves is constrained to increase by a distance $2 \mathrm{D}$, where $\mathrm{D}$ $=0.2,0.4,0.6 \ldots \mathrm{fm}$. The resulting fission barriers are in good agreement with measurements. *Extracted from Ref. [3]

[1] J.M. Blatt and V.F. Weisskopf, Theoretical Nuclear Physics, John Wiley \& Sons, New York, 1952.

[2] W.D. Myers and W.J. Swiatecki, Nucl. Phys. 81 (1966) 1.

[3] W.D. Myers and W.J. Swiatecki, LBL-39224, November, 1995. 


\section{The Excitation of an Independent-particle Gas-Classical or Quantal- by a Time-dependent Potential Well* \\ J. Blocki ${ }^{\dagger}$, J. Skalski ${ }^{\ddagger}$ and W.J. Swiatecki}

A systematic numerical investigation of the excitation of a classical or quantal gas of noninteracting particles in a time-dependent potential well is described. The energy was followed in time for one oscillation around the sphere for six types of deformation: spheroidal shapes and Legendre polynomial ripples $\mathrm{P}_{2}, \mathrm{P}_{3}$, $P_{4}, P_{5}, P_{6}$ with relative $\mathrm{rms}$ amplitudes of 0.2 . Ten different speeds of deformation and eleven different values of the diffuseness of the potential well were studied. In the upper range of deformation speeds the quantal results for the non-integrable shapes $\mathrm{P}_{3}-\mathrm{P}_{6}$ agree approximately with the wall formula for dissipation, the deviations being largely accounted for by the wave-mechanical suppression factor of Koonin et al. For low deformation speeds the dissipation becomes dominated by one or two avoided level crossings.

${ }^{*}$ Condensed from Nucl. Phys. A594 (1995) 137

${ }^{\dagger}$ Inst. for Nucl. Studies, 05-400 Swierk, Poland

FInst. for Nucl. Studies, 00-681 Warsaw, Poland

\section{The Excitation of an Independent-particle Gas by a Time-dependent Potential Well. Part II ${ }^{*}$ \\ J. Blocki ${ }^{\dagger}$, J. Skalski ${ }^{\ddagger}$ and W.J. Swiatecki}

A systematic comparison is carried out between quantal and classical computer simulations of the excitation of independent particles in a time-dependent, diffuse potential well undergoing one cycle of oscillation. Eleven values of the diffuseness, up to 25 values of the oscillation frequency, and five Legendre polynomial deformations $\mathrm{P}_{2}, \mathrm{P}_{3}, \mathrm{P}_{4}, \mathrm{P}_{5}, \mathrm{P}_{6}$ were examined. Oscillations around the spherical shape as well as around a $P_{3}$ deformed shape were considered. The results are compared with the one-body dissipation theory in the form of the wall formula, including corrections for the heating up of the gas, the diffuseness of the surface, the wave-mechanical suppression of dissipation and the reduction in the effective volume available to a quantal gas in a container whose diffuseness is small compared to the particle wavelengths.

*Condensed from LBNL-39225, August, 1996, submitted to Nucl. Phys.

IInst. for Nucl. Studies, 05-400 Swierk, Poland

\#Inst. for Nucl. Studies, 00-681 Warsaw, Poland

\section{The Effect of Dynamical Correlations in a Slowly Pumped Knudsen Gas* J. Blocki ${ }^{\dagger}$, C. Jarzynski ${ }^{\ddagger}$ and W.J. Swiatecki}

In this paper we consider the effect of dynamical correlations on the absorbtion of energy by a gas of mutually noninteracting particles inside a container whose shape changes slowly (and periodically) with time. In principle, such correlations can significantly alter the rate at which energy is absorbed by the gas. We present the results of numerical simulations in which this effect is clearly seen, and compare the results with theoretical estimates.

*Condensed from Nucl. Phys. A599 (1996) 486

${ }^{\dagger}$ Inst. for Nucl. Studies, 05-400 Swierk, Poland

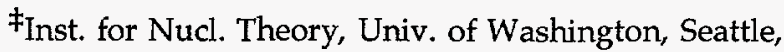
WA 98195 



\section{Seminars}

1995-1996

\section{Special Seminars}

Bikash Sinha, Saha Institute of Nuclear Physics, India

Jay Marx, LBNL

Michael Turner, Fermilab and U of Chicago

Heinz Gaeggeler, $\mathrm{U}$ of Bern and Paul Scherrer Institute,

Switzerland

David Radford, Chalk River, Canada

\section{Heavy Ion Tea}

Rolf Scharenberg, Purdue U

Tamas Csorgo, Budapest

Jørgen Randrup, LBNL

Shin Nan Yang, National Taiwan U

Volker Koch, LBNL

Iwona M. Sakrejda, LBNL

Emil Mottola, LANL

Rob Pisarski, BNL

Ramona Vogt, LBNL

Jim Carroll, UCLA @ LBNL

Nicolo de Groot, NIKHEF/SMC

Volker Koch, LBNL

Jeff Porter, LBNL

Kevin Haglin, MSU

Sean Gavin, BNL

Ramona Vogt and Xin-nian

Wang, LBNL

Dieter Best, GSI

Detlef Irmscher, LBNL

Che-ming Ko, TAMU
Electromagnetic Signals of Quark Gluon Plasma

$10-02-96$

Searching for the Quark-Gluon Plasma

$11-26-96$

Appraising Inflation and Cold Dark Matter

$12-03-96$

Chemistry Gains A New Element: Z=106 (Seaborgium)

$12-05-96$

Coulomb Excitation Studies with the 8-pi array

$12-09-96$

Critical Characteristics

09-28-95

Bose-Einstein Correlations

10-05-95

Extracting Thermodynamic Variables

10-12-95

Pion Production

10-19-95

Chiral Symmetry

10-24-95

$10-25-95$

$10-26-95$

Physics with STAR, Part I

10-26-95

Physics with STAR, Part II

11-02-95

Disoriented Chiral Condensates

11-09-95

Baked Anomalies: Where Does the Omega Go?

11-16-95

Parton Densities in Perturbative QCD

11-30-95

Spin Physics with STAR at RHIC

12-07-95

The Spin of the Nucleon

12-14-95

Electromagnetic Heavy Ion Physics

01-04-96

DLS Dielectron Measurements at the Bevalac

01-16-96

Dileptons from Dense Matter

01-23-96

Return of the J/ $\psi$

01-30-96

$\mathrm{J} / \Psi$ Progress

02-06-96

$\mathrm{K}^{+}$Production in the $1.93 \mathrm{AGeV} \mathrm{Ni+Ni}$ Reaction

02-13-96

CERES/NA45 Measurements of Electromagnetic Probes

02-22-96

Low-Mass Dileptons from Heavy-Ion Collisions

02-27-96 
Klaus Kinder-Geiger, BNL

Xin-nian Wang, LBNL

Richard Seto, UC Riverside

Raju Venugopalan, INT Seattle

Thomas Wienold, LBNL

Spiros Margetis, LBNL

John Harris, LBNL

Tim Hallman, BNL

Fuqiang Wang, Columbia

Ulrich Heinz, Regensburg

Willibrod Reisdorf, GSI

Peter Filip, Bratislava

Craig Ogilvie, MIT

Steffen Bass, U of Frankfurt

Miklos Gyulassy, Columbia

Sergei Panitkin, Stony Brook

Xin-nian Wang, LBNL

Volker Koch, LBNL

Klaus Geiger, BNL

Spencer Klein, LBNL

$\mathrm{Nu} \mathrm{Xu}, \mathrm{LANL}$

Yuri Sinyukov, ITP Kiev

Ziwei Lin, LBNL

Daniel Ferenc, Zagreb
Parton Dynamics

03-05-96

03-06-96

03-07-96

Anatomy of Parton Cascades: Problems and Cures

03-12-96

HIT Forum on Parton Dynamics

$03-19-96$

HIT Forum on Event-by-Event Physics

03-26-96

The PHENIX Experiment at RHIC: Measuring Electrons and Muons

High Parton Densities in Perturbative QCD

04-02-96

Recent Results from NA49 on Stopping and Flow

04-16-96

Aspects of Strangeness Production at CERN Energies

Scaling of Nuclear Stopping in Central Nucleus-

Nucleus Collisions from E(Lab) $=0.2-200 \mathrm{~A}-\mathrm{GeV}$

Measuring the Spin-Averaged and Spin-Dependent

Gluon Structure Function of the Proton with STAR

Kaon Production and K/pi Ratio in Au+Au at 11.1 A GeV/C at the AGS

Hadron Abundancies and Spectra in Relativistic

Nuclear Collisions

Lifetimes and Sizes from Two-Particle Correlations in

Relativistic Nuclear Collisions

Heavy Ion Collisions at 0.1 to $2 \mathrm{~A} \mathrm{GeV}$ : New Results and Plans from the FOPI Collaboration

Azimuthally Sensitive HBT Analysis

04-23-96

04-30-96

05-07-96

06-18-96

06-25-96

07-01-96

07-02-96

07-09-96

07-16-96

Searching for the Quark-Gluon Plasma at the AGS

07-23-96

Ultadense Nuclear Matter, Stopping, and Flow

Excitation Functions with URQMD

07-30-96

Tubulent Glue Scenario at RHIC

07-31-96

Two Particle Correlations: E877 Results

09-10-96

Parton Energy Loss and Jet Quenching

09-17-96

Low-Mass Dilepton Production in Ultrarelativistic Heavy Ion Collisions

09-24-96

Parton Cascades and QCD?

10-01-96

Peripheral Collison Physics at RHIC

10-02-96

Hadronic Freeze-Out Conditions in Ultra-Relativistic Heavy-Ion Collisions

Boson Interferometry at High Multiplicities

10-03-96

Nuclear Shadowing from Dileptons at RHIC

10-08-96

10-15-96

Bose-Einstein Correlations in Relativistic Nuclear Collisions: Current Status and Outlook 
Mark Baker, MIT

Ron Soltz, LLNL

Jørgen Randrup, LBNL

Huan Huang, UCLA

Larry McLerran, Minneapolis

Jay Marx, LBNL

Carlos Lourenco, CERN

Vesa Ruuskanen, Jyväskylä

Joseph Kapusta, Minneapolis

INPA Journal Club

D. Moltz, LBNL

M. Isaac, LBNL

S. Deustua, LBNL

E. Bloom, SLAC

K. Lesko, LBNL

K. Hurley, UCB

L. Rosenberg, MIT

M. Rees, Cambridge

Max Tegmark, Max-Planck Institute

D. Branch, Univ, of Oklahoma

P.J.E. Peebles, Princeton U.

D. Welch, McMaster U.

B. Fujikawa, LBNL

G. Mamon, Institut

d'Astrophysique de Paris

D. Snowden-Ifft, UCB

S. Perlmutter, LBNL

T. Banks, Rutgers U.
What Can We Learn from Two-Particle Correlations in Heavy Ion Collisions?

Calibrating Hadronic Probes of the Quark-Gluon Plasma

Summary of the DCC Workshop at ECT in Trento

What Can Be Learned from Lambda Polarization?

Initial Conditions for Ultra-Relativistic Nuclear

Collisions

Search for the Quark-Gluon Plasma with STAR

Dimuon Production in Heavy Ion Collisions: Recent Results and Future Perspectives

Hydrodynamic Study of Heavy Ion Collisions at the CERN SPS: Any Sensitivity to the Equation of State?

Coulomb Effects on Charged Kaon Distributions from Vlasov Dynamics

A Proposed Measurement of the ${ }^{7} \mathrm{Be}(\mathrm{p}$, gamma)

01-06-95 Reaction Cross Section

First Results from the ${ }^{51} \mathrm{Cr}$ Neutrino Source Experiment with the GALLEX Detector

Some Highlights from the Recent American Astronomical Society Meeting

Gamma-Ray Telescopes

Update on the Sudbury Neutrino Observatory

Cosmic Gamma-Ray Bursts and Soft Gamma Repeaters

A Search for Dark Matter Axions

02-24-95

Theories of Gamma-Ray Bursts Origin

03-01-95

Extracting Power Spectra

03-03-95

Type Ia Supernovae and the Hubble Constant

03-10-95

Numerical Applications of the Action Principle

03-17-95

New Light on Cepheid Variables from MACHO Project Results

03-31-95

The Search for the Origin of VHE Cosmic Rays

04-07-95

A Near-IR Imaging Survey + the Galaxy

Groups/Cosmology Connections

Limits on Dark Matter Using Ancient Mica

04-21-95

On New Supernova Model

04-28-95

Modular Cosmology

05-05-95 
C. Kuo, LBNL

M. Moorhead, LBNL

S. Deustua, LBNL

L. Cayon, LBNL

E. Wasserman, LBNL

B. Tripp, LBNL

D. Lowder, UCB and LBNL

R. Taillet, LAPP (Annecy, France)

D. Weiss, UCB

P. Salati, ENSLAPP (Annecy, France)

S. Bludman, U. Pennsylvania

L. Krauss, Case Western

Reserve U.

S. Perlmutter, LBNL

L. Tenorio, LBNL

E. Gawiser, LBNL

R. Pain, LBNL

C. Baker, UCB

G. Savard, Chalk River Lab.

M. Frank, LLNL

W. Stoeffl, LLNL

M. Bennett, LBNL

L. Krauss, Case Western

Reserve U.

F. Boehm, Caltech

O. Saavedra, CERN

R. Muller, LBNL

A. Westphal, UC Berkeley
Free Oscillations in the Earth

05-12-95

Neutral Current Detection in the Sudbury Neutrino

Observatory

Low Luminosity Active Galactic Nuclei - Do They

Exist?

Topology of Anisotropies in the COBE CMBR Data

Search for $\mathrm{CP} / \mathrm{T}$ Violations

05-19-95

05-26-95

06-02-95

06-09-95

Using Type Ia Supernovae to Measure the Cosmological Expansion Parameters

The HEAT (High Energy Antimatter Telescope)

Experiment and First Flight Results

Dark Matter in the Form of Low Mass Stars in Globular

Clusters

Observation of Bose-Einstein Condensate in Dilute

Atomic Gas

The Gas Deficiency in the Galactic Halo

07-28-95

Big Bang Nucleosynthesis in Crisis

08-04-95

Big Bad Nucleosynthesis; the TRUE Story

08-11-95

An Informal Report on the SN 1a June '95 Conference in Spain

Comments on CMB Parameter Estimation

08-25-95

Microwave Anisotropy from Infrared Galaxies

09-01-95

Supernova at $\mathrm{z}=0.4$

09-08-95

Detecting Neutrino-Induced Radio Signals in the

09-15-95

Antarctic Ice

A New Penning Trap Mass Spectrometer

10-13-95

$\mathrm{X}$ - and Gamma-Ray Detectors with Superconducting Absorber

Anomalous Shape in the Tritium Beta

10-27-95

$\mathrm{H}^{0}$ Search in BNL Experiment 896

11-03-95

The Case for a Cosmological Constant

11-10-95

The S. Onofre Neutrino Oscillation Experiment

$11-17-95$

Correlation of GRBs with Underground Detectors

$12-01-95$

Fresh News from the Ice Ages

12-15-95

Composition of Heavy Isotopes and Ultra-Heavy

Elements in Cosmic Rays Using Detectors on the MIR

01-19-96 
Supernova Group Tag Team

Yuen Dat Chan, LBNL

J. Jochum, UCB

R. Hoffman, UC Santa Cruz

B. Grossan, LBNL

G. Marcy, SFSU and UCB

A. Olinto, $U$ of Chicago

J. Frieman, Fermilab and $U$ of Chicago

C. Alcock, LLNL

B. Price, UCB

M. Isaac, LBNL

B. Fujikawa, LBNL

P. Vetter, LBNL

E. Daw, MIT

P. Mantsch, FNAL

D. Nygren et al., LBNL

M. Kim, LBNL

Dave Nygren et al., LBNL
B. Remington, LLNL
B. Fujikawa, LBNL
S. Elliott, U of Washington
G. Chew, LBNL

J. Marx, LBNL

J. Ellis, CERN

M. Smith, Oak Ridge Natl. Lab.

G. Jernigan, UCB

B. Cabrera, Stanford U.

E. Gawiser, LBNL

R. Pain, LBNL
Journal Club Update on Last Week's American

01-26-96 Astronomical Society Meeting

Getting Ready to Analyze a SNO Event

02-02-96

The Solar Neutrino Problem in the ${ }^{7}$ Be Energy Range

02-09-96

Heavy Element Synthesis in Supernovae

02-16-96

Physics in Mountaineering

02-23-96

Discovery of Planets Orbiting Normal Stars

03-01-96

Forming Primordial Black Holes in Phase Transitions

03-08-96

Weak Lensing and the Measurement of $\mathrm{Q}^{0}$ from Type Ia Supernovae

Cosmic Dark Matter: Recent News from Gravitational Microlensing

High Energy Neutrino Astrophysics: Ice and Oceans

03-29-96

Strange Matter and How To Detect It

04-05-96

Determination of the U-D CKM Matrix Element from Superallowed Fermi Decays

04-12-96

What the $\% * \& ! \# \$$ Is an Anapole Moment?

04-19-96

A Search for Halo Axions

04-26-96

The Pierre Auger Project: A Large Cosmic Ray

Observatory.

05-10-96

Report on: "U.S. Meeting on Future Prospects for kmscale Neutrino Detectors-Arcadia, Apr. 18-19"

The Latest Hubble Constant

05-24-96

Part II of the Report on: "U.S. Meeting on Future

05-31-96

Prospects for km-scale Neutrino Detectors-Arcadia, Apr. 18-19"

Experimental Astrophysics Using Intense Lasers

06-14-96

LSND - Unauthorized

06-21-96

Recent Results from SAGE

07-12-96

Experimental Predictions from Cosmology, with Emphasis on Supernovae Measurements

The Quark-Gluon Plasma and You

07-26-96

Outlook on Neutrino Physics 1996

08-02-96

Nuclear Astrophysics at ORNL

08-16-96

Recent Results from X-ray Timing Explorer 08-23-96

Cryogenic Detection of Dark Matter

08-30-96

Microwave Anisotropy from IR Galaxies

09-01-96

Supernova Rate at $\mathrm{Z}=0.4$

09-02-96 
G. Gwinner, LBNL

T. Culler, LBNL

R. Norman, LBNL

E. Gawiser, LBNL

C. Quigg, Fermilab

N. Glendenning, LBNL

H. Crawford, LBNL

S. Gordon, LBNL

S. Perlmutter, LBNL

S. Freedman, LBNL

R. Puetter, UC San Diego

G. Fisher, Space Sciences Lab, UCB

G. Marcy, SFSU

P. Nugent, LBNL

C. Okada, Boston U

Nuclear Physics Forum

T. L. Khoo, ANL

L. W. Phair, LBNL

R. N. Cahn, LBNL

Luciano Moretto, LBNL

Albert Young, Princeton U

D. Sarantites, Washington $U$

G. Greene, LANL

C. J. Lister, ANL

Bill McCurdy, LBNL

Jorolf Alstad, Oslo U, Norway

S. Yates, U of Kentucky

Vic Viola, Indiana U
Quantum Computing

09-13-96

Measurement of Cratering Rate with Lunar Spherules

09-27-96

You Can See a Lot Just by Looking

09-29-96

Microwave Anisotropy from Infrared Galaxies

10-04-96

Neutrinos

10-06-96

Strange Matter Stars

10-18-96

Status of Experimental Searches for Strange Matter

$10-25-96$

The Radio Properties of an Optically Selected Sample of Supernova Remnants in M33

Latest News from the Supernova Search

11-15-96

The Bloodsucking Vector: A Quest of New Physics in Heavy Ion Collisions

Information, Language and Pixon-Based Image Reconstruction

The Solar Cycle: the Role of Magnetic Flux Tube Dynamics

Detection of a New Planet Around a Nearby Star

12-08-96

Synthetic Spectra of Hydrodynamic Models of Type 1a Supernovae

12-13-96

A Search for Oscillations of Atmospheric Neutrinos with the MACRO Experiment

$12-20-96$

Decay of Superdeformed Bands

02-01-96

Reducible and Statistical Nature of Multifragmentation

02-22-96

Testing $C$ Invariance in Positronium Decay

02-29-96

The Unclear Physics of Phase Transitions

03-14-96

Probing for Physics beyond the Standard Model with

Nuclear Beta Decay

03-21-96

03-28-96

Hyperdeformed Nuclei and How to Make Them

04-04-96

Beer Cans, Neutron Bottles, and the Boeing 777: Neutron Science at LANL

AYE-Ball: A Peep into the Future of Nuclear Structure

04-11-96

The National Energy Research Scientific Computing

Center at LBNL: New Opportunities for Computing

Sciences

SISAK III: Single-Atom Chemical Separations on a OneSecond Timescale

The Search for Two-Phonon Excitations in Nuclei

Delta Resonance-Enhanced Mutlifragmentation
05-16-96

04-18-96

04-25-96

06-03-96 
G. Young, ORNL

V. Flambaum, U of New South Wales, Australia

Wladek Swiatecki, LBNL

Stefan Frauendorf, Institute of Nuclear and Hadronic Physics, Rossendorf, Germany

R. Donangelo, $U$ of Rio de Janeiro, Brazil

Stefan Frauendorf, Institute of Nuclear and Hadronic Physics, Rossendorf, Germany

P. Reiter, ANL

John Hardy, AECL, Chalk River, Canada

Wladek Swiatecki, LBNL

Brian Fujikawa, LBNL

K. Grotowski, Jagellionian U, Poland

John Rasmussen, LBNL

Igor Pavlichenkov, Kurchatov Institute, Moscow

\section{Theory Seminars}

Thomas Schäfer, Stony Brook, NY

David Blaschke, Rostock, Germany

József Zimanyi, Buđapest, Hungary

Biswarup Banerjee, Bombay, India

G. Z. Obrant, St. Petersburg, Russia

Vesteinn Thorsson, INT, Seattle, WA

Jim Huang, Tucson, AZ

Caio Lewenkopf, INT, Seattle, WA
Electromagnetic Studies of Quark-Gluon Plasma Using the PHENIX Detector at RHIC

Experiments with Heavy Nuclei: Anapole Moment

06-20-96

Overview of the Nuclear Thomas-Fermi Model

06-27-96

Persistent Currents: Nuclear Rotation and Cluster

Magnetism

Post-Acceleration Effects in the Direct Break-up of Halo Nuclei

Magnetic Rotation

07-18-96

Gamma-Ray Spectroscopy of Neutron-Rich Nuclei

Using Radioactive Beams at $2.2 \mathrm{MeV} / \mathrm{u}$ and $230 \mathrm{MeV} / \mathrm{u}$

$10-10-96$

Superallowed Beta Decay: A Nuclear Probe of

$11-11-96$

Electroweak Standard Model

Nuclear Dynamics and the Order-to-Chaos Transition

$11-21-96$

Determination of CKM Matrix Elements with

Superallowed Fermi Decays

Study of Reaction Mechanisms and of Exotic Events in Heavy-Ion Collisions

Spontaneous Fission Studies in Gammasphere: What We Have Learned

Non-Adiabatic Mean Field Effects in the Delta $\mathrm{I}=2$

Staggering of Superdeformed Bands

12-05-96

Instantons and Chiral Phase Transition

01-17-95

Thermodynamics of the Quark Hadron Transition In a Nonlocal Chiral Quark Model

ALCOR: A Dynamic Model For Hadronization

01-19-95

02-02-95

02-06-95

Coherent Pion Effect At Large Rapidities In NucleusNucleus Collisions

Kaon Condensation In Neutron Star Matter

02-15-95

Disoriented Chiral Condensate

02-23-95

Electronic Transport In Microstructures and Compound Nucleus Reactions: Similarities and Differences 
Chungsik Song

Carsten Greiner

Igor Dremin, Moscow, Russia

Chris Jarzynski, INT, Seattle, WA

Alex Kovner, Minneapolis, $\mathrm{MN}$

Paul Fallon, NSD/LBNL

Paul Bonche, Saclay, France

Judah Eisenberg, Tel Aviv, Israel

Robert Vinh Mau, Paris, France

Sid Bludman, Philadelphia, PA

Cheuk-Yin Wong, ORNL, TN

Berndt Müller, Duke, NC

Xiao-Ming $X u$, Shanghai, China

Chungsik Song, NSD/LBNL

Shin-Nan Yang, Teipei, Taiwan

Emil Mottola, LANL, NM

Dirk Rischke, Columbia, NY

Krishna Kumar, Tenn Tech U, TN

Engelbert Quack, SLAC, CA

Valeri Emel'yanov, Moscow, Russia

Patrick Talou and Nicolae Carjan, Bordeaux, France

Fridolin Weber, Munich,

Germany

Pawel Danielewicz, MSU, MI

Su Huong Lee, Yonsei U, Korea

Cheuk-Yin Wong
Photon and Dilepton Production From Hot Hadronic Matter In High Energy Nucleus-Nucleus Collisions

Non-Markovian Effects In High-Energy Collisions

Correlation and Statistical Analogies In Multiparticle Production

Can One Do Statistical Mechanics with Only a Few Degrees of Freedom-Case of a Brownian Particle Coupled to Deterministic Chaos

Variation Approach to the Yang-Mills Vacuum

Recent Results in High-Spin Physics

Skyrme Forces Revisited

Proton Spin Content from Skyrmions

Quark Stars and Quark-Hadron Phase Transition

Big Bang Nucleosynthesis in Crisis

Suppression of $\Psi$ ' and $\mathrm{J} / \Psi$ in High-Energy Heavy-Ion Collisions

Interface Tension and Entropy: A Simple Model

$\mathrm{J} / \Psi$ Suppression in an Equilibrating Parton Plasma

Medium Effects on Dilepton Production from Hot

Hadronic Matter

Electroproduction of $f$-Meson and Strangeness Content of the Proton

Non-Equilibrium QCD: The Chiral Phase Transition in Heavy-Ion Collisions

Hot Spots and Turbulent Initial Conditions in AACollisions at $\mathrm{E}_{\mathrm{cm}}=200 \mathrm{AGeV}$

03-03-95

03-09-95

03-30-95

04-24-95

05-18-95

05-23-95

07-18-95

07-25-95

08-01-95

08-04-95

08-14-95

08-17-95

09-19-95

09-26-95

10-03-95

10-31-95

$11-16-95$ Theory

Soft Proton Production from a Quark-Gluon Plasma

11-21-95

Peripheral Heavy Ion Collisions

11-30-95

Time-Dependent Decay Rates

$12-12-95$

Braking of Pulsars

12-14-95

Delays in Elementary Processes

12-19-95

Hadron Properties in Nuclear Medium from QCD Sum Rules

01-09-96

Effect of Initial and Final State Interactions on Cross Sections

$01-30-96$ 
Burkhard Kämpfer, Rossendorf, Germany

Ramona Vogt, NSD/LBNL

Ziwei Lin, Columbia U, NY

Xiaofeng Guo, Iowa State U, IA

Fred Cooper, LANL, NM

Akira Ohnishi, Sapporo, Japan

Peter Valerio, Tucson, AZ

Jürgen Schnack, GSI, Germany

Robert Vinh Mau, Paris, France

J. Zimanyi, KFKI, Budapest, Hungary

Michael Christiansen, U of Århus, Denmark

Yasushi Nara, Sapporo, Japan

Dimitri Kharzeev, U of Bielefeld, Germany

Fridolin Weber, Munich, Germany
Electromagnetic Signals of Parton Matter

02-15-96

Colorful Quarkonium Production

03-14-96

Charm in RHIC

03-21-96

Nuclear Dependence in Direct Photon Production

03-25-96

Time Dependent Variational Methods; From Solitons to Quantum Chaos

Multistep Effects in $\left(\mathrm{K}^{-}, \mathrm{K}^{+}\right)$Reactions: How Are $\mathrm{S}=-2$ Hyperfragments Formed?

Charm and Bottom Production and Decay in UltraRelativistic Heavy-Ion Collisions

Short Range Correlations in Fermionic Molecular Dynamics

Nuclear Forces and Quark Degrees of Freedom

08-15-96

Relativistic Mean Fields, Multipion Correlation, and Hadronization

09-05-96

11-05-96

Study of $\left(\mathrm{K}^{-}, \mathrm{K}^{+}\right)$Reaction within the Quantum

Molecular Dynamics Approach

$J / \psi$ Suppression in Heavy-Ion Collisions

12-11-96

Novel Mechanism in Banyou Stopping

$12-12-96$

Neutron Stars as Probes of Superdense Matter 




\section{Author Index}

\section{A}

Ackermann 85-86

Adams 61, 62-63

Ahmad 85, 145, 148

APEX Collaboration 153

Archer 87

Asztalos 80, 83-86, 88-92, 94, 144

Austin 148

B

Baglin 97

Baldsiefen 89

Bauer 87

Becker 80-81, 83, 87-89, 91, 137

Bennett 166-167

Bernstein 80, 83, 87, 89, 137

Betts 177

Beuville 183

Bieser 177-178

Bilewicz 64

Blocki 240

Blumenthal 85

Bossingham 177

Botlo 177

Bowers 144, 147, 151

Bowman 103, 107

Brinkman 81

Brodsky 204

Browne 136, 140-141

Budker 151

Burks 183

Busse $84-85,88$
C

Canto 94-96

Carlin 103, 107

Carpenter 85

Carroll 166-167

Case 168-170

Cederwall 80-81, 91

Cerny 57-60, 114

Chalmers Collaboration 65

Chan 123-134, 136-139

Cherney 177

Chinowsky 135

Chomaz 225

Chrin 177

Chu 93-95, 97-100

Chung $61-63,71,76$

Cizewski 87

Clark 80-81, 83-90, 92, 113, 117, 144

Colarco 177

Cole 94

Colonna 107, 111, 225

Commins 151

Cooper 219

Crawford 135, 166-167, 177

Cronqvist 166-167

Crowe 168-170

Crystal Barrel Collaboration 168

D

da Cruz 141

Dairiki 35, 142

Dao 177 
Dardenne 94

Davis 150

de Souza 103, 107

Deleplanque 80-92

Deleplanque-Stephens 144

DeMille 151

Dewald 88

Diamond 80-81, 83-92

Diaz 177

Diener 231

DiGregorio 138

Ding 87

Dionisio 82

DLS Collaboration 164

Donangelo 94, 95

Dragowsky 123-134, 138

Draper 81

Duyar 81

$\mathbf{E}$

E878 Collaboration 166

E895 Collaboration 165

E896 Collaboration 167

Ekstrom 98

emiT Collaboration 149

Engel 229

Engelage 166-167

EOS Collaboration 163

Eskola 195

F

Fallon 80-81, 83-92, 144

Farhan 96

Farris 80-81, 83

Ferris 137

Firestone 97-100, 142
Fischer 85

Flores 166-167

Frauendorf 81,91

Freedman 143-153

Fritz 183

Fujikawa 143-147, 149, 152

Fusezy 135

G

Gall 82

Gammasphere Collaboration 147

Gatti 108

Gavin 200, 205

Gavin 206

Gelbke 103, 107

Ghetti 101-102, 105

Glendenning 232-237

Goldman 136, 141

Gong 103, 107

Greene 144-145

Gregorich 61-69, 71-78

Greiner 166-167, 177, 231

Guarnera 225

Gueorguieva 82

Gunion 209

Gwinner 143, 151-152

H

Habib 218-219

Hackman 86

Hallman 166

Hamilton 79, 94

Hancox 156

Hannachi 82, 85

Hanold 111

Harada 229 
Harris 203

Harss 152

Hauschild 80, 87-88

Haustein 114

He 135, 154-156, 176, 184

Heckman 166

Heinsius 168-169

Heiselberg 192

Helsgesson 223

Hendricks $61-63,66-67,69,72-77$

Henry 81, 83, 91

Herskind 86

Hibbert 89

Hindi 138

Hjort 163, 177

Hoffman 61-67, 69-79

Hsu 78

Hua 91

Huang 171, 175, 189-191, 193, 196

Hübel 81-82, 89

Hughes 81

Hunter 178

Hurley 130, 131, 139, 157-159

I

Isaac 123-134, 137-140

Itagaki 230

J

Jackson 135

Jacobs 172,181

Jacobson 177

Janssens 85-86

Jared 177

Jarzynski 240

Jiang 152
Jing 108-111

Joss 80

Judd $167,177,179$

K

Kacher 64, 66, 78

Kaci 82

Kadkhodayan $64,66,78$

Kammel 168-170

Kate 230

Kelly 80, 82-83, 87

Kharraja 82

Kharzeev 194

Khoo 85-86

Kidonakis 202

Kim 103, 107

Klein 177-178, 185

Kluger 219

Kluger, 218

Ko 210, 212

Koch 210-216

Korichi 82, 85

Korten 81

Krane 137-138

Kreek 64

Krücken 84-90, 92

Kuo 166

L

Lakata 168-169

Lane $61-64,66-67,69,72-79$

Larimer 124, 136-138, 140

Laue 61, 62-63

Lauritsen $85-86$

LBNL, GSI, Mainz, Dresden, Krakow,

JAERI, PSI, Bern, Rossendorf, JINR, Oslo,

Chalmers, Collaboration 65 
Lebedev 177

Lee, D.M. 61-67, 69, 71-78

Lee, I.Y. 80-81, 83-92, 141, 144

Lee, S.H. 212

Lesko 123-137, 148

Levine 177

Lhenry 111

Li 210

Lindenstruth 177

Lindstrom 166-167

Ling 135

Lisa 103, 107, 177, 181

Lising 144, 149

Lister 85

Lopez-Martens 82, 85

Lowder 135

Lynch 103, 107

Lyneis $41,113,115-116$

\section{M}

Macchiavelli 80-81, 83-92, 137-138, 141, 144

MacGarth 69

MacLeod 84-90, 92, 137-138, 144

Maier 153

Margetis 173-175, 183

Marks 177

Marx 167

Matis 186

McDonald 130-131, 139-140, 157-159

McGaughey 200

McGrath 61-63, 67, 75-77

McMahan 118-119

McNabb 87

McParland 177

McShane 177

Meier 177
Mohar 64, 78

Moltz 57-60, 114

Moore 85

Moorhead 123-128, 130-132, 135, 137, 140

Moretto 101-112

Mortara 145-146, 148, 150

Mottola 218-219

Müller 195

Myers 238-239

$\mathbf{N}$

NA49 Collaboration 171-172, 174-175

Nakatsukasa 84

Nara 229

Nelson 136

Neu 64, 78

Nguyen 177

Nisius 85

Nolan 80

Nordberg 98

Norman 114, 123-134, 136-141, 157-159

Nygren 135

O

O’Brien 80

Odyniec 175

Ognibene 57, 60

Ohnishi 226-230

Olson 177,180

P-Q

Pardo 152

Paul 152

Paz 218

Peaslee 103, 107

Pei 232, 233-234

Perillo-Isaac 123-134, 137-140 
Peusquens 88

Phair 91, 101-107, 109-110, 112

Pohler 82

Porter 164, 182

Powell 57-60, 114

Price 135, 155, 176, 184

Prindle 180

Przybylski 135

R

Rai 165

Randrup 31, 220-228

Rasmussen 93-96

Rehm 152

Reich 144, 147

Retiere 183

Richards 135

Ring 95-96

Ritter 20

Robinson 138

Ross 146

Rowe 57-60, 114, 143-144

Rubehn 104, 107, 109, 110-112

Rubel 81

Ruuskanen 200

S

Sakrejda 167, 177, 181

Saladin 91

Sarantites 91

Sarcevic 190, 193

Satz 194

Scannapieco 185

Schaab 237

Schaffner-Bielich 231

Schambach 177
Scheetz 177

Schiffer 145, 152

Schmid 84-90, 92

Schmitt 108

Schroeder 1,7

Schück 82

Schuelke 123-134

Schuler 207

Schulze 82

Semple 80

Seweryniak 85

Shang 143-144, 147, 151-152

Shapiro 135

Shaughnessy $61-63,66-67,69,72-77$

Shirley 97

Singh 100

Skalski 240

Skulski 111

Smith 130-131, 139-140, 157-159, 201-203

Smoot 135

Song 212-217

Stalnaker 151

STAR Collaboration 177-178, 181

STAR Trigger Group 179

Stephens 80-92, 144

Stevenson 135

Stöcker 231

Stokstad 14, 123-135, 142

Stone 177, 181

Stoyer $64,66,78,81,93-95$

Strellis $61-63,67,69,72-77,94$

Sui 111

Swiatecki 238-240

Sylwester $61-67,69,72-78$ 
T-U

Teixeira 141

Thews 193

Toy 172

Trentalange 183

Tripp 135

Tsang 103

Tso 101-102, 105-106, 109-111

Tull 177, 180

$\mathbf{V}$

van Severen 82

Veeck 66, 111

Vetter 84-86, 88, 90, 92, 143, 147, 152

Vieu 82

Visser 167,177

Vogt 199-209

von Brentano 88

Vu 177-178

$W$

Wadsworth $80,82,89$

Wallsgrove 72

Wang 189-198

Wasserman 143, 144, 147, 149

Watanabe 64

Weber 232-233, 237
Weigel 237

Wells 183

Wieman 177, 183

Wienold 171

Wierczinski $64,66,77$

Wietfeldt 136

Wilk $61-63,67,69-70,72-76$

Williams 107

Willsau 81

Woosley 142

Wozniak 91, 101-112

Wu 99, 142

Wuosmaa 148

Wutte 146

$\mathbf{X}-\mathbf{Z}$

Xi 113

Xie 115-116

Xu 194

Yang 78

Yee 177

Younes 87

Yu 91, 112

Zaerpoor 137-138

Zeuli 145

Zhu 103

Zipkin 97

Zlimen 136 